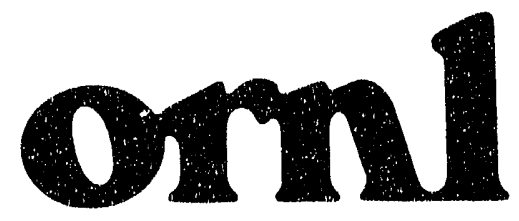

OAK RIDGE NATIONAL LABORATORY

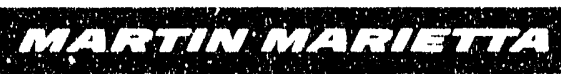

\section{A User's Manual for MASH 1.0-A Monte Carlo Adjoint Shielding Code System}

MANAGED BY

MARTIN MARIETTA ENERGY SYSTEMS, INC.

FOR THE UNITED STATES

DEPARTHENT OF EMERGY

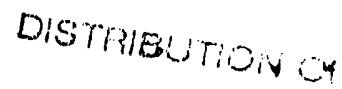
UivLIMETTEO 
Engineering Physics and Mathematics Division

\title{
A USER'S MANUAL FOR MASH 1.0 - A MONTE \\ CARLO ADJOINT SHIELDING CODE SYSTEM
}

Editor:

J. 0. Johnson

\section{Primary Contributors:}

M. B. Emmett, W. A. Rhoades, R. L. Childs, and J. 0. Johnson Oak Ridge National Laboratory

W. H. Scott, Jr., and J. A. Stoddard

Science Applications International Corporation

DATE PUBLISHED - March 1992

This work is sponsored by the Defense Nuclear Agency under DNA Order No. 91-806, Work Unit No. 00440, and DOE Interagency Agreement No. 0046-C083-A1

\author{
Prepared by \\ Oak Ridge National Laboratory \\ Oak Ridge, Tennessee 37831 \\ managed by \\ MARTIN MARIETTA ENERGY SYSTEMS, INC. \\ for the \\ U. S. DEPARTMENT OF ENERGY \\ under Contract No. DE-ACO5-840R21400
}


This report has been reproduced directly from the best avallable copy.

Avallable to DOE and DOE contractors from the Office of Sclentfic and Tectunt cal Information, P.O. Box 62, Oak Ridow, TN 37831; prices avallable from (615) 578-8401, FTS 626-8401.

Avaliabie to the public from the National Technical Imformation Service, U.S. Department of Commerce, 5285 Port Royal Rd., Springfield, VA 22161.

This report was prepared as an account of work sponsored by an agency of the United States Government. Netther the United States Government nor any apency thereof, nor any of their employees, makes any warranty, exprese or implied, or aseumes any legal Hablity or responalbilty for the accuracy, completeness, or usefuineses of any information, apparatus, product, or proceses disolosed, or represents that the weo would not intringe privatety owned rights. Reference hereln to any specific commercial product, proceses, or service by trade name, trademark, manufacturer, or otherwise, does not nocessartly constitute or inply its endorsement, recommendation, or favoring by the United States Government or any agency thereot. The views and opinions of authors expreased herein do not necesearily state or reflect those of the Unted States Government or any agency theraot. 
LIST OF FIGURES . . . . . . . . . . . . . . . . . . ix

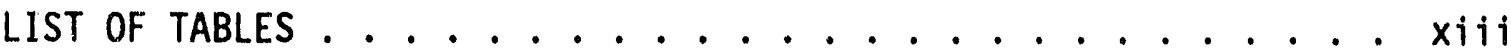

PREFACE .......................... . XV

ABSTRACT . . . . . . . . . . . . . . . . . xvi

1.0 MASH: A MONTE CARLO ADJOINT SHIELDING CODE SYSTEM . . . . . 1.1

1.1 INTRODUCTION TO MASH ................ 1-1

1.1.1 Background ................... 1-1

1.1.2 Summary of Corrections and Improvements to VCS . 1.6

1.2 STRUCTURE OF MASH 1.0 .............. 1-7

1.3 STRUCTURE OF MASH 1.2 USER'S MANUAL.......... 1-10

1.3.1 The Main Text ................ 1-10

1.3.2 The Appendices ................ . 1-10

1.3.3 The Sample Problem .............. 1-10

1.4 REFERENCES ..................... $1-11$

2.0 GIP: A GROUP-ORGANIZED CROSS SECTION INPUT PROGRAM . . . . . 2-1

2.1 INTRODUCTION TO GIP .............. 2-1

2.1.1 Background ................. 2-1

2.1.2 Method Used .................. 2-1

2.2 GIP INPUT REQUIREMENTS ............... $2-2$

2.3 GIP INPUT DATA NOTES ................ $2-4$

2.3.1 Special Diffusion Theory Option ....... 2-7

2.3.2 Upscatter Option ............. 2-8

2.3.3 Adjoint Reordering Example .. . . . . . . . 2-8

2.4 INPUT LIBRARY FORMAT .................. 2 . .

2.4.1 ANISN NUClide Format Cross-Section File... . . 2-10

2.5 GIP OUTPUT FILE ................ 2-12

2.6 LOGICAL UNIT REQUIREMENTS ............. 2-13

2.7 PROGRAMMER INFORMATION ............... 2-14

2.7.1 Inter-machine Adaptability . . . . . . . . . 2-14

2.7.2 Service Subroutines ............. 2-15

2.8 REFERENCES ...................... $2-16$

2.9 SAMPLE PROBLEM .................. $2-17$

3.0 GRTUNCL: AN ANALYYIC FIRST COLLISION SOURCE CODE . . . . . 3-1

3.1 INTRODUCTION TO GRTUNCL .............. 3-1

3.1.1 Background ................ 3-1

3.1.2 Method Used . . . . . . . . . . . . . 3-2

3.2 GRTLINCL INPUT REQUIREMENTS ............ $3-4$

3.3 GRTUNCL INPUT DATA NOTES ............. 3-7 
TABLE OF CONTENTS

(continued)

Page

3.3.1 Adjoint Reordering Example . . . . . . 3-13

3.4 INPUT LIBRARY FORMAT . . . . . . ........... . 3-14

3.4.1 ALC1 Formatted Cross-Section Input File.... . 3-14

3.4.2 ANISN Nuclide Format Cross-Section File . . . . 3-15

3.4.3 GIP Formatted Cross-Section Input File . . . . . 3-17

3.5 GRTUNCL OUTPUT FILE ............... . 3-19

3.6 LOGICAL UNIT REQUIREMENTS . . . . . . . . . . 3-22

3.7 PROGRAMMER INFORMATION . . . . . . . . . . . . 3-22

3.7.1 Inter-machine Adaptability . . . . . . . . . 3-22

3.7.2 Service Subroutines . . . . . . . . . . 3-23

3.8 REFERENCES .................... 3-24

3.9 SAMPLE PROBLEM .................. 3-25

4.0 DORT: A TWO-DIMENSIONAL DISCRETE ORDINATES TRANSPORT CODE . . 4-1

4.1 INTRODUCTION TO DORT .............. 4-1

4.1.1 Background ................. . 4-1

4.1.2 The Problem Solved ............. 4-1

4.1.3 Code Structure .............. 4-3

4.2 THEORETICAL BASIS ................ 4-3

4.2.1 The Integro-Differential Transport Equation . . . 4-3

4.2.2 The Finite Difference Formulation . . . . . . . . 4-4

4.2.3 Fluence Evaluation Strategy ...... . . . . . 4-9

4.2.4 Additional Theory Information . . . . . . . 4-12

4.3 DORT INPUT DATA REQUIREMENTS ............. 4-12

4.3.1 Card-Image Format . . . . . . . . . . . . 4-12

4.3.2 Space Meshes ............... . 4-14

4.3.3 Variable Space Mesh .............. . 4-14

4.3.4 Variable Directional Quadrature ........ 4-15

4.3.5 Variable Legendre Expansion . . . . . . . . . 4-15

4.3.6 Adjoint Data . . . . . . . . . . . . . . . 4-15

4.3.7 Card-Image Input Specifications . . . . . . . . 4-16

4.4 DORT INPUT REQUIREMENTS ............... 4-16

4.5 DORT INPUT DATA NOTES ................ 4-27

4.5.1 Special Geometry Features. . . . . . . . . 4-27

4.5.2 Special Theory Options ............ . 4-30

4.5.3 Cross Section Input and Mixing ........ . 4-30

4.5.4 Activity Edits............... 4-32

4.5.5 Negative Source Removal ........... . 4-33

4.5.6 Problem Type Specification .......... . 4-33

4.5.7 Iteration Limits and Convergence. . . . . . . . 4-34

4.5.8 Search Strategy .. . . . . . . . . . . . 4-36

4.5.9 Upscatter Rebalance ............ 4-37

4.5.10 Fission Rescaling ............. 4-38

4.5.11 Fission Extrapolation .......... . 4-38

4.5.12 Error-Mode Extrapolation ........... 4-38

4.5.13 Fluence Iteration Acceleration........ 4-39 
TABLE OF CONTENTS

(continued)

Page

4.5.14 Stabilization ............. 4-41

4.5.15 Input Source and Fluence Specification.... 4-41

4.5.16 Key Fluence Monitoring ....... . . . . 4-42

4.5.17 Energy Boundary Input ........... 4-42

4.5.18 Fluence Extrapolation Mode1 ........ 4-43

4.5.19 Alternate Zone Map Input .......... 4-44

4.5.20 Note on DORT Mesh for an

Air-Over-Ground Problem ........ 4-44

4.6 DORT INPUT AND OUTPUT FILE FORMATS ...... 4-46

4.7 LOGICAL UNIT REQUIREMENTS . . . . . . . 4-57

4.7.1 Input and Output Data Files ........ 4-. 4-57

4.7.2 Scratch Data Sets ............ . 4-57

4.8 DETAILS OF DORT JOB REQUIREMENTS ......... 4-58

4.8.1 CPU Time Usage ............ 4-58

4.8.2 System Buffer Space (NBUF)

and Memory Requirements .......... 4-60

4.8.3 Problem Printed Output ............ 4- 4-60

4.9 PROGRAMMER INFORMATION ........... 4-64

4.9.1 Inter-machine Adaptability ......... 4-64

4.9.2 Service Subroutines ............ 4-65

4.10 REFERENCES ............. 4-... . . . . .

4.11 SAMPLE PROBLEM ................... 4-68

5.0 VISTA: A VEHICLE INPUT SOURCE TRANSFORMATION

AND ASSEMBLY CODE ................ 5- . . . .

5.1 INTRODUCTION TO VISTA .............. 5-1

5.1 .1 Background .................. 5-1

5.1 .2 Method Used ............. 5-1

5.2 VISTA INPUT REQUIREMENTS ............. 5- . 5

5.3 VISTA INPUT DATA NOTES .......... 5-4

5.4 VISTA INPUT FLUENCE FILE FORMAT . ....... 5-5

5.5 VISTA OUTPUT FILE .............. 5-8

5.6 LOGICAL UNIT REQUIREMENTS .............. . . 5-9

5.7 PROGRAMMER INFORMATION .......... 5-10

5.7.1 Inter-machine Adaptability ........ . 5-10

5.7 .2 Service Subroutines ............ 5-11

5.8 REFERENCES . . . . . . . . . . 5-12

5.9 SAMPLE PROBLEM ....................... 5-13

6.0 MORSE: A MULTIGROUP OAK RIDGE STOCHASTIC EXPERIMENT CODE . . 6-1

6.1 INTRODUCTION TO MORSE .............. . . . . . .

6.1.1 Theoretical Basis........... . 6- 6-2

6.1 .2 In-Group Energy Biasing . . . . . . . . 6-7 
6.2 MORSE CODE STRUCTURE ............ 6-8

6.2 .1 Random Walk Module ........... . 6-10

6.2.2 Multigroup Cross-Section Module . . . . . . . 6-13

6.2.3 The GIFT5 Geometry Package ......... 6-18

6.2.4 Modifications to MORSE for In-Group Biasing. . 6-23

6.2.5 Modifications to MORSE-CG for the

MASH Code System . . . . . . . . . 6-24

6.3 MORSE INPUT REQUIREMENTS ............ 6 6-27

6.3.1 Random Walk Input Instructions . . . . . . 6- 6-27

6.3.2 GIFT5 Geometry Input Instructions ....... 6-33

6.3.3 Cross-Section Module Input Instructions ..... 6-40

6.3.4 Special MASH Input Instructions........ 6-43

6.4 ANISN NUCLIDE FORMAT CROSS-SECTION FILE. ..... 6-43

6.5 MORSE LEAKAGE TAPE OUTPUT FILE FOR DRC ...... 6- 6-45

6.6 LOGICAL UNIT REQUIREMENTS . . . . . . . . . . 6-48

6.7 REFERENCES .............. 6- 6- . . . . .

6.8 SAMPLE PROBLEM ............... 6-...... 62

7.0 DRC: A DETECTOR RESPONSE CODE ........... $7-1$

7.1 INTRODUCTION TO DRC ............ 7-1

7.1.1 Background ................ 7-1

7.1 .2 Method Used . . . . . . . . . . . . 7-2

7.2 DRC INPUT REQUIREMENTS $\ldots \ldots \ldots \ldots$

7.3 DRC INPUT DATA NOTES . . .......... $7-9$

7.3.1 Common Block Information ........ 7-10

7.4 DRC INPUT FILE FORMATS .......... 7-10

7.5 DRC OUTPUT FILES . . . . . . . . . . 7-18

7.6 LOGICAL UNIT REQUIREMENTS ........... 7-18

7.7 REFERENCES .............. . 7-19

7.8 SAMPLE PROBLEM ................... 7-.19

APPENDIX A: FIDO INPUT ................ A-1

APPENDIX B: IN-GROUP ENERGY BIASING . . . . . . . . . B-1

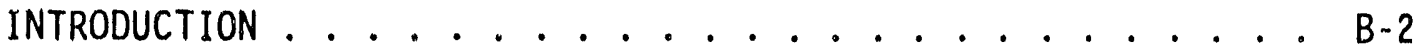

BACKGROUND . . . . . . . . . . . . B-3

THEORETICAL BASIS FOR IN-GROUP ENERGY BIASING . . . . . . B-4

Markov Chain Analysis of the Forward

Three-Group Infinite Medium Problem ......... B-4

Adjoint Three-Group Analysis ......... B-.

In-Group Energy Bias Analysis ............ B-11

IN-GROUP BIASING CODE MODIFICATIONS FOR MORSE ...... B-16 


\section{TABLE OF CONTENTS}

(continued)

Page

DEMONSTRATION CALCULATIONS ........... B-17

REFERENCES ................... . . . . . . . . .

APPENDIX C: THE GIFT5 GEOMETRY PACKAGE ........... c-1

BACKGROUND .................. C-2

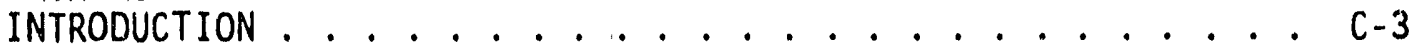

TARGET DESCRIPTION DATA ...............

PRELIMINARY STEPS .................

SOLIDS . . . . . . . . . . . . . . . . C C-4

SOLID TABLE . . . . . . . . . . . . . C-27

"COMBINATION" OF SOLIDS . . . . . . . . . . . . . . . . . C-27

REGION TABLE . . . . . . c-30

RECOMMENDED PROCEDURES FOR THE REGION TABLE ....... c c-33

REGION IDENTIFICATION TABLE . . . . . . . . . . . . C-34

SPECIAL REGION IDENTIFICATION NUMBERS ........ C-36

USING IDENTIFICATION NUMBERS TO "COMBINE" REGIONS ..... C-37

RULES FOR REGIONS ................ . . . . . . . . .

GIFT5 GEOMETRY PACKAGE MEMORY REQUIREMENTS

AND REGION TOLERANCES .............. c . . . . . 40

REGION RPP TABLE .................... . . . . .

THE TITLE AND TARGET SPECIFICATION CARDS ......... .41

CARD ORDER FOR THE TARGET DESCRIPTION INPUT ....... $\quad . .44$

REFERENCES . . . . . . . . . . . . . . . . . .

APPENDIX D: MASH 1.0 CODE SYSTEM ABSTRACT . . . . . . . . D- 1

APPENDIX E: USER NOTES . . . . . . . . . . . E E-1

INTRODUCTION . . . . . . . . . . . . . . E E 2

QUESTIONS AND ANSWERS ON AIR-OVER-GROUND
ENVIRONMENT CALCULATIONS WITH GRTUNCL/DORT ......... E $\cdots 2$

QUESTIONS AND ANSWERS ON ADJOINT MONTE CARLO 
(This page is intentionally left blank.) 


\section{LIST OF FIGURES}

Figure $\quad$ Page

1-1. Typical VCS Problem Involving an Armored Vehicle . . . 1-2

1-4. Typical VCS Problem Involving an Enclosed Structure . . . 1-3

1-3. General Structure of the MASH Code System . . . . . . 1-8

1-4. Data Flow Diagram of the MASH Code System . . . . . . 1-9

2-1. Samplle GIP Input for the Two-Meter Box Air-over-Ground Analysis............ 2-18

2-2. Sample GIP Output for the Two-Meter Box Air-Over-Ground Analysis ............ 2-20

3-1. Schematic Diagram of the Air-Over-Ground Geometry Model Used in the GRTUNCL Analysis . . . . . 3-27

3-2. Sample GRTUNCL Input for the Two-Meter Box Air-Over-Ground Analysis ........... . 3-28

3-3. Sample GRTUNCL Output for the Two-Meter Box Air-Over-Ground Analysis ............. . 3-30

4-1. Triangular Geometry Options.... . . . . . . . 4-29

4-2. Schematic Diagram of the Air-Over-Ground Geometry Model Used in the DORT Analysis . . . . . . . . 4-70

4-3. Sample DORT Input for the Two-Meter Box Air-Over-Ground Analysis ............... 4-71

4-4. Sample DORT Output for the Two-Meter Box Air-Uver-Ground Analys is ............ . 4-74

5-1. Schematic Diagram of the Air-Over-Ground Geometry Model Used in the VISTA Analys is . . . . . . 5-14

5-2. Sample VISTA Input for the Two-Meter Box Air-Over-Ground Analysis............. 5-15

5-3. Sample VISTA Output for the Two-Meter Box Air-Over-Ground Analysis .............. . 5-16

6-1. Schematic Diagram of the Two-Meter Box Geometry Model Used in the MORSE Analysis . . . . . . 6-56

6-2. Sample MORSE Input for the Two-Meter Box Analysis . . . 6-57 


\section{LIST OF FIGURES \\ (continued)}

Figure Page

6-3. Sample GIFT5 Input for the Two-Meter Box Analysis . . . 6-59

6-4. Sample MORSE Output for the Two-Meter Box Analysis . . . 6-62

7-1. Sample DRC Input for the Two-Meter Box

Protection Factor Analysis........... 7-21

7-2. Sample DRC Output for the Two-Meter Box

Protection Factor Analysis ......... 7-22

B-1. Characteristics of Functions in Equation (27) ..... B-15

C-1. Four-faced, Four Vertices, Convex Polyhedron

(ARB4) Input .................... C-6

C-2. Five-faced, Five Vertices, Convex Polyhedron

(ARB5) Input .................... C-7

C-3. Five-faced, Six Vertices, Convex Polyhedron

(ARB6) Input .................... C-8

C-4. Six-faced, Seven Vertices, Convex Polyhedron

(ARB7) Input .................. C-9

C-5. Six-faces, Eight Vertices, Convex Polyhedron

(ARBB) Input ................. C-10

C-6. N-faces, Convex Poiyhedron (ARBN) Input ....... C-11

C-7. Triangular Surfaced (ARS) Polyhedron Input . . . . . . C-12

C-8. Box (BOX) Input ................... C-14

C-9. Ellipsoid of Revolution (ELL) Input .......... C-15

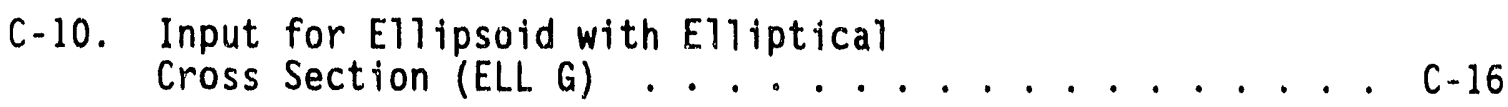

C-11. Half Space (HAF) Input ............. C-17

C-12. Right Angle Wedge (RAW) Input ........... C-18

C-13. Right Circular Cylinder (RCC) Input ......... C-19

C.14. Right Elliptical Cylinder (REC) Input ......... C-20 


\section{LIST OF FIGURES}

(continued)

Figure

C-15. Rectangular Parallelepiped (RPP) Input ......... C-21

C-16. Sphere (SPH) Input ................ C-22

C-17. Truncated Elliptical Cone (TEC) Input .......... C-23

C.18. Truncated General Cone (TGC) Input . . . . . . . . . . C-24

C-19. Torus (TOR) Input ................ C -25

C-20. Truncated Right Angle Cone (TRC) Input . . . . . . . C-26

C-21. Intersection, Subtraction, and Union

Between Two Solids .............. C-29

C-22. Intersection, Subtraction, and Union

Between Three Solids............... C-31

C-23. Card Input for the Region Cards and the

Region Table for the Sample Target ......... . L -32

C-24. Card Input for the Region Identification Table and a

Region Identification Table for the Sample Target . . . C-36

C-25. Illustrations for Region Rules............. C-38

C-26. Card Input for the Region RPP Table and the
Region RPP Table for the Sample Target........ C -42

C-27. Card Input for the Title Card and

Target Specification Card ............ C-43 
(This page is intentionally left blank.) 


\section{LIST OF TABLES}

Table

Page

4-1. Directional Properties of the Geometries . . . . . . . 4-6

4-2. Typical Stabilization Values.............. . 4-41

4-3. Radial Mesh Away from Source.............. 4-45

4-4. Mesh Upward from Source ................ 4-45

4-5. Mesh Downward from Source .............. 4-46

4-6. Mesh Upward from Ground ................ 4-46

6-1. BANKR Arguments .................. 6-11

6-2. Variables that may be Written on Collision Tape (NBIND) . 6-12

6-3. Examples of the Relationship Between Random Walk and Cross-Section Module Group Input Parameters . . . . . . 6-14

6-4. Location of MASH Arrays in BLANK COMMON . . . . . . . . 6-25

6-5. Definition of Variables in Common IVCS . . . . . . 6-26

6-6. Summary Description of Input for Solids Table . . . . . 6-35

6-7. Definition of Variables in USER COMMON ......... 6-49

7-1. Detector Response Definitions in DRC . . . . . . . . 7-6

7-2. Definitions of Parameters and Protection Factors used to Characterize the Effectiveness of Shields . . . 7-7

7-3. Location of DRC Arrays in BLANK COMMON . . . . . . . 7-11

7-4. Definitions of Variables in Common TDT1 ........ 7-12

7-5. Definitions of Variables in Common IDRC ....... 7-13

7-6. Definitions of Variables in USER COMMON ........ 7-18

C-1. List of 20 Geometric Solids Used by the GIFT5
Geometry Package................. . . . . . . .

C-2. Solid Table for the Sample Target . . . . . . . . . C-28

C-3. Identification Numbers Used for Vehicles and Air Regions
Within the Vehicles in the GIFT5 Geometry Package. . . . C-35 
(This page is intentionally left blank.) 


\section{PREFACE}

The development of the MASH code system has been due to the efforts of numerous people over a period of several years. All of those involved in the MASH effort would 1 ike to express their gratitude to the Defense Nuclear Agency who has continuously supported and monitored the project. Since MASH represents a collection of codes, and parts of this manual are extracted from other documents supporting these various codes, the major contributors to the MASH effort deserve mention. Ballistic Research Laboratory (BRL) contributed the GIFT5 geometry package (a) ong with much of its documentation) utilized in the MORSE component of MASH. Science Applications International Corporation (SAIC) contributed the in-group energy biasing methodology incorporated in MORSE (along with much of its documentation) and the programming required to couple the GIFT5 geometry package into the MORSE component of MASH. Furthermore, SAIC collaborated with the extensive programming changes, error debugging, and code testing throughout the development phase of the MASH project. Oak Ridge National Laboratory (ORNL) contributed most of the codes which make up the MASH code system. The ORNL investigators have incorporated the many contributions from within ORNL along with the contributions from the other investigators at BRL and SAIC into the MASH code system and are responsible for the final version of MASH to be released along with the supporting documentation.

Each section within this manual contains its own set of references to acknowledge the principal sources of the contributions to that particular section. These references acknowledge the contributions of those not directly involved with the MASH project, but without the ir efforts MASH would not exist. Here it is sufficient to state that the efforts of all those who aided this project through technical consultation, code programming and testing, review, manuscript preparation, and administrative support are gratefully acknowledged.

J. 0. Johnson, editor 
(This page is intentionally left blank.) 


\section{ABSTRACT}

The Monte Carlo Adjoint Shielding Code System, MASH, calculates neutron and gamma-ray environments and radiation protection factors for armored military vehicles, structures, trenches, and other shielding configurations by coupling a forward discrete ordinates air-over-ground transport calculation with an adjoint Monte Carlo treatment of the shielding geometry. Efficiency and optimum use of computer time are emphasized. The code system includes the GRTUNCL and DORT codes for air over-ground transport calculations, the MORSE code with the GIFT5 combinatorial geometry package for adjoint shielding calculations, and several peripheral codes that perform the required data preparations, transformations, and coupling functions. MASH is the successor to the Vehicle Code System (VCS) initially developed at Oak Ridge National Laboratory (ORNL).

The discrete ordinates calculation determines the fluence on a coupling surface surrounding the shielding geometry due to an external

neutron/gamma-ray source. The Monte Carlo calculation determines the effectiveness of the fluence at that surface in causing a response in a detector within the shielding geometry, i.e., the "dose importance" of the coupling surface fluence. A coupling code folds the fluence together with the dose importance, giving the desired dose response. The coupling code can determine the dose response as a function of the shielding geometry orientation relative to the source, distance from the source, and energy response of the detector.

This user's manual includes a short description of each code, the input required to execute the code along with some helpful input data notes, and a representative sample problem (input data and selected output edits) for each code. 
(This page is intentionally left blank.) 


\subsection{MASH: A MONTE CARLO ADJOINT SHIELDING CODE SYSTEM}

\subsection{INTRODUCTION TO MASH}

\subsubsection{Background}

An armored vehicle can provide important protection against the effects of a nuclear weapon. In the case of a tactical weapon, prompt radiation may be the dominant effect over a considerable area surrounding the detonation. The Vehicle Code System (VCS) was developed by Oak Ridge National Laboratory (ORNL) to facilitate the calculation of radiation protection factors for a given vehicle; i.e., the ratio by which the free-field radiation is reduced due to the presence of the vehicle. 1,2 VCS was constructed by linking together a discrete-ordinates air-overground transport calculation and an adjoint Monte Carlo vehicle doseimportance calculation. A typical problem involving an armored vehicle is shown in Figure 1-1. While the title implies the code system was to be used only for vehicles, VCS could also be used for other shielded structures. A typical problem involving a shielded structure, such as a house, is shown in Figure 1-2.

The typical VCS problem involves the detonation of a low-kiloton yield tactical nuclear weapon within a kilometer of the target (vehicle). The air-over-ground transport calculation determines the neutron and photon fluence as a function of energy on a coupling surface surrounding the target (vehicle). The dose-importance calculation determines the effectiveness of particles at the coupling surface in contributing to dose at a detector position within the target (vehicle). A coupling code folds the fluence together with the dose importance, giving the dose response. The coupling code can also rotate the vehicle, move it to different distances from the source, and perturb the energy response of the detector.

VCS was initially developed as a four-code system including MORSE, DOT, DRC, and VISA. MORSE ${ }^{3}$ is both a forward and an adjoint Monte Carlo transport code which can calculate the fluence in the complicated threedimensional vehicle geometry. $D \mathrm{OT}^{4}$ is a two-dimensional discrete ordinates radiation transport code which calculates the energy- and angle-dependent free-field initial radiation environment. DRC couples the adjoint fluences from MORSE with the free-field environments from DOT to calculate the radiation environments or protection factors at "detector" locations in the vehicle or structure. VISA is used to format the DOT angular fluence into a data file for use by DRC.

VCS has been and still is regularly used at ORNL, the Ballistic Research Laboratory (BRL), Science Applications International Corporation (SAIC), Lawrence Livermore National Laboratory (LLNL), and Wehrwissenschaftliche Dienststelle der Bundeswehr fur ABC-Schutz (WWD), West Germany for the 


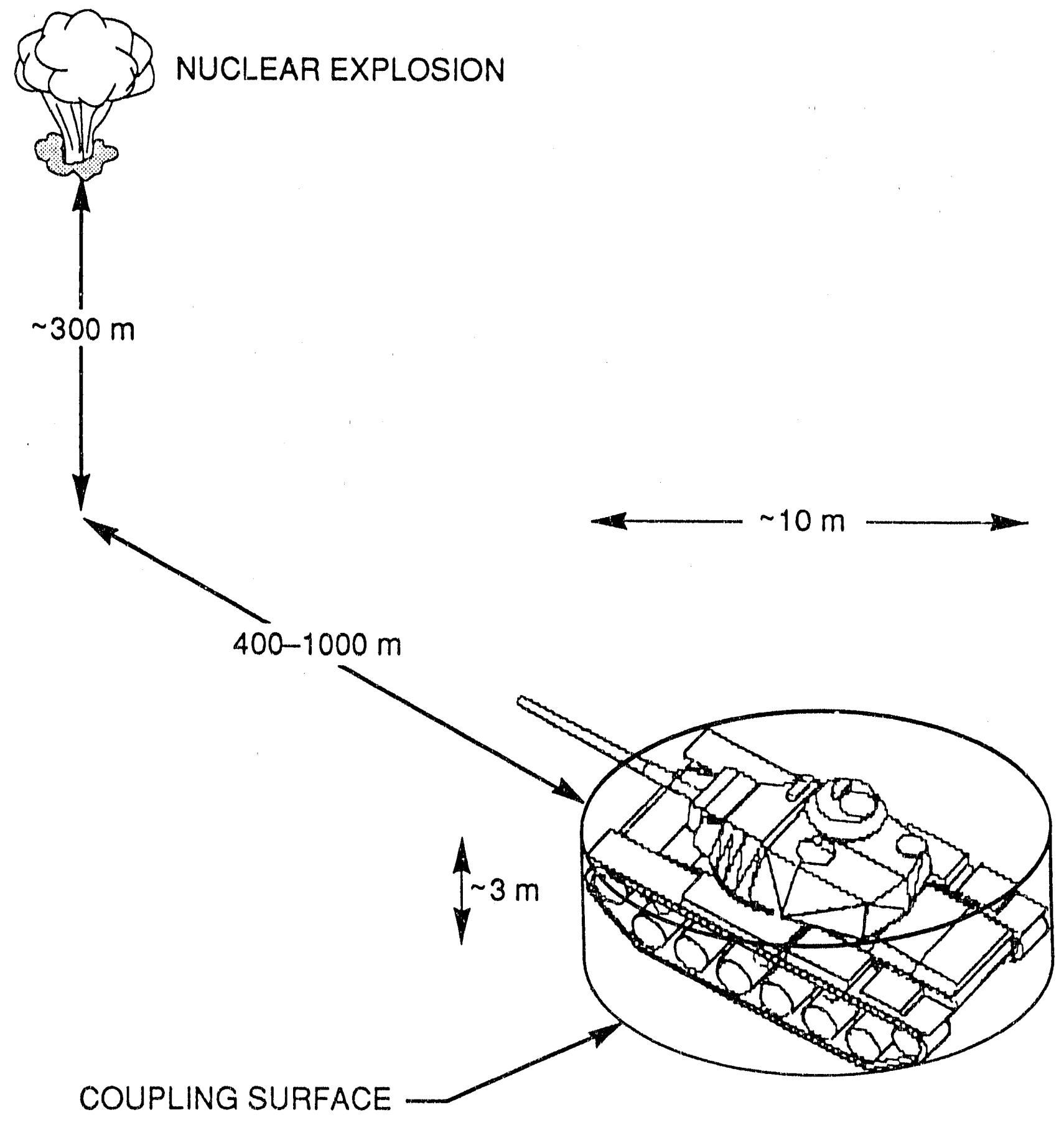

Figure 1-1. Typical VCS Problem Involving an Armored Vehicle. 


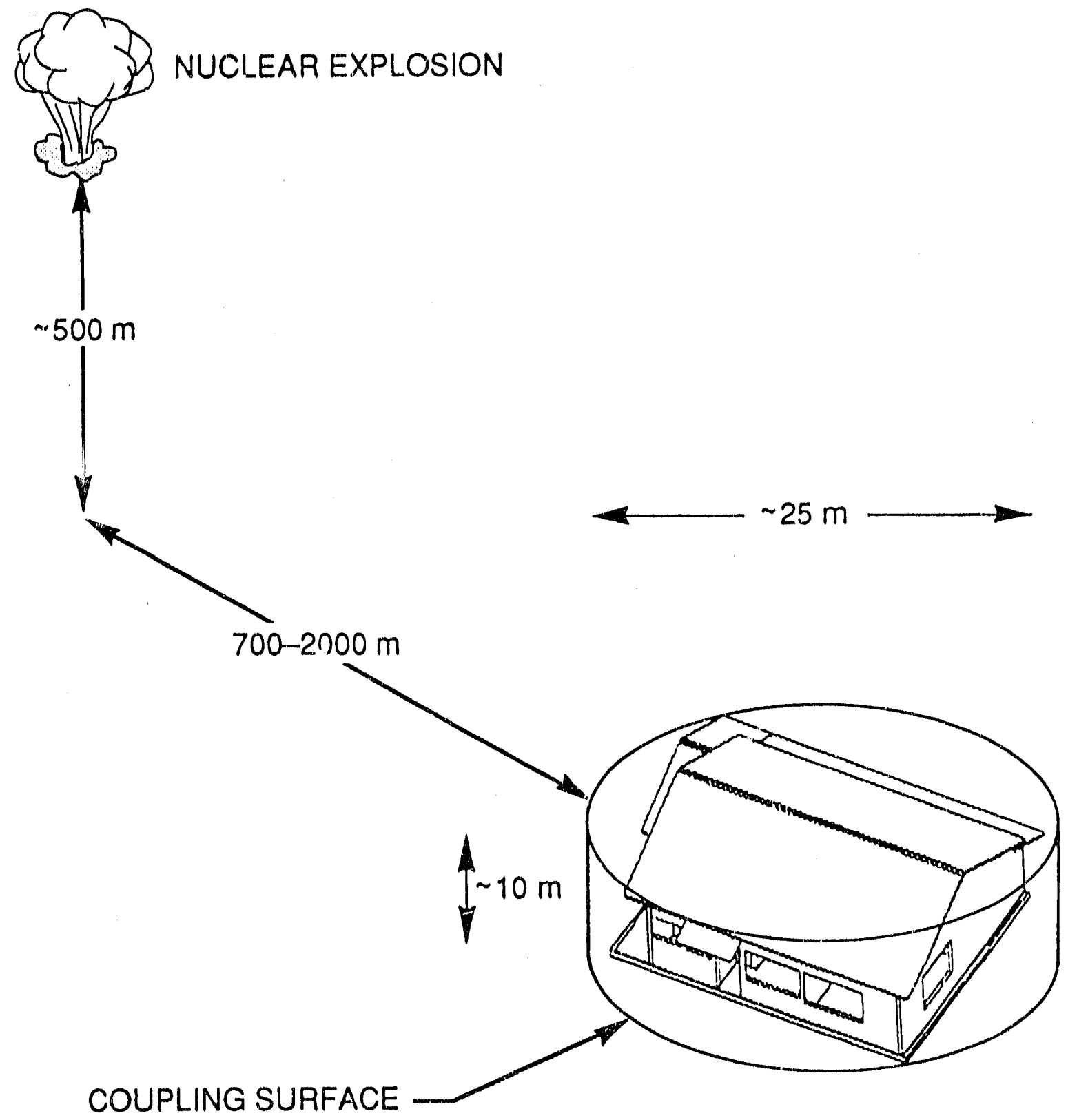

Figure 1-2. Typical VCS Problem Involving an Enclosed Structure. 
calculations of neutron and gamma-ray protection factors of military vehicles, structures, trenches, and other shield configurations. VCS represents a powerful tool for coupling complex geometry calculations into single free-field environments, and as such, has had applications to a great many shielding problems. Past and present applications include a wide variety of Army vehicles, house shielding at Hiroshima and Nagasaki, trench shielding factors at the Nevada Test Site, and inmissile radiation environments.

Over the course of time, different versions of VCS were created due to the different installations tailoring the code to suit their particular purpose. At ORNL, the DOT III code was replaced with the DOT IV6,7 code, and VISA was repleced with VISTA. BRL, which has been a major user and source of funding for the VCS code, made a number of modifications to the MORSE and DRC parts of VCS in 1976-1977. The major change to MORSE was the replacement of the combinatorial geometry mode1, which SAIC had previously installed in MORSE, ${ }^{8}$ with GIFT combinatorial geometry, which was similar to the MORSE combinatorial geometry but which had been further developed at BRL. ${ }^{9-11}$ After those modifications, BRL contracted SAIC to evaluate and document some aspects of the VCS. That effort concluded that "VCS is a sophisticated and well-planned code package capable of accurate, state-of-the-art radiation transport in complex, three-dimensional vehicles" and suggested a new "within-group" energy biasing scheme for adjoint MORSE. ${ }^{12}$ In two subsequent efforts, one for the Wehrwissenschafti iche Dienststelle der Bundeswehr fur ABCSchutz (WWD), West Germany, ${ }^{13}$ and the other for BRL, ${ }^{14}$ SAIC installed and tested the new in-group energy bias methodology in the adjoint MORSE part of VCS. The study for WWD demonstrated that this scheme could provide a factor of four increase in the efficiency of VCS for a shielding calculation with $10 \mathrm{~cm}$ of steel.

The result of all this independent developinent of VCS was a proliferation of different versions of the code, of which most were not compatible. The Defense Nuclear Agency (DNA) expressed concern over the potential for disagreement between independent analyses of the same armored vehicle. Consequently, BRL, ORNL, and SAIC were tasked to identify the problems associated with the "current" version(s) of VCS, incorporate the various modifications (which would improve the VCS code) that have been added to the different versions, and create a singularly referenced version of VCS. In particular, the purpose of this project was to establish and verify a version of VCS which is generally acceptable to the user community, and to place this version of VCS under responsible custody on the Defense Nuclear Agency (DNA) computing network for use on the CRAY computer system at LOS Alamos National Laboratory (LANL) under the CRAY Time Sharing System (CTSS). In this effort, SAIC was to take the lead in the selection of the geometry model and in the development and testing of the in-group energy biasing; ORNL was to develop a new reference cross section data set, modify DRC to couple MORSE and DOT calculations made with different energy group sets, and compile the new version of VCS. SAIC and ORNL were to share the task of checking out the new coding. 
In years prior to this effort, three problems with VCS and the primary cross section library use in VCS analysis were identified, all of which have been addressed in this effort. These three problems included:

1. The biasing treatment used in the MORSE adjoint calculation of VCS did not favorably sample the most important regions of energy space, especially for calculations involving steel and polyethylene. These two materials are potentially important shielding components of armored vehicles. Furthermore, theoretical analyses of the standard adjoint scattering treatment indicated inherent large variances for adjoint Monte Carlo analyses of thick media.

2. The energy group structure of the 58 group ( 37 neutron groups, 21 gamma groups) DLC 31 cross section library, 15 had suspected deficiencies for transport in steel and air, especially near $1 \mathrm{MeV}$ and at epithermal energies.

3. The more recent GIFT5 ${ }^{16}$ geometry routines used by BRL for constructing vehicle models have certain incompatibilities with the geometry routines in. MORSE. Vehicle models developed by BRL for various analyses other than nuclear survivability/shielding effectiveness had to be revised, at a significant cost in man power and elapsed time, for VCS analyses.

As a result of this effort, SAIC installed a "user community compatible" geometry package and in-group energy biasing into VCS by incorporating the GIFT5 geometry package and a revised in-group energy bias package into the BRL MIFT version of MORSE. After a significant checkout effort, this package was released to ORNL for further checkout and testing. SAIC called this version of MORSE "MIFT2", 17 indicating that it was an update of the BRL MIFT code. ORNL developed the new Defense Applications Broad-group Library (DABL69), ${ }^{18}$ with 46 neutron energy groups and 23 gamma-ray groups, made several modifications to many of the code system modules, and incorporated the SAIC changes to the MORSE component into the new version of VCS called MASH (Monte Carlo Adjoint Shielding). This final version "MASH 1.0" has been compiled and tested and placed under responsible custody of DNA on the DNA computing network for use on the CRAY computer system at Los Alamos National Laboratory (LANL) under the CRAY Time Sharing system (CTSS). Future efforts wit1 involve making a version of MASH 1.0 operational on an IBM RISC system 6000 workstation. 


\subsubsection{Summary of Corrections and Improvements to VCS}

The VCS code was outdated in several respects as well as being deficient in others. Among the changes made to VCS to produce version 1.0 of the MASH code system are:

1. The replacement of the MORSE module with the latest version of MORSE-CG.

2. The incorporation of in-group energy biasing into the MORSE component of MASH.

3. The replacement of the combinatorial geometry package in MORSE with the new GIFT5 geometry package.

4. The modifications to DRC to couple MORSE and DORT calculations with different energy group structures, and read the new VISTA formatted records.

5. The replacement of the DOT component with the newer DORT ${ }^{19}$ component.

6. The replacement of VISA with the newer VISTA code.

7. The replacement of the DLC-31 $(37 n-21 \gamma)$ group coupled cross-section library with the new DABL69 $(46 n-23 \gamma)$ group library as the reference cross-section data library.

A11 components of MASH 1.0, except the GIFT5 input module to MORSE, use the FIDO free-form input data described in detail in Appendix $A$. The GIFT5 geometry input for the MORSE component currently is in fixed format, however, future efforts will convert this input over to the FIDO free-form method.

Additional improvements to MASH 1.0 include programming upgrades to the MORSE and DRC modules. These two codes now have all arrays variably dimensioned, variable tape unit numbers, revised common and subroutine structures, a new leakage tape format, and orderly termination of cases. Several errors in choosing bins for the coupling surface and in determining detector number for the storage of results have been corrected. Because traceability or quality assurance is increasingly important, some changes were made to tape formats to provide more definitive information about the computer runs. 


\subsection{STRUCTURE OF MASH 1.0}

The general structure of the MASH code system is shown in Figure 1-3, while the data flow is detailed in Figure 1-4. The GIP code is used to create and mix the materfals required to run the air-over-ground transport analysis. The compositions and source data for the afr-overground transport calculation are input into both the GRTUNCL and DORT codes. In order to maintain particle balance and reduce ray effects, GRTUNCL calculates the uncollided fluence and first-collision source throughout the problem space. This information is input to DORT which calculates the resulting collided fluence, and merges the two results. All fluence information is passed to VISTA, where it is condensed and reordered for convenient processing. Only a few ground ranges from the radiation source are normally processed at this step. Additional ground ranges may be obtained by rerunning the VISTA code at a minimal cost.

The complex three-dinensional target (vehicle) geometry description and material compositions are input to the MORSE code. MORSE records the required information for each adjoint track leaving the coupling surface on a leakage data set which is input to the Detector Response Code, DRC. DRC folds the adjoint leakage data together with the reformatted DORT fluence at the coupling surface, i.e., the VISTA code output fluence file, for the selected orientation, range, and detector response. It also provides edits of estimated variances of the results as requested by the user. The protection factor itself is obtained by dividing the free-field dose, avallable from DORT, by the dose response at the detector position within the target (vehicle) given by DRC.

Special advantage is taken of the fact that the target (vehicle) is small compared to the separation distance between the source (weapon) and target (vehicle). This means that the fluence in a given direction and at a given elevation and energy is essentially independent of the azimuthal variable about the target (vehicle). This reduces the volume of data processed by DRC, minimizes the cost of both VISTA and DRC, and simplifies the construction of both codes. [Note: This assumption could lead to error for some large structures such as house clusters, large buildings, etc., where the distance from the detector location to the coupling surface is significant relative to the gradient in the external fluence field.]

Most of the cost of MASH is incurred in the DORT and MORSE codes. Therefore, it is advantageous to save the VISTA output for use with many MORSE runs. Likewise, it is practical to use a single MORSE leakage tape for a target (vehicle) to examine a number of target orientations, source ranges and types, and detector response functions. 


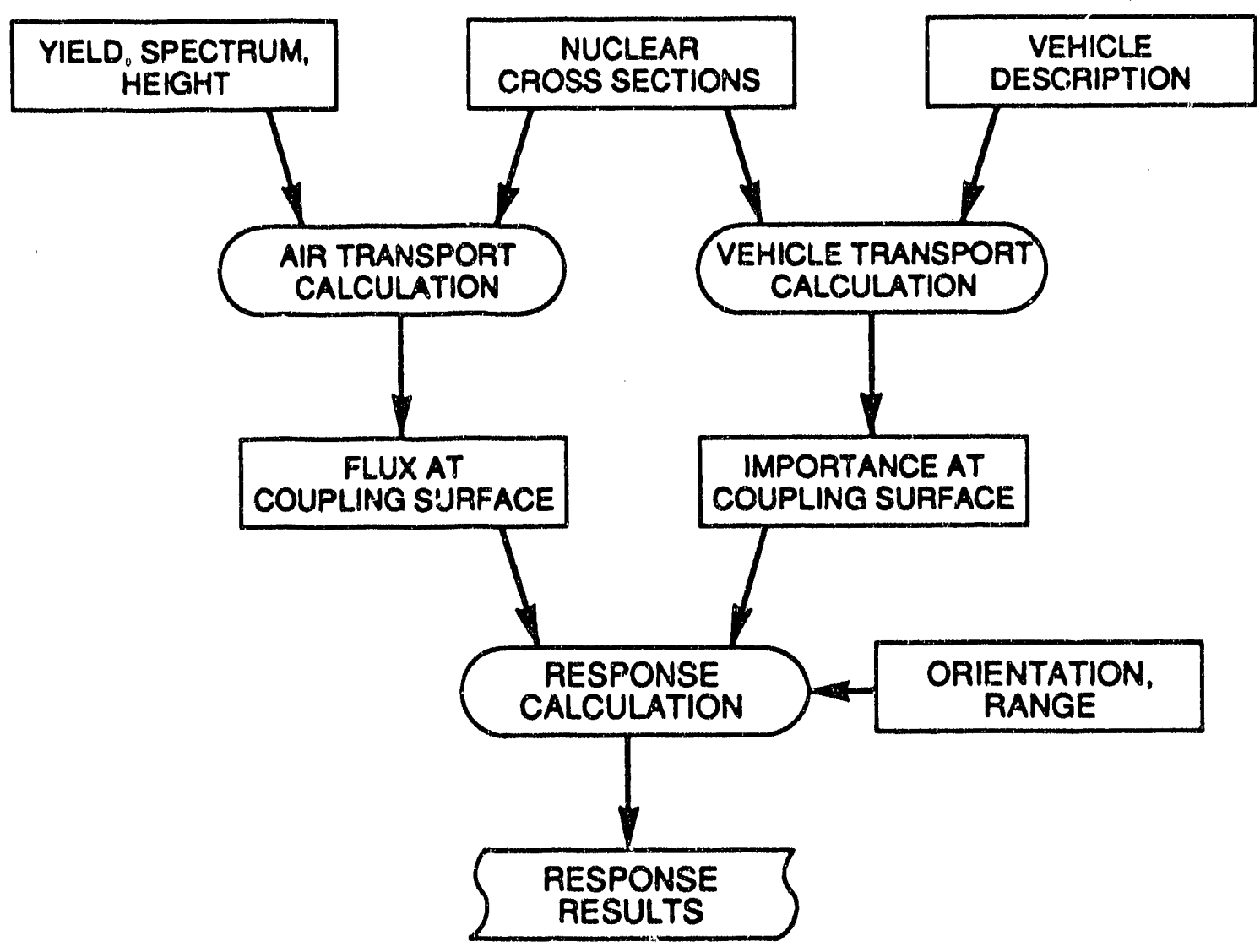

Figure 1-3. General Structure of the MASH Code System. 


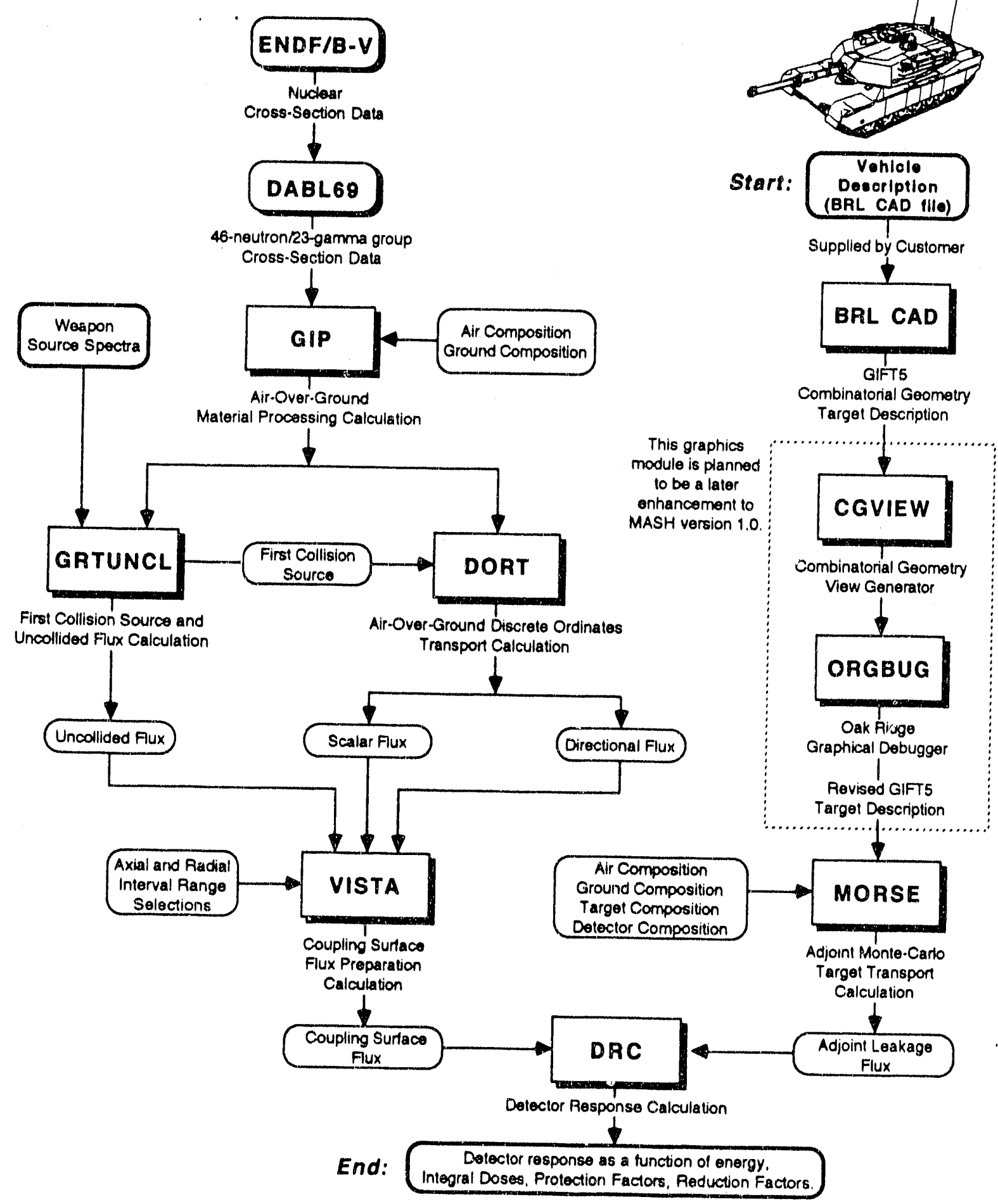

Figure 1-4. Data Flow Diagram of the MASH Code System. 


\subsection{STRUCTURE OF MASH USER'S MANUAL}

\subsubsection{The Main Text}

The remainder of the main text of this manual (Sections 2.0 through 7.0 ) gives a detailed description of each of the codes in MASH. Included in each section is a brief description of the code and its theory (if applicable), the input requirements, detailed input data notes, the input and output file formats, the logical unit requirements, a list of references pertaining to that particular section, and a sample problem. For the two major codes within MASH, i.e., DORT and MORSE, the section is not intended to be a complete description and the interested user might consider obtaining the reference documents for these two codes. However, the information in each of the sections should be sufficient for understanding and using MASH.

\subsubsection{The Appendices}

The Appendices include detailed descriptions of FIDO input (Appendix A), the theory behind in-group energy biasing (Appendix B), a detailed description for the GIFT5 geometry package (Appendix C), a 1isting of the MASH 1.0 computer code abstract (Appendix D), and a short, informal "user motes" section (Appendix E) which addresses some of the caveats of performing the MASH analysis of the sample problem as determined through the experience of the MASH 1.0 manual's editor. The inclusion of the in-group biasing theory in the appendix (rather than in the MORSE section) was due to the format chosen for the "user's" manual. The detailed description of the GIFT5 geometry package was included for completeness since this package is unpublished.

\subsubsection{The Sample Problem}

The sample problem represents a "benchmark" calculation of MASH 1.0 on the Two-Meter Box Test Bed experiments performed at APRF.20,21 The results of the MASH analysis were generally within $15 \%$ of the experimental measurements for both differential and integral free-field and in-box neutron and gamma-ray dose. There was al so excellent agreement in the protection factor analys is for the box. This sample problem was independently analyzed by both SAIC and ORNL with $C / C$ ratios, i.e., SAIC calculations/ORNL calculations, typically with in $5 \%$. The sample problem input and output for each code is given in the respective sections of the report. However, each part of the sample problem fits into the data flow shown on Figure 1-4, and the user should take time to see which parameters in one code depend on parameters from the previous code, i.e., the axial and radial mesh between DORT and GRTUNCL. In the discussion of the sample problem in each section, most of these dependencies are identified. 


\subsection{REFERENCES}

1. W. A. Rhoades and M. B. Emmett et al., "Vehic.le Code System (VCS User's Manual)," ORNL/TM-4648, Oak Ridge National Laboratory (August 1974).

2. W. A. Rhoades et al., "Development of a Code System for Determining Radiation Protection of Armored Vehicles (The VCS Code)," ORNL/TM-4664, Oak Ridge National Laboratory, (October $1974)$.

3. M. B. Emmett, "The MORSE Monte Carlo Radiation Transport Code system," ORNL-4972 (1975), ORNL-4972/R1 (1983), ORNL-4972/R2 (1984), Oak Ridge National Laboratory.

4. W. A. Rhoades and F. R. Mynatt, "The DOT III Two-Dimensional Discrete Ordinates Transport Code, "ORNL-TM-4280, Oak Ridge National Laboratory, (September 1973).

5. L. F. Schanzler and F. W. Bucholz et al., "Protection Factors of the Leopard IAl Comparison of Calculated Results with Measured Values," Wehrwissenschaftliche Dienststelle der Bundeswehr fur ABC-Schutz, 3042 Munster, Federal Republic of Germany. Paper presented at the meeting on Protection Factors of Armored Vehicles, German Ministry of Defense, Bonn, Germany (22-25 June 1982).

6. W. A. Rhoades, D. B. Simpson, R. L. Childs, and W. W. Engle, Jr. "The DOT IV Two-Dimensional Discrete Ordinates Transport Code with Space-Dependent Mesh and Quadrature," ORNL/TM-6529, Oak Ridge National Laboratory (January 1979).

7. W. A. Rhoades and R. L. Childs, "An Updated Version of the DOT 4 One- and Two-Dimensional Neutron/Photon Transport Code," ORNL-5851, Oak Ridge National Laboratory (1982).

8. E. A. Straker, W. H. Scott, Jr., and N. R. Byrn, "The MORSE Code with Combinatorial Geometry, " Defense Nuclear Agency DNA 2860T, SAI-72-511-LJ, Science Applications International Corporation, (May 1972).

9. Lawrence W. Bain, Jr. and Mathew J. Reisinger, "The GIFT Code User Manual; Volume I. Introduction and Input Requirements," BRL 1802, Ballistic Research Laboratory, (JuTy 1975).

10. Gary G. Kueh1, Lawrence W. Bain, Jr. and Mathew J. Reisinger, "The GIFT Code User Manual; Volume II. The Output Options," ARBRLTR-02189, Ballistic Research Laboratory, (September 1979).

11. A. E. Rainis and Ralph E. Rexroad, "MIFT: GIFT Combinatorial Geometry Input to VCS Code," BRL Report No. 1967, Ballistic Research Laboratory, (March 1977). 
12. W. H. Scott, Jr., "Vehicle Code System (VCS) Documentation and Uncertainty Analysis," SAI Report SAI-133-79.977-LJ, Science Applications International Corporation, (December 1979).

13: W. H. Scott, Jr., and V. E. Staggs, "Adjoint Energy Biasing and Thermal Neutron Diffusion in the MORSE and VCS Codes," SAI-133-81-384-LJ Science Applications International Corporation, (November 1981).

14. W. H. Scott, Jr., et al., "Predictive Algorithm for Radiation Protection," Volume 1, "VCS In-Group Energy Bias, The DACM Code and DOT Calculations," SAIC-85/1710, Science Applications International Corporation, (May 1985).

15. D.E. Bartine, et al., "Production and Testing of the DNA Few-Group Coupled Neutron-Gamma Cross-Section Library," ORNL/TM-4840, Oak Ridge National Laboratory, (March 1977).

16. "The GIFT5 Geometry Package", Ballistic Research Laboratory, (No formal documentation exists for GIFT5.)

17. J. A. Stoddard, S. D. Egbert, and W. H. Scott, Jr., "The Vehicle Code System with In-Group Energy Bias and GIFT5 Geometry, "DNATR-87-23, Science Applications International Corporation, (January 1987).

18. D. T. Ingersol1, R. W. Roussin, C. Y. Fu, and J. E White, "DABL69: A Broad-Group Neutron/Photon Cross-Section Library for Defense Nuclear Applications," ORNL/TM-10568, Oak Ridge National Laboratory, (June 1989).

19. W. A. Rruades and R. L. Childs, "The DORT Two-Dimensional Discrete- Ordinates Transport Code," Nuclear Science \& Engineering 99,1 , pp. 88-89, (May 1988).

20. J. 0. Johnson, J. D. Drischler, and J. M. Barnes, "Analysis of the Fal1-1989 Two-Meter Box Test Bed Experiments Performed at the Army Pulse Radiation Facility (APRF)," ORNL/TM-11777, Oak Ridge National Laboratory, (May 1991).

21. R. T. Santoro et a1., "DNA Radiation Environments Program Fall 1989 2-Meter Box Experiments and Analysis," ORNL/TM-11840, Oak Ridge National Laboratory, (May 1991). 


\subsection{GIP: A GROUP-ORGANIZED CROSS SECTION INPUT PROGRAM*}

\subsection{INTRODUCTION TO GIP}

\subsubsection{Background}

GIP is an auxiliary code used to pre-mix the material cross-section sets for very large discrete ordinates calculations (relative to available core space). The motivation for developing GIP was due to complications arising when the amount of core space required for the rlixing table exceeded the available core storage. GIP was written to treat each component of the Legendre expansion, i.e., $P_{L}$ term, of a cross-section set as a separate nuclide. This data processing treatment was seen as a requirement in the $1960^{\prime} \mathrm{s}$, when the code originated. Although certain features of the mixing table expedite the mixing of $P_{L}$ sets, there is little doubt the code would be more convenient for present-day problems if all of the components of the Legendre expansion for a cross-section set were treated as a unit.

GIP accepts nuclide-organized microscopic cross-section data from either the input stream in card-image format or from a data library prepared by the $\mathrm{ALCl}^{1}$ program. Macroscopic cross section mixtures can be prepared as specified by a mixing table similar to that of DORT $^{2}$. The total upscatter cross section can be calculated and inserted into the set. The result is a "GIP" cross section library file (defined in Section 2.5). Microscopic and/or macroscopic cross-section data can be included in the output cross-section library file and/or printed output file.

The chief virtues of GIP are its extreme simplicity and its high compatibility with DORT, ANISN ${ }^{3}$, and related codes. It is operable on virtually any type computer that provides sequential scratch files. It uses the FIDO card-image input processor described in Appendix $A$, and shares many subroutines with DORT. Its major disadvantage is the inability to translate complex input library files such as ISOTXS. ${ }^{4}$

\subsubsection{Method Used}

The cross sections specified in the input stream are selected, and the number of energy groups which can be processed at one time is decided. The groups for the amount of core allocated, i.e., "one coreful1," are selected as each nuclide is read and dumped onto a source file. The remaining groups, if any, are written to a holding file. When all nuclides have been located, the data are read from the solirce file into

\footnotetext{
"W. A. Rhoades and M. B Emmett, "DOS: The Discrete Ordinates System," ORNL/TM-8362, Cak Ridge National Laboratory, (September 1982).
} 
core and written in group-ordered format onto a sorting file. A new corefull of data is then moved from the holding file to the source file, and then to the sorting file as before. Unprocessed groups, if any, are spilled to a second holding file, which is processed like the first holding file. This sequence continues until all groups have been processed. Efficiency is improved when one group of data can be written directly to the sorting file each time the source file is loaded. In the final step, the group-organized data are read, mixed, edited, and written to the output file.

The advaniages of the GIP system are that it can process a cross section volume of several corefulls with inconsequential cost, using only sequential files, and that it is easily adapted to new or different computing environments. Unfortunately, if the cross section volume increases to ten or more corefulls, for example, the sorting process may become inefficient.

The GIP method was originated by $W$. W. Engle, Jr. in a program called TAPEMAKER ${ }^{3}$, the predecessor to the ALCl program, which operated on the IBM 7090 before the days of random access files. The basic procedure has changed little since then.

\subsection{GIP INPUT REQUIREMENTS}

The following input cards are required to execute GIP. Default values are in brackets ([]).

Title Card (72 alphanumeric character description)

INPUT DATA BLOCK I

$1 \$$ Array - Integer Control Parameters

IGM number of energy groups

IHT position of total cross section in cross-section table

IHS position of self-scatter cross section in cross-section table

IHM cross-section table length per energy group

MS cross-section mixing table length

$-5-$.

MCR number of materials read from cards

MTP number of materials read from input cross-section library tape (nuclide or GIP format)

MTM total number of materials (MCR+MTP+MIXTURES) 
ITH output cross-section library flag

$$
0 / 1=\text { forward/adjoint cross-section library }
$$

ISCT maximum order of Legendre expansion of cross sections

$-10 \cdots$

IPRT output cross-section print control flag $0 / 1 / 2=$ print all materials/none/mixtures only

IOUT output cross-section library control flag

IDOT output cross-section library format flag

NBUF number of $k$-bytes of core

E (terminate array with "E")

$T$ (terminate block with "T") [T always required]

\section{INPUT DATA BLOCK 2}

$10 \$$ Array - Material Numbers (MS entries)

$11 \$$ Array - Component Numbers (MS entries)

Mixing Table

12* Array - Density Multipliers (MS entries)

$7 \$$ Array - Mix Code (optional - MTM entries)

$13 \$$ Array - Nuclide ID Numbers on Library File (MTP entries required only if IDATl is not equal to 2)

$T$ (terminate block with "T") [T always required]

INPUT DATA BLOCK 3

14* Array - Cross Sections for Nuclide 1, ANISN format, all groups

[each new nuclide must start on a new card]

- Cross Sections for Nuclide MCR, ANISN format, all groups (IHM*IGM*MCR entries required)

$T$ (terminate block with " $T$ ") [ $T$ required if $M C R>0$ ] 


\subsection{GIP INPUT DATA NOTES}

Except for the title card, all data are read using the FIDO input system. A detailed description of the FIDO input system is given in Appendix $A$. Data arrays are entered in blocks, each terminated by a "T". Unused data arrays (e.g., los if $M S=0$ ) are not entered, but a "T" must still be entered to signal the termination of each block. Multiple problems can be stacked with a blank card separating cases. Input data are not saved between stacked cases.

1 Array The meaning of the first eight input parameters of the 1 \$ array are compatible with GRTUNCL (Section 3.0) and DORT (Section 4.0).

IHT, IHS, IHM The discussion of these input parameters is given in Section 2.4.1.

MCR, MTP, MTM GIP assigns storage space (IHM*IGM words/set) for MTM sets of cross sections, called "materials." The first MCR spaces are filled with data read from cards in the $14^{*}$ array. The next MTP material spaces are filled with nuclide data read from the data set mounted on the input library logical unit (default $=8$ ) and stored in the order indicated by the $13 \$$ array. The remaining spaces between MCR+MTP and MTM are available for preparing mixtures.

NOTE: If $M C R=0$, the $14^{*}$ array and corresponding data block terminator is not required.

ITH If ITH=1, two major reordering steps are carried out to produce an adjoint cross-section library:

1. The inscatter matrix is transposed, i.e., the table position associated with group $g$ describing scattering from $\mathrm{g}^{\prime}$ to $\mathrm{g}$ is changed to describe scattering from $g$ to $\mathrm{g}^{\prime}$.

2. The ordering of the groups is reversed, i.e., data for group IGM appear first in the output file, followed by IGM-1, etc.

A simple three group example is illustrated in Section 2.3.3.

ISCT When the order of scatter is greater than zero, GIP expects the $P_{1}$ cross section to be material. M+1, the $P_{2}$ cross section to be $M+2$, etc., where $M$ is the $P_{0}$ material number:

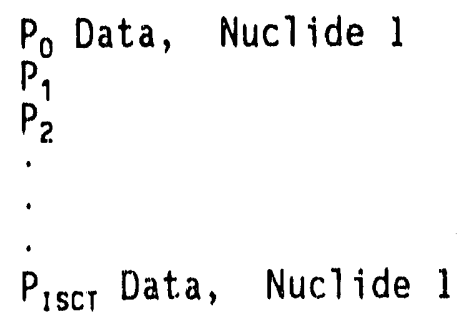




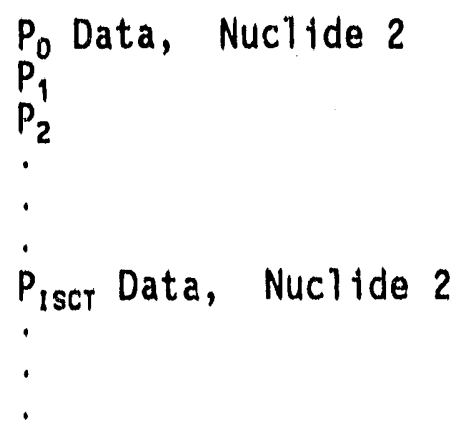

For components other than $P_{0}$, cross section positions other than positions IHT+1 through IHM may be zero.

IPRT, IOUT If IOUT=0, the output includes MCR+MTP microscopic cross sections, plus (MTM-MCR-MTP) mixtures. If IOUT $=2$, only the mixtures will be output. Printed cross-section edits are similarly controlled by IPRT.

For most DORT problems, I/0 manipulation can be reduced by asking only for the output of the mixtures from GIP. They are then referred to in DORT such that material one is the first GIP mixture, etc. Any microscopic data to be used in DORT activity edits must be included as a mixture, as must nuclides whose concentrations are to be modified in a search.

IDOT This parameter identifies three possible output cross section library formats. Only the ANISN and DOT IV formats are discussed since they are used in present day applications. The DOT III format was retained from earlier versions of GIP, and althougn still operational, should be avoided, unless DOT III will be used for the final calculation.

NBUF This parameter is only applicable for IBM machines and must allow enough buffer space for the data sets which are listed in Section 2.6.

10\$, 11\$, and 12* Arrays The cross section mixing table is used to combine elements into macroscopic mixtures. Experience will reveal that only the imagination limits its flexibility. As with DORT, the integers in the mixing table refer to a continuous array of MTM cross section sets of which the first MCR+MTP are nuclide data read from card-image input and a library file, respectively. All of the sets beyond MCR+MTP are preset to zero before the mixing table is executed. In non-search problems where cross-section storage is difficult, i.e., large problems that do not involve material buckling or concentration searches, material spaces originally used for input nuclides can be reused for mixtures. While the general rules for creating mixtures are equally applicable for GIP, GRTUNCL, and DORT, it should be noted that reference to eigenvalue modification in the DORT mixing table description has no application to GIP mixing. The interpretation rules applicable to GIP are as follows: 
a. If the mixture number is $N$ and the component number is 0 , then the cross sections in mixture $N$ will be multiplied by the number entered in the density column:

$$
\sigma_{\text {MIXTURE N(NEW) }}=\sigma_{\text {MIXTURE N(OLD) }} \text { * DENSITY }
$$

b. If the mixture number is $N$ and the component number is $M$, then the cross sections in component $M$, multiplied by the density, wil1 be added to the cross sections in mixture $N$ :

$$
\sigma_{\text {MiXTURE N(NEW) }}=\sigma_{\text {MIXTURE N(OLD) }}+\sigma_{\text {COMPONENT } M} \text { * DENSITY }
$$

Note: Either a mixture or a component may be made up of microscopic or macroscoptc cross sections for a single isotope or mixture of isotopes.

A sample mixing table showing the various options is given below.

\begin{tabular}{ccc}
$\begin{array}{c}\text { MIXTURE } \\
(10 \$ \text { ARRAY) }\end{array}$ & $\begin{array}{c}\text { COMPONENT } \\
\text { (11\$ ARRAY) }\end{array}$ & $\begin{array}{c}\text { DENSITY } \\
\text { (12* ARRAY) }\end{array}$ \\
\hline 6 & 1 & 0.4 \\
6 & 3 & 0.5 \\
7 & 2 & 0.4 \\
7 & 4 & 0.5 \\
7 & 0 & 3.0
\end{tabular}

The mixture table given above would do the following:

(1) Add components 1 and 3 with densities 0.4 and 0.5 , respectively, to form mixture 6 :

$\sigma_{6}=\sigma_{1} \star 0.4+\sigma_{3} * 0.5$

(2) Add components 2 and 4 with densities 0.4 and 0.5 , respectively, to form mixture 7 :

$\sigma_{7}=\sigma_{2} * 0.4+\sigma_{4} * 0.5$

(3) Multiply mixture 7 by 3.0: $\sigma_{7}=\sigma_{7} \star 3.0$

$10 \$$ Array If the 10 s entry is tagged with a negative, the next ISCT components will be treated as $P_{L}$ components. For example:

$\frac{10 \$ 11 \$ 12 *}{-13 \quad 1.0} \quad$ (with ISCT $\left.=3\right)$

will be equivalent to 


\begin{tabular}{ccc}
$10 \$$ & $11 \$$ & $12 *$ \\
\hline 13 & 1 & 1.0 \\
14 & 2 & 1.0 \\
15 & 3 & 1.0 \\
16 & 4 & 1.0
\end{tabular}

$7 \$$ Array The numbers in the mixing table normally refer to positions in the material block, which contains MCR nuclides from cards, followed by MTP nuclides from tape and MTM-MCR-MTP mixtures. It is often conventent, however, when constructing the mixing table to refer to fictitious "mix code" numbers. The $7 \$$ array can be entered to define the itst of fictitious $m+x$ codes corresponding to each materiat. If the $7 \$$ array is omitted, the mix code is equal to the material number.

13\$ Array A negative entry in the $13 \$$ array replaces the following ISCT entries with successive ID's. Thus:

$13 \$$

$-101$

$-101$

$-101$
$13 \$$

101

102

103

$14^{*}$ Array The input of the $14^{*}$ array is performed by subroutines whicil have certain restrictions in addition to those of normal FIDO input:

a. The nuclide spaces are preset to 0.0 , so that "E" or "FO" will have the effect of filling the remaining space with 0.0 , but "E" and "F" must not be used in the input foi" the last nuclide.

b. The $14^{*}$ or $14^{* *}$ designation may precede each nuclide block, but is not required except in the first block.

c. Entries, including operators such as "T", following the last data item for nuclides other than the last, and on the same card as the last item, will be ignored.

d. The "T" which terminates this block must appear alorie in column 3 of a separate card.

\subsubsection{Special Diffusion Theory Option}

If the cross-section set contains a transport cross section in position $\mathrm{N}$, then setting ISCT $=-\mathrm{N}$ causes the total cross section (position IHT) to be replaced by the transport cross section and the self-scatter cross section (position IHS) to be reduced such as to maintain a constant 
absorption cross section. The resulting cross-section set is suicable for use in a $P_{0}$ DORT calculation, especially one using the diffusion package.

NOTE: Negative entries in the 10 s array must not be used with this option.

\section{3 .2 Upscatter Option}

If the self-scatter cross section position (IHS) is greater than IHT+1, the upscatter processor calculates the total upscattering cross section from each group and places it in the appropriate table position. No user action is required.

\subsubsection{Adjoint Reordering Example}

A three-group example showing the relationship between a forward and adjoint cross-section set is shown below. In this example, there is no upscatter, one activation cross section, IHT $=4$, IHS $=5$, and IHM $=7$.

\begin{tabular}{|c|c|}
\hline Direct & Adjoint \\
\hline $\begin{array}{r}\sigma^{F}(1) \\
\sigma^{A}(1) \\
\nu \sigma^{F}(1) \\
\sigma^{\top}(1)\end{array}$ & $\begin{array}{c}\sigma^{F}(3) \\
\sigma^{\wedge}(3) \\
\nu \sigma^{F}(3) \\
\sigma^{\top}(3)\end{array}$ \\
\hline $\begin{array}{l}\sigma(1+1) \\
0 \\
0\end{array}$ & $\begin{array}{l}\sigma(3-3) \\
0 \\
0\end{array}$ \\
\hline $\begin{array}{r}\sigma^{F}(2) \\
\sigma^{A}(2) \\
\nu \sigma^{F}(2) \\
\sigma^{\top}(2)\end{array}$ & $\begin{array}{c}\sigma^{F}(2) \\
\sigma^{A}(2) \\
\nu \sigma^{*}(2) \\
\sigma^{\top}(2)\end{array}$ \\
\hline $\begin{array}{l}\sigma(2-2) \\
\sigma(1+2) \\
0\end{array}$ & $\begin{array}{l}\sigma(2+2) \\
\sigma(2+3) \\
0\end{array}$ \\
\hline $\begin{array}{c}\sigma^{F}(3) \\
\sigma^{A}(3) \\
\nu \sigma^{F}(3) \\
\sigma^{\top}(3)\end{array}$ & $\begin{array}{r}\sigma^{F}(1) \\
\sigma^{A}(1) \\
\nu \sigma^{F}(1) \\
\sigma^{\top}(1)\end{array}$ \\
\hline $\begin{array}{l}\sigma(3-3) \\
\sigma(2+3) \\
\sigma(1-3)\end{array}$ & $\begin{array}{l}\sigma(1+1) \\
\sigma(1-2) \\
\sigma(1+3)\end{array}$ \\
\hline
\end{tabular}




\subsection{INPUT LIBRARY FORMAT}

The input library format from which MTP nuclides are taken is defined in the following manner:

\section{ALC Cross Section Library Format}

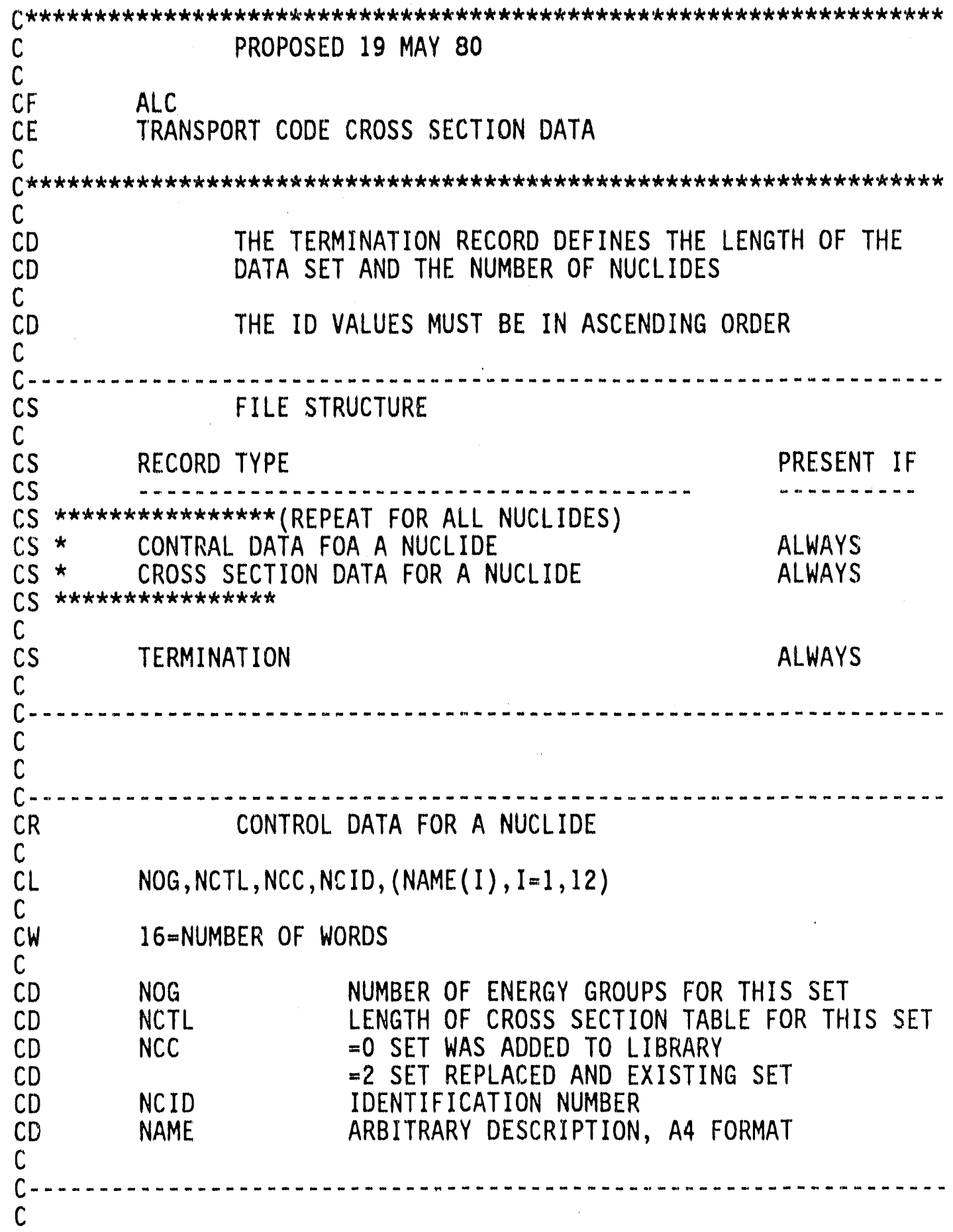




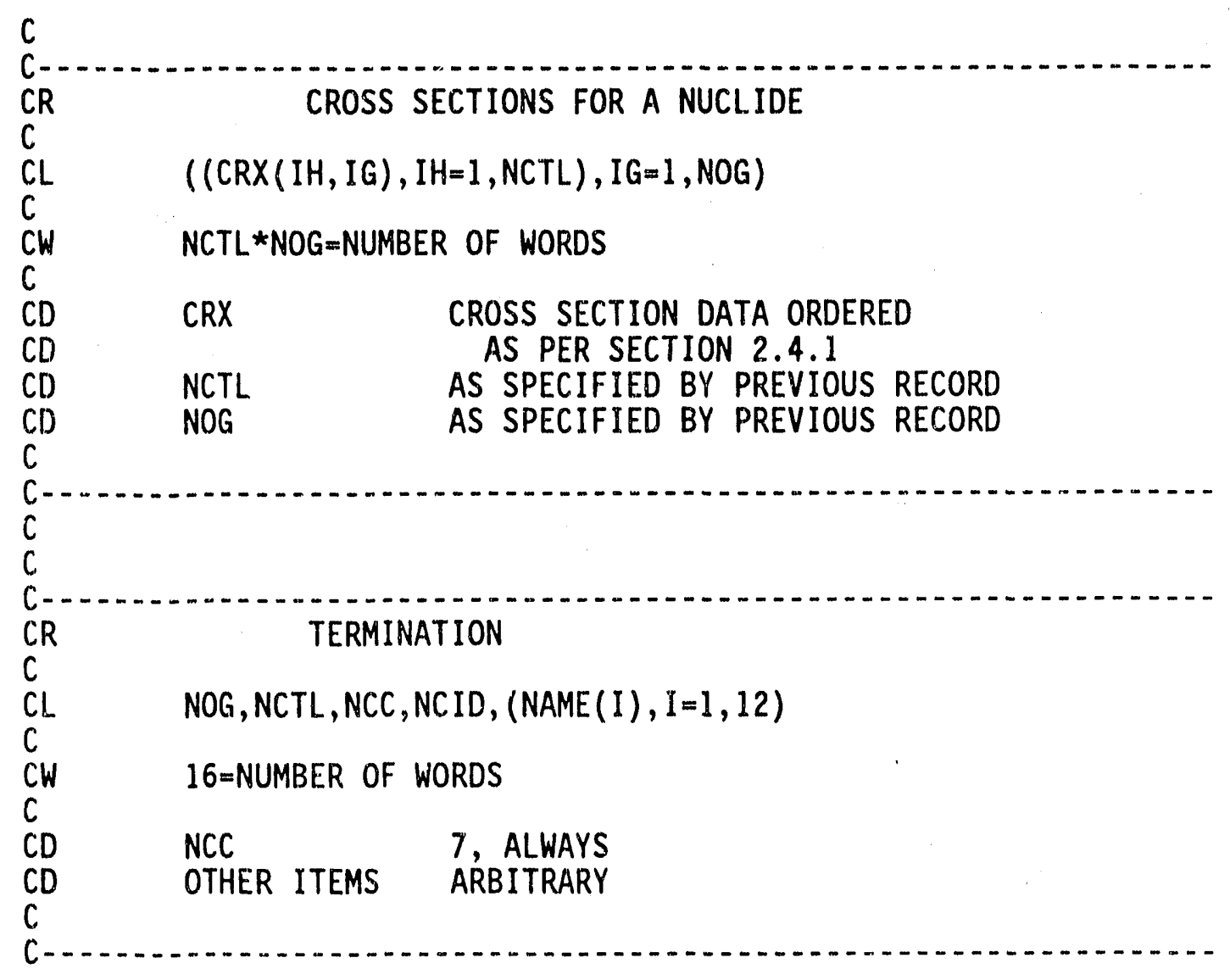

\subsubsection{ANISN Nuclide Format Cross-section File}

In a normal GIP problem, cross sections are read from card input and/or from an external data set in nuclide-organized format, i.e., all the cross sections for one group of one nuclide, followed by other groups for that nuclide, and finally followed by data for other nuclides. Within a group, GIP expects the ordering of data in the cross section table in the following format:

\begin{tabular}{ccc} 
Table Position & Entry & \multicolumn{1}{c}{ Cross Section Type } \\
\hline 1 & $\sigma^{\mathcal{G}}(\mathrm{g})$ & $\begin{array}{l}\text { First activation cross section } \\
\text { (if any) }\end{array}$ \\
$\vdots$ & & \\
IHT-3 & $\sigma^{\mathrm{L}}(\mathrm{g})$ & $\begin{array}{l}\text { Last activation cross section } \\
\text { (if any) }\end{array}$ \\
IHT-2 & $\sigma^{\mathrm{A}}(\mathrm{g})$ & Absorption
\end{tabular}




\begin{tabular}{|c|c|c|}
\hline IHT-1 & $\nu \sigma^{F}(g)$ & Neutron Production \\
\hline IHT & $\sigma^{\top}(g)$ & Total removal \\
\hline IHT+1 & $\sigma^{\text {TUS }}(g)$ & $\begin{array}{l}\text { Total upscatter cross section from } \\
\text { group } g \text { (omit this entry if } N U S=0 \text { ). }\end{array}$ \\
\hline IHS-NUS & $\sigma(g+N U S \rightarrow g)$ & Scattering from group $\mathrm{g}+\mathrm{NUS}$ to $\mathrm{g}$ \\
\hline$\dot{b}$ & - & \\
\hline IHS-1 & $\sigma(g+1 \rightarrow g)$ & Scattering from group $\mathrm{g}+1$ to $\mathrm{g}$ \\
\hline IHS & $\sigma(g+g)$ & Scattering from group $\mathrm{g}$ to $\mathrm{g}$ \\
\hline IHS+1 & $\sigma(g-1 \rightarrow g)$ & Scattering from group $\mathrm{g}-1$ to $\mathrm{g}$ \\
\hline . & . & \\
\hline IHM & $\sigma(g-N D S \rightarrow g)$ & Scattering from group $\mathrm{g}$-NDS to $\mathrm{g}$ \\
\hline
\end{tabular}

From this, it can be seen that:

$$
\begin{aligned}
\text { NUS } & =\text { IHS-IHT-2 = number of upscatter groups, if greater than } 0, \\
\text { NDS } & =\text { IHM-IHS }=\text { number of downscatter groups, } \\
L & =I H T-3=\text { number of activation cross sections. }
\end{aligned}
$$

Special illustrations of interest are:

$$
\begin{aligned}
& I H M=I G M+3 \\
& I H S=4 \\
& I H T=3
\end{aligned}
$$

Full downscatter; no upscatter; no activation cross section

and, 


$$
\begin{aligned}
& I H M=I G M+5+\text { NUS } \\
& I H S=6+\text { NUS } \\
& I H T=4
\end{aligned} \quad \begin{aligned}
& \text { Full downscatter; NUS upscatter; } \\
& \text { room for an activation cross } \\
& \text { section in position } 1 .
\end{aligned}
$$

Thus the parameters IHT, IHS, and IHM completely describe the format of the cross sections. If there are no activity cross sections, IHT=3. If there is no upscatter IHS $=$ IHT +1 . If there is no downscatter IHM $=$ IHS (i.e., a one group problem). If there is upscatter, GIP will compute a total upscatter cross section for each group of each material and place that cross section in position IHT +1 . The activity cross sections are only used for activity calculations and not used in the transport process itself.

\subsection{GIP OUTPUT FILE}

The GIP output file is group-ordered for transport codes and this format is compatible with both ANISN and DORT requirements. The groups are normally ordered from high to low neutron energy, then from high to low photon energy, if any photon groups are present.

\section{GIP Cross Section Library Format}

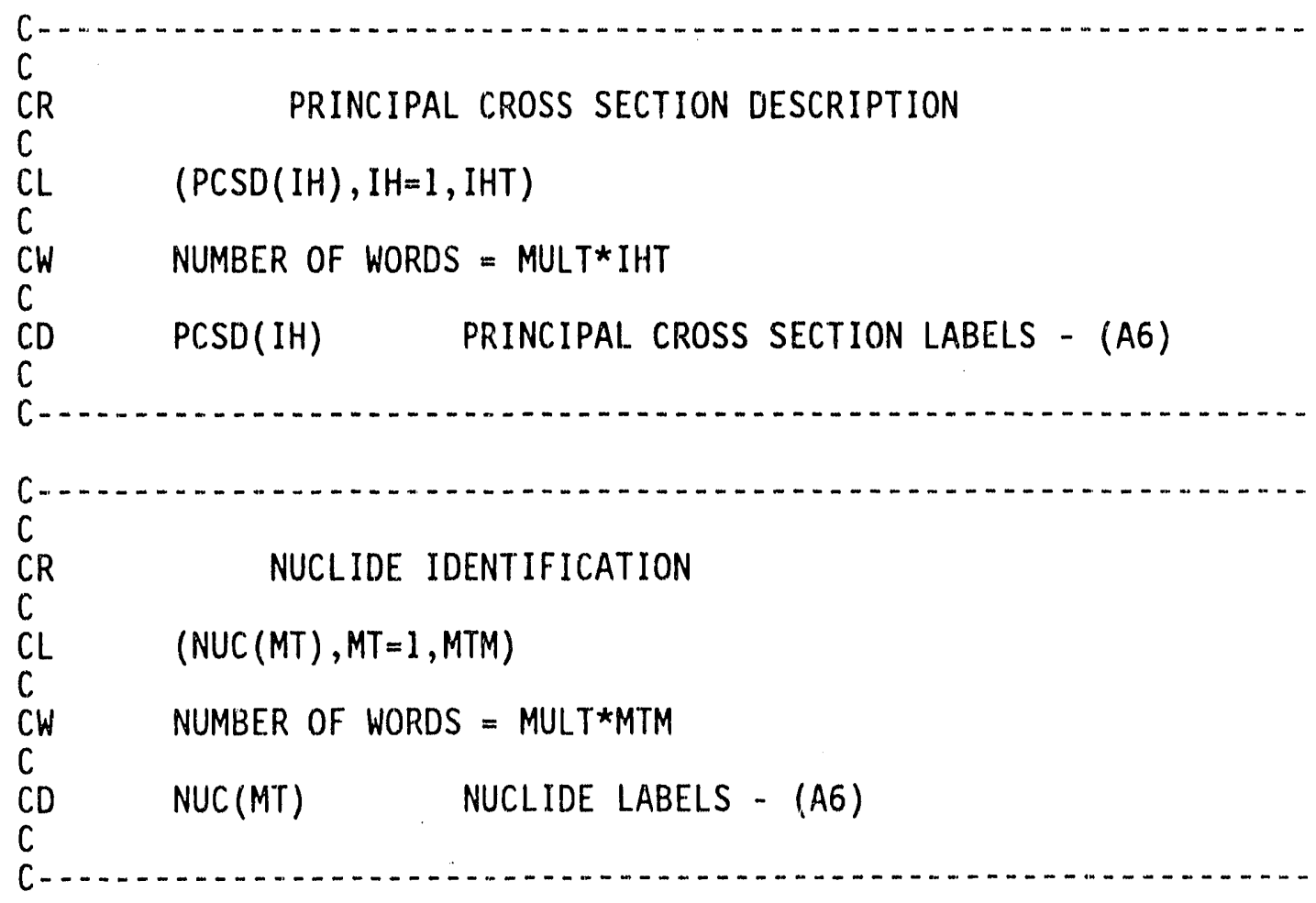




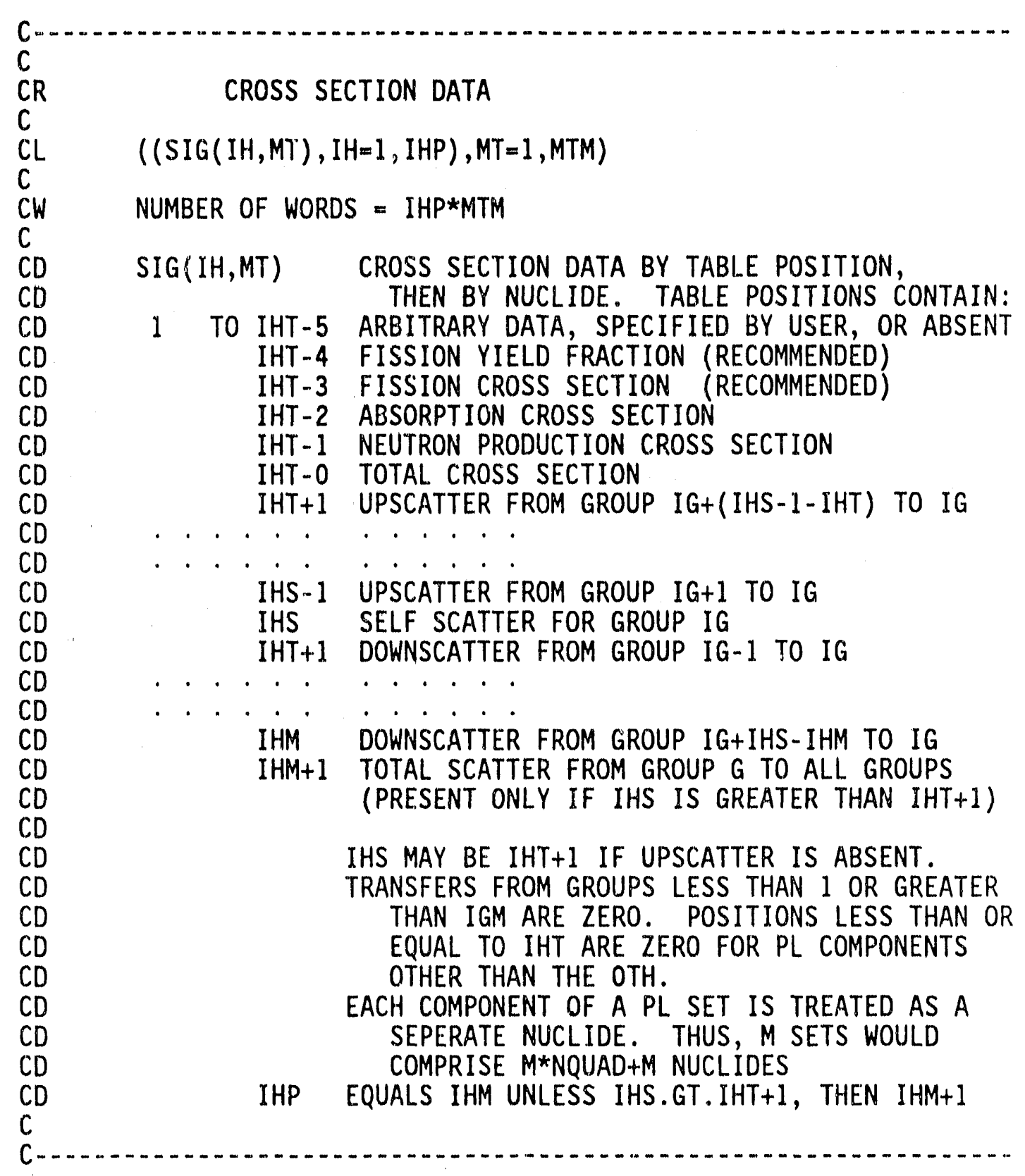

\subsection{LOGICAL UNIT REQUIREMENTS}

Below is a listing of the files required to execute a GIP case along with the default values used in the code. In setting up a GIP case, efforts must be made for these units to be available.
1. Logical Unit 1 - SCRATCH
2. Logical Unit 2 - SCRATCH
3. Logical Unit 3 - SCRATCH 
4. Logical Unit 8 - GIP formatted Cross-section Library Output

5. Logical Unit 9 - ANISN formatted Cross-section Library Input

6. Logical Unit 4 - SCRATCH

7. Logical Unit 6 - Printed Output

8. Logical Unit 5 - Card Input

Logical unit assignments for the first six data files can be modified by altering locations 15-20 of the 1 s array (not shown. in Section 2.2) to the new logical unit numbers. The new values will not be 1 isted in the input edit, however.

\subsection{PROGRAMMER INFORMATION}

\subsubsection{Inter-machine Adaptability}

The GIP code is intended to be easily adaptable to any type of sophisticated computer, and yet to take advantage of certain localized structural features which may be machine-dependent.

In general, the guidelines of ANS-STD.3-19715 are followed. This requires general adherence to a simple, standard FORTRAN language except where deviations provide important improvements in capability and can be documented. In addition, machine-dependent features have been kept localized and have not been included in the subroutines which perform actual computation.

The recommended procedures of the Committee on Computer Code Coordination (CCCC $)^{4,6}$ have been followed where practical: Compliance with these standards has been incomplete where cost was prohibitive, the nature of the codes did not allow it, or the expected level of performance could not be obtained in that fashion.

Where minor or certain machine-dependent features are required, alternative features are enclosed in pairs of 3-character "language flags." The alternative statements remain in the source program, with inappropriate sections transformed into comment statements. If the code is set up for IBM operation, for example, it might contain:

$\begin{array}{ll}\text { CIB } & \text { ENTRY IBCDC }(H, E, L, P) \\ C I B & \\ C D C & \\ C & \text { ENTRY IBCDC } \\ C D C & \end{array}$


The corresponding $C D C$ configuration would be:

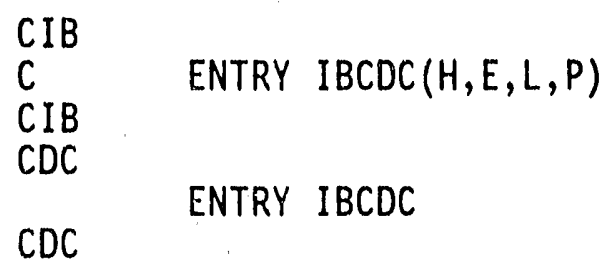

All versions of a given procedure are thus available for inspection by all users. The selection of options is made by a computer program at distribution time. A listing of machine-dependent sections can also be prepared in that process.

The language flags achieve the major objective of having a single, unified source for each program which is maintained for all users. With these provisions, the basic FORTRAN programs are operable on IBM, CDC, UNIVAC, CRAY, Amdah1, and other computers, except that system-dependent service routines as specified by CCCC must be provided.

Optional packages and procedures which provide enhanced convenience are also available. The optional run-time storage allocation requires system-dependent routines. Instructions for installing these options are distributed with the source programs. All known users at this time use all of the optional enhancements available to them. Even so, the basic operation with FORTRAN routines plus the CCCC package remains available if needed.

\subsubsection{Service Subroutines}

Certain standard service subroutines are specified by the CCCC for use in reactor physics codes. The service routines used in these codes include:

TIMER Provides timing and job identification data

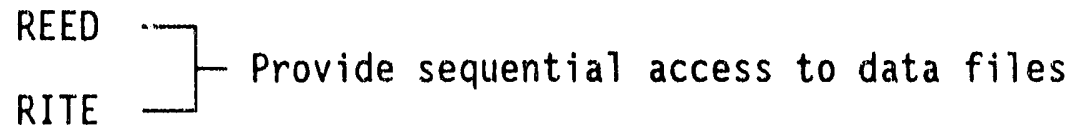

DRED 7 Provide random access to data files

DOPC Provides initiating, closing, and certain repositioning of data files 
CRIT $7 \begin{aligned} & \text { Provide block transfer of data between fast and slow } \\ & \text { memory. }\end{aligned}$

Many of these subroutines also call other subroutines. Many are entirely system dependent. Each configuration to be distributed contains an appropriate set of service subroutines, insofar as possible. The realities of computing environments may require local modification or substitution. The specifications given in Reference 4 , together with extensive in-stream comments, provide guides for such modification.

\subsection{REFERENCES}

1. W. A. Rhoades, "The ALC1 Program for Cross-Section Library Management," ORNL-TM-4015, Oak Ridge Nationa1 Laboratory, (December 1972).

2. W. A. Rhoades and R. L. Childs, "The DORT Two-Dimensional Discrete Ordinates Transport Code," Nuclear Science \& Engineering 99, 1, pp. 88-89, (May 1988).

3. W. W. Engle, Jr., "A USER'S MANUAL FOR ANISN, A One-Dimensional Discrete-Ordinates Transport Code with Anisotropic Scattering," K-1693, Oak Ridge National Laboratory, (March 1967).

4. R. Douglas 0'Dell, "Standard Interface Files and Procedures for Reactor Physics Codes, Version IV," LA-6941-MS, Los Alamos National Laboratory, (September 1977).

5. ANS Standard "Recommended Programming Practices to Facilitate the Interchange of Digîal Computer Programs," prepared by Subcommittee 10, ANS Standards Committee (Apri1 1971).

6. B. M. Carmichael, "Standard Interface Files and Procedures for Reactor Physics Codes, Version III," LA-5486-MS, Los Alamos National Laboratory, (February 1974).

7. J. 0. Johnson, J. D. Drischler, and J. M. Barnes, "Analys is of the Fal1-1989 Two-Meter Box Test Bed Experiments Performed at the Army Pulse Radiation Facility (APRF)," ORNL/TM-11777, Oak Ridge National Laboratory, (May 1991).

8. R. T. Santoro et al., "DNA Radiation Environments Program Fall 1989 2-Meter Box Experiments and Analysis," ORNL/TM-11840, Oak Ridge National Laboratory, (May 1991). 


\subsection{SAMPLE PROBLEM}

A complete listing of the input cards for the sample problem is given in Figure 2-1, and some selected output is shown in Figure 2-2. The sample problem demonstrates the processing of the materials for the air-overground analysis of the two-meter box experiments.7,8

In viewing Figure 2-1, the input illustrates; 69 energy groups (IGM), a cross-section table length (IHM) of 72, no upscatter (IHT=3, and IHS=4), and a mixing table length (MS) of 558. No material data was read in from cards $(M C R=0)$ and 144 materials were read in from tape (MTP=144). Since ISCT $=5$, there were actually 24 different nuclides read in from tape, each with a $P_{0}$ through $P_{5}$ component. The total number of mixtures (MTM) was 198, and only mixtures were output (IOUT=2) to the GIP library. No print was requested for the printed output (IPRT=1), and a DOT IV formatted cross-section file was output (IDOT=2). All comments after the slash (/) are ignored in FIDO input and are only useful for quick identification of the input parameters. The GIP sample input in Figure 2-1 shows multiple mixtures of the same material, j.e., ground and air, with different number densities. This allows mixing the cross sections for several different air-over-ground calculations in only one GIP case.

The selected GIP output shown in Figure 2-2 first illustrates the input parameters read in the first data block, followed by the memory requirements to run this particular GIP case, the input library record length, and an indication that the second data block has been successfully read. The output then produces the mixing table $(10 \$, 11 \$$, and $12 *$ arrays) along with the mixing codes ( $7 \$$ array) and nuclide identification numbers (13\$ array). This output is useful for checking the input to make sure there are no errors in nuclides selected for mixtures, number densities used in mixtures, and nuclides selected from the input library. The mixing table is followed by a listing of the nuclides extracted from the input cross-section library file. This table is also useful for verifying the elements pulled off of the input cross-section library file. In this table, the user can also verify the $P_{L}$ components used in the mixing. The output then shows some messages informing the user of the cross-section sorting process discussed in Section 2.1.2. Finally, the output lists the titles for the grouporganized cross sections written to the output unit. If the print flag had been turned on (IPRT $=0$ or 2), the cross sections would have been printed after the titles. It should be noted that setting the print option to print either all elements and mixtures (IPRT=0) or only mixtures (IPRT=2) will produce a considerable amount of output for large group structures and high orders of scattering. 
gip mixtures for the $10 / 89$ two meter box experiments - p5 forward

$1 \$ \$ 693472558$ /igm, iht, ihs, ihm, ms

014419805 /mcr,mtp, mtm, ith, isct

e $t$

122120 /iprt, iout, idot, nbuf

$10 \$ \$ 4 i 145 \quad 150 \quad 19 q 6 \quad 4 i 151 \quad 156 \quad 10 q 6 \quad 4 \$ 157 \quad 162 \quad 3 q 6 \quad 4 i 163 \quad 168 \quad 5 q 6$

/ aprf ground borated concrete air 1020 steel

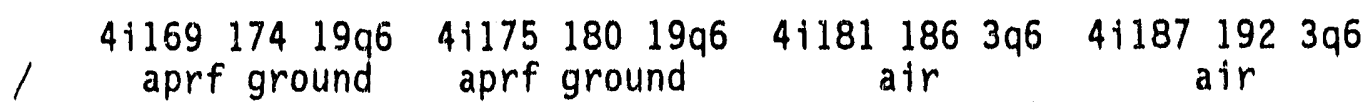

$411931983 q 6$

1 air

$11 \$ 58116010 i 677840 i 851264 i 139144$ / aprf ground

$\begin{array}{llllllll}16 i 1 & 18 & 4 i 31 & 36 & 16 i 43 \quad 60 & 4191 & 96\end{array}$

$4 i 10310810 i 127138$ / borated concrete

$4 i 1610 i 25364 i 7984$ / air

$4 i 192416 i 557210 i 97108$ / 1020 steel

$58 i 16010 i 677840 i 851264 i 139144$ / aprf ground

$58 i 16010 i 677840 i 851264 i 139144$ / aprf ground

$411610 i 25364 i 7984$ / air

$\begin{array}{llllll}4 i 1 & 6 & 10 i 25 & 36 & 4 i 79 & 84\end{array}$, / air

$411610 i 25 \quad 36417984 \quad /$ air

12 ** /number densities (atoms/b-cm)

6r4.236-02 6r9.586-09 6r3.509-08 6r3.775-04

$6 r 4.752-056 r 4.178-026 r 1.427-046 r 9.126-05$

$6 r 1.377-036 r 9.514-036 r 3.610-06 \quad 6 r 3.537-06$

$6 r 2.048-046 r 2.599-056 r 7.199-066 r 3.213-04$

$6 r 2.783-076 r 2.793-076 r 4.858-076 r 7.315-08$ /aprf ground $-34 \%$ h20

6r7.020-03 6r2.887-04 6r1.168-03 6r5.908-02

$6 r 1.659-036 r 4.656-036 r 1.080-02 \quad 6 r 3.486-03$

$6 r 1.431-036 r 7.667-046 r 6.709-04$

/borated concrete

Figure 2-1. Sample GIP Input for the Two-Meter Box Air-Over-Ground Analys is . 
$6 r 6.122-076 r 3.985-056 r 1.100-056 r 2.383-07 / a 1 r-10-24-89(a)$

$6 r 8.078-046 r 4.213-046 r 6.113-056 r 7.381-05$

$6 r 3.877-046 r 8.391-02$

$/ 1020$ steel

$6 r 2.653-026 r 1.162-086 r 4.254-086 r 4.576-04$

$6 r 5.760-056 r 3.823-026 r 1.729-046 r 1.106-04$

$6 r 1.669 .036 r 1.153-026 r 4.376-066 r 4.288 .06$

$6 r 2.482-046 r 3.151-056 r 8.727-06 \quad 6 r 3.895-04$

$6 r 3.373-076 r 3.386-076 r 5.889-076 r 8.867-08$

/aprf ground $-20 \%$ h20

$6 r 5.819-026 r 7.553-096 r 2.765-086 r 2.974-04$

$6 r 3.744-056 r 4.532-02.6 r 1.124-046 r 7.190-05$

$6 r 1.085-036 r 7.496-036 r 2.845-06 \quad 6 r 2.787-06$

$6 r 1.613-046 r 2.048-056 r 5.672-06 \quad 6 r 2.532-04$

$6 r 2.193-076 r 2.201-076 r 3.828-076 r 5.763-08$

/aprf ground $-48 \%$ h2o

6r3.278-07 6r4.021-05 6r1.095-05 6r2.405-07/atr - 11-01-89(h)

6r9.350-07 6r3.858-056r1.082-05 6r2,308-07/air - 10-30-89(a)

6r5.596-07 6r3.948-05 6r1.087-05 6r2.361-07 /air - 10-25-89(a)

$\begin{array}{lllllllllllllllllllllllll}13 \$ \$ 1 & 2 & 3 & 4 & 5 & 6 & 43 & 44 & 45 & 46 & 47 & 48 & 49 & 50 & 51 & 52 & 53 & 54 & 55 & 56 & 57 & 58 & 59 & 60\end{array}$

$\begin{array}{llllllllllllllllllllll}61 & 62 & 63 & 64 & 65 & 66 & 67 & 68 & 69 & 70 & 71 & 72 & 79 & 80 & 81 & 82 & 83 & 84 & 85 & 86 & 87 & 88\end{array}$

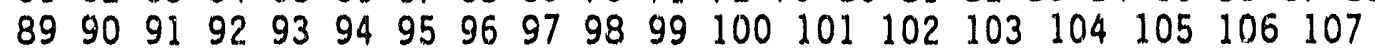

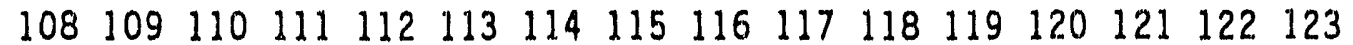

$\begin{array}{llllllllllllllll}124 & 125 & 126 & 127 & 128 & 129 & 130 & 131 & 132 & 133 & 134 & 135 & 136 & 137 & 138 & 157\end{array}$

$\begin{array}{llllllllllllllll}158 & 159 & 160 & 161 & 162 & 163 & 164 & 165 & 166 & 167 & 168 & 169 & 170 & 171 & 172 & 173\end{array}$

$\begin{array}{llllllllllllllll}174 & 175 & 176 & 177 & 178 & 179 & 180 & 181 & 182 & 183 & 184 & 185 & 186 & 199 & 200 & 201\end{array}$

202203204205206207208209210223224225226227228

/ $h, b 10, b 11, c, n, 0, n a, m g, a l, s i, p, s, c l, a r, k, c a, m n, f e, c o, n i, c u, z r, n b, s n$

Figure 2-1.' (continued) 


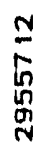

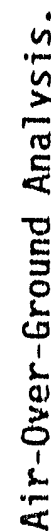

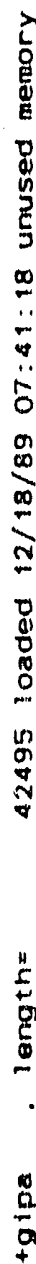

$\stackrel{5}{4}$

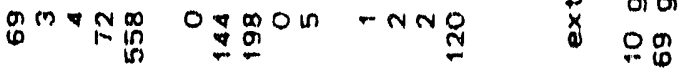

䒽

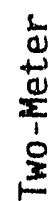

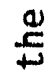

安

ï

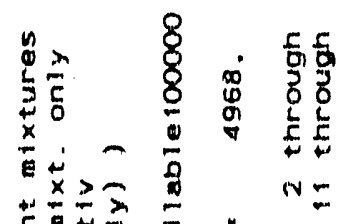

䓂

总

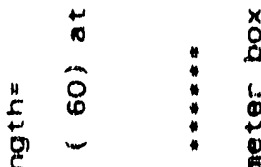

要名

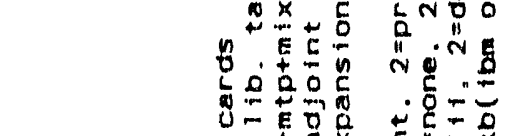

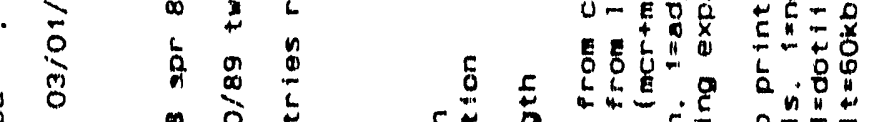

ก

"

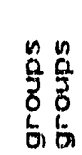

尊

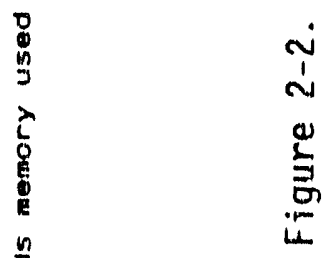

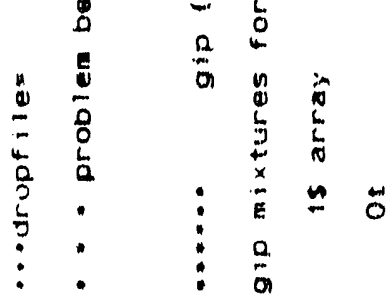

等

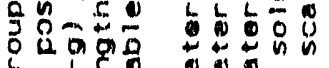

I.

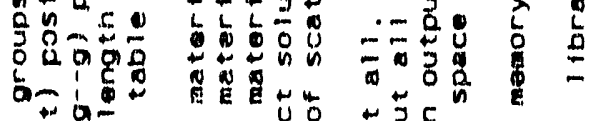

5 क

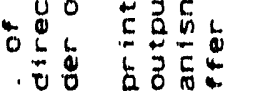

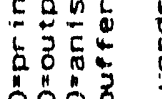

og 


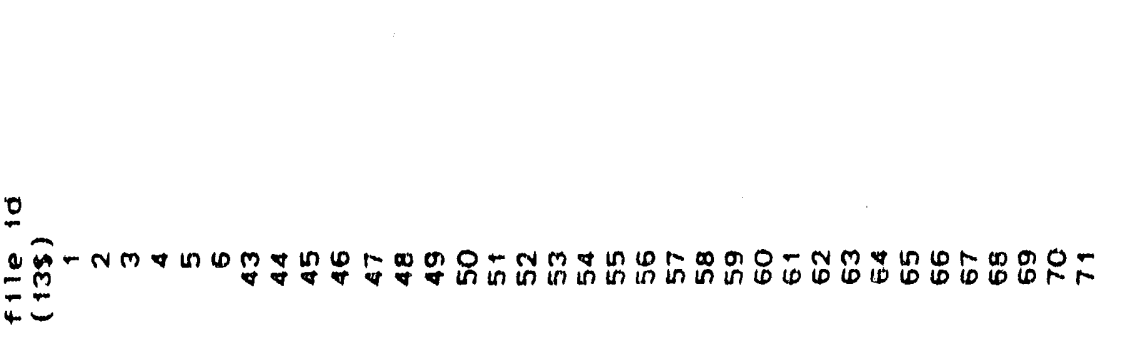

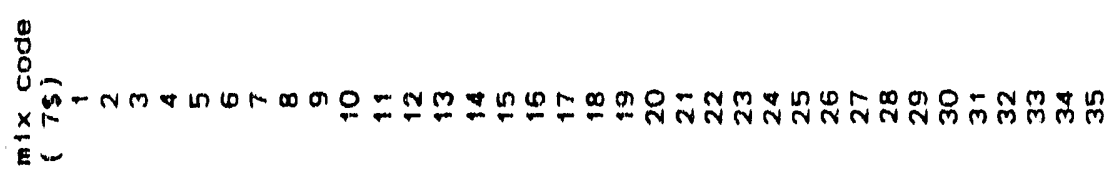

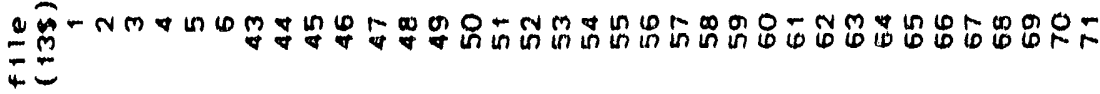

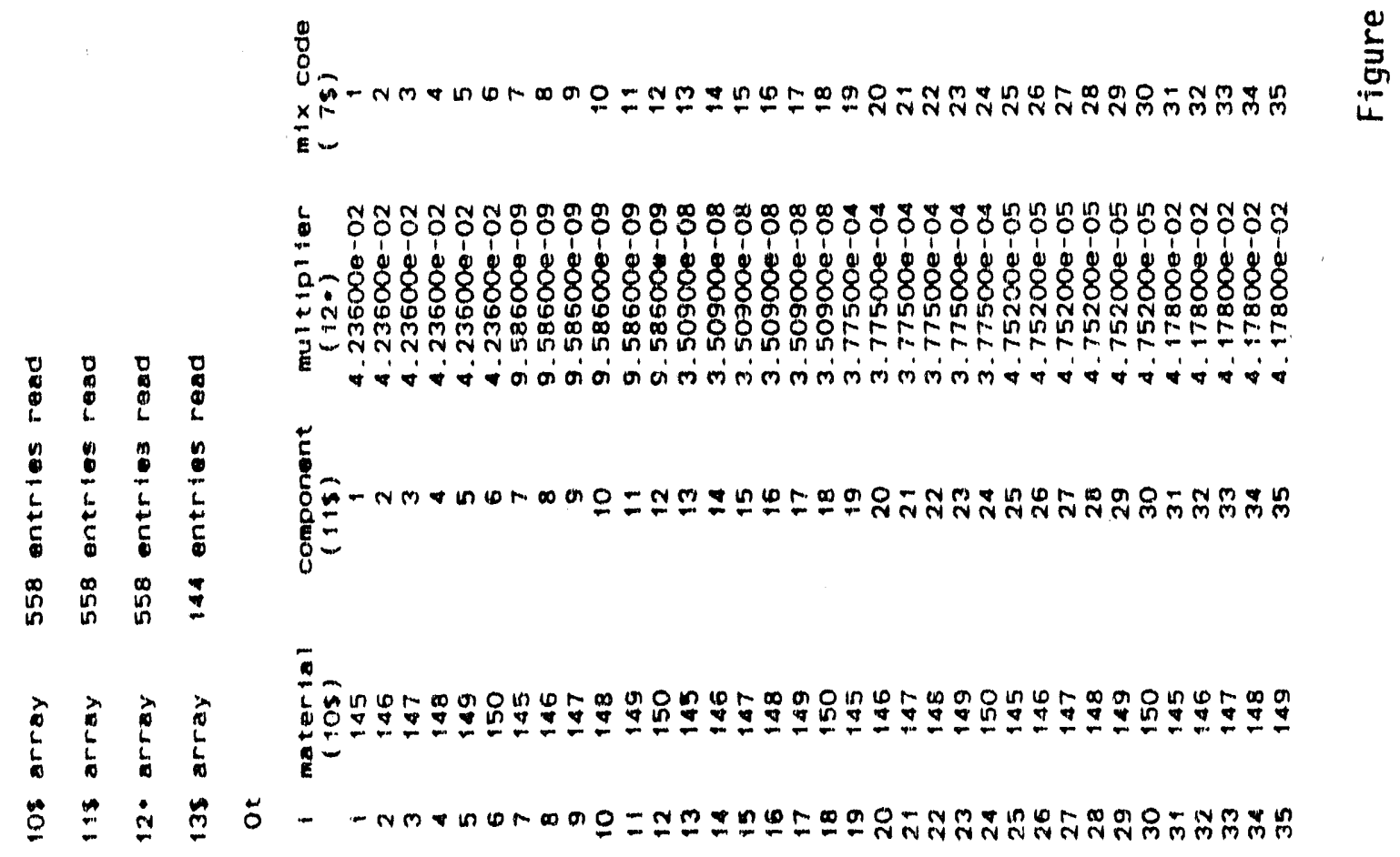




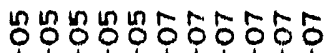


in $\ln$ in $\ln$ in

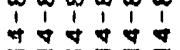

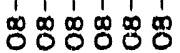

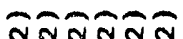

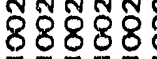

等

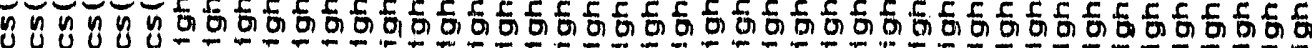

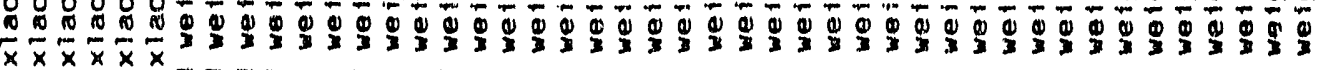

二= =

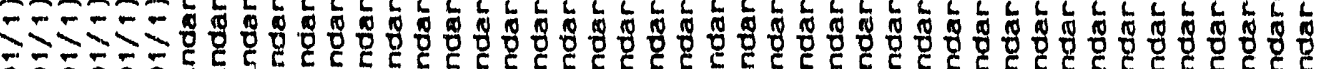

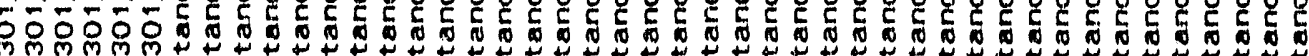

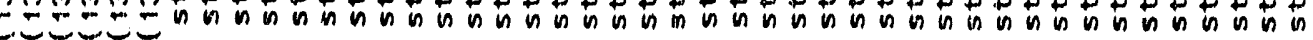

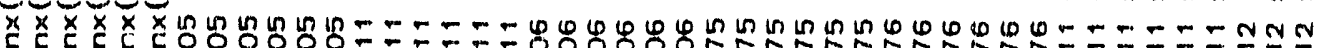

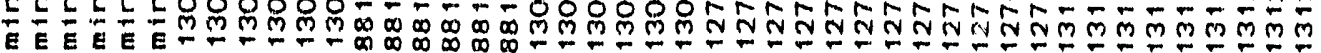

-

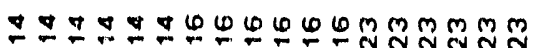

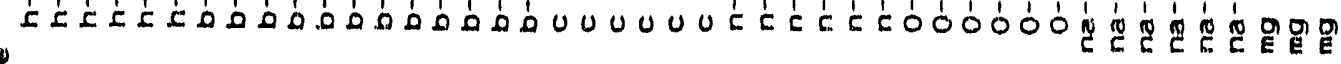

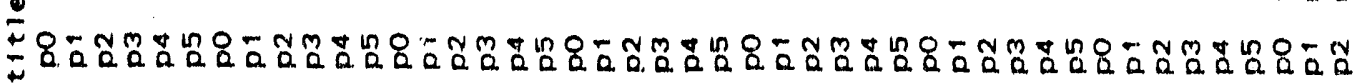

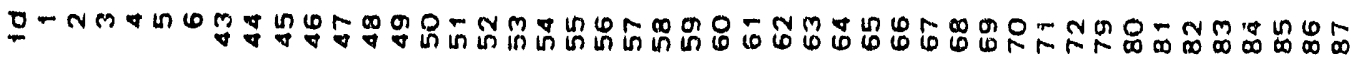

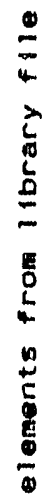

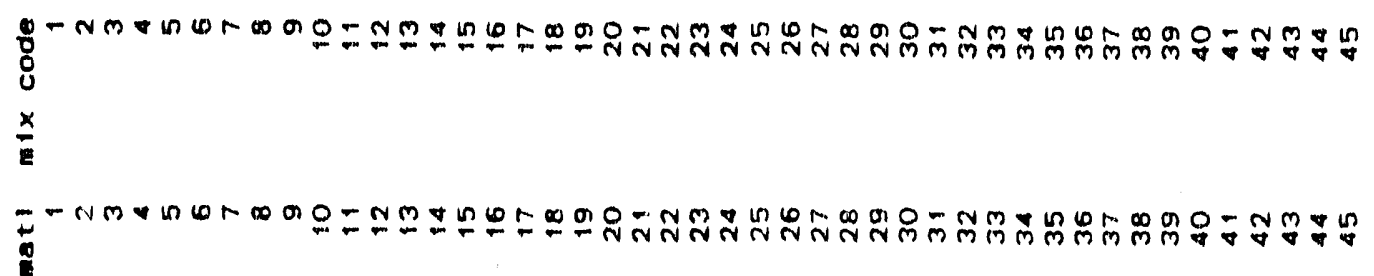




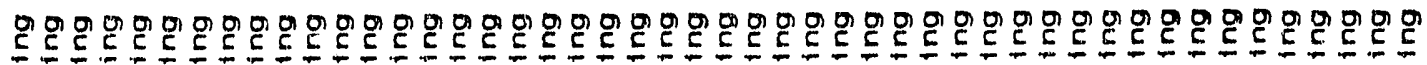
F

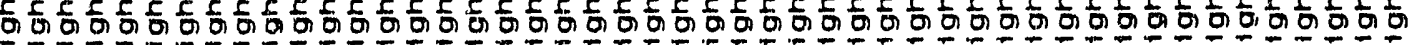

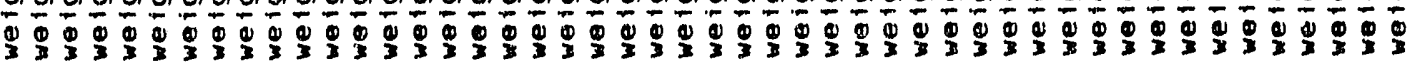

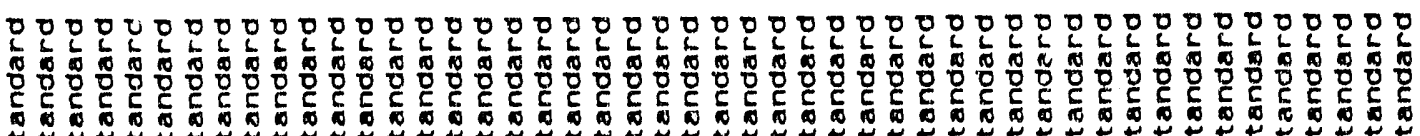

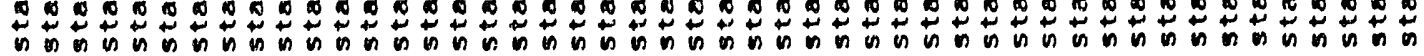
ำำm

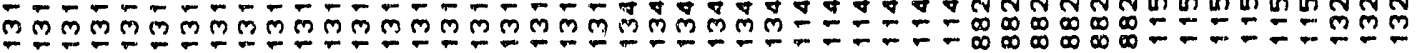

nNANA

ज̆ $\bar{m} \bar{m} \bar{m} \bar{m}$

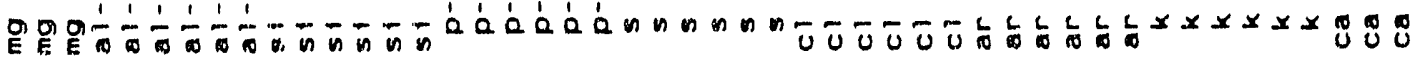

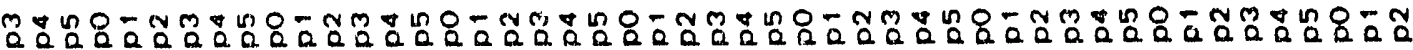

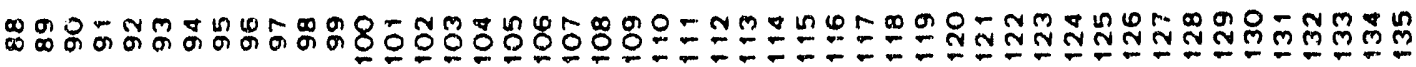

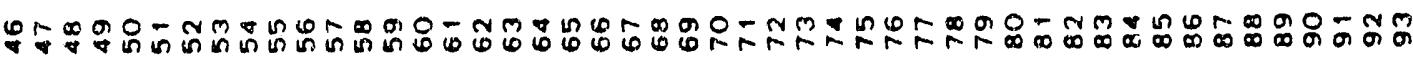

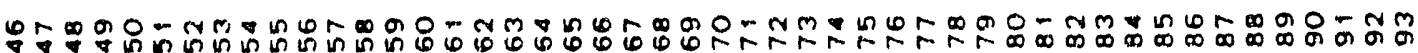




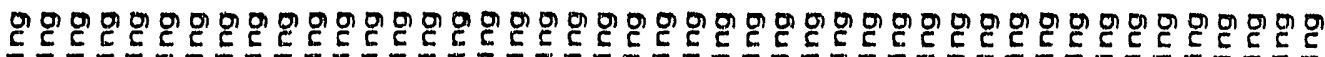

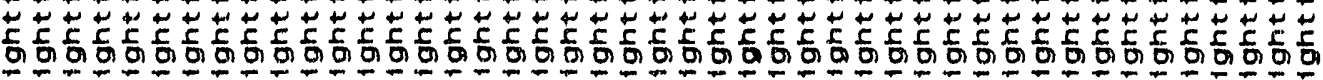

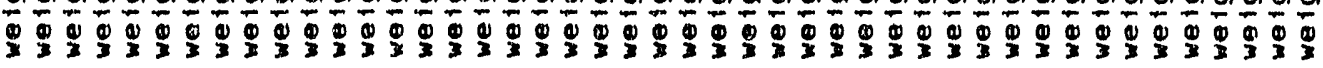

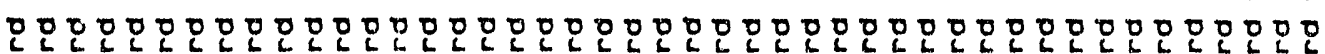

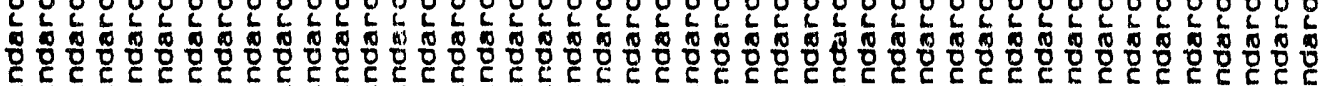

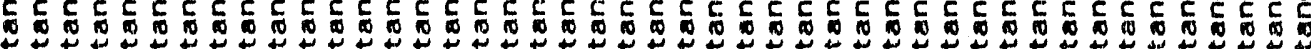

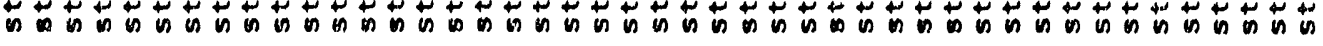

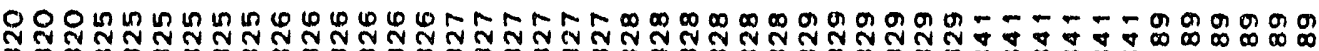

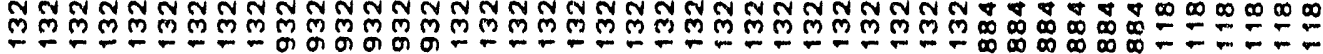

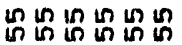

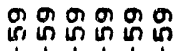

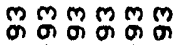

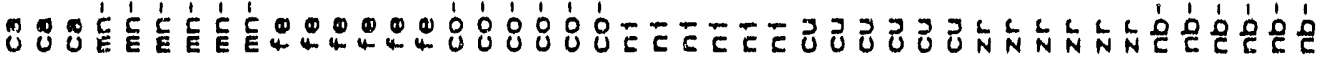

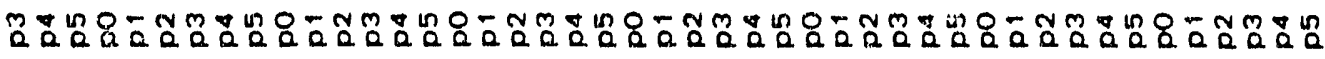

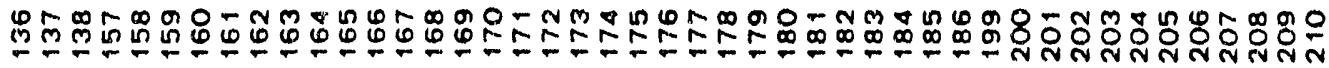

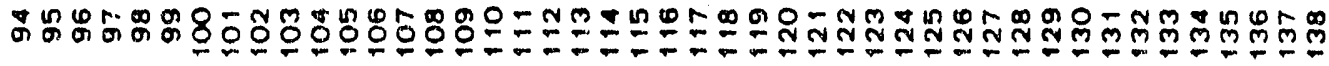

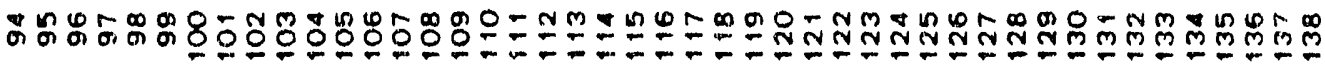




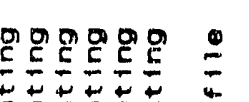

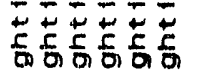

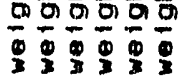

000000 帛

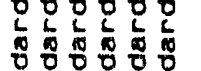

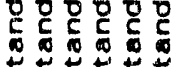

知落落

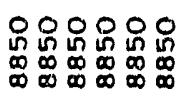

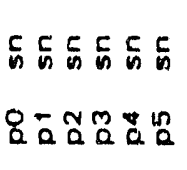

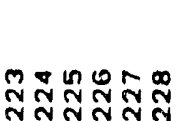
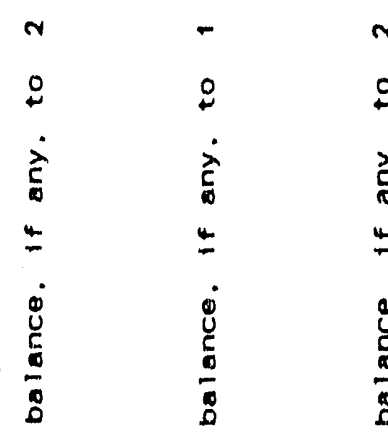

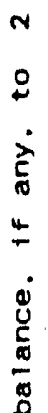

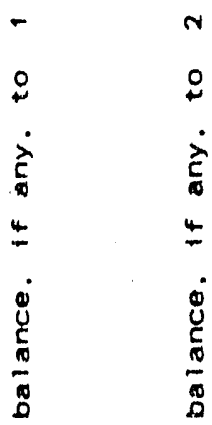

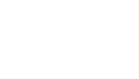

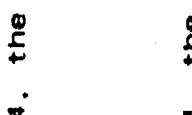

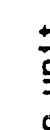

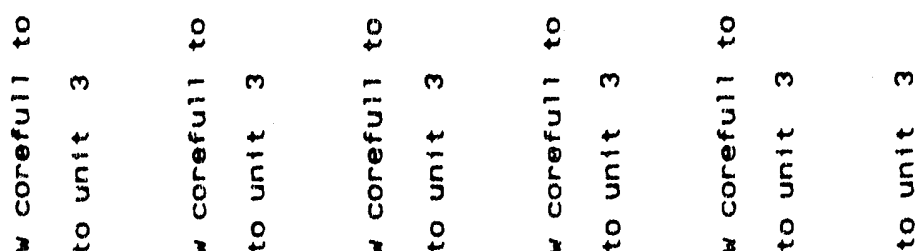

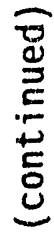

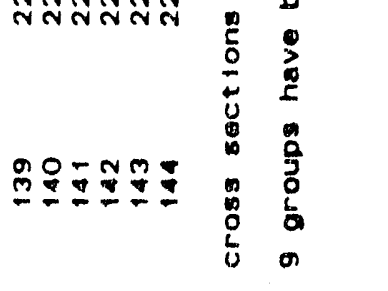

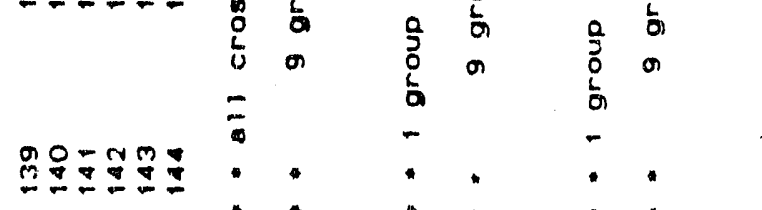




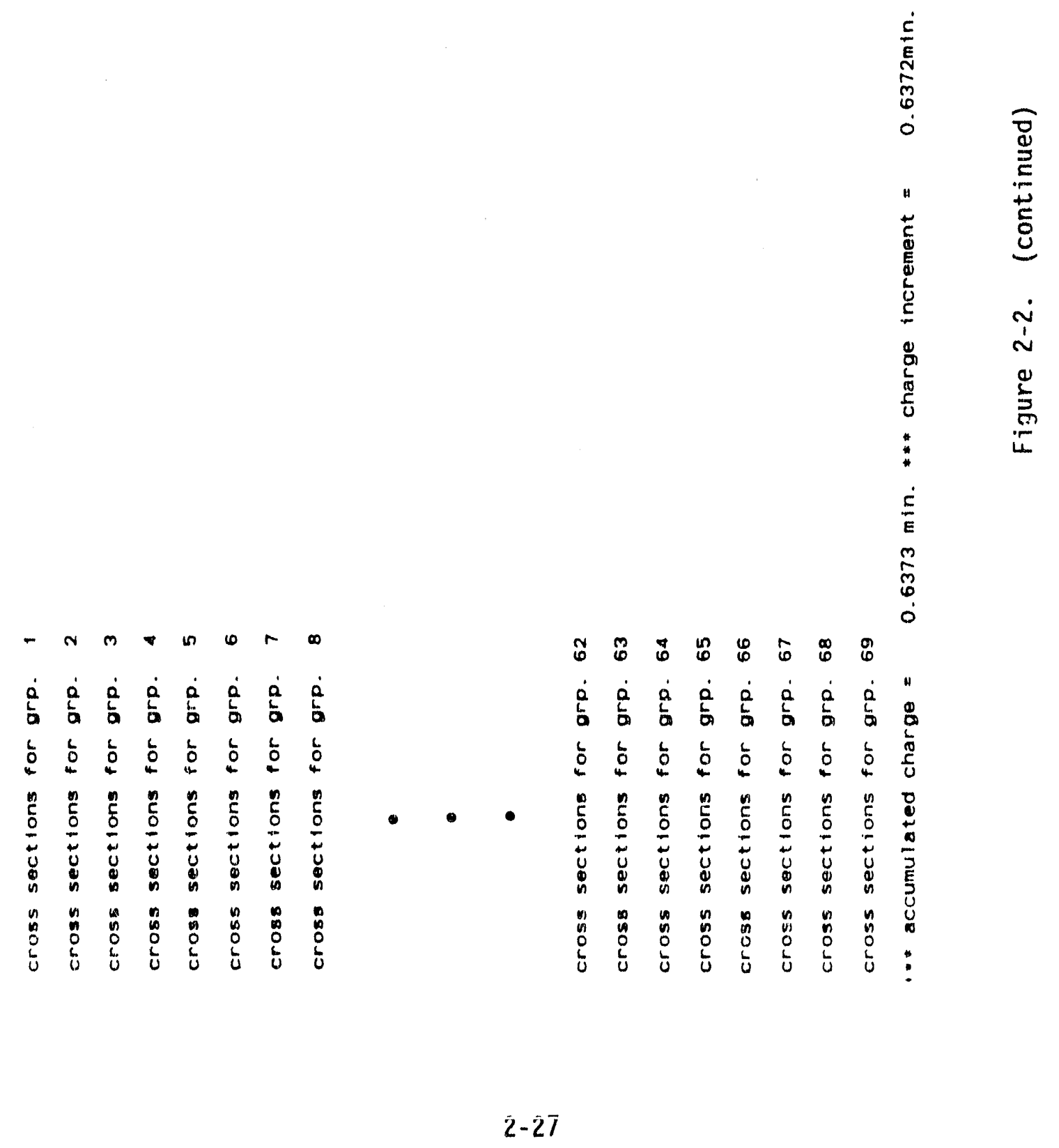


(This page is intentionally left blank.) 


\subsection{GRTUNCL: AN ANALYTIC FIRST COLLISION SOURCE CODE*}

\subsection{INTRODUCTION TO GRTUNCL}

\subsubsection{Background}

In the development of DORT', a general method was obtained which was inherently capable of solving most two-dimensional neutron and gamma-ray transport problems, including deep penetration. The approximations of discrete ordinates theory are not limiting since, at least in principal, the number of energy groups, solid angle segments and space intervals, and the order of expansion of the differential scattering cross sections may be increased as necessary to obtain the desired solution. However, the problems which can be solved in practice are limited by the finite computer speed, memory size, and costs. In order to extend the capabilities of DORT, a combination of efficient programming and analytical "tools" were used. One of the analytical "tools" developed was the analytic first collision source code - GRTUNCL.

In two-dimensional $r-z$ cylindrical geometry, the finite number of angles may result in an anomaly called the "ray effect." The problems in which ray effects are noticed are characterized by sources and detectors which are small compared to the total geometry, and a scattering mean free path which is long compared to the space mesh. Hence, if a problem such as a point source or point detectar in a highiy absorbing medium is approximated by the discrete ordinates difference equations, the fluences along a spherical surface centered about the source are observed to rise and fall in a wave-like pattern instead of being constant. Furthermore, the peaks in the distribution are observed to fall along rays following the polar angles of the quadrature centered at the source.

In the discrete ordinates solution of the two-dimensional cylindrical transport equation, the angles in the quadrature are arranged in levels which are comprised of directions having the same polar direction cosine, $\eta$. Within these levels, particles change angles due to both the curvature and scattering derivatives. However, transfers from a direction in one $\eta$ level to one in another level occur only through scattering. Particles that start out in an $\eta$ level tend to stay in the same level until scattered. Since there are only a finite number of $\eta$ levels, particles tend to appear only at space intervals which are oriented with the source along a discrete $\eta$ level. If the source region

\footnotetext{
"R. L. Childs and J. V. Pace, III, "GRTUNCL: First Collision Source Program," Unpublished documentation (1982), included in the documentation of the Radiation Shielding Information Center CCC-484/DORT Code Package, Oak Ridge National Laboratory.
} 
is distributed or if scattering is dominant, no ray effects appear. However, if the source is localized and spatial convection is dominant (i.e., the change in the particle fluence is governed by geometric attenuation), ray effects may be significant.

An obvious way to mitigate ray effects would be to increase the number of $\eta$ levels. However, this approach is uneconomical and usually only slightly effective. Another technique is to employ an analytic first collision source. This method essentially removes ray effects since the source is now distributed continuously in angle. For points within a mean free path or so of the source, the analytic uncollided fluence dominates. For intermediate points a few mean free paths from the source, the fluence is dominated by the source from first collisions which, although emitted into the discrete mesh, is distributed smoothly in space due to the analytic uncollided fluence. For points many mean free paths from the source, the fluence is due to particles which have suffered many collisions, a situation in which ray effects would not normally appear.

Unfortunately, the technical problems involved in implementing the analytic first collision source in the general problem are prohibitive. For an arbitrary source distribution, the contribution to each space point from each source interval must be calculated. Furthermore, the cylindrical symmetry in $r-z$ geometry must be accounted for by an azimuthal integration, for each source interval. For example, a problem with 3000 space points and only 10 azimuthal intervals would require up to $9 \times 10^{7}$ calculations to determine the uncollided fluence. The point source on the cylindrical axis problem which produces the worst ray effects involves only $3 \times 10^{3}$ calculations for the same problem. Problems which have a co-axial parallel beam source may also be treated. Although these problems do not normally show severe ray effects, the source is usually input in the directions nearest the axis, which are usually $13^{\circ}$ or more from the axis. This causes a slightly incorrect uncollided fluence and also makes it difficult to determine the collided fluence in the near axis angles.

\subsubsection{Method Used ${ }^{2}$}

An analytic first collision source technique has been developed for use with the two-dimensional code, DORT, for the simple sources described above. Basically, GRTUNCL is used to obtain the uncollided fluences and to generate space, angle, and energy dependent first collision source distributions for input into the DORT code. The actual calculational scheme in GRTUNCL for an isotropic source consists of first calculating the uncollided fluence given by; 


$$
\begin{array}{r}
\phi_{u, g}\left(\mathbf{r}_{i}\right)=\sum_{k} S_{g}\left(\mathbf{r}_{k}\right) \exp \left[-\beta_{g}\left(\mathbf{r}_{k}, \mathbf{r}_{i}\right)\right] \\
\times \frac{\Delta A_{k}}{4 \pi\left|\mathbf{r}_{i}-\mathbf{r}_{k}\right|^{2}}
\end{array}
$$

where $\Phi_{u, g}\left(r_{i}\right)$ is the uncollided fluence in energy group $g$ at detector

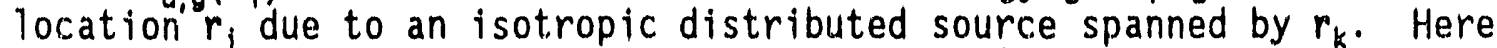
$S_{g}\left(r_{k}\right)$ is the number of source particles emitted at space point $r_{k}$ in energy group $g$ per unit time per unit surface area, $\Delta A_{k}$ is the area on the source disk associated with $S_{q}\left(r_{k}\right)$, and $\beta_{g}\left(r_{k}, r_{j}\right)$ is the number of mean-free-paths along the vector from $r_{k}$ to $r_{i}$. The evaluation of Equation (3-1) produces estimates of the direct and transmitted contributions, providing $r_{k}$ spans the resolved and background sources, respectively.

GRTUNCL then generates a space-, angle- and energy-dependent first collision source given by

$$
\begin{aligned}
S_{g}\left(\mathbf{r}_{i}, \Omega\right)= & \sum_{l} \sum_{m} \frac{A^{l, m}(\Omega)}{4 \pi} \sum_{g^{\prime}} \Sigma_{g^{\prime} \rightarrow g}^{l}\left(\mathbf{r}_{i}\right) \\
& \times \sum_{k} S_{g^{\prime}}\left(\mathbf{r}_{k}\right) \frac{\exp \left[-\beta_{g^{\prime}}\left(\mathbf{r}_{k}, \mathbf{r}_{i}\right)\right]}{4 \pi\left|\mathbf{r}_{i}-\mathbf{r}_{k}\right|^{2}} \\
& \times A^{l, m}\left(\Omega_{k}\right) \Delta A_{k}
\end{aligned}
$$

where $\Omega_{k}$ represents the unit vector from $r_{k}$ to $r_{j}$, the $A^{1, m}(\Omega)$ are reduced surface harmonics that result from two-dimensional symmetry, and the $\Sigma^{\prime}\left(g^{\prime} \rightarrow g, r_{i}\right)$ are the $l^{\prime}$ th coefficients in Legendre expansions of the energy group-to-group scattering cross sections. The DORT code uses the first collision source distribution to calculate the distributions of the collided fluence moments throughout the geometry mesh. 
The above procedure is implemented in GRTUNCL in the following manner:

1. The geometry is described with the same space mesh and material zones for the DORT problem.

2. The uncollided fluence (magnitude and angle) is calculated for each space point and energy group.

3. The first collision source, $S$, a function of radial interval, $I$, axial interval, $J$, angular moment, $L$, and group, $G$, is calculated for each space point, angular moment, and energy group.

4. The source array, $S$, and the scalar uncollided fluence are written to an output file.

5. DORT has been modified to use, as an option, the anisotropic source array, $S$, as the fixed source. The result of such a calculation is the collided fluence $\Phi_{c, g}(r, \Omega)$ where the arguments are as before except that $\Omega$, for angle, is added. At the end of the calculation, an option is avallable to produce either the collided fiuence or the total fluence (collided plus uncollided).

\subsection{GRTUNCL INPUT REQUIREMENTS}

The following input cards are required to execute GRTUNCL. Default values are in brackets:([]).

Titlo Card (72 alphanumeric character description)

\section{INPUT DATA BLOCK 1}

1\$ Array - Integer Control Parameters

ITH input cross-section library flag

$$
0 / 1=\text { forward/adjoint cross-section library }
$$

ISCT maximum order of Legendre expansion of cross sections

IZM number of material zones

IM number of radial intervals

JM number of axial intervals

-5 -..

IGM number of energy groups

IHT position of total cross section in cross-section table 
IHS position of self-.scatter cross section in cross-section table

IHM cross-section table length per energy group

MS cross-section mixing table length

MCR number of materials read from cards

MTP number of materials read from input cross-section Tibrary tape (nuclide or GIP format)

MT total number of materials (MCR+MTP+MIXTURES)

IDAT1 cross-section storage control flag

- o implies store cross sections in core

$=1$ implies store cross sections on tape

- 2 implies cross sections on input GIP tape

NOA number of quadrature points in azimuthal spatial integration for ring source

$\left\langle 6^{*}\right.$ and $7 *$ arrays required if $\left.>0\right)$

IMODE output fluence flle format flag

$3 / 4=$ DOT III/DOT IV format

IPRTC output cross-section print control flag

$0 / 1$ print all materials/none

NFLSV uncollided fluence moments output control flag

a 0 implies no effect.

$>0$ implies write uncollided fluence moments on NFLSV

NPSO first collision source output control flag

$=0$ implies no effect

$>0$ implies write first collision source on NPSO

IPRTF number of groups of uncollided fiuence to be printed

$-20-.$.

IPRTS number of groups of first collision source to be printed

IZ3 source distribution table control flag

= 0 implies no effect

$>0$ implies lerigth of source distribution table

IDFAC density factor control flag

- 0 implies no effect

$>0$ implies density factors specifted in $3^{*}$ array

NBUF number of $k$-bytes of core

NTNPR $=0$ implies no effect

$>0$ implies NTNPR is large scale print output unit 
2* Array - Real Control Parameters

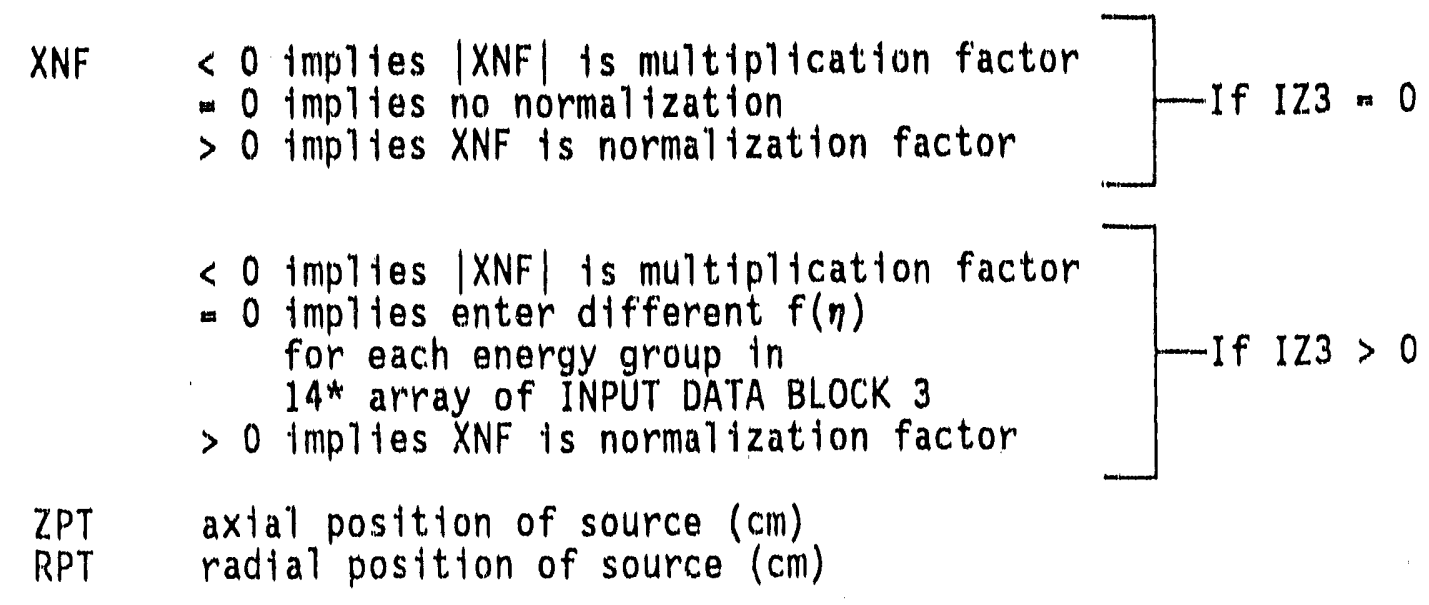

$T$ (terminate block with "T") [T always required]

\section{INPUT DATA BLOCK 2}

$13 \$$ Array - Nuclide ID Numbers on Library Flle (MTP entries required only if IDATI is not equal to 2)

14* Array - Cross Sections for Nuclide 1, ANISN3 format, all groups

[each new nuclide must start on a new card]

- Cross Sections for Nuclide MCR, ANISN format, all groups (IHM*IGM*MCR entries required)

$T$ (terminate block with "T") [T always required]

\section{INPUT DATA BLOCK 3}

1* Array - fission spectrum (IGM entries)

2* Array - axial interval boundartes (JM+1 entries)

3* Array - density factors by interval (IM*JM entries if IDFAC $>0$ )

4* Array - radial interval boundaries (IM+1 entries)

6* Array - angular quadrature weights for azimuthal spatial integration (NDA entries)

$7^{*}$ Array - discrete values of integrand for azimuthal spatial integration (NOA entries) 


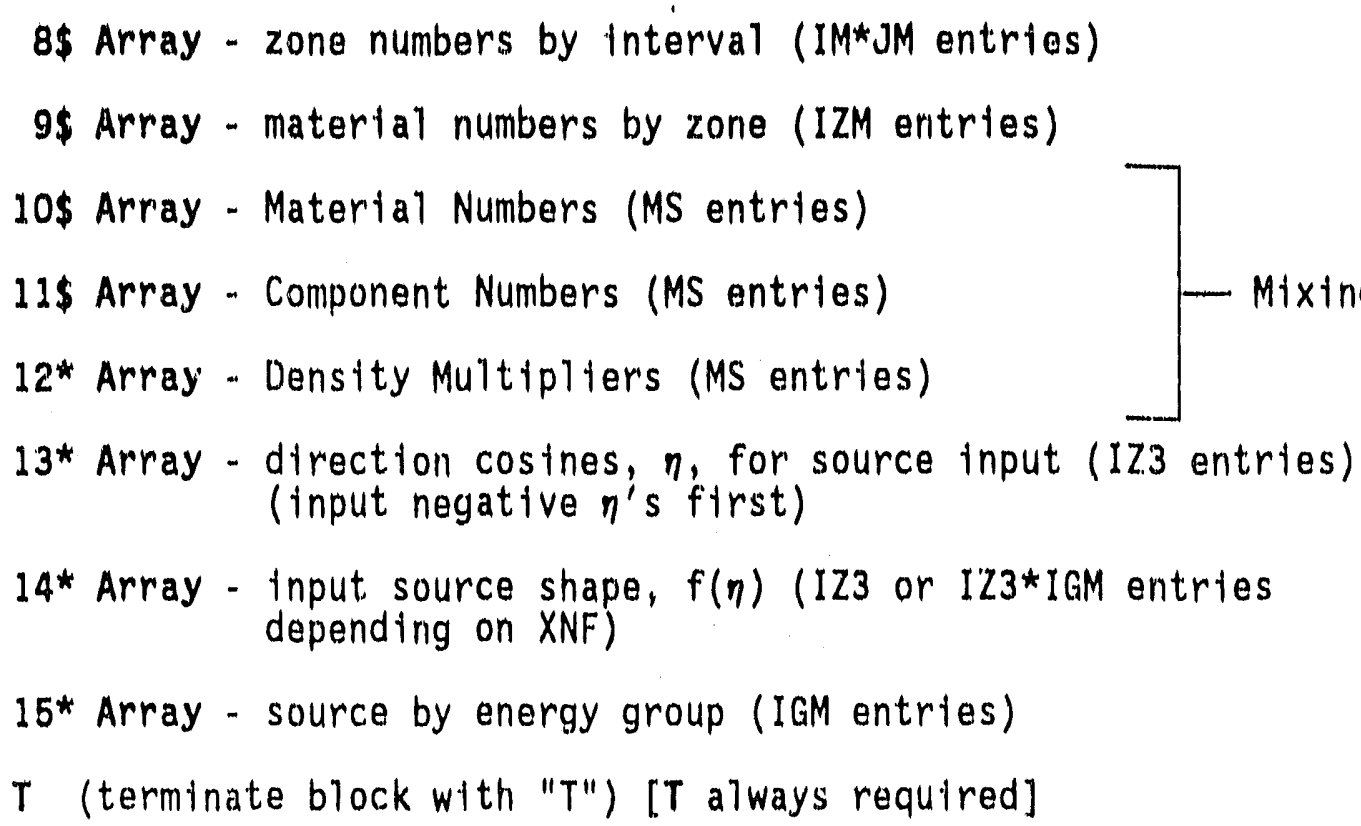

\subsection{GRTUNCL INPUT DATA NOTES}

Except for the title sard, all data are read using the FIDO input system. A detailed description of the FIDO input system is given in Appendix $A$. Data arrays are entered in blocks, each terminated by a " $T$ ". Unused data arrays (e.g., $10 \$$ if $M S=0$ ) are not entered, but a " $T$ " must still be entered to signal the termination of each block.

1 Array The definitions of many of the parameters in this array are compatible with GIP (Section 2.0) and DORT (Section 4.0).

ITH This flag only applies to the cross sections. If ITH=1, the source must be input in reverse order by energy group. If ITHal, two major reordering steps are carried out to produce an adjoint cross-section library:

1. The inscatter matrix is iransposed, i.e., the table position associated with group $g$ describing scattering from $\mathrm{g}^{\prime}$ to $\mathrm{g}$ is changed to describe scattering from $g$ to $\mathrm{g}^{\prime}$.

2. The ordering of the groups is reversed, i.e., data for group IGM appear first in the output file, followed by IGM-1, etc.

A simple three group example is illustrated in Section 3.3.1.

ISCT When the order of scatter is greater than zero, GRTUNCL expects the $P_{1}$ cross section to be matertal $M+1$, the $P_{2}$ cross section to be $M+2$, etc., where $M$ is the $P_{0}$ material number: 


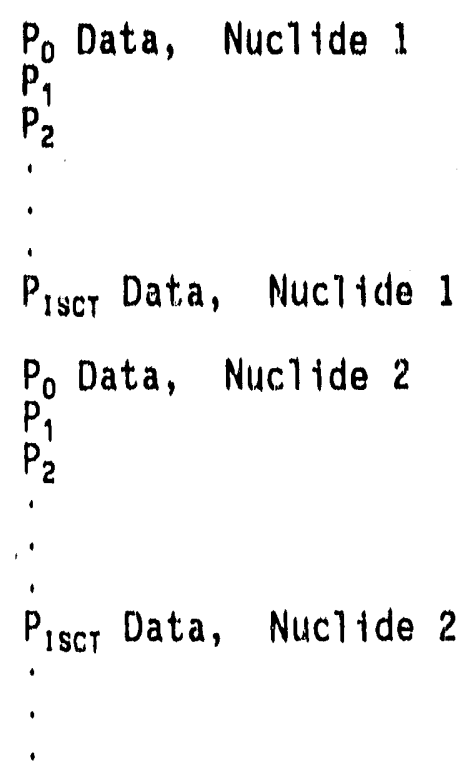

For components other than $P_{0}$, cross section positions other than positions IHT+1 through IHM may be zero.

IZM The number of material zones specifled in GRTUNCL should correspond to the number of material zones specified in DORT. This is not a requirement, however, because in DORT the user has the option of spectfying the convergence by zone and consequently may have more zones defined than are required in GRTUNCL. It is generally useful to set the number of material zones according to the DORT requirements and ut 11 ize the same input for GRTUNCL.

IM, JM The number of radial (IM) and axial (JM) intervals must correspond to the number of intervals utilized in DOR'T. Since GRTUNCL calculates the uncollided fluence and first collision source by interval, the number of intervals and interval boundaries must correspond to the DORT intervals. In setting up the interval boundaries, the position of the source (both axially and radially) must be considered so that the fluence does not differ by more than a factor of two between adjacent intervals. It should be noted that the interval next to the source interval will always exhibit greater than a factor of two difference in the fluence. A good rule of thumb for an air-overground calculation is to assume an initial "source" interval size and apply a factor of 1.3 to that interval size to determine the interval boundaries. For example, assume the source is positioned at $r=0.0$ arid the source interval is $10 \mathrm{-cm}$ wide. The radial interval boundaries will be $0.0,10.0,13.0(10.0 \times 1.3), 17.0(13.0 \times 1.3), 22.0(17.0 \times 1.3)$, $29.0(22.0 \times 1.3)$, etc., until the interval size reaches some predetermined maximum size, such as 25 meters for air. A simflar sequence could be used for axial interval boundartes.

NOTE: GRTUNCL cannot handle a vartable mesh. Therefore, in model ing the atr-over-ground problem, ignore the DORT variable mesh option. 
IHT, IHS, IHM The discussion of these input parameters is given in Section 3.4.2.

MCR, MTP, MT GRTUNCL assigns storage space (IHM*IGM words/set) for MT sets of cross sections, called "materials." The first MCR spaces are fllled wtth data read from cards in the 14* array in INPUT DATA BLOCK 2. The next MTP material spaces are filled with riuclide data read from the data set mourited on the input library logical unit (default=4) and stored in the order indicated by the $13 \$$ array. The remaining spaces between MCR+MTP and MT are avallable for preparing mixtures.

NOTE: If MCR $=0$, and IDATI $=2$, the $13 \$$ and $14^{*}$ arrays in INPUT DATA BLOCK 2 and corresponding data block terminator are not required. This corresponds to pre-mixing the material cross sections in GIP and is the recommended method of supplying cross-section data to GRTUNCL.

IDAT1 This parameter controls the cross-section data storage. If IDATI $=0$, MCR materials are read in from cards, MTP materials are read in from an ANISN formatted library on logical unit 4, aid MT materials are stored in core. If IDATI $=1$, MCR cross sections are read in from cards, MTP materials are read in from an ANISN formatted library on logical unit 4, and MCR+MTP materials are stored on logical unit one prior to mixing. MT materials (GIP formatted) are then stored on logical unit two after inixing. If IDATI $=2$, MTP materials are read in from a GIP formatted library on logical unit four and stored on logical unit two. IDAT] $=2$ is the recommended option for cross-section storage

NOA This parameter can be zero for on-centerline sources. Further discussion is given in the data notes for the $6^{*}$ and $7^{*}$ arrays.

IMODE This parameter identifies two possible output fluence file formats. Oniy the DOT IV format is discussed since it is used in present day applications. The DOT III format was retained from earlier versions of GRTUNCL, and although st 111 operational, should be avoided, unless DOT III will be used in the final calculations.

IPRTC If IPRTC $=0$, printed cross-section edits for all materials will be output. It is recommended this parameter be set to one to avoid a large amount of output.

NFLSV If NFLSV is greater than zero, the uncollided fluence moment data are written on NFLSV. If IMODE $=4$, the output is written in the VARSOR format, (see Section 3.5) which is suitable for use with DORT.

NPSO If NPSO is greater than zero, the first collision source data are written on NPSO. If IMODE $=4$, the output is written in the VARSOR format, (see Section 3.5) which is sultable for use as DORT input. If NPSO equals zero, the input parameters IPRTF and IPRTS are ignored. 
IPRTF If IPRTF is greater than zero, IPRTF groups of uncollided fluence edits will be output. For a large number of intervals (IM*JM), it is recommended this parameter be set to a small number (less than five). Analyzing the first non-zero group of the incollided fluence will indicate the adequacy of the interval bounciaries in the spatial mesh.

IPRTS If IPRTS is greater than zero, IPRTS groups of first collision source edits will be output. It is recommended this parameter be set to zero because it generates a large volume of output.

IZ3 This parameter controls the number of direction cosines, $\eta$, used to describe the source input in the 13* array and the number of points used to describe the source shape, $f(\eta)$, input in the $14^{*}$ array. If IZ:3=0, only an energy dependent source distribution is entered in the $15^{*}$ array, and the $13^{*}$ and $14^{*}$ arrays are omitted.

NBUF This parameter times 1000 is the initial memory objective of GRTUNCL on the CRAY. GRTUNCL divides the calculation into blocks where the number of blocks ranges from one to JM depending on how much memory has been allocated. A smaller number of blocks requires more memory. Consequently, a trade off between memory and $1 / 0$ requirements results from the choice for NBUF. The minimum memory requirements (for JM blocks) will appear or the printed edits.

XNF This parameter is typically used as a normalization factor. For example, if the source distribution is per source particle, XNF can be used to obtain the absolute normalization if the total number of source particles is entered here. The meaning of the value of XNF is dependent on the parameter IZ3 and impacts additional input to GRTUNCL.

ZPT and RPT If RPT=0, the source will be a point source located axially at ZPT. If RPT>0, the source will be a ring source located axially at ZPT.

$13 \$$ Array A negative entry in the $13 \$$ array replaces the following ISCT entries with successive ID's. Thus:

$\begin{array}{lll}\frac{13 \$}{-101} & \frac{13 \$}{101} \\ -101 & & 102 \\ -101 & \text { is equivalent to } & 103\end{array}$

14* Array The input of the $14^{*}$ array is performed by subroutines which have certain restrictions in addition to those of normal FIDO input:

a. The nuclide spaces are preset to 0.0 , so that "E" or "FO" will have the effect of filling the remaining space with 0.0 , but "E" and "F" must not be used in the input for the last nuclide. 
b. The $14^{*}$ or $14^{* *}$ designation may precede each nuclide block, but is not required except in the first block.

c. Entries, including operators such as " $T$ ", following the last data item for nuclides other than the last, and on the same card as the last item, will be ignored.

d. The "T" which terminates this block must appear alone in column 3 of a separate card.

7* Array The integration limits are assumed to be from -1 to 1 so ${ }_{-1} \int^{1} f(X) d X$ is assumed to be $\Sigma f\left(X_{i}\right) W_{i}$ where the $W_{i}{ }^{\prime} s$ are entered in the $6^{*}$ array and the $X_{i}$ 's are entered in the $7^{*}$ array.

8\$ Array The material zone numbers by interval are entered by IM entries by JM entries. For example, the material zone numbers are entered for the first axial interval, then the second axial interval, etc.

9\$ Array Neyative numbers entered in this array indicates an anisotropic material and ISCT higher order moments must follow imnediately on the cross-section unit. For example if a -1 and -7 are entered in this array for the first two material zones, and ISCT=5, GRTUNCL will expect to read the $P_{0}$ through $P_{5}$ components of the first material then the $P_{0}$ through $P_{5}$ components of the second material on the cross-section unit.

10\$, 11\$, and 12* Arrays The cross section mixing table is used to combine elements into macroscopic mixtures. Experience will reveal that only the imagination limits its flexibility. As with DORT, the integers in the mixing table refer to a continuous array of MT cross section sets of which the first MCR+MTP are nuclide data read from card-image input and a library file, respectively. All of the sets beyond MCR+MTP are preset to zero before the mixing table is executed. In non-search problems where cross-section storage is difficult, i.e., large problems that do not involve material buckling or concentration searches, material spaces originally used for input nuclides can be reused for mixtures. While the general rules for creating mixtures are equally applicable for GIP, GRTUNCL, and DORT, it should be noted that reference to eigenvalue modification in the DORT mixing table description has no application to GRTUNCL mixing. The interpretation rules applicable to GRTUNCL are as follows:

a. If the mixture number is $N$ and the component number is 0 , then the cross sections in mixture $N$ will be multiplied by the number entered in the density column:

$$
\sigma_{\text {MIXTURE N(NEW) }}=\sigma_{\text {MIXTURE N(OLD) }} * \text { DENSITY }
$$


b. If the mixture number is $N$ and the component number is $M$, then the cross sections in component $M$, multiplied by the density, will be added to the cross sections in mixture $N$ :

$$
\sigma_{\text {MIXTURE N(NEW) }}=\sigma_{\text {MIXTURE N(OLD) }}+\sigma_{\text {COMPONENT } M} * \text { DENSITY }
$$

Note: Either a mixture or a component may be made up of microscopic or macroscopic cross sections for a single isotope or mixture of isotopes.

A sample mixing table showing the various options is given below.

\begin{tabular}{ccc}
$\begin{array}{c}\text { MIXTURE } \\
(10 \$ \text { ARRAY })\end{array}$ & $\begin{array}{c}\text { COMPONENT } \\
\text { (11\$ ARRAY) }\end{array}$ & $\begin{array}{c}\text { DENSITY } \\
\text { (12* ARRAY) }\end{array}$ \\
\hline 6 & 1 & 0.4 \\
6 & 3 & 0.5 \\
7 & 2 & 0.4 \\
7 & 4 & 0.5 \\
7 & 0 & 3.0
\end{tabular}

The mixture table given above would do the following:

(1) Add components 1 and 3 with densities 0.4 and 0.5 , respectively, to form mixture 6 : $\sigma_{0}=\sigma_{1} \star 0.4+\sigma_{3} \star 0.5$

(2) Add components 2 and 4 with densities 0.4 and 0.5 , respectively, to form mixture 7 :

$\sigma_{7}=\sigma_{2} * 0.4+\sigma_{4} * 0.5$

(3) Multiply mixture 7 by 3.0: $\sigma_{7}=\sigma_{7} * 3.0$

$10 \$$ Array If the 10 entry is tagged with a negative, the next ISCT components will be treated as $P_{L}$ components. For example:

$\frac{10 \$ 11 \$ 12^{*}}{-13} \quad$ (with ISCT $=3$ )

will be equivalent to

$\begin{array}{lll}10 * & 11 \$ & 12^{*}\end{array} \quad$ (with ISCT $=0$ )


$13^{*}, 14^{*}$, and $15^{*}$ Arrays These arrays control the source description in GRTUNCL. If $I Z 3=0$, the source is entered only as a function of energy in the $15^{*}$ array and the $13^{*}$ and $14^{*}$ arrays are omitted. This would be indicative of an isotropically emitted source spectrum. If IZ3>0, then the direction cosines, $\eta$, of the source are entered in the 13* array (IZ3 entries with negative $\eta^{\prime}$ s entered first), and the source shape $f(\eta)$ is entered in the $14^{*}$ array (IZ3 or IZ3*IGM entries depending on the value of XNF), and the energy dependence is entered in the $15^{*}$ array (IGM entries). The units of the entries in the $14^{*}$ array are particles/unit weight, i.e., $4 \pi^{*}$ particles/steradian. Typically, if IZ3>0 and $X N F=0$, the $13^{*}$ array would contain IZ3 entries, the $14^{*}$ array would contain IZ3*IGM entries, and the 15* array would be filled with IGM ones.

\subsubsection{Adjoint Reordering Example}

A three-group example showing the relationship between a forward and adjoint cross-section set is shown below. In this example, there is no upscatter, one activation cross section, IHT $=4, I H S=5$, and IHM $=7$.

\begin{tabular}{cc} 
Direct & Adjoint \\
\hline$\sigma^{\mathrm{F}}(1)$ & $\sigma^{\mathrm{F}(3)}$ \\
$\sigma^{\mathrm{A}}(1)$ & $\sigma^{\mathrm{A}}(3)$ \\
$\nu \sigma^{\mathrm{F}}(1)$ & $\nu \sigma^{\mathrm{F}}(3)$ \\
$\sigma^{\top}(1)$ & $\sigma^{\top}(3)$ \\
$\sigma(1 \rightarrow 1)$ & $\sigma(3+3)$ \\
0 & $\sigma$ \\
0 & 0 \\
$\sigma^{\mathrm{F}}(2)$ & $\sigma^{\mathrm{F}}(2)$ \\
$\sigma^{\mathrm{A}}(2)$ & $\sigma^{\mathrm{A}}(2)$ \\
$\nu \sigma^{\mathrm{F}}(2)$ & $\nu \sigma^{\mathrm{F}}(2)$ \\
$\sigma^{\top}(2)$ & $\sigma^{\top}(2)$ \\
$\sigma(2 \rightarrow 2)$ & $\sigma(2 \rightarrow 2)$ \\
$\sigma(1 \rightarrow 2)$ & $\sigma(2 \rightarrow 3)$ \\
0 & 0 \\
$\sigma^{\mathrm{F}}(3)$ & \\
$\sigma^{\mathrm{A}}(3)$ & $\sigma^{\mathrm{F}}(1)$ \\
$\nu \sigma^{\mathrm{F}}(3)$ & $\sigma^{\mathrm{A}}(1)$ \\
$\sigma^{\top}(3)$ & $\nu \sigma^{\mathrm{F}}(1)$ \\
$\sigma(3 \rightarrow 3)$ & $\sigma^{\top}(1)$ \\
$\sigma(2 \rightarrow 3)$ & $\sigma(1+1)$ \\
$\sigma(1+3)$ & $\sigma(1+2)$ \\
& $\sigma(1+3)$
\end{tabular}




\subsection{INPUT LIBRARY FORMAT}

GRTUNCL inputs two different types of cross section files depending on the value of IDAT1. If IDATI $=0$ or 1 , the $A C L 1^{4}$ prepared input 1 ibrary format is expected. If IDATI $=2$, the GIP formatted library format (see Section 3.4.3) is expected.

\subsubsection{ACL1 Formatted Cross-section Input File}

If IDATI $=0$ or 1 , the ACL1 prepared input 1ibrary format from which MTP nuclides are taken is defined in the following manner:

ALC Cross Section Library Format

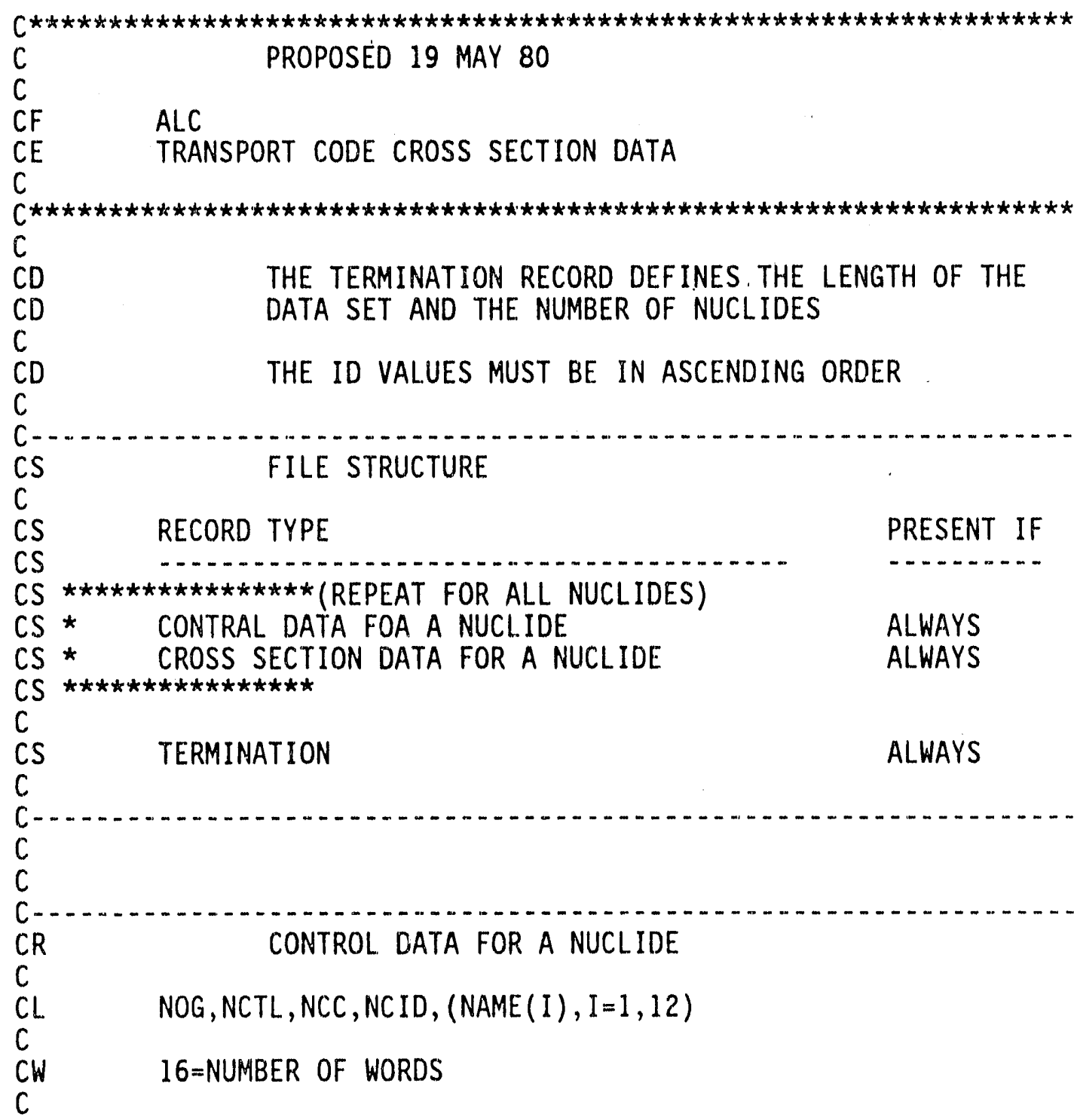




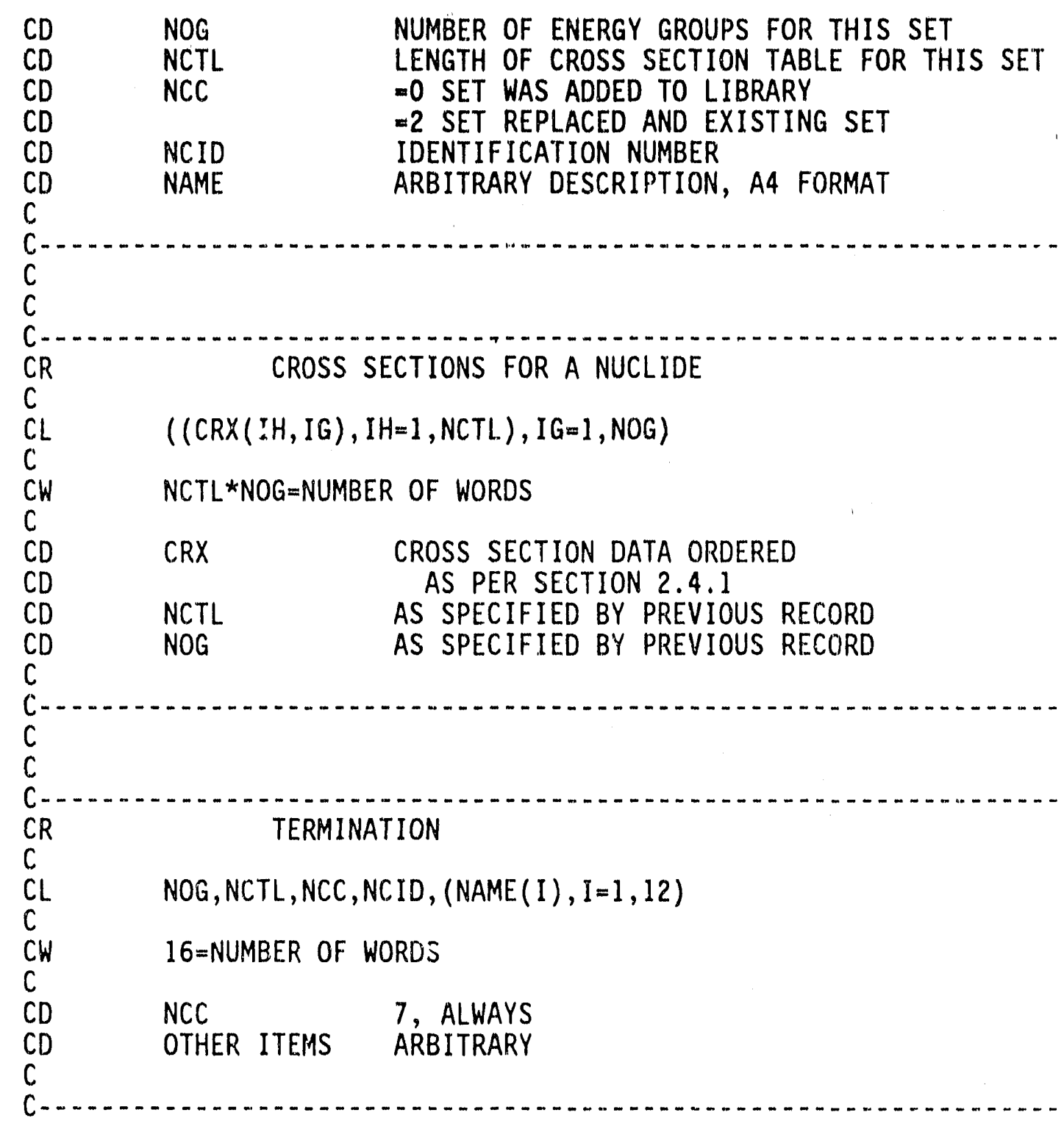

\subsubsection{ANISN Nuclide Format Cross-section File}

In a normal GRTUNCL problem, cross sections are read from card input and/or from an external data set in nuclide-organized format, i.e., all the cross sections for one group of one nuclide, followed by other groups for that nuclide, and finally followed by data for other nuclides. Within a group, GRTUNCL expects the ordering of data in the cross section table in the following format: 


\begin{tabular}{|c|c|c|}
\hline Table Position & Entry & Cross Section Type \\
\hline 1 & $\sigma^{4}(g)$ & $\begin{array}{l}\text { First activation cross section } \\
\text { (if any) }\end{array}$ \\
\hline IHT-3 & $\sigma^{L}(g)$ & $\begin{array}{l}\text { Last activation cross section } \\
\text { (if any) }\end{array}$ \\
\hline IHT - 2 & $\sigma^{A}(g)$ & Absorption \\
\hline IHT -1 & $\nu \sigma^{F}(g)$ & Neutron Production \\
\hline IHT & $\sigma^{\top}(\mathrm{g})$ & Total removal \\
\hline $\mathrm{IHT+1}$ & $\sigma^{\text {TUS }}(g)$ & $\begin{array}{l}\text { Total upscatter cross section from } \\
\text { group } g \text { (omit this entry if } N \cup S=0 \text { ) }\end{array}$ \\
\hline IHS-NUS & $\sigma(g+N \cup S \rightarrow g)$ & Scattering from group $g+N U S$ to $g$ \\
\hline$\dot{.}$ & & \\
\hline IHS-1 & $\sigma(g+1+g)$ & Scattering from group $\mathrm{g}+1$ to $\mathrm{g}$ \\
\hline IHS & $\sigma(g \rightarrow g)$ & Scattering from group $\mathrm{g}$ to $\mathrm{g}$ \\
\hline IHS+I & $\sigma(g-1+g)$ & Scattering from group $\mathrm{g}-1$ to $\mathrm{g}$ \\
\hline $\begin{array}{l}\cdot \\
.\end{array}$ & $\dot{.}$. & \\
\hline IHM & $\sigma(g-N D S+g)$ & Scattering from group $\mathrm{g}$-NDS to $\mathrm{g}$ \\
\hline
\end{tabular}

From this, it can be seen that:

NUS $=$ IHS-IHT-2 = number of upscatter groups, if greater than 0 ,

NDS $=I H M-I H S=$ number of downscatter groups,

$L=I H T-3=$ number of activation cross sections.

Special illustrations of interest are: 


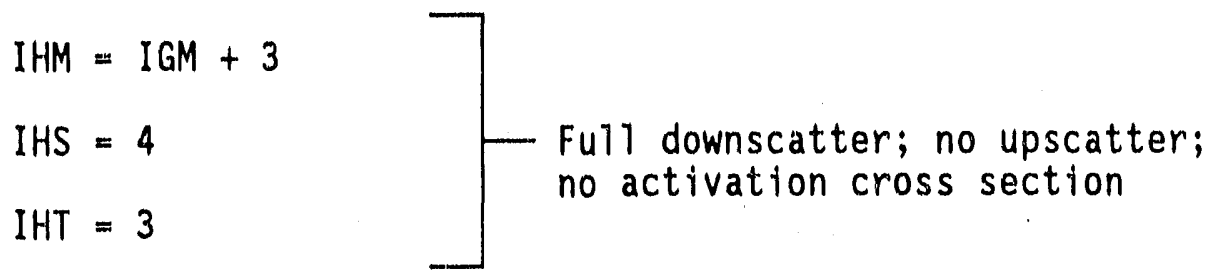

and,

$$
\begin{aligned}
& I H M=I G M+5+\text { NUS } \\
& I H S=6+\text { NUS } \\
& I H T=4
\end{aligned} \quad \begin{aligned}
& \text { FU11 downscatter; NUS upscatter; } \\
& \text { room for an activation cross } \\
& \text { section in position } 1 .
\end{aligned}
$$

Thus the parameters IHT, IHS, and IHM completely describe the format of the cross sections. If there are no activity cross sections, IHT=3. If there is no upscatter IHS $=$ IHT +1 . If there is no downscatter IHM $=$ IHS (i.e., a one group problem). If there is upscatter, GRTUNCL will compute a total upscatter cross section for each group of each material and place that cross section in position IHT +1 . The activity cross sections are only used for activity calculations and not used in the transport process itself.

\subsubsection{GIP Formatted Cross-section Input File}

If IDAT1 $=2$, GRTUNCL expects to read a GIP formatted 1 ibrary on logical unit four. Also, if IDATI=0 or 1 , GRTUNCL will create a GIP formatted cross-section file. The GIP input file is group-ordered for transport codes and this format is compatible with both ANISN, GRTUNCL, and DORT requirements. The groups are normally ordered from high to low neutron energy, then from high to low photon energy, if any photon groups are present.

GIP Cross Section Library Format

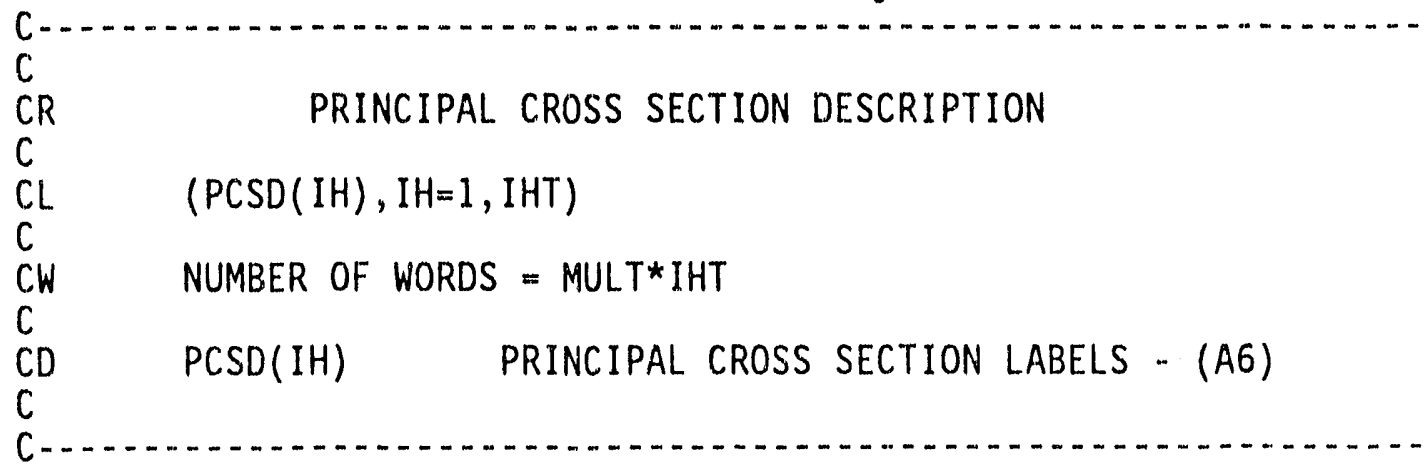




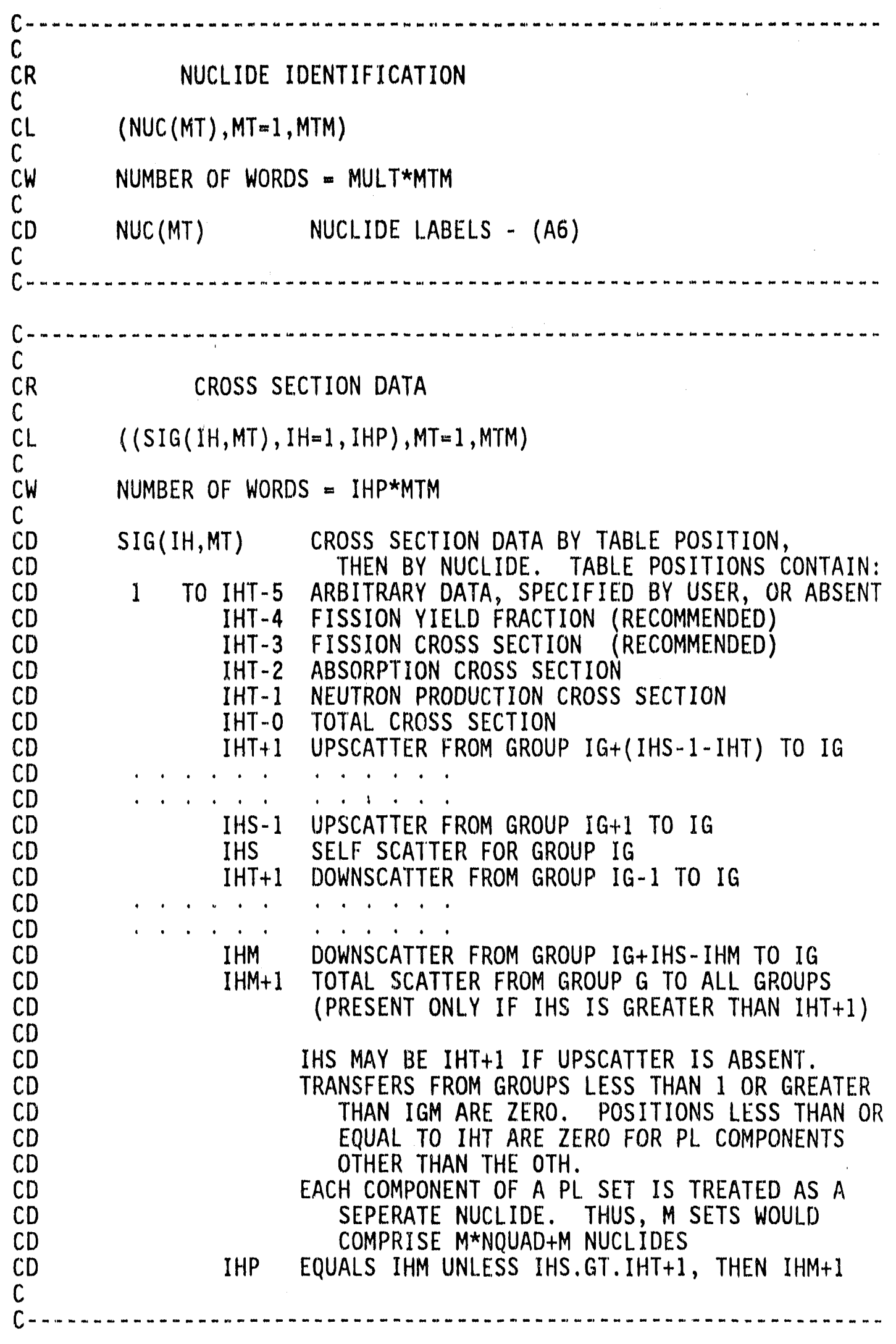




\subsection{GRTUNCL OUTPUT FILE}

GRTUNCL can output efther a DOT III or DOT IV formatted file depending on the value of IMODE. Current applications only use the DOT IV format - referred to as the VARSOR format in this document. Both the first colliston source and uncollided fluence moment data are written in this format. The VARSOR format is defined in the following manner:

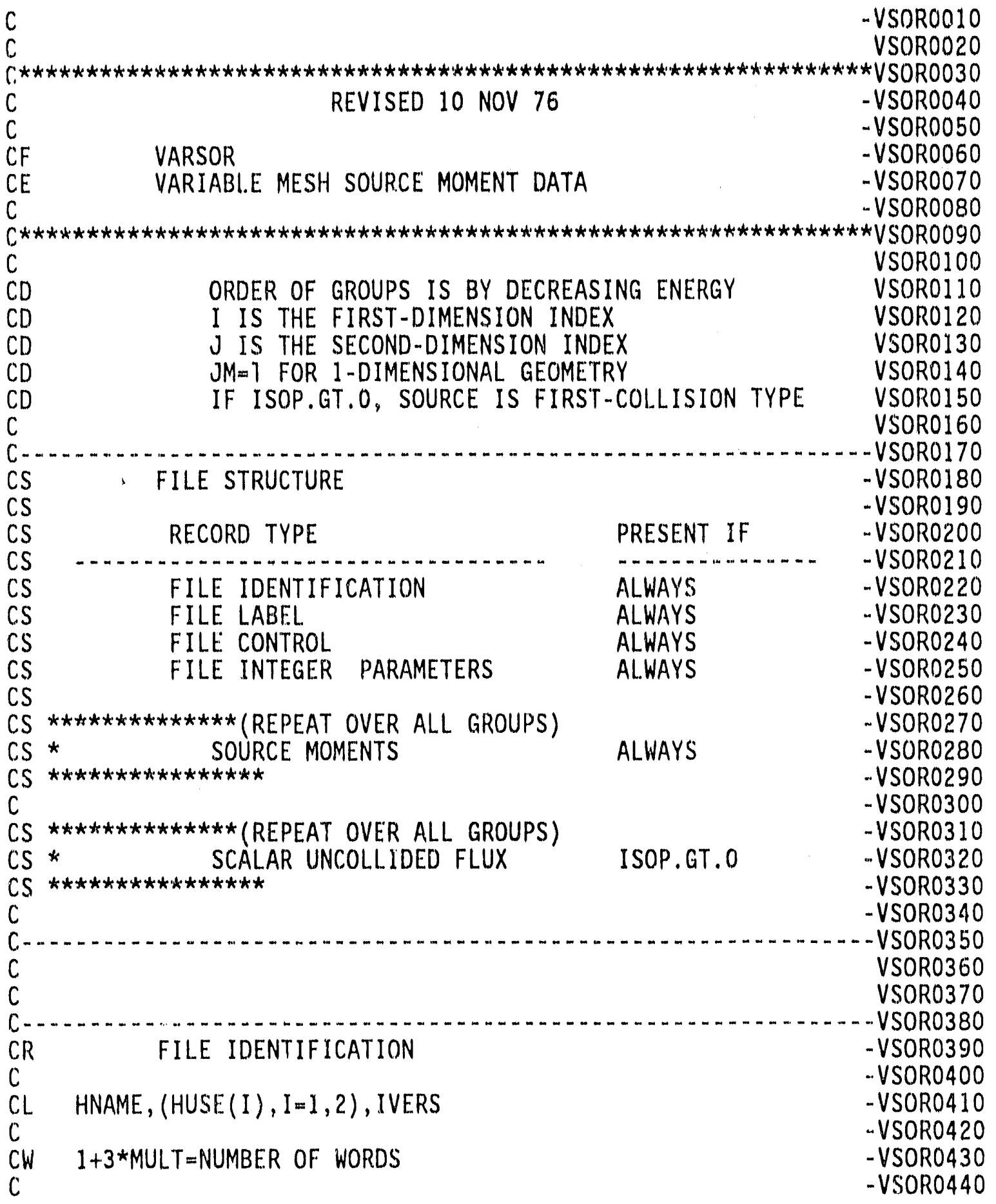




\begin{tabular}{|c|c|c|c|}
\hline $\begin{array}{l}C \\
C R \\
C \\
C L \\
C \\
C W \\
C \\
C D \\
C D \\
C D \\
C D \\
C D \\
C D \\
C \\
C-- \\
C \\
C \\
C-. \\
C R \\
C \\
C D \\
C \\
C W \\
C \\
C D \\
C D \\
C D \\
C D \\
C D \\
C D \\
C D \\
C D \\
C D \\
C D \\
C D \\
C D \\
C D \\
C\end{array}$ & $\begin{array}{l}\text { FILE } \\
\text { IGM, NEUT, JM, } \\
\text { 25=NUMBER } \\
\text { IGM } \\
\text { NEUT } \\
\text { JM } \\
\text { LM } \\
\text { IMA } \\
\text { MMA } \\
\text { ISM } \\
\text { IMSISM } \\
\text { ISOP } \\
\\
\text { IDUM(I) }\end{array}$ & $\begin{array}{l}\text { OF WORDS } \\
\text { NUMBER OF ENERGY GROUPS } \\
\text { LAST NEUTRON GROUP } \\
\text { (IGM IF ALL NEUTRONS, O IF ALL GAMMA) } \\
\text { NUMBER OF SECOND-DIMENSION (J) INTERVALS } \\
\text { MAXIMUM LENGTH OF MOMENT EXPANS ION } \\
\text { MAXIMUM NUMBER OF FIRST-DIMENSION INTERVALS } \\
\text { NUMBER OF BOUNDARY DIRECTIONS } \\
\text { NUMBER OF I -BOUNDARY SETS } \\
\text { TOTAL NUMBER OF I - INTERVALS, ALL. I-SETS } \\
\text { UNCOLLIDED FLUX FLAG } \\
\text { O - NO UNCOLLIDED FLUX RECORDS PRESENT } \\
\text { I - UNCOLLIDED FLUX RECORDS PRESENT } \\
\text { ARRAY SET TO O }\end{array}$ & 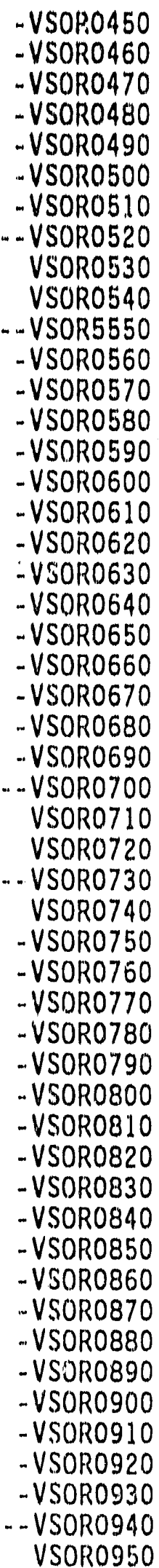 \\
\hline
\end{tabular}




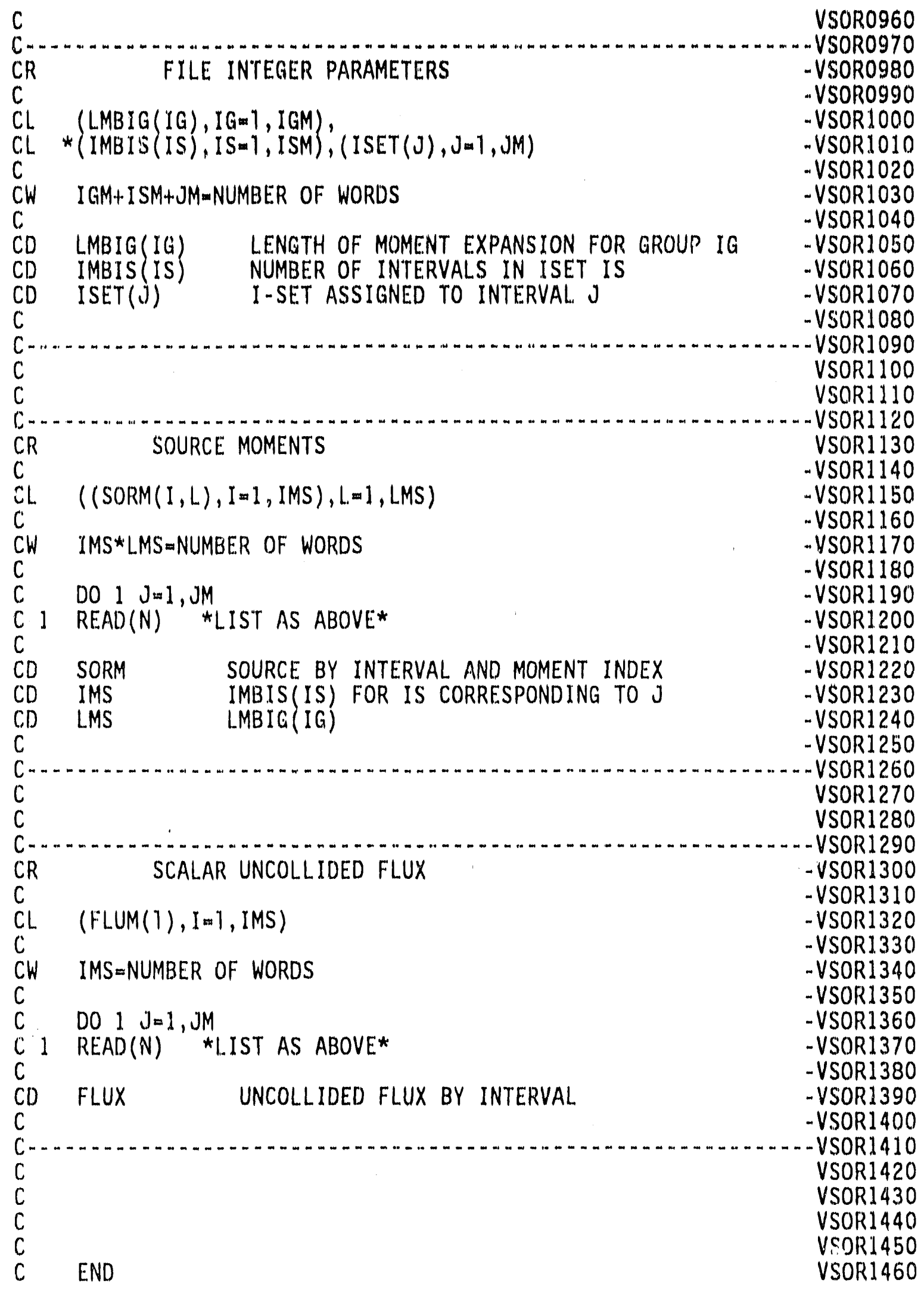




\subsection{LOGICAL UNIT REQUIREMENTS}

Below is a listing of the files required to execute a GRTUNCL case along with the default values used in the code. In setting up a GRTUNCL case, efforts must be made for these untts to be avallable.
1. Logical Unit
1 - SCRATCH, (holds group independent cross sections from input if logical untt 4 is not a group-independent (GIP) tape
2. Logical Unit
2 - SCRATCH, (holds GIP cross sections after mixing)
3. Logical Unit
4 - ANISN/GIP formatted Cross-section Library Input depending on value for IDAT1 (required if MTP>0)
5. Lngical Unit NPSO - First Colliston Source Output
6. Logical Unit NFLSV - Uncollided Flux Moments Output
7. Logical Unit NTNPR - Large Scale Print Output
8. Logical Unit 91 - SCRATCH
9. Logical Untt 92 .. SCRATCH
10. Logical Unit 6 - Printed Output
11. Logical Untt 5 - Card Input

\subsection{PROGRAMMER INFORMATION}

\subsubsection{Inter-machine Adaptability}

The GRTUNCL code is intended to be easlly adaptable to any type of sophisticated computer, and yet to take advantage of certain localized structural features which may be machine-dependent.

In general, the guidelines of ANS-STD.3-19716 are followed. This requires general adherence to a simple, standard FORTRAN language except where deviations provide important improvements in capability and can be documented. In addition, machine.deperident features have been kept localized and have not been included in the subroutines which perform actual computation.

The recominended procedures of the Committiee on Computer Code Coordination (CCCC $)^{5.7}$ have been followed where practical. Compliance with these standards has been incomplete where cost was prohibitive, the nature of the codes did not allow $1 t$, or the expected level of performance could not be obtained in that fashion.

Where minor or certain machine-dependent features are required, alternative features are enclosed in pairs of 3-character "language flags." The alternative statements remain in the source program, with inappropriate sections transformed into cominent statements. If the code is set up for IBM operation, for example, it might contain: 


$\begin{array}{ll}\text { CIB } & \text { ENTRY } \operatorname{IBCDC}(H, E, L, P) \\ C I B & \\ C D C & \\ C & \text { ENTRY IBCDC } \\ C D C & \end{array}$

The corresponding CDC configuration would be:

$\begin{array}{ll}C I B & \\ C & \text { ENTRY IBCDC }(H, E, L, P) \\ C I B & \\ C D C & \\ C D C & \text { ENTRY IBCDC }\end{array}$

A11 versions of a given procedure are thus avallable for inspection by all users. The selection of options is made by a computer program at distribution time. A listing of machine-dependent sections can also be prepared in that process.

The language flags achieve the major objective of having a single, unified source for each program which is maintained for all users. With these provisions, the basic FORTRAN prograins are operable on IBM, CDC, UNIVAC, CRAY, Aindahl, and other computers, except that system-dependent service routines as specified by CCCC must be provided.

Optional packages and procedures which provide enhanced conventence are also avallable. The optional run-time storage allocation requires system-dependent routines. Instructions for installing these options are distributed with the source programs. All known users at this time use all of the optional enhancements available to them. Even so, the basic operation with FORTRAN routines plus the CCCC package remains available if needed.

\subsubsection{Service Subroutines}

Certain standard service subroutines are specified by the CCCC for use in reactor physics codes. The service routines used in these codes include:

TIMER Provides timing and job identification data

REED RITE Provide sequential access to data files 


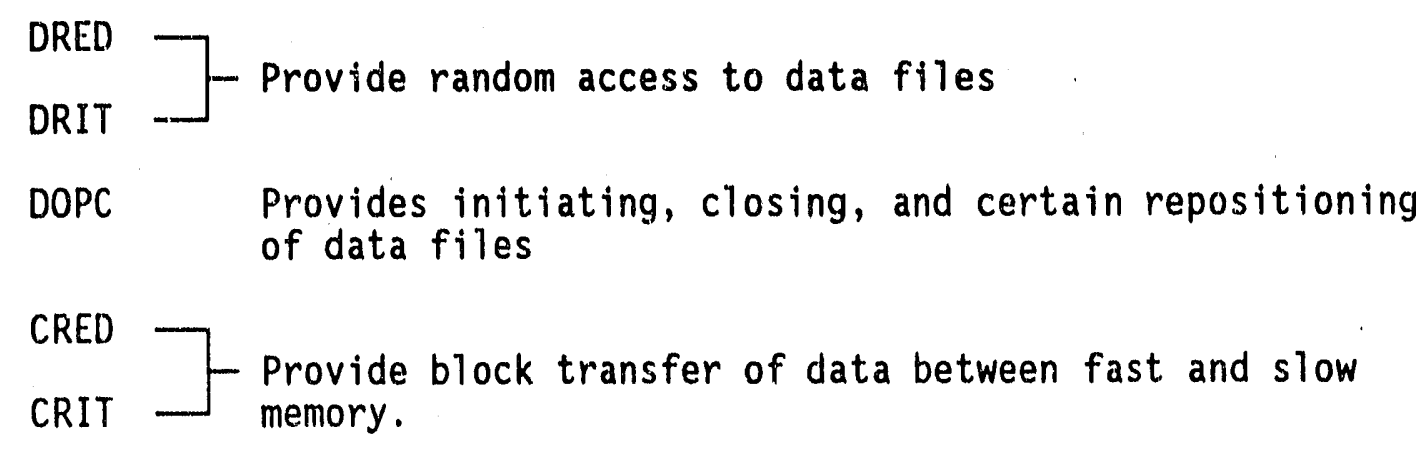

Many of these subroutines also call other subroutines. Many are entirely system lependent. Each configuration to be distributed contains an appropriate set of service subroutines, insofar as possible. The realities of computing environments may require local modification or substitution. The specifications given in Reference 4 , together with extensive in-stream comments, provide guides for such modification.

\subsection{REFERENCES}

1. W. A. Rhodses and R. L. Childs, "The DORT Two-Dimensional Discrete Ordinates Transport Code," Nuclear Science \& Engineering 99, 1, pp. 88-89, (May 1988).

2. R. A. Lillie, R. G. Alsmiller, Jr., and J. T. Mihalczo, "Design Calculations for a $14-\mathrm{MeV}$ Neutron Collimator, "Nuclear Technology V01. 43, pp. 373-377, (May 1979).

3. W. W. Engle, Jr., "A USER'S MANUAL FOR ANISN, A One-Dimensional Discrete-Ordinates Transport Code with Anisotropic Scattering," K-1693, Oak Ridge National Laboratory, (March 1967).

4. W. A. Rhoades, "The ALC1 Program for Cross-Section Library Management," ORNL-TM-4015, Oak Ridge National Laboratory, (December 1972).

5. R. Douglas 0'Dell, "Standard Interface Files and Procedures for Reactor Physics Codes, Version IV," LA-6941-MS, Los Alamos National Laboratory, (September 1977).

6. ANS Standard "Recommended Programming Practices to Facilitate the Interchange of Digital Computer Programs," prepared by Subcommittee 10, ANS Standards Committee (Apri1 1971).

7. B. M. Carmichael, "Standard Interface Files and Procedures for Reactor Physics Codes, Version III," LA-5486-MS, LoS Alamos National Laboratory, (February 1974). 
8. J. 0. Johnson, J. D. Drischler, and J. M. Barnes, "Analys is of the Fal1-1989 Two-Meter Box Test Bed Experiments Performed at the Army Pulse Radiation Facility (APRF)," ORNL/TM-11777, Dak Ridge National Laboratory, (May 1991).

9. R. T. Santoro et al., "DNA Radiation Environments Program Fall 1989 2-Meter Box Experiments and Analys is," ORNL/TM-11840, Dak Ridge National Laboratory, (May 1991).

\subsection{SAMPLE PROBLEM}

The sample problem demonstrates the calculation of the air-over-ground environment for the two-meter box experiments. ${ }^{8,9}$ Figure 3-1 shows a simple diagram of the geometry modeled in the GRTUNCL input. A complete listing of the input cards for the sample problem is given in Figure 3-2, and some selected output is shown in Figure 3-3.

In viewing Figure $3-2$, the input illustrates; 8 material zones (IZM), 66 radial intervals (IM), 84 axial intervals (JM), 69 energy groups (IGM), a cross-section table length (IHM) of 72 , no upscatter (IHT=3, and IHS =4), and a mixing table length (MS) of 0 . No material data was read in from cards $(M C R=0)$ and 54 materials ( 9 different mixtures, each with a $P_{0}$ through $P_{5}$ component) were read in from a GIP tape (MTP=54, and IDAT $1=2$ ). Thus, the tota 7 number of materials (MT) was 54 , and no print was requested for the printed output (IPRTC=1). A DOT IV formatted first collision source file was output (IMODE $=4$ ) on logical unit 23 (NPSO=23) and the first five groups of uncollided fluence were printed (IPRTF=5). The input source was a point source located 16.143 meters above the $z=0.0 \mathrm{plane}(\mathrm{ZPT})$, at $r=0.0$ (RPT), and was entered as an angular/energy dependent source with 40 directions specified $(I Z 3=40)$. Eighteen materials ( 3 mixtures) were used in the calculation which correspond to the first eighteen materials output in the GIP sample problem shown in the last section (ground, borated concrete, and air). The three mixtures and eight material zones illustrates the multiple zone option per material eluded to in Section 3.3. All comments after the slash (/) are ignored in FIDO input and are only useful for quick identification of the input parameters. The GRTUNCL sample input in Figure 3-2 shows an angular and energy dependent source distribution with units $4 \pi^{*}$ particles/steradian per leaking neutron. The energy dependence (15* array) is already represented in the shape function (14* array) and is therefore entered as ones.

The selected GRTUNCL output shown in Figure 3-3 first illustrates the input parameters read in the first data block, followed by the blocking and memory requirements to run this particular GRTUNCL case with this order of blocking, and an indication that the second data block has been successfully read. The output then produces the zone number by interval map, the material number by interval map, and a table listing the input in the $2^{*}, 3^{*}, 4^{*}, 6^{*}, 7^{*}, 9^{*}, 10 \$, 11 \$$, and $12^{*}$ arrays. This output is uc ful for checking the input to make sure there are no errors. This 
table is followed by a listing of the fission spectrum (1* array), then the source distribution $\left(13^{*}, 14^{*}\right.$, and $15^{*}$ arrays). The output then shows some messages informing the user of the completion of the calculations (by block) and the time required to perform each block. After the calculation is complete (all blocks calculated), GRTUNCL prints the balance table. This table is useful for checking the source normalization, and leakages for the system. Errors in the mode1, cross sections, or mesh should make themselves evident in this table.

Finally, the output lists the fission density by interval and the first collision source by interval (for the number of groups specified in the input). 


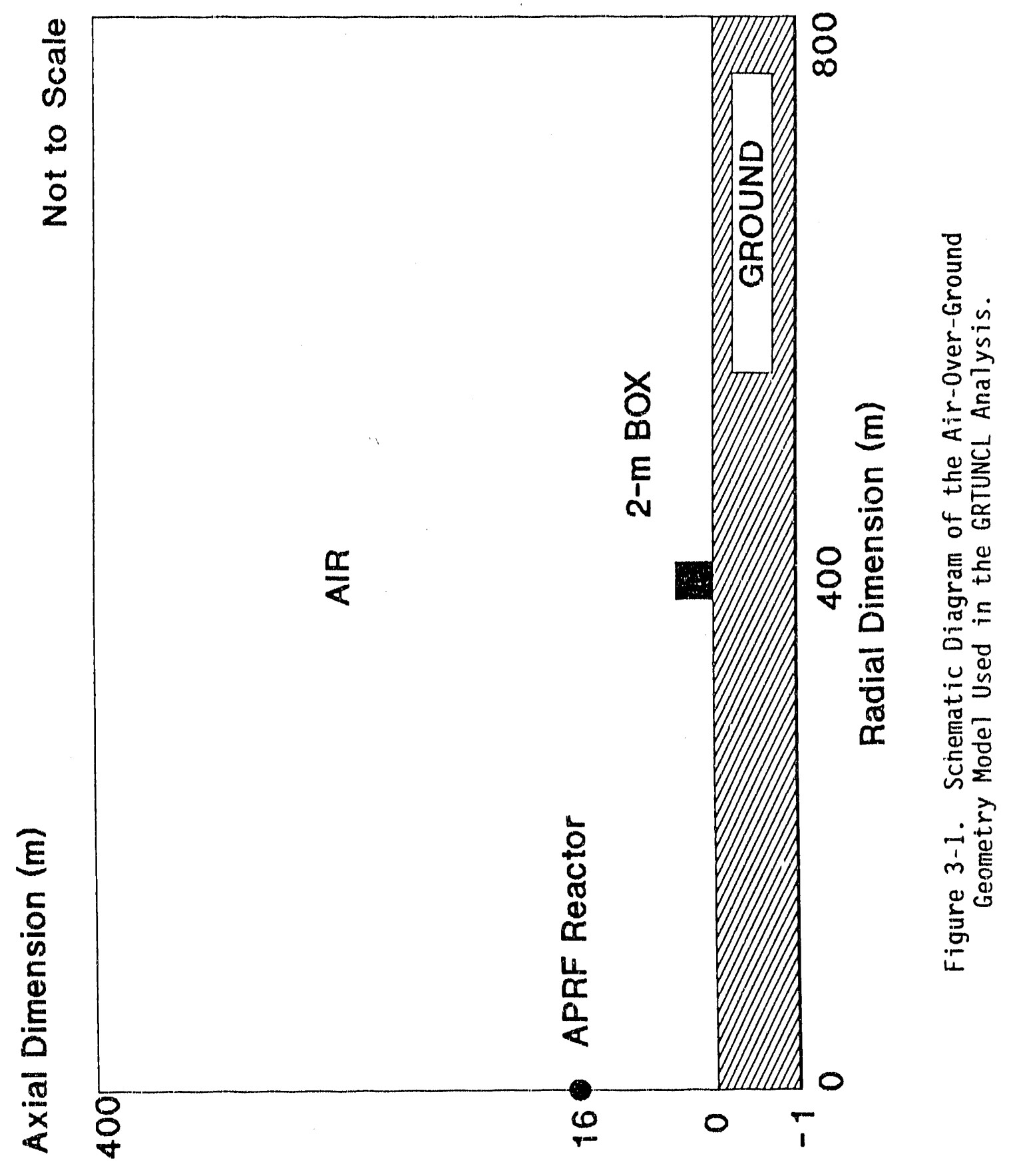


1 aprf air-over-ground using new saic 1989 angle-energy leakage source

$16.143 \mathrm{~m}$ source height, simple topography out to $400 \mathrm{~m}$ test site

' $34 \%$ ground moisture, $10 / 24 / 89$ (a) air parameters

$1 \$ \$ 586684$ / ith, isct, izm, im,jm

6934720 / igm, iht, ihs, ihm, ms

0545420 / mcr, mtp, mt, idail, noa

$\begin{array}{lllll}4 & 1 & 0 & 235 & 5\end{array}$

04003000 / iprts, iz3, idfac, nbuf, ntnpr

2** 01614.30 / xnf,zpt,rpt

$t$

1** fo /fission spectrum

$2 * * / a x i i(j m+1)$

$\begin{array}{lllllllllllllllll}-80 & -75 & -70 & -65 & -60 & -55 & -50 & -45 & -40 & -35 & -30 & -25 & -20 & -15 & -10 & -5 & -2.5\end{array}$

$\begin{array}{llllllllllllllll}-1 & 0 & 50 & 150 & 250 & 489 & 749 & 949 & 1102 & 1220 & 1311 & 1381 & 1435 & 1476 & 1508 & 1532\end{array}$

$\begin{array}{lllllllllllll}1551 & 1566 & 1577 & 1585 & 1592 & 1597 & 1601 & 1604 & 1607 & 1608.5 & 1610 & 1614.3 & 1619\end{array}$

$1620.5 \quad 1622 \quad 1624 \quad 1627 \quad 1631 \quad 1636 \quad 1643 \quad 1651 \quad 1662 \quad 1677 \quad 1696 \quad 1720 \quad 1752$

$1793 \quad 1847 \quad 1917200821262279247927393076 \quad 3514 \quad 40854826 \quad 57897042$

86701078713000160001900022000250002800031000340003700040000

$4 * * / \operatorname{radii}(\mathrm{im+1})$

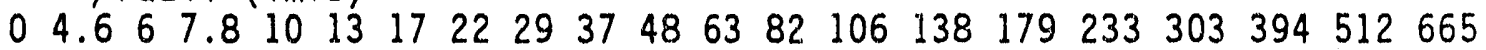

$\begin{array}{llllllllllllll}865 & 1125 & 1462 & 1900 & 2471 & 3212 & 4175 & 5428 & 7056 & 9000 & 11000 & 13000 & 15000\end{array}$

1650017500190002100023000250002700028500295003050031500

3300035000370003850039500405004150043000450004700049000

5100054000570006000063000660006900072000750007800080000

$8 \$ \$$ / zone numbers by interval

1 zones 1, 2, \& 3-aprf ground, 4 \& 5-borated concrete, 6, 7, \& 8-air $66 r 18 q 66 / j$-ints $1-9$

$19 r 547 r 12 q 66 / j$-ints $10-12$

19r4 $3 r 3 \quad 8 r 13 r 3 \quad 3 r 2 \quad 5 r 3 \quad 3 r 2 \quad 4 r 3 \quad 3 r 2314 r 15 q 66 / j$-ints $13-18$

$33 r 7 \quad 3 r 6 \quad 5 r 7 \quad 3 r 6 \quad 4 r 7 \quad 3 r 67$ 14r8 $3 q 66$ /j-ints $19-22$

$52 r 714 r 83 q 66 \quad / j$-ints 23-26

$66 r 871 q 66 / j$-ints $27-98$

$9 \$ 3 r-12 r-73 r-13$ / mat by zone

Figure 3-2. Sample GRTUNCL Input for the Two-Meter Box Air-Over-Ground Analys is. 
$13 * *$ / angular directions of source ( -1 . is down +1 . is up)

$-.99794-.98973-.97337-.94900-.91680-.88117-.84355-.80122$

$-.75441-.70316-.64809-.58978-.52822-.46383-.39684-.32761$

$-.25670-.18443-.11105-.03705+.03705+.11105+.18443+.25670$

$+.32761+.39684+.46383+.52822+.08978+.64809+.70316+.75441$

$+.80122+.84355+.88117+.91680+.94900+.97337+.98973+.99794$

14 **

$\rightarrow$ aprf angular leakage source, s(angle, energy)/40 angles/dabl69/(3-1-90) ' units are $4^{*}$ pi*particles/steradian per leaking neutron

$\begin{array}{llllllllll}1.41 \mathrm{e}-06 & 1.43 \mathrm{e}-06 & 1.65 \mathrm{e}-06 & 1.85 \mathrm{e}-06 & 2.02 \mathrm{e}-06 & 2.37 \mathrm{e}-06 & 2.49 \mathrm{e}-06 & 2.60 \mathrm{e}-06\end{array}$

2.67e-06 2.74e-06 2.70e-06 2.75e-06 2.81e-06 2.86e-06 2.91e-06 3.00e-06

3.03e-06 3.05e-06 3.07e-06 3.07e-06 3.07e-06 3.07e-06 3.07e-06 3.07e-06

[IZ3*IGM entries]

$\begin{array}{lllllllll}3.05 e-07 & 2.88 e-07 & 2.60 e-07 & 2.21 e-07 & 1.88 e-07 & 1.88 e-07 & 1.92 e-07 & 1.94 e-07\end{array}$

$\begin{array}{lllllllll}2.00 e-07 & 1.99 e-07 & 2.02 e-07 & 2.04 e-07 & 2.04 e-07 & 2.02 e-07 & 2.02 e-07 & 2.09 e-07\end{array}$

2.11e-07 2.11e-07 2.04e-07 1.34e-07 9.76e-08 8.75e-08 7.01e-08 6.14e-08

$15^{* *}$ / source multiplier for each energy group $69 r 1.0$

$t$

Figure 3-2. (continued) 


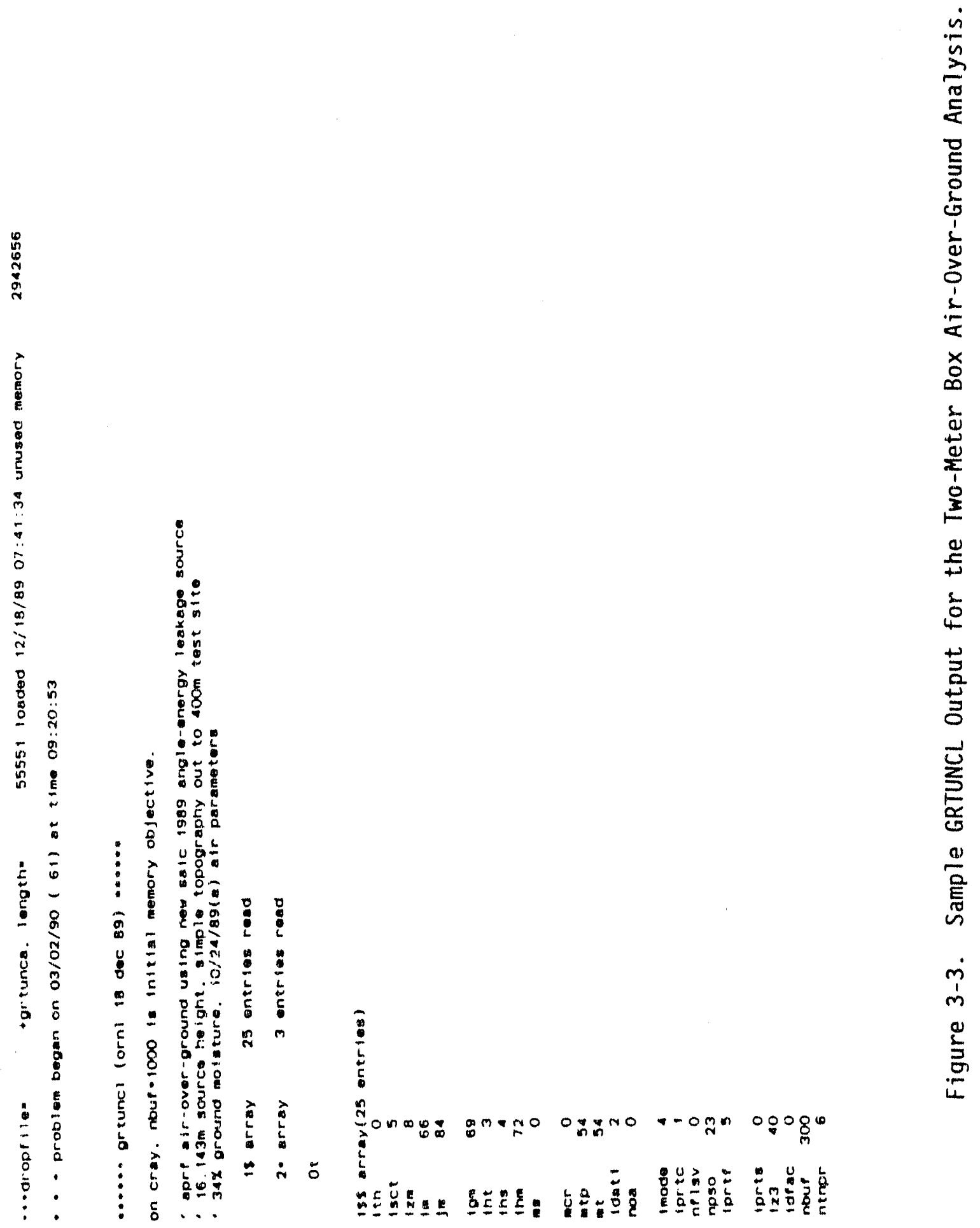





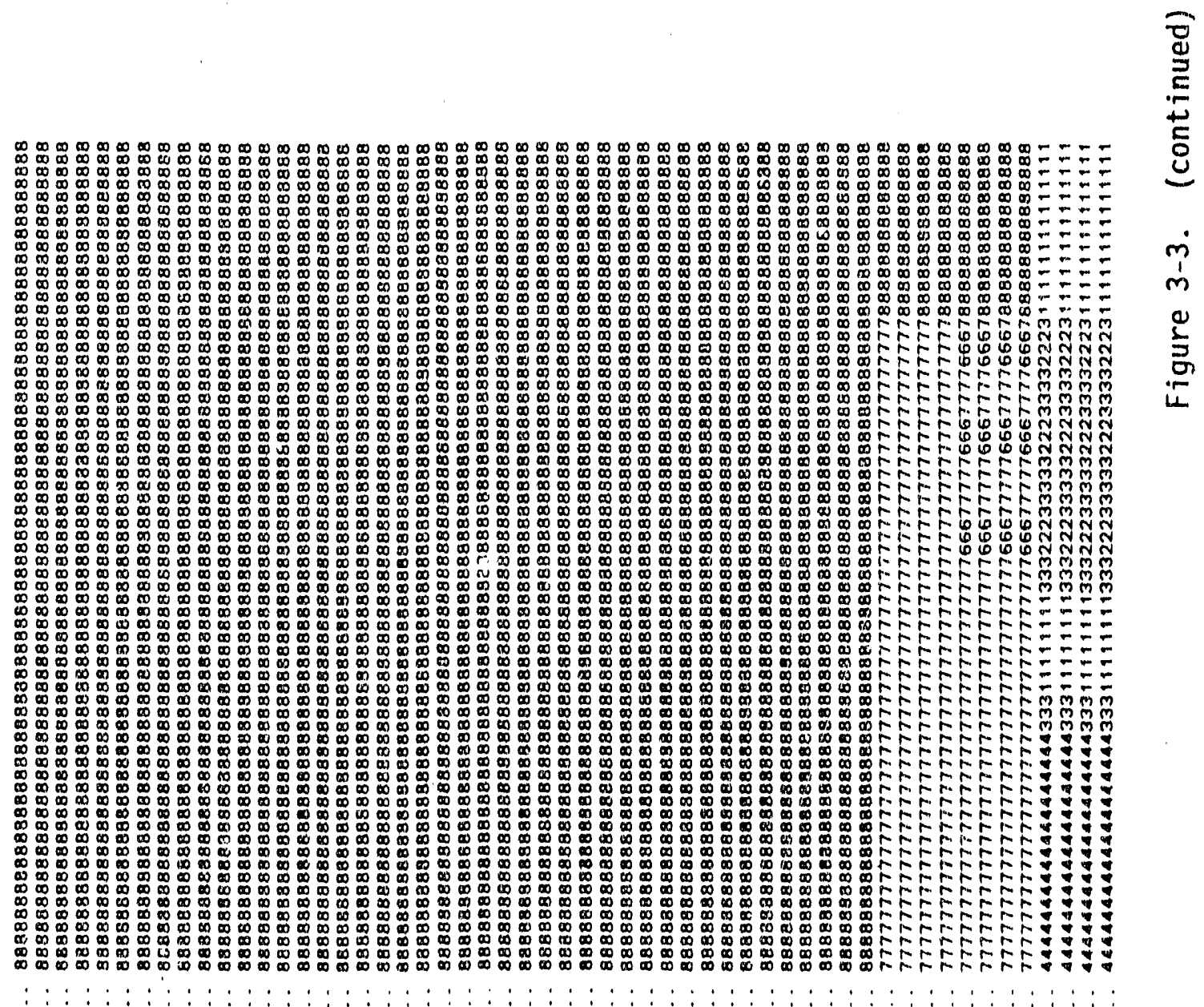

ดิ 


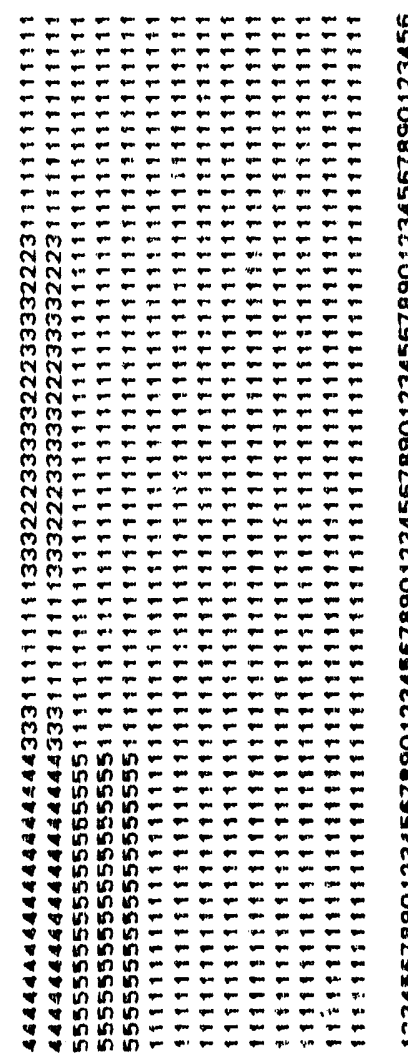

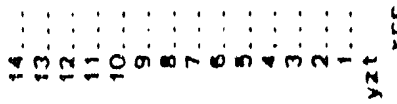

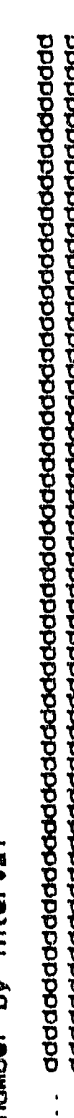

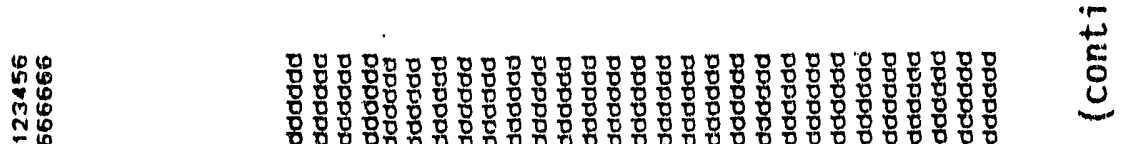

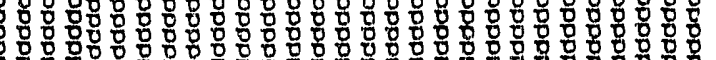

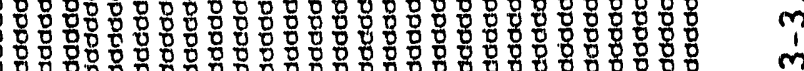

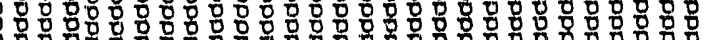

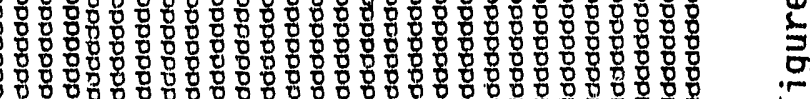
作

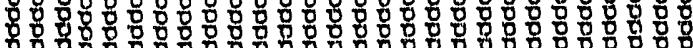

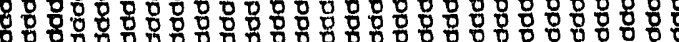

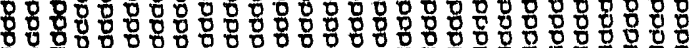

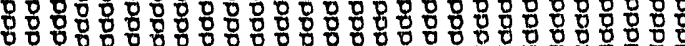
0.5
0

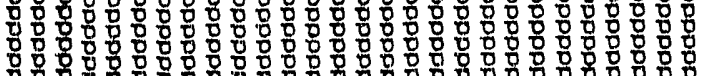

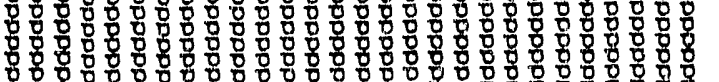

$\widehat{\mathrm{d}}$

.

U. $\dot{m}$

疍

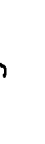




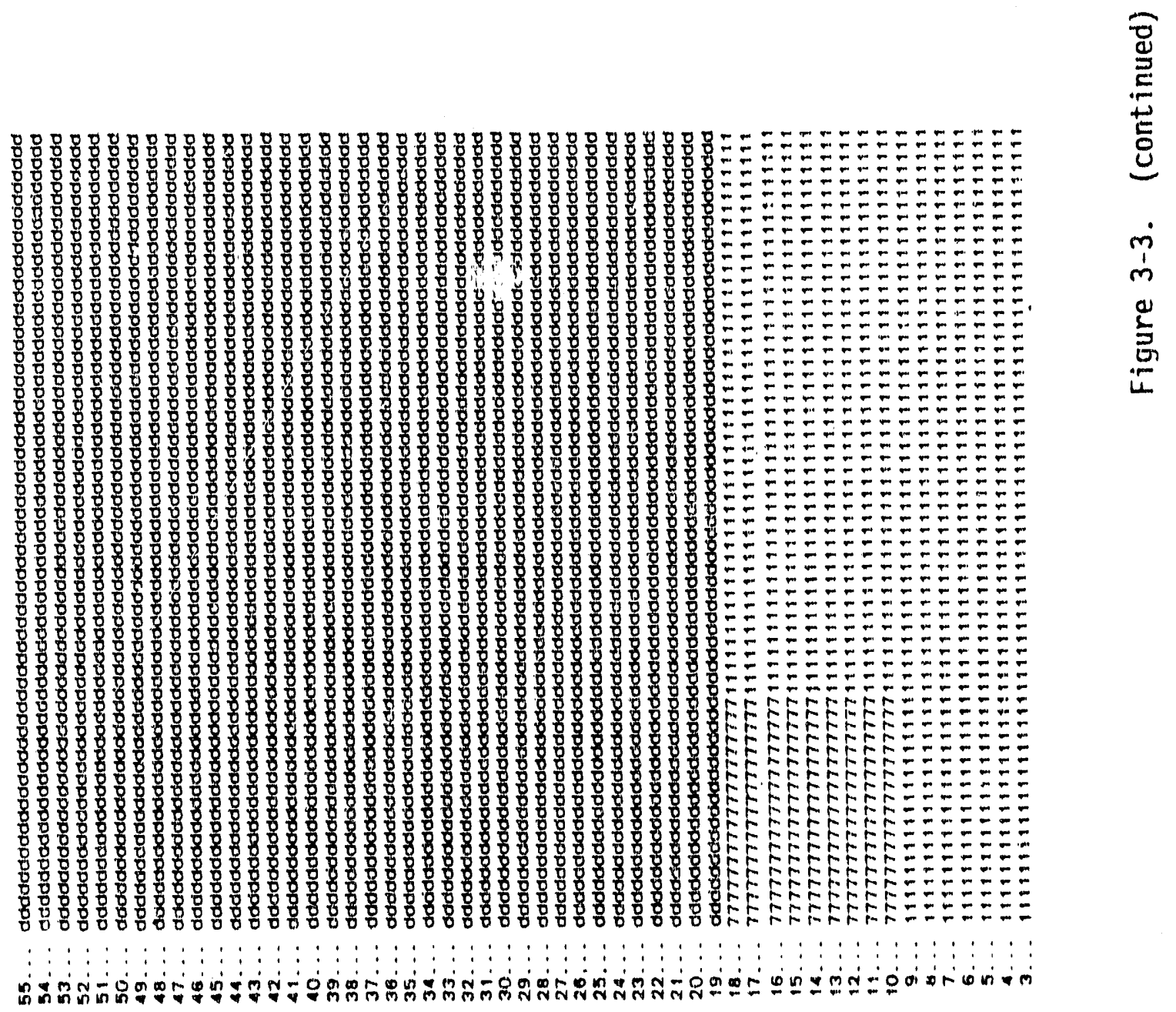




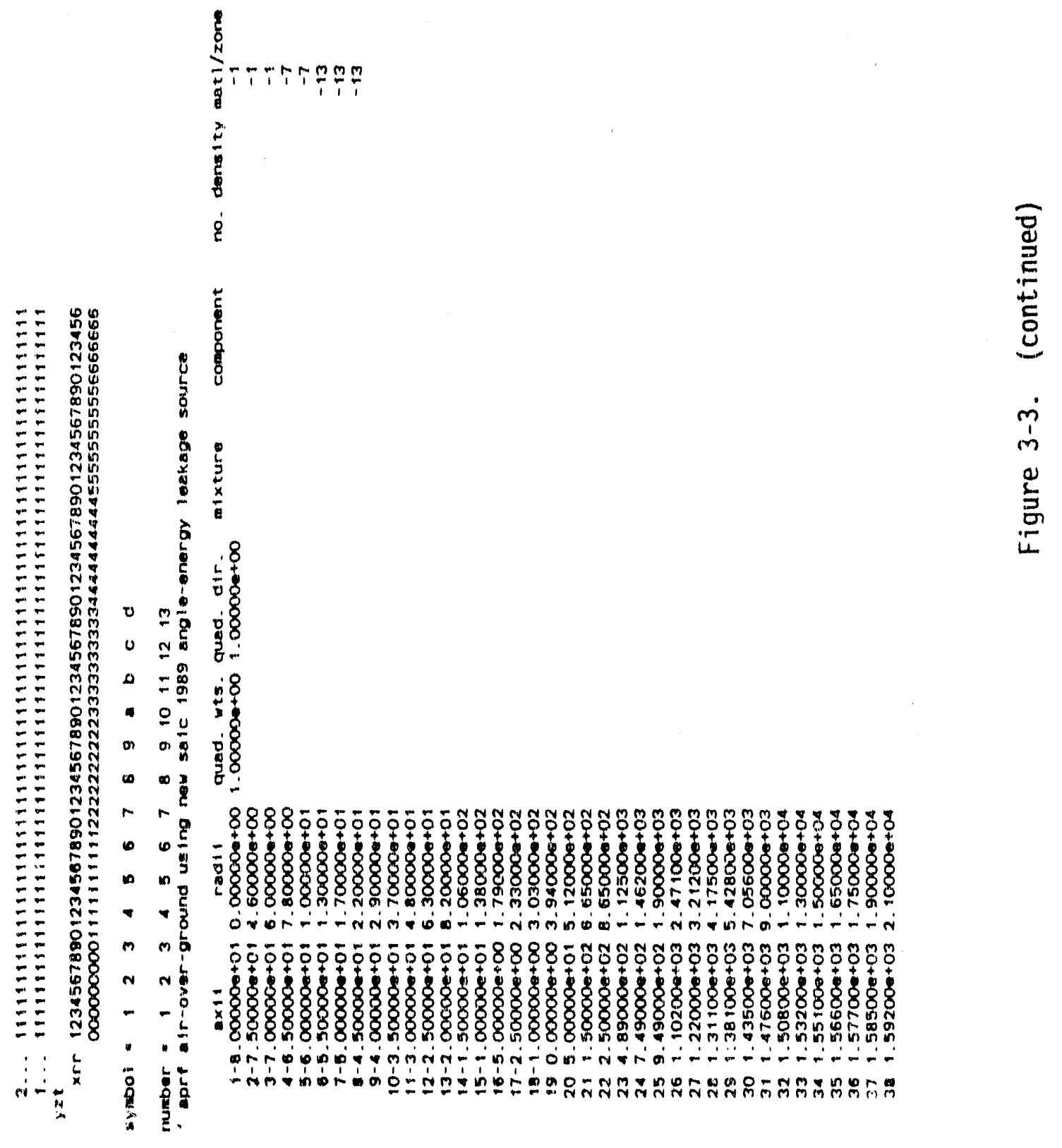




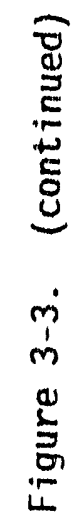
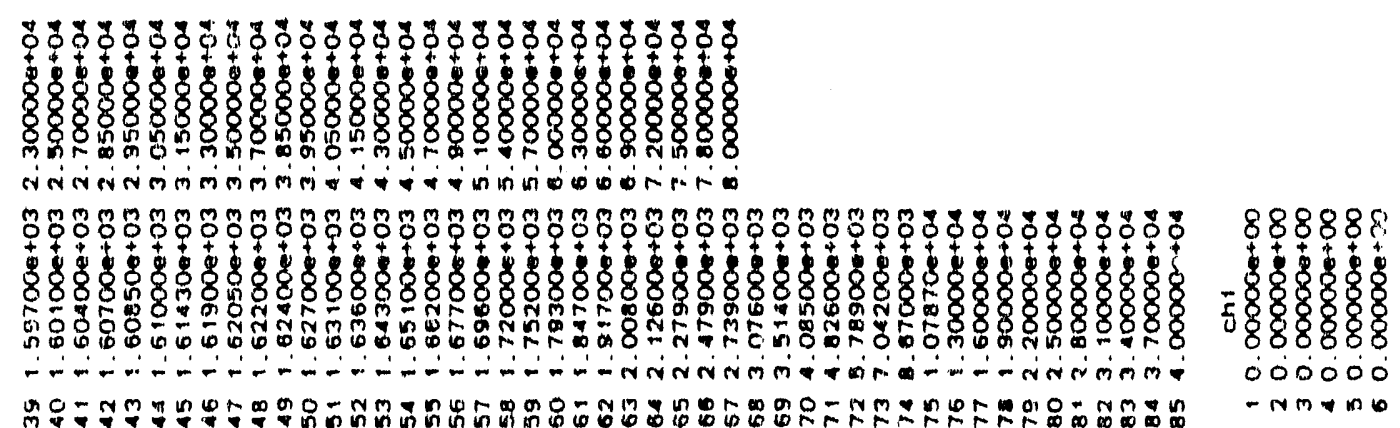
导

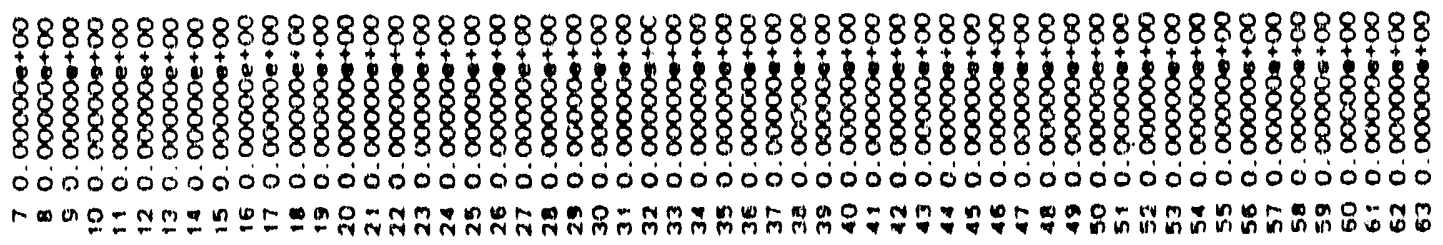




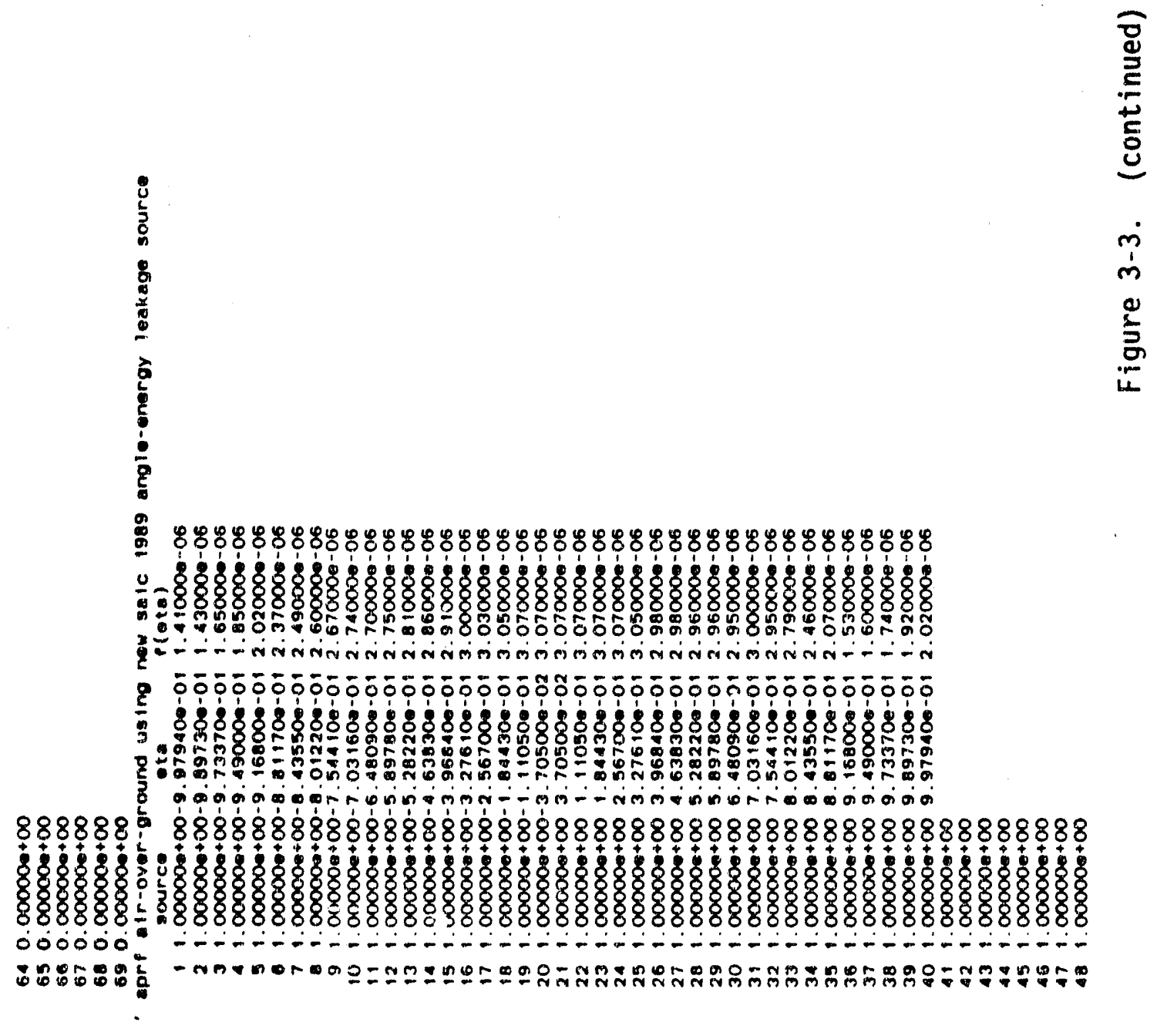

ฮั

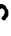

5 


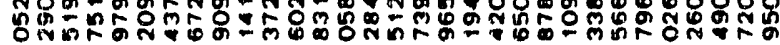

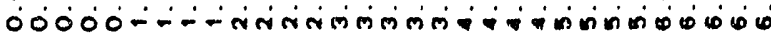

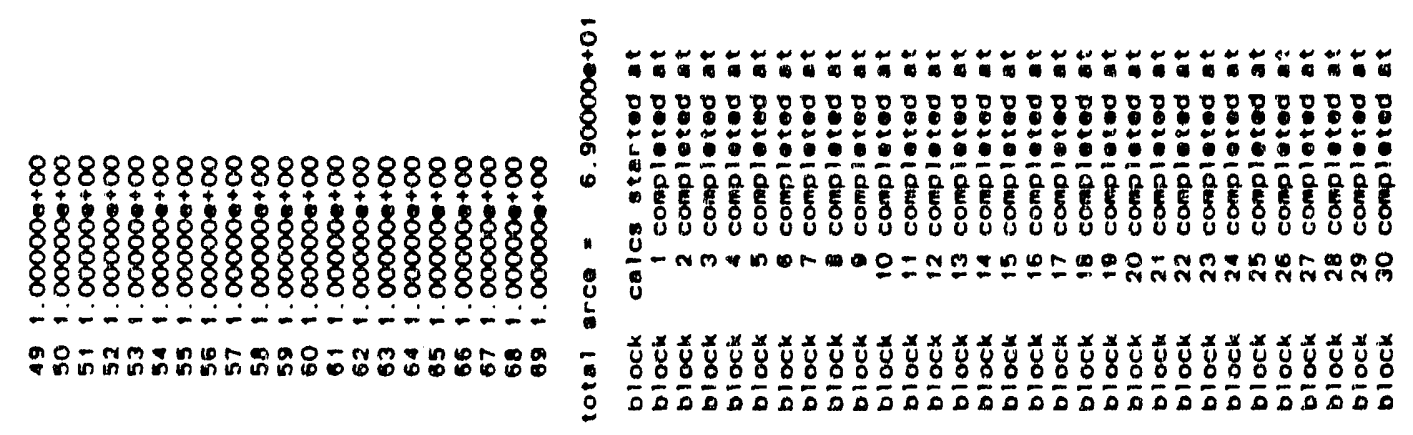




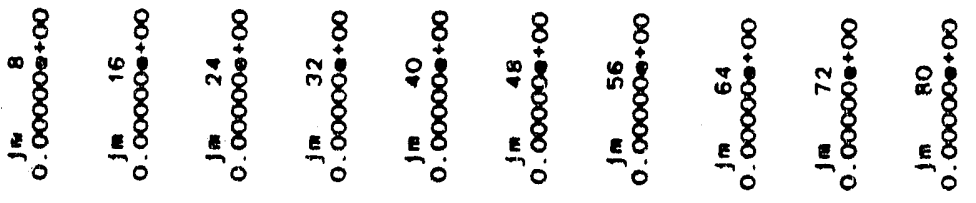

||

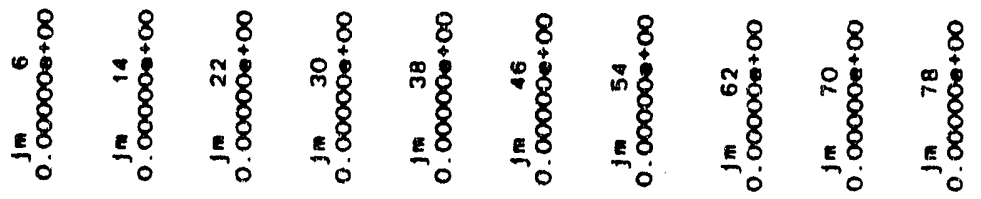

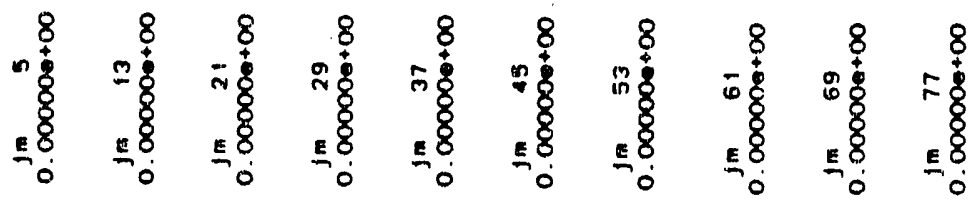

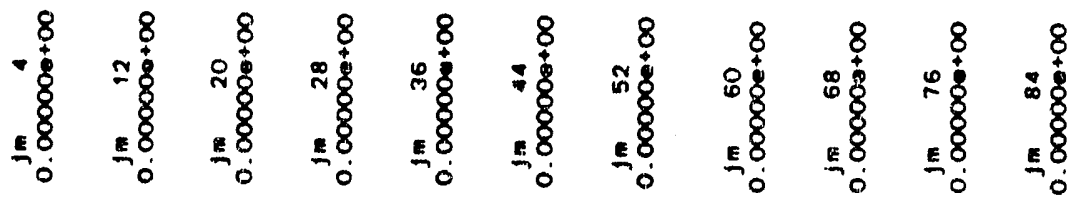

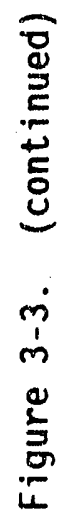

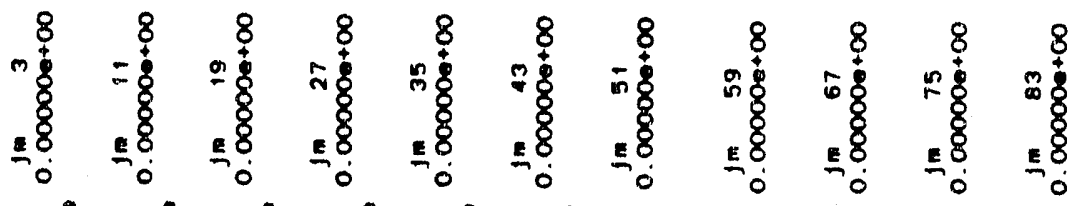

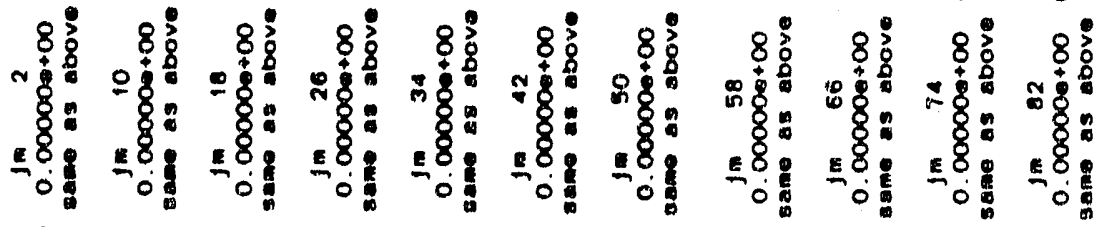

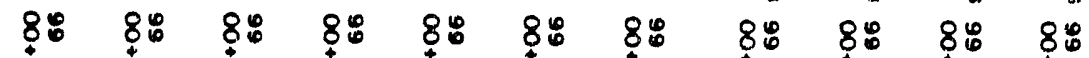

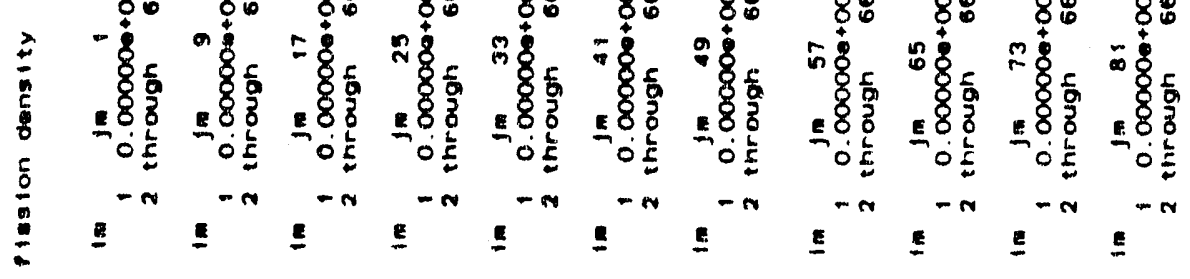




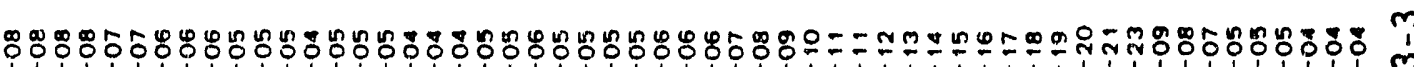
等

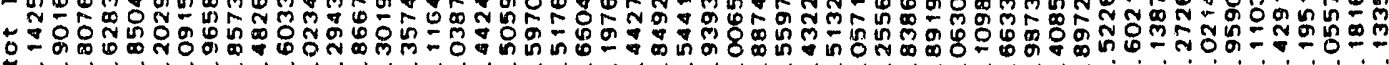

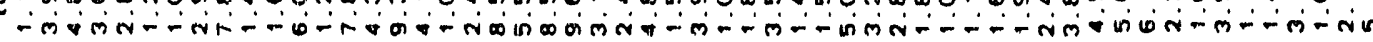

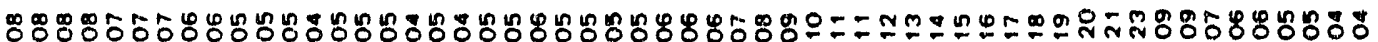

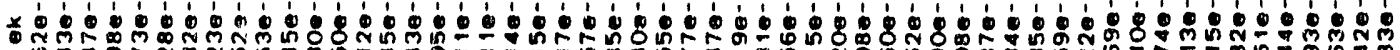

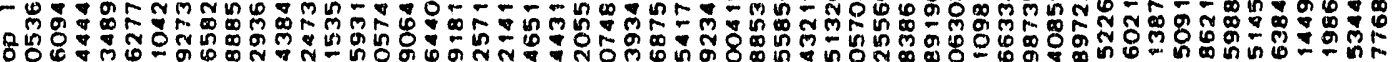
- mañ

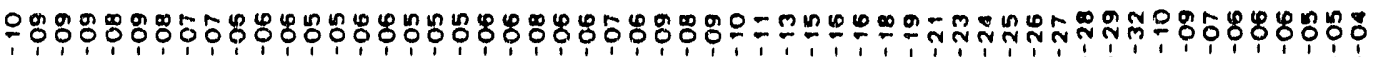

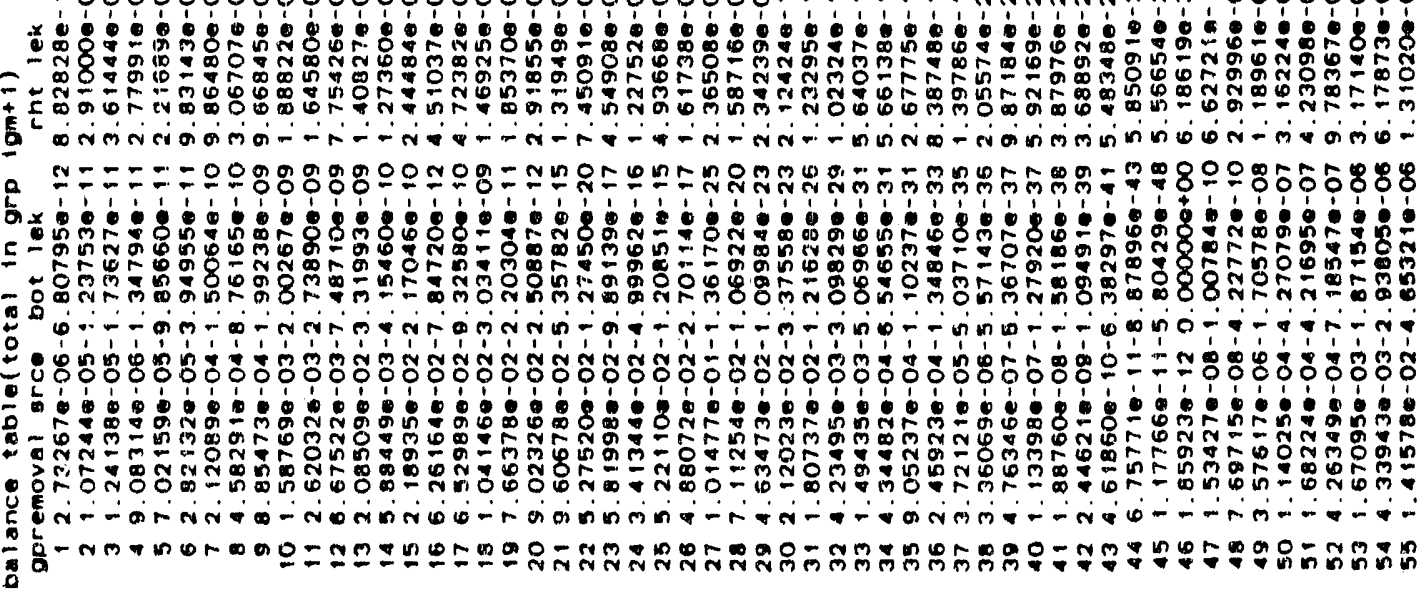




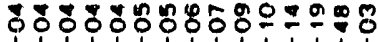

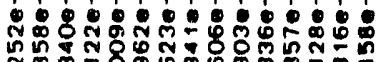

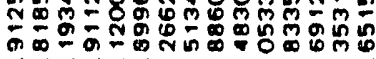

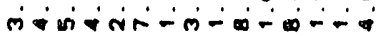

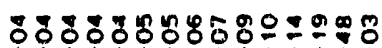

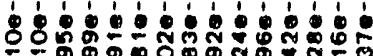

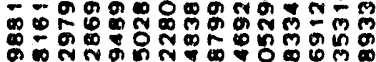

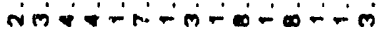

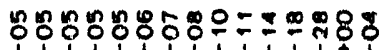

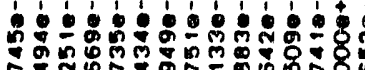

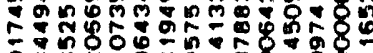
.

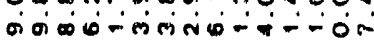

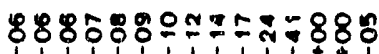
-

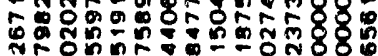

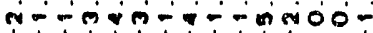

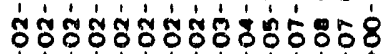
-

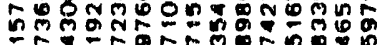

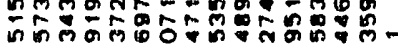

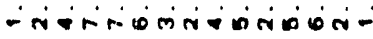

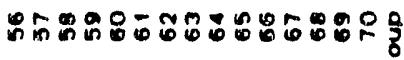

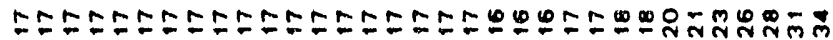

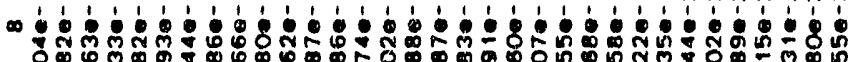
N कம ம் ம்

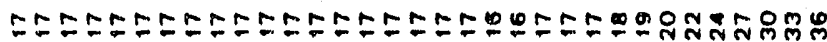

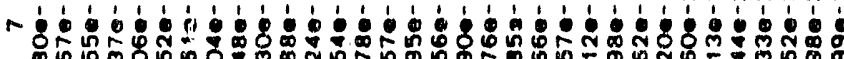

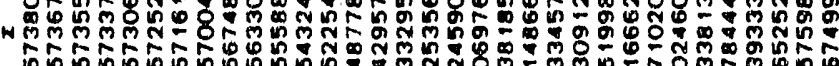

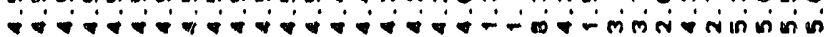

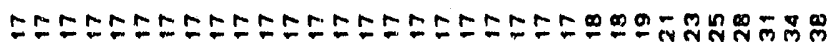
๑

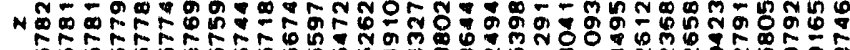

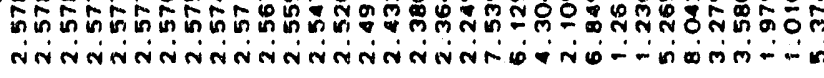

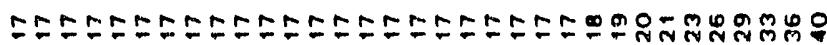
๓

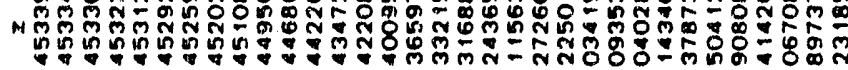

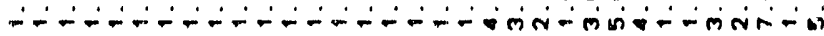

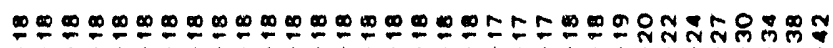
-

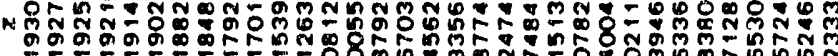

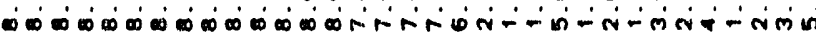
๑ฒ m N

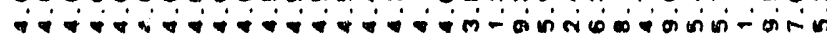

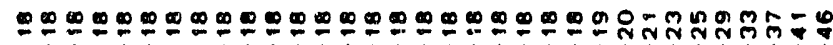
พ

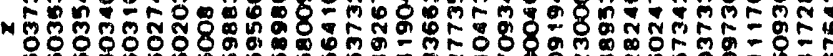
तथं

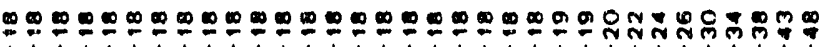
-

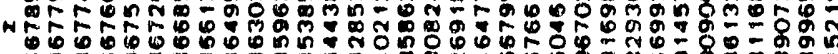

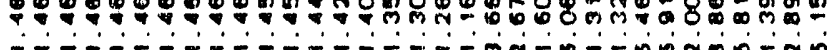

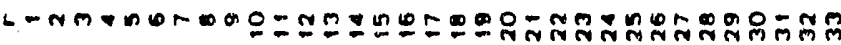




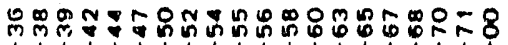

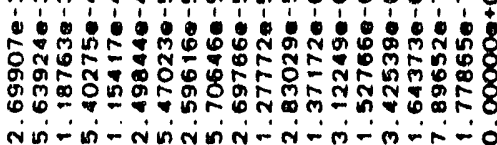

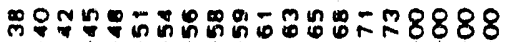

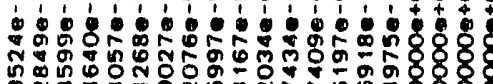

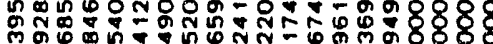

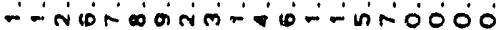

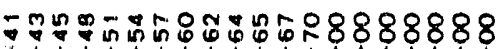

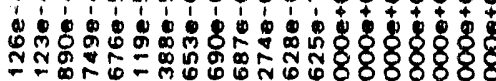
¥

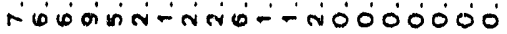

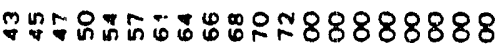

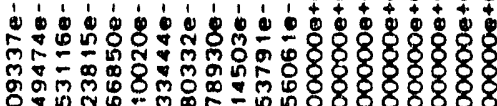

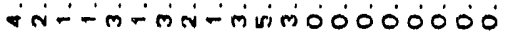

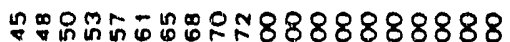

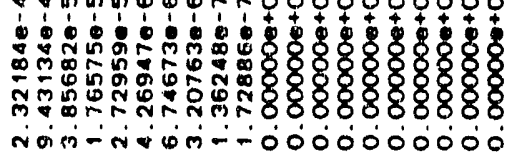

$F=5=$
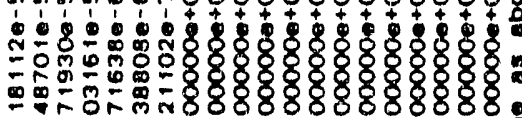

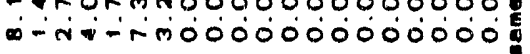

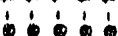

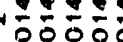

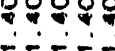

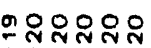

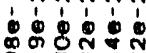

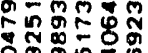

$\therefore$ rivini

กิ์

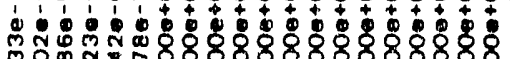

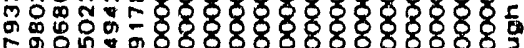

20

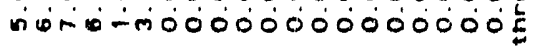

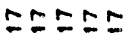

$\therefore \dot{0} \div \dot{0}$

10

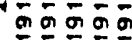

तinivin

우윰유유

I

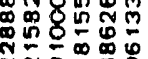

-

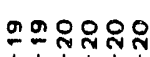

S.

两

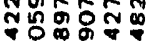

$\therefore$-rin-

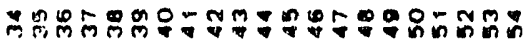

으무융ำ

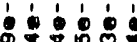

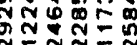

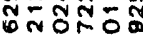

$\therefore$ कivinis

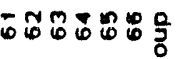




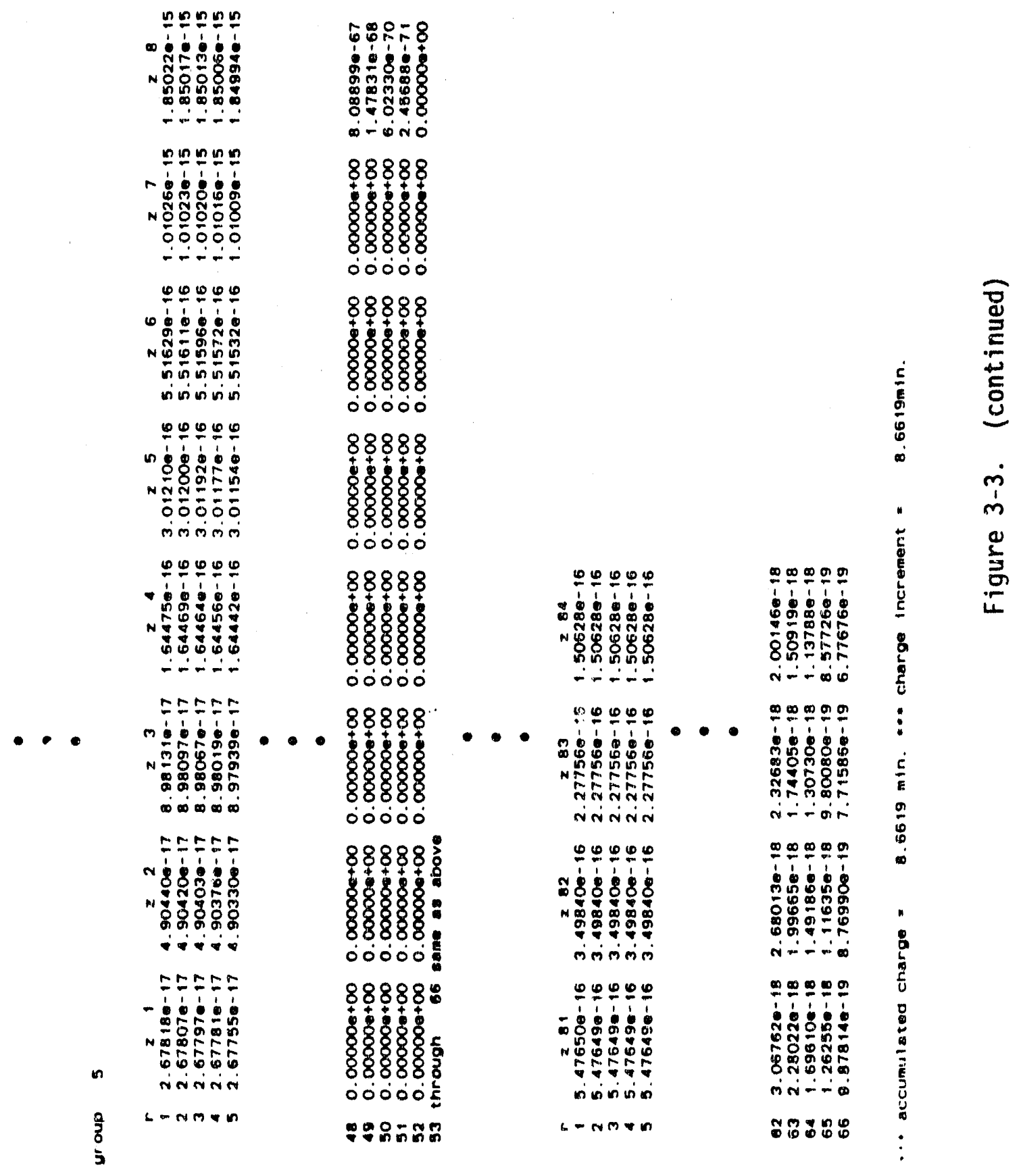




\subsection{DORT: A TWO-DIMENSIONAL DISCRETE ORDINATES TRANSPORT CODE*}

\subsection{INTRODUCTION TO DORT}

\subsubsection{Background}

A summary of the mathematical basis for the method of discrete ordinates as we use in DORT ${ }^{1}$ today is given by Lathrop and Brinkley. ${ }^{2}$ Mynatt et a1. ${ }^{3}$ give a full mathematical development, applications information, and comparisons of results with experiments. Carlson and Lathrop ${ }^{4}$ give a full development of the method with emphasis on physical principles and evaluation techniques. They also give an annotated bibliography of early work in the field including a very early report by Carlson in 1953.5 A 1958 paper by Carlson and Bel16 may have been the first widely-distributed description of the "discrete $S_{n}$ method" as they called it. In 1960, Carlson, Lee, and Worlton published a description of the DSN code, which used their method. ${ }^{7}$ Davison, $:$ in 1957 , gave a thorough review of fundamental discrete-ordinates theory, and a review of Carison's work in an Appendix. Davison acknowledges a 1953 book by Chandrasekhar as the origin of the method as he presents it, and a 1943 paper by Wick ${ }^{10}$ as the origin of the basic idea.

In this section, we follow the notation of Lathrop and Brinkley to some extent. Their TWOTRAN II code was useful to us in constructing DOT $4^{11}$, and it may be worthwhile to comment on the relationship between TWOTRAN and DOT. The earlier versions of DOT actually descended from the LASL codes DTK and DDK, and from the LASL/UNC code DDF/2DF. A verston of DOT was distributed informally in 1966, although formal publication was not made until the DOT III version in 1973.12 Early versions of TWOTRAN were published in a $1968 \mathrm{GA}$ report ${ }^{13}$ and elsewhere.

Descendants of both TWOTRAN and DOT remain in widespread use today, and their very different capabilities apparently justify this dual

existence. Numerous other writings pertinent to DOT development. appear in ANS Transactions and elsewhere. A reasonably complete bibliography of related material is give in Appendix $C$ of Reference 14.

\subsubsection{The Problem Solved}

DORT is primarily intended to solve large neutron and photen transport problems on a wide variety of computer types using the method of discrete ordinates. Most DORT problems deal with the calculation of

\footnotetext{
*W. A. Rhoades and R. L. Chiids, "The DORT Two-Dimensional Discrete Ordinates Transport Code," Nuclear Science \& Engineering 99 , pp. 88-89, (May 1988).
} 
radiation resulting from a given extraneous source, i.e., "fixed-source" problems. If such a system has fissile material and is subcritical, the multiplication can be calculated. The code also has $K_{\text {eff }}$ capability and various types of searches. A diffusion theory section is valuable for initiating such problems or as an alternative to the primary discrete ordinates section where applicable.

Special remeshing features allow the number of first-dimension (i) mesh intervals to vary with the second dimension (j) index. An arbitrary coarse mesh can be used for fluence acceleration, saving memory and CPU time. The directional quadrature set can be chosen from an arbitrary number of input sets. The choice can vary with spatial option and with energy group. The flexibility has proven quite effective in concentrating CPU effort in areas needing attention, such as streaming Gaps. Biased direction sets can be used when streaming is primarily in the upward/downward directions.

A variety of options allow sources to be specified at internal or external boundaries, distributed by space and energy, or determined from an input fluence guess. "First-collision-source" data, actually an analytical first-flight scattering source, can be accommodated. Output files for an "analytical-last-flight" integration can be obtained. These features provide increased accuracy when dealing with out-ofsystem or localized sources or detector locations.

A single output file contains both distributed fluence moments and boundary directional fluences, so that this file, plus the original input data, provide an "exact" restart. This allows a large problem to be solved in several computer runs without loss of efficiency.

Both 1-D and 2-D geometries can be treated. Discrete ordinates geometries include 1-D plane or slab and 2-D XZ, RQ, or RZ. The diffusion-theory section has triangular-mesh capability as well. A powerful slab reflection/transmission feature is available. A variety of acceleration options are available. Extensive use of input options and output edits give the user very direct control over the iteration process. While this places a burden of decision-making on him, it is essential to the solution of large and difficult problems. Default and recommended values assist the uninitiated in solving problems without much prior use of the code.

Output source information to be used in coupling to other problems can be obtained. Typical applications include "bootstrapping," in which a very large problem is solved in several segments; core-vessel combinations, in which a core is solved in an eigenvalue calculation and then represented by internal boundary conditions in a fixed-source vessel calculation; coupled discrete-ordinates-Monte Carlo calculations; and RZ air-ground calculations coupled to 3-D discrete ordinates analysis of local features. 
Portions of this section were extracted from Reference 14 for completeness. The interested user should consult that document for a full description of the DORT code. An extensive problem set and a tutorial description is available to demonstrate the various features and problem setup techniques.

\subsubsection{Code Structure}

This code and its predecessors have been adapted to a large variaty of computers from the IBM 7090 to modern Crays. In order to maintain compatibility without undue confusion, a "unified source file" concept is used; only a single version of the basic code is kept, with final configuration details supplied when a file is prepared for compilation. Standard language and programming practices are used throughout the main body of the code. In-stream "language flags" make minor language adjustments as required when a specific configuration is tailored. Interchangeable interface packages provide coupling with systemdependent library features.

On all computers, direct (random) access scratch files are used as an extension of memory. Data are retrieved from the files into large buffers controlled by the I/O manager routine. The working program then uses and updates the buffers before their return to the scratch files. Since communication with the buffers is processed entirely through the inter face package, the buffers can be located in a large cache memory such as the LCM on the late CDC 7600 or, presumably, on a Cray SSD device. The code attempts to make the maximum use of available memory in order to minimize $1 / 0$ costs and delays. Reversion to more extensive use of scratch-files is automatic when required.

The flexibility provided, together with the close control of the solution process, requires an extensive and complicated input data list. Most of these data can be ignored for most problems. A diverse set of sample problems serves as a guide to actual requirements for practical problems.

\subsection{THEORETICAL BASIS}

\subsubsection{The Integro-Differential Transport Equation}

The Boltzmann transport equation, as applied to static neutron and photon transport, ${ }^{2}$ can be expressed: 
$\underline{\nabla} \boldsymbol{\Omega} \psi(\underline{\underline{r}}, \mathrm{E}, \boldsymbol{\Omega})+\sigma^{T}(\underline{r}, \mathrm{E}, \boldsymbol{\Omega}) \psi(\underline{r}, \mathrm{E}, \boldsymbol{\Omega})=$

$$
\begin{aligned}
& \iint \sigma^{\mathbf{s}}\left(\underline{\underline{r}}, \mathrm{E}, \mathrm{E}^{\prime}, \underline{\Omega}, \underline{\Omega}^{\prime}\right) \psi\left(\underline{\boldsymbol{\Omega}}, \mathrm{E}^{\prime}, \underline{\Omega}^{\prime}\right) \mathrm{d} \boldsymbol{\Omega}^{\prime} \mathrm{d} \mathrm{E}^{\prime} \\
& +\frac{1}{4 \pi} \chi(\underline{r}, E) \iint \nu\left(\underline{r}, E^{\prime}\right) \sigma^{F}\left(\underline{r}, E^{\prime}\right) \psi\left(\underline{r}, E^{\prime}, \Omega^{\prime}\right) d \underline{\Omega}^{\prime} d E^{\prime}+Q(\underline{r}, E, \Omega)
\end{aligned}
$$

In this, oh is a differential solid angle about the direction vector $\Omega$, $d E$ is a differential energy about $E$, and $r$ is a position vector with reference to an arbitrary origin. The directional fluence function, $\psi$, is defined such that the number of particles moving in volume dr about $r$ with energies falling within $d E$ and directions of motion within ds will be $\psi$ chd $2 d r$. Also, $\sigma$ and $\sigma^{F}$ are macroscopic cross sections for total interaction and fission, respectively, and $\sigma^{\mathbf{s}}$ is the cross section for scattering from energy $E^{\prime}$ and direction $\Omega^{\prime}$ to energy $E$ and direction $\Omega$. The functions $\nu$ and $\chi$ represent the total fission yield of secondary particles and the corresponding energy distribution. $Q$ represents an extraneous source, if any.

Although the general Boltzmann equation includes time dependence, removal of that dependence in the static form presented here is an important simplification. Certain other simplifications will also be made later. As a practical matter, scattering is generally independent of initial direction, so that it can be expressed in terms of the single scattering angle:

$$
\mu_{0}=\underline{\Omega}^{\prime} \cdot \underline{\Omega}
$$

rather than as a function of two direction vectors. Although some applications exist for the dependence of $\sigma^{\top}$ on $\Omega$, they are unusual, and this dependence is not allowed in DORT. A somewhat weaker justification exists for the removal of the dependence of $\chi$ on space. In fact, if more than one fissile species exists in the system, and if the species do not always fission in the same proportion, $\chi$ will depend on space. Nonetheless, the code does not presently allow such dependence.

\subsubsection{The Finite Difference Formulation}

The procedures for obtaining a finite-difference equation from Equation 4-1 are discussed thoroughly in References 3 and 4, aithough the form presented here follows Reference 2: 


$$
\begin{aligned}
W_{m} \mu_{m} & \left(A_{i+\frac{1}{2}, j} N_{i+\frac{1}{2}, j, m, g}-A_{i-\frac{1}{2}, j} N_{i-\frac{1}{2}, j, m, g}\right) \\
& +W_{m} \tau_{m}\left(B_{i, j+\frac{1}{2}} N_{i, j+\frac{1}{2}, m, g}-B_{i, j-\frac{1}{2}} N_{i, j-\frac{1}{2}, m, g}\right) \\
& +\left(A_{i+\frac{1}{2}, j}-A_{i-\frac{1}{2}, j}\right)\left(\alpha_{m+\frac{1}{2}} N_{i, j, m+\frac{1}{2}, g}-\alpha_{m-\frac{1}{2}} N_{i, j, m-\frac{1}{2}, g}\right) \\
& +\sigma_{i, j, g}^{T} W_{m} V_{i, j} N_{i, j, m, g}=W_{m} V_{i, j} S_{i, j, m, g}
\end{aligned}
$$

It can be shown that the formulation of Mynatt et al. ${ }^{3}$ is equivalent to this.

In this notation, subscripts $i$ and $j$ represent mesh intervals in the first and second space dimensions. The subscript $m$ refers to the direction in a ordered set of directions along which fluence is to be evaluated. Subscripts such as $i+1 / 2$ refer to the interval boundaries, e.g., boundary $i+1 / 2$ is the boundary separating interval $i$ from interval $i+1$. In the case of $m$, this is somewhat artificial, since each $m$ represents a discrete direction with no defined sector of solid angle associated. In fact, directions represented by successive $m^{\prime}$ s may not even be "adjacent" in direction space. Even so, the equation requires coupling between certain fluences having consecutive $m$ values, and the coupling terms, $\alpha$, will be defined in a consistent manner. Subscripts such as $m+1 / 2$, then, merely denote artificial intermediate values. The subscript $g$ refers to eneigy group.

It may be noted at this point that obvious subscripts are frequently omitted in discrete-ordinates writings, e.g., the first term of Equation 4-3 might later be written:

$$
W \mu\left(A_{i+\frac{1}{2}} N_{i+\frac{1}{2}}-A_{i-\frac{1}{2}} N_{i-\frac{1}{2}}\right)
$$

In practice, this approach is probably less confusing than the inclusion of long lists of redundant subscripts.

In certain discussions, $I$ and $J$ are understood to be the upper limits of $i$ and $j$. It may also be noted that space mesh positions are discussed such that boundaries having $i=1 / 2, i=I+1 / 2, j=1 / 2$, and $j=J+$ $1 / 2$ are spoken of as "left," "right," "bottom," and "top," boundaries, regardless of geometry. 
The ordered set of directions of particle travel are characterized by their direction cosines, $\left(\mu_{m}, \xi_{m}, \eta_{m}\right)$. In all geometries, $\mu_{m}$ is the cosine of the angle which the direction of travel makes with the firstdimension axis, i.e., $X$ or $R$, while $\eta_{m}$ is the cosine of the angle with the $Z$ axis (Table 4-1). In each case, $\xi_{m}$ is the cosine with the remaining direction vector, either along the $Y$ axis, or in the azimuthal direction in the case of $R \theta$.

Due to symmetry, no flow is associated with one of the direction cosines, either $\eta$ or $\xi$. Thus, $\boldsymbol{q}$ is used to represent. either $\xi$ or $\eta$, as shown in Table 4-1. Also, $\omega$ is allowed to represent the remaining cosine. The parameters $A$ and $B$ are cell areas perpendicular to the axes from which $\mu$ and, are measured.

Each direction has an associated weight, $W_{m}$. Quantities involving integrals over all directions are to be evaluated by sums with $W_{m}$ as the weighting function. $N$ represents the directional fluence, $\boldsymbol{\phi}$, in direction, $m$, although it will be seen that $N$ has different units. $V$ and $S$ are the volume and source in a given mesh cell, and $\sigma^{\top}$ is the macroscopic total cross section. It is assumed that $S$ includes extraneous sources, scattering from other energy groups, scattering at the given energy from other directions, fission, and any other particle sources, e.g., $(n, 2 n)$.

Table 4-1. Directional Properties of the Geometries.

\begin{tabular}{|c|c|c|c|c|c|c|}
\hline & & $1-0$ & & & $2-0$ & \\
\hline Geometry & $x Z$ & RZ & $R \theta$ & $x Z$ & $R Z$ & $k \theta$ \\
\hline Dimensions Solved & $x$ & $\mathbf{R}$ & $R$ & $x, z$ & $R, Z$ & $R, \theta$ \\
\hline Dimension Corresponding to $\mu$ & $x$ & $R$ & $R$ & $x$ & R & $R$ \\
\hline Dimension Corresponding to & Y & $\theta$ & $\theta$ & $Y$ & $\theta$ & $\theta$ \\
\hline Dimension Corresponding to $\eta$ & 2 & $\mathbf{z}$ & 2 & 2 & Z & $z$ \\
\hline Cosine Corresponding to & $\eta$ & $\eta$ & $\xi$ & $\eta$ & $\eta$ & $\xi$ \\
\hline Cosine Corresponding to & $\xi$ & $\xi$ & $\eta$ & $\xi$ & $\xi$ & $\eta$ \\
\hline Dimension Perpendicular to $\mathrm{A}$ & $x$ & $R$ & $R$ & $x$ & $R$ & $R$ \\
\hline Dimension Perpendicular to $B$ & 2 & $Z$ & $\theta$ & Z & Z & $\theta$ \\
\hline
\end{tabular}


As indicated in Table 4-1, the 1-D geometries evaluate fluence dependence in only the first dimension. In such cases, the usual evaluation procedures are used, but $B=0$.

It can be observed that, except for the terms in $\alpha$, Equation $4-1$ is simply a statement of particle balance for a given cell, and could have been written down directly. From this, it is evident that the discreteordinates equation conserves particles inherently, if the a terms are defined such as to conserve.

The terms in a are effective only in curved geometry, RZ or RO. Their physical meaning can be made apparent by considering a particle moving inward along a path which will take it closer to the axis of a cylinder. While $\eta$ is a constant for such a path, $\mu$ constantly increases from negative, through zero, and ultimately toward $\mu=+1$ as the particle moves away from the centerline. Thus, the discrete-ordinates equation must show a comparable flow toward directions of larger $\mu$ for comparable particle paths.

In order to allow manageable evaluation of the terms in $\alpha$, an ordering discipline is imposed upon the directions represented by $m$. Since the $\alpha$ terms must couple directions of like $\eta$, directions of like $\eta$ are grouped contiguously, in order of increasing $\mu$. The groups of directions with like $\eta$ (" $\eta$ levels") are ordered such that all directions with $\eta<0$ precede all directions with $\eta>0$. Thus, the $\alpha$-flow, following an actual path inward and then outward, is always toward directions of increasing $m$ within an $\eta$ level. It can also be seen that there must be no flow between $\eta$ levels in order to be consistent with the physical model.

The recursion relationship for the $\alpha^{\prime} s$ can be obtained at this point by observing that Equation 4-3 must be valid in a source-free region where $N$ is uniform. Since no constraint may be placed upon $\left(\sigma^{\top}, A, B\right.$, or $V$, then it must follow that, for a given $\eta$ level:

$$
-W_{m} \mu_{m}=\alpha_{m+\frac{1}{2}}-\alpha_{m-\frac{1}{2}}
$$

Since it is required that a coupling be only between directions within an $\eta$ level, then $a_{m-1 / 2}=0$ for the first direction of the level. It is essential that, for each level:

$$
\sum_{m} W_{m} \mu_{m}=0
$$


so that $a_{n+1 / 2}$ will be 0 for the last $m$ of that level, and there will be no net flow into or out of the $\eta$ level. This insures that particle conservation is rigorously met. Symmetrical quadrature sets always obey this condition, and it is important that unsymmetrical sets obey this condition, also.

There is certain advantage to be gained by substituting a new variable, $\beta$ for $\alpha$ :

$$
\beta_{m}=\frac{1}{W_{m}}\left(\alpha_{m+\frac{1}{2}}+\alpha_{m-\frac{1}{2}}\right)
$$

The recursion relationship for the $\beta_{m}$ can be obtained by applying its definition to the recursion relationship for $\alpha$ :

$$
\begin{gathered}
W_{m} \beta_{m}=\alpha_{m+\frac{1}{2}}+\alpha_{m-\frac{1}{2}}, \\
W_{m+1} \beta_{m+1}=\alpha_{m+\frac{3}{2}}+\alpha_{m+\frac{1}{2}}, \\
\alpha_{m+\frac{1}{2}}=\alpha_{m-\frac{1}{2}}-W_{m} \mu_{m},
\end{gathered}
$$

and

$$
\alpha_{m+\frac{3}{2}}=\alpha_{m+\frac{1}{2}}-W_{m+1} \mu_{m+1} .
$$

Therefore

$$
W_{m+1} \beta_{m+1}=W_{m} \beta_{m}-\left(W_{m+1} \mu_{m+1}+W_{m} \mu_{m}\right) . \quad(4-11)
$$

Recalling that $a_{m-1 / 2}=0$ for the first direction of each $\eta$ level, then it follows that $\beta_{m}=-\mu_{m}$ for such levels; at least if $W_{m} \neq 0$. As will be discussed later, this relationship is assumed even when $W_{m}=0$.

It is important, both in applying boundary conditions and in obtaining proper stability, to "follow the flow" in writing the recursion formulas; i.e., all directions with $\mu<0$ must be evaluated from large $X$ or $R$ values toward small, and so with the other dimension. Similarly, positive $\mu$ values require evaluation from small $X$ to large, etc. These procedures can be written in a single formula by use of subscript increments defined in terms of the signum function $(\mathrm{Sg})$; which has value \pm I according to the sign of its argument: 


$$
c=\frac{1}{2} S g\left(\mu_{m}\right) ; d=\frac{1}{2} S g\left(\tau_{m}\right)
$$

Written in terms of $\beta, c$, and $d$, Equation $4-3$ can be simplified to:

$$
\begin{gathered}
|\mu|\left(A_{i+c} N_{i+c}-A_{i-c} N_{i-c}\right)+|\tau| B\left(N_{j+d}-N_{j-d}\right) \\
+\frac{1}{2} \Delta A\left\{\left(\beta_{m}-\mu_{m}\right) N_{m+\frac{1}{2}}-\left(\beta_{m}+\mu_{m}\right) N_{m-\frac{1}{2}}\right\}+V \sigma^{T} N=V S
\end{gathered}
$$

where

$$
\Delta A=A_{i+\frac{1}{2}}-A_{i-\frac{1}{2}}
$$

and where $N$ is simply a simplified representation of $N_{i, j, m, g}$, etc.

\subsubsection{Fluence Evaluation Strategy}

Evaluation "following the flow," as discussed above, is also evaluation "sweeping away from boundary conditions into the mesh." Since evaluation always begins with $m=1$, the first sweep must be toward smaller values of $i$ and $j$. Where $I$ and $J$ represent the largest values of $i$ and $j$, then the boundary values $N_{1}+1 / 2$ and $N_{j}+1 / 2$ are decided $a$ priori based on physical considerations at the outer boundaries of the system. No such physical considerations govern the boundary fluence at $m=1 / 2$, however, as will be seen.

Before dealing with $N_{1 / 2}$, let us observe that, even with $N_{\text {f-c }}$ and $N_{\text {J-d }}$ known from boundary considerations and $N_{m-1 / 2}$ to be defined by a yetunspecified process, Equation 4-3 would still involve 4 unknowns. The three advance boundary values, $N_{1+c}, N_{d+d}, N_{m+1 / 2}$ have been treated as completely independent, and are, therefore, not completely defined by Equation 4-3. In fact, all of the values of $N$ represent values of a single continuous function $\phi$ at adjacent lncations, and cannot vary more freely than $\phi$ does. If we suspect that $\phi$ can be approximated adequately by straight-line segments between adjacent boundaries, then the following "linear" or "diamond" difference model results: 


$$
\begin{aligned}
N & =\frac{1}{2}\left(N_{i+c}-N_{i-c}\right), \\
N & =\frac{1}{2}\left(N_{j+d}-N_{j-d}\right),
\end{aligned}
$$

and

$$
N=\frac{1}{2}\left(N_{m+\frac{1}{2}}-N_{m-\frac{1}{2}}\right),
$$

As previously discussed, $\beta_{1}=-\mu_{1}$. Accordingly, Equations 4-13 and 4-15 cannot yield direct information as to an appropriate value of $N_{1}$, and the other results are independent of this value. Accordingly, $N_{1 / 2}^{2}$ is arbitrary and unknown. If $N$, is to be related to $N_{1 / 2}$ and $N_{3 / 2}$, the only acceptable assumption is :

$$
N_{\frac{3}{2}}=N_{1} .
$$

Since each $\eta$ level begins with a $\beta_{m}=-\mu_{m}$, each must start with an "Initiating direction" having:

$$
N_{m+\frac{1}{2}}=N_{m} ; \beta=-\mu .
$$

If an initiating direction occurs for $m>1$, and $m^{\prime}$ is the predecessor to $m$, then Equation 4-15 defines values for $m^{\prime}+1 / 2$ and $m-1 / 2$ which do not agree. In fact, the fluence is not required to be continuous between directions on different $\eta$ levels, and they do not, in general, describe adjacent directions. Thus, there is neither physical nor mathematical requirement for continuity of fluence between them.

It has been traditional in the early DOT codes to allow these initiating directions to have $W_{m}=0$, i.e., "zero-weight directions, " and to adjust their directions such that $\xi=0$. Carlson and Lathrop describe this procedure. ${ }^{4}$ On the other hand, TWOTRAN II uses directions having small, finite weights and having $W_{m}$ small, but not 0 . Toml inson et al. ${ }^{3}$ showed that, other factors being equal, this choice does not affect the results of test problems significantiy. Both types of calculations are available in DORT, al though the direction sets input to the code must always contain directions with $W_{m}=0$ to signal the beginning of a new $\eta$ level. When THOTRAN-like calculations are done, or in XZ geometry, fluence is not calculated in directions having $W_{m}=0$. 
Having decided on boundary values for initiating the sweeps, Equations 4-15 and 4-17 define the remaining unknowns in terms of the single unknown, $N$, for the initial direction of each $\eta$ level:

$$
\begin{aligned}
& N_{i+c}=2 N-N_{i-c} \\
& N_{j+d}=2 N-N_{j-d}
\end{aligned}
$$

Defining, as a matter of convenience:

$$
\bar{A}=\frac{1}{2}\left(A_{i+\frac{1}{2}}+A_{i-\frac{1}{2}}\right)
$$

observing that:

$$
2|\mu| A_{i+c}+\Delta A(\beta-\mu)=2|\mu| \bar{A}+\Delta A \beta
$$

and recalling that $\beta=-\mu$ for the initial direction of each $\eta$-level, the value of $N$ can be written explicitly:

$$
N=\frac{V S+2|\mu| \bar{A} N_{i-c}+2|\tau| B N_{j-d}}{V \sigma^{T}+2|\mu| \bar{A}+2|\tau| B} ; \beta=-\mu
$$

This equation applies to the initiating directions, whether $W=0$ for such directions or not. For all other directions, Equation 4-17 does not apply, and $N_{m-1 / 2}$ is obtained by applying Equation $4-15 \mathrm{c}$ to the preceding directions:

$$
N_{m+\frac{1}{2}}=2 N-N_{m-\frac{1}{2}} ; \beta \neq-\mu .
$$

Since $\beta=-\mu$ for such directions, terms in $\beta$ appear in the formulation for $N$ :

$$
N=\frac{V S+2|\mu| \bar{A} N_{i-c}+2|\tau| B N_{j-d}+\Delta A \beta N_{m-\frac{1}{2}}}{V \sigma^{T}+2|\mu| \bar{A}+2|\tau| B+\Delta A \beta} \quad ; \quad \beta \neq-\mu
$$


At this point, an explicit sweep pattern has been found. Determining $N_{m=1 / 2}, N_{1}+1 / 2$, and $N_{J+1 / 2}$ as described, Equation 4-22 gives $N_{1, d}$ explicitly. Equation 4-18 gives $N_{1-1 / 2}$ explicitly, recalling that $\mu<0$ for the first direction, and thus $c=-1 / 2$. This evaluation can proceed for all i's down to the boundary value at $i=1 / 2$. With these values in storage, values for $m=11 / 2$ can be found using Equation 4-17. If that $\eta$-level contains additional directions with $\mu<0$, values for $m=2$ are evaluated from Equation 4-24, and extrapolated with Equation 4-23. This process continues for all $\mathrm{m}^{\prime} \mathrm{s}$ for which $\eta<0$ and $\mu<0$. Having these values, the values for directions having $\eta<0$ and $\mu>0$ at $i=1 / 2 \mathrm{can}$ be determined from physical boundary conditions. The sweep proceeds as before, except, of course, that evaluation is from $i=1 / 2$ to $i=I+$ $1 / 2$. From these values, Equation 4-19 determines $N_{J-1 / 2}$, and the process proceeds as before downward to $j=1 / 2$. Boundary values at $j=1 / 2$ for directions having $\eta>0$ are determined from physical boundary conditions at the bottom boundary, and the sweep resumes, this time from $j=1 / 2$ to $j=J+1 / 2$.

\subsubsection{Additional Theory Information}

There is an excellent description of the detailed inner workings of the DORT code given in Reference 14. In this document, the interested user will find the detailed information on the various fluence weighting models, sweep strategies, source terms, boundary conditions, iteration strategies, rebalance and acceleration techniques, spatial and directional remeshing techniques, directional quadrature sets, and other information relevant to a full understanding of the DORT code. This information will not be repeated here.

\subsection{DORT INPUT DATA SPECIFICATIONS}

\subsubsection{Card-Image Format}

The card-image input data for a problem consist of a title card followed by a variable number of blocks of data separated by "T" delimiters. Within each block, a variable number of data arrays are specified, keyed according to an array number, and identified as to real or integer type.

A separator card must be placed between successive problem decks. If it contains the word "DIAG" in the first four columns, a non-fatal

diagnostic will be given by ERRO. If the word "DUMP" appears, a fatal diagnostic is given. If the word "mEND" appears, execution will stop. otherwise, the next problem begins immediately. No data are retained in memory between problems. At the end of execution, all data files are closed. Control is then returned to the driver, if the code was invoked by a driver, or else to the operating system. 
All of the card-image input except the title card and separator card are read by the FIDO input processor. The format required for FIDO data entries is described in Appendix $A$. The data arrays and blocks to be read are described in following sections. The first four blocks of data are permanent, and their " $T$ " delimiter must be supplied whether array entries are required or not.

Blocks, after the first four, containing arrays numbered 91 and higher, are options supplied to allow certain simple problems to be solved without the labor of preparing formal fluence and source input files. The conditions under which these blocks are required are included in their description.

As each array of data is read, its length is compared with the required length, and an error message is given if appropriate. After each block of data, a call to subroutine ERRO is made if any of the arrays in the block were of improper length, or if the data were otherwise unsuitable. ERRO prints an explanation of the error and sets an error flag. Data are edited as soon as possible after each block is completed.

Processing of input continues for as long as possible, even after an error. In most cases, this allows the user to test all of his data blocks in spite of an early error. Execution is terminated at the end of input processing if a sufficiently severe error has been encountered.

It is possible that one error will cause subsequent data to be erroneously flagged as wrong. If data which appear correct are flagged by an error message, this possibility should be considered. An error can also cause subsequent system related fallures, such as addressing or storage errors.

The first four blocks of data contain, in general terms:

1. control parameter input arrays (61-63) which set array lengths explicitiy and control execution options.

2. primary input arrays (71-78) whose lengths depend upon arrays $(61-63)$, and whose contents determine later array lengths. These arrays describe variable-mesh, variable quadrature, variable $P_{L}$, and super-mesh features.

3. secondary input arrays (81-87), whose length depend upon arrays $(61-78)$, and whose contents determine later array lengths. These include the directional quadrature specification set, coarse mesh specification, assignment of direction sets to super-mesh cells, and assignment of material zones to edit regions.

4. general input arrays $(1-30)$, which do not determine the lengths of other input arrays. These include space-mesh specifications, cross-section mixing instructions, specification of activity edits, and much more. 
In the remainder of Section 4.3 of this report, the broad concepts required to understand the input data are discussed, followed by a detailed specification of the input data, and finally, clarification of several input-related topics.

\subsubsection{Space Meshes}

Five different subdivisions of the problem space are determined from the input data. Two sets of fine-mesh interval boundaries $\left(2^{*}\right.$ and $4^{*}$ arrays) are input, corresponding to the two spatial dimensions. The intersection of these boundaries forms the fine-mesh grid cells. (In RO geometry, the $2 *$ array is entered in units of revolutions, not radians.)

A coarse mesh, specified by an additional 2 sets of intersecting boundaries (85* and $86^{*}$ arrays), is used in the fluence acceleration routines. A good choice of coarse mesh can save both memory space and execution time without impairing the convergence of a problem. In some cases, proper application of the coarse mesh can help the convergence rate as well. A coarse mesh of one mean-free-path is ideal for many problems. Default options allow the coarse mesh to be identical to the fine mesh if the variable space mesh feature (described later) is not used. A coarse-mesh I-boundary must also be a boundary in each of the fine-mesh I-boundary sets. A coarse-mesh J-boundary must al so be a fine-mesh J-boundary.

A super mesh is similarly specified $\left(75^{*}\right.$ and $76^{*}$ arrays) for use with the variable quadrature feature (described later), and with certain search options. A super-group representation (74\$ array) is also required when variable quadrature is used. The location of the supermesh and super-group boundaries is chosen by the user as desired, except that the super-mesh boundaries must also be fine-mesh boundaries.

Material zones ( $8 \$$ array) are always specified. A material zone must contain only one cross section set, although many zones may share a single cross section set. In addition to cross section assignment, certain input and edit features depend upon material zones.

Edit regions ( $84 \$$ array) are groupings of material zones used for the purpose of condensing certain output tables. Certain input and edit features depend upon edit regions.

\subsubsection{Variable Space Mesh}

The fine mesh can be specified such that the I mesh depends upon J. To accomplish this, several "I-sets" are specified, with different numbers of intervals and different interval boundaries. The ISET(J) array then relates the proper set of boundaries to the J-level. Prudent use of this feature can save computation time and storage by concentrating work in areas requiring a fine mesh. I-direction coarse-mesh and super-mesh boundaries must be contained in all fine-mesh I-sets. Left and right 
boundaries of all I-sets must coincide. A "standard" I-set, which is as large as any of the I-sets, must be chosen. Boundary fluences and sources are kept in the standard I-set format.

\subsubsection{Variable Directional Quadrature}

The directional quadrature sets, "M-sets," are specified by super-zone and super-group. For example, this allows the user to specify a highly biased quadrature in the area of a streaming crack at high energy without wasting such detail elsewhere in the problem. A standard M-set must be chosen which:

1. has as many directions up and as many down as any set,

2. has exactly $|M M|$ directions, and

3. has as many $\eta$ levels upward and as many downward as any set.

\subsubsection{Variable Legendre Expansion}

The order of moment expansion $\left(P_{L}\right)$ may be specified by cross section set (78\$) and by energy group (77\$). The former option allows selective examination of anisotropic effects, while the group dependence can save computation time in lower energy groups, where anisotropy is less pronounced.

NOTE: These features have not been made operable in current versions of the code.

\subsubsection{Adjoint Data}

If an adjoint problem is to be solved, all data in the first four bilocks are entered in the normal manner. All input files supplied by the user, and any arrays numbered 91 and higher, must be supplied in adjoint form, i.e., reversed with respect to energy. For files having directional information, the user should remember that an adjoint problem is solved as a function of $-\Omega$, rather than $\Omega$ where $\Omega$ is a direction specified by the quadrature set. In other words, if $\mu>0$ and $\eta>0$ for a given direction, the adjoint data calculated for that direction will be that appropriate to a particle moving in the $(-\mu,-\eta)$ direction. Directional input files must be similarly reversed, and directional output files will be. Output files have the energy groups in the order calculated, i.e., reversed from the usual order. 


\subsubsection{Card-Image Input Specifications}

In the following section, the symbol "en" indicates that a detailed discussion is given elsewhere in this region. The symbol "\#" denotes the word "number," and "RV" means "recommended value." After each array, the length is specified in parentheses. If an array or block is not always supplied, the conditional requirement is given in brackets. The symbol "*" denotes a feature not presently operable.

\subsection{DORT INPUT REQUIREMENTS}

The following input cards are required to execute DORT. Default values are specified in parentheses.

Title Card (72 alphanumeric character description)

INPUT DATA BLOCK 1

61\$ Array - Data Set Logical Unit Reference Numbers 0

(length subject to change)

NOTE: Leaving a unit as 0 disables the corresponding feature.

NTFLX fluence guess input unit

NTFOG fluence output unit

NTSIG cross-section unit (default $=8$ )

NTBSI external boundary source input

NTDSI distributed source input unit

(must supply scratch unit if INPSRM >0)

$-5 \cdots$

NTFCI reserved (enter 0 )

NTIBI internal boundary source input unit

NTIBO internal boundary fluence output unit

NTNPR large-scale print unit

NTOIR (all print goes on standard output unit if NTNPR $=0$ )

$-10 \ldots$.

NTDSO distributed source output unit

E (terminate array with "E") 
62\$ Array - Integer Control Parameters (length subject to change)

IADJ $\quad 0 / 1=$ forward/adjoint calculation

ISCTM maximum urder of scattering ( $L$ of $P_{L}$ ) (negative indicates $P_{L}$ calculation is specified by

IZM energy group (77\$ array))*

IM maximum number of lst-dimensional (I) spatial intervals in any I-set (negative indicates number of intervals

varies with 2nd dimension, f.e., variable mesh
JM number of 2nd-dimension ( $J$ ) spatial intervals

$-5 \ldots$

IGM number of energy groups

IHT position of total cross section in cross-section table 0

IHS position of self-scatter cross section in cross-section table 0

IHM length of cross-section table for each energy group 0

MIXL cross-section mixing table length 0

$-10-.$.

MMESH number of material zone bodies ( $0=$ no effect)

MTP number of cross-section sets from NTSIG

( 0 has the effect of MTP = MTM) 0

MTM total number of materials, including mixtures 0

IDFAC density factor input option

0 implies feature not used

1 implies DNIJ $(I, J)$ input as $3^{*}$, used to modify cross sections

MM maximum number of directions in any $M$-set

(negative indicates variable quadrature is used)

$-15-.$.

INGEOM geometry option 0

$0 \quad x-z$ siab

1 R-Z cylinder

2 R- $\theta$ circle

$3180^{\circ}-360^{\circ}$ triangular

$460^{\circ}$ triangular

$590^{\circ}$ triangular

$6120^{\circ}$ triangular

IBL left boundary condition
0 vold
1 reflected
2 periodic
3 cylindrical
4 fixed boundary source
5 albedo 
IBR right boundary condition $0 / 1 / 2 / 3 / 4 / 5$ (see IBL)

IBB bottom boundary condition $0 / 1 / 2 / 3 / 4 / 5$ (see IBL)

IBT top boundary condition $0 / 1 / 2 / 3 / 4 / 5$ (see IBL)

ISRNX source (outer) iteration maximum (default $=1$ )

IFXMI inftial fluence (inner) iteration maximum per group (default $=20$ )

(negative indicates maximum given by group (28\$ array))

IFXMF alternate fluence (inner) iteration maximum per group 0 (no effect if 0 )

MODE fiuence extrapolation model (default = 4)

0 linear with negatives set to 0

1 inear with no fixup of negatives

2 scalar weighted

3 zero weighted

4 theta weighted

5 vector weighted

KTYPE calculation type o

0 fixed source

1 K eigenvalue

2 DB2 search

3 concentration search

4 dimension search

$-25-.-$

IACC rebalance method in discrete ordinates section (default $=2$ ) 0

0 single groupwise rebalance factor

1 diffusion acceleration

2 partial current rebalance

KALF rebalance stabilization 0

0 standard method

1 alternate method

IGTYPE solution method 0

0 discrete ordinates used always

$N$ method selected by group ( $7 \$$ array) for first

$N$ outers, then finishing with discrete ordinates

INPFXM fluence input option

0 fluence set to 0 , or read from unit NTFLX if NTFLX >0

$1 F I J(I, J)$ read as 93* array for each group

$2 F I J(I, J) * F G(G)$, uses $93^{*}$ and $95^{*}$ arrays

$3 F I(I) * F J(J) * F G(G)$, uses $93 *, 94 *$, and $95 *$ arrays

INPSRM distributed source input options $0 / 1 / 2 / 3$ (options are analogous to INPFXM; use $96^{\star}, 97 *$, $98^{*}$ arrays) (NTDSI $>0$ is required if INPSRM $>0$ ) 
NJNTSR interior boundary source at NJNTSR J-boundaries wiil be input from NTIBI (may be 0 )

NINTSR interior boundary source at NINTSR I-boundaries will be input from NTIBI (may be 0 )

NJNTFX interior boundary fluence at NJNTFX J-boundaries will be written on NTIBO (may be 0 )

NINTFX interior boundary fluence at NINTFX I-boundaries will be written on NTIBO (may be 0 )

IACT number of region and pointwise activities calculated 0 (negative indicates region activities only)

$-35-\ldots$

IRED balance table output control

In general, IRED is the largest number in the $84 \$$ array, except that:

IRED $=I Z M$ one region for each zone, balances for IZM regions

IRED $=1$ combines all zones into a single region

IRED $=0$ no balance table output, but one region for each zone

IRED $=-1$ provides one region per zone, balance tables will be given for the entire system only

IPDB2 punch $J$-direction $D^{2}$ from balance $t a b l e$ output

0 no $D B^{2}$ produced

1 single average $D^{2}$

2 IGM groupwise $D B^{2}$ values

3 IGM*NREG groupwise and regionwise $\mathrm{DB}^{2}$ values

(use negative entry to bypass balance table print)

IFXPRT scalar fluence print option (default = 1)

0 scalar fluence for all groups printed in output phase

1 scalar fluence not printed

2 scalar fluence printed as calculated

ICSPRT cross section print option (default $=1$ )

0 cross sections printed

1 cross sections not printed

IDIRF directional fluence

0 directional fluence not saved

1 directional fluence saved and printed

2 directional fluence saved but not printed

(directional fluence will be written onto NTDIR

if IDIRF $>0$ and NTDIR $>0$ )

$-40-\cdots-$

JDIRF first $J$-interval for directional fluence output

JDIRL last $J$-interval for directional fluence output

NBUF system buffer space 0

IBM: I/0 system block space, kbytes (default $=90$ )

CRAY: no effect 
IEPSBZ zone importance convergence (uses $24^{*}$ array)

0 feature not used

1 zone importance used

11 as 1 \& zone convergence printed after convergence

21 as 1 \& zone convergence printed after each iteration

MINBLK minimum $J$-blocking for fluence moment storage ( $r v=0)$

0 l space block per group, all groups in memory allowed

11 space block per group, 1 group in memory allowed

$N$ at least $N$ space blocks required, 1 group in memory

$45 \ldots$

MAXBLK maximum J-blocking

0 JM space blocks per group allowed

1 only 1 space block per group allowed

$N$ maximum of $N$ space blocks per group allowed

ISBT standard I-set for boundary fluences (default $=1$ )

MSBT standard $M$-set for boundary fluences (default $=1$

MSDM standard $M$-set for dimensioning (default $=1$ (MSDM = MSBT aiways in this version)

IBFSCL number of fluence iterations before first rebalance

in first source iteration (default $=1$ ) 4

$-50-.-$

INTSCL minimum number of rebalance iterations (default $=4$ ) 0

ITMSCL maximum number of rebalance iterations (default $=50$ ) 0

NOFIS fission spectrum option (default $=1$ )

0 fission calculated with $X$ normalized to 1.0

1 fission calculated with input $X$ values

2 fission calculation bypassed, saving memory space

(NOFIS will be set to 2 if the $1^{*}$ array is 0 )

IFDB2Z $\mathrm{DB}^{2}$ input option

0 option not used

1 DR2Z(IG,IZ) entered as $6^{*}$ array by group, then by region

ISWP type of diffusion theory sweep ( $r v=4$ or 5 )

0 line inversion in diraction of largest number of mesh intervals

1 alternating direction (line-column) on consecutive outer iterations

2 line inversion

3 column inversion

4 alternating direction (1ine-column) on consecutive inner iterations

5 alternating direction (column-line) on consecutive inner iterations

6 line inversion ou'tward from center $J$ interval 
KEYJN J-interval for key fluence print (ignored if 0 ) (default = 1) 0

KEYIN I-interval for key fluence print (ignored if 0 ) (default = 1) e

NSIGTP cross section input file (NTSIG) format 0

0 GIP format

1 ORDOSW format

NORPOS cross section table position for output normalization (option not used if NORPOS $=0$ )

NORMAT cross-section material number for output normalization ( 0 implies use macro cross section set; negative implies do not use density factor in normalization)

$-60 \ldots$

MSTMAX maximum number of M-sets ( 0 implies JM sets allowed)

NEGFIX negative scattering source fixup (default $=-1$ )

0 option not used

1 full source fixup used

2 initial fixup used

-1 economy fixup used

LOCOBJ fast memory objective into which problem

will try to fit a

IBM: no effect

CRAY: fast memory objective, words*1000

( 0 implies use default)

LCMOBJ slow memory objective into which problem

will try to fit o

CRAY CTSS: sequential file size, kwords

( 0 implies use of system default)

NKEYFX length of key fluence arrays $(29 \$, 30 \$)$

( 0 bypasses feature, negative causes print only at convergence or iteration limit for each group)

$-65-.-$

NCNDIN maximum user condition code allowed (default $=4$ )

NEUT last neutron group number $(0$ implies NEUT $=I G M)$

E (terminate array with "E")

63* Array - Real Control Parameters (1ength subject to change)

TMAX maximum minutes of CPU time for this problem ( 0 ignored) a

XNF value to which source is normalized ( 0 ignored if

KTYPE $=0$, otherwise 0 implies XNF $=1.0$ )

EPS eigenvalue convergence criterion (source iterations)

(default $=1.0 \mathrm{E}-4$ ) $\mathrm{C}$

EPP pointwise fluence convergence criterion

(fluence iterations) (negative: source iterations can halt without meeting EPP) (default $=1.0 \mathrm{E}-3$ ) 0 
EPV volumetric fluence convergence criterion (fluence iterations)

$-5 \cdots$

$\begin{array}{ll}\text { EPF } & \begin{array}{l}\text { Pointwise fission convergence criterion } \\ \text { (default }=1.0 E-3) 0\end{array} \\ \text { EKOBJ } & \text { k-effective sought in search (KTYPE }>1) \text { or initial } \\ & \text { k-effective (KTYPE }=1 \text { ) (default }=1.0) 0 \\ \text { EVTH } & \text { k-effective convergence ratio (default }=0.2) 0 \\ \text { EVCHM } & \text { maximum EV change ratio per iteration (default }=1.5) \\ \text { EVMAX } & \text { maximum EV change ratio, overall (default }=10.0)\end{array}$

$-10-$.

EVKMX maximum allowed $\mid \dot{K}$-effective - EKOBJ $\mid$ (default $=1.0)$

EVI initial eigenvalue (default $=1.0$ )

DEVDKI initial eigenvalue slope (default $=-1.0$ )

EVDELK initial eigenvalue increment (default $=0.3$ )

SORMIN maximum source iteration acceleration (default $=10.0$ ) 0

$-15-$.

CONACC fluence acceleration acceptance criterion (default $=1.0$ )

CONSCL fluence acceleration convergence criterion (default $=1.0 \mathrm{E}-4$ )

CONEPS fluence acceleration convergence ratio (default $=0.01$ )

WSOLOI reserved (enter 0 )

WSOLII fluence acceleration damping increment (default $=-1.5$ ) (negative indicates use only as required)

$-20--$

WSOLCN fluence acceleration damping constant (default $=1.5$ )

ORF diffusion theory fluence acceleration factor (defautt $=0.6$ )

FSNACC reserved (enter 0 )

FLXMIN minimum fluence for convergence tests (default $=1.0 E-60)$

SMOOTH reserved (enter 0 )

$-25-\cdots$

EPO source iteration fluence convergence criterion o

EXTRCV source iteration extrapolation convergence criterion $a$ (default $=0.2$ )

THETA theta-weighted extrapolation model parameter (default $=0.9$ )

E (terminate array with "E")

$T$ (terminate block with "T") [T aiways required] 
INPUT DATA BLOCK 2

Primary Dimension-Setting Arrays (Omit arrays not needed)

\begin{tabular}{|c|c|}
\hline $71 \$$ Array & $\begin{array}{l}\operatorname{ISET}(J) \quad(\#=J M) \quad[I M<0] \\
\text { Index of radial mesh set to use at each } J \text { level. } \\
\text { (default }=\text { Set } 1)\end{array}$ \\
\hline $72 \$$ Array & $\begin{array}{l}\text { IMBIS (ISET) }(\#=J M) \quad[I M<0] \\
\text { Number of intervals in each I set; } \\
\text { then fill array with } 0^{\prime} s \text {. }\end{array}$ \\
\hline $3 \$$ Array & $\begin{array}{l}\text { MMBMS }(\text { MSET }) \quad(\#=\text { MSTMAX) } \quad[M M<0] \\
\text { Number of directions in each } M \text { set; } \\
\text { then fill array with } 0^{\prime} s \text {. }\end{array}$ \\
\hline I\$ Array & $\begin{array}{l}\text { ISZNG }(I G) \quad(\#=I G M) \quad[M M<0] \\
\text { Super group number by group } \\
\text { (default }=\text { a single supergroup) }\end{array}$ \\
\hline 5* Array & $\begin{array}{l}\text { SZNBZ (JSZ) } \quad(\#=J M) \quad[M M<0 \text { or KTYPE }>1] \\
\text { J super mesh boundaries } \\
\text { Enter as many boundaries as desired. Then fill with } 0 . \\
\text { (default }=\text { a single superzone) }\end{array}$ \\
\hline 76* Array & $\begin{array}{l}\text { SZNBR(ISZ) }(\#=|I M|) \\
\text { I super mesh boundaries } \\
\text { Enter as in } 75^{*}\end{array}$ \\
\hline $77 \$$ Array & $\begin{array}{l}\text { ISCTG }(I G) \quad(\#=\text { IGM) } \quad \text { [ISCTM }<0] \\
\text { Order of Legendre expansion used in calculation }\end{array}$ \\
\hline $3 \$$ Array & $\begin{array}{ll}\text { NSIG(MT) } \quad(\#=M T M) & {[M C R<0]} \\
\text { Order of Legendre expansion of cross section sets }\end{array}$ \\
\hline
\end{tabular}

From these, the following are determined:

\begin{tabular}{|c|c|}
\hline $\begin{array}{l}\text { MGSZN } \\
\text { NJSZN } \\
\text { NISZN } \\
\text { ISM } \\
\text { MSM } \\
\text { IMSISM } \\
\text { MMSMSM } \\
\text { IMSJM } \\
\text { IMA }\end{array}$ & 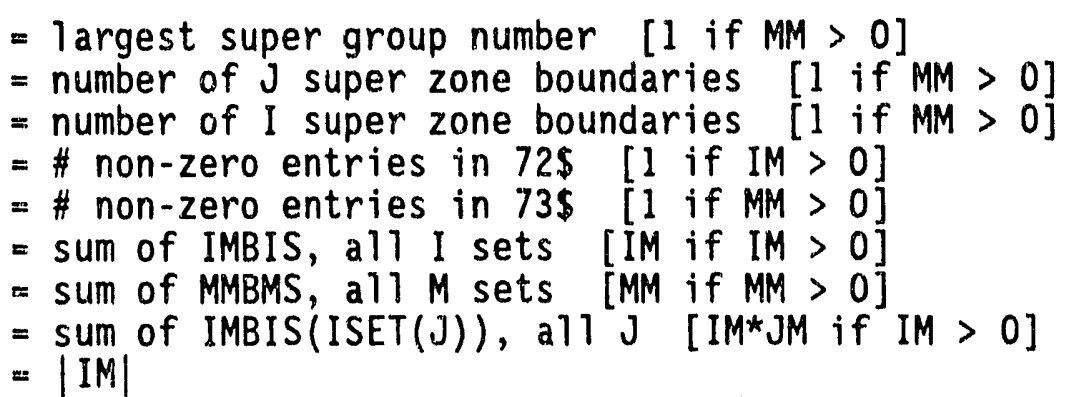 \\
\hline
\end{tabular}




$$
\begin{array}{ll}
\text { MMA } & =|M M| \\
\text { MMSIMS } & =\text { MMA*IMA } \\
\text { MMSJM } & =\text { MMA*JM } \\
\text { IHP } & =\text { IHM }+1 \text { if IHS >IHT + I; otherwise = IHM }
\end{array}
$$

\section{INPUT DATA BLOCK 3}

Secondary Dimension-Setting Arrays (Omit arrays not needed)

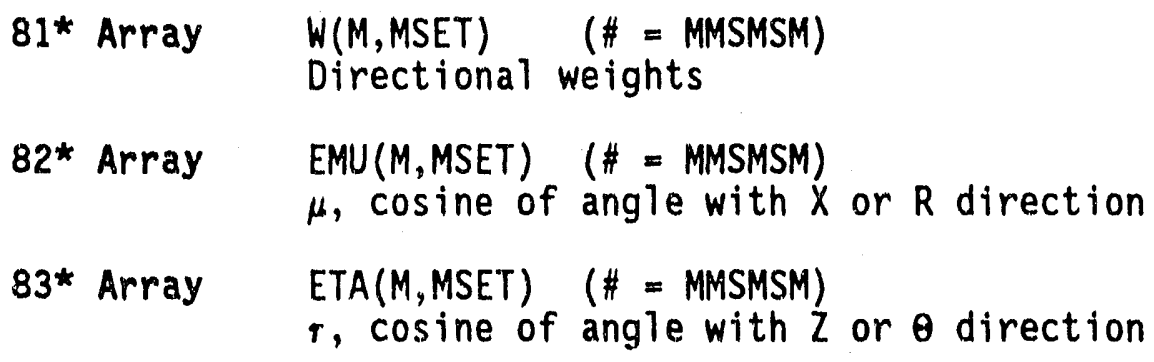

84\$ Array IZNRG(IZ) (\#=IZM) [IRED $\neq 0$ or IACT $\neq 0$ ]

Region number by zone (default controlled by value of IRED, one region per zone if IRED $=I Z M, 0$, or -1 ; one region if $I Z M=1$; otherwise IZNRG must be specified in full)

85* Array ZCMB (JC) (\#= JM)

$J$ coarse mesh boundaries

Enter as many boundaries as desired up to the limit, JM. Then fill with any number at least as large as the last $\mathrm{J}$ fine-mesh boundary. (default $=1$ course mesh for each interval)

86* Array RCMB(IC) (\# = IMA)

I coarse mesh boundaries, as in $85^{*}$

$87 \$$ Array IJGSZ (ISZ, JSZ, IGSZ) (\#=NISZN*NJSZN*NGSZN) [MM $<0]$

$M$ set by I super mesh, then by $\mathrm{J}$ super mesh, then by super group

$T$ (terminate block with "T") [T always required]

From these, the following are determined:

$$
\begin{array}{ll}
\text { JCM } & =\text { \# of coarse mesh boundaries entered in the ZCMB array } \\
\text { ICM } & =\# \text { of coarse mesh boundaries entered in the RCMB array } \\
\text { NREG } & =\text { maximum of IZNRG (IZ) array } \\
\text { ICMJCM } & =I C M * J C M \\
I C P J C P & =(I C M+1) *(J C M+1)
\end{array}
$$




\section{INPUT DATA BLOCK 4}

General Input Arrays (Omit arrays not needed)

1* Array CHI(IG) fission spectrum fractions, $\chi$, by group (\# =IGM)

2* Array $\operatorname{ZIN}(J) Z$ or $\theta$ fine-mesh boundaries $(\#=J M+1)$

3* Array DNIJ $(I, J)$ density factor (\# = IMSJM) [IDFAC >0] (default $\mathrm{m}$ 1.0)

4* Array RIN(I,ISET) $X$ or $R$ fine-mesh boundaries

(\# = IMSISM + ISM)

5* Array ENER(IG) top neutron energy group boundaries + bottom energy of last neutron group + top photon energy group boundaries + bottom energy of last group (\#=IGM + 2) [NTFOG > 0] 0

6* Array $D B 2 Z(I G, I Z) D B^{2}$ by group, then by region $(\#=I G M * N R E G) \quad[$ IFDB2Z $>0]$

7\$ Array ITHYG(IG) theory by group (\#= IGM) [IGTYPE >0]

8\$ Array IJZN $(I, J)$ material zone by fine space mesh (\# = IMSJM) 0

9\$ Array IZMT(IJZN) material number by material zone (\#=IZM)

$10 \$$ Array MIXT(MIX) mixture ID (\#= MIXL)

11s Array NUCL(MIX) nuclide ID $(\#=M I X L)$

12* Array DE!SS(MIX) number density (\#= MIXL)

$13 \$$ Array MATL(MT) ID number by material (\#= MTM)

(defautt MATL(MT) $=$ MT)

14* Array ZNTSR(JNTSR) $Z$ or $\theta$ boundary positions for J-boundary source input $(\#=$ NJNTSR)

15* Array RNTSR(INTSR) $X$ or $R$ boundary positions for I-boundary source input $\quad(\#=$ NINTSR $)$

16* Array ZNTFX(JNTFX) $Z$ or $\theta$ boundary positions for J-boundary fluence output (\#= NJNTFX)

17* Array RNTFX(INTFX) $X$ or $R$ boundary position: for I-boundary fluence output (\#= NINTFX)

18* Array FJSRZ(JSZN) J-Super zone search fraction (\#= NJSZN) 0 $[K T Y P E=4]$ 
19* Array FISRZ(ISZN) I-Super zone search fraction (\# = NISZN) 0 [KTYPE $=4$ ]

20* Array ABDOL (IG,J) left boundary albedo (\# = IGM*JM) $[I B L=5]$

21* Array $A B D O R(I G, J)$ right boundary albedo (\#=IGM*JM) $[I B R=5]$

22* Array $A B D O B(I G, I)$ bottom boundary albedo (\# = IGM*IMA) $[I B B=5]$

23* Array ABDOT(IG,I) top boundary albedo (\#= IGM*IMA) $[I B T=5]$

24* Array EPSBZ(IZ) fluence error importance by material zone $(\#=I Z M) \quad[$ if IEPSBZ >0] 0

25\$ Array ICMAT(IAC) material to be used in activity calculations $(\#=|I A C T|)$ Q

26\$ Array ICPOS (IAC) cross section table position for activity $(\#=|I A C T|)$ Q

27* Array ACMUL(IAC) activity multiplier $(\#=\mid$ IACT $\mid)$

28\$ Array ITMBG(IG) initial iteration limit by group (\# = IGM) [IFXMI < 0] 0

29\$ Array KEYAJ(NKEY) J positions of key fluences in ascending order $(\#=\mid$ NKEYFX $\mid)$ e

30 Array KEYAI(NKEY) I positions of key fluences in ascending order for like $\mathrm{J} \quad(\#=\mid$ NKEYFX $)$ ?

$T$ (terminate block witi, "T") [T always required]

\section{INPUT DATA BLOCK 5}

External Boundary Source Input

[required if INGEOM $<20$ and IBL, IBR, IBB, or IBT $=4$ ]

91* Array SII $(M, J) \quad(\#=M M A * J M) \quad[I B L=4$ or $I B R=4]$

I boundary source for a group

92* Array SJI $\left.(M, I) \quad(\#=M M)^{*} I M A\right) \quad[I B B=4$ or $I B T=4]$

$J$ boundary source for a group

$T$ (terminate block with "T") [T required if any arrays are required] 
Left and right sources are intermingled in the same array according to direction. If $\mu>0$, the source applies to the left boundary; if $\mu<0$, to the right. Likewise, if $r>0$, a J-boundary source applies to the bottom; if $r<0$, the source applies to the top boundary. If any blocks of this type are required, IGM are required.

If IADJ > 0, the energy groups must be entered in reversed order. Omit block and delimiter if INGEOM $\geq 20$, or if no arrays are required.

\section{INPUT DATA BLOCK 6}

Fluence Guess Input [required if INPFXM >0]

93* Array $F I J(I, J), F I J(I, J)$ or $F I(I)$ as $\operatorname{INPFXM}=1,2$, or 3 $(\#=$ IMSJM, IMSJM, or IM) [INPFXM >0]

94* Array FJ $(J) \quad(\#=$ JM) $\quad[$ INPFXM = 3]

95* Array $F G(I G) \quad(\#=$ IGM) [INPFXM > 1]

Note: Each array of this block must be followed by "T". If no arrays are required, no "T" is required. If IADJ >0, energy groups must be specified in reversed order.

\section{INPUT DATA BLOCK 7}

Distributed Source Input [required if INPSRM $>0$ ]

96* Array as 93* Array above

97* Array as 94* Array above

98* Array as 95* Array above

\subsection{DORT INPUT DATA NOTES}

\subsubsection{Special Geometry Features}

If INGEOM $\geq 10$, a one-dimensional problem will be solved with vertical flow suppressed. Use void boundary condition at top and bottom. RZ direction sets must be used with INGEOM $=11$, while RO sets must be used with INGEOM $=12$. Solutions with INGEOM $=11$ or 12 yield essentially the same result. 
If INGEOM $=20$, KTYPE $=0$, and IBR $=4$, a combined reflection/transmission problem in slab geometry is solved. Omit the 91* and 92* array blocks, and supply the input spectrum as CHI. The code will generate a boundary source in the leftmost direction of nonzero weight of each downward q level. The value of JM must be the number of downward $\eta$ levels. In this case, $J$ corresponds to the incident $\eta$ level, not to a $Y$-direction mesh. The $2 *$ array should be filled with the values $0.0,1.0,2.0, \ldots . J M$. The first $\eta$ level will correspond to the last J level. |CHI(IGM)| particles will enter each level in each group. If $\mathrm{CHI}(\mathrm{I})<0$, the emerging fluence is printed by direction, then by space interval.

If INGEOM $=30$, the calculation proceeds as with INGEOM $=20$, except that emerging fluence will be calculated for a source entering each group JDIRF $\leq$ IGI $\leq$ JDIRL in turn, and leaving in al1 groups IGI $\leq I G O \leq$ IGM. The total source per group in each $\mathrm{J}$-level is $\mid \mathrm{CHI}$ (IGI)|. If CHI $<0$, the fluence from sources entering that group will be printed. The problem is "double differential," in that the fluence leaving each group resulting from a source in each individual input group is calculated.

If $3 \leq$ INGEOM $\leq 6$, and if all iterations are to be performed in diffusion theory, equilateral-triangular geometry is avallable. Geometry options, together with the required value of IM, are:

\begin{tabular}{llc} 
INGEOM & TRIANGULAR GEOMETRY OPTION & VALUE OF IM \\
\hline 3 & $180^{\circ}-360^{\circ}$ Symmetry & User's option \\
4 & $60^{\circ}$ Symmetry & $2 * J M-1$ \\
5 (not operable) & $90^{\circ}$ Symmetry & $2 *$ JM+1 \\
6 & $120^{\circ}$ Symmetry & $2 * J M$
\end{tabular}

The resulting geometry is illustrated in Figure 4-1.

The mesh input data involve certain unusual requirements. In most cases, the value of IM is a function of JM as shown above. Also, the radial dimensions used for input do not correspond directly to the actual dimensions of the mesh. For all but $90^{\circ}$ symmetry, every mesh interval is an equilateral triangle with sides having a length $S$. 

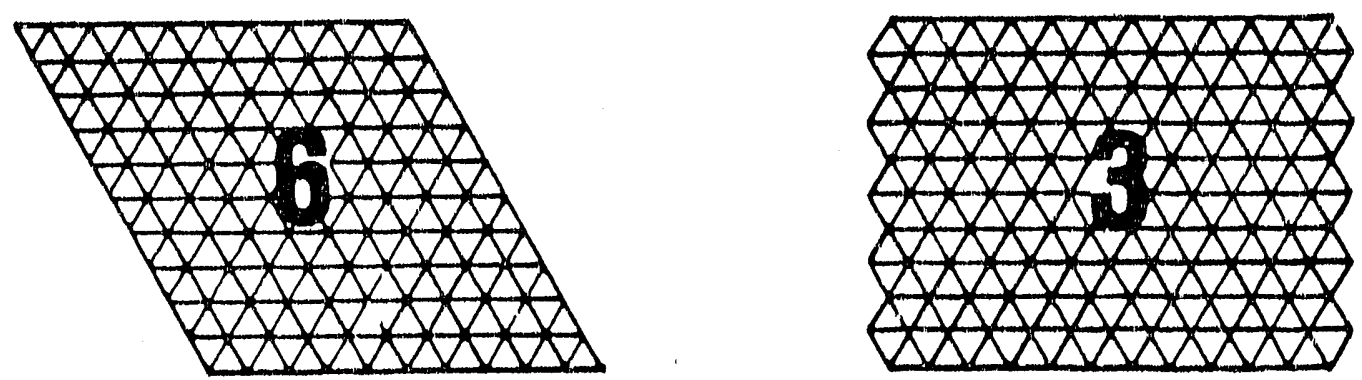

\section{INGEOM SYMMETRY}

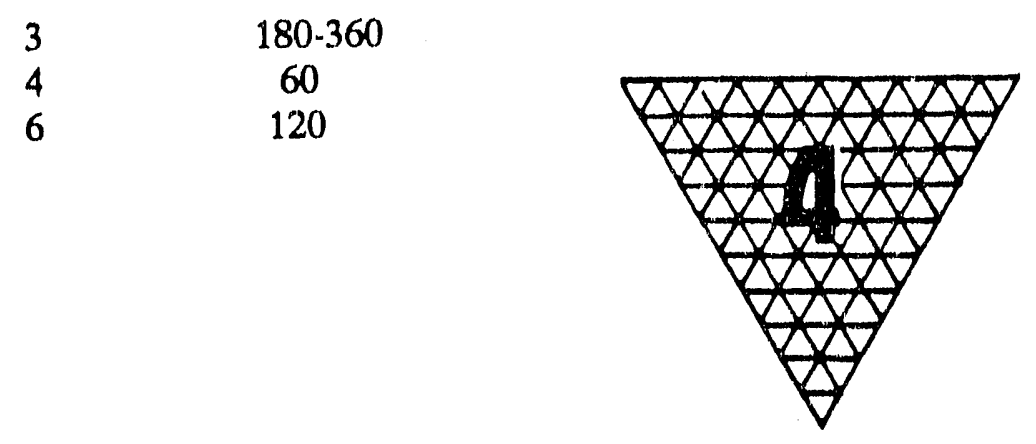

Figure 4-1. Triangular Geometry Options.

$J M+1 J$-interval boundaries are entered, beginning with 0 , and with spacing $\sqrt{3} \mathrm{~S} / 2$. With $120^{\circ}, 180^{\circ}$, or $360^{\circ}$ symmetry, IM +1 I-interval boundaries are entered, beginning with 0 , and with spacing $S / 2$. With $60^{\circ}$ symmetry, the code will automatically set IM to negative, and require the entry of JM I-sets. The jth I-set has $2 j$ boundaries with spacing $S / 2$. The description applicable to $90^{\circ}$ symmetry is not available.

For all cases, the $(1,1)$ mesh interval is a triangle with vertex down. Example meshes are shown for the various triangular geometry options.

For $60^{\circ}$ symmetry, the parameter ISBT should be set equal to JM, and the bottom boundary condition must be reflected. For $120^{\circ}$ symmetry, the left and bottom boundaries must be periodic if periodic boundary conditions are selected. 


\subsubsection{Spectal Theory Options}

If IGTYPE $>0$, then the first IGTYPE outer 1terations will be performed using alternate theory specifted by group in the $7 \$$ array. If the gth entry is $N_{g}$ then the first $\left|N_{g}\right|$ inner iterations of the first IGTYPE outer iterations on group, $g, w+11$ be performed using diffusion theory if $N_{g}>0$. If $\left|N_{0}\right|$ is less than the applicable maximum number of iterations, the iterations will be completed in transport theory, even though the alternate theory may have achleved convergence.

\subsubsection{Cross Section Input and Mixing}

The internal cross section storage comprises MTM sets of cross sections for each energy group. The first MTP sets are read from logical unit NTSIG, which is always required. The remainder are prepared using a "mixing table," described below. [It is intended that the first MTP "materials" will be microscopic nuclide data, and the remainder will be macroscopic mixtures, although other uses are possible.] The material numbers entered in the IZMT array (9\$) must be integers between 1 and MTM, corresponding to the appropriate data.

Each material consists of IHP cross sections as indicated in the description of the ORDOSW input file. If ISCTM $>0$, then ISCTM sets of Legendre expansion data must follow each set designated in the IZMT array. If MCR < 0 , then the required number of expansion sets is indicated in the NSIG array (78\$) (this feature is not presentiy operable).

If MIXL >0, cross sections are to be modified by a mixing table, specified by the MIXT, NUCL, DENS, and MATL arrays $\left(10 \$, 11 \$, 12^{\star}\right.$, and 13\$). The MATL array assigns an arbitrary ID number to each material. If it is not entered, the ID's $1,2, \ldots \ldots$. MTM are assumed. Each integer entered in the MIXT and NUCL arrays must be one of these ID's, or 0 . In the following:

$m$ number of the material having ID $|p|$ if $p \neq 0$; otherwise 0

$n=$ number of the material having ID $|q|$ if $q \neq 0$; otherwise 0

and the interpretation of the table is as follows:

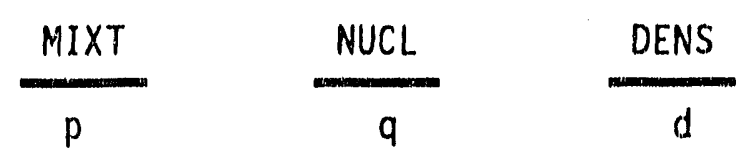

(a) If $q=0$, then the data of material $m$ will be multiplied by $d$.

(b) If $q>0$, then the data of material $n$, multiplied by $d$, will be added tie that of material $m$. 
(c) If $q=p$, the data of material $m$ will be modified by the eigenvalue in concentration searches.

(d) If $p<0$, the appropriate Legendre expansion components will be treated as was the principal set.

Each set of entries is executed in sequence. In searches, the table may be executed repeatediy. As an example, with MIXL $=6$, MTM $=11$, and ISCTM $=3$.

\begin{tabular}{rrrrr}
$I$ & MIXT & NUCL & DENS & MATL \\
\hline 1 & 1000 & 0 & 0 & 10 \\
2 & 1000 & 10 & .1 & 20 \\
3 & 1000 & 20 & .2 & 30 \\
4 & 1000 & 1000 & 0 & 40 \\
5 & -2000 & 0 & 0 & 50 \\
6 & -2000 & 30 & .3 & 60 \\
7 & & & & 1000 \\
8 & & & & 2000 \\
9 & & & & 3000 \\
10 & & & & 5000 \\
11 & & & &
\end{tabular}

Material 7 will consist of:

Material $1 * .1+$ material $2 * .2$

and will be modified by the eigenvalue.

Materials 8, 9, 10, 11 will be:

Materials $3,4,5$, and $6 * 3$.

Although the value of $\sigma^{\wedge}$ does not affect the fluence in a calculation directly, it must be used to obtain correct balance tables. It should meet the condition:

$$
\sigma_{g}^{T}=\sigma_{g}^{A}+\sum_{g \prime} \sigma_{g \rightarrow g^{\prime}}
$$

If $\sigma^{\top}(g)$ is replaced by a "transport cross section" in reactor core problems, a must be $\sigma_{\mathrm{g} \cdot \mathrm{g}}$ reduced such as to maintain $\sigma^{\wedge}$ constant.

It may be important to note that many standard cross section files such as ISOTXS and MATXS have Legendre expansion data which must be multipl fed by $21+1$, where 7 is the expansion index, for use in DORT. It is intended that this be done in the code which prepares the ORDOSW (or optional GIP input file). 


\subsubsection{Activity Edits}

A very general provision for obtalning energy-integrated reaction rates is provided. The arrays ICMAT, ICPOS, and ACMUL define an "activity table," interpreted as follows:

$$
\begin{aligned}
& \frac{\text { ICMAT }}{r} \quad \frac{\text { ICPOS }}{s} \frac{\text { ACMUL }}{t} \\
& A_{i, j}=t d_{i, j} C_{i, j, r, s} \sum_{g} \phi_{g, j, i} \sigma_{g, r, s} \\
& B_{z}=\sum_{i, j} X_{i, j, z} A_{i, j} V_{i, j}
\end{aligned}
$$

where

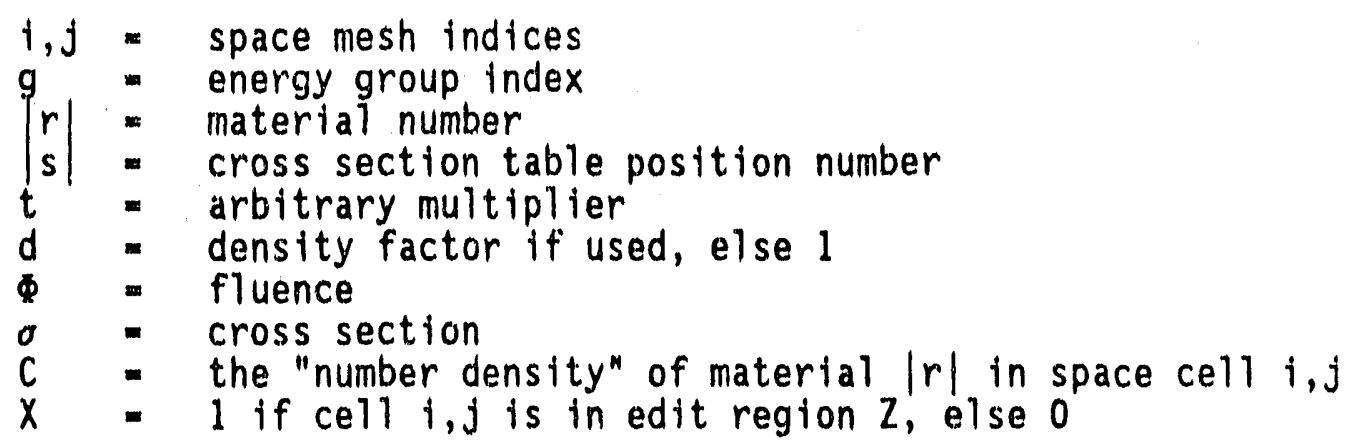

The value of $C$ is determined as follows:

(a) If $r$ is the macro material used in cell $i, j$, then $c m 1$,

(b) If $r$ is 0 , then $C=1$, and the cross sections for the macro material in material in $c$ : $i, j$ are used,

(c) If $r<0$, then $c=1$,

(d) If $r>0$ and is not the macro, then $C$ is the sum of all assignments of $r$ to the macro according to the mixing table. Suppose IACT $=4$ and the following table is entered, referring back to the mixing tabie fllustrated above; and suppose material 7 is named as a macro in a portion of the problem:

\begin{tabular}{crrr} 
I & ICMAT & ICPOS & ACMUL \\
\hline 1 & 7 & 3 & 1. \\
2 & -7 & 3 & 1 \\
3 & 0 & 4 & 500. \\
4 & 1 & 5 & 1. \\
5 & 1 & -5 & 1.
\end{tabular}


Activity 1 will use position 3 of material 7 , but will be 0 where 7 is not the macro. Activity 2 will be similar, but will be calculated whether 7 is the macro or not. Activity 3 will use position 4 of the macro in each space cell, and will multiply the result by 500 . Activity 4 will use position 5 of material 1 and a "number density" of .1 wherever 7 is the macro, since. $1^{*}$ material 1 was added to material 7 in the mixing table.

As a special feature, if $|s|>$ IHP, then the value of CHI (1*) for the group will be used to replace $\sigma$, and $c$ and $d$ will be everywhere 1 . This can be useful in adjoint problems.

If $s<0$, the activity will be written on the punch data set. This can be used for fission density output, for example, as in Activity 5 of the illustration.

\subsubsection{Negative Source Removal}

Any problem using ISCTM > 0 may incur negative source generation. This is simply due to the failure of a truncated $P_{L}$ expansion to describe the cross section adequately. If the problem has no areas of monodirectional flow or void, this effect will probably not be noticed, and will usually cause no harm. Voids or monodirectional flow, however, can result in severe distortions, or even negative scalar values for the fluence. The NEGFIX $>0$ option will remove these distortions in most cases. The penalty in CPU time is usually in the range of $15-30 \%$, although it can be higher if a major portion of the problem is so affected.

\subsubsection{Problem Type Specification}

Five major types of calculation are available, chosen by KTYPE:

KTYPE = 0 M calculation - fixed source or subcritical multiplication

$=1$ K calculation - determines $K_{\text {eff }}$

$=2$ B search - modify $D B^{2}$ to produce specified $K_{e f f}$

$=3 C$ search - modify concentrations to produce specified $K_{\text {eff }}$

$=4 \mathrm{D}$ search - modify dimensions to produce specified $K_{\text {eff }}$

When KTYPE $=0$ and CHI $=0$ ( $1^{*}$ array) or NOFIS $=2$, a simple fixedsource problem is solved. Fluence from the input source is determined in one source iteration unless upscatter cross sections are used. Upscatter requires ISRMX $>1$ and EPO $>0$ for satisfactory results.

If some values of CHI $\neq 0$ and NOFIS $\neq 2$, fissions resulting from the fixed source and the input fluence guess, if any, are calculated in the first source iteration. These are added to the fixed source to jeg in the second iteration, and so on until subsequent fission generations have been accounted for. Since these subcritical multiplication problems tend to be slowly convergent, source iteration acceleration, 
discussed elsewhere, is used. If the system is, by itself, supercritical, this calculation will surely fail; a reflection of the fact that the fluence would be divergent in such a physical situation. The number of fission neutrons produced per source neutron is determined by iteration. Several source iterations are required. When KTYPE $=1$, a calculation of $K_{\text {eff }}$ is performed. Acceleration of the source iterations is discussed elsewhere.

$K$ calculations can be improved somewhat be setting EVI equal to the best-available estimate of $K$. This is especially important in the case of restarts.

When KTYPE > 1, properties of the system configuration are changed in order to drive $K_{\text {eff }}$, calculated as described above, to a specified value, EKORJ. For each configuration, the value of $K_{\text {eff }}$ is partially converged, ine configuration is changed, and the process continues until $K_{\text {eff }}$ has stabilized and $i t$ is near the objective value. Additional details of this process are given in another section.

\subsubsection{Iteration Limits and Convergence}

In general, source iterations will be performed until the time TMAX is exceeded (IXX $=8$ ), the iterations limit ISRMX is exceeded (IXX $=1$ ), or convergence is reached. Source iteration convergence has not been reached if:

$(I X X=2)$ the problem uses diffusion-theory source iterations followed by transport theory, and iransport theory has not begun.

$(I X X=3)$ the search has not converged; i.e., KTYPE > 1 , EPS > 0 , and:

$$
\epsilon^{*}=\left|\frac{K^{N}-E K O B J}{K^{N}}\right|>E P S
$$

where: $K^{*}$ is the value of $K_{\text {eff }}$ for the latest (Nth) source.

$(I X X=4)$ iteration fluence iterations for some group have not converged; i.e., EPO >0 and:

$$
\epsilon^{F}=\left|\frac{\phi_{i, j, g}^{N}-\phi_{i, j, g}^{N-1}}{\phi_{i, j, g}^{N}}\right|>E P O
$$


$(I X X=5)$ fission density has not converged; i.e., EPF $>0$ and:

$$
\epsilon^{D}=\left|\frac{F_{i, j}^{N}-F_{i, j}^{N-1}}{F_{i, j}^{N}}\right|>E P F
$$

$(I X X=6) \quad K_{e f f}$ has not converged; i.e., EPS $>0$ and:

$$
\epsilon^{K}=\left|\frac{K^{N}-K^{N-1}}{K^{N}}\right|>E P S
$$

$(I X X=7) \quad$ No source iterations have been completed.

The value of IXX is printed at each iteration, together with each of the above measures of convergence.

In general, problems with $K T Y P E>0$ require several outer iterations for convergence, while problems with KTYPE $=0$ require only 1 . In the latter case, performing several outer iterations can be very wasteful. Exceptional cases are fixed-source $(\mathrm{KTYPE}=0)$ problems with non-zero fission or with upscatter. In these cases, several outer iterations are required.

The fluence iteration limit for each source iteration will be IFXMI or, if IFXMI < 0 , the groupwise value from input array 28\$. After $K^{N}$ and fission density have converged, the iteration limit is changed to IFXMF if IFXMF $>0$. Fluence iterations proceed until the time TMAX is exceeded, the iteration limit is exceeded, or convergence is reached. Fluence convergence has not been reached if:

- Pointwise fluence convergence has not been reached; i.e., EPP > 0 and:

$$
\begin{gathered}
\epsilon^{P}\left|\frac{\phi_{i, j, g}^{n}-\phi_{i, j, g}^{n-1}}{\phi_{i, j, g}^{n}}\right| W_{i, j}>E P O \\
n=\text { fluence iteration index }
\end{gathered}
$$

Where $W_{i, j}$ is the value of the importance function (24* array) for the position $i, j$ if IEPSBZ $>0$, and otherwise 1.0 . The largest $\epsilon^{p}$ for any group after the last fluence iteration is called "group convergence," $\epsilon^{G}$. 
- Volumetric fluence convergence has not been reached; i.e., EPV > 0 and:

$$
\frac{\sum_{i, j}\left|\phi_{i, j, g}^{n}-\phi_{i, j, g}^{n-1}\right| W_{i, j} V_{i, j}}{\sum_{i, j} \phi_{i, j, g}^{n} W_{i, j} V_{i, j}}>E P V
$$

Fluences less than FLXMIN are not considered in the tests. This prevents values near zero, which are badly affected by roundoff, from thwarting convergence. FLXMIN also is used as a minimum coarse-mesh fluence by the fluence acceleration routines, preventing similar degeneracy there.

\section{M.5.8 Search Strategy}

The searches proceed by successive adjustment of the eigenvalue, EV. EV is constrained to be positive, and extensive checks prevent oscillation and overextrapolation. Each search seeks a value of EV which adjusts $K_{\text {eff }}$ to an input value, EKOBJ. EV is used to adjust the system parameters as follows:

$$
\begin{aligned}
& \text { B search: }\left(D B^{2}\right)_{\text {eff }}=\left(D B^{2}\right)_{\text {input }} * E V \\
& C \text { search: }(C)_{\text {eff }} *(C)_{\text {input }} *(1+f(E V-1))
\end{aligned}
$$

To adjust material $m$ in this way, an entry is made in the mixing table:

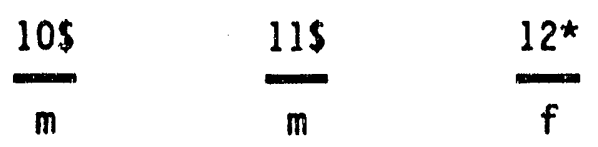

D search: $(\Delta d)_{\text {eff }}=(\Delta d)_{\text {input }}{ }^{*}(1+f(E V-1))$

Here, $d$ represents either dimension and $f$ is the superzone search fraction input $\left(18^{*}\right.$ or $19 *$ array)

The initial estimate of EV is EVI. Defining search convergence as:

$$
\text { CONVSR }=\left(K^{N}-E K O B J\right) / K^{N}
$$


and eigenvalue convergence as:

$$
C V K=\left(K^{N}-K^{N-1}\right) / K^{N}
$$

the initial value of EV is used until:

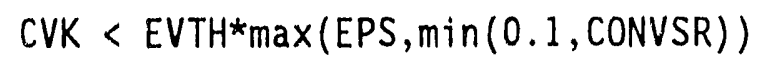

The second value of EV is determined by linear extrapolation if EVDELK $=0$, using the input value of eigenvalue dependence on $K$, DEVDKI. If EVDELK $\neq 0$, the second $E V$ is:

$$
\text { EVI } \pm \text { EVDELK }
$$

where the sign of DEVDKI is used to determine the correct step direction. Subsequent values of EV are obtained by nonlinear interpolation if possible, and linear when nonlinear fails.

Each new value of EV is constrained to the range between EV*EVCHM and EV/EVCHM. EV is always limited to the range between EVI*EVMAX and EVI/EVMAX. If EV moves to its limit and stays there, or if $K$ exceeds the range between EKOBJ+EVKMAX and EKOBJ-EVKMAX, the search is stopped.

\subsubsection{Upscatter Rebalance}

If upscatter is present in a problem, upscatter rebalance is performed after source iteration 2 and successive iterations. All fluences within the upscatter energy range are multiplied by a factor DVUPS:

$$
\text { DVUPS }=\text { UPSS } /(\text { UPSS }-(\text { UPSP }- \text { UPS }))
$$

where: IGUPS = first group into which upscatter is non-zero

UPS = previous upscatter sum

UPSP $=$ new upscatter sum

UPSS = sum of all scatter into groups $\geq$ IGUPS from groups < IGUPS

This factor is applied to fluences for all energy groups $\geq$ IGUPS, bringing the system into global balance. This procedure is usualiy helpful, and has not been observed to cause erratic behavior. The value of $\epsilon^{u}=$ DVUPS-1 is printed in the output data as upscatter convergence. 


\subsubsection{Fission Rescaling}

An approach analogous to upscatter rebalance is used when a fixed source is used with a multiplying medium. In these cases, fluences for all energy groups are multiplied by FSNORM:

$$
\text { FSNORM }=F X S U M /(F X S U M-(\text { SRNEW }- \text { FSOLD }))
$$

where: $\quad$ FXSUM $=$ total fixed source

SRNEW = new fission source estimate

FSOLD = previous fission source estimate

Since the upscatter rebalance is applied before fission sums are calculated, there is no conflict between these procedures, and one supplements the other.

\subsubsection{Fission Extrapolation}

After these rebalance and rescaling procedures, a feature is provided to use a fixed fission acceleration factor:

$$
(F S N I J)_{\text {extrap }}=(F S N I J)_{\text {calc }}+f\left[(F S N I J)_{c a l c}-(F S O I J)_{\text {final }}\right]
$$

where: FSNIJ = new fission distribution

FSOIJ = previous fission distribution

$f=$ FSNACC, an input parameter, adjusted downward as necessary to insure that no fission value is changed more than $20 \%$

No extrapolation of fluences is attempted with this procedure. Tests have shown that reasonably chosen values of FSNACC speed the convergence of certain problems, especially $M$ calculations, and cause oscillation in others. Its use is not generally recommended. The option of FSNACC has been removed from DORT.

\subsubsection{Error-Mode Extrapolation}

To accomplish error-mode extrapolation, DORT accumulates the sum FERAH and the eigenvalue estimate $\lambda$ :

$$
\begin{aligned}
\text { FERAH } & =\Sigma \mid(\text { FSNIJ })_{\text {extrap }}-(\text { FSOIJ })_{\text {extrap }} \mid \\
\lambda & =(\text { FERAH })_{\text {new }} /(\text { FERAH })_{\text {previous }}
\end{aligned}
$$


and an error-mode removal parameter $\boldsymbol{\theta}$ :

$$
\theta=\lambda /(1-\lambda)
$$

When estimates of $\theta$ in three successive iterations are in fractional agreement within EXTRCV, the fission is extrapolated according to:

$$
(\text { FSNIJ })_{\text {final }}=(\text { FSNIJ })_{\text {extrap }}+\theta^{\prime}\left[(\text { FSNIJ })_{\text {extrap }}-(\text { FSOIJ })_{\text {\&inal }}\right]
$$

where $\theta^{\prime}$ is $\theta$ adjusted so that the fission density will change by no more than |SORMIN|, where SORMIN is a input value. The fluences are similarly extrapolated by the factor $\theta^{\prime}$; with each fluence limited by the |SORMIN| ratio.

Tests show this procedure to be safe and effective. It is compatibie with upscatter rebalance and can be used together with fission rescaling.

If fission rescaling is not used, $\theta$, in certain cases, may be 100 or larger. In such cases, single-precision IBM calculations may not be sufficient to provide a stable value of $\theta$, and so the extrapolation is not effective. Rescaling may lower $\theta$ in such cases to a manageable value, i.e., to 25 or less.

As a counter example, if a system is driven by a source at its periphery, the unmultiplied fluence due to the source is so different in shape from the final fluence solution that fission rescaling may slow or thwart convergence. A negative value of SORMIN must be used in such cases, so that fission rescaling is disabled.

\subsubsection{Fluence Iteration Acceleration}

\begin{tabular}{|c|c|c|}
\hline IACC & KALF & Result \\
\hline $\begin{array}{l}0 \\
1 \\
1 \\
2\end{array}$ & $\begin{array}{c}\text { any } \\
0 \\
1 \\
0\end{array}$ & $\begin{array}{l}\text { Groupwise rebalance } \\
\text { Diffusion acceleration, corner mesh } \\
\text { Diffusion acceleration, centered mesh } \\
\text { (Conventional) Partial current }\end{array}$ \\
\hline 2 & 1 & $\begin{array}{l}\text { (Conventional) Partial current } \\
\text { rebalance, } \Phi \text { stabilized }\end{array}$ \\
\hline
\end{tabular}

Five types of fluence iteration acceleration can be selected: 
Corner-mesh diffusion acceleration (CNRDA) is usually best for "normal" problems. High-energy neutron groups in problems having complex geometry may be better solved by partial-current rebalance (PCR). The choices of stabilization with PCR are of roughly equal merit. In the case of problems having two facing reflected or periodic boundaries, such as cell or $R \theta$ problems, it is often helpful to use the coarse-mesh feature to specify only one interval in the reflected (or periodic) direction, and to specify PCR. (In such a case, the code will arrange one interval as a default unless the $85^{*} / 86^{*}$ array is entered explicitly.)

All of the fluence iteration acceleration schemes except the groupwise rebalance require the performance of a certain number of rebalance iterations after each fluence iteration. Default input parameters constrain this number between 4 and 50 (INTSCL and ITMSCL). In 1-D problems, or if the coarse mesh has only 1 interval in either direction, INTSCL $=1$ is appropriate. In $K$ calculations and searches, a lower value of ITMSCL (e.g., 8) can lower costs. Within the iteration limits, the iterations are terminated when:

$$
M A X_{i, j}\left|\frac{\phi_{i, j}^{K}-\phi_{i, j}^{K-1}}{\phi_{i, j}^{K}}\right|<C O N S C L
$$

where $K$ is the iteration index.

If the upper iteration limit is reached without satisfying CONSCL, the improved data are accepted for use if convergence has reached CONACC. otherwise, unaccelerated data are used.

It Is often effective to make CONSCL less than EPP by roughly a factor of 10, and to leave CONACC at its default value. Other combinations are often used, however.

If IBFSCL >0, rebalance iterations are bypassed on the first fluence iteration of the first source iteration. This sometimes saves CPU time on very difficult problems, and sometimes prevents certain types of early iteration failure. On less difficult problems, especially those using diffusion acceleration, it may lower efficiency slightly.

If WSOLOI > 0, fluence acceleration will not be performed on those energy groups for which, for all materials of a problem:

$$
\sigma^{S} / \sigma^{T}<W S O L O I
$$

This can save unneeded CPU effort without impairing convergence rate. 


\subsubsection{Stabilization}

The partial-current rebalance method requires choice of a "stabilization factor," F. The effect of this factor is to adjust the rebalance matrix in such a way as to insure positive factors if $F>1.0$. The user inputs an initial value, WSOLCN, and an increment, WSOLII. If WSOLII $>0, F$ is increased by WSOLII after each rebalance. If WSOLII $<0, F$ is increased by |WSOLII| only when incipient oscillation is detected.

The choice of these factors has a very large effect on the running time of difficult problems. Some combinations are shown in Table 4-2. If diffusion acceleration can be used, this difficuit choice is avoided.

Table 4-2. Typical Stabilization Values

\begin{tabular}{ccl}
\hline WSOLCN & WSOLII & \multicolumn{1}{c}{ Comments } \\
\hline 1.0 & 0 & $\begin{array}{l}\text { Satisfactory for many we11-behaved core } \\
\text { problems which are probably better } \\
\text { solved by diffusion acceleration. }\end{array}$ \\
1.0 & 1.0 & $\begin{array}{l}\text { Satisfactory for many difficult fixed.- } \\
\text { source problems }\end{array}$ \\
1.0 & 1.0 & $\begin{array}{l}\text { One solver of very difficult problem } \\
\text { uses this }\end{array}$ \\
1.0 & -1.5 & This may be a "best-buy" single choice. \\
\hline
\end{tabular}

\subsubsection{Input Source and Fluence Specification}

For $K_{e f f}$ and configuration search problems, the initial source is generated internally based on the fission cross sections and the fluence guess. For fixed-source problems, the user must supply an external source of particles. This can be done by supplying an external source file containing an internal or external boundary source, a distributed source, or a first-collision source. The external boundary source and distributed source can be generated internally by card-image input in a few simple cases. At least one non-zero source type must always be supplied.

An external boundary source is indicated by the approrriate choice of boundary condition. If input parameter NTBSI $>0$, the logical unit NTBSI is to contain the information. Otherwise, it is to be found in the card-image input. Formats for these inputs are explained elsewhere in this region. 
A distributed source is to be supplied on logical unit NTDSI if NTDSI > 0 and INPSRM $=0$. If INPSRM $>0$, NTDSI must point to a scratch device onto which a source generated from card images will be placed.

A first-collision source is to be supplied on NTFCI if NTFCI $>0$. This source is simply a special type of distributed source, and is specified by a special type of distributed source file format. In such a case, the file also gives the "uncollided fluence" the scattering of which will lead to the source. This fluence is added to the fluence calculated by the code in some of the output results. (It is subtracted from a fluence guess on NTFLX, if any.)

Internal boundary sources are indicated by NJNTSR $>0$ and/or NINTSR $>0$. In these cases, internal boundaries are specified in the $14^{*}$ and $15^{*}$ arrays at which sources input from logical unit NTIBI are located.

In principle, several types of sources can coexist. Little actual experience is available in these areas, however, and such results should be carefully checked.

If KTYPE > $>$, a non-zero fluence guess is always required. If NTFLX $>0$ and $I N P F X M=0$, the fluence is to be supplied on logical unit NTFLX. If INPFXM $>0$, the fluence guess is to be generated from card images. Problems with KTYPE $=0$ are usually started without a fluence guess, unless the problem has been partially solved in a previous execution.

\subsubsection{Key Fluence Monitoring}

In many cases, the convergence of all of the fluence data is of less interest than convergence of one or a few key fluences. If KEYJN $>0$ and KEYIN $>0$, the value of a single key fluence will be printed in the fluence-iteration monitor line. If not, the position of maximum fluence change at the second fluence iteration will be so monitored. In addition, an arbitrary array of key fluences can be specified by adjusting NKEYFX. If desired, these fluences can be printed only after the convergence of each energy group.

\subsubsection{Energy Boundary Input}

The energy boundaries are used only for transfer to the output files. They may be ignored for most applications. The ordering is: 


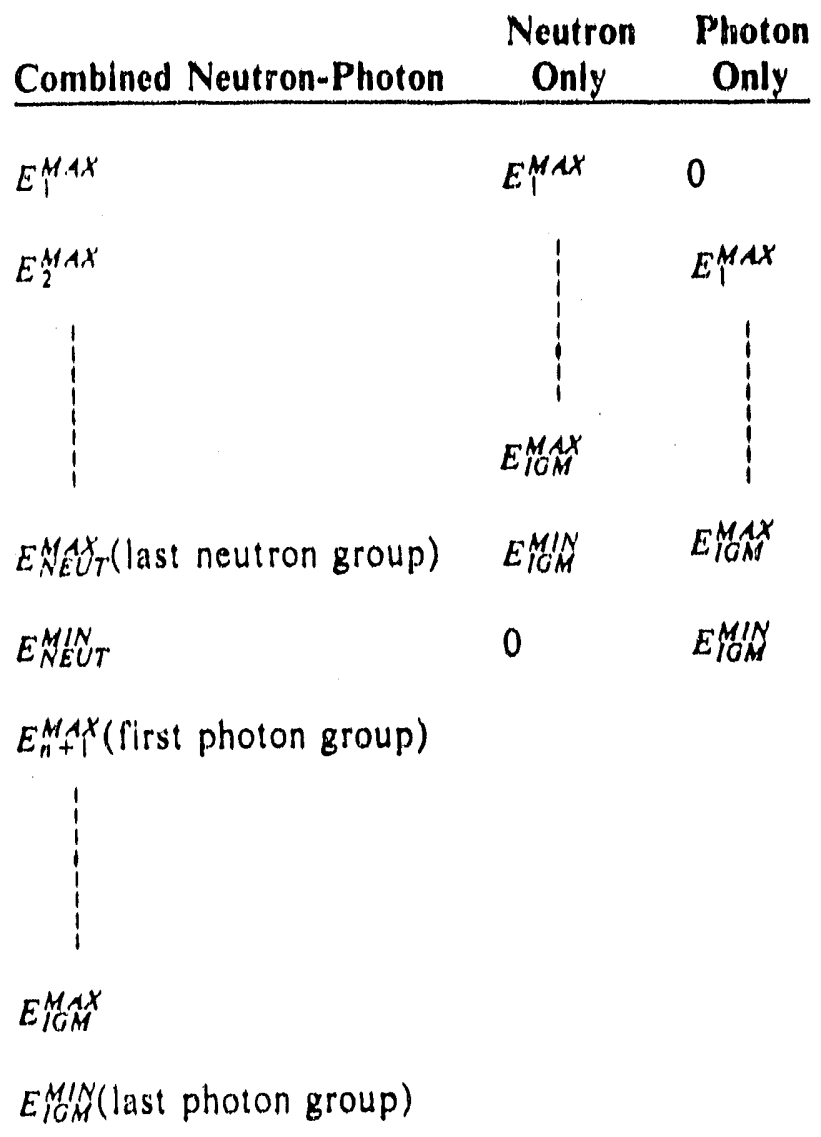

\subsubsection{Fluence Extrapolation Model}

The choice of mode can have a important influence on both CPU time requirements and the accuracy of the solutions. In general, the $\theta$ weighted model gives reasonable results for a wide range of problems. The linear-zero model is similar to the tried-and-proven method used in TWOTRAN-II. It gives well-accepted and rapid solutions to problems such as a reactor core not containing severe heterogeneities. It will often fail to converge or give inaccurate results for difficult deep penetration problems, however. The ordinary weighted model almost always converges better than the other models. Its results are usually not suitable for $K_{\text {eff }}$ calculations, but it is used exclusively by many deep-penetration analysts. 


\subsubsection{Alternate Zone Map Input}

An al ternate "combinatorial-geometry" option is avatlable. Rather than the usual $8 \$$ description, supply the following:

$$
\begin{array}{lllll}
-z_{1} & I_{1} & J_{1} & I_{1} & J_{1} \\
-z_{2} & I_{2} & J_{2} & I_{2} & J_{2} \\
\text { etc. } & & & & \\
\text { F } 0 & & & &
\end{array}
$$

Each set of five integers contains:

$$
\begin{aligned}
& -z \text { : The zone number, tagged with a negative } \\
& j] \text { Indices of lower, lefthand corner of zone in } \\
& j \text { zone map } \\
& \text { I ] Indices of upper, righthand corner of zone in } \\
& \text { zone map }
\end{aligned}
$$

As many descriptors as required to a 11 mit of IMSJM are used, and the remainder of the array is filled with 0 . Zones can be overlaid as destred. This type of $8 \$$ array must not be intermingled with the traditional type within a given problem. When used with vartable mesh, the zones are understood to be cylindrical in space, although they may be discontinuous in the zone map printout.

\subsubsection{Notes on DORT Mesh For An AirmOver-Ground Problem}

When setting up an air-over-ground DORT problem, both radial and axial mesh near the source must be chosen to restrict $1 / R^{2}$ attenuation between boundaries to about a factor of 2 . In the sample problem given in below, a basic 100-crm exclusion radius is used, and only one interval is needed within that. Then, mesh is chosen such that:

$$
\begin{aligned}
& R_{i}= R_{0}+d_{i-1} \quad(i=1,2, \ldots .) \\
& d_{i}= d_{i-1}+\Delta d \\
& \Delta d= \gamma d_{i-1} \text { or in other words, so that no } r_{i} \text { is more than } \\
& 1+\gamma \text { times } d_{i-1} \quad 0<\gamma \leq 1 \\
& \text { (A } r \text { of } .3 \text { is a good choice for all air-over-ground problems.) }
\end{aligned}
$$


Table 4-3 shows the radial mesh, where $R_{0}=0, d_{0}=100$, and $y=0.3$. When the calculated $\Delta d$ exceeds 3000,3000 should be used. The same approach is used above the source, as shown in Table 4-4. The mesh downward from the source (Table 4-5) is begun the same way, but it will meet the mesh upward from the ground (Table 4-6).

The basic mesh upward from the ground uses a larger value of $d_{0}$, and proceeds upward until the $\Delta d$ it predicts is larger than $\Delta \gamma$ predicted by the downward mesh. The two sets are joined at 6500 in this

11 lustration, a compromise between 6096 and 6943 . In the actual problem, additional intervals are added below 550 to account for ground effects. A small interval near the vehicle may be desired, also. Meshes for other source heights can be deduced from this lilustration.

Table 4-3. Radlal mesh away from source.

\begin{tabular}{rrr}
\hline$R$ & $\Delta d$ & $d$ \\
\hline 0 & $n$ & 100 \\
100 & 30 & 130 \\
130 & 39 & 169 \\
169 & 51 & 220 \\
220 & 66 & 286 \\
286 & 86 & 371 \\
371 & 111 & 483 \\
483 & 145 & 627 \\
627 & 188 & 816 \\
816 & 245 & 1,060 \\
1,060 & 310 & 1,379 \\
1,379 & 414 & 1,792 \\
1,792 & 538 & 2,330 \\
2,330 & 699 & 3,029 \\
3,029 & 909 & 3,937 \\
3,937 & 1,181 & 5,119 \\
5,119 & 1,536 & 6,654 \\
6,654 & 1,996 & 8,650 \\
8,650 & 2,595 & 11,246 \\
11,246 & 3,374 & 14,619 \\
\hline
\end{tabular}

Table 4-4. Mesh upward from source.

\begin{tabular}{crr}
\hline$z$ & $\Delta d$ & $d$ \\
\hline 12,750 & & 100 \\
12,850 & 30 & 130 \\
12,880 & 39 & 169 \\
12,919 & 51 & 220 \\
12,970 & 66 & 286 \\
13,036 & 86 & 371 \\
13,121 & 111 & 483 \\
13,233 & 145 & 627 \\
13,377 & 188 & 816 \\
13,566 & 245 & 1,060 \\
13,810 & 310 & 1,379 \\
14,129 & 414 & 1,792 \\
14,542 & 538 & 2,330 \\
15,080 & 699 & 3,029 \\
15,779 & 909 & 3,937 \\
16,687 & 1,181 & 5,119 \\
17,869 & 1,536 & 6,654 \\
19,404 & 1,996 & 8,650 \\
21,400 & 2,595 & 11,246 \\
23,996 & 3,374 & 14,619 \\
\hline
\end{tabular}


Table 4-5. Mesh downward from source.

\begin{tabular}{ccc}
\hline$z$ & $\Delta d$ & $d$ \\
\hline 12,750 & - & -100 \\
12,650 & -30 & -130 \\
12,620 & -39 & -169 \\
12,581 & -51 & -220 \\
12,530 & -66 & -286 \\
12,464 & -86 & -371 \\
12,379 & -111 & -483 \\
12,267 & -145 & -627 \\
12,113 & -188 & -816 \\
11,934 & -245 & $-1,060$ \\
11,690 & -318 & $-1,379$ \\
11,371 & -414 & $-1,792$ \\
10,958 & -538 & $-2,330$ \\
10,420 & -699 & $-3,029$ \\
9,721 & -909 & $-3,937$ \\
8,813 & $-1,181$ & $-5,119$ \\
7,631 & $-1,536$ & $-6,654$ \\
$\rightarrow 6,096$ & $-1,996$ & $-8,650$ \\
\hline 4,100 & $-2,595$ & $-11,426$ \\
\hline
\end{tabular}

Table 4-6. Mesh upward from ground.

\begin{tabular}{rrr}
\hline$z$ & $\Delta d$ & $d$ \\
\hline 50 & - & 500 \\
550 & 150 & 650 \\
700 & 195 & 845 \\
895 & 254 & 1,098 \\
1,148 & 330 & 1,428 \\
1,478 & 428 & 1,856 \\
1,906 & 557 & 2,413 \\
2,463 & 724 & 3,137 \\
3,187 & 941 & 4,079 \\
4,128 & 1,224 & 5,302 \\
5,352 & 1,591 & 6,893 \\
$+6,943$ & 2,068 & 8,961 \\
\hline 9,011 & 2,688 & 11,649 \\
11,699 & 3,495 & 15,144 \\
\hline
\end{tabular}

\subsection{DORT INPUT AND OUTPUT FILE FORMATS}

The DORT user input and output data files are expressed in formats designed according to recommendations of DOE's Committee on Computer Code Coordination (CCCC). 17 The particular files and which format they use are listed in Section 4.7. Two exceptions, retained for backward compatibility, are an optional capability to process a "GIP" cross section input file and to produce a "DOT Angular Fluence Tape" as output.

The GIP file is defined exactly as the records labeled "CROSS SECTION DATA" in the ORDOSW file (of which only the cross-section portion is 1 isted), and has no other record types. The "DOT III Angular Fluence Tape" is defined in the DOT III User's Manual, ${ }^{12}$ and is simulated by DORT as accurately as possible. The user should consult Reference 14 for the full description of the ORDOSW file. 


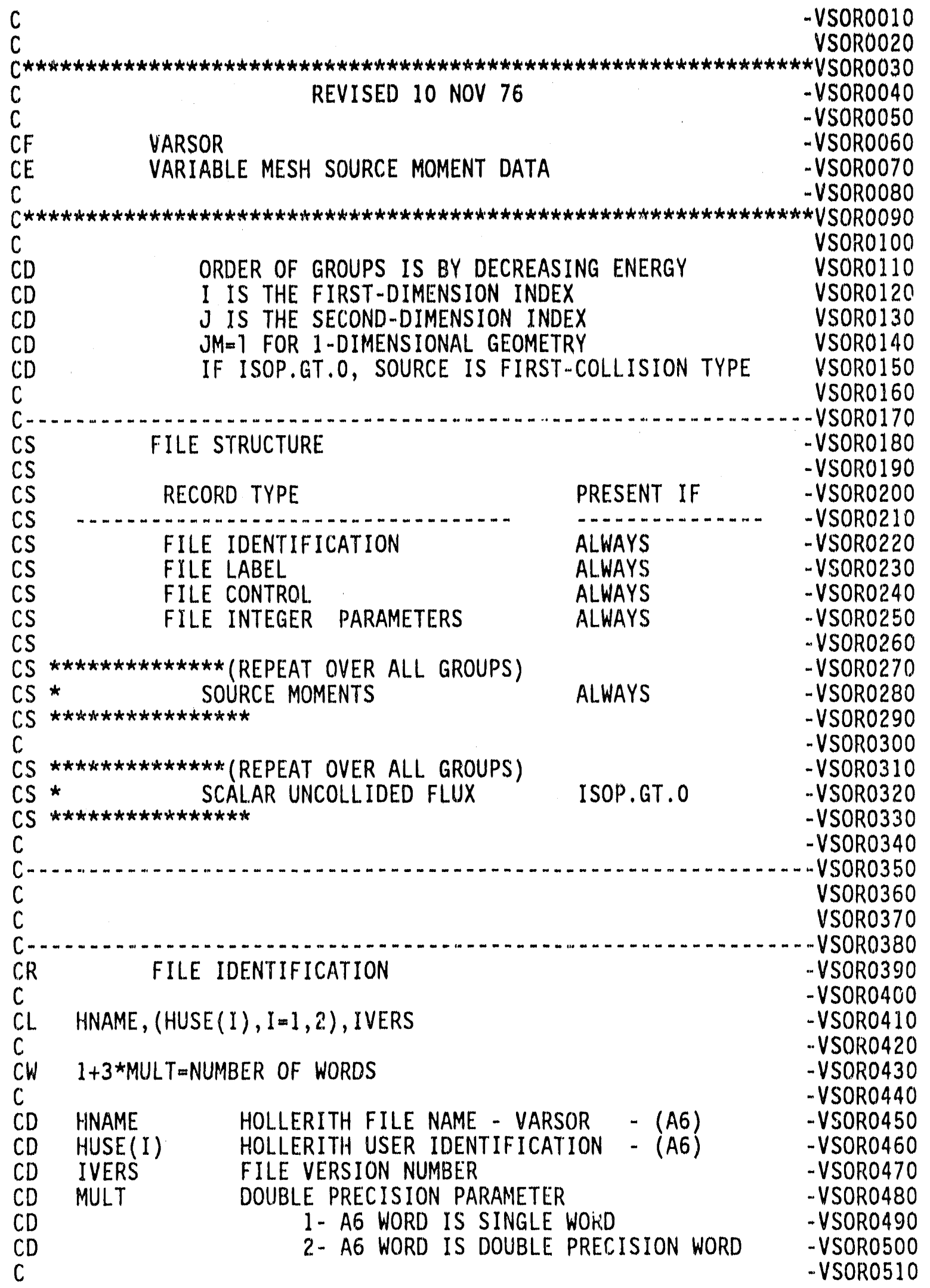




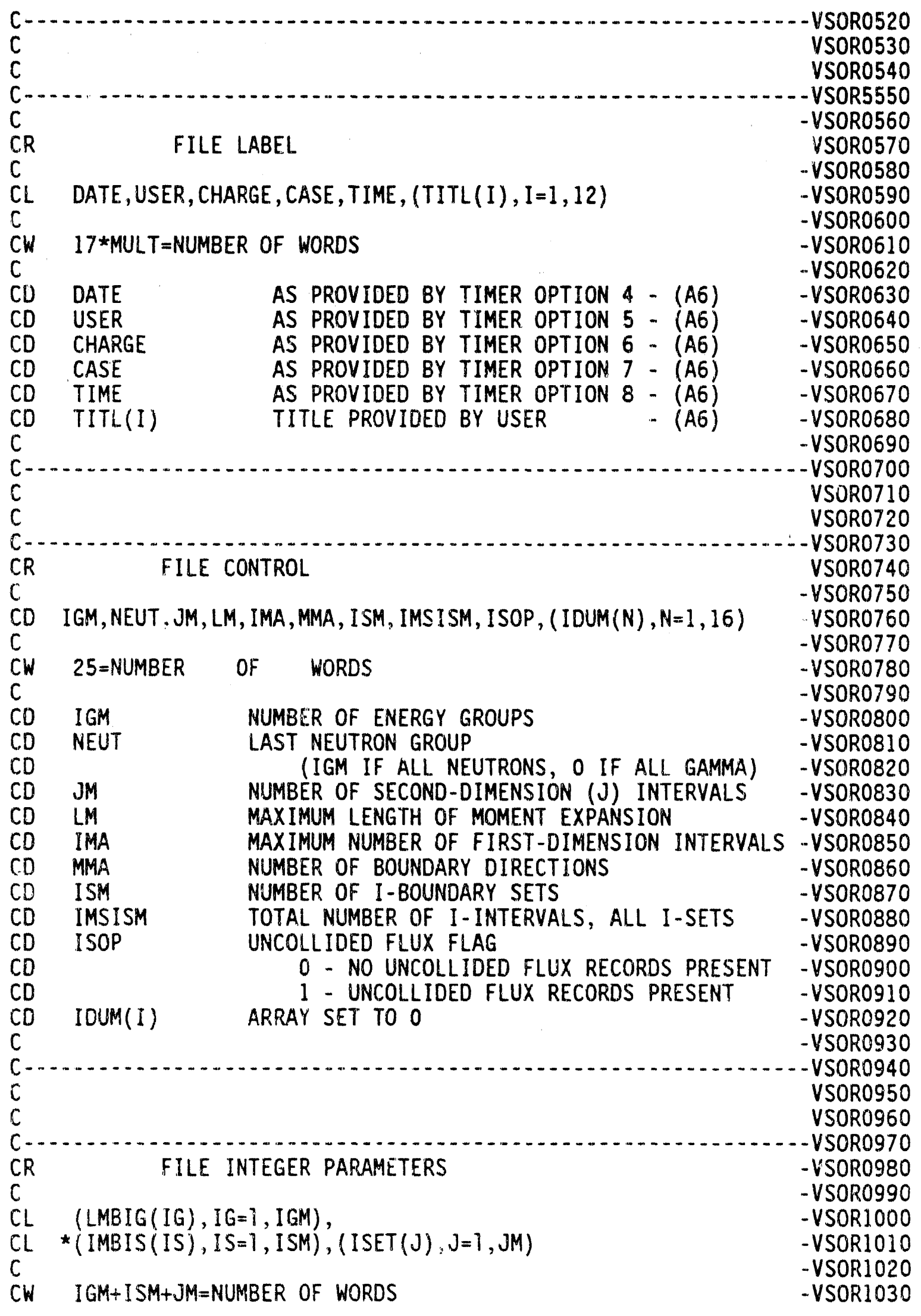




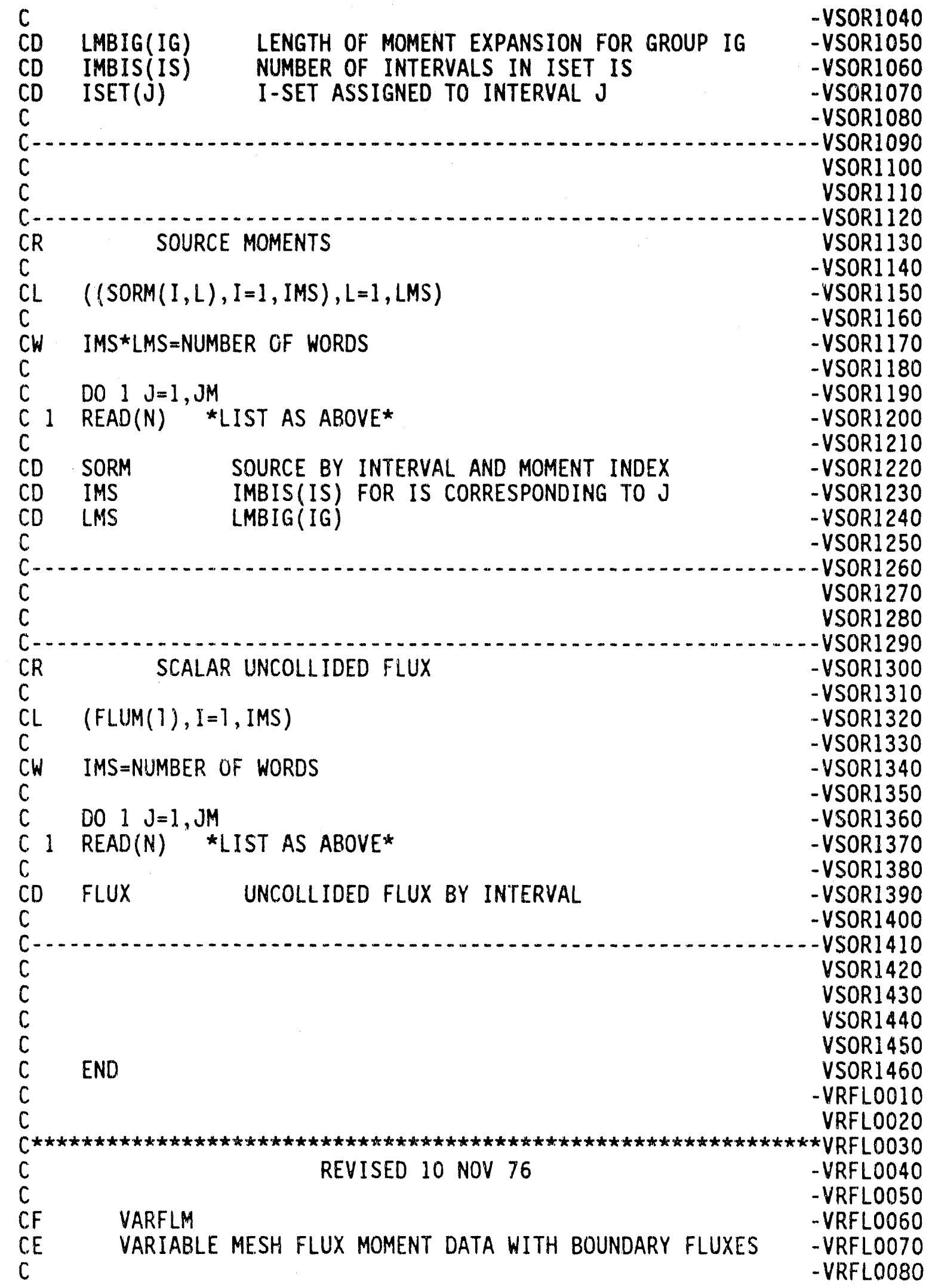




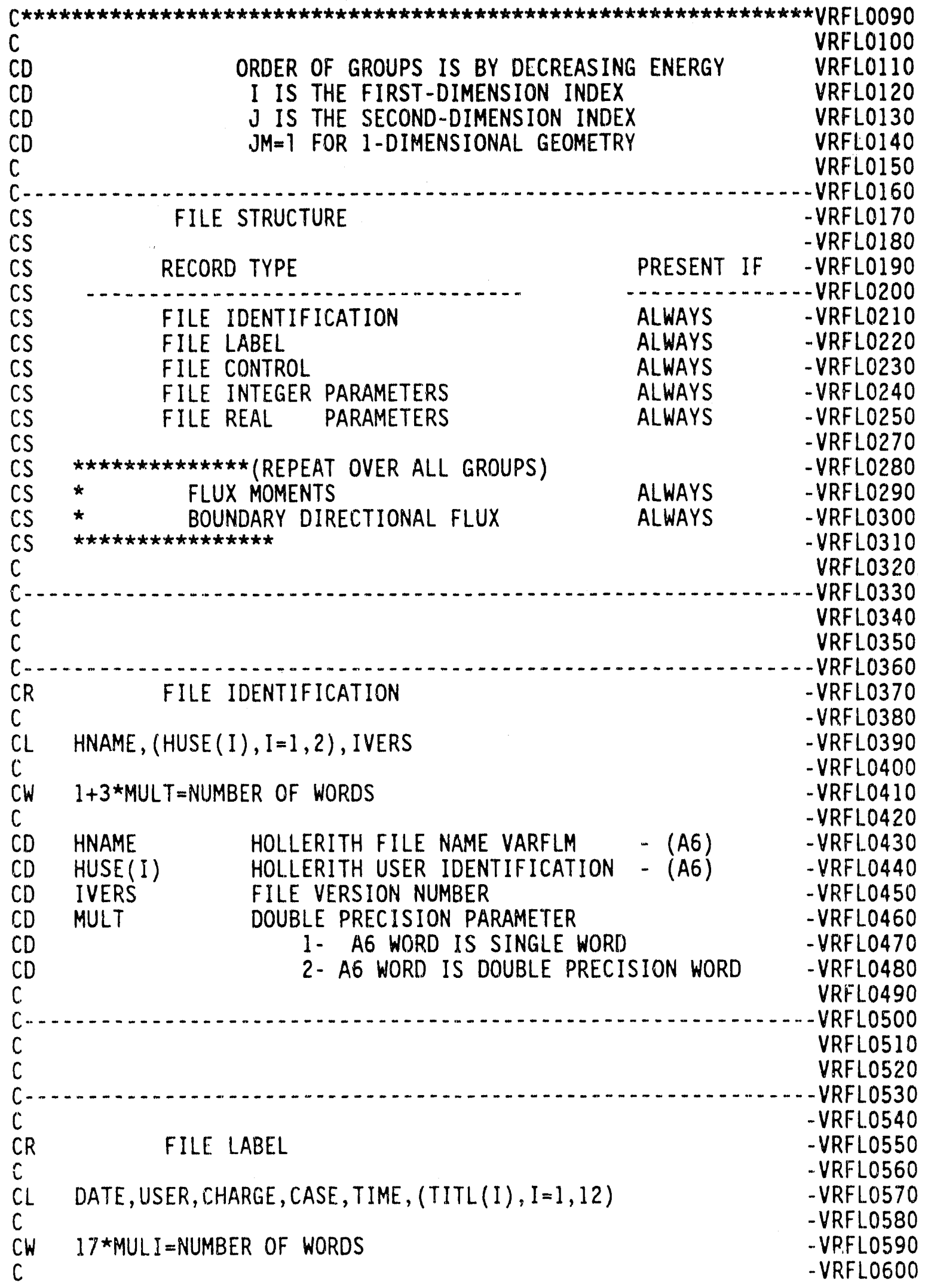




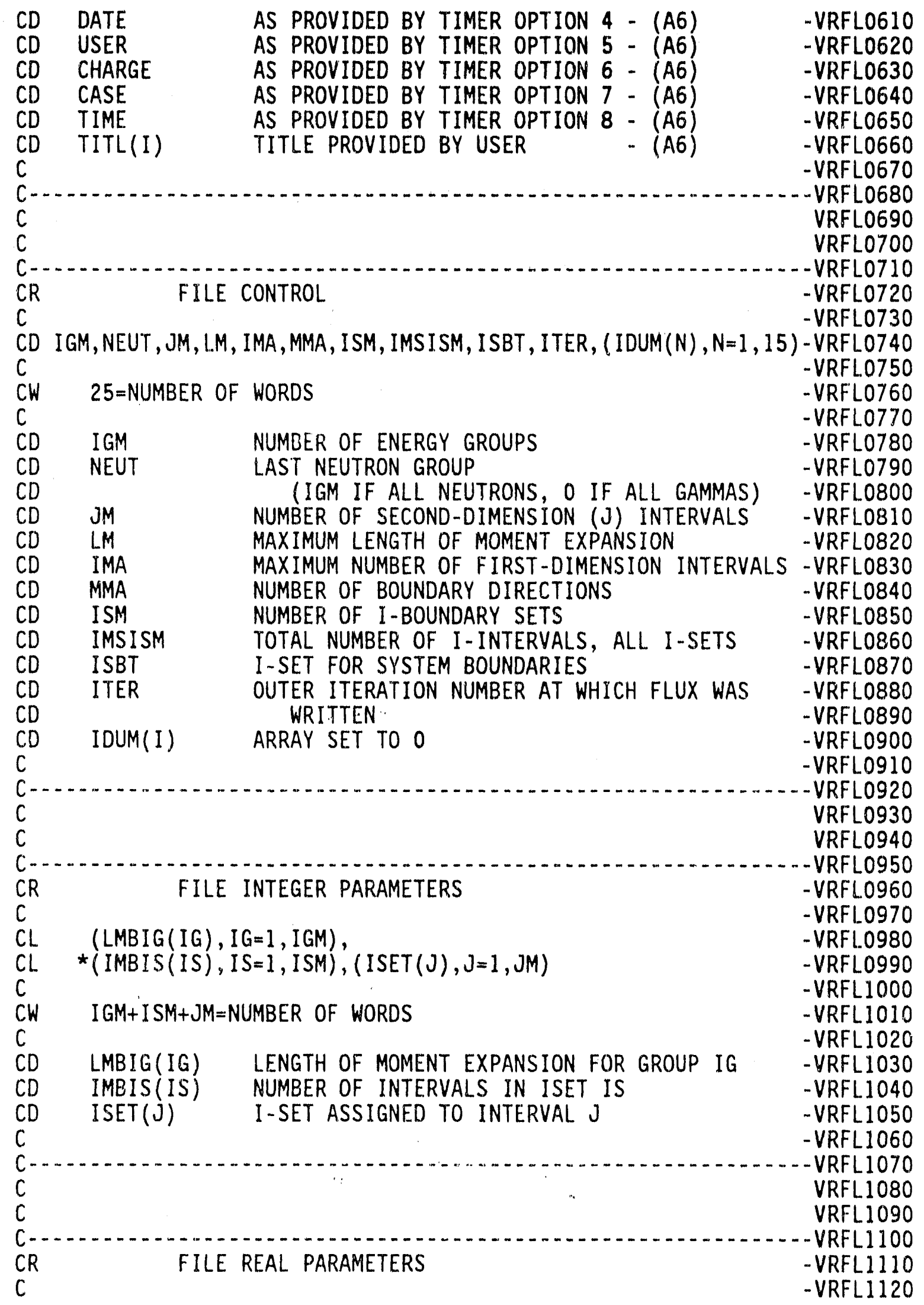




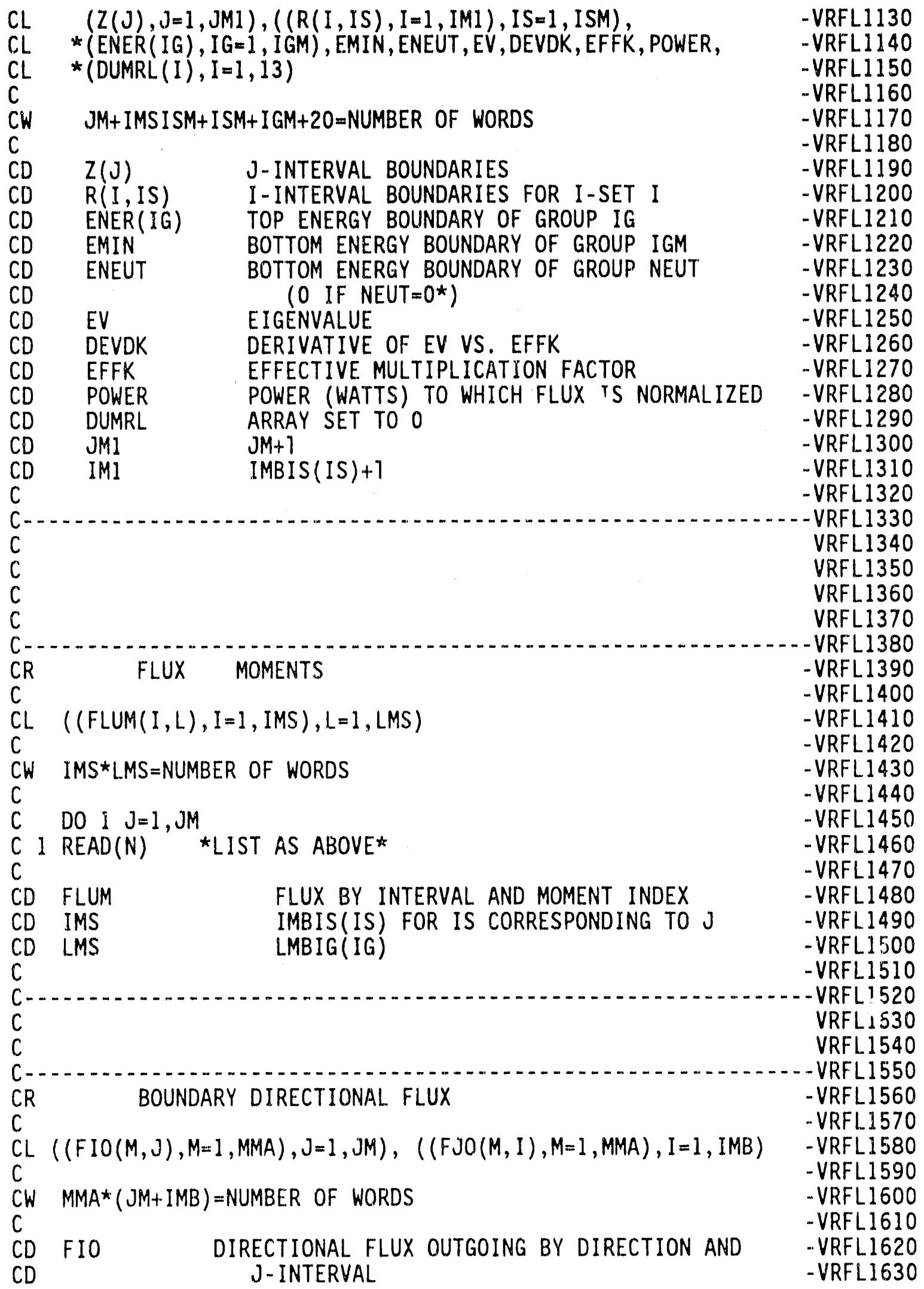




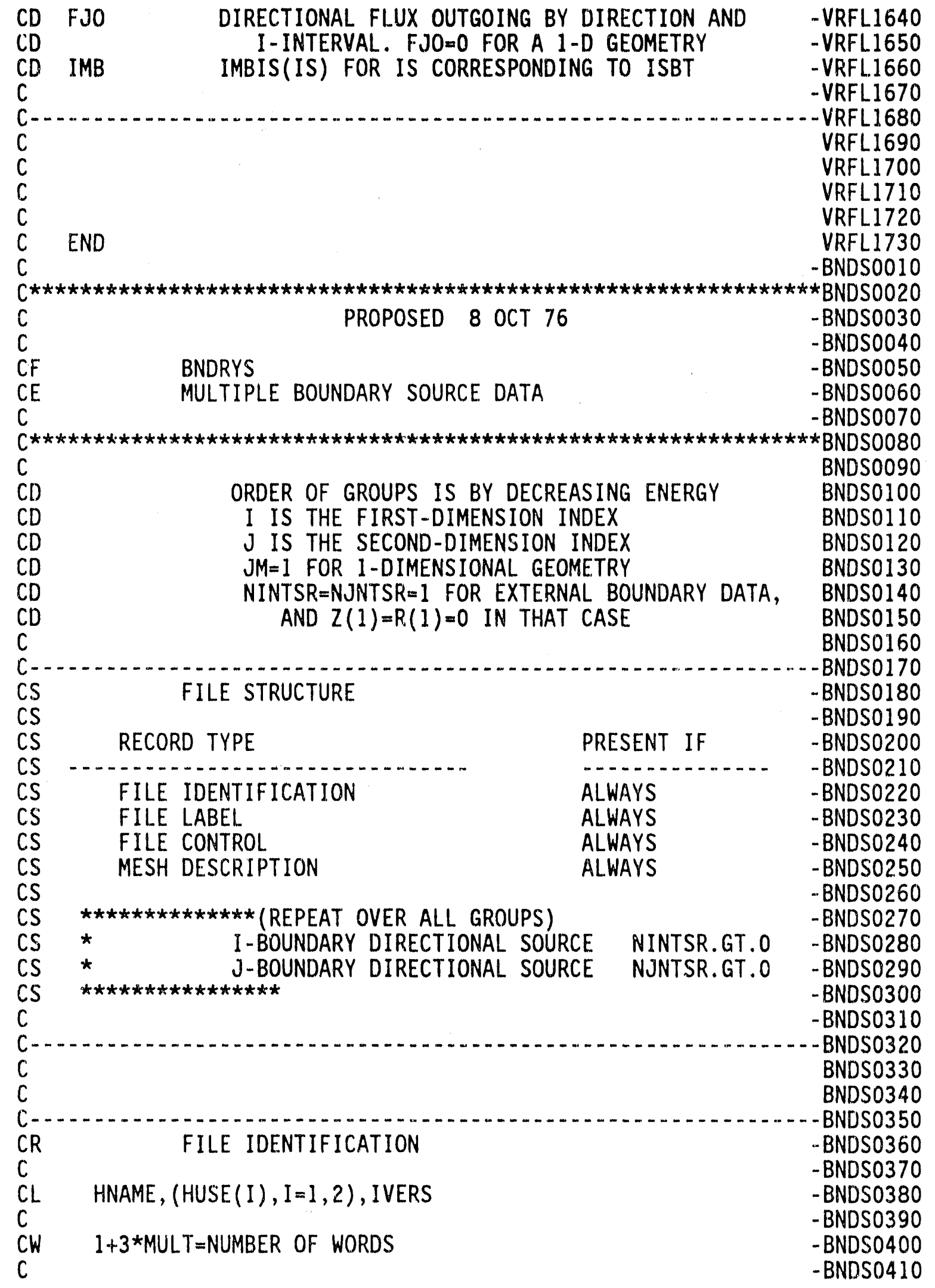




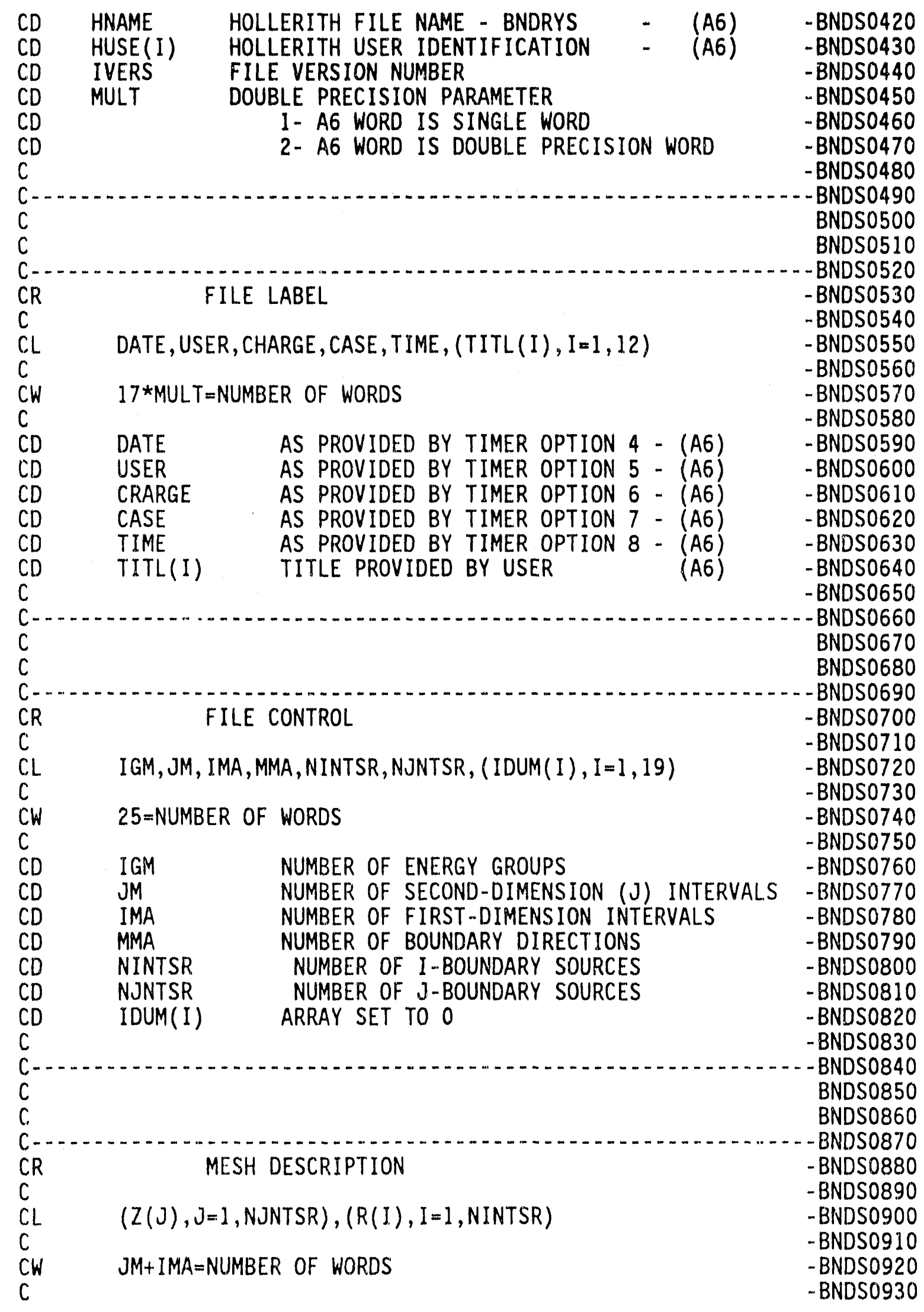




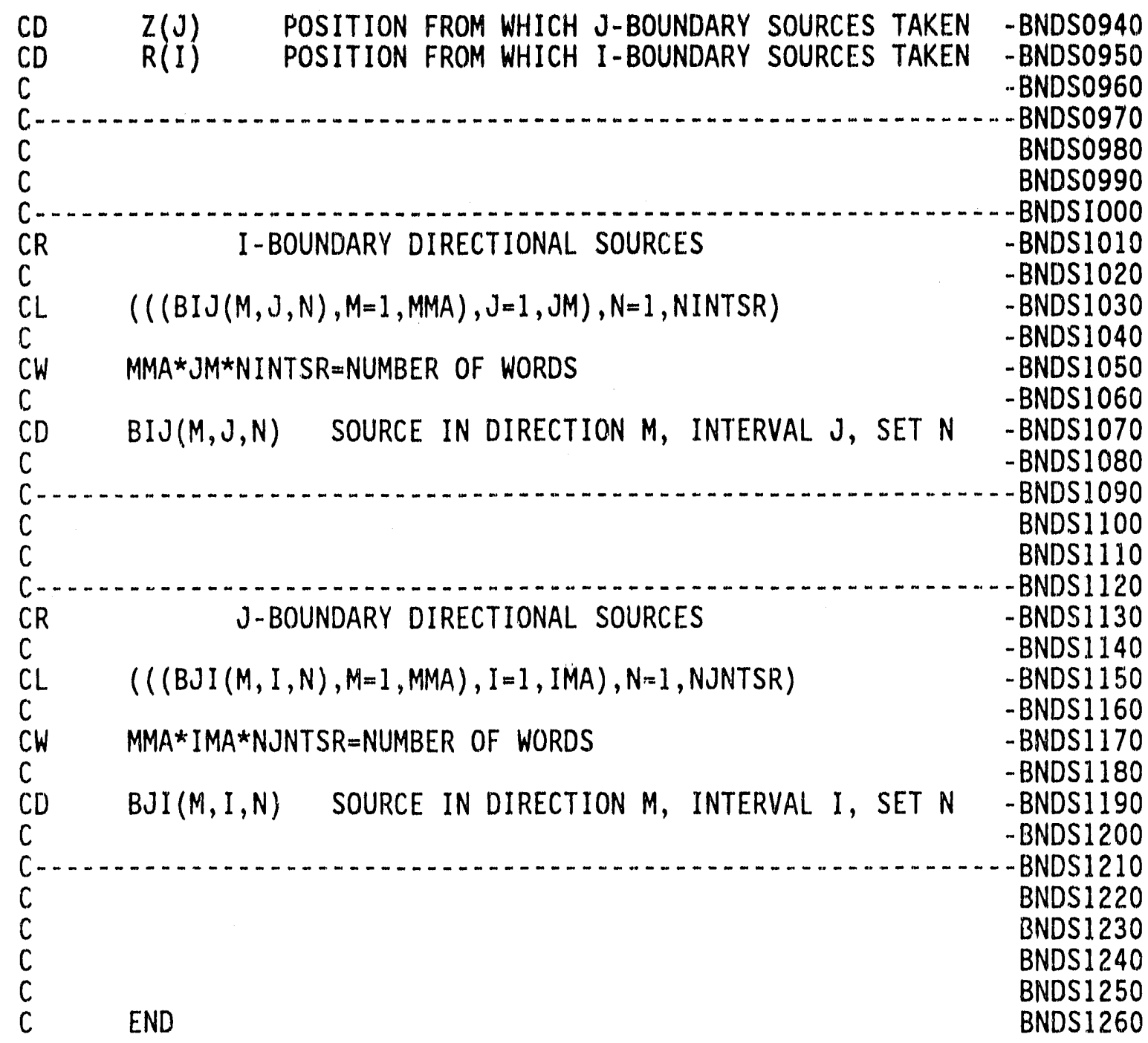

NOTE: This is only the cross section portion of the ORDOSW file.

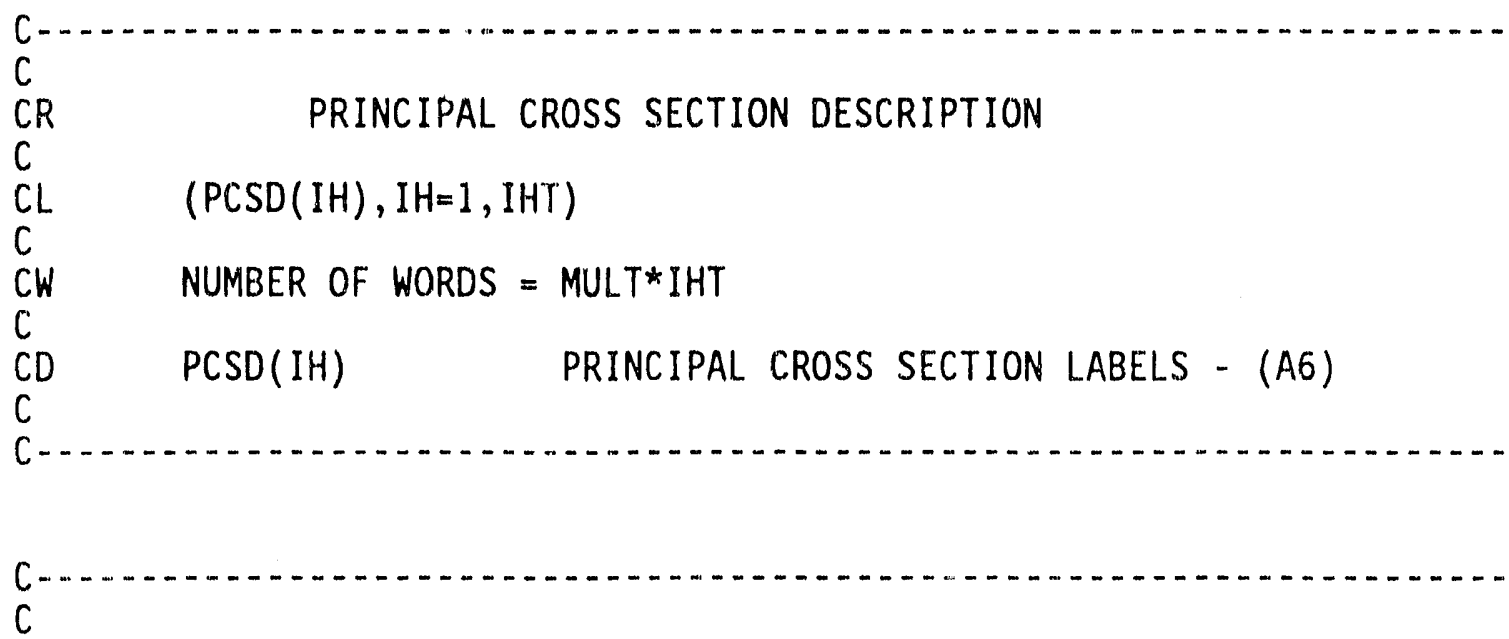



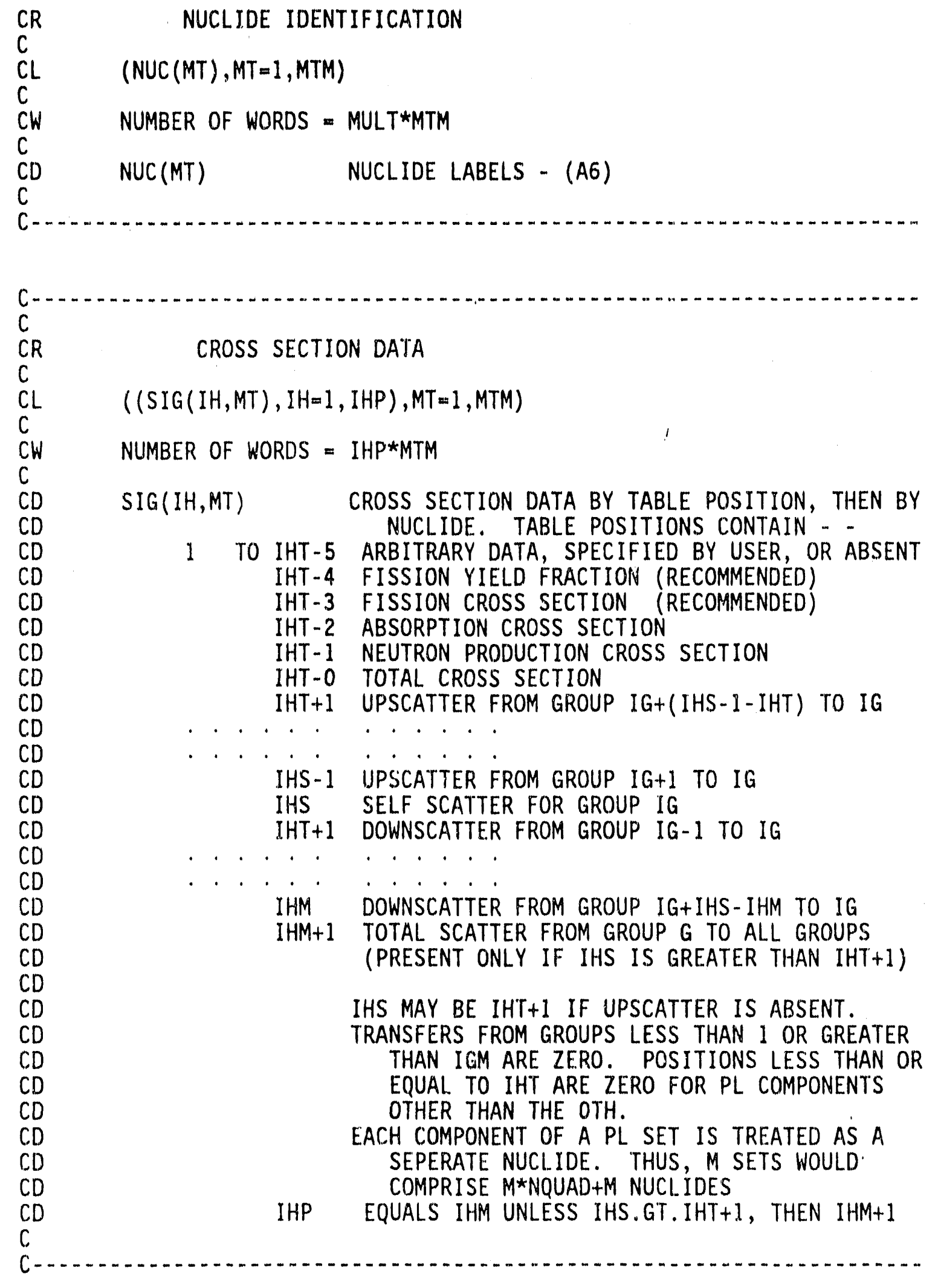


\subsection{LOGICAL. UNIT REQUIREMENTS}

\subsubsection{Input and Output Data Flles}

All of the user input and output data flles are expressed in formats designed according to recommendations of DOE's Comittee on Computer Code Coordination (CCCC). 17 The spectfications of these files are contained in Section 4.6. Two exceptions, retained for backward. compatibllity, are an optional capability to process a "GIP" cross section input file and to produce a "DOT Angular Fluence Tape" as output.

The GIP file is defined exactly as the records labeled "CROSS SECTION DATA" in the ORDOSW file, and has no other record types. The "DOT I1I Angular Fluence Tape" is defined in the DOT III User's Manual, ${ }^{12}$ and is simulated by DORT as accurately as possible. An alternate format is used when the albedo generation option is used.

The correspondence of file type to use in the code is:

\begin{tabular}{lll}
\hline SYMBOL & FILE TYPE & USE \\
\hline NTFLX & VARFLM & Fluence and moment input \\
NTFOG & VARFLM & Fluence and moment output \\
NTSIG & ORDOSW or GIP & Cross section input \\
NTBSI & BNDRYS & External boundary source input \\
NTDSI & VARSOR & Distributed source moment input \\
NTFCI & VARSOR & First collision source input \\
NTIBI & BNDRYS & Internal boundary source input \\
NTIBO & BNDRYS & Internal boundary fluence output \\
NTDIR & DOT III "Angular & Directional fluence output \\
& FIuence Tape" & Directional fluence moment output \\
NTDSO & VARSOR &
\end{tabular}

Logical units 5 and 6 are also used by DORT as the standard card image input and printed output units respectively.

\subsubsection{Scratch Data Sets}

The following lists the scratch data sets, the length of each record, and the number of records: 


\begin{tabular}{|c|c|c|c|c|c|}
\hline $\begin{array}{l}\text { Logleal } \\
\text { Unit } \\
\text { Number }\end{array}$ & $\begin{array}{l}\text { Loglcal } \\
\text { Unit } \\
\text { symbol }\end{array}$ & Desoription & $\begin{array}{l}\text { Record Length } \\
\text { (1 reoord) }\end{array}$ & $\begin{array}{l}\text { Number of } \\
\text { Records } \\
\text { (nrec) }\end{array}$ & Requil rend if: \\
\hline 81 & & Scalar Flivende scratch & IMA*JM & INH & Not Used at \\
\hline $\begin{array}{l}91 \\
92 \\
93 \\
94 \\
95\end{array}$ & $\begin{array}{l}\text { NDFLX } \\
\text { NDSOR } \\
\text { NDSIN } \\
\text { NDEFX } \\
\text { NOFIJ }\end{array}$ & $\begin{array}{l}\text { Fluence scratch } \\
\text { Tutal source scratch } \\
\text { iN-Source serateh } \\
\text { Boundary Fluerco soratch } \\
\text { Scalar Fl wence Scratch }\end{array}$ & 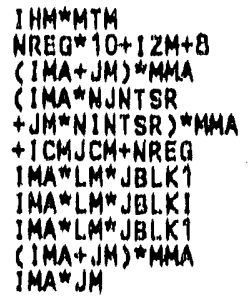 & $\begin{array}{l}\text { IOM } \\
\text { IOM } \\
\text { IOM } \\
\text { IOM } \\
\text { NJGLK"IOM } \\
\text { NJGLK } \\
\text { NJGLK } \\
\text { IOM } \\
\text { IOM }\end{array}$ & 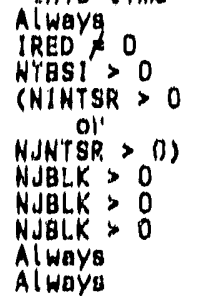 \\
\hline
\end{tabular}

NOTE: The symbol "\#" denotes the word "number."

$J B L K 1=\# J$ levels per $J$ block, $1 \leq J B L K 1 \leq J M$

NJBLK = \#J blocks, MINBLK $\leq$ NJBLK $\leq$ MAXBLK (NJBLK = 0 if fluences are stored in working memory)

LM - maximum number of moments (including $0^{\text {th }}$ ) in spherical harmonic expansion, LM $=1+\mid$ ISCT $\mid *((\mid$ ISCT $\mid+3)) / 2$

NREG = number of edit regions

$I Z M=$ number of material zones

For unit 84, If NTBSI $>0$ and NINTSR $>0$, for example, this file will have IGM + IGM records.

The number of $J$ blocks, NJBLK, is controlled by input data. When default values of MINBLK and MAXBLK are used, the code will attempt to hold all fluence and source data in fast memory, t.e., NJBLK $=0$. Failing in this, it will attempt to allocate space for the entire mesh to be held as 1 block in the user-buffer area, 1.e., NJBLK = 1. Falling in this, the space mesh is broken into as many blocks as required. NJBLK is restricted to limits specified by MINBLK and MAXBLK.

\subsection{DETAILS OF DORT JOB REQUIREMENTS}

\subsubsection{CPU Time Usage}

A reasonably accurate time limit should be calculated and entered as TMAX for all problems which run more than a few minutes. The maximum time given to the operator or operating system should be somewhat larger than TMAX. This allows DORT to complete Its $I / 0$ and data summaries if the time is exceeded during the iteration phase. The CPU time irequired, $c$, can be estimated by the following formula: 


$$
\begin{gathered}
C=A+\left[\frac{F W}{F R}\right] * \frac{150}{S F} \\
F W=\sum_{P} \sum_{G} \sum_{F} \sum_{J} \sum_{I} M M
\end{gathered}
$$

where

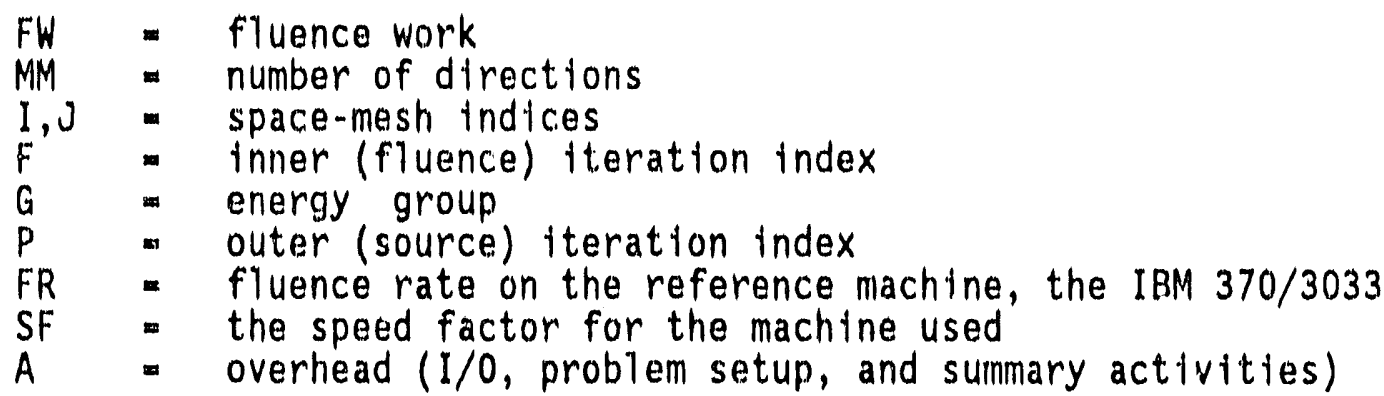

The value of $A$ is typically 0 to 2 minutes on the IBM 360/91. Suggested speed factors are shown in Table 4-7.

\begin{tabular}{ll}
$\begin{array}{c}\text { Table 4-7. } \\
\text { (Based on IBM }\end{array}$ 709 Factor \\
(B) \\
\hline CDC 7600 & 250 \\
IBM $360 / 195$ & 250 \\
IBM $370 / 3033$ & 150 \\
IBM $360 / 91$ & 150 \\
CDC 6600 & 30 \\
IBM 7094 & 10 \\
IBM 7090 & 5 \\
\hline
\end{tabular}

The value of FR depends somewhat upon the class of problem solved. FR is about 1.0 milliton fluences per minute for large $P_{3}$ problems using the weighted difference model. The linear-zero model sometimes runs a bit faster, and a $P_{0}$ calculation runs as much as $40 \%$ faster. The IBM speeds assume the use of optional assembler language fluence routines. Assembler language routines are not avatiable for CDC use. Expert opintons offered to us differ as to how much the CDC performance would beneftt from the use of assembler language. 


\subsubsection{System Buffer Space (NBUF) and Memory Requirements}

A discussion on the system buffer space is given in Reference 14 for many of the machines on which the DORT code is operable. This parameter is machine dependent and the interested user should consult this reference. The use of the default given in the input will usually give the user adequate buffer space.

The user must understand the various storage arrangements in order to provide enough space for data storage. An exact formula for the amount of storage required is not practical. Numerous small arrays are assigned, and arrays overlap in storage in many cases. Approximate methods have proven satisfactory, however. A detalled presentation of the memory requirements can be found in Reference 14.

\subsubsection{Problem Printed Output}

Each DORT Job starts with a notice of the date and time, followed by a record of the particular version of the code being used, and messages from the programmer lriforming the user of warnings or spectal features. The title supplied by the user as input data is next. As each array of input data is read, a notice of the array number and length is made.

The first array edits are of the 61\$ (logical unit assignments), $62 \$$ (integer control parameters), and $63^{*}$ (real control parameters). The inttial allocation of fast memory and (CDC only) slow memory are listed, followed by primary dimenston-setting arrays, mixing table information, material assignment, importance, fission spectrum, and search fractions.

The next section is a set of data describing the directional remesh process, MM $<0$. These tables are largely for the benefit of programmers in diagnosing troublesome problems. The quadrature set assignment by super-zone is an input array. The transition table indicates which transition set governs the transition from the row $M-S e t$ to the column M-Set.

The unpacking arrays follow; one for each M-Set. These integers simply indicate places in a full |MM|-direction array where data corresponding to a smaller M-Set can be kept without being overwritten.

The actual transition sets are given next. Each set consists of two columns of integers. Each position in each column corresponds to a direction in the corresponding M-Set. The remeshing rout ine decodes the integers in order to determine how to construct fluences in a new M-Set which corresporid to a previous $M$-Set. Since the tables are given in unpacked form, as needed by the code, unused positions are filled with "-1."

The direction sets are listed, together with "BETA" (the $\mu$-flow coupling term), $\mu$-mates, $r$-mates, and level-mates. The $\mu$-mate of direction $(\mu, \tau)$ is direction $(-\mu, \tau)$. The $r$-mate is direction $(\mu,-q)$. The level-mate 
is the first direction in the $\eta$-level containing the $r$-Mate. Unsymmetric direction sets may not possess all of these mates. If a mate required for a boundary condition can not be found, an error flag is set.

The values of the coupling set $C_{n i j}$ are edited, followed by the $\mathrm{J}$ finemesh and I fine-mesh sets. The real values of super-mesh and coarsemesh boundaries are interpreted into integar assignment tables edited at this point. The total size of these meshes, number of super groups, and of edit zones are given, together with related input array edits.

Maps of the zone and material assignments are given, together with the integral values of the unnormalized input sources by group, with the total as group IGM +1 . A table of storage usage gives data useful to the programmer, as well as items needed in adjusting the input data, as discussed el sewhere.

A source iteration monitor line ends each source iteration, and initial values are indicated in an "iteration 0" line. Entries on this line are:

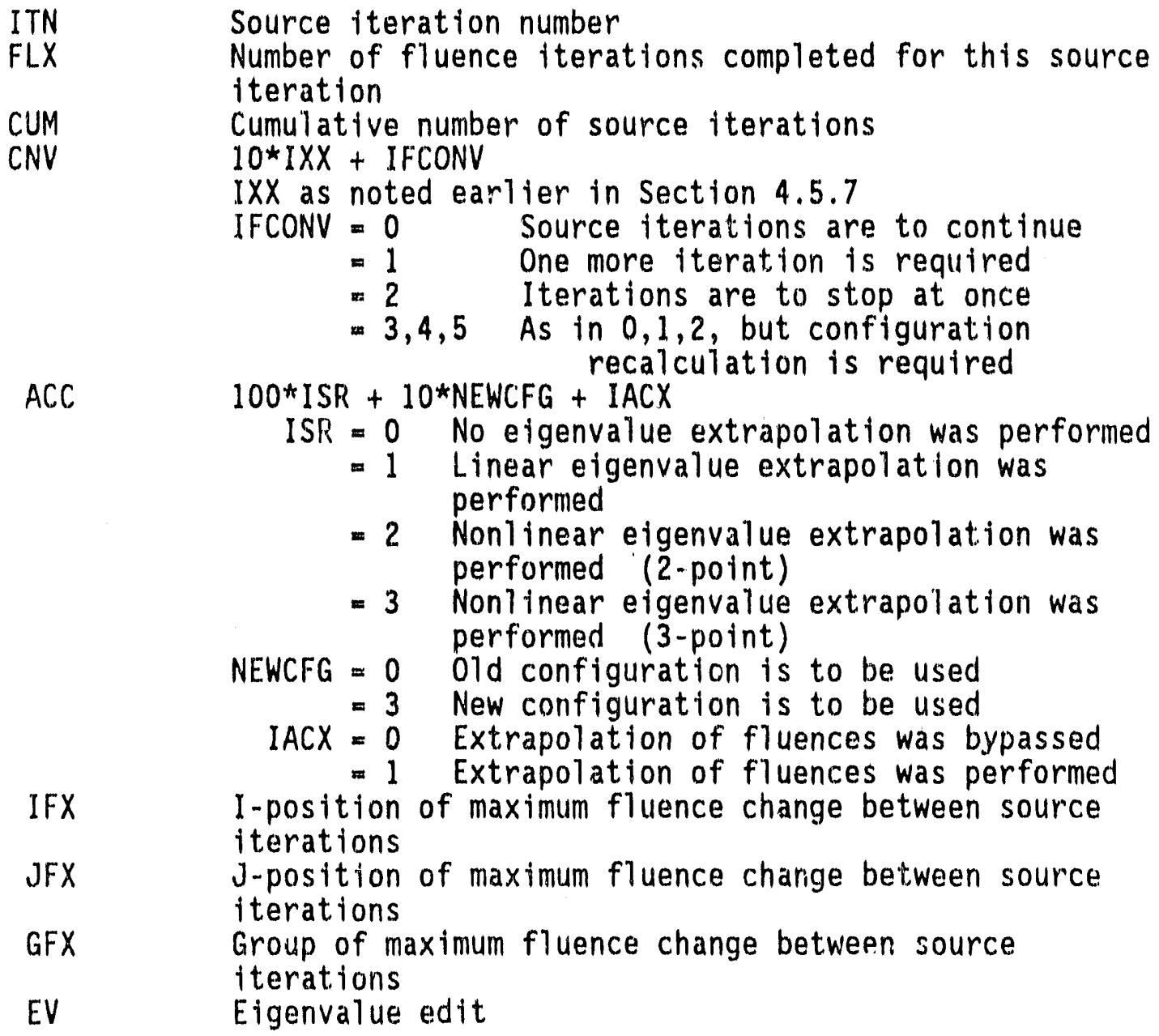




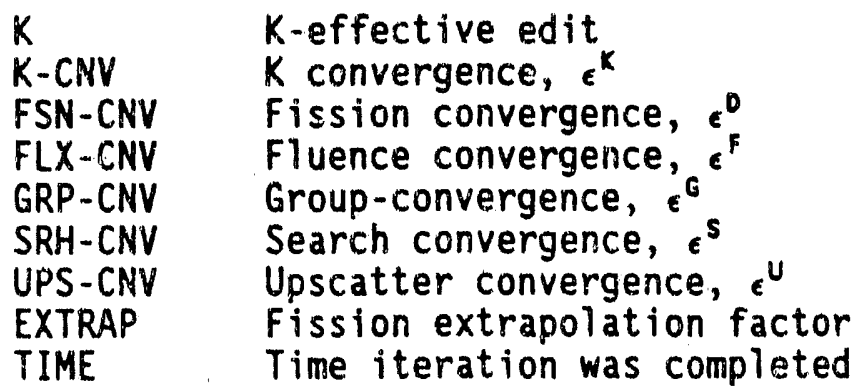

The interpretations of $E V$ and $K$ are:

\begin{tabular}{ccc} 
KTYPE & EV edit & Kedit \\
\hline 0 & $\Sigma V_{\nu \sigma} \sigma_{\Phi}(\Sigma X V \Phi$ for adjoint $)$ & $\Sigma X V \nu \sigma^{F} \Phi$ \\
1 & $"$ & $"$ \\
$2,3,4$ & search eigenvalue & $"$
\end{tabular}

If the $\chi^{\prime} s$ do not sum to $1, K$ will differ from $E V$ in a $K$-calculation by a ratio equal to the sum of $\chi$. Even if $X$ is precisely normalized to 1 by selecting the input value NOFIS $=0$, EV and $K$ may be slightly different due to roundoff.

As each fluence iteration is performed on each energy group, another monitor line is printed. It contains:

GRP Energy group number

ITN Fluence iteration number

IMFD I-position of maximum fluence change

JMFD J-position of maximum fluence change

MX FX DV Relative maximum fluence change

MX DV FX Most rapidly changing fluence value

REBL Number of rebalance iterations - a negative value means the rebalance factors were not used

REBL ERR Maximum relative change in rebalance factors in the last iteration 
MAX REBL Largest amount by which any factor deviates from 1

GRP REBL Amount by which groupwise rebalance factor (whether used or not) deviates from 1

VOLM ERR Volumetric convergence, discussed earlier, except that absolute value is not used

KEY FLUX Value of single key fluence requested by input data, after acceleration

NEGFIX Maximum fraction of negative total source at any position, if NEGFIX $=1$

SOURCE Total of all sources into a group, including external boundary sources

TIME The time at which group iteration is begun is given at the end of the heading column. Arbitrary diagnostic information is given below the time indication.

Iterations performed using diffusion theory will provide only the first six items. Edits of key fluence values and/or convergence by region may follow each iteration or the set of iterations for each group, according to the options chosen. The interpretation of fluence convergence also depends on options chosen, as discussed earlier.

If a dimension search has been performed, the final dimensions are edited. Fluence and fission source edits follow, together with activity edits by interval and region.

System balance tables follow by group and region. Group and system totals are given as IGM +1 and NP.EG +1 . The columns are largely selfexplanatory. One may note that an external boundary source is external to the system, and is, therefore, included in these tables as a flow at the corresponding boundary. Flow and leakage signs are such that a positive value is outward from the region. Fission source is source used to calculate the fluence, while fission rate is the fission which. results from the fluences. They will tend to differ by $K$ times the sum over $\mathrm{g}$ of the $\chi_{\text {in }}$ a $\mathrm{K}$-calculation. Inscatter and outscatter are scatter to and from the energy group. They should, in general, be equal in neutron-only problems. This will not be true in coupled $n-\gamma$ problems. The outscatter is calculated from:

$$
\text { outscatter }=\text { collisions }- \text { selfscatter }- \text { absorption }
$$

and will be valid only if the absorption cross section meets the balance condition. 
The edit of selfscatter per collision is valuable in judging the difficulty of converging fluences for a given energy group. In general, values larger than 0.85 may give the PCR fluence acceleration difficulty. The average density factor is collision weighted.

The value of OUT/IN approaches unity for well-converged problems. It is:

Collision $+D B^{2}$ Loss + Positive Boundary Outflow

Fixed Source + Fission Source + Inscatter + Negative Boundary Outflow

Sources, flows, leakage, and OUT/IN may not be correct in the case of groups in which no iterations are performed.

The fluences, as well as balance table information which depend on thein, are not divided by $K_{e f f}$, and will, in general, tend to vary according to $K_{e f f}$. Some users speak of this condition as "fluences normalized according to $K$."

Each problem ends with a summary of clock and CPU time binned according to major sections of the code. Depending on configuration, some bins may not have labels. These are for diagnostic information only.

Some output may not appear in certain problems, depending on the options chosen. The use of NTPRT can alter the order of printing on some systems, or can transfer some output items to alternate output devices.

\subsection{PROGRAMMER INFORMATION}

\subsubsection{Inter-machine Adaptability}

The DORT code is intended to be easily adaptable to any type of sophisticated computer, and yet to take advantage of certain localized structural features which may be machine-dependent.

In general, the guidelines of ANS-STD.3-1971 16 are followed. This requires general adherence to a simple, standard FORTRAN language except where deviations provide important improvements in capability and can be documented. In addition, machine-dependent features have been kept localized and have not been included in the subroutines which perform actual computation.

The recommended procedures of the Committee on Computer Code Coordination (CCCC $)^{15,17}$ have been followed where practical. Compliance with these standards has been incomplete where cost was prohibitive, the nature of the codes did not allow it, or the expected level of performance could not be obtained in that fashion. 
Where minor or certain machine-dependent features are required, alternative features are enclosed in pairs of 3-character "language flags." The alternative statements remain in the source program, with inappropriate sections transformed into comment statements. If the code is set up for IBM operation, for example, it might contain:

$\begin{array}{ll}\text { CIB } & \\ \text { CIB } & \text { ENTRY IBCDC }(H, E, L, P) \\ \text { CDC } & \\ C & \text { ENTRY IBCDC } \\ \text { CDC } & \end{array}$

The corresponding $\operatorname{CDC}$ configuration would be:

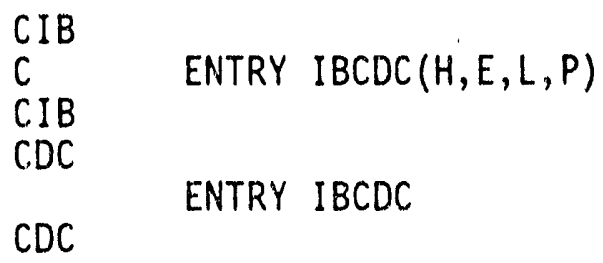

All versions of a given procedure are thus available for inspection by all users. The selection of options is made by a computer program at distribution time. A listing of machine-deperident sections can also be prepared in that process.

The language flags achieve the major objective of having a single, unified source for each program whic' is maintained for all users. With these provisions, the basic FORTRAN programs are operable on IBM, CDC, UNIVAC, CRAY, Amdah1, and other computers, except that system-dependent service routines as specified by CCCC must be provided.

Optional packages and procedures which provide enhanced convenience are also available. The optional run-time storage allocation requires system-dependent routines. Instructions for installing these options are distributed with the source programs. All known users at this time use all of the optional enhancements available to them. Even so, the basic operation with FORTRAN routines plus the CCCC package remains available if needed.

\subsubsection{Service Subroutines}

Certain standard service subroutines are specified by the CCCC for use in reactor physics codes. The service routines used in these codes include: 
TIMER Provides timing and job identification data

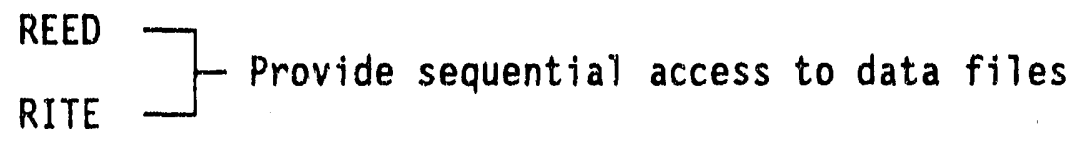

DRED - Provide random access to data files

DOPC Provides initiating, closing, and certain repositioning of data files

CRED
CRIT $\longrightarrow \begin{aligned} & \text { Provide block transfer of data between fast and siow } \\ & \text { memory. }\end{aligned}$

Many of these subroutines also call other subroutines. Many are entirely system dependent. Each configuration to be distributed contains an appropriate set of service subroutines, insofar as possible. The realities of computing environments may require local modification or substitution. The specifications given in Reference 16, together with extensive in-stream comments, provide guides for such modification.

\subsection{REFERENCES}

1. W. A. Rhoades and R. L. Childs, "The DORT Two-Dimensional Discrete Ordinates Transport Code," Nuclear Science \& Engineering 99, 1, pp. 88-89, (May 1988).

2. K. D. Lathrop and F. W. Brinkley, "TWOTRAN-II, An Interfaced, Exportable Version of the TWOTRAN Code for Two-Dimensional Transport," LA-4848-MS, (JuTy 1973).

3. F. R. Mynatt, et al., "Development of Two-Dimensional Discrete Ordinates Transport Theory for Radiation Shielding," CTC-INF-952, (August 1969).

4. B. G. Carlson and K. D. Lathrop, "Transport-Theory - The Method of Discrete Ordinates," LA-3251-MS, (Rev. October 1965).

5. B. G. Carson, "Solution of the Transport Equation by $S_{n}$ Approximations," LA-1599, (October 1953).

6. B. G. Carlson and G. I. Bell, "Solution of the Transport Equation by the $S_{n}$ Method," Proc. U.N. Intern. Conf. Peaceful Uses At. Energy, 2nd, Geneva P/2386, (1958). 
7. B. Carlson, C. Lee, and J. Worlton, "The DSN and TDC Neutron Transport Codes," LAMS-2346, (February 1960).

8. B. Davison, Neutron Transport Theory, Oxford University Press, 174 (1957).

9. S. Chandrasekhar, Radiative Transfer, Oxford University Press, (1950).

10. G. C. Wick, "Uber Ebene Diffusionsprobleme," Z. Phys., 121, 702 (1943).

11. W. A. Rhoades, D. B. Simpson, R. L. Childs, and W. W. Engle, Jr., "The DOT-IV Two-Dimensional Discrete Ordinates Transport Code with Space-Dependent Mesh and Quadrature," ORNL/TM-6529, Oak Ridge National Laboratory, (January 1979).

12. W. A. Rhoades and F. R. Mynatt, "The DOT III Two-Dimensional Discrete Ordinates Transport Code," ORNL-TM-4280, Oak Ridge National Laboratory, (September 1973).

13. K. D. Lathrop, "TWOTRAN - A FORTRAN Program for Two-Dimensional Transport," GA-8747, (JuTy 1968).

14. H. A. Rhoades and R. L. Childs, "An Updated Version of the DOT 4 One- and Two-Dimensional Neutron/Photon Transport Code," ORNL-5851, Oak Ridge National Laboratory, (January 1979).

15. R. Douglas O'Dell, "Standard Interface Files and Procedures for Reactor Physics Codes, Version IV," LA-6941-MS, Los Alamos National Laboratory, (September 1977).

16. ANS Standard "Recommended Programming Practices to Facilitate the Interchange of Digital Computer Programs," prepared by Subcommittee 10, ANS Standards Committee (Apri1 1971).

17. B. M. Carmichae1, "Standard Interface Files and Procedures for Reactor Physics Codes, Version III," LA-5486-MS, Los Alamos National Laboratory, (February 1974).

18. J. O. Johnson, J. D. Drischler, and J. M. Barnes, "Analys is of the Fal1-1989 Two-Meter Box Test Bed Experiments Performed at the Army Pulse Radiation Facility (APRF)," ORNL/TM-11777, Oak Ridge National Laboratory, (May 1991).

19. R. T. Santoro et al., "DNA Radiation Environments Program Fall 1989 2-Meter Box Experiments and Analysis," ORNL/TM-11840, Oak Ridge National Laboratory, (May 1991). 


\subsection{SAMPLE PROBLEM}

The sample problem demonstrates the calculation of the air-over-ground environment for the two-meter box experiments. ${ }^{18,19}$ Figure $4-2$ shows a simple diagram of the geonetry modeled in the DORT input. (It should be noted again that the DORT and GRTUNCL geometry models are equivalent for the analys is performed in MASH.) A complete listing of the input cards for the sample problem is given in Figure 4-3, and some selected output is shown in Figure 4-4.

The DORT input stream shown in Figure 4-3 includes a tremendous amount of control parameters in the first data block (62\$, 62\$, and 63* arrays). The user will note that most of these parameters are system or code defaults. Consequently, only those parameters of particular importance to the sample problem will be emphasized. The $61 \$$ array parameters indicate the input and output files utilized in this calculation. It should be noted that this particular DORT case utilized an input fluence guess (NTFLX). This was because this calculation was restarted from a previous one. If no input fluence guess is used, this unit would be zero. The scalar fluences were written on unit 21 (NTFOG), the cross sections (from GIP) were input on unit 4 (NTSIG), the directional fluences were written on unit 22 (NTDIR), and the distributed source was read in from unit 23 (NTDSI). The distributed source represents the output file from the GRTUNCL sample problem discussed in Section 3.0, and the cross sections are from the GIP sample problem discussed in Section 2.0. The user should compare the parameters used in these two problems with the ones used in the DORT case to see how the different programs interact.

In viewing Figure $4-3$, the input illustrates; 8 material zones (IZ.M), 66 radial intervals (IM), 84 axial intervals (JM), 69 energy groups (IGM), a cross-section table length (IHM) of 72, no upscatter (IHT $=3$, and $I H S=4$ ), and a mixing table length (MS) of 0.54 materials ( 9 different mixtures, each with a $P_{0}$ through $P_{5}$ component) were read in from a $G I P$ tape $(M T M P=54)$. The total number of materials (MT) was 54, and no print was requested for the printed output (ICSPRT $=1)$. The problem included; 240 directions in the quadrature set $(M M=240)$, RZ geometry (INGEOM=1), group dependent fluence iteration (IIXMI $=-4$ ), theta weighted fluence extrapolation model $(M O D E=4)$, directional fluences saved (IDIRF $=2$ ), and 20 key fluences printed at the group convergence (NKEYFX=-20). The group dependent fluence iteration scheme is given in the $24 \$$ array. The axial and radial intervals for the key fluences are given in the $29 \$$ and $30 \$$ arrays. The first directional fluence axial interval saved was 13 (JDIRF), and the last interval saved was 25 (JDIRL). The user should note these two parameters match parameters used in the VISTA sample problem given in the Section 5.0. A zone dependent convergence criterion was chosen (IEPSBZ $=11$ ) and the convergence parameters for each material zone are in the $24^{\star}$ array. This parameter is useful when performing a large air-over-ground problem, and the user does not want to converge the entire mesh to a fine convergence criteria when only a portion of the mesh is really important. In the sample problem, there 
was a tight convergence criteria used at the locations of importance in the mesh, a less strict criteria used around these areas, and then a minimal criteria used for the areas of lee importance. The zone numbers by interval are entered in the $8 \$$ array, and the material by zone is entered in the $9 \$$ array. These arrays along with the axial intervals (2* array) and radial intervals (4* array) match the same arrays in the GRTUNCL sample problem, with the exception that GRTUNCL requires a negative entry in the $9 \$$ array for to indicate the higher order of cross section scattering.

A full description of the DORT printed output is given in Section 4.8.3 above. The user should consult this section for viewing the sample problem output. The selected DORT output shown in Figure 4-4 first illustrates the input parameters read in the first data block, followed by the initial memory requirements to run this particular DORT case. The output then produces the directional remesh arrays, followed by indicators that the second, third, and fourth data blocks have been successfully read. A listing of some of the parameters in the third data block $\left(10 \$, 11 \$, 12^{*}, 9 \$, 24^{*}\right.$, etc.) are next in tabular form. This table is followed by the quadrature information and Legendre coefficients for scattering integrals. This information is followed by the fine mesh sets, the zone number and material number by interval maps. This table is followed by a listing of storage information usefur to the programmer. Next, the source iteration information followed by the key fluence array is printed for each group. This is an important table in that the problem convergence is illustrated here. Finally, the system balance tables, depending on the option chosen, (IRED) are printed. In this sample problem, a balance table was chosen for each zone in the problem along with a system table. The last line printed on the sample problem output is a summary of clock and CPU time used. 


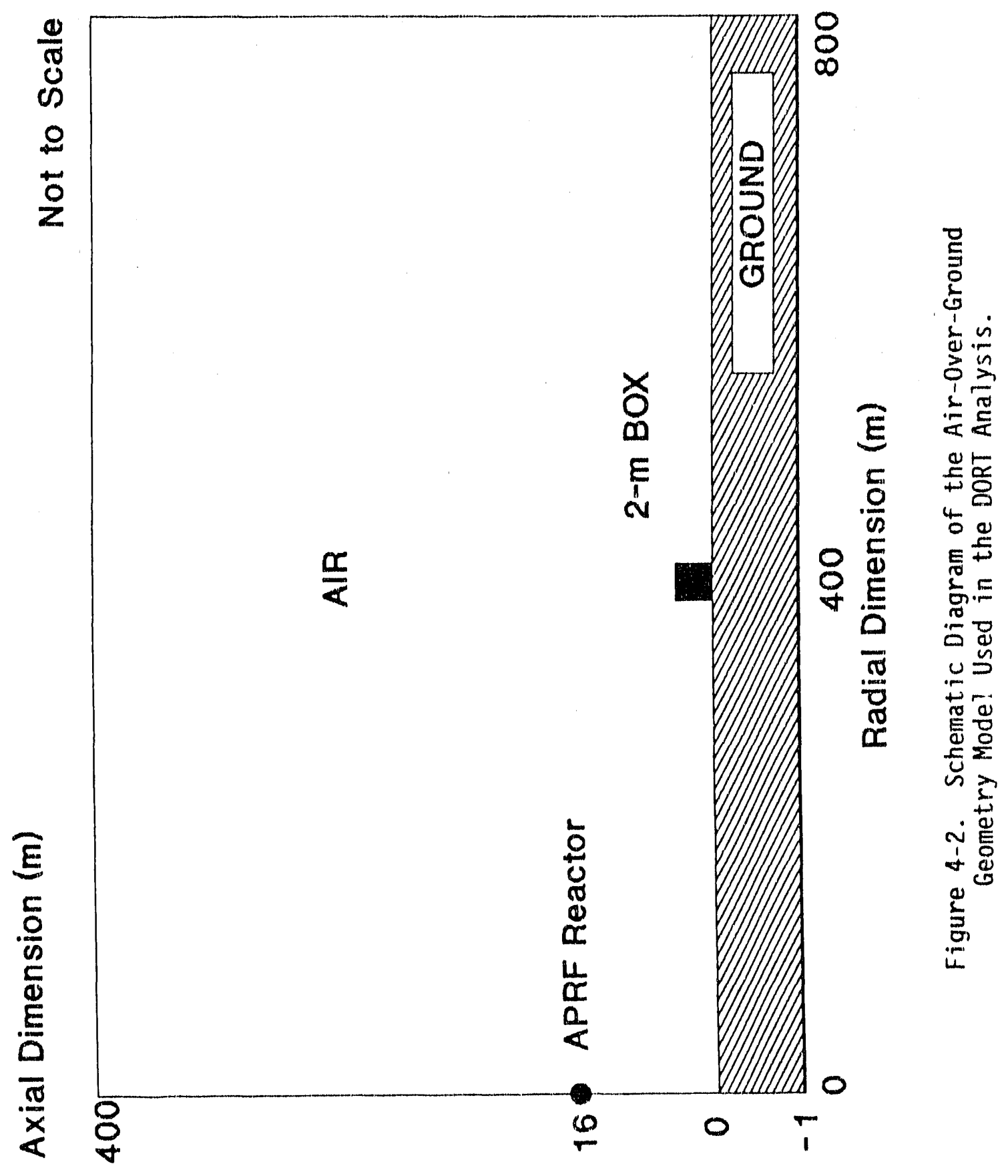


1 dort - aprf aog using saic 1989 angle-energy leakage source, $\mathrm{mm}=240$

$116.143 \mathrm{~m}$ source height, simple topography out to $400 \mathrm{~m}$ test site

' $34 \%$ ground moisture, $10 / 24 / 89$ (a) a ir parameters

$61 \$ 20214023$ / ntfix,ntfog,ntsig,ntbsi,ntdsi

000022 / ntfci,ntibi, ntibo, ntnpr, ntdir

0 e / ntdso

$62 \$ \$ 0586684$ / iadj, isctm, $\{\mathrm{zm}, i \mathrm{~m}, j \mathrm{~m}$

6934720 / igm, iht, ihs, ihm, $m|x|$

054540240 / mer,mtp,mtm, idfac,mm

110000 / ingeom, ibl, ibr, ibb, ibt

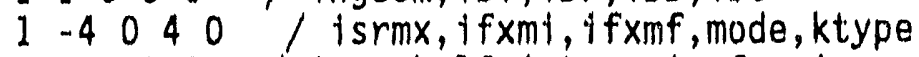

200000 / lacc,kalf, Igtype, inpfxm, inpsrm

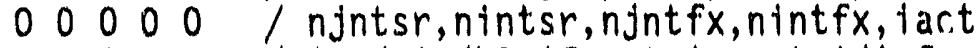

80112 / ired, ipdb2, ifxprt,icsprt,idirf

1325120111 / jdirf,jdirl, nbuf, lepsbz, minblk

11111 / maxblk, isbt,msbt,msdm, ibfscl

450200 / intscl, itmscl, nofis, ifdb2z, iswp

1928000 / keyjn, keyin, nsigtp, norpos, normat

$00250250-20$ / mstmax, negfix, locobj, 1cmobj, nkeyfx

446000 / ncndin, neut, itally, isp1, isp2

e

$63 * * 1200.01-41-20.0$ / tmax, xnf, eps, epp, epv

1-3 $1.00 .2 \quad 1.5 \quad 10.0$ /epf, ekobj, evth, evchm, evmax

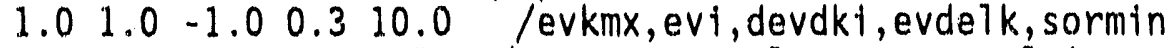

$1.01 .4 \quad 1-20.3-1.5 \quad$ / conacc, conscl, coneps, wsolot, wsolit

$\begin{array}{lllll}1.5 & 0.6 & 0.0 & 1-60 & 0.0\end{array}$ /wsolcn, orf, fsnacc, fl xmin, smooth

1-2 0.20 .9 / epo, extrcv, theta

e $t$

t

81 ** / wts mm240

02 2r102900-8 0 2r307825-8 02 2r510200-8 02 2r708425-8 0

$2 r 901350-8 \quad 0 \quad 563869-8 \quad 316131-8$ n2 $0 \quad 641385-8 \quad 359590-8$ n2 0

714976-8 $400849-8$ n2 0 784547-8 $439853-8$ n2 0 857529-8

480771-8 n2 $0 \quad 642875-8 \quad 293289-8 \quad 479164-8$ n3 0 681415-8

310872-8 $507890-8$ n3 0 716550-8 $326901-8 \quad 534077-8$ n3 0

745915-8 $340298-8 \quad 555965-8$ n3 0 7755565-8 $353825-8 \quad 578064-8$

n3 0 489468-8 $3862.82-8 \quad 513536-8 \quad 364389-8$ n4 0 500102-8

394674-8 $524693-8 \quad 372306-8$ n4 0 508580-8 $401365-8 \quad 533587-8$

378617-8 n4 0 515474-8 $406806-8 \quad 540820-8 \quad 383750-8$ n4 0

$517107-8 \quad 408094-8 \quad 542534-8 \quad 384965-8$ n4 q120

Figure 4-3. Sample DORT Input for the

Two-Meter Box Air-Over-Ground Analys is. 


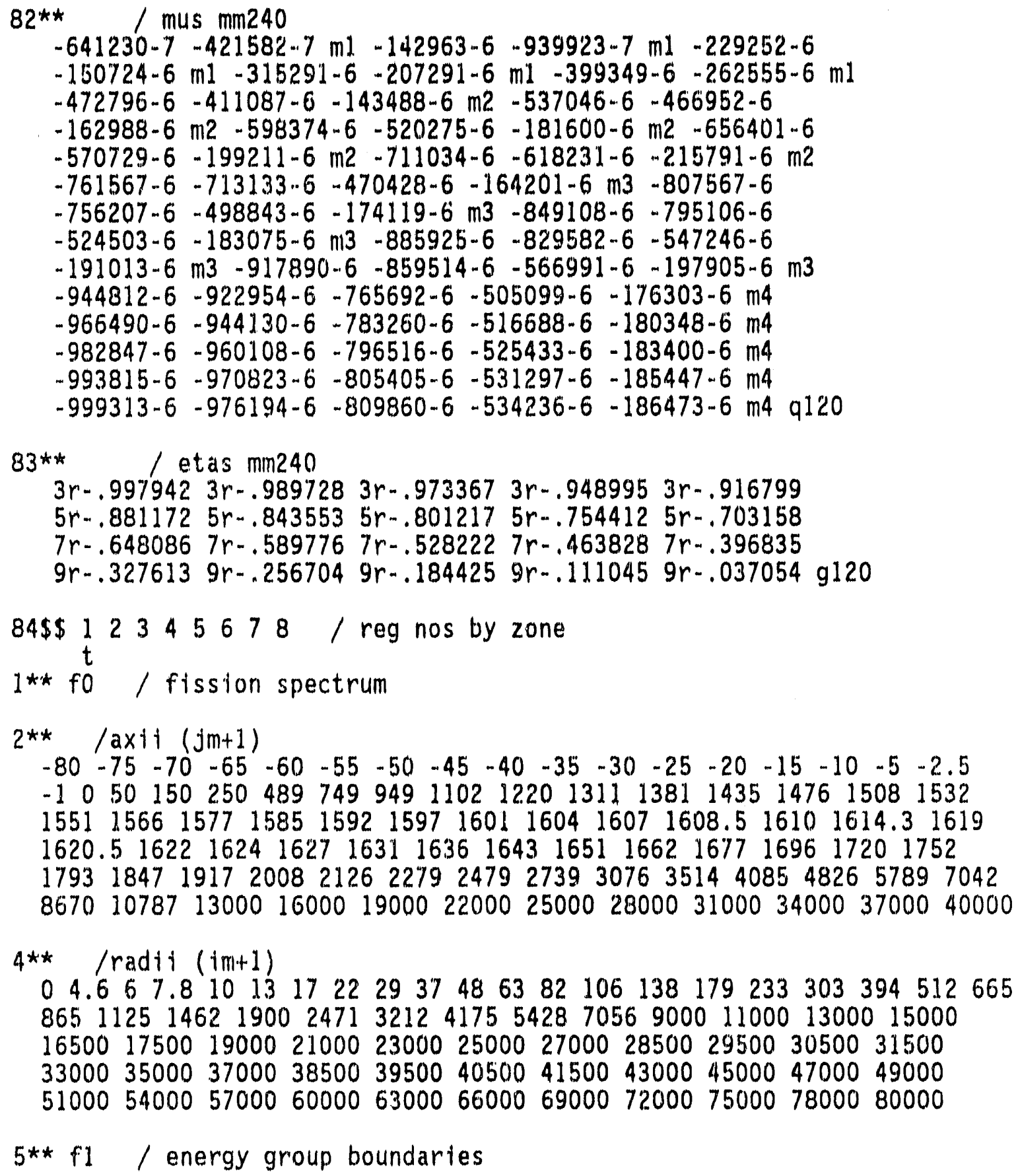

Figure 4-3. (continued) 


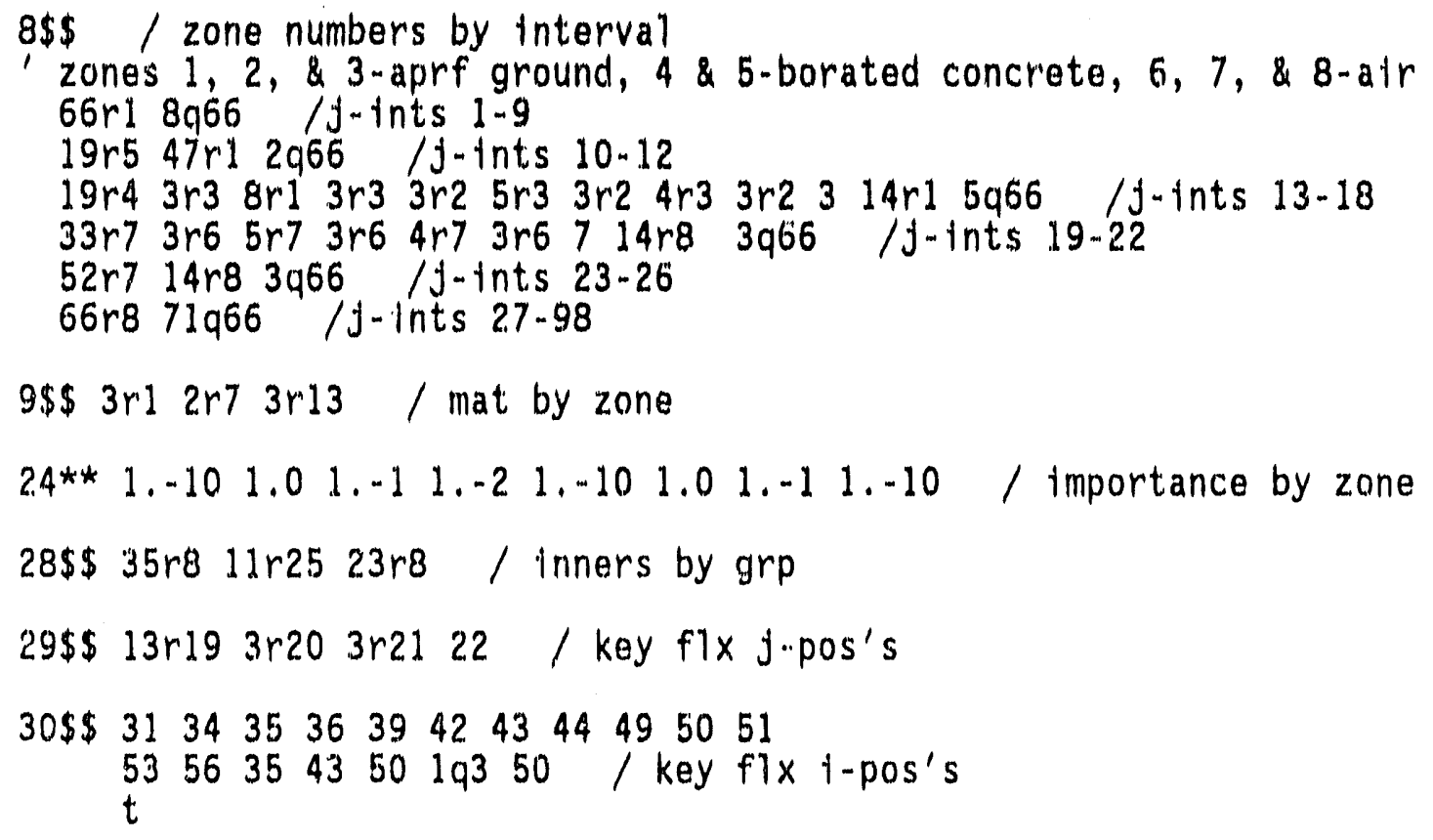

$9 \$ \$ 3 r 12 r 73 r 13$ / mat by zone

$2.4 * * 1,-101.01 .-11 .-21,-101.01 .-11 .-10$ / importance by zone

$28 \$ 35 r 811 r 2523 r 8 /$ inners by grp

$29 \$ 13 r 193 r 203 r 2122$ / key f1x J.pos's

$30 \$ \$ 31343536394243 \quad 44495051$

5356354350 lq3 50 / key fix i-pos's

$\mathrm{t}$

Figure 4-3. (continued) 


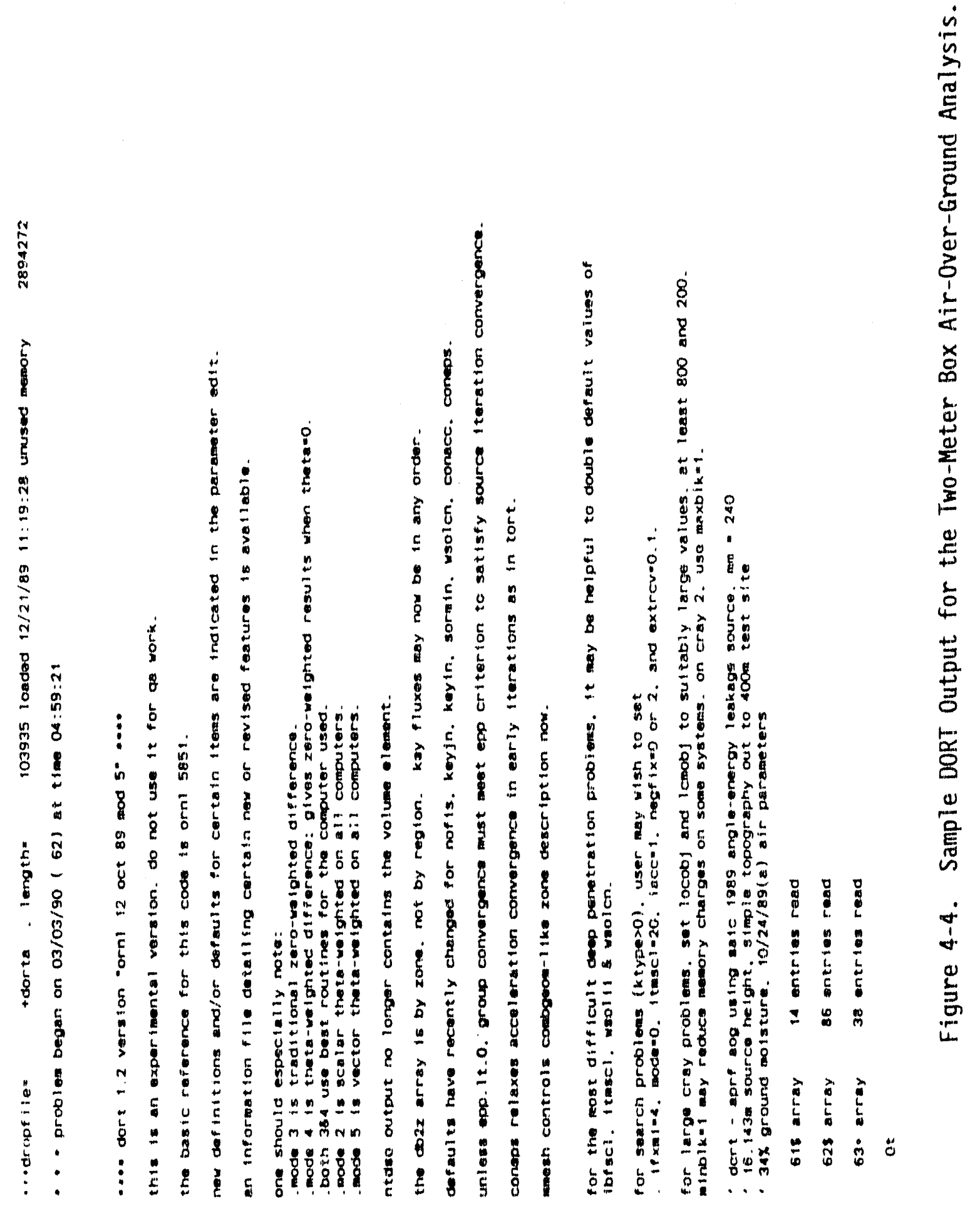



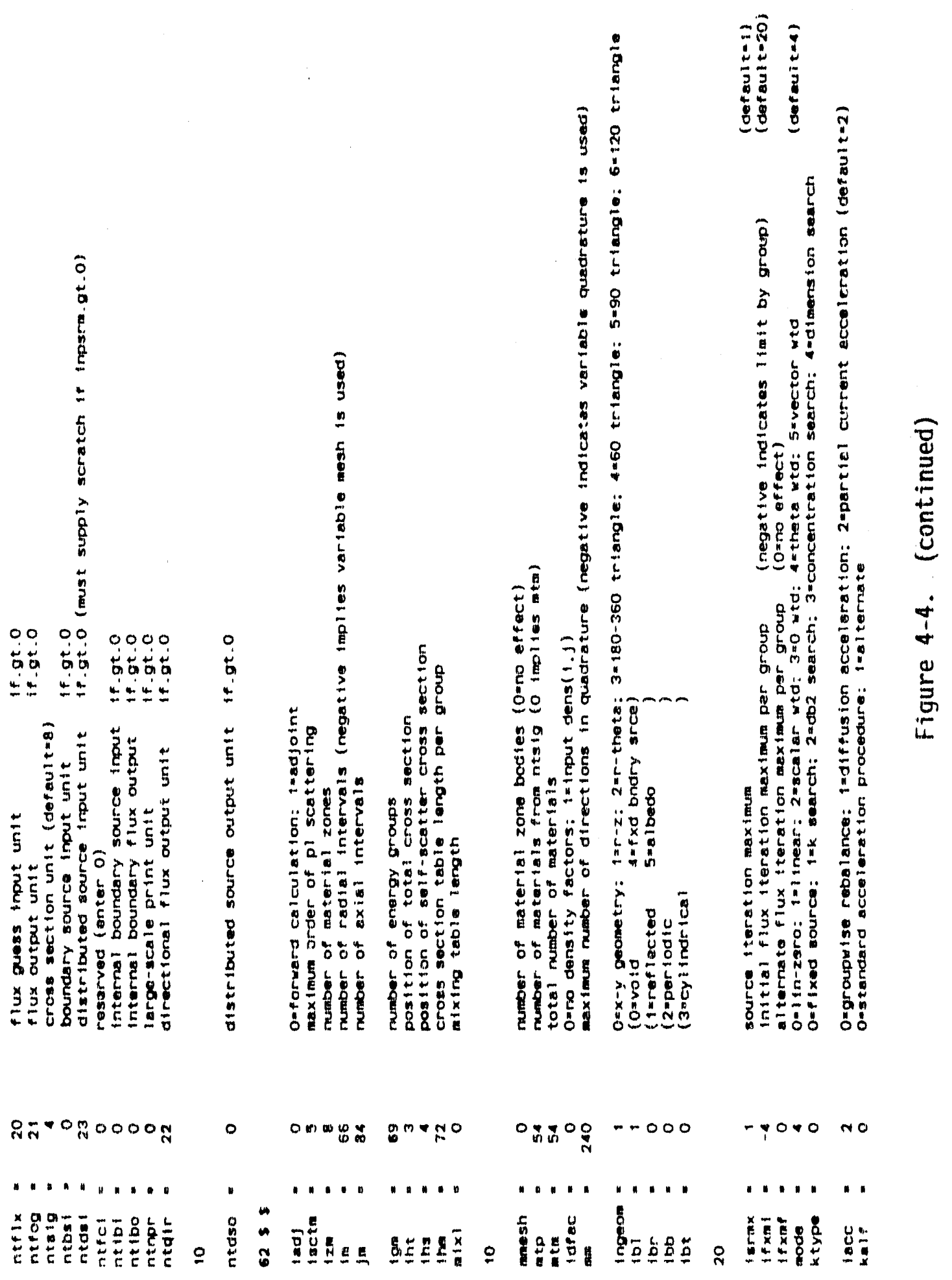

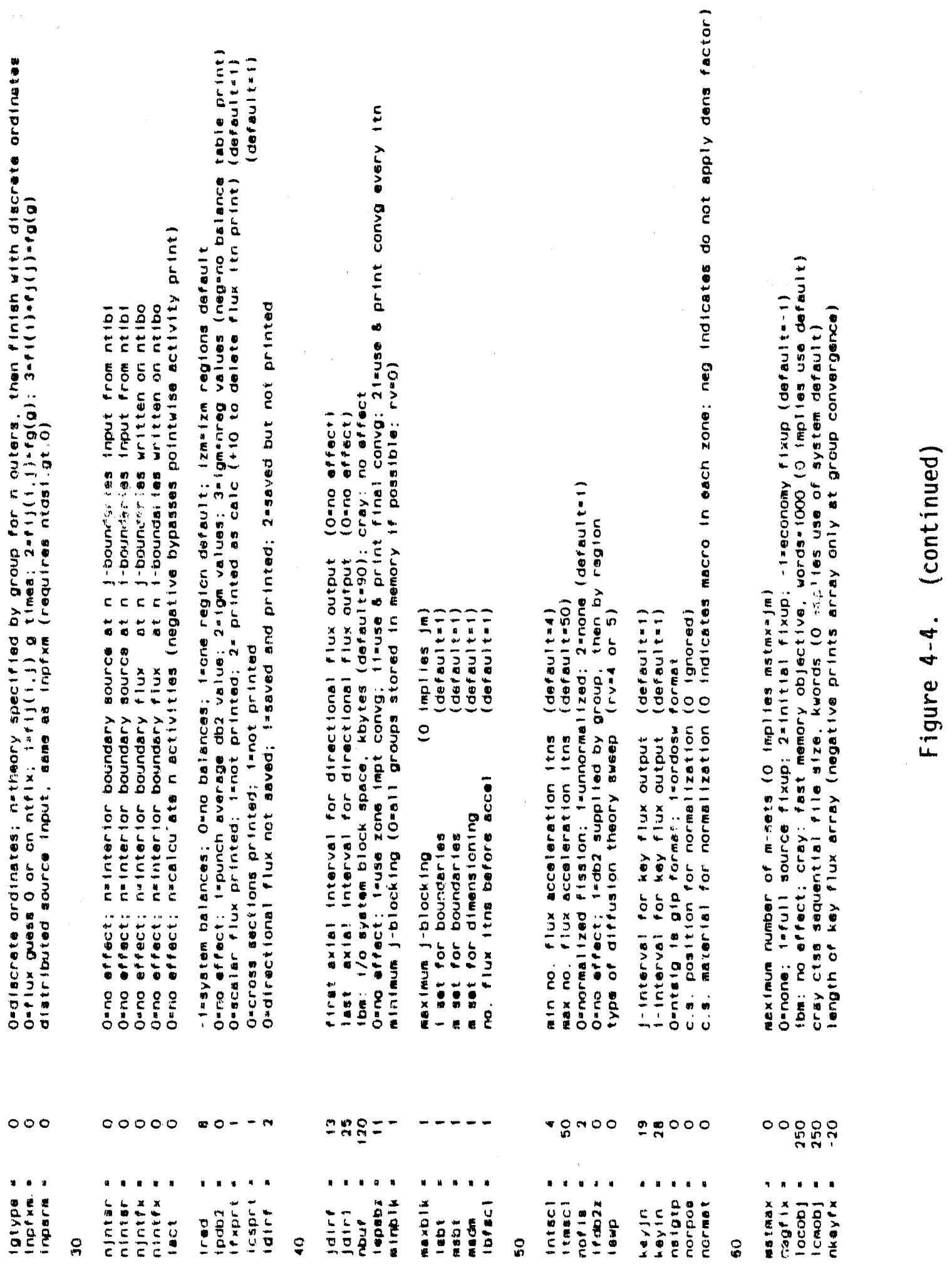

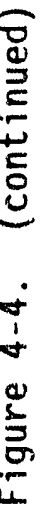

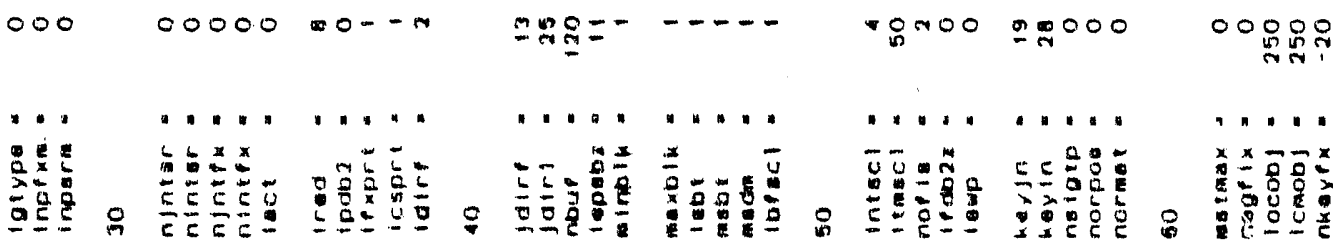




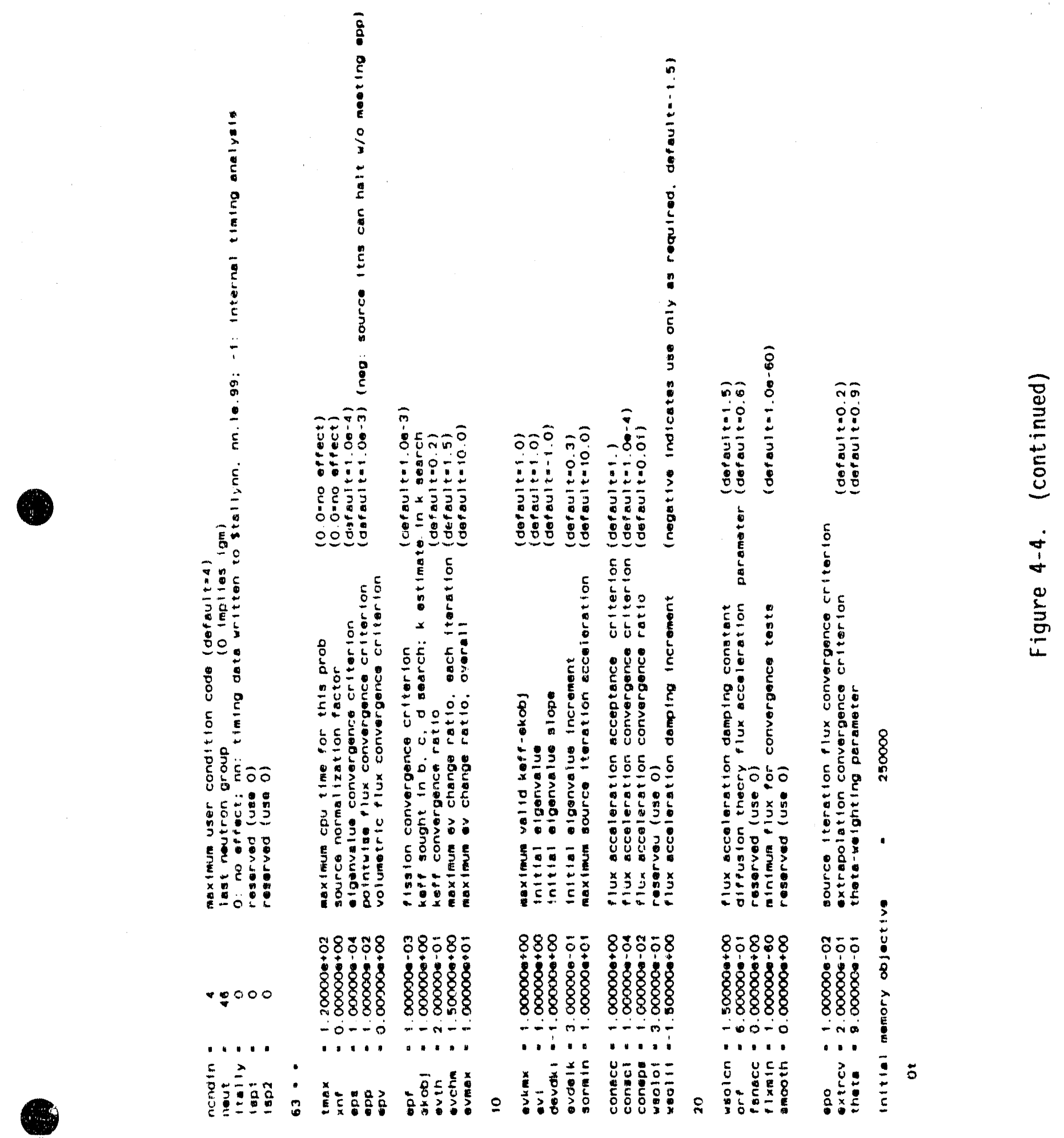





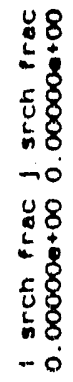

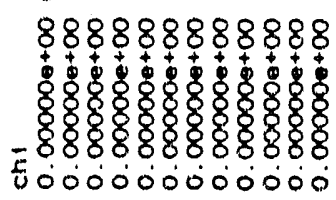

웅ㄷㅇ응

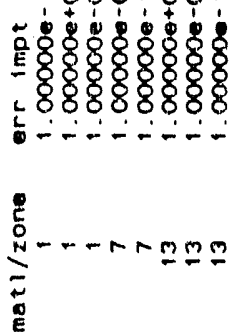

ㅁ

ฮั

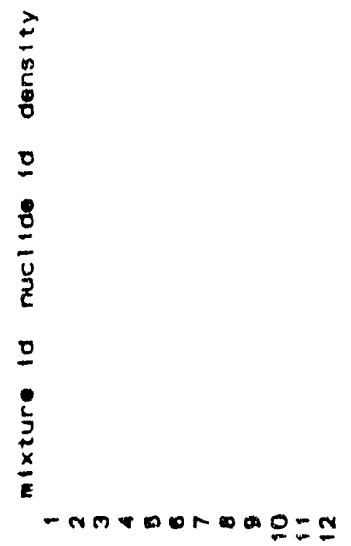

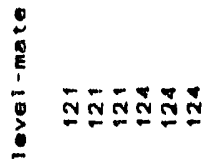

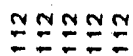
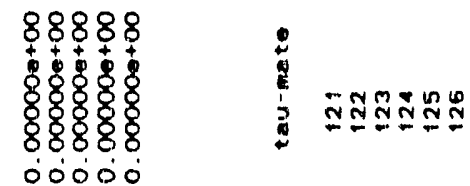

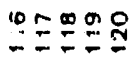

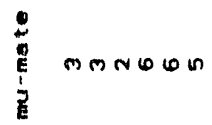

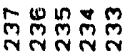
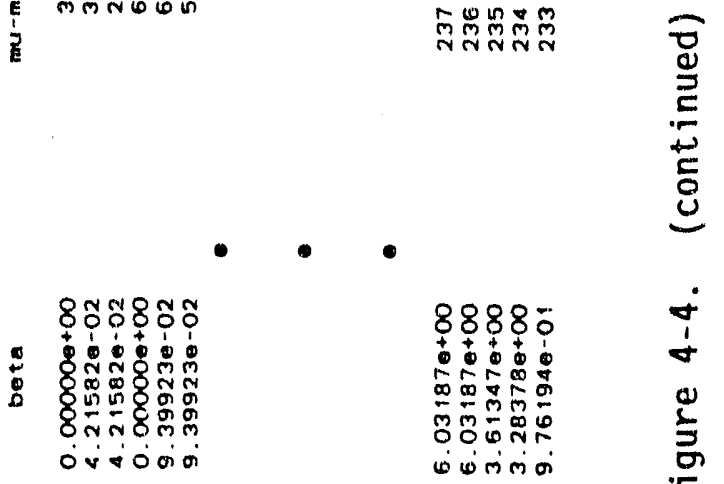

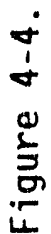

ํํํํํำ

88888

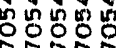

minm

ए人̄o-

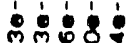

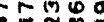

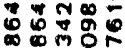

- nino

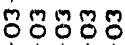

$\therefore$

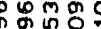

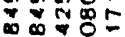

minotion

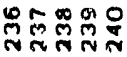




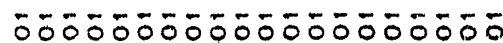

๘

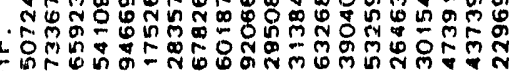
-

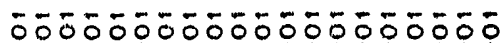

一

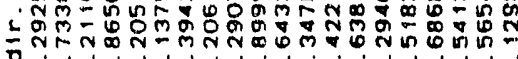

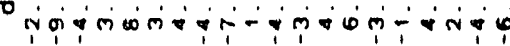

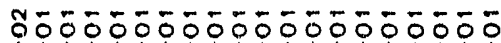

๑

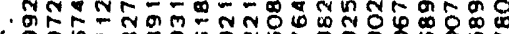

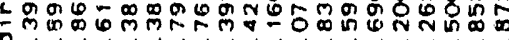
op

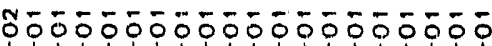

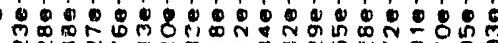

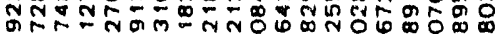

* L

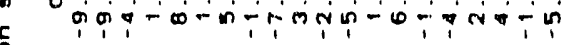

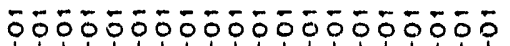

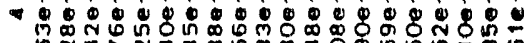
, ᄂ พั\%

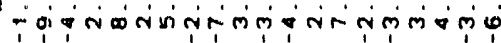

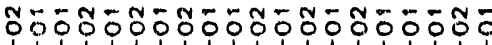

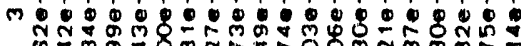
ñm

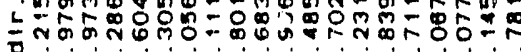
-

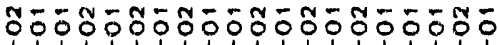
ง ผำำ ถั

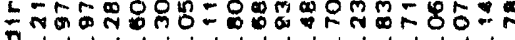

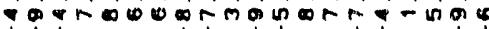

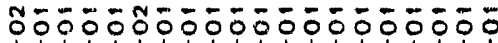

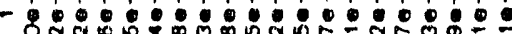
ल

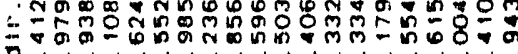

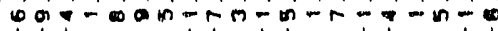
-

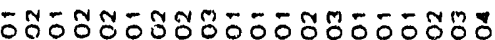

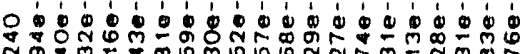
ง

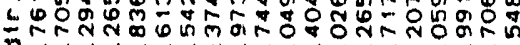

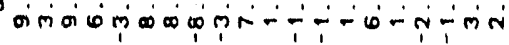

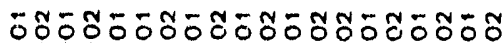

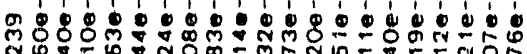

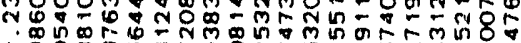

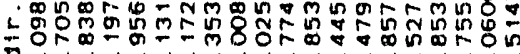
-

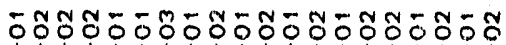

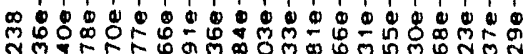

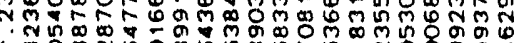

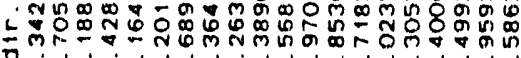

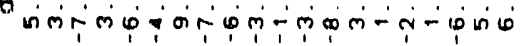

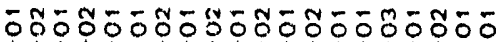

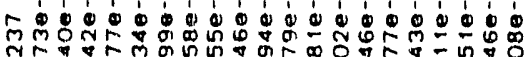

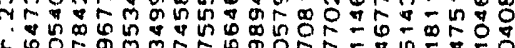

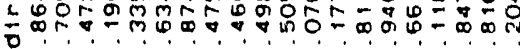
-mi-

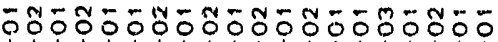

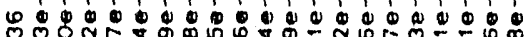

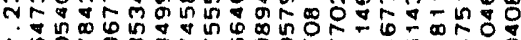

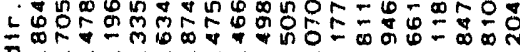

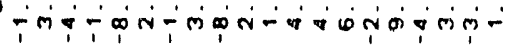

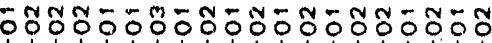
舟 $808 \div$ సกำ

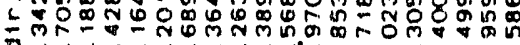

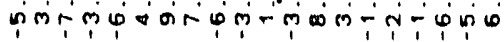

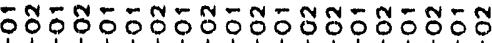
× $800 \%$

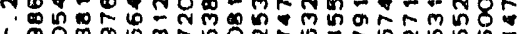

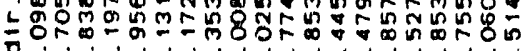

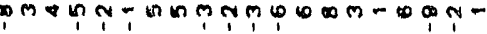

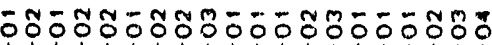

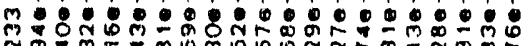
ง ฮำ

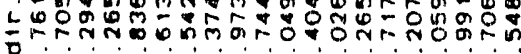

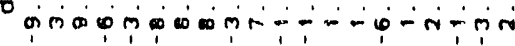

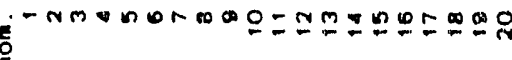




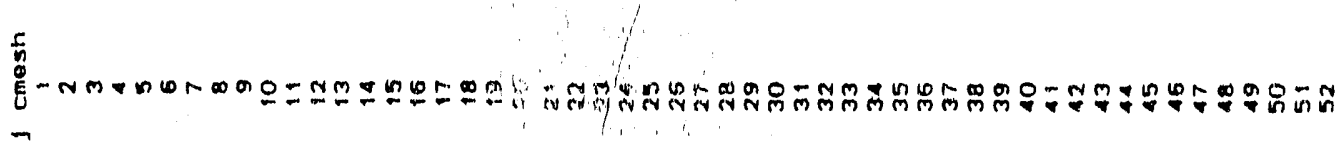

$\frac{5}{5}$
0
0

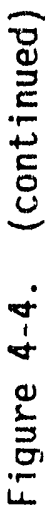

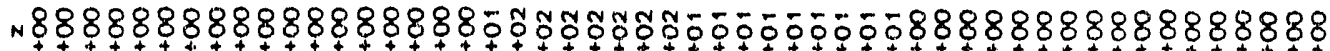

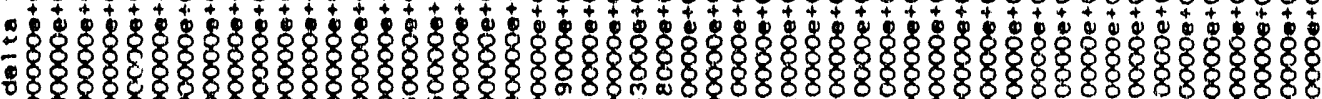

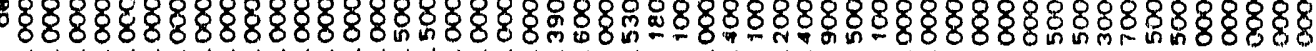

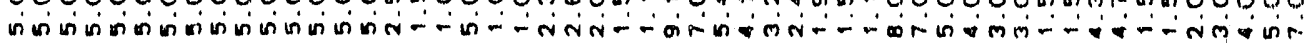

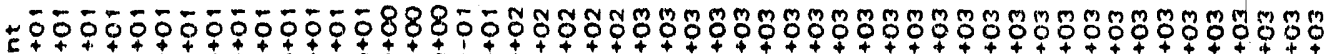

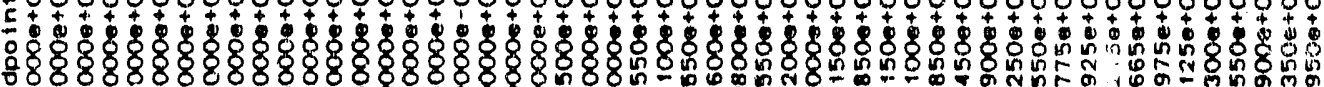

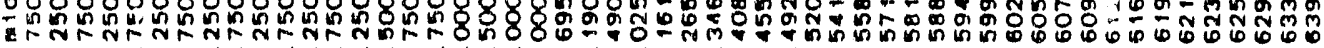

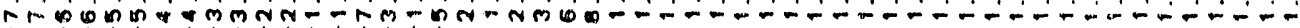

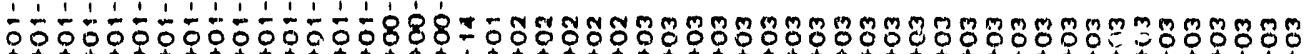



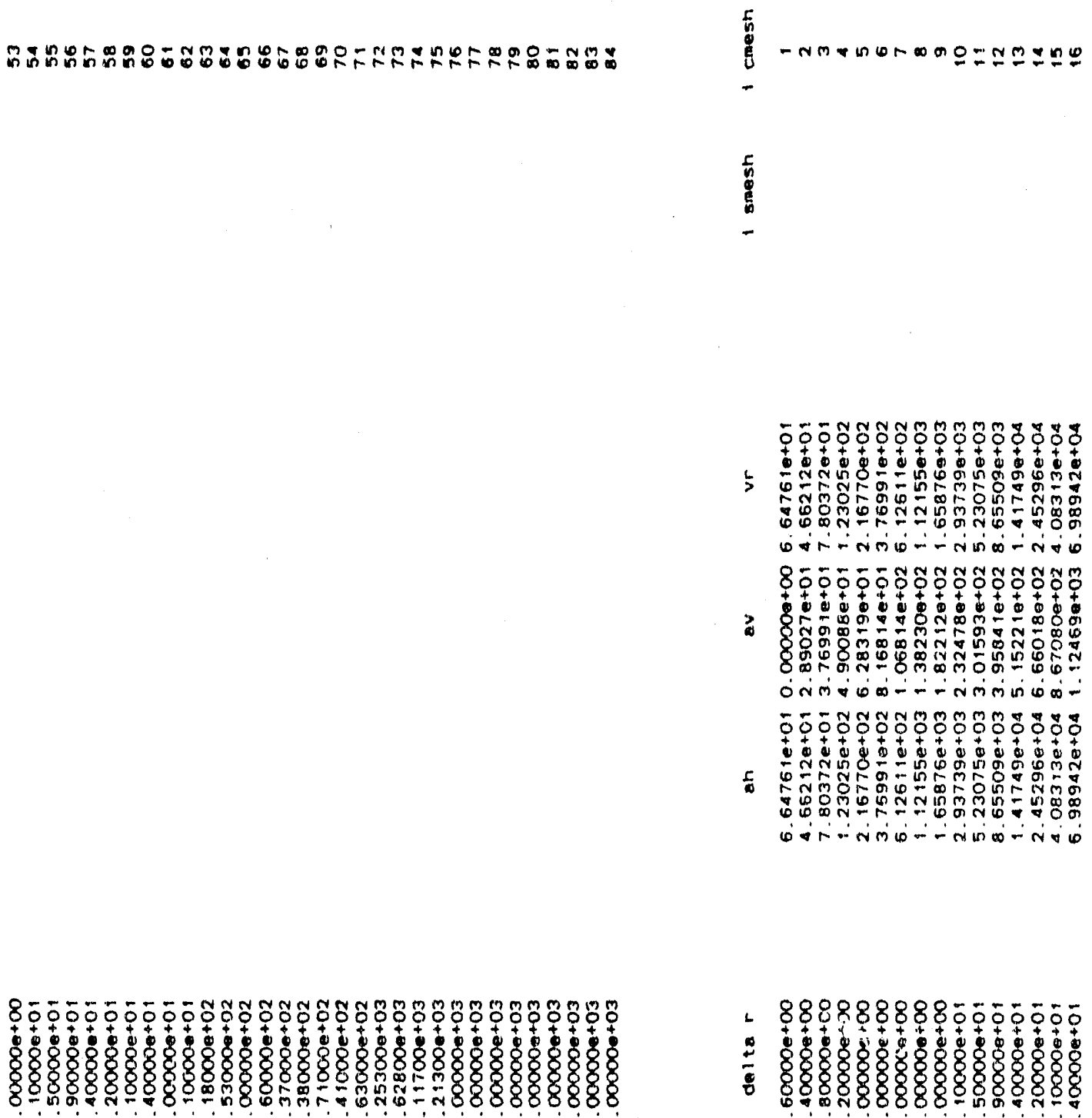

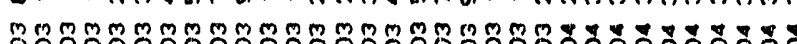

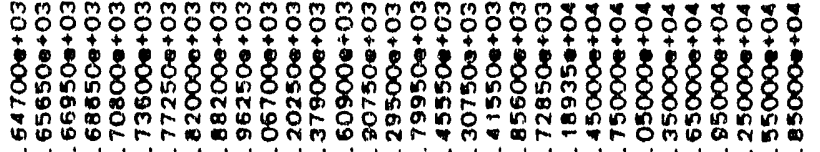

$\therefore-\therefore-1-\therefore$ -
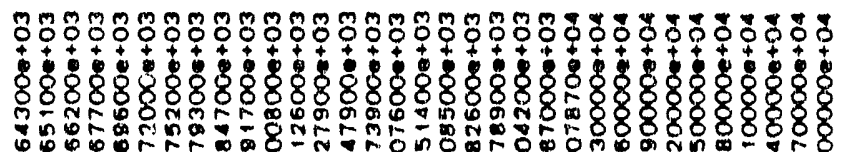

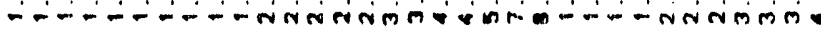

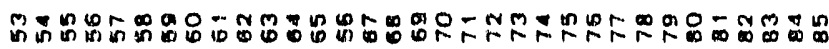
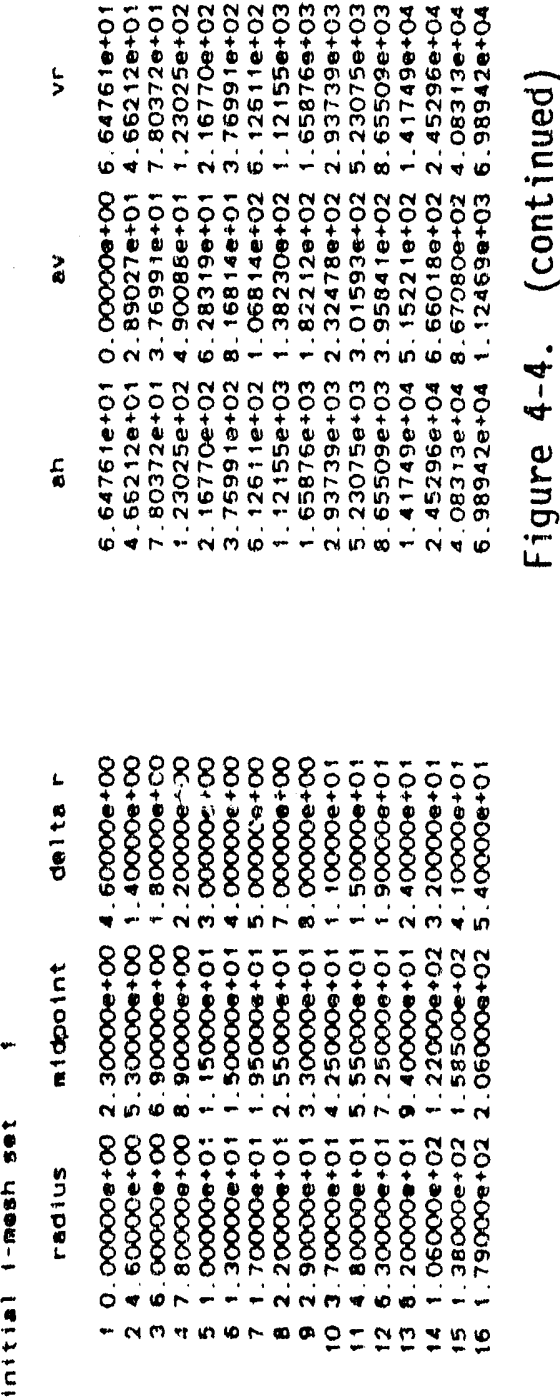


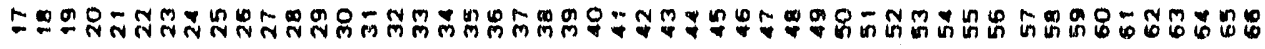

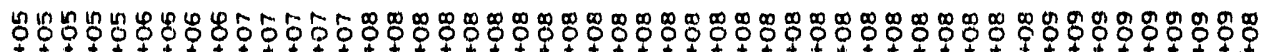

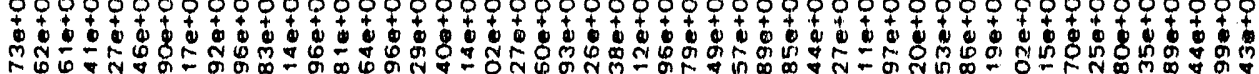

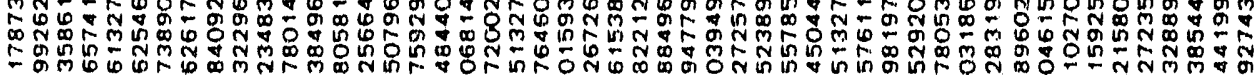
$\therefore$ minar

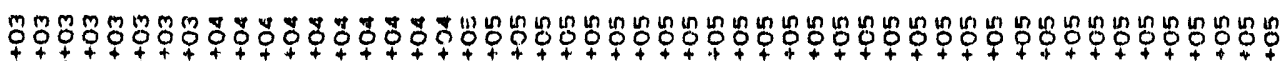

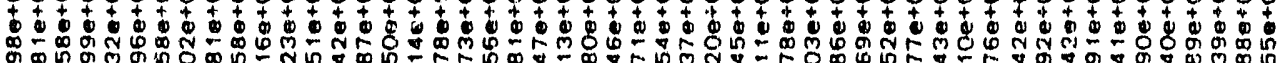

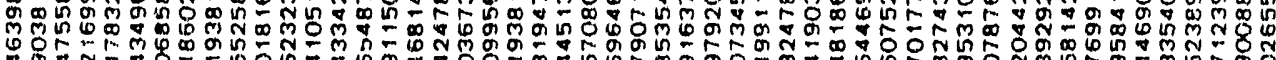
$\therefore$ -

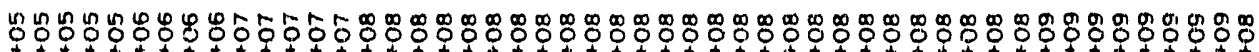

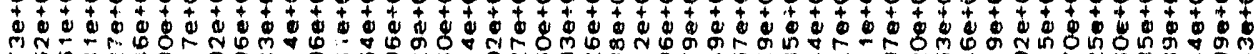

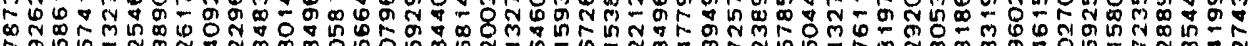

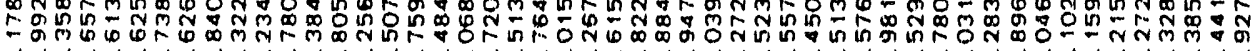

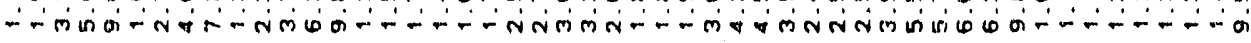

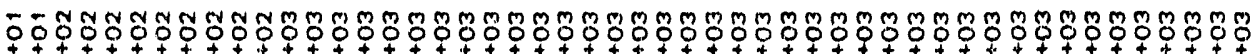

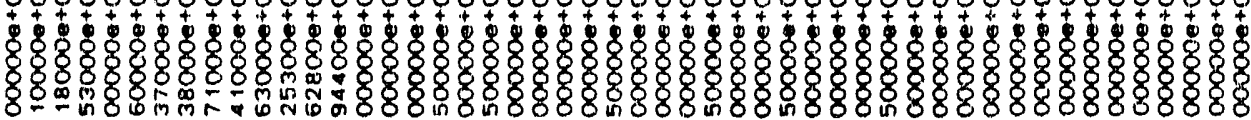

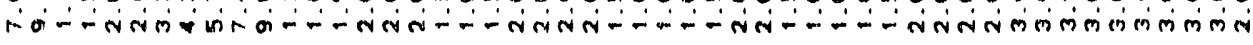

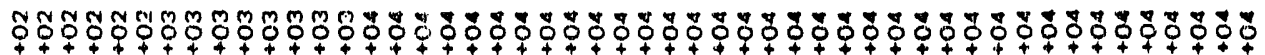

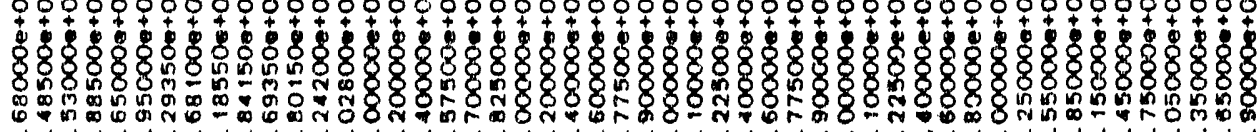

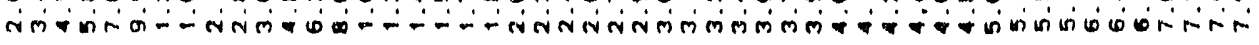

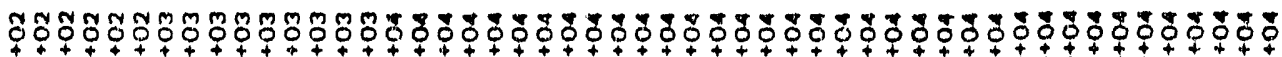

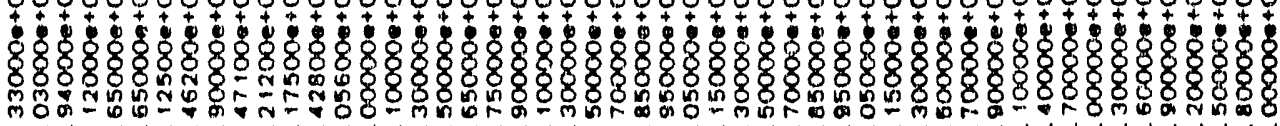
तm

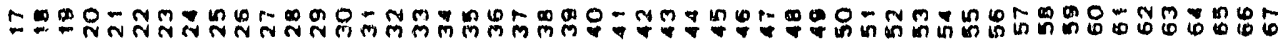



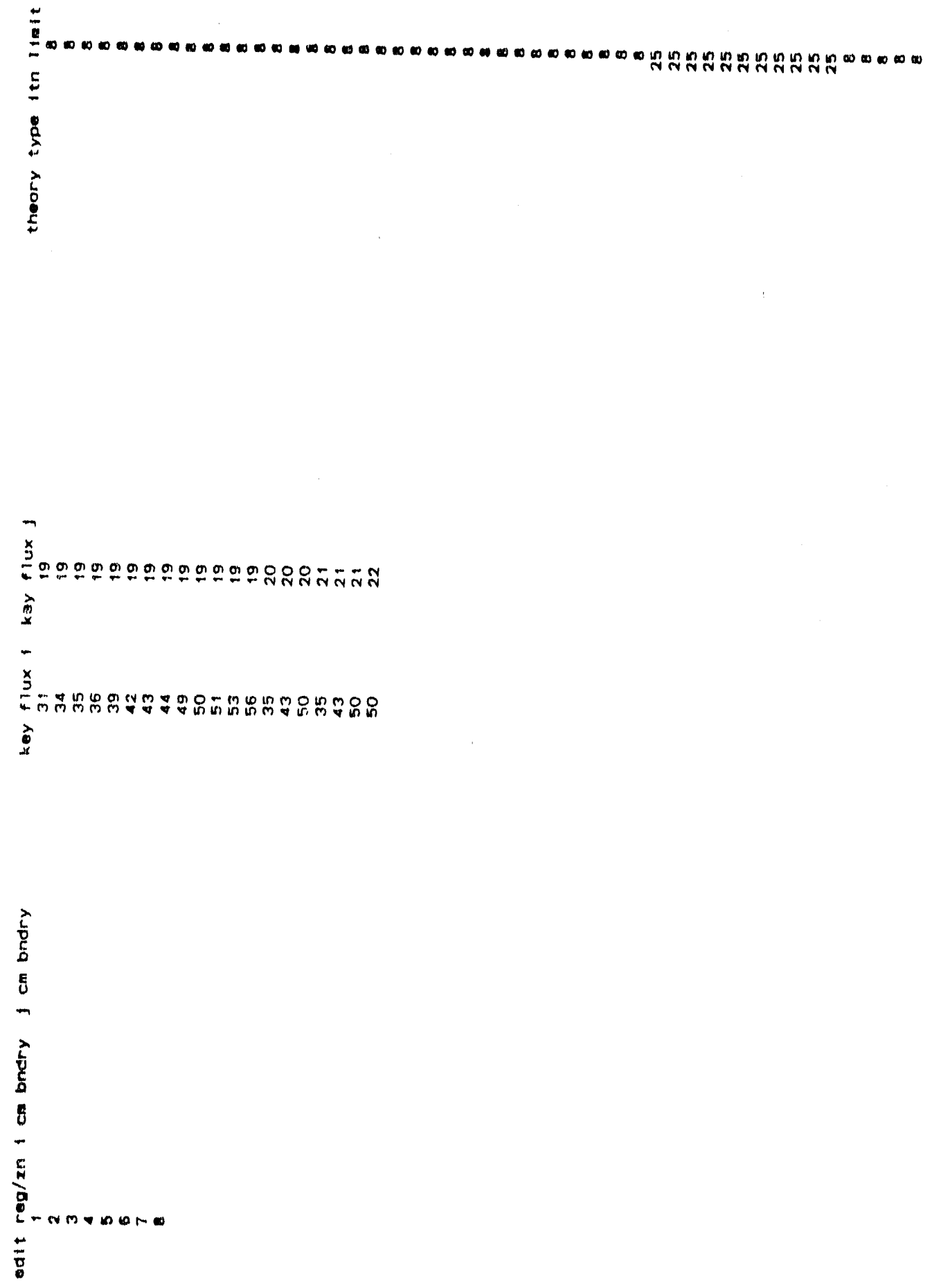

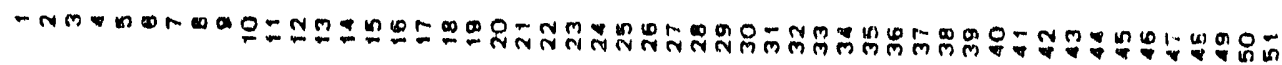




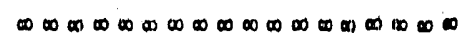

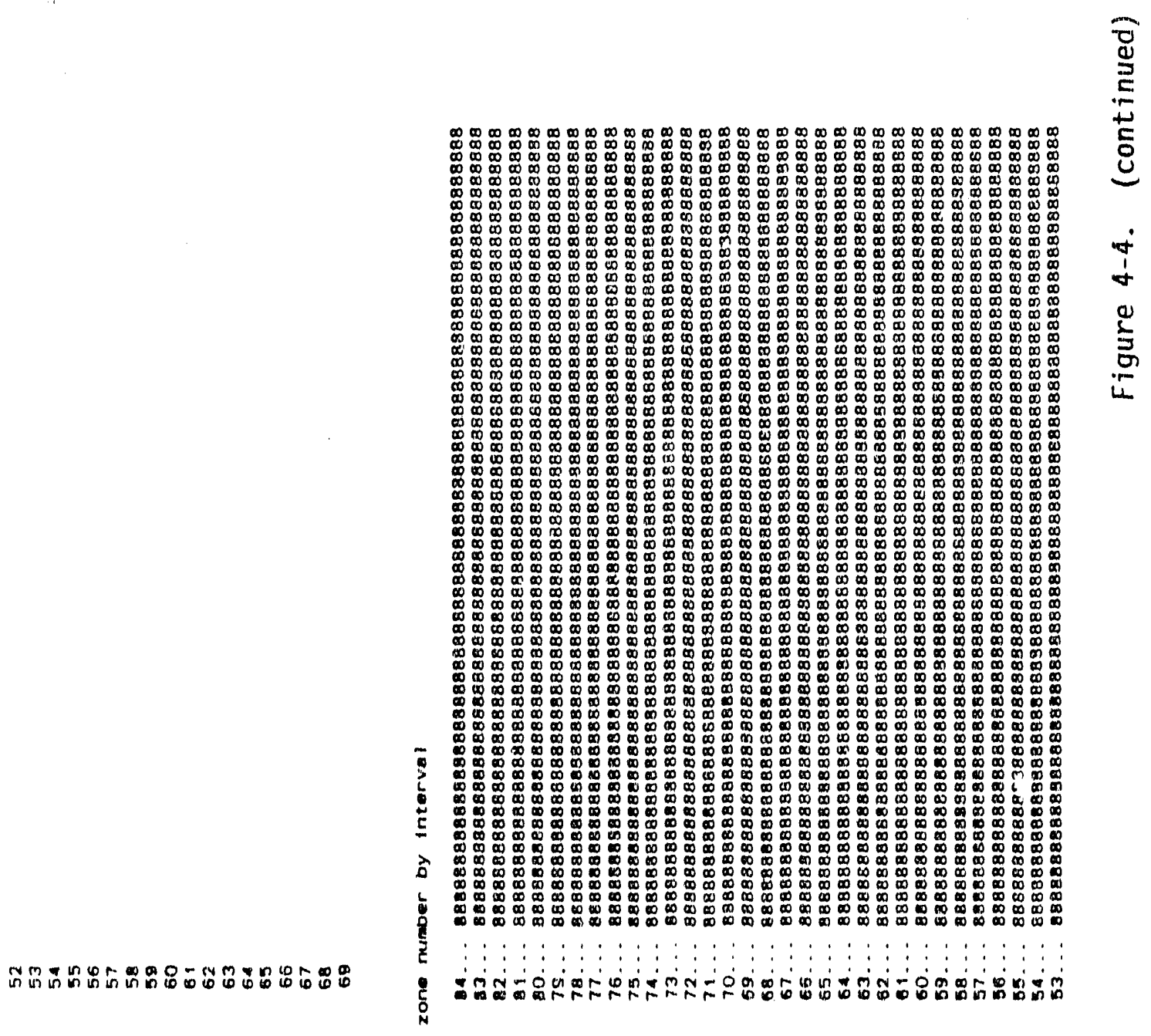




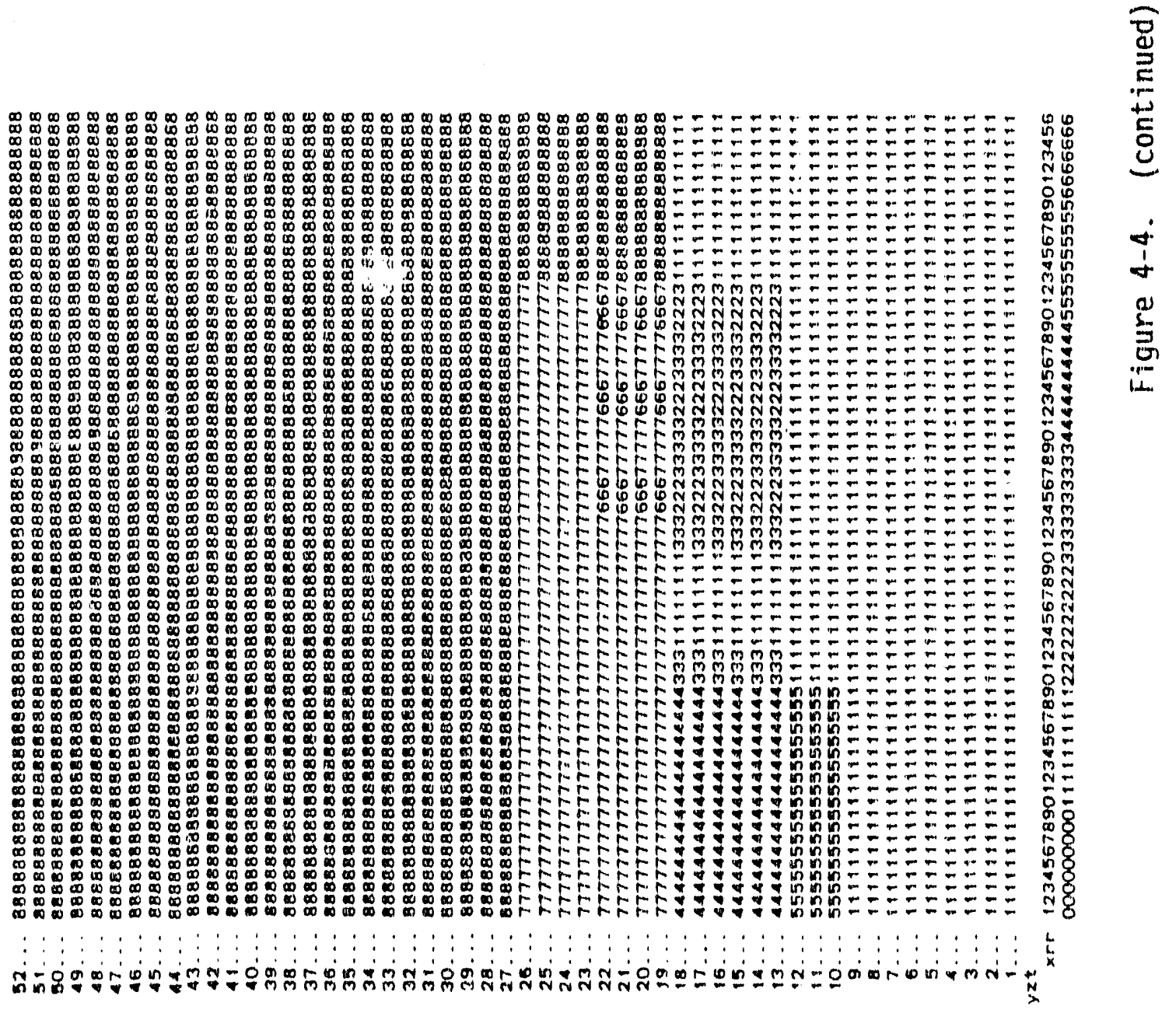




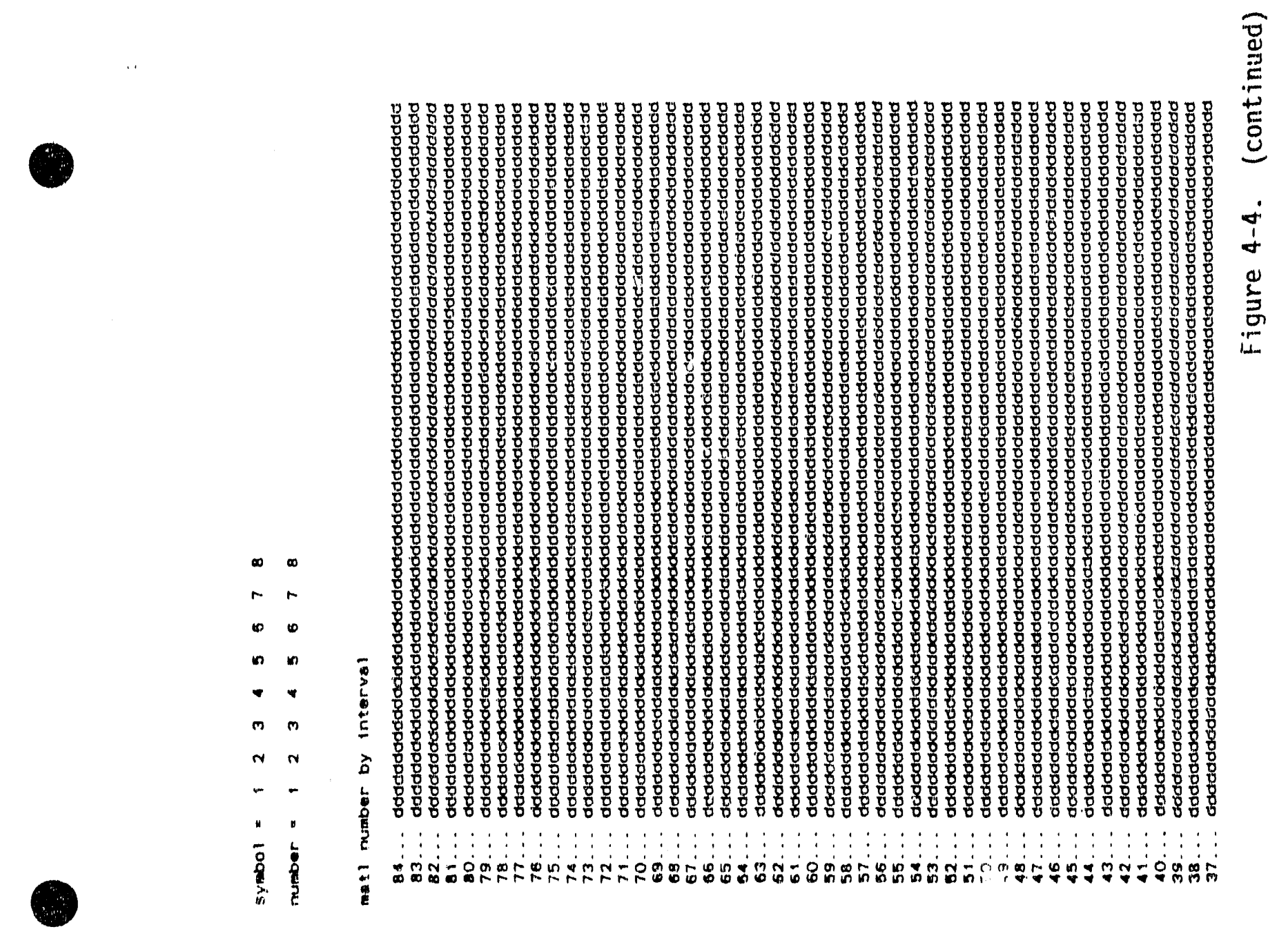



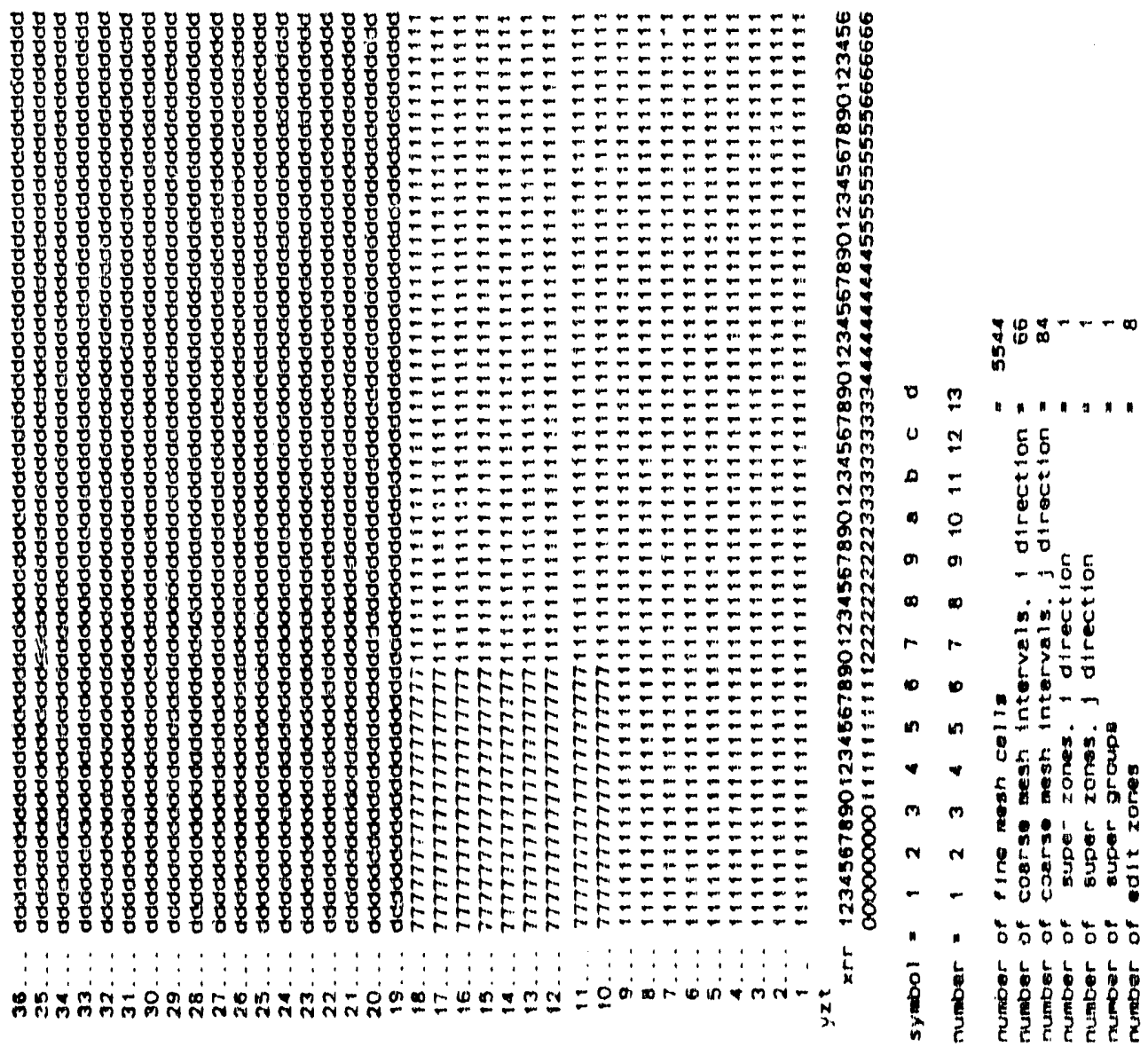

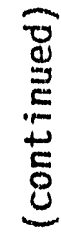

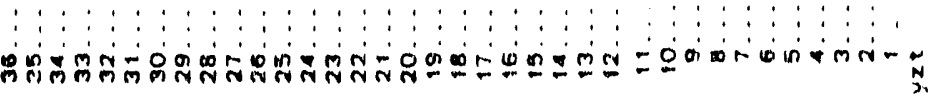




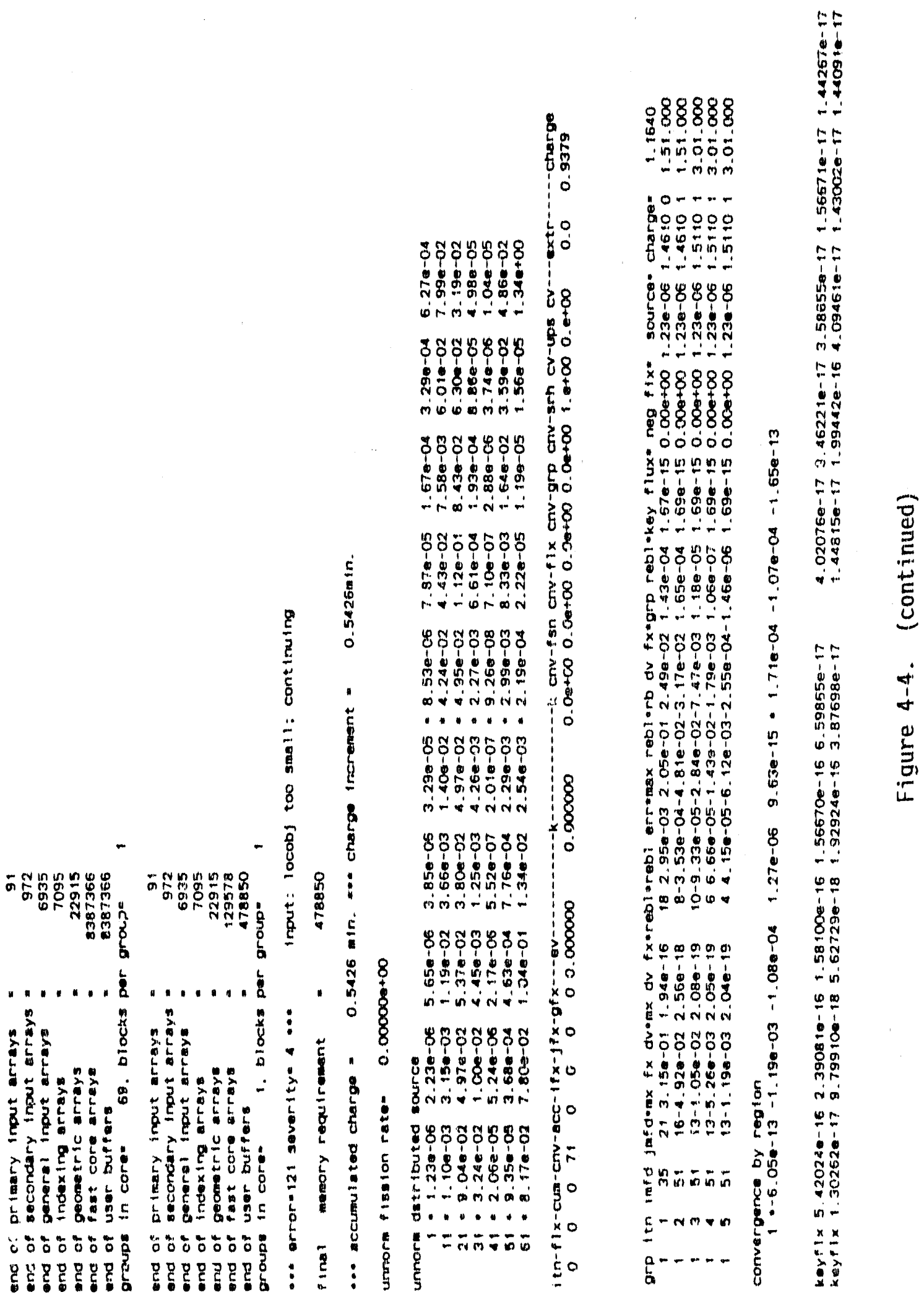




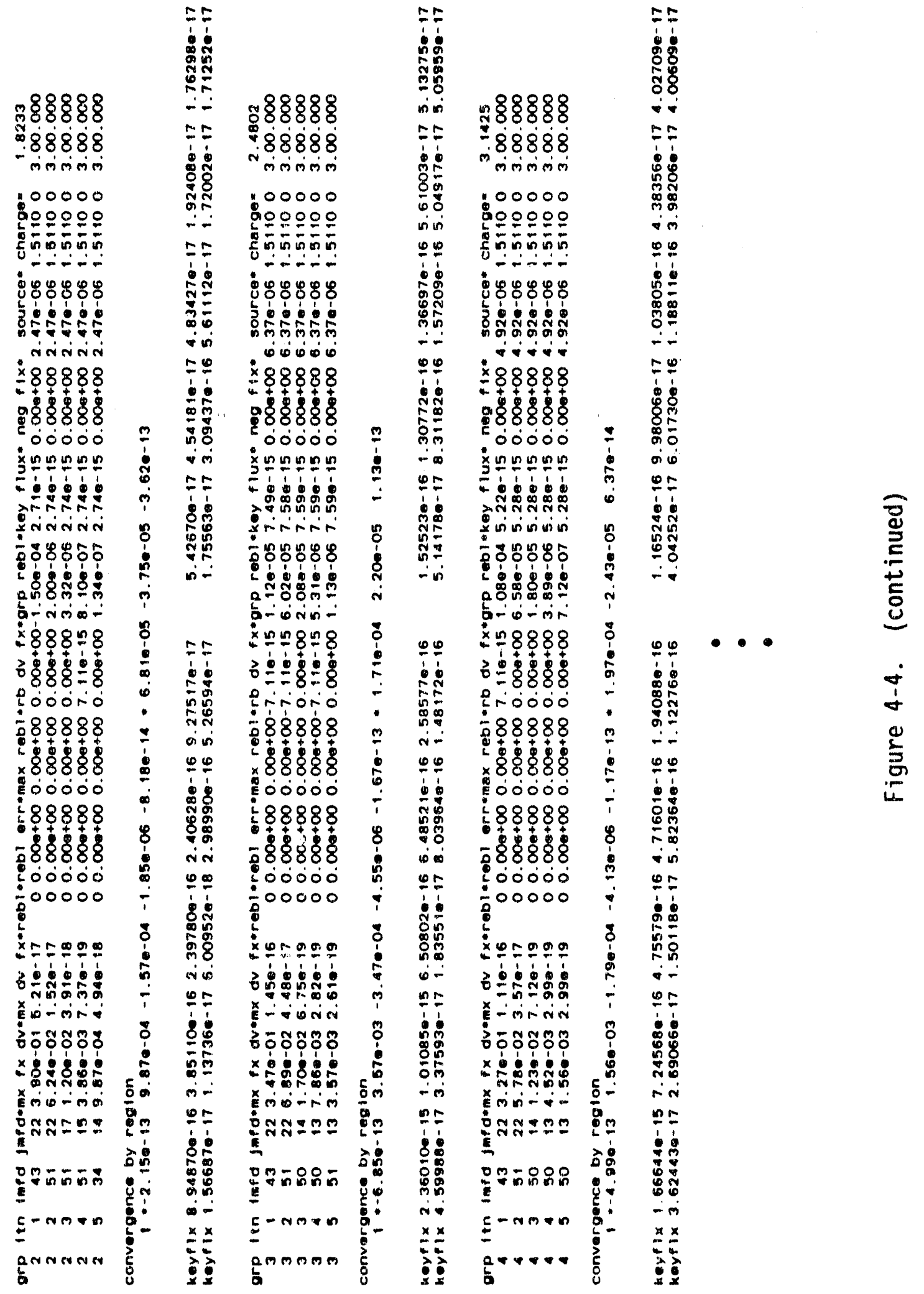




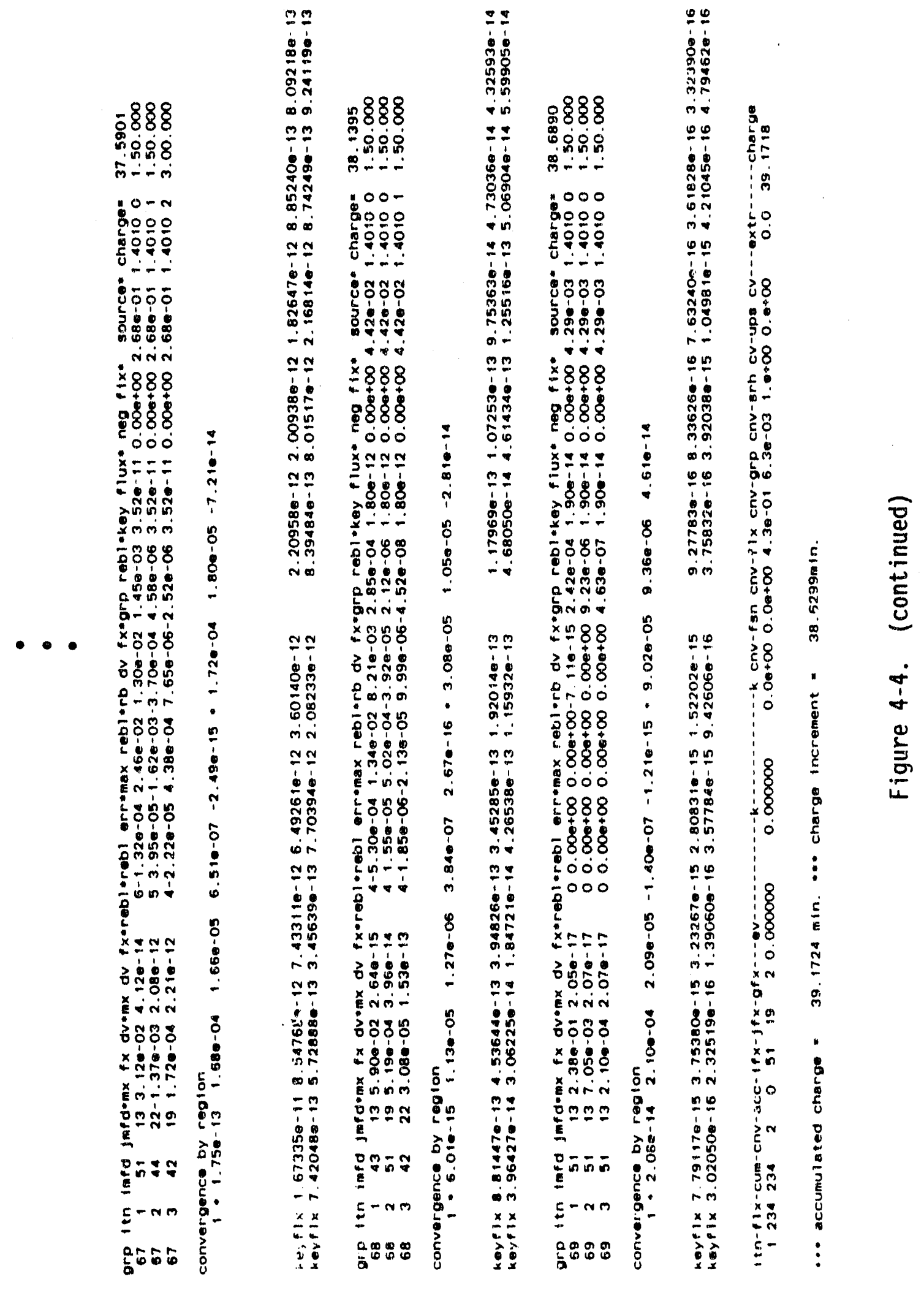




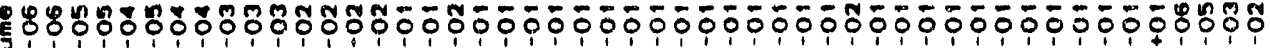

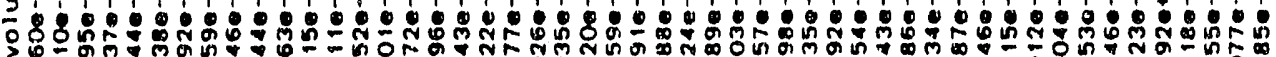

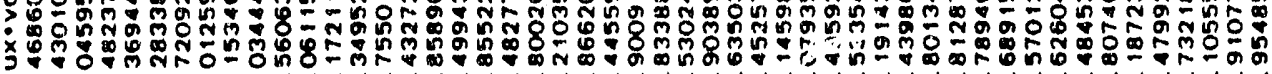

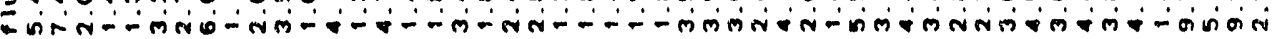

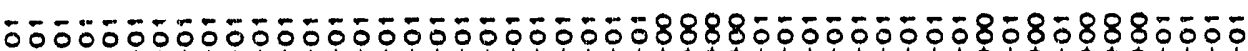

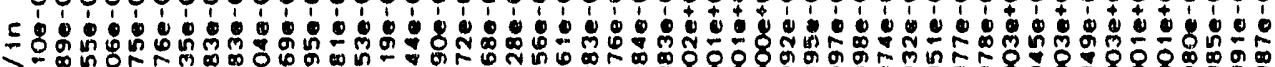

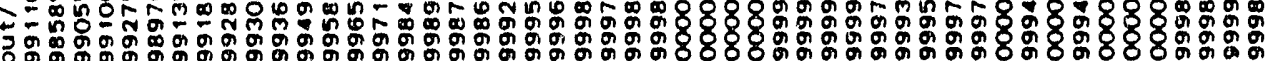

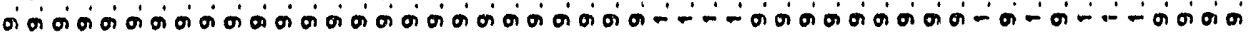
88888888888888888888888888888888888888888888888888

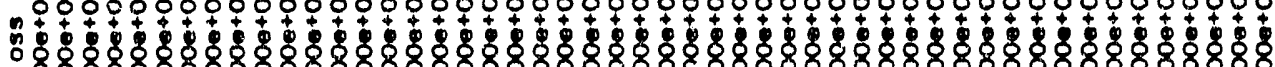

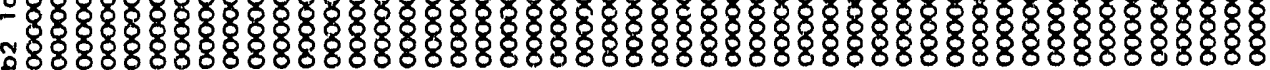

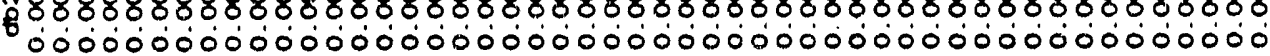

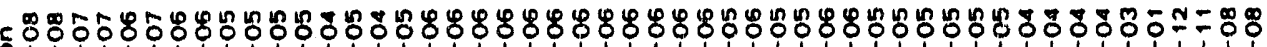

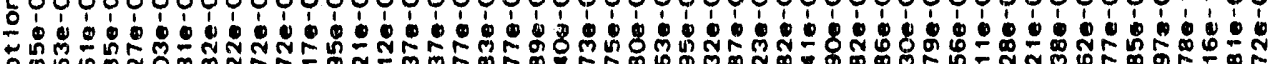

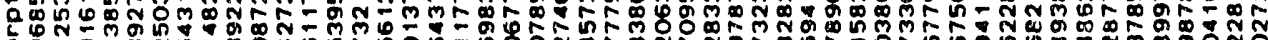

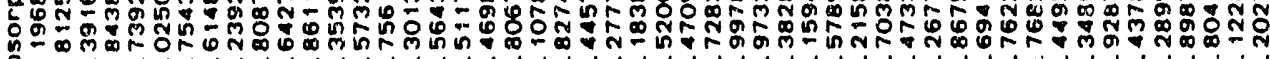

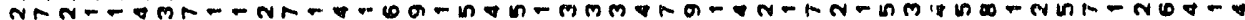

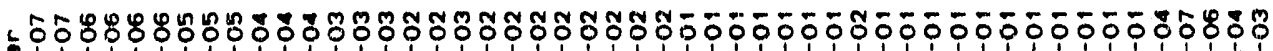

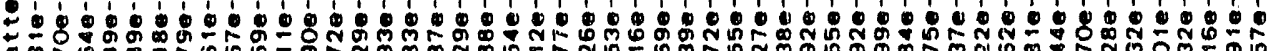

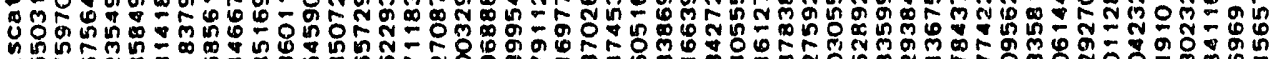

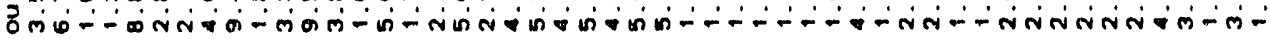
行

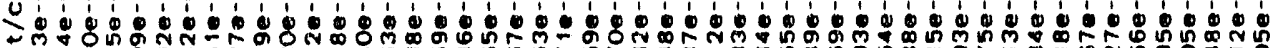
S̄m

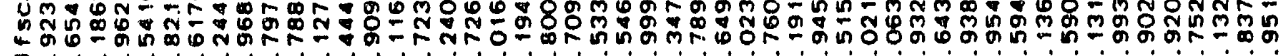

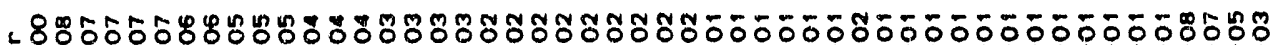
o

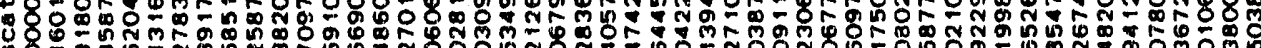

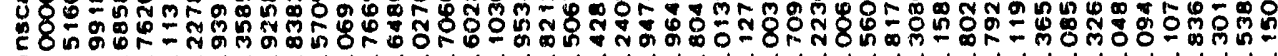

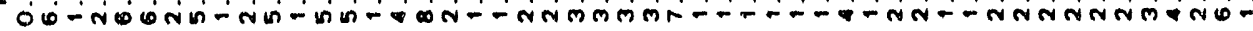
88888888888888888888888888888888888888888888888888

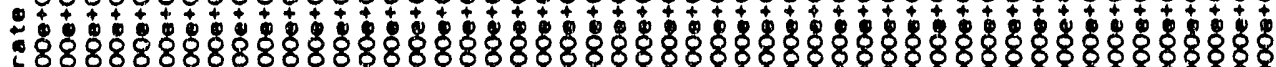
传

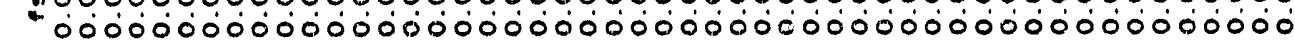
. 88888888888888888888888888888888888888888888888888

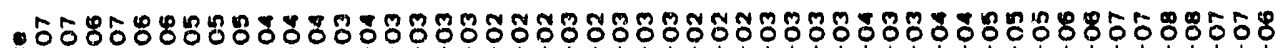

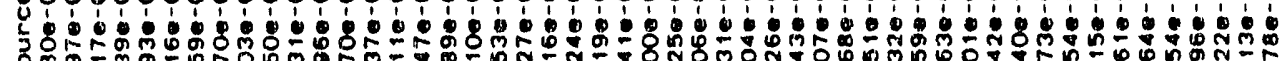

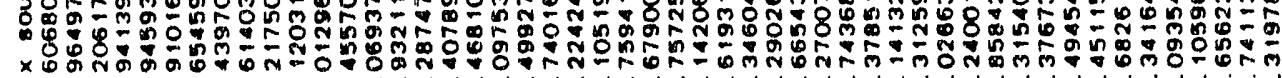

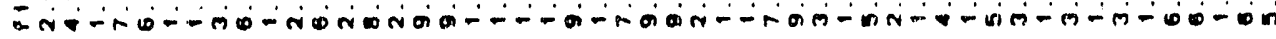

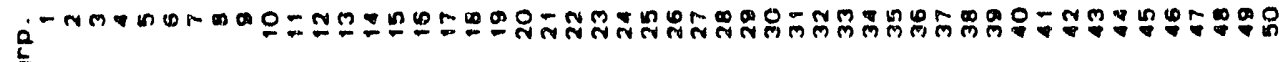
$\frac{0}{6}$ 
0-50-0̄0805088888858\%-

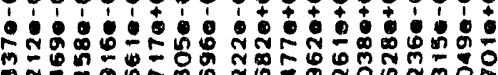

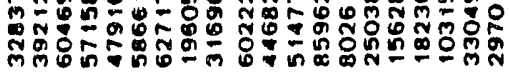

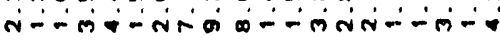

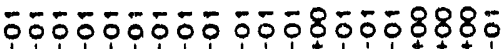

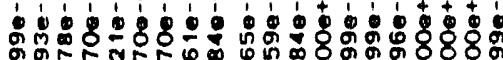

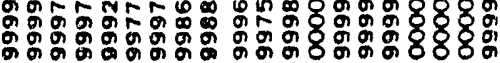

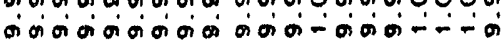
88888888888888888888

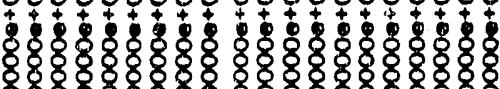
88888888888888888888 00000000000000000000

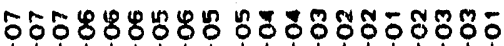

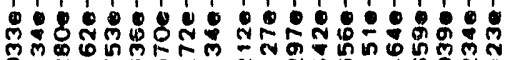

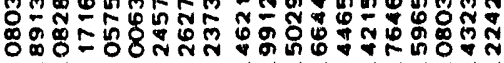

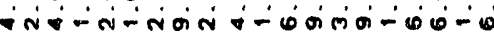

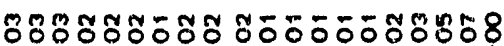

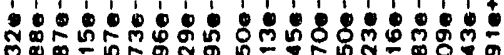

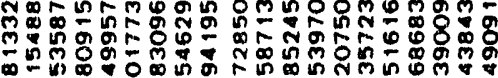

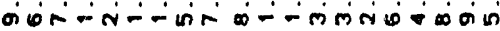
สํํํํํํํํํํํำ 舟若

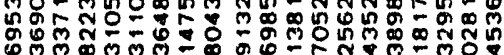

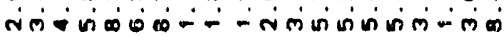

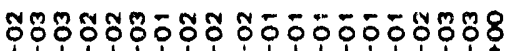

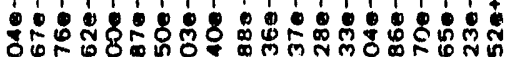

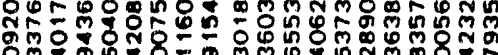

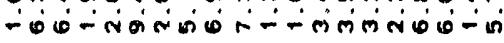
88888888888888888888

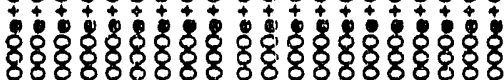
$888888888 \% 8 \%$ 00000000000000000000 88888888888888888888

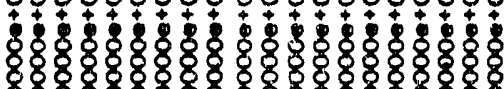
88888888888488888888 ó000000000000000000

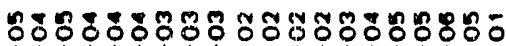

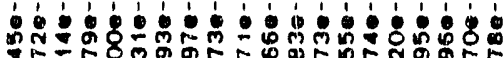

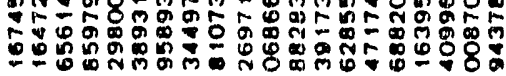

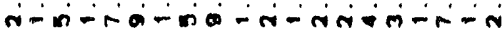

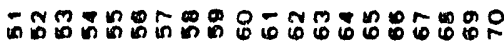

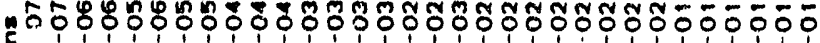

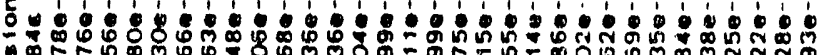

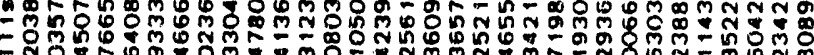

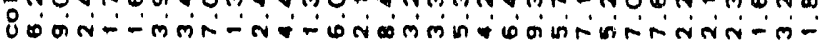

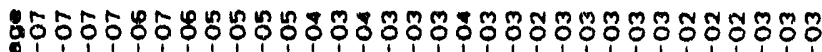
ฮั -

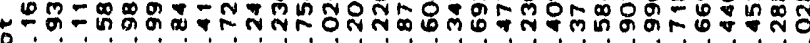

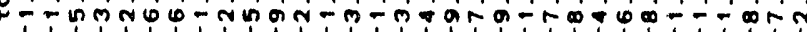

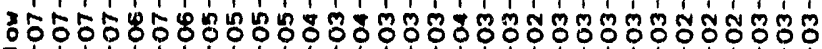

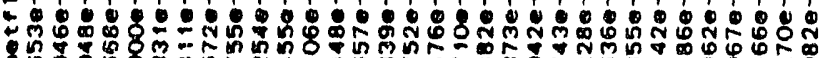

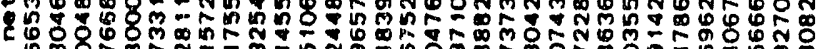

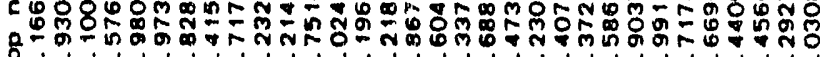

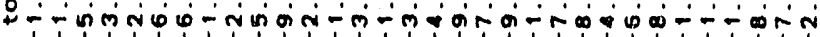

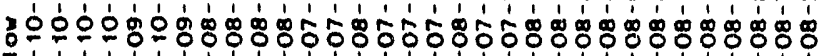
†。

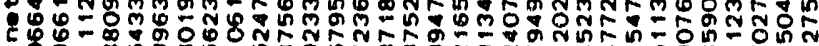

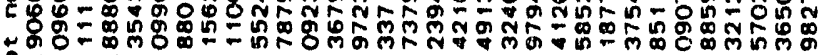

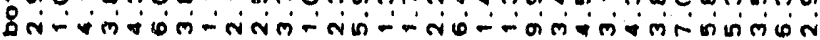

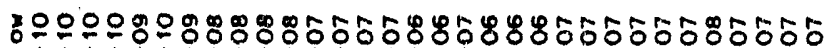

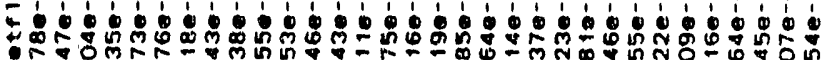

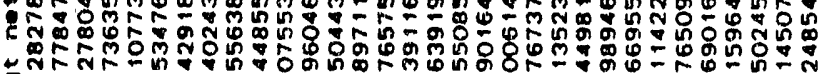

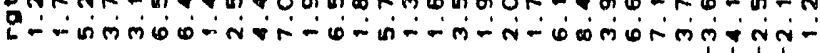

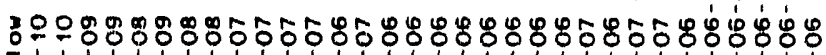

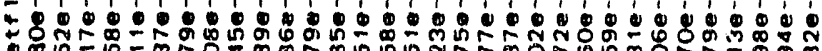
0.

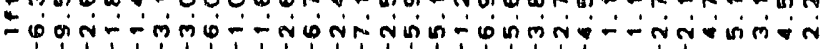

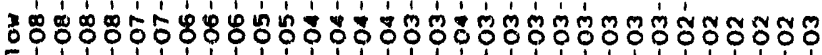
造

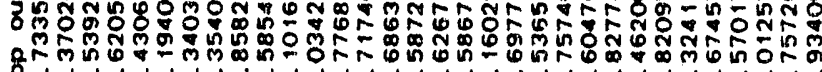
Q-

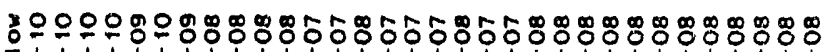

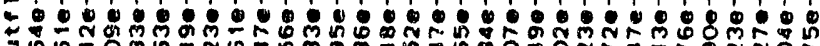

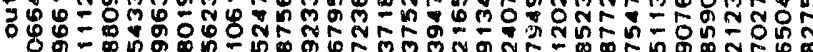

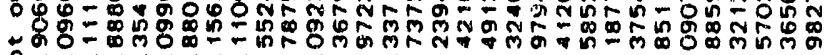

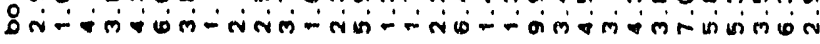

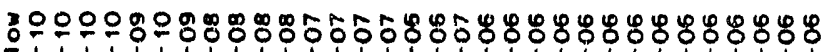

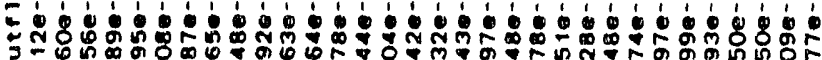

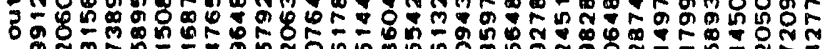

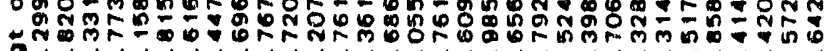

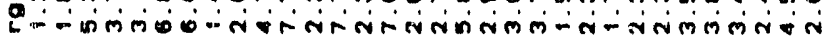

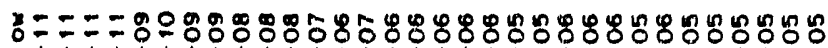
Fon

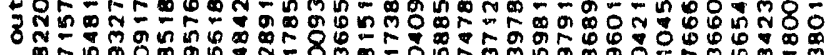

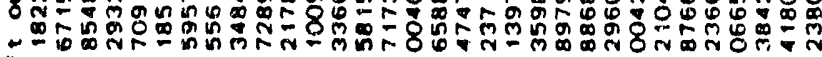

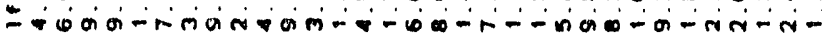

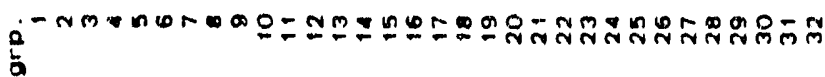




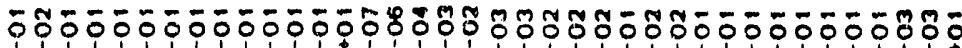

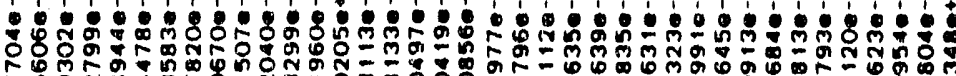

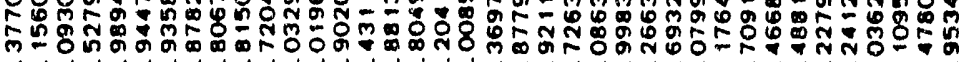

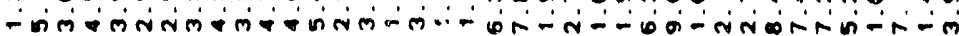

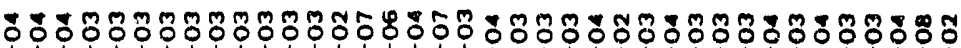

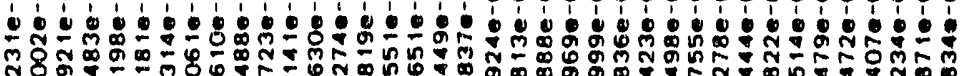

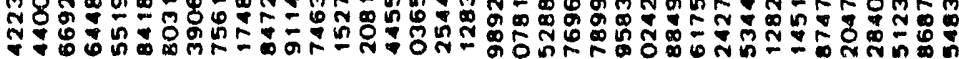

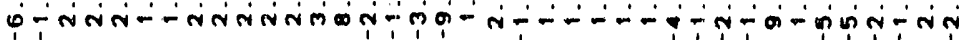

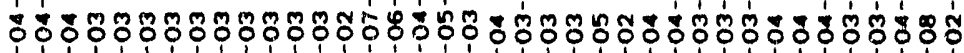

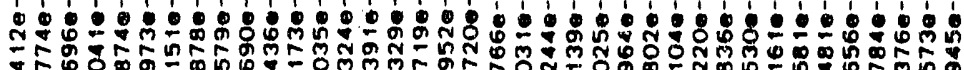

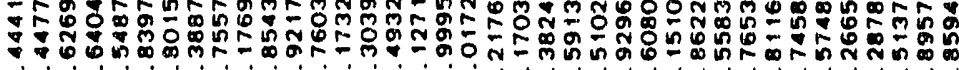
- -

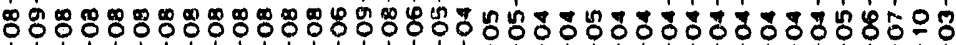
Ð

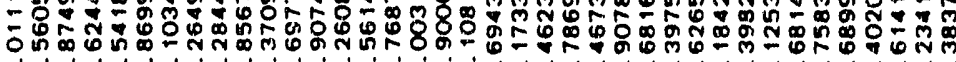

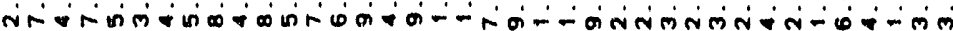

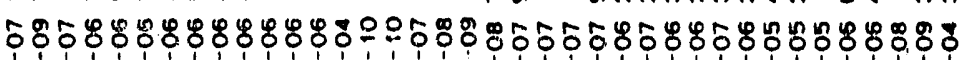

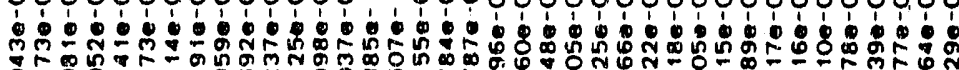

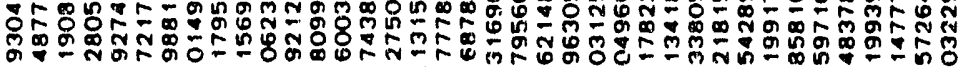

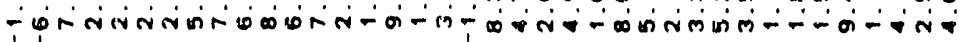

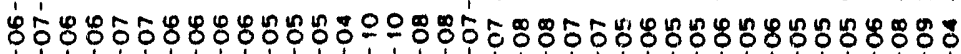
ớñ

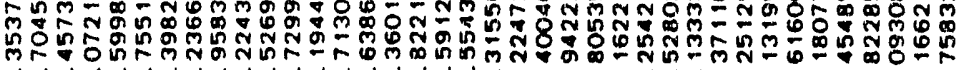

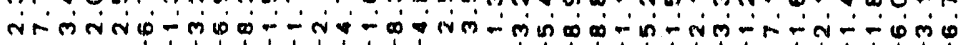

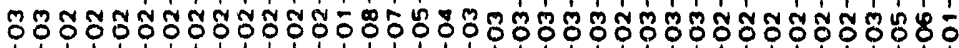

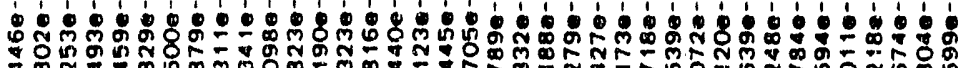

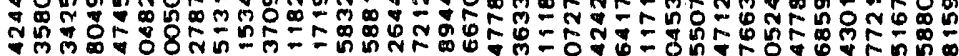

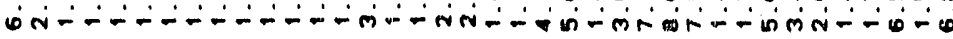

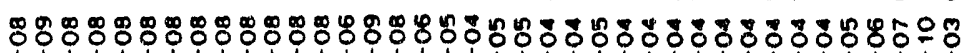

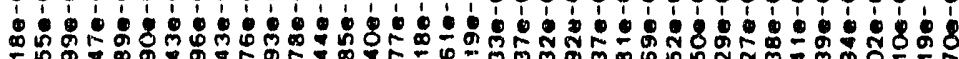

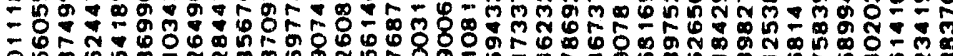

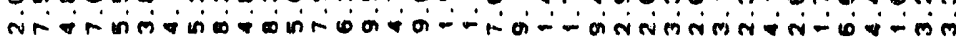

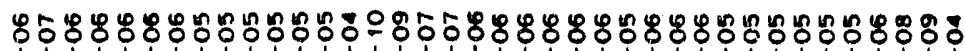

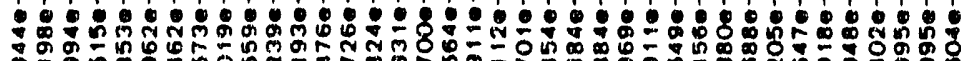

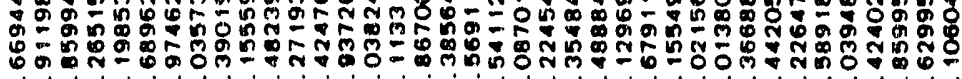
- 0 - का

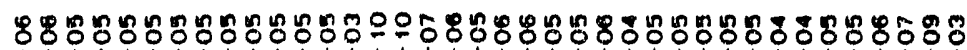
等

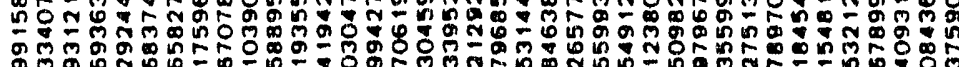

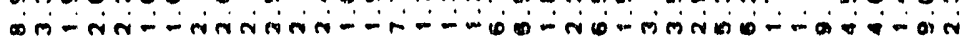

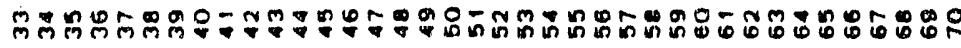




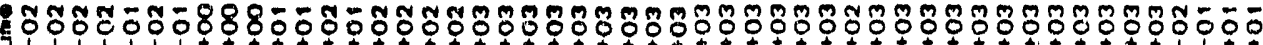

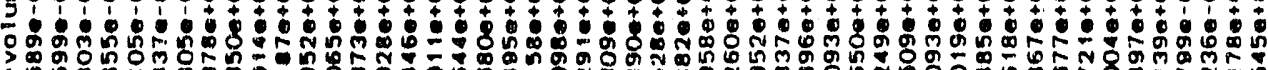

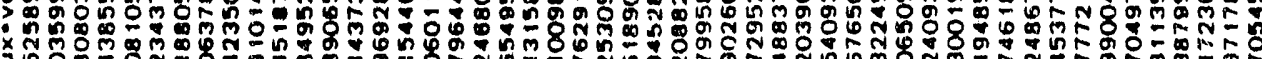
20nm-

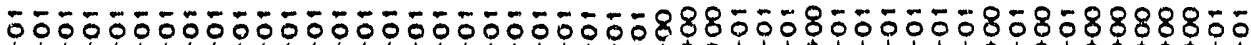

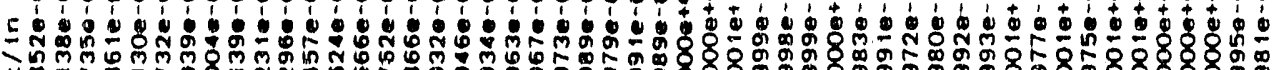

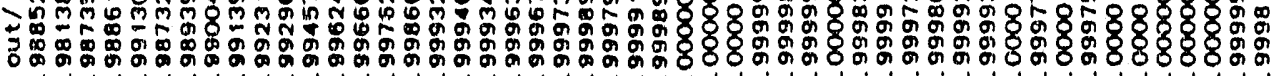

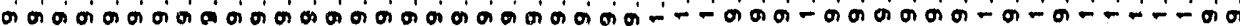
88888888888888888888888888888888888888888888888888

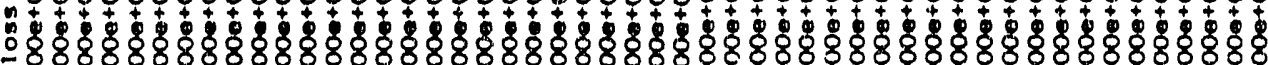

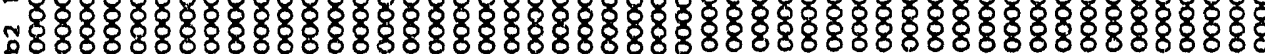

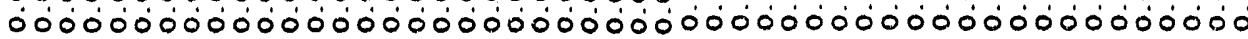

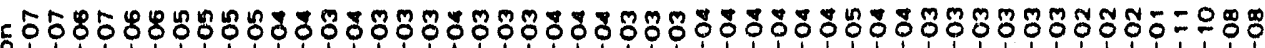

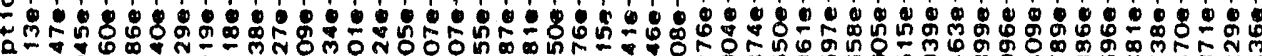

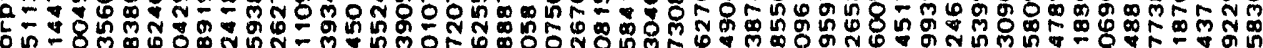
的市 ᄂ

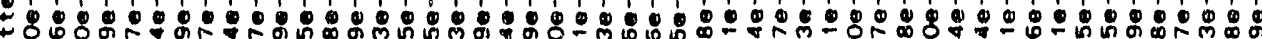

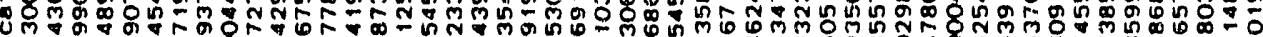

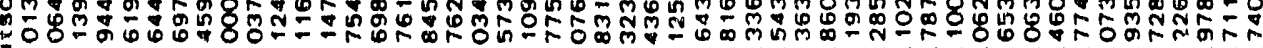

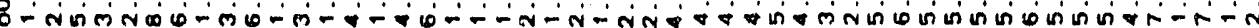

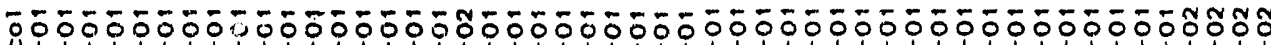

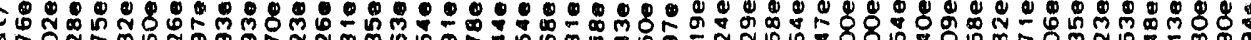

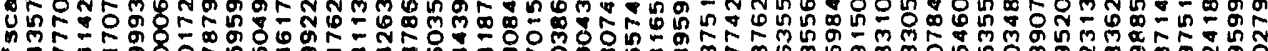
- 7 -

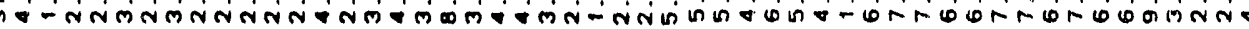

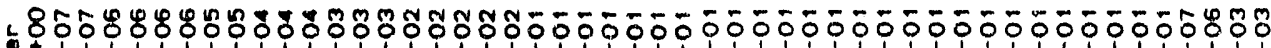

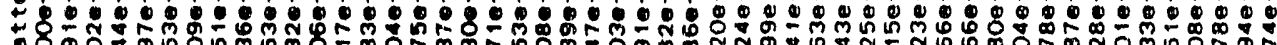

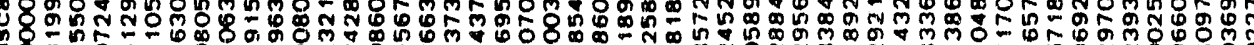
c. 8 m.

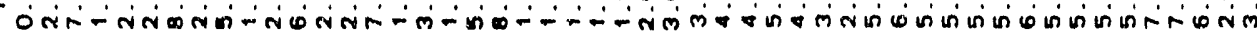
88889888888888888888888888888888888888888888888888

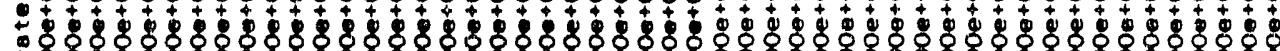

L000000000000000000000000000000000000000000000000000

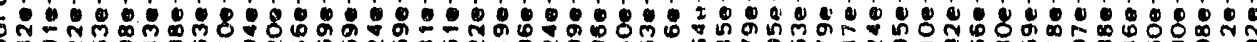

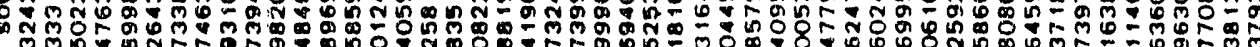
๓ - - vinmor-io- m-

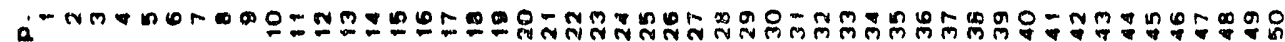
宫 


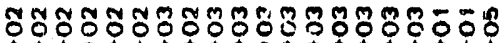

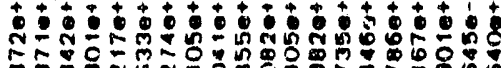

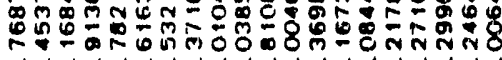

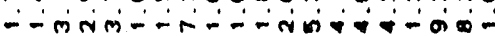
$\overline{0} 0 \overline{0} \overline{0} \overline{0} 0 \overline{0} 0 \overline{0} 08888888 \overline{0}$

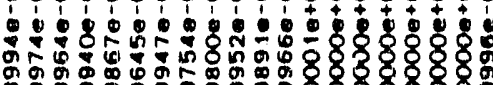

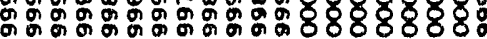

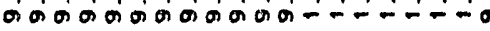
88888888888888888888

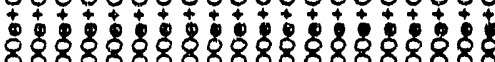

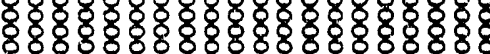
00000000000000000000

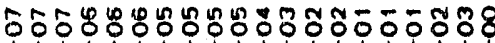

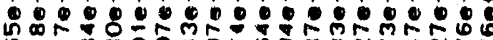

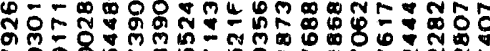
ono in

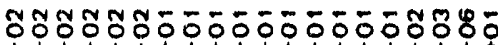

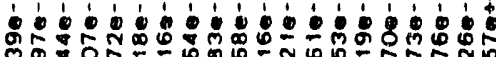

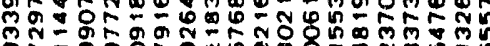

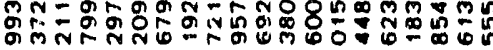
$\therefore$ -

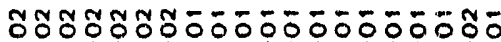
ฌ́ สูกิ

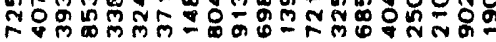

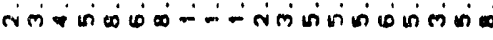

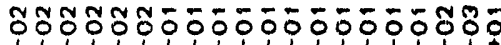

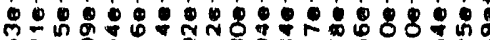

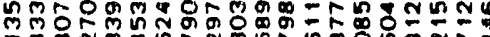

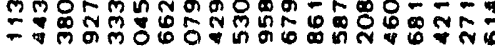
वं88888888888888888888

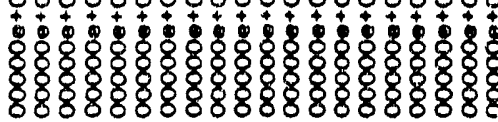
00000000000000000000 88888888888888888888

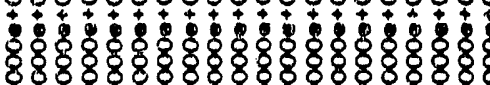
888888888888888808888 00000000000000000000

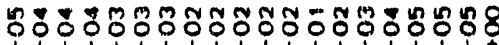

Eํ.

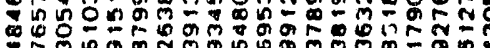

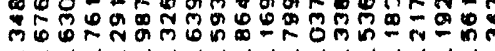

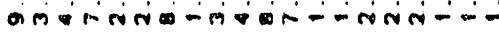

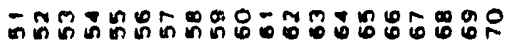

4

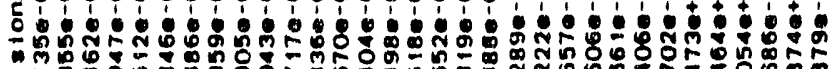

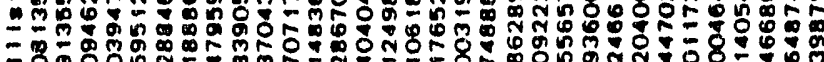

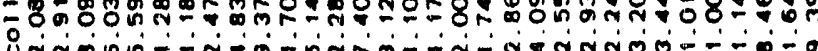

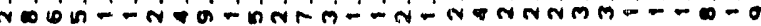

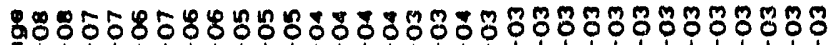

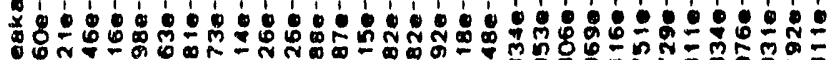

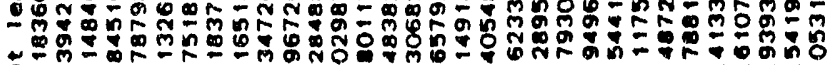

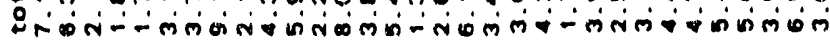

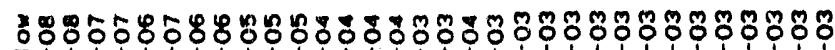
†!

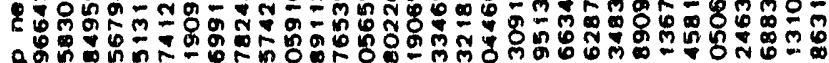

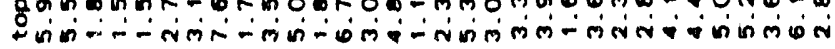

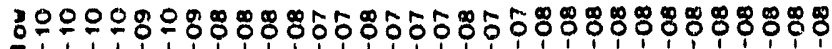

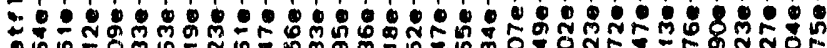

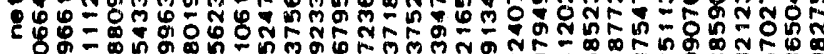

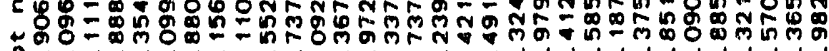

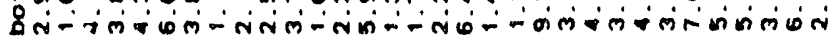

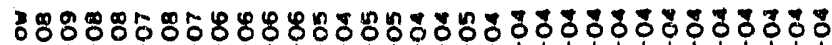

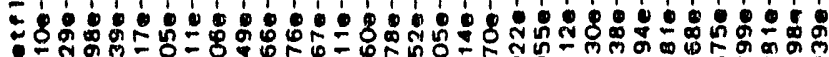

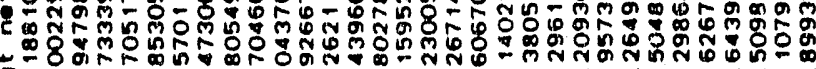
a वै88888888888888888888888888888888

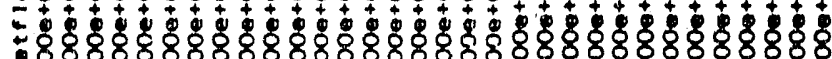

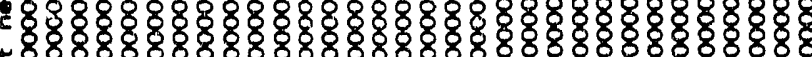

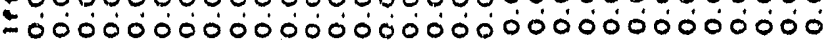

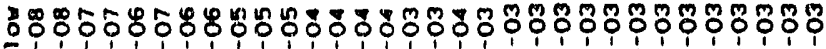
*

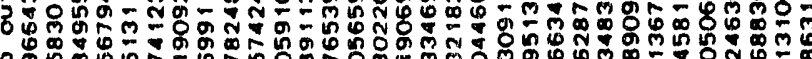
o की

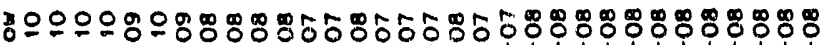

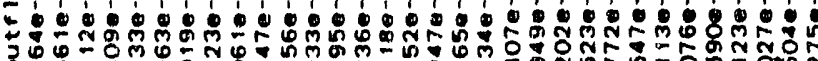
ว 舟-

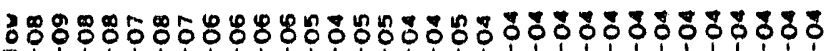
† \$ ช Q\%88888888888888888888888888888888

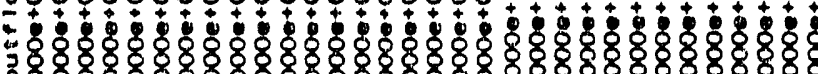

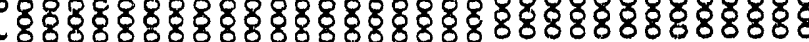

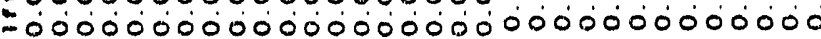

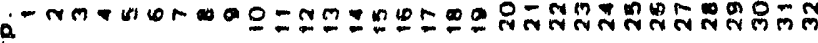
$\bar{a}$ 


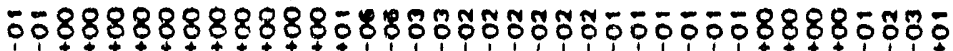

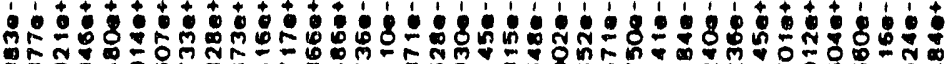

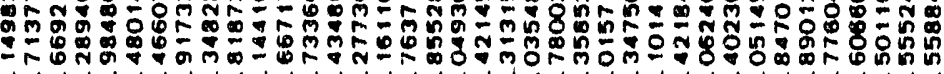

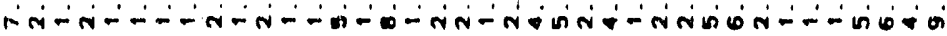

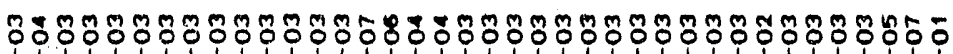
ด!

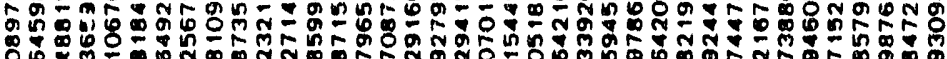

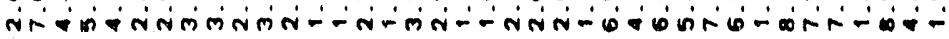

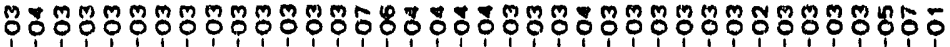

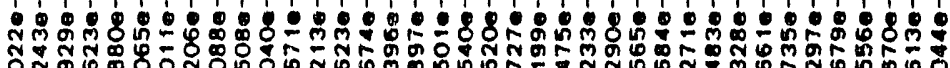

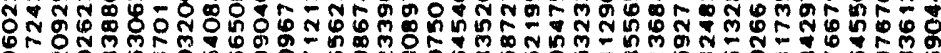
- - in

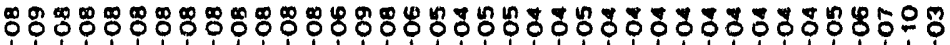

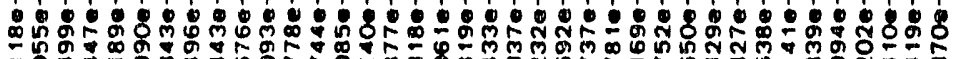
二

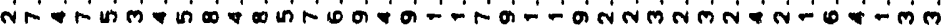

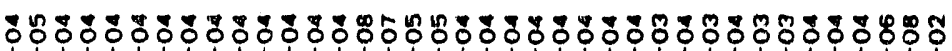

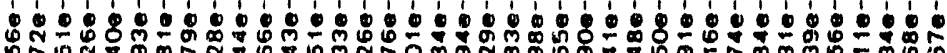

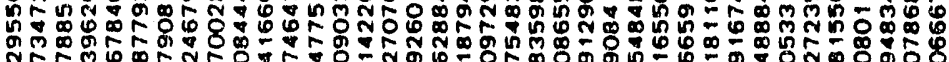

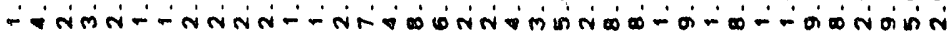
88888888888888888888888888888888888888

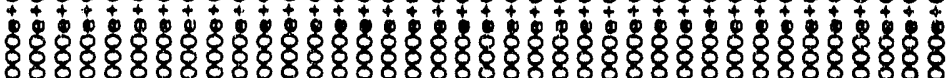

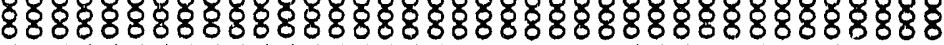
ó000000000000000010000000000000000000

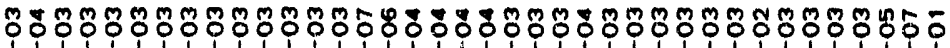

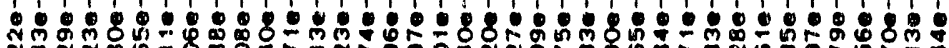
สำ สู่

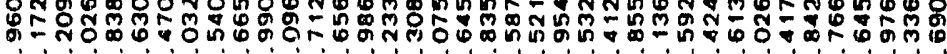

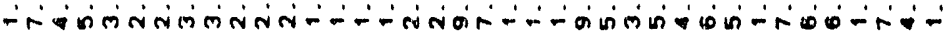

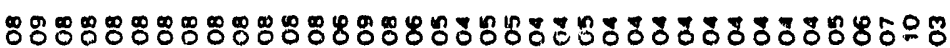
D.

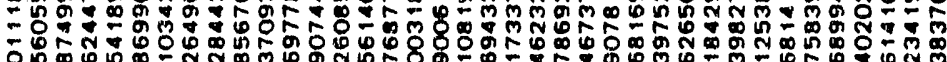
ति-

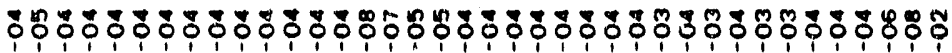

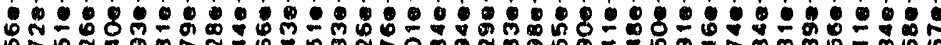
คำ

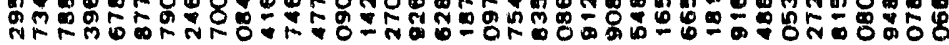
$\therefore$ तm - 88888888888888888888888888888888888888

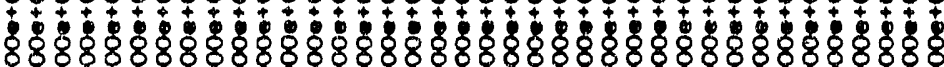

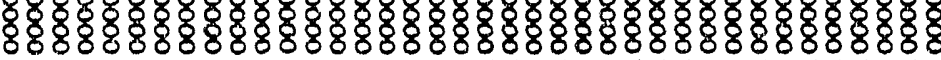
00000000000000000000000000000000000000

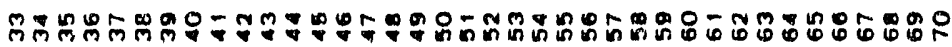


(This page is intentionally left blank.) 


\subsection{VISTA: A VEHICLE INPUT SOURCE TRANSFORMATION AND ASSEMBLY CODE*}

\subsection{INTRODUCTION TO VISTA}

\subsubsection{Background}

The Vehicle Input Source Iransformation and Assembly (VISTA) code processes directional fluences from a two-dimensional directional fluence file produced by DORT ${ }^{1}$. These fluences in the DORT fluence file are nodified and reformatted for use in the forward-adjoint folding process of the DRC module in MASH or for use in DOTTOR2 to produce a boundary sour"ce for TORT ${ }^{3}$.

VISTA was developed by W. A. Rhoades to replace the VISA module of the Vehicle Code System (VCS) 4,5 as the code for processing the scalar and angular fluences from DORT and the uncollided fluence file from GRTUNCL. An additional incentive for the development of VISTA was to have a code capable of processing the DORT directional flux files for eventual use in the TORT code. VISTA represents an improvement to VISA through increased gelneralization, and the addition of several new output options. In particular, VISTA can produce a file formatted for use in the DRC module of MASH, or a file formatted for use in DOTTOR (a code which transforms 2-D fluence information into a 3-D boundary source at or near the coupling surface for use in TORT). Furthermore, VISTA can copy old MASH or DOTTOR formatted files to new files, convert MASH formatted output files to DOTTOR formatted output files, and combine two MASH or DOTTOR formatted files into new DRC or DOTTOR formatted files.

The basic approach in VISTA is similar to that of an unpublished code from the 1960's named LIMBO. The exact authors of LIMBO are unknown, but they may have included R. D. Rodgers, F. R. Mynatt, and/or M. L. Gritzner. Credit for the preservation and availability of LIMBO belongs to J. V. Pace, III.

\subsubsection{Method Used}

Because of the large volume of data, it is not practical to produce properly-normalized directional fluence files from DORT. Further, when the GRTUNCL code is used to produce a first-collision source for DORT, the uncollided fluence, i.e., the fluence resulting from the particle flight before the first collision, is not included in the DORT output.

\footnotetext{
"VISTA is an updated and undocumented version of the VISA program found in the following reference: W. A. Rhoades, M. B. Emmett, G. W. Morrison, J. V. Pace, III, and L. M. Petrie, "Vehicle Code System (VCS) User's Manual," ORNL-TM-4648, Oak Ridge National Laboratory, (Âugust 1974).
} 
VISTA repairs the normalization problem by calculating the scalar fluence for each mesh cell, comparing this with the scalar fluence file produced by DORT, and normalizing the directional fluence such that it corresponds exactly to the DORT result. Then, the uncollided fluence from GRTUNCL is added to the directional fiuence in the direction applicable at each miesh cell. The processing can be restricted to a subset of the space mesh, reducing the volume of the output file.

As the new data are calculated, they are dumped to a random-access scratch file. Then, the data are retrieved in the order required by the requested output format and transferred to the output file. On some computer systems, reading and writing in the sorting step can proceed concurrentiy. For convenience, limited ability to transform between formats is included. Certain input and output files can be either formatted or unformatted as a convenience in using data shipped between computers through a network.

\subsection{VISTA INPUT REQUIREMENTS}

The following input cards are required to execute VISTA. Default values are in brackets ([]).

Title Card (72 alphanumeric character description)

\section{INPUT DATA BLOCK 1}

$1 \$$ Array - Integer Control Parameters
NIP number of radial intervals output
$J P L$ first axial interval output
JPU last axial interval output
NED print edit control parameter
$=0$ implies no effect
$>0$ implies print NED source groups
NORM scalar fluence normalization control flag
$0 / 1=$ normalize/do not normalize to scalar fluence

$-5 \ldots$
ISGRP number of groups on uncollided fluence file
$=0$ implies use collided fluence only
$<0$ implies use uncollided fluence only
NFLSV logical unit number of scalar fluence input, [1]
NAFT logical unit number of angular fluence input, [2]
NUNCL logical unit number of uncollided fluence input, [3]
NDATA logical unit number of source output, [4] 
N5 logical unit number for card input, [5]

N6 logical unit number for printed output, [6]

NJ1 first axial interval input control flag

$=0$ implies set $\mathrm{NJI}=1$

$>0$ implies first axial interval input

NJM last axial interval input control flag

$=0$ implies set NJM - JM

$>0$ implies last axial interval input

NAFTI NAFT missing groups control flag

$<0$ implies first NAFTI groups $=1.0$, all others $=0.0$

$=0$ implies no effect

$>0$ implies number of groups missing at beginning of NAFT

$-15-$.

NTYPE fluence file output option control flag

$=0$ implies process DORT files on NFLSV, NAFT, NUNCL into MASH output on NDATA

$=1$ implies process DORT files on NFLSV, NAFT, NUNCL into DOTTOR output on NDATA

$=10$ implies copy MASH output file on NFLSV to a new MASH output file on NDATA

- 11 implies copy DOTTOR output file on NFLSV to a new DOTTOR output file on NDATA

- 21 implies convert MASH output file on NFLSV to a DOTTOR output file on NDATA

$=30$ implies combine MASH output files on NUNCL and NFLSV to a new MASH output file on NDATA

$=31$ implies combine DOTTOR output files on NUNCL and NFLSV to a new DOTTOR output file on NDATA

NEUI last neutron group control flag

(required if NGAMX $>0$ and NTYPE = 0 or 1 )

$<0$ implies garmma output only

$>0$ implies last reutron group on input fluence files

NGAMX initial missing gamma group control flag

$=0$ implies no effect

$>0$ implies NGAMX initial gamma groups missing on all input files (NFLSV, NAFT, and NUNCL)

$=0$ implies no effect

$>0$ implies NGAMX initial gamma
groups missing on input file NUNCL

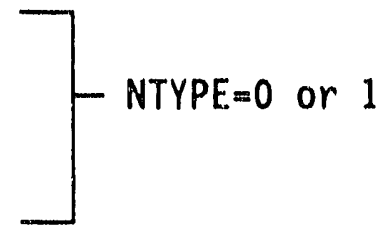

E (terminate array with "E")

$T$ (terminate block with "T") [T always required] 
INPUT DATA BLOCK 2 [omit data block if NTYPE $\geq 10$ ]

\author{
2* Array \\ SH height of point source \\ HSA cosine of angle with $z$ axis into which source is emitted \\ XNEUT NFLSV fluence file multiplier (if NTYPE $=30$ or 31 ) [1.0] \\ XGAM NUNCL fluence file multiplier (if NTYPE $=30$ or 31) [1.0] \\ E (terminate array with "E")
}

\author{
$4 \$$ Array \\ IVAL values of radial intervals to be output (NIP entries) \\ $T$ (terminate block with "T") [T always required]
}

\title{
5.3 VISTA INPUT DATA NOTES
}

Except for the title card, all data are read using the FIDO input system. A detailed description of the FIDO input system is given in Appendix A. Data arrays are entered in blocks, each terminated by a "T". Unused data arrays (e.g., $2^{*}$ and $4 \$$ if NTYPE $z=10$ ) are not eritered, but a "T" must still be entered to signal the termination of each block.

NIP The number of radial intervals output should encompass the TORT geometry for a DOTTOR formatted source file. At present, the DRC module in MASH only uses one radial interval for the source. Therefore, the value for NIP is usually set to one, although it is not required to be just one. It should be noted that a new version of DRC is in the testing stage that will allow interpolation in the DORT radial mesh and then NIP will have the same meaning for MASH as it does for DOTTOR.

NFLSV, NAFT, and NUNCL Logical unit numbers for these input fluence files specified less than zero (i.e., negative entries) imply FORMATTED or BCD files instead of binary files.

JPL and JPU The output axial intervals must be equal to, or a subset of, the input axial intervals (NJI and NJM) from the DORT file. These intervals should also encompass the TORT or MASH geometry in the $Z$ direction.

NED This parameter controls the print of the output source file by group. Care should be taken in using this control flag because it could produce large amounts of output. 
NJ1 and NJM These parameters must correspond to the parameters JDIRF and JDIRL in the $62 \$$ array of DORT. These parameters in DORT give the axial intervals that are written out on the directional fluence file. Consequently, the same $N J 1$ and $N J M$ values must be read as input into VISTA.

NAFTI This parameter is primarily used to tell the number of initial groups on NAFT which had a zero source. For instance, if the initial source distribution used in GRTUNCL and DORT did not have any values in the first eight groups, then NAFTI would equal eight (assuming no upscatter).

NEUI This parameter is used to output a gamma only file from a coupled neutron-gamma file if input negative.

NOTE: If you execute a coupled neutron-gamma DORT problem with all neutron source groups zero and with or without gamma source groups zero, you must output a full neutron-gamma coupled source file. You cannot output a gamma only source file.

NGAMX This parameter is only used to indicate missing (or deleted) gamma groups from the input fluence files.

SH The source height entered here must correspond to the source height used in GRTUNCL and/or DORT.

IVAL This parameter should correspond to the NIP interval(s) of the

DORT mesh required to encompass the TORT geometry, or the radial distance for the source for MASH required by the DRC module.

\subsection{VISTA INPUT FLUENCE FILE FORMAT}

As stated earlier, VISTA's primary function is to process, modify, and reformat directional fluence files from the DORT code into a suitable format for use in either MASH or DOTTOR. Also, VISTA can perform a number of utility functions (i.e., copy, convert, and combine) on previously processed VISTA files. Consequently, VISTA will read several different types of input file formats depending on the value of NTYPE. For this section, only the primary function of VISTA will be addressed. The MASH formatted source file will be addressed in the next section, and the interested reader should consult the DOTTOR manual (Ref. 2) for information on the DOTTOR formatted source file.

To accomplish its primary function, VISTA reads a DOT IV formatted file - referred to as the VARSOR format in this document. Both the uncollided fluence on unit NUNCL and the scalar and directional fluence files on NFLSV and NAFT, respectively, are written in this format. The VARSOR format is defined in the following manner: 


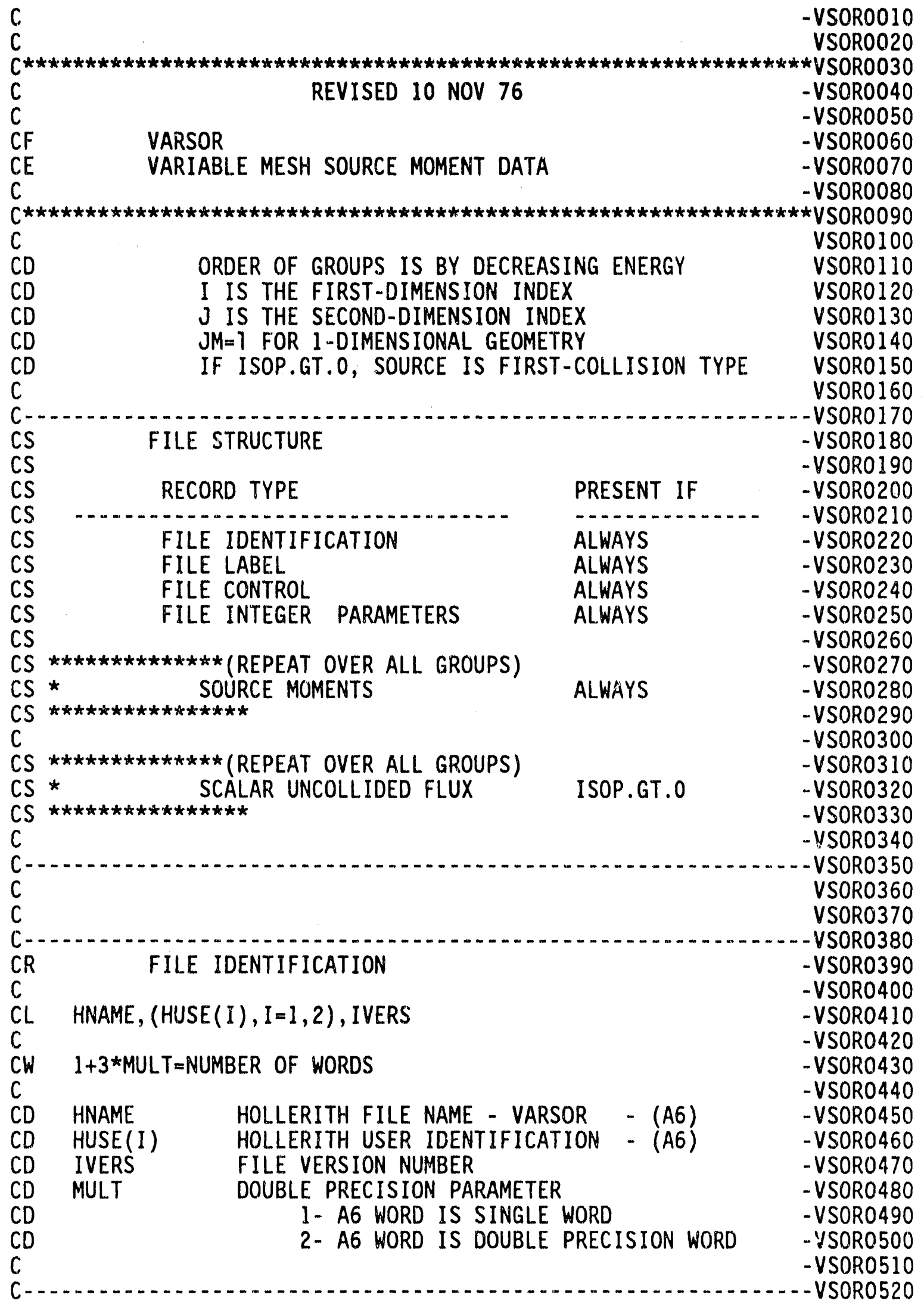




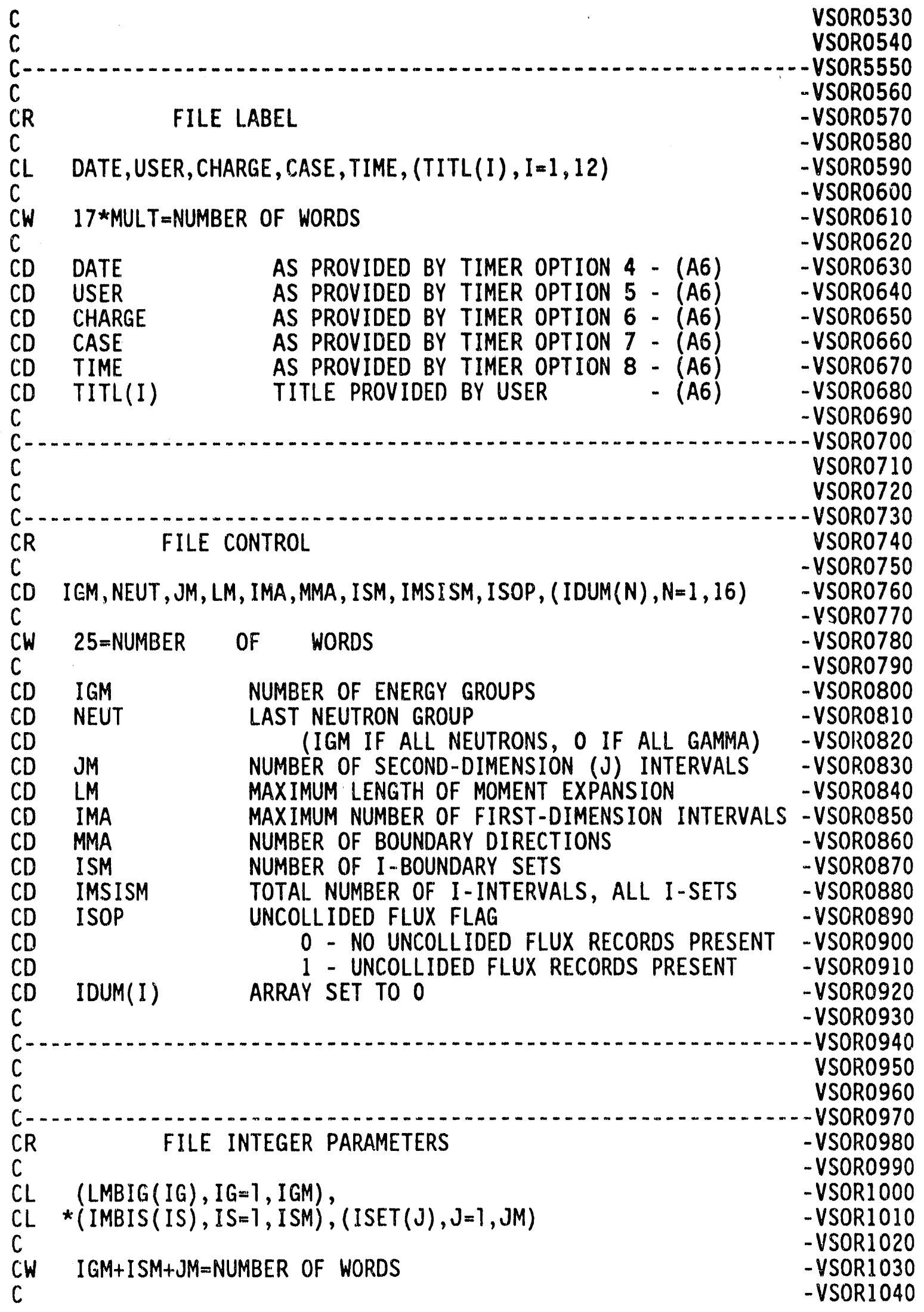




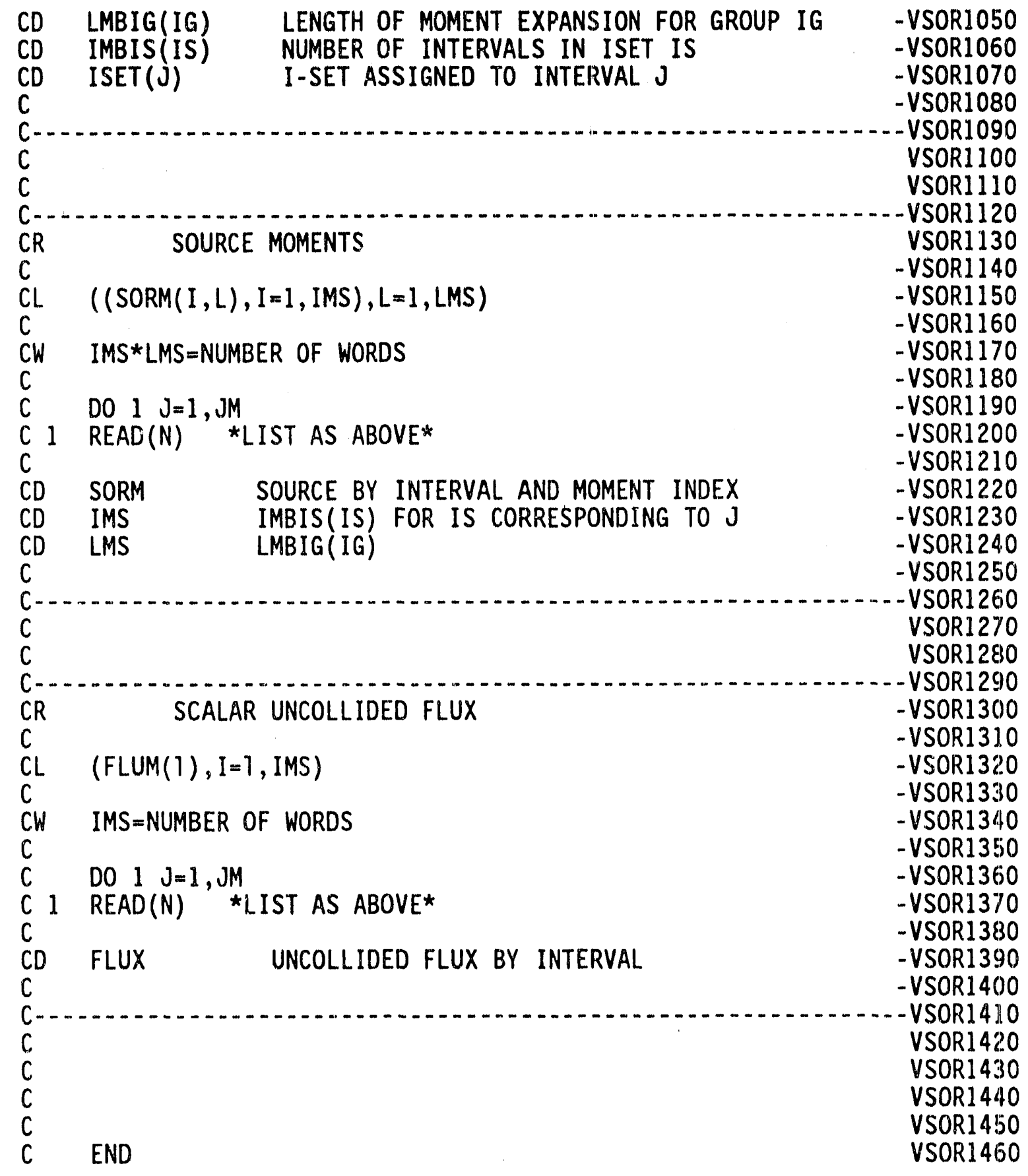

\subsection{VISTA OUTPUT FILES}

The VISTA code produces two types of output file - a DOTTOR formatted source file and a MASH formatted source file. The DOTTOR formatted source file will not be addressed in this manual, and the interested reader should consult Reference 2. The MASH formatted source file is as follows: 
Record 1: TITLE, TDOT - 144 Alphanumeric Characters

Record 2:

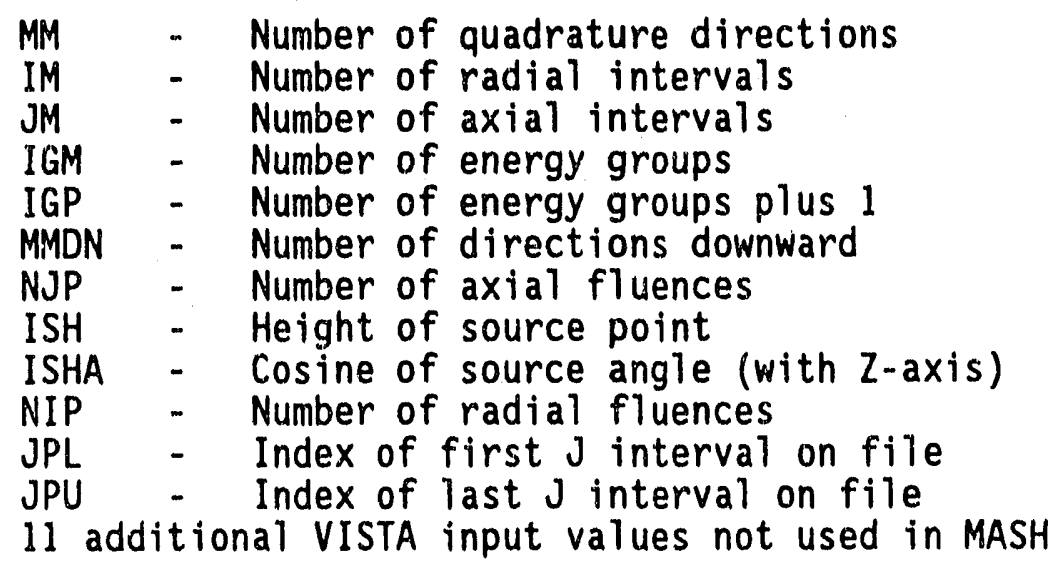

Record 3: IVAL - Array of radial interval numbers being output

Record 4: [MM values per array; 3*MM total values]

WT - Weights from DORT quadrature

AMU - Mu's from DORT quadrature

ETA - Eta's from DORT quadrature

Record 5: $\quad[$ [NIP + NJP values]

$R \quad$ - Midpoints of radial bins

$Z$ - Midpoints of axial bins

Record 6 to END: [NANG*NJFLX values per record]

[IGM records per radius, and NIFLX radii]

FLUX - Directional fluences

It should be noted that much of the information on the DOTTOR formatted source file is similar to the information given above in the MASH formatted source file.

\subsection{LOGICAL UNIT REQUIREMENTS}

Below is a listing of the files required to execute a VISTA case along with the default values used in the code. In setting up a VISTA case, efforts must be made for these units to be available. 
1. Logical Unit NFLSV - DORT Scalar fluence input file

2. Logical Unit NAFT - DORT directional fluence input file

3. Logical Unit NUNCL - GRTUNCL uncollided fluence input file

4. Logical Unit NDATA - MASH or DOTTOR formatted output source

5. Logical Unit 91 - SCRATCH

6. Logical Unit 6 - Printed Output

7. Logical Unit 5 - Card Input

Default logical unit assignments for the first four data files are 1, 2, 3 , and 4 .

NOTE: Logical unit numbers for the input fluence files

(NFLSV, NAFT, NUNCL) specified less than zero

(i.e., negative entries) imply FORMATTED or BCD

files instead of binary files.

\subsection{PROGRAMMER INFORMATION}

\subsubsection{Inter-machine Adaptability}

The VISTA code is intended to be easily adaptable to any type of sophisticated computer, and yet to take advantage of certain localized structural features which may be machine-dependent.

In general, the guidelines of ANS-STD.3-1971 ${ }^{7}$ are followed. This requires general adherence to a simple, standard FORTRAN language except where deviations provide important improvements in capability and can be documented. In addition, machine-dependent features have been kept localized and have not been included in the subroutines which perform actual computation.

The recommended procedures of the Committee on Computer Code Coordination (CCCC $)^{6,8}$ have been followed where practical. Compliance with these standards has been incomplete where cost was prohibitive, the nature of the codes did not allow it, or the expected level of performance could not be obtained in that fashion.

Where minor or certain machine-dependent features are required, alternative features are enclosed in pairs of 3-character "1anguage flags." The alternative statements remain in the source program, with inappropriate sections transformed into comment statements. If the code is set up for IBM operation, for example, it might contain:

CIB

CIB

$$
\text { ENTRY } \operatorname{IBCDC}(H, E, L, P)
$$


CDC

C ENTRY IBCDC

CDC

The corresponding CDC configuration would be:

$\begin{array}{ll}C I B & \\ C & \text { ENTRY } \operatorname{IBCDC}(H, E, L, P) \\ C I B & \\ \text { CDC } & \\ \text { CDC } & \text { ENTRY IBCDC }\end{array}$

All versions of a given procedure are thus available for inspection by all users. The selection of options is made by a computer program at distribution time. A listing of machine-dependent sections can also be prepared in that process.

The language flags achieve the major objective of having a single, unified source for each program which is maintained for all users. With these provisions, the basic FORTRAN programs are operable on IBM, CDC, UNIVAC, CRAY, Amdahi, and other computers, except that system-dependent service routines as specified by CCCC must be provided.

Optional packages and procedures which provide enhanced convenience are also available. The optional run-time storage allocation requires system-dependent routines. Instructions for installing these options are distributed with the source programs. All known users at this time use all of the optional enhancements available to them. Even so, the basic operation with FORTRAN routines plus the CCCC package remains available if needed.

\subsubsection{Service Subroutines}

Certain standard service subroutines are specified by the CCCC for use in reactor physics codes. The service routines used in these codes include:

TIMER Provides timing and job identification data

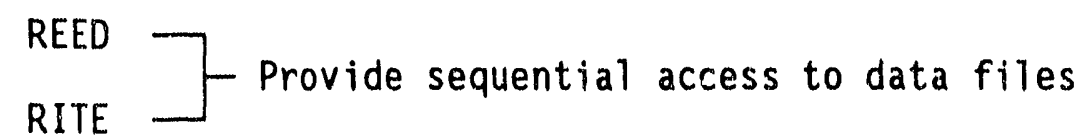

DRED - Provide random access to data files 
DOPC

Provides initiating, closing, and certain repositioning of data files

CRED 7

Provide block transfer of data between fast and slow memory.

Many of these subroutines also call other subroutines. Many are entirely system dependent. Each configuration to be distributed contains an appropriate set of service subroutines, insofar as possible. The realities of computing environments may require local modification or substitution. The specifications given in Reference 6 , together with extensive in-stream comments, provide guides for such modification.

\subsection{REFERENCES}

1. W. A. Rhoades and R. L. Childs, "The DORT Two-Dimensional Discrete Ordinates Transport Code," Nuclear Science \& Engineering 99, 1, pp. 88-89, (May 1988).

2. J. L. Thompson, M. B. Emmett, and H. L. Dodds, Jr., "Development and Evaluation of DOTTOR, A Computer Code to Couple Two-

Dimensional to Three-Dimensional Discrete Ordinates Calculations," ORNL/TM-9919, Oak Ridge National Laboratory, (April 1986).

3. W. A. Rhoades and R. L. Childs, "The TORT Three-Dimensional Neutron/Photon Transport Code," ORNL-6268, Oak Ridge National Laboratory, (November 1987).

4. W. A. Rhoades, "Development of a Code System for Determining Radiation Protection of Armored Vehicles (The VCS Code)," ORNLTM-4664, Oak Ridge National Laboratory, (October 1974).

5. W. A. Rhoades, M. B. Emmett, G. W. Morrison, J. V. Pace, III, and L. M. Petrie, "Vehicle Code System (VCS) User's Manual," ORNLTM-4648, Oak Ridge National Laboratory, (August 1974).

6. R. Douglas O'Dell, "Standard Interface Files and Procedures for Reactor Physics Codes, Version IV," LA-6941-MS, LOs Alamos National Laboratory, (September 1977).

7. ANS Standard "Recommended Programming Practices to Facilitate the Interchange of Digital Computer Programs, " prepared by Subcommittee 10, ANS Standards Committee (April 1971).

8. B. M. Carmichael, "Standard Interface Files and Procedures for Reactor Physics Codes, Version III," LA-5486-MS, LOS Alamos National Laboratory, (February 1974). 
9. J. O. Johnson, J. D. Drischler, and J. M. Barnes, "Analysis of the Fal1-1989 Two-Meter Box Test Bed Experiments Performed at the Army Pulse Radiation Facility (APRF)," ORNL/TM-11777, Oak Ridge National Laboratory, (May 1991).

\subsection{SAMPLE PROBLEM}

The sample problem demonstrates the processing of the DORT and GRTUNCL fluence files from the calculation of the air-over-ground environment for the two-meter box experiments at 400 meters. 9 Figure 5-1 depicts a simple diagram of which portion of the DORT geomeiry is being processed for output from VISTA. A complete listing of the input cards for the sample problem is given in Figure 5-2, and some selected output is shown in Figure 5-3.

In viewing Figure $5-2$, the input illustrates; 1 radial interval output (NIP), DORT interval 13 as the first axial interval output (JPL), and DORT interval 25 as the last axial interval output (JPU). No source groups were printed (NED) and the output fluences were to be normalized to the scalar fluence (NORM). There were 69 energy groups (IGM), DORT interval 13 was the first axial interval input (NJ1), and DORT axial interval 25 was the last axial interval input (NJM). There were no groups missing in the input directional fluence file (NAFTI), 46 neutron energy groups (NEUI), and no initial gamma groups missing (NGAMX). A MASH formatted output source file was requested (NTYPE=0). All comments after the slash (/) are ignored in FIDO input and are only useful for quick identification of the input parameters. Finally, the VISTA sample input in Figure 5-2 shows a source height (from GRTUNCL) of 16.143 meters (SH) and a value of 50 for the DORT radial interval (IVAL).

The selected VISTA output shown in Figure 5-3 first illustrates the input parameters read in the first two data blocks along with some of the parameters obtained from the DORT input directional fluence files. The memory requirements are also given in this output. This output is followed by an "angle number by DORT interval" array which represents the particular direction in the DORT quadrature of the uncollided component of the fluence. This array is followed by more information from the DORT directional fluence file, i.e., quadrature information, radial position of the interval IVAL (400 meters), and the axial positions corresponding to the axial intervals input (NJ1 and NJM) and output (JPL and JPU). Finally, the output illustrates the progress of the source ouiput reformatting (and normalization if selected) by group. The source is written to the SCRATCH unit (91) and after completion written to the output unit NDATA. If source group edits (NED>0) had been requested, they would appear at this point. One check for proper execution of VISTA is by examining the scaling adjustment factors printed by group. These factors should be on the order of $10^{-2}$ or less if correct information (input) has been given and there is a reasonable degree of corivergence in the DORT directional fluence files. 


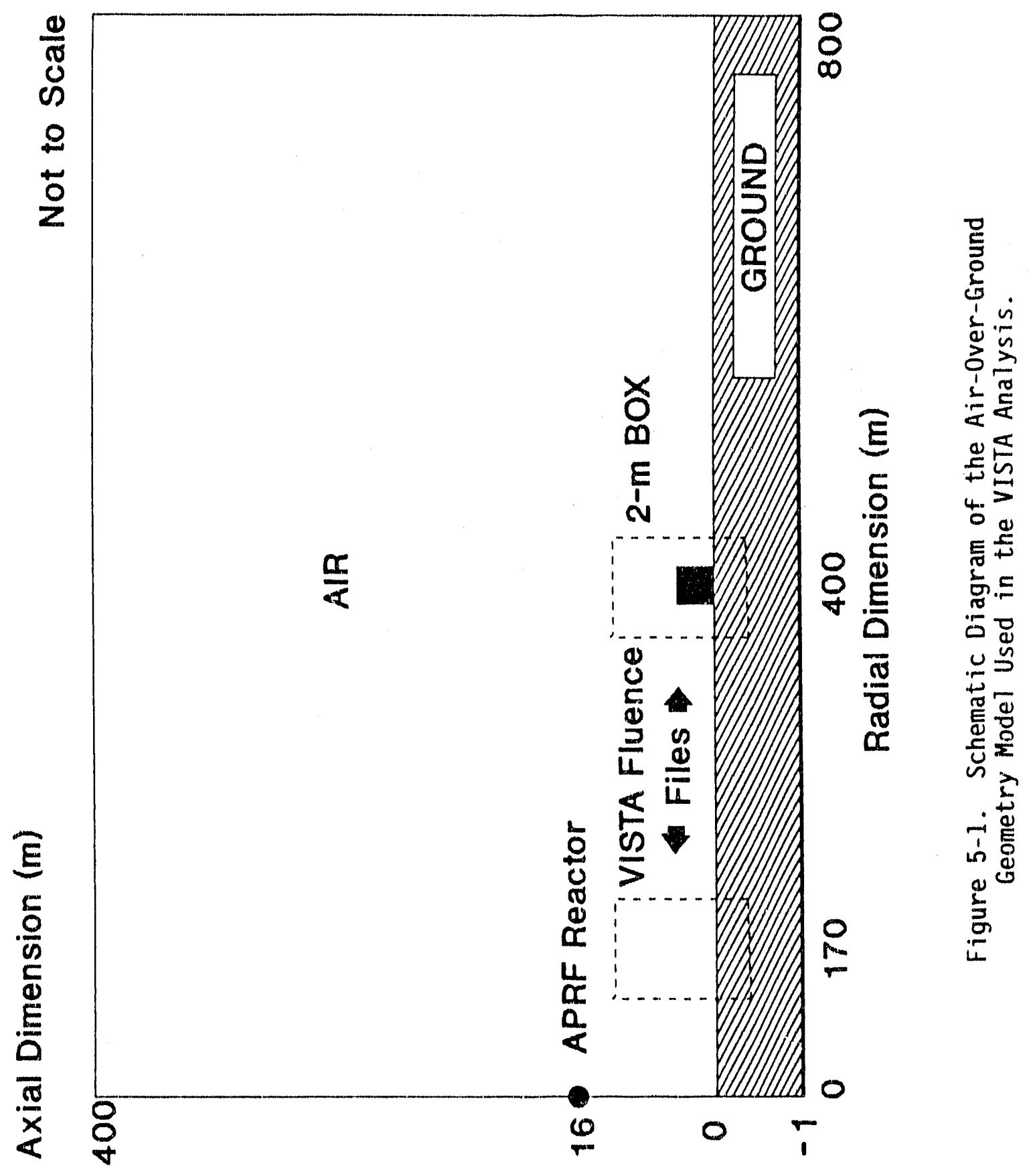




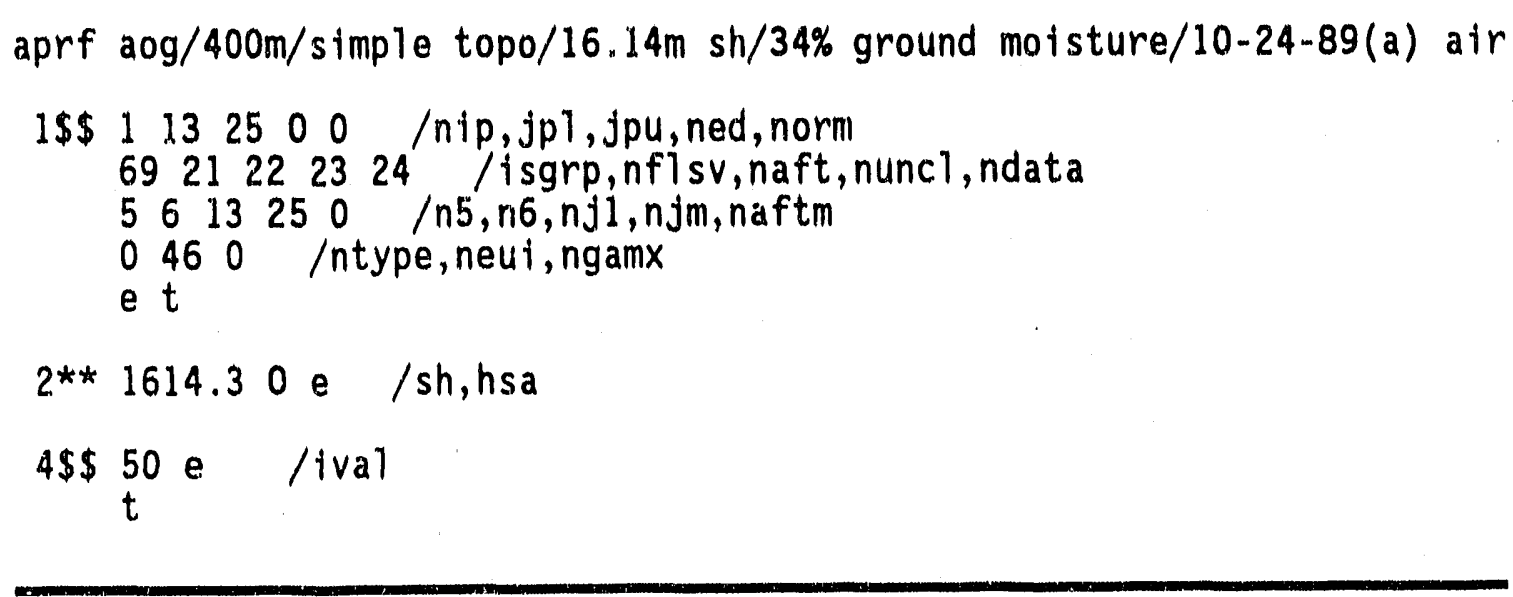

Figure 5-2. Sample VISTA Input for the Two-Meter Box Air-Over-Ground Analysis. 


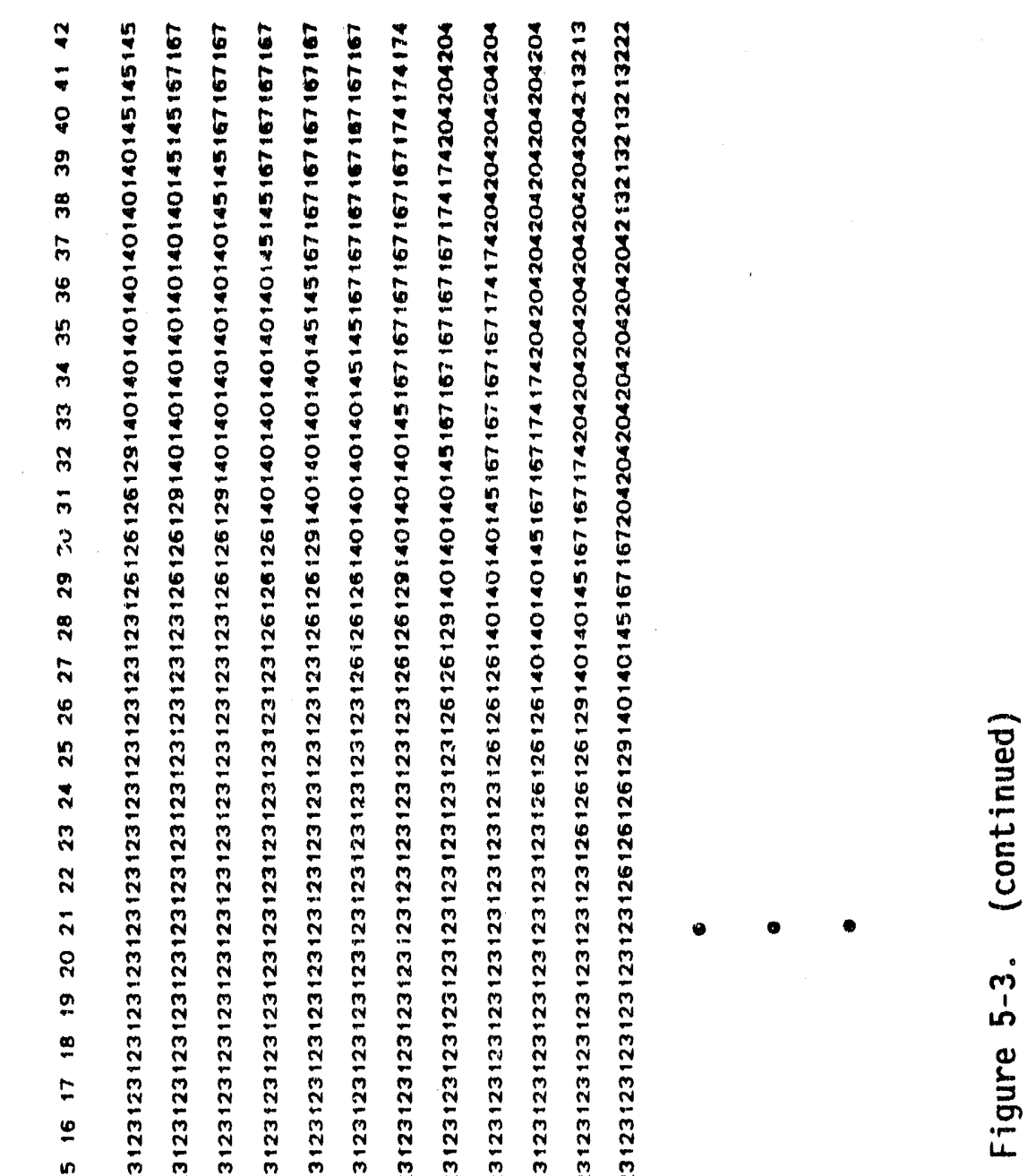

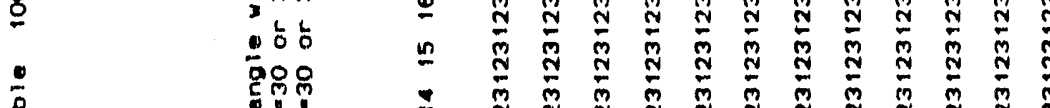

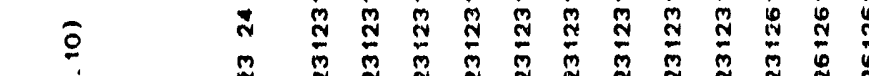

ऽ) ¿.

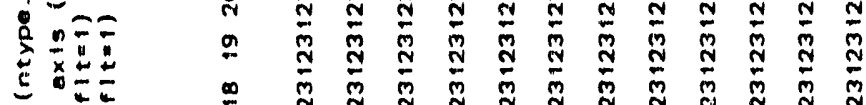

in

离

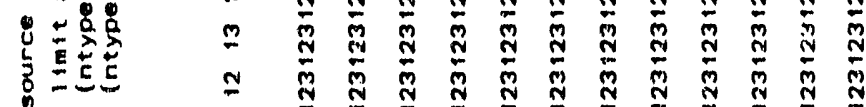

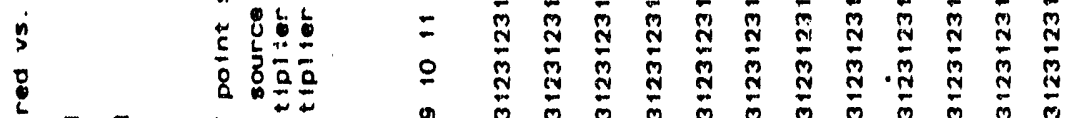

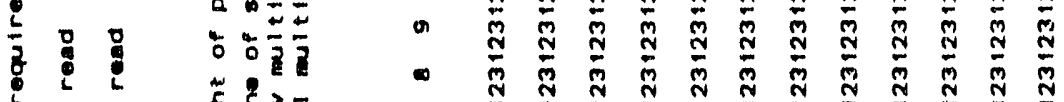

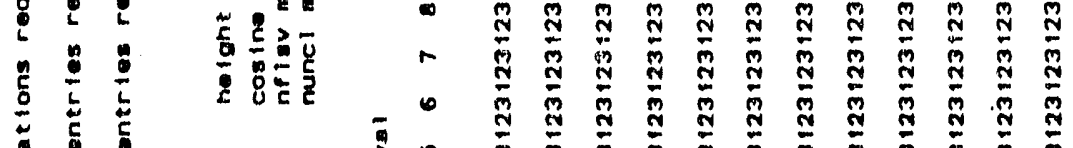

ర)

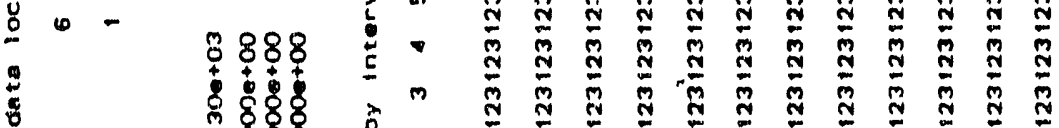

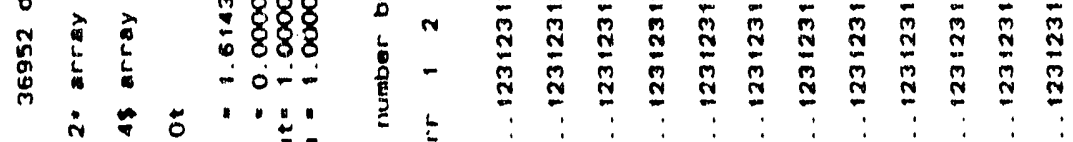

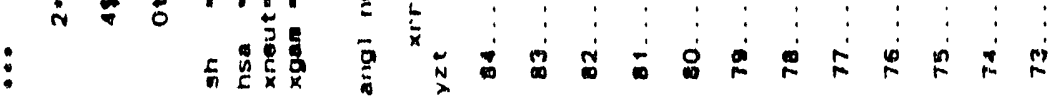




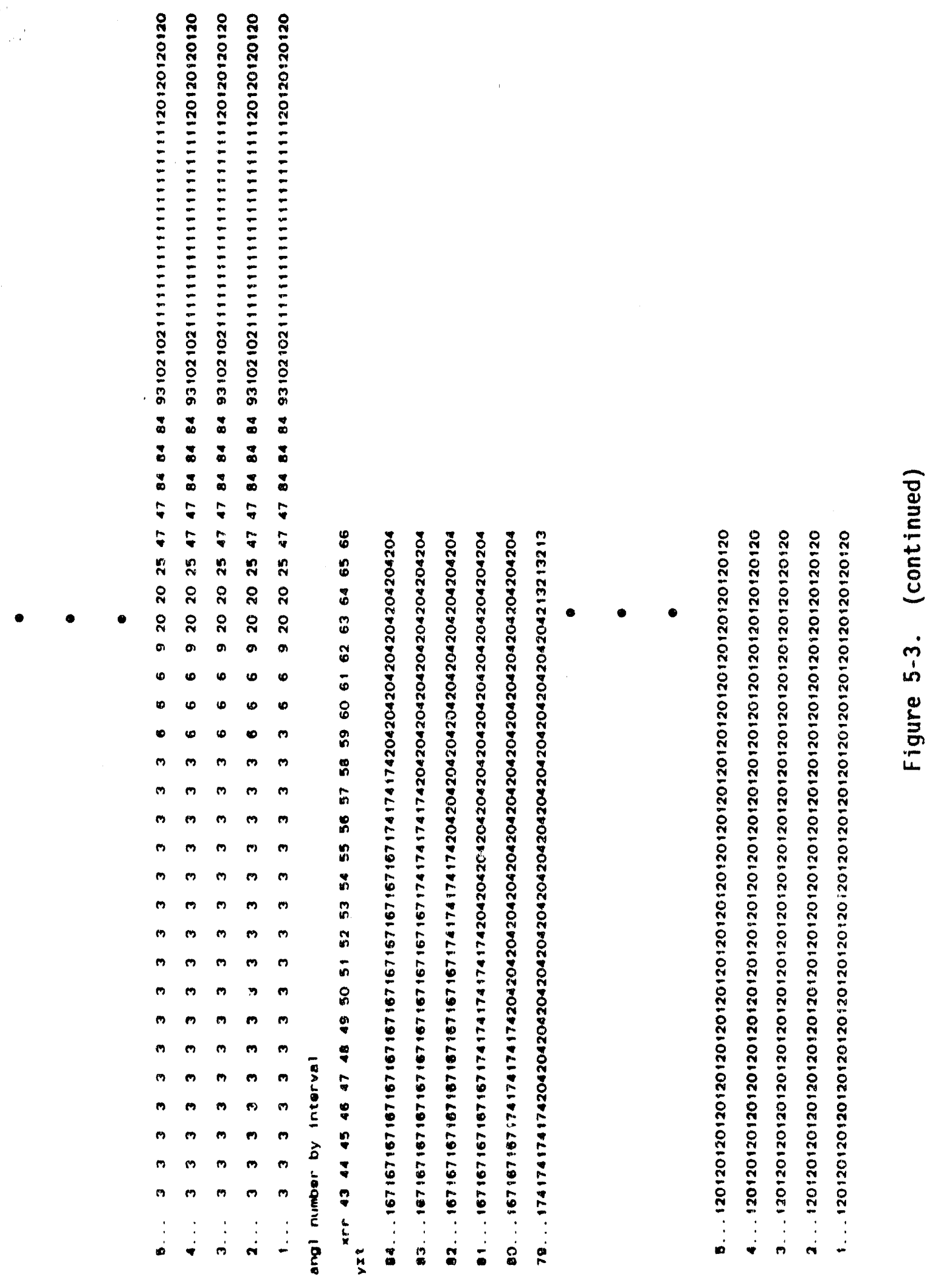

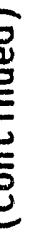

$n$
1
0
0
0

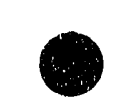



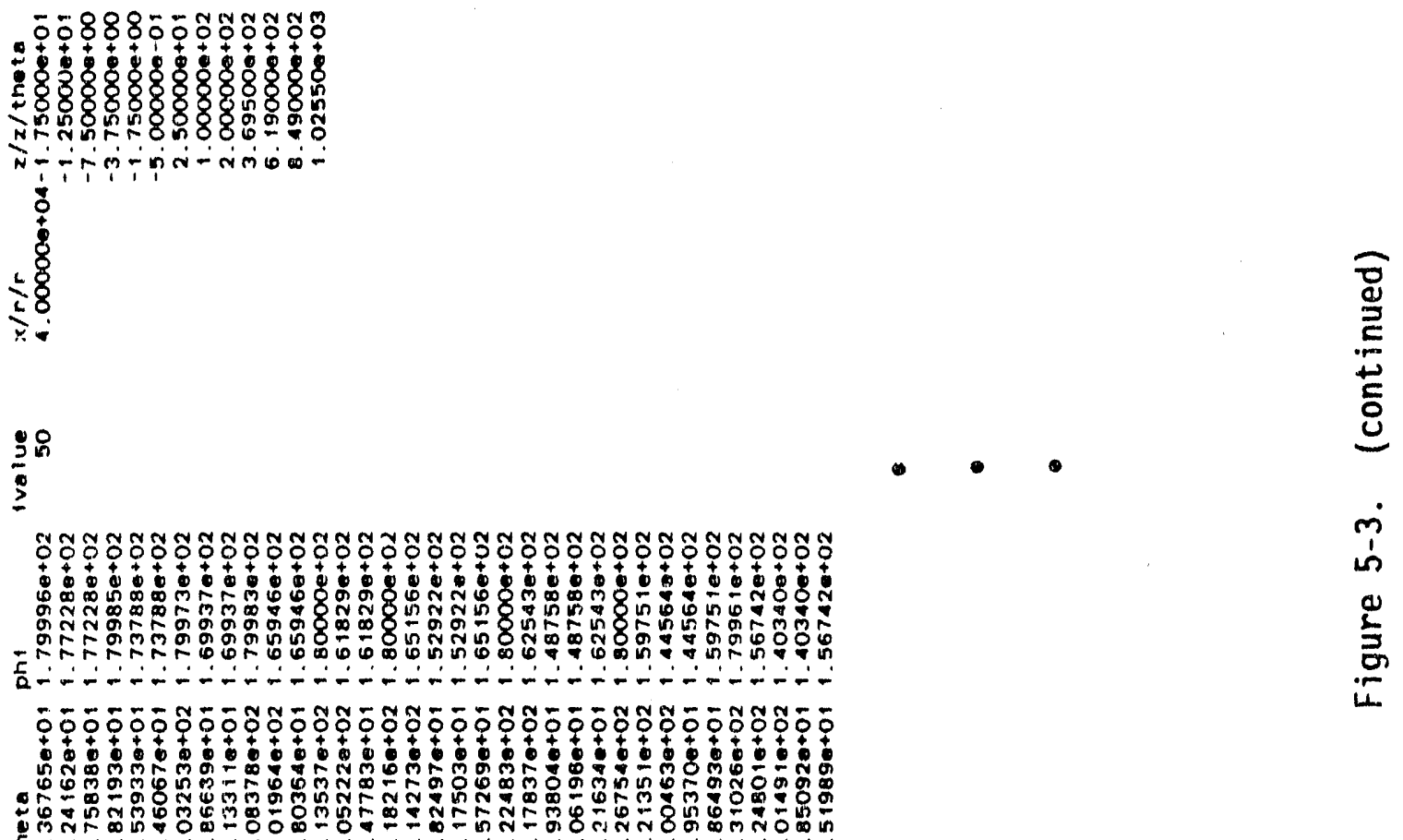

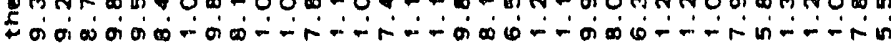

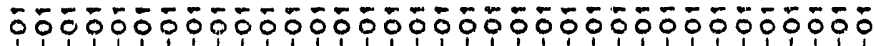
ำ

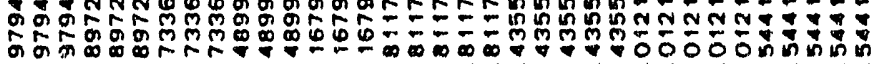

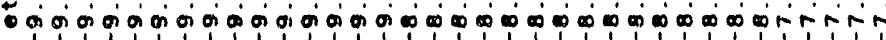

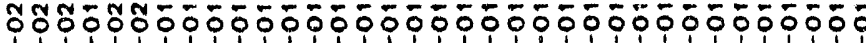
\& 8 舟

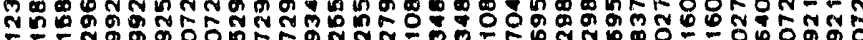

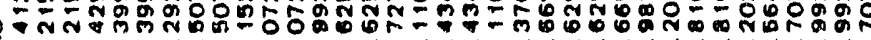

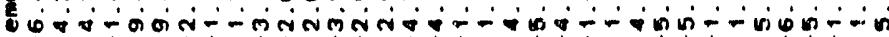

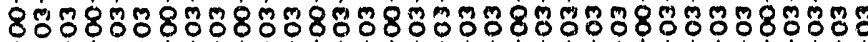

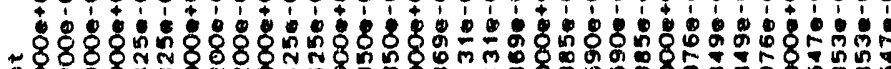

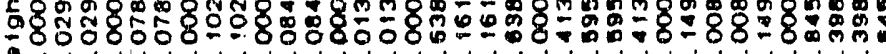

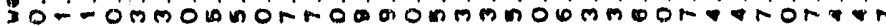
- 


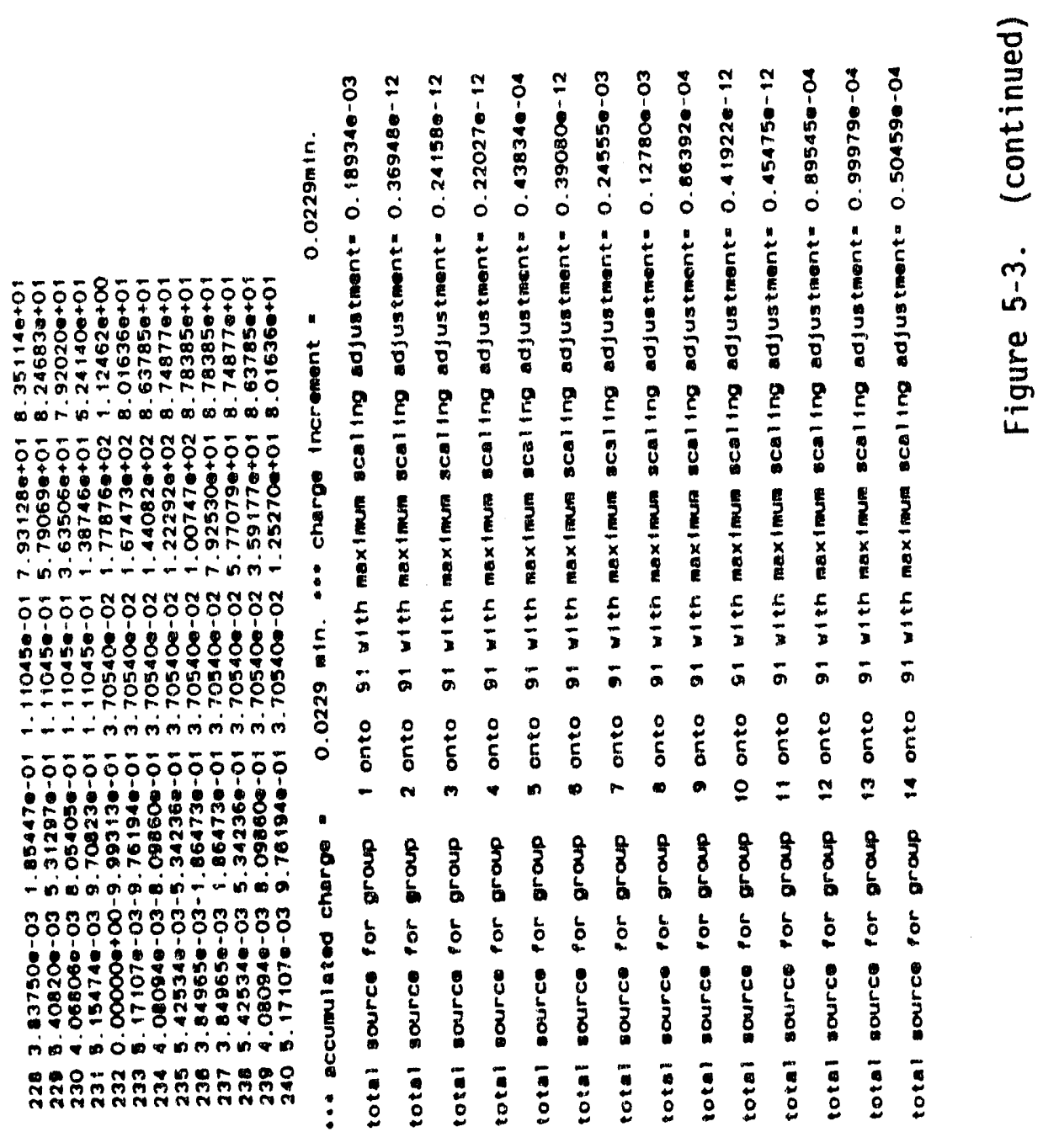

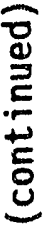

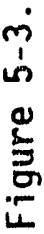




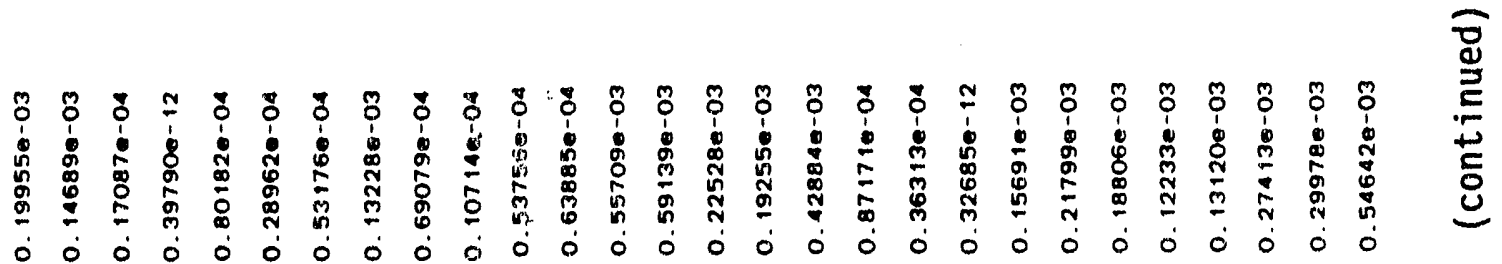

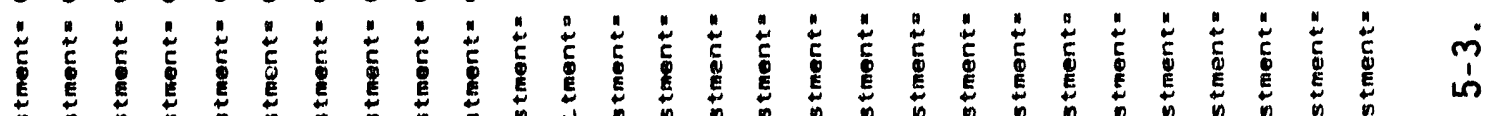

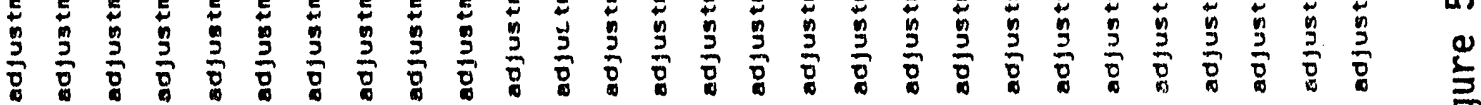

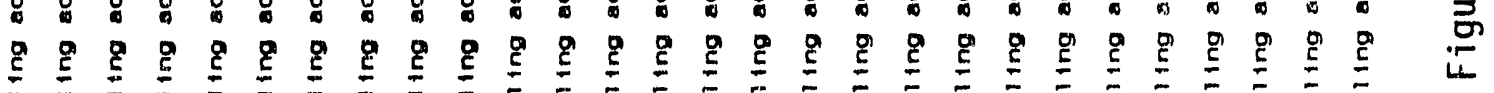

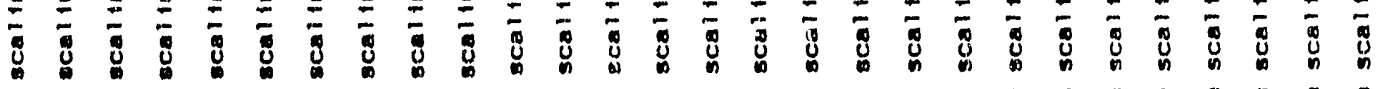

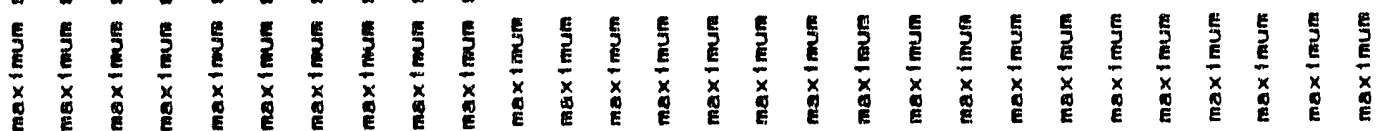

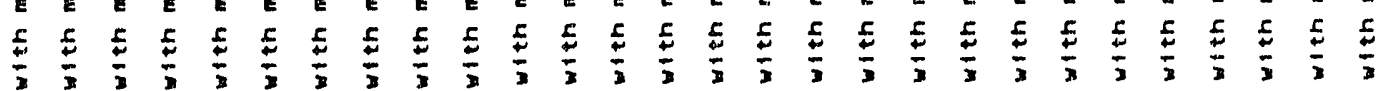

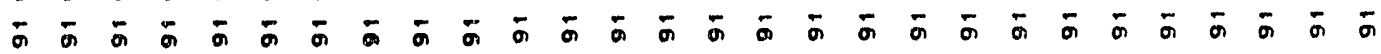
站 ก

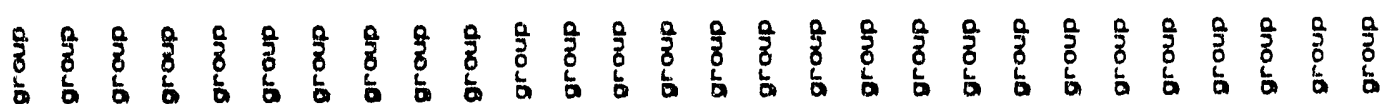
b

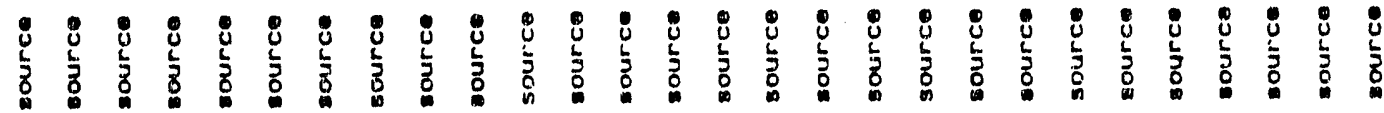

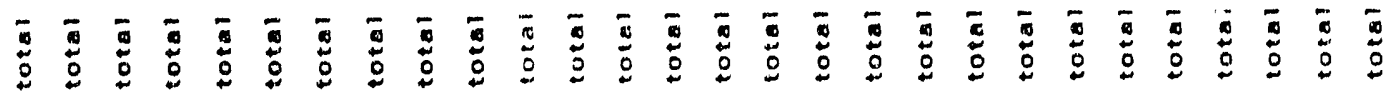




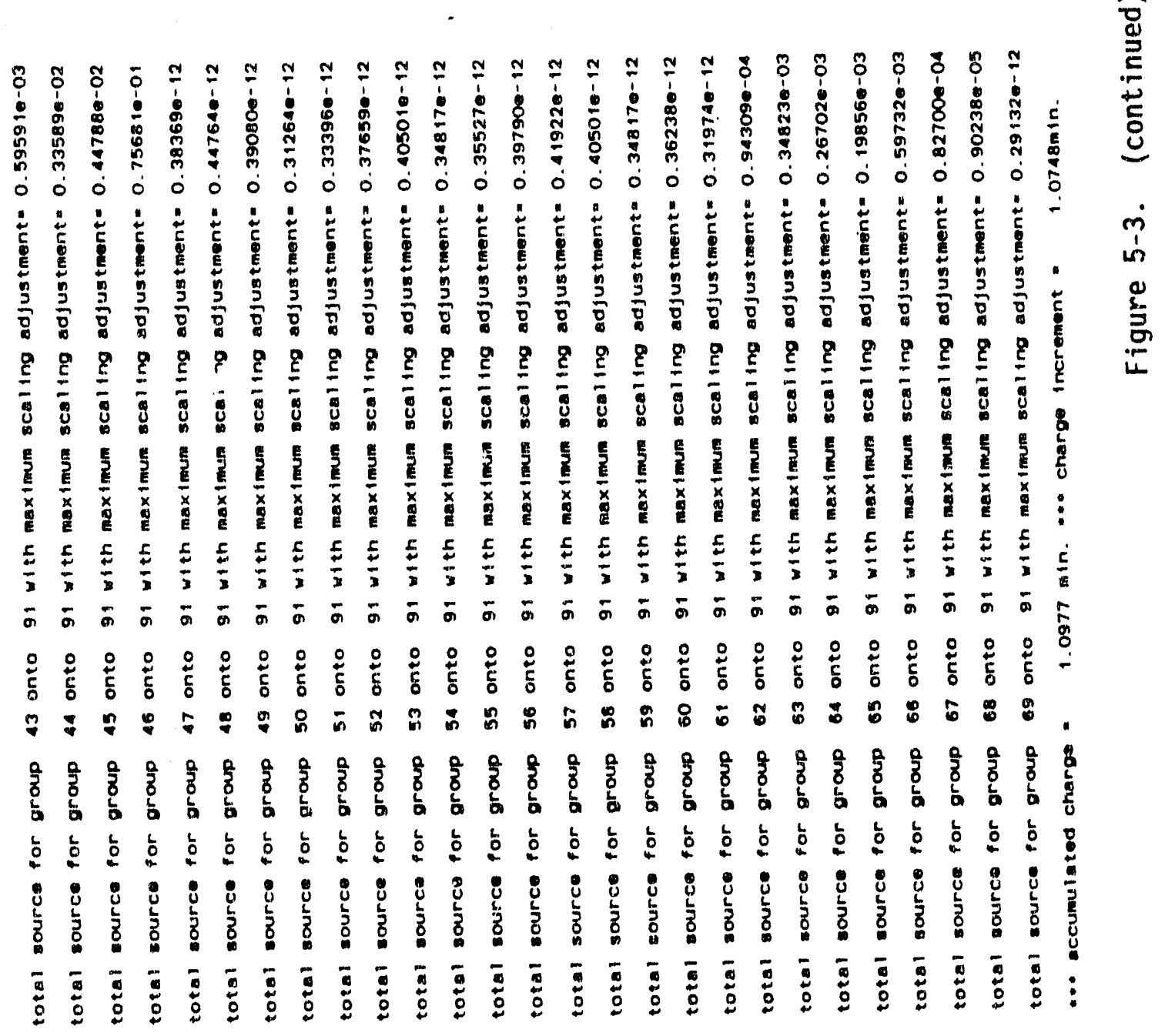

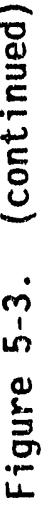




\subsection{MORSE: A MULTIGROUP OAK RIDGE STOCHASTIC EXPERIMENT CODE*}

\subsection{INTRODUCTION TO MORSE}

The Multigroup Dak Ridge Stochastic Experiment code (MORSE) ${ }^{1}$ is a mult ipurpose neütron and gamma-ray transport Monte Carlo code. Some of its features include the ability to treat the transport of either neutrons or gamma rays or a coupled neutron and secondary gamma-ray problem. The MORSE code also incorporates multigroup cross sections, an option of solving either the forward or adjoint problem, and modular input-output, cross section, analys is, and geometry modules. MORSE contains time dependence for both shielding and criticality problems, an albedo option at any material boundary, a three-dimensional combinatorial geometry package, and several types of optional importance sampling. Finally, MORSE includes a set of debugging routines accessible from anywhere in the program.

Traditionally, Monte Carlo codes for solving neutron and gamma-ray transport problems have been separate codes. This has been due to the physics of the interaction processes and the corresponding cross-section information required. However, when multigroup cross sections are employed, the energy group to energy group transfers contain the cross sections for all processes. Also, for anisotropic scattering each group-to-group transfer has an associated angular distribution which is a weighted average over the various cross sections involved in the energy transfer process. Thus, these multigroup cross sections have the same format for both neutrons and gamma rays. In addition, the generation of secondary gamma rays may be considered as just another group-to-group transfer. Therefore, using multigroup cross sections, the logic of the random walk process (the process of being transported from one collision to another) is identical for both neutrons and gamma rays.

The use of multigroup cross sections in a Monte Carlo code means that the effort required to produce cross-section libraries is reduced. Coupled neutron gamma-ray cross-section libraries are available from the Radiation Shielding Information Center at Oak Ridge National Laboratory.

Cross sections may be read in either the DTF-IV format or ANISN ${ }^{3}$ and DOT4-6 format. The ANISN/DOT formatted cross-section file may be in either fixed or free form. The auxiliary information giving the number of groups, elements, Legendre coefficients, etc., is used to produce the necessary probability tables needed by the random walk module. The possible transport cases that can be treated are neutron only, gamma ray only, coupled neutron-gamma ray, gamma ray from a coupled set, and

"M. B. Emmett, "The MORSE Monte Carlo Radiation Transport Code System," ORNL-4972 (1975), ORNL-4972/R1 (1983), ORNL-4972/R2 (1984), Oak Ridge National Laboratory. 
fission, with all of the above options for either a forward or adjoint case and for isotropic or anisotropic scattering up to a $P_{16}$ expansion of the angular distribution. The option of storing the Legendre coefficients for use in a next-event estimator is also provided.

While the capabiliiies and versatility of MORSE are well documented for applications to a wide variety of problems in both the forward and adjoint mode of operation, in the MASH code system, MORSE is only used in adjoint mode for the analysis of the complex target description (vehicle) and to create the leakage tape for input to DRC.

\subsubsection{Theoretical Basis}

Monte Carlo like discrete ordinates solves the Boltzmann transport equation to obtain the estimates of the neutron or gamma-ray fluence. The general time-dependent integro-differential form of the Boltzmann transport equation, the derivation of which can be regarded as a bookkeeping process that sets the net storage of particles within a differential element of phase space (drdEdR) equal to the particle gains minus the particle losses in (drdEdn) and leads to the following useful form:

$$
\begin{aligned}
& \frac{1}{v} \frac{\partial}{\partial t} \Phi(\mathrm{r}, \mathrm{E}, \Omega, t)+\Omega \cdot \nabla \Phi(\mathrm{r}, \mathrm{E}, \Omega, \mathrm{t})+\Sigma_{t}(\mathrm{r}, \mathrm{E}) \Phi(\mathrm{r}, \mathrm{E}, \Omega, t) \\
& =S(\mathrm{r}, \mathrm{E}, \Omega, \mathrm{t})+\iint \mathrm{d} \mathrm{E}^{\prime} \mathrm{d} \Omega^{\prime} \Sigma_{s}\left(r, \mathrm{E}^{\prime} \rightarrow \mathrm{E}, \Omega^{\prime} \rightarrow \Omega\right) \Phi\left(\mathrm{r}, \mathrm{E}^{\prime} \Omega^{\prime} \mathrm{t}\right)
\end{aligned}
$$

where

$(r, E, \Omega, t)$ denotes the general seven-dimensional phase space, $r=$ position variable,

$E$ = the particle's kinetic energy, $v=$ the particle's speed corresponding to its kinetic energy $E$,

$\Omega=$ a unit vector which describes the particle's direction of motion,

$t=$ time variable,

$$
\Phi(r, E, \Omega, t)=
$$

the time dependent angular fluence, 


$$
\Phi(r, E, \Omega, t) d E d \Omega=
$$

the number of particles that cross a unit area normal to the $\Omega$ direction per unit time at the space point $r$ and time $t$ with energies in $d E$ about $E$ and with directions that lie within the differential solid angle $d \Omega$ about the unit vector $\Omega$,

$$
\frac{1}{v} \frac{\partial}{\partial t} \Phi(r, E, \Omega, t) d E d \Omega=
$$

net storage (gains minus losses) per unit volume and time at the space point $r$ and time $t$ of particles with energies in $\mathrm{dE}$ about $\mathrm{E}$ and with directions which lie in $\mathrm{d} \Omega$ about $\Omega$,

$$
\Omega \cdot \nabla \Phi(r, E, \Omega, t) \mathrm{dEd} \Omega=
$$

net convective loss per unit volume and time at the space point $r$ and time $t$ of particles with energies in $d E$ about $E$ and directions which lie in $\mathrm{d} \Omega$ about $\Omega$,

$$
\Sigma_{t}(r, E)=
$$

the total cross section at the space point $r$ for particles of energy $E$,

$$
\Sigma_{\mathfrak{t}}(r, E) \Phi(r, E, \Omega, t) d E d \Omega=
$$

collision loss per unit volume and time at the space point $r$ and time $t$ of particles with energies in $d E$ about $E$ and directions which lie in $\mathrm{d} \Omega$ about $\Omega$, 


$$
\Sigma_{s}\left(r, E^{\prime} \rightarrow E, \Omega^{\prime} \rightarrow \Omega\right) d E d \Omega=
$$

the differential scattering cross section which describes the probability per unit path that a particle with energy $E^{\prime}$ and an initial direction $\Omega^{\prime}$ undergoes a scattering collision at the space point $r$ which places it into a new direction that lies in $d \Omega$ about $\Omega$ with a new energy in $d E$ about $E$,

$$
\left[\iint \Sigma_{\vartheta}\left(r, E^{\prime} \rightarrow E, \Omega^{\prime} \rightarrow \Omega\right) \Phi(r, E, \Omega, t) d E^{\prime} d \Omega^{\prime}\right] d E d \Omega=
$$

inscattering gain per unit volume and time at the space point $r$ and time $t$ of particles with energies in dE about $E$ and directions which lie in $d \Omega$ about $\Omega$,

$$
S(r, E, \Omega, t) d E d \Omega=
$$

source particles emitted per unit volume and time at the space point $r$ and time $t$ of particles with energies in $\mathrm{dE}$ about $E$ and directions which 1 ie in $\mathrm{d} \Omega$ about $\Omega$.

An effect of interest such as biological dose, energy deposition, or particle fluence (denoted by $\lambda$ ) for a given problem can be expressed in terlis of the fluence field $\Phi(r, E, \Omega, t)$ and an appropriate response

function $P^{(}(r, E, \Omega, t)$ due to a unit angular fluence and is given by:

$$
\lambda=\iiint \int P^{\Phi}(r, E, \Omega, t) \Phi(r, E, \Omega, t) d r d E d \Omega d t .
$$

Consistent with the MORSE code, the energy dependence of Equation (6-1) is represented in terms of energy groups. A "group" form of Equation $(6-1)$ is obtained by integrating each term with respect to the energy variable over the energy interval $\triangle E_{g}$. MORSE solves the integral form of the Boltzmann transport equation. Section 4.10 of Reference 1 details the derivation of many integral forms of the Boltzmann transport equation and its adjoint. In this report only a summary of the two 
particular equations used for the forward and adjoint analys is will be given, and the interested user should consult Reference 1 for further detalls.

The solution of the forward integral transport equation by Monte Carlo generally involves a solution for $\chi_{g}(r, \Omega, t)$, referred to as the integral emergent particle density, i.e., the density of particles with phase space coordinates $(r, \Omega, t)$ leaving collisions. Quantities of interest are then obtained by summing the contributions over all collisions, and frequently over most of phase space. The "Integral Emergent Particle Density Equation" is written (in operator notation) as:

$\chi_{g}(r, \Omega, t)=S_{g}(r, \Omega, t)+C_{g^{\prime}-g}\left(r, \Omega^{\prime} \rightarrow \Omega\right) T_{g^{\prime}}\left(r^{\prime} \rightarrow r, \Omega^{\prime}\right) \chi_{g^{\prime}}\left(r^{\prime} \Omega^{\prime}, t^{\prime}\right) \quad(6-3)$

where

$$
\begin{aligned}
T_{g^{\prime}}\left(r^{\prime} \rightarrow r, \Omega^{\prime}\right) & =\text { the transport integral operator, and } \\
C_{g^{\prime}-g}\left(r, \Omega^{\prime} \rightarrow \Omega\right) & =\text { the collision integral operator. }
\end{aligned}
$$

The "Integral Emergent Particle Density Equation" was selected as the basis for the forward random walk since the source particles would be introduced according to the natural distribution.

The random walk based on the above Equation (6-3) would introduce a particle into the system according to the source function. The particle travels to the site of its first collision as determined by the transport kernel (operator). Its weight is modified by the nonabsorption probability and a new energy group and flight direction are selected from the collision kernel (operator). The transport and collision kernels are applied successively determining the particle's emergent phase space coordinates corresponding to the second, third, etc., collision sites until the random walk is terminated due to the reduction of the particle's weight below some cut-off value or because the particle escapes from that portion of phase space associated with a particular problem (for example, escape from the system, slowing down below and energy cut-off, or exceeding some arbitrarily specified age cut-off).

In some cases, particularly in MASH, it is of interest to solve the adjoint problem. This involves solving the "Adjoint IntegroDifferential Boltzmann Equation" given below. 
$-\frac{1}{v} \frac{\partial}{\partial t} \Phi^{*}(r, E, \Omega, t)-\Omega \cdot \nabla \Phi^{*}(r, E, \Omega, T)+\Sigma_{t}(r, E) \Phi^{*}(r, E, \Omega, t)$

$=S^{*}(r, E, \Omega, t)+\iint \Sigma_{s}\left(r, E \rightarrow E^{\prime}, \Omega \rightarrow \Omega^{\prime}\right) \Phi^{*}\left(r, E^{\prime}, \Omega^{\prime}, t\right) d E^{\prime} d \Omega^{\prime}$

where

$$
\Phi^{*}(r, E, \Omega, t)=
$$

the time dependent angular adjoint fluence,

and the other terms defined for the forward transport equation have equivalent meaning for the adjoint equation. The adjoint fluence is sometimes referred to as the particle's importance.

The solution of the adjoint integral transport equation by Monte Carlo generally involves a solution for $G_{0}(r, \Omega, t)$, referred to as the integral emergent adjuncton density, f.e., the densit.y of adjunctons with phase space coordinates $(r, \Omega, t)$ leaving collisions. Quantities of interest are then obtained by summing the contributions over all collisions, and frequently over most of phase space. The "Integral Emergent Adjuncton Density Equation" is written (in operator notation) as:

$G_{g}(r, \Omega, t)=P_{g}^{\phi}(r, \Omega, t)+C_{g^{\prime}-g}\left(r, \Omega^{\prime} \rightarrow \Omega\right) T_{g^{\prime}}\left(r^{\prime} \rightarrow r, \Omega^{\prime}\right) G_{g^{\prime}}\left(r^{\prime}, \Omega^{\prime}, t^{\prime}\right) \quad(6-5)$

where

$$
\begin{aligned}
T_{g^{\prime}}\left(r^{\prime} \rightarrow r, \Omega^{\prime}\right) & =\text { the transport integral operator, } \\
C_{g^{\prime}-g}\left(r, \Omega^{\prime} \rightarrow \Omega\right) & =\text { the collision integral operator, and } \\
P_{g}^{\Phi}(r, \Omega, t) & =\text { the source of adjunctons. }
\end{aligned}
$$

Equation (6-5) is almost identical to the forward equation given in Equation (6-3) which defines the forward emergent particle density and serves as the formal basis of the random walk in MORSE. Consequently, 
the same logic in MORSE can be used for both the forward and adjoint mode of solution. The Monte Carlo solution of Equation (6-5) will generate data from which the adjuncton fluence $\chi_{g}^{*}(r, \Omega, t)$ and other quantities of interest can be determined. The general use of $\chi_{a}^{*}(r, n, t)$ must take into account the reversal of directions between adjunctons and real particles, 1.e. $\Omega$ (adjoint) - $\Omega$ (forward). Further, if outward boundary crossings would be scored in the forward problem, the corresponding source adjunctons would be introduced in the inward direction. Likewise, adjunctons would be scored for entering a volume from which the source particles in the forward problem would be emitted. It should be noted that many sources and response functions are isotropic, and the problem of directional reversal need not be considered. The various relationships between the adjoint and forward quantities are derived in Section 4.10 of Reference 1.

\subsubsection{In-Group Energy Biasing}

One feature of MORSE alluded to in the introduction was that several types of optional importance sampling techniques are avallable to the user. In standard MORSE, these include splitting, Russian roulette, exponential transform, and source energy biasing to name a few. In the MASH version of MORSE, there is an additional biasing scheme referred to as in-group energy biasing. In-group energy biasing is a methodology developed by $W$. Scott of SAIC ${ }^{16-18}$ to adjust for irregularities observed in adjoint Monte Carlo analyses in thick media where the weights of adjoint particles could, in undergoing multiple scattering events, become very large, i.e., "the fat particle" problem. Such particles may be scored with a wide variety of weight values. This results in a very inefficient calculation with poor statistical precision, which is not improved by longer run times. The procedure was first implemented in the MIFT2 ${ }^{19}$ version of the VCS20,21 MORSE code by SAIC. After testing by both SAIC and ORNL, the procedure was installed into this version of MASH. A detalled description of the theorotical basis for in-group biasing is presented in Appendix B. The user should examine this section to understand the physics of the biasing technique. Here only a cursory explanation of how in-group biasing works is given.

In-group energy biasing is easy to use. Unlike other Monte Carlo blasing methods, in-group biasing does not require that the user set up and input, based on his judgment, arrays of data to improve the efficiency of the Monte Carlo calculation. This means that a person less skilled in adjoint Monte Carlo can often successfully use MASH or adjoint MORSE, since use of in-group blas requires only the setting of a switch in MASH. Success in the use of the other biasing methods generally requires considerable adjoint Monte Carlo experience.

The major objective of the in mgroup biasing scheme is to eliminate the instability in the variance for random walks. This is equivalent to eliminating the problem in which the weight of an adjoint particle can grow unbounded by many repeated in-group scatterings. 
The defining feature of in-group energy biasing is that for all hut the last group, a particle's weight only changes when it scatters is a new group. If the particle stays in the same group, its weight does not change. Thus, when an adjoint particle has a probability of nonabsorption (PNAB) greater than 1.0, its weight will not increase so long as it scatters in the same group. When it finally upscatters to a new group, its weight will be increased by a fixed amount that is a function only of the cross sections and not of how many times the particle scattered in the same group. With standard adjoint MORSE, such a particile would have its weight increased by PNAB each time that it scattered so that its final weight upon upscatter would depend upon how many in-group scatterings that it had.

\subsection{MORSE CODE STRUCTURE}

Iriput to MORSE is read in five separate modules: (1) random walk; (2) cross section; (3) user; (4) source; and (5) geometry. The random walk input is read in subroutines INPUT1 and INPUT2 and includes all variables needed for the random walk process. The cross-section input is read in cross-section module subroutines XSEC, JNPUT, and READSG. The parameters needed to set aside storage are read in XSEC, the mixing parameters are read in JNPUT, and the actual cross sections are read by READSG. Cross sections may be efther on card or on tape. Input information required for analys is of the histories is read by subroutine SCORIN of the analysis package which is called from INPUT2. Since the source varies from problem to problem, input may also be read in by subroutine SORIN for the definition of the source. The geometry input is read by subroutine JOMIN. Any additional input required by the user may be read in by subroutine INSCOR which is called from SCORIN.

In general, output of input parameters occurs in the same routine in which the input was read. In addition, there are two routines (OUTPT and OUTPT2) for the output of results of the random walk process. output of analysis results is generally performed in subroutine NRUN, but may also be done in a user-written routil. ENDRUN which is called by NRUN.

The input section takes care of setting up all variables needed in the transport process. It should be noted that initial calculations by the cross-section module stem from subroutine calls in XSEC. The analys is portion of the code is interfaced with MORSE through BANKR with several uses made of cross-section routines in making estimates of the quantity of interest. With the exception of output from the random walk process, the rest of the code consists of subroutine calls by MORSE. The geometry module is interfaced through GOMST, and the source is interfaced through MSOUR. The diagnostic module is independent and any part of it may be executed from any routine.

The diagnostic module provides an easy means of printing out, in useful form, the information in the variaus labelled comrnons and any part of 
blank common. The IBM-360 version also has the following features: special routine is provided for printing out the particle bank; by loading parts of core with a Junk word, the diagnostic package can determine which variables have been used; a "repeating line" feature is also included.

The usual geometry module for MORSE is the combinatorial geometry package $(C G)^{7}$. However, the MORSF code in MASH contains the GIFT5 geometry package developed at BRL ${ }^{8-11}$. The GIFT5 geometry package is simflar to CG; however, it contains a significant increase in the number of primitive bodies or "solids" and some added advantages relative to Monte Carlo tracking in complex geometries. The GIFT5 geometry package was first installed into the MIFT2 ${ }^{19}$ code by SAIC. After considerable testing, the package was installed into this version of MORSE for the MASH code system.

An albedo scattering may be forced to occur at every entry into a specifled medium. A sample subroutine is provided for specular reflection and a subroutine call is provided (ALBIN, called from XSEC) for reading and storing albedo data of any degree of complexity. Thus transport of particles may be carried out in parts of the problem and an albedo scattering treated for other parts of the problem.

Time dependence is included by keeping track of the chronolngical age of the particle. For neutrons the age is incremented by the time needed to travel the distance between collisions if it traveled at a velocity corresponding to the average energy of the group. Provision is made for inputting a thermal group velocity separately. Nonrelativistic mechanics are assumed. The age of secondary gamma rays is determined from the neutron age at the collision site and is incremented by determining the time required to travel between collisions at the speed of light. For fission problems the age of the parent is given to the daughters at birth.

There are several types of importance sampling techniques included in the code. The Russian roulette and splitting logic of $05 R^{12}$ is an option in MORSE. Also, the exponential transform is provided with parameters allowed as a function of energy and importance region. Source energy blasing is an option as well as energy biasing at each collision. In fission problems the fission weights may be renormalized as a function of an estimate of $K_{\text {eff }}$ so that the number of histories per generation remain approximately constant. If desired, all importance sampling may be turned off.

Some other general features include the ability to run problems without the use of magnetic tapes, the ability to terminate a job internally after a set elapsed CPU time and obtain the output based on the number of histories treated up to that time, batch processing for the purpose of determining statistics for groups of particles, and a repeat run feature so that results for a time-dependent fission problem may be obtained with statistical estimates. The output of numerous counters permits one to obtain an insight into the physics of the problem. 


\subsubsection{Random Halk Module}

The basic random walk process of choosing a source particle and then following it through its history of events is governed by the routines in this module of MORSE. A given problem is performed by following a number of batches of particles which then constitute a run. Multiple runs are also permitted. The baich process feature is used so that statistical variations between groups of particles can be determined. Thus, a batch of source particles is generated and stored in the bank. The random walk for this batch of particles is determined by picking one particle out of the bank and transporting it from collision to collision, splitting it into two particles, killing by Russian roulette, and generating secondary particles (either gamma rays or fission neutrons) and stroming them in the bank for future processing. Termination of a history occurs when a particle leaks from the system, reaches an energy cutoff, reaches an age limit, or is killed by Russian roulette.

The random walk module performs the necessary bookkeeping for the bank and the transportation and generation of new particles and relays this information to the analysis module for estimation of the desired quantities. Use is made of the cross-section module and the geometry module during the random walk process and the input-output routines for the reading and printing of pertinent information about the problem.

In this module the main program is used to set aside the storage required in blank common and to pass this information to subroutine MORSE which is the executive routine for the random walk process. After performing the necessary input operations and setting up storage requirements, the walk process consists of three nested loops: one for runs, ane for batches, and the inner-most is for particles. After each termination of the batch loop, some bookkeeping is required before the generation of a new batch of source particles. After the termination of a run, a summary of the particle terminations, scattering counters, and secondary production counters are output, as well as the results of Russian roulette and splitting for each group and importance region.

There are only two main labelled commons (APOLLO and NUTRON) in the random walk routines. Tables 4.4 and 4.5 in Reference 1 1ist the definitions of the variables in these two commons. The parameters stored in these commons are labelled by "current" and "previous" descriptors which refer to values of parameters leaving the current and previous event sites, respectively (WTBC. is the exception, being the weight entering the current event site). Also note that "event" includes boundary crossings, albedo collisions, etc., as well as real collisions. The user is afforded access to these parameters through several different calls to subroutine BANKR throughout the random walk. The calling routine and location in the random walk where BANKR is called is given in Table 6-1. The user can then interact with the random walk through additional programming only in subroutine BANKR and not need to work with the random walk rout ines themselves. The user also has an input option to write a collision tape for further analysis. 
Table 6-1. BANKR Arguments.

\begin{tabular}{|c|c|c|}
\hline $\begin{array}{l}\text { BANKR } \\
\text { Argument }\end{array}$ & $\begin{array}{l}\text { Called } \\
\text { From }\end{array}$ & $\begin{array}{l}\text { Location of BANKR } \\
\text { Call in Random Walk }\end{array}$ \\
\hline-1 & MORSE & $\begin{array}{l}\text { After call to INPUT - to set parameters } \\
\text { for new problem. }\end{array}$ \\
\hline-2 & MORSE & $\begin{array}{l}\text { At the beginning of each batch of NSTRT } \\
\text { particles. }\end{array}$ \\
\hline-3 & MORSE & At the end of each batch of NSTRT particles. \\
\hline-4 & MORSE & $\begin{array}{l}\text { At the end of each set of NITS batches } \\
\text { a new problem is about to begin. }\end{array}$ \\
\hline 1 & MSOUR & At a source event. \\
\hline 2. & TESTW & After a splitting has occurred. \\
\hline 3 & FPROB & After a fission has occurred, \\
\hline 4 & GSTORE & $\begin{array}{l}\text { After a secondary particle has been } \\
\text { generated. }\end{array}$ \\
\hline 5 & MORSE & $\begin{array}{l}\text { After a real collision has occurred } \\
\text { post-collision parameters are available. }\end{array}$ \\
\hline 6 & MORSE & $\begin{array}{l}\text { After an albedo collision has occurred- } \\
\text { post-collision parameters are available. }\end{array}$ \\
\hline 7 & NXTCOL & $\begin{array}{l}\text { After a boundary crossing occurs (the } \\
\text { track has encountered a new geometry medium } \\
\text { other than the albedo or void media). }\end{array}$ \\
\hline 8 & NXTCOL & $\begin{array}{l}\text { After an escape occurs (the geometry has } \\
\text { encountered medium zero). }\end{array}$ \\
\hline 9 & MORSE & $\begin{array}{l}\text { After the post-collision energy group exceeds } \\
\text { the maximum desired. }\end{array}$ \\
\hline 10 & MORSE & $\begin{array}{l}\text { After the maximum chronological age has } \\
\text { been exceeded. }\end{array}$ \\
\hline 11 & TESTW & After a Russian roulette kill occurs. \\
\hline 12 & TESTW & After a Russian roulette survival occurs. \\
\hline 13 & GSTORE & $\begin{array}{l}\text { After a secondary particle has been generatec } \\
\text { but no room in the bank is available. }\end{array}$ \\
\hline
\end{tabular}


The input allows the user to specify which of the thirteen positive BANKR Arguments to put on the tape as well as which of the 36 parameters listed in Table 6-2. These features of MORSE prove useful in applying the code to new and different problems.

Table 6-2. Variables That May be Written on Collision Tape (NBIND).

\begin{tabular}{|c|c|c|c|}
\hline $\begin{array}{l}\text { Variable } \\
\text { Number }\end{array}$ & $\begin{array}{c}\text { Variable } \\
\text { Name }\end{array}$ & $\begin{array}{c}\text { Variable } \\
\text { Number }\end{array}$ & $\begin{array}{c}\text { Variable } \\
\text { Name }\end{array}$ \\
\hline 1 & NCOLL & 19 & WTBC \\
\hline 2 & NAME & 20 & ETAUSD \\
\hline 3 & $I G$ & 21 & ETA \\
\hline 4 & $U$ & 22 & AGEC \\
\hline 5 & V & 23 & OLDAGE \\
\hline 6 & $W$ & 24 & NREG \\
\hline 7 & $x$ & 25 & NMED \\
\hline 8 & $Y$ & 26 & NAMEX \\
\hline 9 & $z$ & 27 & WATEF \\
\hline 10 & WATE & 28 & BLZNT \\
\hline 11 & IGO & 29 & BLZON \\
\hline 12 & UOLD & 30 & $\operatorname{VEL}(I G)$ \\
\hline 13 & VOLD & 31 & VEL (IGO) \\
\hline 14 & WOLD & 32 & TSIG \\
\hline 15 & $X O L D$ & 33 & PNAB \\
\hline 16 & YOLD & 34 & NXTRA \\
\hline 17 & ZOLD & 35 & EXTRA1 \\
\hline 18 & OLDWT & 36 & EXTRA2 \\
\hline
\end{tabular}




\subsubsection{Muitigroup Cross-Section Moduln}

The function of this module in the multigroup Monte Carlo code is to read ANISN-type 3 cross sections for media or elements, mix several elements together to obtain media cross sections, determine group-togroup transfer probabilities and determine the probabilities and angles of scattering for each group-to-group transfer. All variables are flexibly dimensioned and are part of blank common.

Various types of cross sections can be processed by the cross-section module. Neutron only, gamina ray only, neutron gamma-ray coupled, or gamma rays from coupled neutron gamma-ray cross sections can be processed for either a forward or an adjoint problem with or without fission. The Legendre coefficients are stored if a next-event estimator is to be used. Table 6-3 indicates the relationship between the various input parameters in the random walk module and the cross section module. The user must be careful that these particular parameters match.

The cross sections are read for one coefficient and one element into a buffer area. Then these cross sections are decomposed into total, fission, and downscatter matrix and stored in temporary arrays so that they may be mixed to form media cross sections. The total and fission cross sections are stored only once for an element, but the downscatter matrix is stored for each coefficient.

The cross sections are transposed as stored if an adjoint problem is being solved. Two major reordering steps are carried out to produce an adjoint cross-section library:

1. The inscatter matrix is transposed, i.e. the table position associated with group $g$ describing scattering from $\mathrm{g}^{\prime}$ t.o $\mathrm{g}$ is changed to describe scattering from $g$ to $\mathrm{g}^{\prime}$.

2. The ordering of the groups is reversed, i.e. data for group IGM appear first in the output file, followed by IGM-1, etc.

After all cross sections are stored, the contribution of each element to the cross section for the media is determined. Also at this time the sum of the downscatter vector for each group is determined for the future calculation of the nonabsorption probability; the gammaproduction cross section is also determined by summing the transfers to the gamma groups. After the cross sections for the medium have been determined, the nonabsorption probability, fission probability, and gamma-production probabilities are formed by dividing by the total cross section. The downscatter matrix is converted to a probability table by dividing by the scattering cross section. 
Table 6-3. Examples of the Relationship Between Random Walk and Cross Section Module Group Input Parameters.

Case A - Neutron Only Cross Sections (22 groups)

Case B - Gamma-Ray Only Cross Sections (18 groups)

Case C - Neutron Gamma-Ray Coupled Cross Sections (22-18 groups)

\begin{tabular}{|c|c|c|c|c|c|c|}
\hline \multicolumn{7}{|c|}{ Problem Type } \\
\hline $\begin{array}{l}\text { Input } \\
\text { Variable }\end{array}$ & $\begin{array}{l}\text { Case A } \\
\text { Top 14 } \\
\text { groups }\end{array}$ & $\begin{array}{l}\text { Case B } \\
\text { Top } 17 \\
\text { groups }\end{array}$ & $\begin{array}{c}\text { Case C } \\
n \text { only } \\
\text { Top } 14 \text { gps }\end{array}$ & $\begin{array}{c}\text { Case C } \\
\gamma \text { only } \\
\text { Top } 17 \text { gps }\end{array}$ & $\begin{array}{c}\text { Case C } \\
n+\gamma \\
14 n+17 \gamma\end{array}$ & \\
\hline $\begin{array}{l}\text { MORSE } \\
\text { Input }\end{array}$ & & & & & & \\
\hline NGPQTN & 14 & 0 & 14 & 0 & 14 & \\
\hline NGPQTG & 0 & 17 & 0 & 17 & 17 & CARD B \\
\hline NMGP & 22 & 18 & 22 & 18 & 40 & Variables \\
\hline NMTG & 22 & 18 & 22 & 18 & 40 & \\
\hline NGP & 22 & 18 & 22 & 0 & $22^{a}$ & CARD XB \\
\hline$N G G$ & 0 & 0 & 0 & 18 & 18 & - Variables \\
\hline INGP & 22 & 18 & 40 & 40 & 40 & \\
\hline
\end{tabular}

aMust be equal to total number of neutron groups in the data; otherwise it picks up the gammas from the wrong location.

Note: For Cross sections with full downscatter, NDS = NGP, $N D S G=N G G$, INDS = INGP, and ITBL = number of downscatters + number of upscatters +3 . Usually, $I S G G=$ number of upscatters +4 ; i.e., NUS +4 . 
A simple three group example showing the relationship between a forward and adjoint cross-section set is shown below. In this example, there is no upscatter, one activation cross section, and the cross-section input parameters (defined in Sectfor, 6.3.3) are IHT $=4$, ISGG $=5$, and ITBL = 7.

\begin{tabular}{|c|c|}
\hline Direct & Adjoint \\
\hline $\begin{array}{r}\sigma^{\mathrm{F}}(1) \\
\sigma^{A}(1) \\
\nu \sigma^{\mathrm{F}}(1) \\
\sigma^{\top}(1)\end{array}$ & $\begin{array}{c}\sigma^{F}(3) \\
\sigma^{\wedge}(3) \\
\nu \sigma^{F}(3) \\
\sigma^{\top}(3)\end{array}$ \\
\hline $\begin{array}{l}\sigma(1+1) \\
0 \\
0\end{array}$ & $\begin{array}{l}\sigma(3+3) \\
0 \\
0\end{array}$ \\
\hline $\begin{array}{c}\sigma^{F}(2) \\
\sigma^{A}(2) \\
\nu \sigma^{F}(2) \\
\sigma^{\top}(2)\end{array}$ & $\begin{array}{r}\sigma^{F}(2) \\
\sigma^{A}(2) \\
\nu \sigma^{F}(2) \\
\sigma^{\top}(2)\end{array}$ \\
\hline $\begin{array}{l}\sigma(2+2) \\
\sigma(1-2) \\
0\end{array}$ & $\begin{array}{l}\sigma(2+2) \\
\sigma(2+3) \\
0\end{array}$ \\
\hline $\begin{array}{r}\sigma^{F}(3) \\
\sigma^{A}(3) \\
\nu \sigma^{F}(3) \\
\sigma^{\top}(3)\end{array}$ & $\begin{array}{l}\sigma^{F}(1) \\
\sigma^{\wedge}(1) \\
\nu \sigma^{F}(1) \\
\sigma^{\top}(1)\end{array}$ \\
\hline $\begin{array}{l}\sigma(3 \rightarrow 3) \\
\sigma(2 \rightarrow 3) \\
\sigma(1 \rightarrow 3)\end{array}$ & $\begin{array}{l}\sigma(1+1) \\
\sigma(1+2) \\
\sigma(1+3)\end{array}$ \\
\hline
\end{tabular}

The Legendre coefficients for each group-to-group transfer are converted to angles and probabilities of scattering at those angles by the use of a generalized Gaussian quadrature using the angular distribution as a weight function. That is,

$$
\int_{-1}^{+1} f(\mu) \omega(\mu) d=\sum_{i=1}^{n} f\left(\mu_{i}\right) \omega_{i}
$$


where

$f(\mu)$ is any polynomial of order $2 n-1$ or less,

$\omega(\mu)$ is the angular distribution for $\mu$, the cosine of the scattering angle,

$\mu_{i}$ is a set of discrete cosines, and

$\omega_{i}$ is the probability of the corresponding cosine.

Thus, a set of $\mu_{i}^{\prime}$ s and $\omega_{i}$ 's that satisfy the equation must be found. To do this, a set of polynomials, $Q_{1}$, which is orthogonal with respect to the angular distribution, is defined such that

$$
\int_{-1}^{+1} Q_{i}(\mu) Q_{j}(\mu) \omega(\mu) d \mu=\delta_{i j} N_{i}
$$

where $N_{i}$ is a normalization constant.

The moments of the angular distribution $M_{i}, i=1,2 n-1$, determine the orthogonal polynomials, $Q_{i}, i=1, n$. The desired cosines, $\mu_{i}$, are given by the roots of $Q_{n}$,

$$
Q_{n}\left(\mu_{i}\right)=0
$$

and the corresponding probabilities are

$$
\omega_{i}=\left(\sum_{k=1}^{n-1} \frac{Q_{k}^{2}\left(\mu_{i}\right)}{N_{i}}\right)^{-1}
$$

In the process of deriving the orthogonal polynomials, some restrictions on the moments of the angular distribution are obtained. These restrictions arise if both the original distribution and the derived point distribution are to be everywhere non-negative. The restrictions are: 
1) $N_{i}>0$ for $i=1, n$.

This restriction may be written in terms of the determinant of the determinant of the moments:

$$
\left|\begin{array}{ccccc}
1 & M_{1} & M_{2} & \cdots & M_{i} \\
M_{1} & M_{2} & M_{3} & \cdots & M_{i+1} \\
\vdots & & & & \\
\dot{M}_{i} & M_{i+1} & M_{i+2} & \cdots & M_{2 i}
\end{array}\right|>0
$$

2) The roots of $Q_{i}(\mu)$ must all lie in the interval

$$
-1 \leq \mu_{i} \leq 1
$$

It must be emphasized that the restriction arising from the original distribution being everywhere positive (or zero) does not restrict the truncated expansion of the distribution to be everywhere positive. That is, moments from a truncated distribution that is not necessarily everywhere positive are used to derive a discrete distribution with positive probabilities.

Other characteristics of this representation are that the information is compact, the angles are clustered where the angular distribution is peaked, and because of the restrictions, cross sections that have blunders in them are rejected because they produce angles outside the range of -1 to +1 .

An option on input makes it possible to write a tape containing the processed cross sections and all variables from Common LOCSIG needed during the random walk process. In starting a new case, the normal cross-section input, except for the cross sections and the mixing cards, is required. Both the information from input and from tape are printed for compatibility checks. Note that if an adjoint problem is being solved, the input information and the information for tape will not be identical.

One of the more important options provided for in this package is the ability to treat upscattering. Thus, multigroup cross sections with many thermal groups may be utilized. (In a crude way photoneutron production may be treated as an upscatter process from a gamma-ray group to a neutron group.) Also, the capability of using cross sectioris with partial downscatter is present. 
The downscatter array made it possible to have a feature that is helpful in reducing cross-section storage requirements for neutron problems involving materials other than hydrogen when a cross-section tape is used. For non-hydrogeneous media there is very seldom complete downscatter to thermal energy, so a rout ine was added to search the $P_{0}$ array to determine the minimum downscattered energy group for any element and for each group. The zero cross sections for downscatters below this energy group are not stored, and thus the storage requirements are reduced.

Another important feature of this module is the ability to use a point cross-section representation for the total, scattering, and fission cross sections. Either an $05 R^{12}$ or an $06 R^{13}, 14$ (variabie supergroup boundaries) format tape (CODE7) may be used over a specified energy range. The energy of a particle is chosen uniformly within an energy group for the purpose of selecting the particle track lengths or calculating a nonabsorption probability. For gamma rays or for neutron energies outside the specified range, the total cross section from the multigroup cross sections is used. (Point, total, and scattering cross sections for the adjoint case can also be used.) For some applications, detailed nonabsorption probabilities and fission probabilities may also be required. The logic was included in the new module for the crosssection manipulation required for the use of the point cross sections. Up to 16 point cross-section media with up to 100 supergroups are allowed.

One major change in philosophy has been made for the case in which point cross sections are used. The modular framework of MORSE is broken in this case in that Subroutine GTSCT uses the energies corresponding to the multigroup boundaries in order to set up an array of indices. It is assumed by GTSCT that the energies exist in the first NTG cells of Blank Common.

An executive program $X C_{C H E R} 15$ may be used in conjunction with this cross-section module independent of MORSE for the purpose of checking and editing multigroup cross sections. Also, this executive program may be used to generate a processed cross-section tape independent of a MORSE calculation.

\subsubsection{The GIFT5 Geometry Package}

GIFT5 geometry is similar to the older MORSE combinatorial geometry in that specified geometric shapes (spheres, cylinders, parallelepipeds, etc.) may be combined to describe complex geometry configurations. As with MORSE combinatorial geometry, a GIFT5 geometry file is comprised largely of a "body" or "solids" table followed by a "zone" or "region" table in which the bodies are combined by $(+),(-)$, or (OR) operators. In GIFT geometry, however, "bodies" (MORSE terminology) are called "solids" and "zones" (MORSE terminology) are called "regions". In the GIFT5 graphics code there is no equivalent to the "regions" as used in MORSE. In MASH, where the term "region" could have a double meaning, 
the MORSE equivalent of "region" is called "importance region" to minimize any confusion. In this section the GIFT terminology, i.e., solids and regions, equivalent to bodies and zones in MORSE, will be used.

Installation of the GIFT5 geometry in MASH was greatly facilitated by BRL's initial installation of GIFT into MORSE to derive MIFT, in which they (BRL) had developed the necessary interfaces between MORSE and the GIFT geometry, and SAIC's installation of GIFT5 into MIFT2. SAIC's Installation of GIFT5 in MIFT consisted largely of replacing GIFT geometry routines with GIFT5 routines. The subsequent installation of GIFT5 into the MORSE code in MASH involved mainly a transfer of the MIFT2 logic into the MORSE logic with a few changes necessary to accommodate the minor differences in the two codes.

MASH interfaces with the GIFT5 geometry routines in four major locations: INPUT1 for processing of the input geometry data, LOOKZ to identify the region (zone) for starting particles, and GOMST and NXTCOL to track particies during the random walk. INPUTI calls GIFTM, which sets a few switches and calls GENI.

GENI calls SOLIN, which processes the solids (bodies) table, REGIN, which processes the regions (zones) table, IDIN, which processes the identification table, and RPPIN which processes any boundary RPP information. SOLIN calls ALBERT, which processes ARB input data, and ARIN, which processes ARS input data. Severay other routines, including DOT, CROSS, EQUIV, UNITV, UN2, ARANG2, CPOINT, and PLANE, are called by these routines to perform miscellaneous operations such as vector dot products, cross products and sorts. GENI and routines called by GENI analyze the geometry for obvious errors, such as XMAX smaller than XMIN for an RPP or non-coplanar points on an ARB, and print error messages when such errors are found. GENI then stores the geometry data with XMAX, XMIN, YMAX, YMIN, ZMAX, and ZMIN bounding values for each region (zone) for use in subsequent analyses.

LOOKZ calls RAY to identify the starting region location for starting particles. RAY calculates the region numbers and intercept distances for all regions on a line-of-sight from the particle start point to exit from the defined geometry.

GOMST and NXTCOL determine the particle coordinates at the next collision site by calculating the expected flight path distance in mean free paths, then tracking the particle through the different regions and materials of the geometry until the mean free path is "expended" or until the particle escapes from the geometry.

First, in NXTCOL, the mean free path for the particle path is calculated with a call to GETETA, a flag, MARK, is set to 1 , and the call to GOMST is made. In GOMST the distance DISTO for the particle mean free path in the current medium is calculated. Then the particle starting

coordinates and direction cosines, $X B$ and $W B$ are stored. $A$ call to the GIFT5 REGION subroutine is made to determine the distance along the 
particle line-of-sight to exit from the current region, and a test is made to determine whether the particle does, in fact, extt the regton. If it does exit the region, a call to GIFTS subroutine RAY is made to calculate intercepts of the particle line-of-sight, MARK is set to 0 (indicating a regton boundary crossing) and the distance traveled is cumulated. A test on MARK is then made. If MARK is equal to 1, the collision site is in the same region as before and GOMST and NXTCOL are exited. If MARK is zero, a boundary crussing occurred and tests are made first for the extended path scoring option (If "Yes", a score is made), then for particle escape (if "Yes", an escape is scored). If there is no escape, the flight distance in the new medium is calculated.

As seen in the foregoing paragraph, particle tracking uses calls to GIFT5 subroutines RAY and REGION. Given a particle ine-of-sight defined by a starting point and a direction, REGION calculates the distance along the ray to all entry and exit points for a specifled region. Because a region can have complicated shapes, it is possible for a ray to have several entry and exit points. While REGION calculates entry and exit points for only one specified region, RAY does so for all regions or the linemof-sight.

The particle tracking logic with GIFT5 routines is considerably different from the tracking logic in combinatorial geometry MORSE-CG. In MORSE, each time a particle exits a region, MORSE-CG searches an "experience" table to determine candidate neighboring regions which the particle might be entering. If analysis shows that the particie is not entering any of the regions in the experience table, or if there are no candidate regions in the table, all regions are checked sequentially until the new region is found. When the new region is found, it is added to the experience table.

MASH, during the processing of the input geometry data, performs three steps which facilitate later particle tracking. First, bounding $X, Y$, and $Z$ values are found for each region, and the resulting XMIN, XMAX, YMIN, YMAX, ZMIN, ZMAX are stored in the MASTER/ASTER array (the Region RPP Table discussed in Appendix C). Second, these XMIN, XMAX, YMIN, YMAX, etc., data sets are each sorted, high value first, carrying along the region number, and stored in the MASTER/ASTER array. Third, the bounding RPP for the entire problem geometry is defined and stored.

During particle tracking, MASH first derives the RPP which bounds the particle flight path line-of-sight (ray) from its starting point to escape from the defined geometry. Next, it compares the XMIN, XMAX, ..., ZMAX data for this bounding RPP to the region min-max table to derive the shortest list of candidate regions that the ray might intercept. Then for each region on the 1ist, it compares the region $\mathrm{m} / \mathrm{n} / \mathrm{max}$ values with the ray RPP, eliminating any region RPP that doesn't intersect the ray RPP. Next a "fly by" test is performed, then a check of whether the ray actually penetrates the region bounding RPP. Last, if all of the above tests are passed, a check is made, via a call to REGION, to determine whether the ray penetrates the region. 
The above procedure is performed in RAY for all candidate regions. When it is found that the ray does penetrate a region, the RIN and ROUT distances (distances along the ray to region entry and exit, respectiveiy) are added to a list (RAYIN and RAYOUT), in common RAYINT, along with the region number, IREGON, and a count of the number of entries on the 1ist, NINT. Last, the RAYIN/RAYOUT ilst is checked for overlapping regtons. If overlapping regtons are found, an error message is written.

In initial testing of MIFT2 after installation of the GIFT5 geometry routines, SAIC found that particle tracking resulted in undetected errors whenever there were undefined regions in the geometry. While vehicle geometries for VCS analysis have not intentionally been developed with undefined regions, undefined regions could result from errors in developing the complex vehicle geometry. The result of undefined regions in MIFT, before corrections were implemented, was that particles got lost in the sense that their $X, Y, Z$ coordinates did not correspond to the reglons. In the analys is of a test case vehicle, for example, in which the interior air region was intentionally left undefined, the effect was to 111 the interior with the steel boundary matertal.

In MORSE-CG, entry of particles into an undefined region also results in the particle getting lost. MORSE-CG, however, kills the particles by setting the weight (WATE) to zero and picks up a new particle after writing a message.

The error in the treatment of undefined regions was corrected (by SAIC) in MIFT2 by the addition of subroutine RAYCHK, which is called by NXTCOL immediately after the call to subroutine RAY. Forturiately the RAYIN and RAYOUT arrays from RAY contain all the information necessary to reconstruct the ray penetration parameters for the undefined region. When a particle encounters an undefined region, RAYCHK reconstructs the ray parameters and assigns a region number of -99 and a material number of 1000 (void) to the undefined region. RAYCHK then prints a message for each of the first 25 undefined region encounters, giving the region numbers of adjacent regions to help locate the problem area. The RAYCHK subroutine was included in the installation of GIFT5 into the MASH version of MORSE.

There is, of course, a danger of analyzing an erroneous geometry with a code that treats geometric undefined regions or overlapping regions or both. The best solution appears to have the code print a limited number (e.g., 20) of messages indicating that it has found undefined or overlapping regions and then to continue the calculation. Users could a)ter the logic to terminate after the message, if they wish. In any event, the user must be responsible to generate the correct code input.

The bulk of the data used in GIFT5 is stored in a single large array in COMMON GIFTCM. This array has various names in different routines, but usual Ty appears as MASTER or ASTER. MASTER and ASTER are equivalenced for storage of both real and integer data. This array is currently 
dimensioned to 5,000 in the GIFT5 geometry routines and subroutine GOMST of the MORSE random walk interface routines. The value of 5,000 defines the storage used throughout the code, and a user who wishes to redimension this value either upward or downward needs to change this common size throughout the code. This size is sufficient for a medium size problem. SAIC used a MASTER/ASTER array size of 15,000 for the analysis of some very large problems. It was, for example, suffictent for the analysis of the MPGS (Mobile Position Gun System) test case, which consists of 281 solids and 233 regions, and the Long Is land Barracks, whtch conststed of 308 solids and 311 regions.

There are 12 sets of data stored in the MASTER/ASTER array as follows:

1. Solid pointers, one word per solid plus 1, integer data. Two values are packed in each word, ITYPE in the right 15 bits and LOCDA in the next 16 bits. ITYPE is the solid type, and LOCDA is the beginning location at which spectfic data for the solid are stored.

2. Solid data, as required to describe the solids, real.

3. Region pointers, integer, one word for each region. Two values are packed in each word, NUMDES in the right 15 bits and LOCDES in the next 16 bits. NUMDES is the number of solids in the region description and LOCDES is the beginning location at which the region descriptors for the specific region are stored.

4. Region descriptors, integer, one word per descriptor, NUMDES descriptors per region. Two numbers are packed in each word, the solid number in the right 15 bits and an operator code number in the 1 eft 16 bits. The operator code is 1 for $\mathrm{ORt}, 2$ for OR-, 3 for $R G+, 4$ for $R G-, 5$ for + and 6 for -.

5. Region circumscribing RPP data (XMIN, XMAX, YMIN, YMAX, ZMIN, ZMAX limits for each region). Six words of real data are stored for each region (zone).

6. Ordered region numbers, integer, sorted into the order large to smal1, first based on XMIN values for circumscribing RPP, then an XMAX, on YMIN, on YMAX, on ZMIN, and last on ZMAX. The total size of this part of' the MASTER/ASTER array is six times the number of regions, beginning at storage location LREGON. 
7. XMIN, XMAX, YMIN, YMAX, ZMIN, and ZMAX for RPP that circumscribes the overall defined geometry, Six words, real, beginning at storage location LENRPP.

8. Identification table, integer, one word for each region. Two values are packed in each word, ISPACE in the right 15 bits and ITEM in the next 16 bits. ISPACE is the space code number (columns 11-15 of the input identification table) and ITEM is the component code number (columns 6-10 of the input identification table).

9. Region ray table, integer, one word per region. Three values are packed in each word. The right 15 bits stores the ray number, the next 10 bits stores the location where the ray end is stored and the next 10 bits stores the location where the ray start is stored.

10. Ray intersection table storing distance (RIN) from start of ray to first intersection of solid. Real, not packed. One word per solid.

11. Ray intersection table storing distance (ROUT) from start of ray to last intersection of solid. Real, not packed. One word per solid.

12. Surfaces and ray count, one word per solid. Three integers packed per word, the ray number is the right 14 bits, the surface number at ray exit in the next 9 bits, and the surface number at ray entry in the next 9 bits.

\subsubsection{Modiffcations to MORSE for In-Group Biasing}

Several routines in the MORSE module of MASH were modified to incorporate the changes to add the in-group biasing option. Subroutines COLISN, GPROB, GSTORE, GTIOUT, and INPUT1 were modified, and a new subroutine GETLOC was added. Also, invoking the in-group bias option changes the definition of the quantity GWLO from the probability of secondary particle production to the fraction of the probability of secondary particle production specifled by the cross sections (natural probability).

Subroutine INPUT1 was modified to read the in-group biasing switch INGB as the eighth parameter on CARD I. Variable INGB turns on the in-group when equal to 1 ; otherwise, the option is off. INGB is in COMMON/IGBABS/. Subrout ine COLISN handles scattering probabilities $P_{\text {if }}$ which give the probability of scattering to group $j$ when a scattering 
has occurred in group $i$. Originally the particle weight WATE was multiplied by $P N A B_{i}$, the non-absorption probability for group $i$ where $i$ was the group coming into the collision; and then a random number was compared with the probabilities $P_{i j}$ to select the outgoing group. This sampling scheme was modified for in-group biasing. The same codinc is used except that the random number is compared to $F * P_{i j}$, where $F$ is a bias factor as follows:

$$
\begin{aligned}
& \text { if } i=j=1 \text {, then } F=1.0 \\
& \text { if } i=j \neq 1 \text {, then } F=\text { PNAB }_{i} \\
& \text { if } i \neq j, \quad \text { then } F=\frac{1-P_{i i} \text { PNAB }_{i}}{1-P_{i j}}
\end{aligned}
$$

After the outgoing group has been sampled, the weight is corrected by

$$
\text { WATE }=\text { WATE * PNAB/F. }
$$

The method implemented correctly treats in-group biasing with or without energy biasing and includes logic for splitting when WATE exceeds WTMAX. The splitting game actually resamples the upscatter distribution for each splitting secondary particle. In this way each secondary is independent of the others so that the probability transition is more completely represented than in the original scheme where all splitting secondaries had the same energy group and the same direction cosines.

\subsubsection{Modifications to MORSE-CG for the MASH Code System}

The current version of MASH contains the newest MORSE-CG routines. The main differences between this and the previous VCS have been explained elsewhere in this report. The routines that are peculiar to MASH have been rewitten with the following characteristics:

1. All arrays are now variably dimensioned. This required the addition of several arrays of data to blank common. Table 6-4 defines these variables.

2. All tape unit numbers are variables.

3. Common structure is revised, having fewer commons with more variables in each. A labelled common IVCS was added for this purpose, and it is defined in Table 6-5. 
4. Subroutine structure is slightly different. One of the changes was to split subroutine BANKR into two routines, BANKR and NBANKR.

5. Problems terminate in an orderly manner. If a job fails because the particle name exceeds the maximum storage allowed (IMAXN), data generated for all but the last batch is copied from the scratch unit to the user's save unit. A message is printed by the new routine EREND to notify the user of this and of how many batches were actually completed.

6. A new MORSE leakage tape format is used in order to provide more definitive information on the case that was run.

Table 6-4. Location of MASH Arrays in BLANK COMMON.

\begin{tabular}{|c|c|c|}
\hline $\begin{array}{l}\text { Mnemonic } \\
\text { variable } \\
\text { name }\end{array}$ & $\begin{array}{l}\text { Location in } \\
\text { blank common } \\
B C(I) \text { or } N C(I)\end{array}$ & Purpose \\
\hline $\operatorname{ILEAK}(N B)$ & $I=N L A S T+N B-1$ & $\begin{array}{l}\text { Number of particles/batch } \\
\text { that leak from system }\end{array}$ \\
\hline ILEAK (NITS+1) & $I=N L A S T+N I T S$ & $\begin{array}{l}\text { Number of records of leakage } \\
\text { parameters }\end{array}$ \\
\hline ASORC (NB) & $I=L O C L K+N B-1$ & $\begin{array}{l}\text { Total source weight for } \\
\text { batch NB }\end{array}$ \\
\hline BUF (IPOS) & $I=$ LOCASC + IPOS - I & $\begin{array}{l}\text { Temporary storage of leakage } \\
\text { parameters }\end{array}$ \\
\hline IGRUP (IN) & $I=L O C B U F+I N-1$ & $\begin{array}{l}\text { Group number of source } \\
\text { energy in forward mode }\end{array}$ \\
\hline $\operatorname{IDET}(\mathrm{IN})$ & $I=L O C I G P+I N-1$ & $\begin{array}{l}\text { Detector number where response } \\
\text { for escaping particle IN will } \\
\text { be stored }\end{array}$ \\
\hline IVEH(IN) & $I=$ LOCIDT $+I N-1$ & $\begin{array}{l}\text { Signal indicating where }(\gamma, n) \\
\text { conversion occurred }\end{array}$ \\
\hline RDEN(NR) & $I=L O C I V H+N R-1$ & $\begin{array}{l}\text { Density factor for altering } \\
\text { total cross section for } \\
\text { importance region NR }\end{array}$ \\
\hline
\end{tabular}


Table 6-5. Definition of Variables in Common IVCS.

\begin{tabular}{|c|c|}
\hline Variable & Definition \\
\hline SWATE & Total source weight for current batch \\
\hline TOTS & $\begin{array}{l}\text { Total particle weight for the entire run } \\
\text { (i.e., the summation from } 1 \text { to NITS batches of SWATE) }\end{array}$ \\
\hline IMAXN & The size of arrays IVEH, IGRUP, IDET - defined as $5 *$ NMOST \\
\hline MAXSZ & $\begin{array}{l}\text { The size of the buffer for the leakage parameters } \\
\text { (set at } 1000 \text { ) }\end{array}$ \\
\hline IGRND & Media number of ground \\
\hline IAIR & Media number of air \\
\hline NREC & Number of records written to scratch unit ISCU \\
\hline NAME 1 & The name of the particle that split \\
\hline ISCU & $\begin{array}{l}\text { Logical unit number of scratch unit for leakage parameter } \\
\text { storage (set to } 23 \text { ) }\end{array}$ \\
\hline ICTU & Logical unit number of MORSE leakage tape (set to 24) \\
\hline IPOS & Counter for number of leakage parameters in buffer \\
\hline LOCLK & Starting location in blank common of ILEAK array \\
\hline LOCASC & Starting location in blank common of ASORC array \\
\hline LOCBUF & Starting location in blank common of BUF array \\
\hline LOCIGP & Starting location in blank common of IGRUP array \\
\hline LOCIDT & Starting location in blank common of IDET array \\
\hline LOCIVH & Starting location in blank common of IVEH array \\
\hline LOCRDN & Starting location in blank common of RDEN array \\
\hline TTL (10) & Title of problem (will be written to leakage tape) \\
\hline
\end{tabular}




\subsection{MORSE INPUT REQUIREMENTS}

This section describes the input data for the MORSE program. The data is free-form. Most of the rules governing FIDO free-form input in Appendix $A$ are appropriate. MORSE free-form input requires that the ' $\$$ ' and the "**' not begin in column 1. Error messages will be printed in this case, but users often misinterpret them. Therefore, beg in the "\$\$" and "**" parameters in column 2. MORSE input also does not require data block terminators "T". Standard MOR.SE includes the SAMBO input (after the cross-section module input). The MORSE component in MASH does not include the SAMBO routines since the analysis of the adjoint leakage tape is performed in DRC. Consequently, the additional input data after the cross-section input are special data required by MASH. This data replaces the usual SAMBO analysis input.

\subsubsection{Random Walk Input Instructions}

This input is read by subroutine INPUT1.

CARD A Job Titie Card [20A4]

TITLE 80 alphanumeric character description

[NOTE: Any character other than a blank or alphanumeric in column one will terminate the job.]

CARD B Job Control Parameters [Begin array with "\$"]

$\$ \$$

NSTRT number of particles per batch

NMOST maximum number of particles allowed for in the bank(s); may equal NSTRT if no splitting, fission, and secondary generation

NITS number of batches

NQUIT number of sets of NITS batches to be run without calling subroutine INPUT

NGPQTN number of neutron groups being analyzed

$-5-$.

NGPQTG number of gamma-ray groups being analyzed

NMGP number of primary particle groups for which cross sections are stored; should be same as NGP (or the same as $N G G$ when $N G P=0$ ) on Card $X B$ read by subroutine XSEC NMTG total number of groups for which cross sections are stored; should be same as NGP+NGG as read on Card $X B$ read by subroutine XSEC 
NCOLTP set greater than zero if a collision tape is desired; the collision tape is written by the user routine BANKR

IADJM set greater than zero for an adjoint problem

[NOTE: For MASH analysis, IADJM is always set $>0$ ]

$-10-.$.

MAXTIM maximum clock time in minutes allowed for the problem to be on the computer; [15.5 hrs is max]

MEDIA number of cross-section media; should agree with NMED on Card XB read by subroutine XSEC

MEDALB albedo scattering medium is absolute value of MEDALB; MEDALB $=0$, no albedo information to be read in, MEDALB < 0 , albedo only problem - no cross sections are to be read, MEDALB > 0, coupled albedo and transport problem.

[NOTE: For MASH analysis, MEDALB is always set $=0$ ]

[NOTE: See Table 6-3 in Section 6.2.2 for examples on parameters NGPQTN, NGPQTG, NMGP, and NMTG.]

CARD C Source Control Parameters [Use "\$\$" and "**" as indicated]

$\$ \$$

ISOUR source energy group if $>0$

[ if ISOUR $<0$ or if ISOUR $=0$ and NGPFS $\neq 0$, SORIN is called for input of Cards EI and E2.]

NGPFS number of groups for which the source spectrum is to be defined. [If ISOUR $<0$, NGPFS $\geq 2$ ]

ISBIAS no source energy biasing if set equal to zero; otherwise the source energy is to be biased, and Cards $\mathrm{E} 2$ are required.

NOTUSD an unused variable. Must specify a "0".

WTSTRT weight assigned to each source particle

EBOTN lower energy limit of lowest neutron group (eV) (group NMGP)

EBOTG lower energy limit of lokiest gamma-ray group (eV) (group NMTG)

TCUT age in sec at which particles are retired; if TCUT $=0$, no time kill is performed

VELTH velocity of group NMGP when NGPQTN $>0$; i.e., thermal-neutron velocity $(\mathrm{cm} / \mathrm{sec})$ 
CARD D Source Control Parameters [Begin with "**".]

$\star *$

$\left.\begin{array}{l}\text { XSTRT } \\ \text { YSTRT } \\ \text { ZSTRT }\end{array}\right]-X, Y, Z$ coordinates for source particles
AGSTRT starting age for source particles.
$\left.\begin{array}{l}\text { UINP } \\ \text { VINP } \\ \text { WINP }\end{array}\right] \begin{aligned} & \text { source particle direction cosines; } \\ & \text { if all are equal to zero, } \\ & \text { isotropic directions are chosen in MORSE }\end{aligned}$

[NOTE: Source data on Cards $C$ and $D$ will be overridden by any changes in subroutine SOURCE.]

CARDS El Input Source Distribution [Begin with "**"]

[NOTE: Omit if ISOUR on Card $C>0$ or if ISOUR $=$ NGPFS $=0$ ]

FS NGPFS values of FS, where FS equals the unnormalized fraction of source particles in each group

CARDS E2 Relative Importance of Group Source [Begin with "**"]

[NOTE: Omit if ISOUR $>0$ or if ISOUR $\leq 0$ and ISBIAS $=0$ ]

$\star *$

BFS NGPFS values of BFS, the relative importance of a source in group I. (If ISBIAS >0)

CARDS F Energy Group Boundaries [Begin with "**"]

$\star \star$

ENER NMTG values of ENER, the energies (in eV) at the upper edge of the energy group boundaries

[NOTE: The lower energies of groups NMGP and NMTG were read on Card $C_{0}$ ] 
CARD G Collision Tape Control Parameters [2I5,5X,36I1,5X,13I1]

[NOTE: Omit if NCOLTP on Card B $\leq$ ]

NHISTR

NHISMX

$\operatorname{NBIND}(\mathrm{J}), \mathrm{J}=1,36$

$\operatorname{NCOLLS}(J), J=1,13$ logical tape number for the first collision tape. the highest logical number that a collision tape may be assigned.

an index to indicate the collision parameters to be written on tape.

an index to indicate the types of collisions to be put on tape.

[NOTE: See Tables 6-1 and 6-2 in Section 6.2.1 for information concerning NBIND and NCOLLS.]

CARD H Random Number

RANDOM starting random number.

\begin{tabular}{|c|c|}
\hline $\begin{array}{l}\text { IBM } 3033 \\
\text { CRAY } \\
\text { CDC } 6600 \\
\text { UNIVAC } 1108\end{array}$ & $\begin{array}{l}-(212 \text { Format }) \\
-(4 X, 020 \text { Format }) \\
-(4 X, 020 \text { format }) \\
-(4 X, 012 \text { Format })\end{array}$ \\
\hline
\end{tabular}

\section{CARD I Biasing Control Parameters [Begin with "\$\$"]}

$\$ \$$

NSPLT index indicating that splitting is allowed (if $>0$ )

NKILL index indicating that Russian roulette is allowed (if $>0$ )

NPAST index indicating that exponential transform is invoked (if $>0)$ (Subroutine DIREC required)

NOLEAK index indicating that non-leakage is invoked (if $>0$ )

IEBIAS index indicating that energy biasing is allowed (if $>0$ )

MXREG number of importance regions described by geometry input (will be set to one if $\leq 0$ )

MAXGP group number of last group for which Russian roulette or splitting or exponential transform is to be performed. [Note: For adjoint, set = NMTG or overstoring results.]

INGB in-group bias switch (INGB $=1$ recommended)

= I implies use in-group biasing

- 0 implies do not use in-group biasing 


\section{CARD $J$ Biasing Parameters [use " $\$ \$ "$ and "**" as indicated] \\ [NOTE: Omit if NSPLT + NKILL + NPAST = 0]}

\section{$\$ \$$}

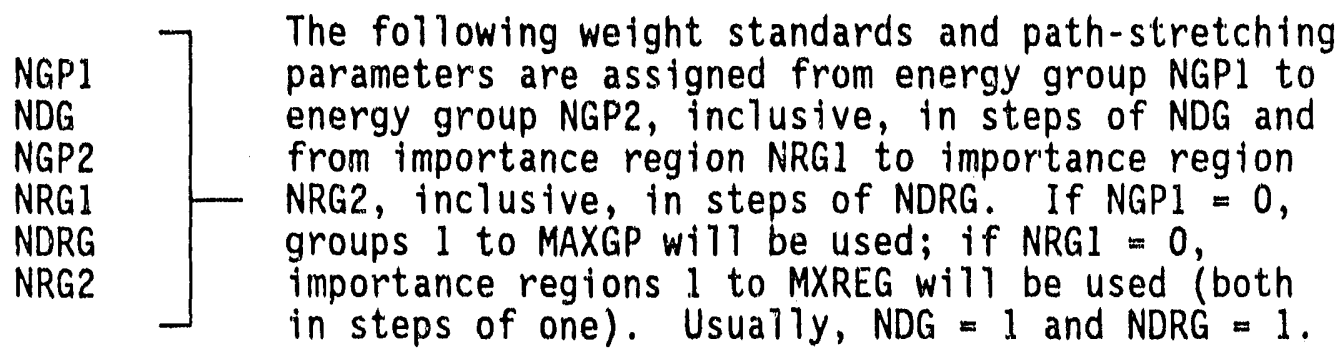

$\star \star \star$

WTHIHl weight above which splitting witl occur.

WTLOW weight below which Russian roulette is played.

WTAVE1 weight given those particles surviving Russian roulette.

PATH path-length stretching parameters for use in

exponential transform (usually $0 \leq$ PATH $\leq 1$ ).

[NOTE: The above information is repeated until data for all groups and importance regions are input.]

[NOTE: End Card(s) $J$ with a negative value of NGP1, i.e., NGP1 $=-1$

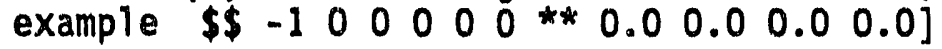

\section{CARDS $K$ Energy Biasing Parameters [Begin wiln "**"]}

[NOTE: Omit if IEBIAS on Card $I \leq 0$ ]

$\star *$

( (EPROB (IG,NREG), IG $=1$, NMTG), NREG $=1$, MXREG)

Values of the relative energy importance of particles leaving a collision in importance region NREG. Input for each region must start on a new card.

\section{CARD L Problem Identification [Begin with "\$\$"]}

\section{$\$ \$$}

NSOUR set $\leq 0$ for a fixed source problem; otherwise the source is from fissions generated in a previous batch

MFISTP index for fission problem, if $\leq 0$ no fissions are allowed

NKCALC the number of the first batch to be included in the estimate of $\mathrm{K}_{\text {eff }}$; if $\leq 0$ no estimate of $\mathrm{K}_{\text {eff }}$ is made 
NORMF the weight standards and fission weights are unchanged if $\leq 0$; otherwise fission weights will be multiplied, at the end of each batch, by the latest estimate of $K_{\text {eff }}$ and the weight standards are multiplied by the ratio of fission weights produced in previous batch to the average starting weight for the previous batch. For time-dependent decaying systems, NORMF should be $>0$.

CARDS $M$ Fission Neutron Weights [Begin with "**"]

[NOTE: Omit if MFISTP on Card $L \leq 0$ ]

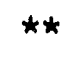

(FWLO(I), I = I, MXREG)

values of the weight to be assigned to fission neutrons.

CARDS $\mathbf{N}$ Fission Induced Source Particle Fractions [Begin with "**"]

[NOTE: Omit if MFISTP on Card $L \leq 0$ ]

(FSE(IG,IMED), IG $=1$, NMGP), IMED $=1$, MEDIA)

the fraction of fission-induced source particles in group IG and medium IMED.

[NOTE: Input for each medium must start on a new card.]

CARDS 0 Secondary Particle Production Probability [Begin with "**"]

[NOTE: Omit if NGPQTN $=0$ or NGPQTG $=0$, i.e., include if coupled neutron gamma-ray problem]

$\star \star$

( (GWLO(IG,NREG) IG $=1$, NMGP or NMTG - NMGP), NREG $=1$, MXREG)

values of the probability of generating a secondary particle. The secondary particle will be a gamma ray in the forward problem and a neutron in the adjoint problem. NMGP groups are read for each importance region in a forward problem and NMTG-NMGP for an adjoint problem. 
[NOTE: The definition of GWLO depends on whether or not in-group biasing is specifled (Card I, variable INGB), as follows:

INGB=1 (in-group biasing invoked) GWLO is the fraction of the natural production probability as specified by the cross sections and may be assigned values greater than, equal to, or less than one.

INGBmO (no in-group biasing) GWLO is the probability of generating a gama ray (adjoint neutron).]

[NOTE: Input for each importance region must start on a new card.]

\subsubsection{GIFT5 Geometry Input Instructions}

This input card is read in subroutine GIFTM.

CARD GA GIFT5 Program Control Card [10I1, 2F10.0, 315 format]

[NOTE: Option is not set if 0 ; otherwise the option is set]

IPRNT print MASTER-ASTER array of processed geometry

ITEMPR print identification table by item

IMNMAX print ordered region rpp equivalents

ISOLEQ print solid rpp equivalents

NOPRNT suppress GENI printing

$-5-$.

IWSOL print solid table

IWREG print region table

IWRPP print region rpp table

IWID print region identification table

IWSUM print summary table

$-10-.-$

$\begin{array}{ll}\text { TOL } & \text { geometry tolerance } \\ \text { TOLLOS } & \text { ine-of-sight tolerance } \\ \text { IVOID } & \text { void space code } \\ \text { IFANTM phantom item code } \\ \text { IEND signal end of components along ray }\end{array}$

The control specifications card consists of first, a series of ten print control switches on 10I1 format, for which a 0 usually means "do not print" and a 1 means "print". "0000100000" appears useful for this card. Next are tolerances, for which 0.001 appears adequate. The last 
inputs on the card are void, phantom and ray end codes, 1, 0, and 0 , respectively.

The GIFTs input geometry consists of five parts as follows:

1. Titie Card

2. Target Specification Card

3. Soltds Table

4. Regions Table

5. Region Identification Table

Detalled descriptions of all these sections of input are given in Appendix $C$. Here, only the input instructions will be presented.

NOTE: The next six sections of input (Cards GB through Cards GG) for MASH are read from LOGICAL UNIT 8 (as currentiy configured). This is noted because the rest of the input is read from LOGICAL UNIT 5.)

\section{CARD GB GIFTS Title Card [A2, 3X, A60 format]}

TGTUN target unit specification [(cm) only for MASH]

ITITLE target geometry description titie

This input card is read in subroutine GENI.

\section{CARD GC GIFT5 Target Specification Card [2I5 format]}

NSOLID number of geometry solids in Solids Table

NRMAX number of geometry regions in Reglons Table

This input card is read in subroutine GENI.

CARD GD GIFT5 Solids Table [A5, A3, A1, 1X, 6F10.0, A10 format]

These input cards are read in subroutine SOLIN.

The solids table defines the basic geometric shapes. Table 6-6 summarizes the input for the different solid types. The basic format for all cards in the solids table is (A5, A3, A1, IX, 6F10.0, A10) with the first field (A5) in the format normally used for the sequence number of the solid in the solid table, the second field (A3) used to designate solid type (e.g., RPP for rectangular parallelpiped or SPH for sphere) 
Table 6-6. Summary Description of Input for Solids Table.

\begin{tabular}{|c|c|c|c|c|c|c|c|c|c|}
\hline 1.5 & 6.8 & $9-10$ & $11-20$ & $21-30$ & $31-40$ & $41-50$ & $51-60$ & $61-70$ & $71-80$ \\
\hline NO. & RPP & & XMIN & XMAX & YMIN & YMAX & ZMIN & ZMAX & COMMENTS \\
\hline $\begin{array}{l}\text { No. } \\
\text { NO. }\end{array}$ & BOX & & $\begin{array}{l}v X \\
W X\end{array}$ & $\begin{array}{l}\text { VY } \\
W Y\end{array}$ & $\begin{array}{l}V Z \\
W Z\end{array}$ & $\begin{array}{l}H X \\
D X\end{array}$ & $\begin{array}{l}\text { HY } \\
\text { DY }\end{array}$ & $\begin{array}{l}\mathrm{HZ} \\
\mathrm{DZ}\end{array}$ & $\begin{array}{l}\text { COMMENTS } \\
\text { COMMENTS }\end{array}$ \\
\hline $\begin{array}{l}\text { No. } \\
\text { NO. }\end{array}$ & RAW & & $\begin{array}{l}V X \\
W X\end{array}$ & $\begin{array}{l}\text { VY } \\
W Y\end{array}$ & $\begin{array}{l}V Z \\
W Z\end{array}$ & $\begin{array}{l}H X \\
D X\end{array}$ & $\begin{array}{l}\text { HY } \\
\text { DY }\end{array}$ & $\begin{array}{l}\mathrm{HZ} \\
\mathrm{DZ}\end{array}$ & $\begin{array}{l}\text { COMMENTS } \\
\text { COMMENTS }\end{array}$ \\
\hline NO. & SPH & & $\forall x$ & VY & VZ & $R$ & & & COMMENTS \\
\hline $\begin{array}{l}\text { NO. } \\
\text { NO. }\end{array}$ & ELL. & & $\begin{array}{l}V X \\
B\end{array}$ & VY & VZ & $A X$ & AY & $A Z$ & $\begin{array}{l}\text { COMMENTS } \\
\text { COMMENTS }\end{array}$ \\
\hline No. & ELL & $G$ & $\begin{array}{l}V X \\
B X\end{array}$ & $\begin{array}{l}V Y \\
B Y\end{array}$ & $\begin{array}{l}V Z \\
B Z\end{array}$ & $\begin{array}{l}A X \\
C X\end{array}$ & $\begin{array}{l}\text { AY } \\
C Y\end{array}$ & $\begin{array}{l}A Z \\
A Z\end{array}$ & $\begin{array}{l}\text { COMMENTS } \\
\text { COMMENTS }\end{array}$ \\
\hline NO. & TOR & & $\begin{array}{l}V X \\
\text { RI }\end{array}$ & $\begin{array}{l}V Y \\
R 2\end{array}$ & $V Z$ & $N X$ & NY & $N 2$ & COIMMENTS \\
\hline $\begin{array}{l}\text { No. } \\
\text { NO. }\end{array}$ & RCC & & $\begin{array}{l}V X \\
R\end{array}$ & VY & $V Z$ & $H X$ & HY & $H Z$ & $\begin{array}{l}\text { COMMENTS } \\
\text { COMMENTS }\end{array}$ \\
\hline $\begin{array}{l}\text { No. } \\
\text { NO. }\end{array}$ & REC & & $\begin{array}{l}V X \\
A X\end{array}$ & $\begin{array}{l}V Y \\
A Y\end{array}$ & $\begin{array}{l}V Z \\
A Z\end{array}$ & $\begin{array}{l}H X \\
B X\end{array}$ & $\begin{array}{l}H Y \\
B Y\end{array}$ & $\begin{array}{l}\mathrm{HZ} \\
\mathrm{BZ}\end{array}$ & $\begin{array}{l}\text { COMMENTS } \\
\text { COMMENTS }\end{array}$ \\
\hline $\begin{array}{l}\text { NO. } \\
\text { NO. }\end{array}$ & TRC & & $\begin{array}{l}V X \\
R 1\end{array}$ & $\begin{array}{l}\text { VY } \\
\text { R2 }\end{array}$ & $V Z$ & $H X$ & HY & $H Z$ & COMMENTS \\
\hline $\begin{array}{l}\text { NO. } \\
\text { NO. } \\
\text { NO. }\end{array}$ & TEC & & $\begin{array}{c}\text { VX } \\
\text { AX } \\
\text { RATIO }\end{array}$ & $\begin{array}{l}\text { VY } \\
\text { AY }\end{array}$ & $\begin{array}{l}V Z \\
A Z\end{array}$ & $\begin{array}{l}H X \\
B X\end{array}$ & $\begin{array}{l}\text { HY } \\
B Y\end{array}$ & $\begin{array}{l}\mathrm{HZ} \\
\mathrm{BZ}\end{array}$ & $\begin{array}{l}\text { COMMENTS } \\
\text { COMMENTS } \\
\text { COMMENTS }\end{array}$ \\
\hline $\begin{array}{l}\text { NO. } \\
\text { NO. } \\
\text { NO. }\end{array}$ & TGC & & $\begin{array}{c}V X \\
A X \\
T(A)\end{array}$ & $\begin{array}{c}V Y \\
A Y \\
T(B)\end{array}$ & $\begin{array}{l}V Z \\
A Z\end{array}$ & $\begin{array}{l}H X \\
B X\end{array}$ & $\begin{array}{l}\text { HY } \\
\text { BY }\end{array}$ & $\begin{array}{l}\mathrm{HZ} \\
\mathrm{BZ}\end{array}$ & $\begin{array}{l}\text { COMMENTS } \\
\text { COMMENTS } \\
\text { COMMENTS }\end{array}$ \\
\hline NO. & HAF & & $A$ & B & $c$ & $D$ & & & COMMENTS \\
\hline $\begin{array}{l}\text { NO. } \\
\text { NO. } \\
\text { NO. }\end{array}$ & ARS & NVEX & $\begin{array}{l}\text { NC } \\
\times 1 \\
\times 3 \\
\text { (STAR } \\
\text { S HAS }\end{array}$ & $\begin{array}{l}\text { NP } \\
Y 1 \\
Y 3 \\
\text { NEW C } \\
\text { AME IN }\end{array}$ & $\begin{array}{c}\text { NR } \\
Z 1 \\
Z 3 \\
\text { ZI } \\
\text { D WITH } \\
\text { IT EXCEF }\end{array}$ & $\begin{array}{l}\times 2 \\
\text { ETC. } \\
\text { EACH CUR }\end{array}$ & Y2 & BLANK & $\begin{array}{l}\text { COMMENTS } \\
\text { COMMENTS } \\
\text { COMMENTS }\end{array}$ \\
\hline
\end{tabular}


Table 6-6. Summary Description of Input for Solids Table (Continued).

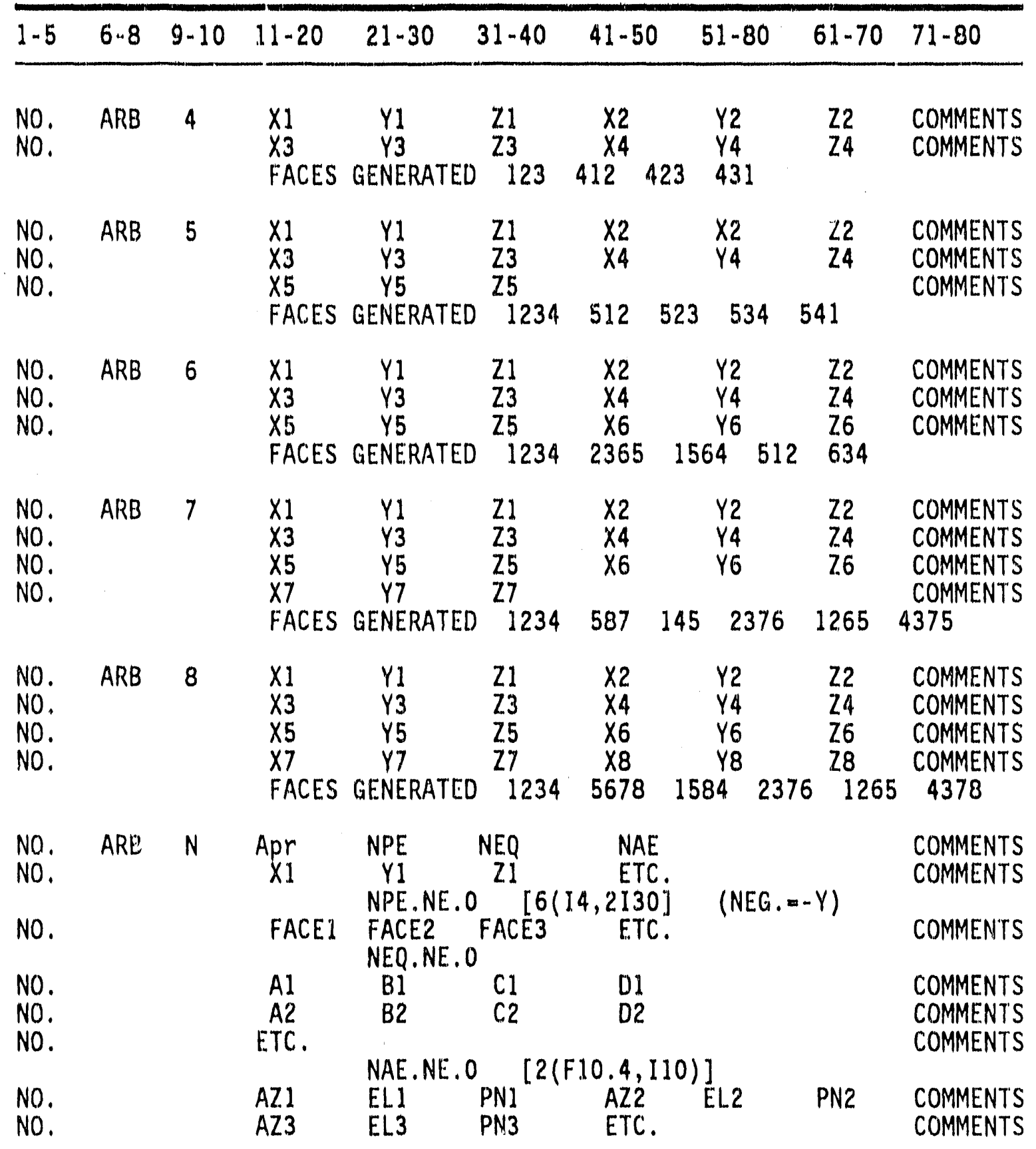


Table 6-6. Summary Description of Input for Solids Table (Continued).

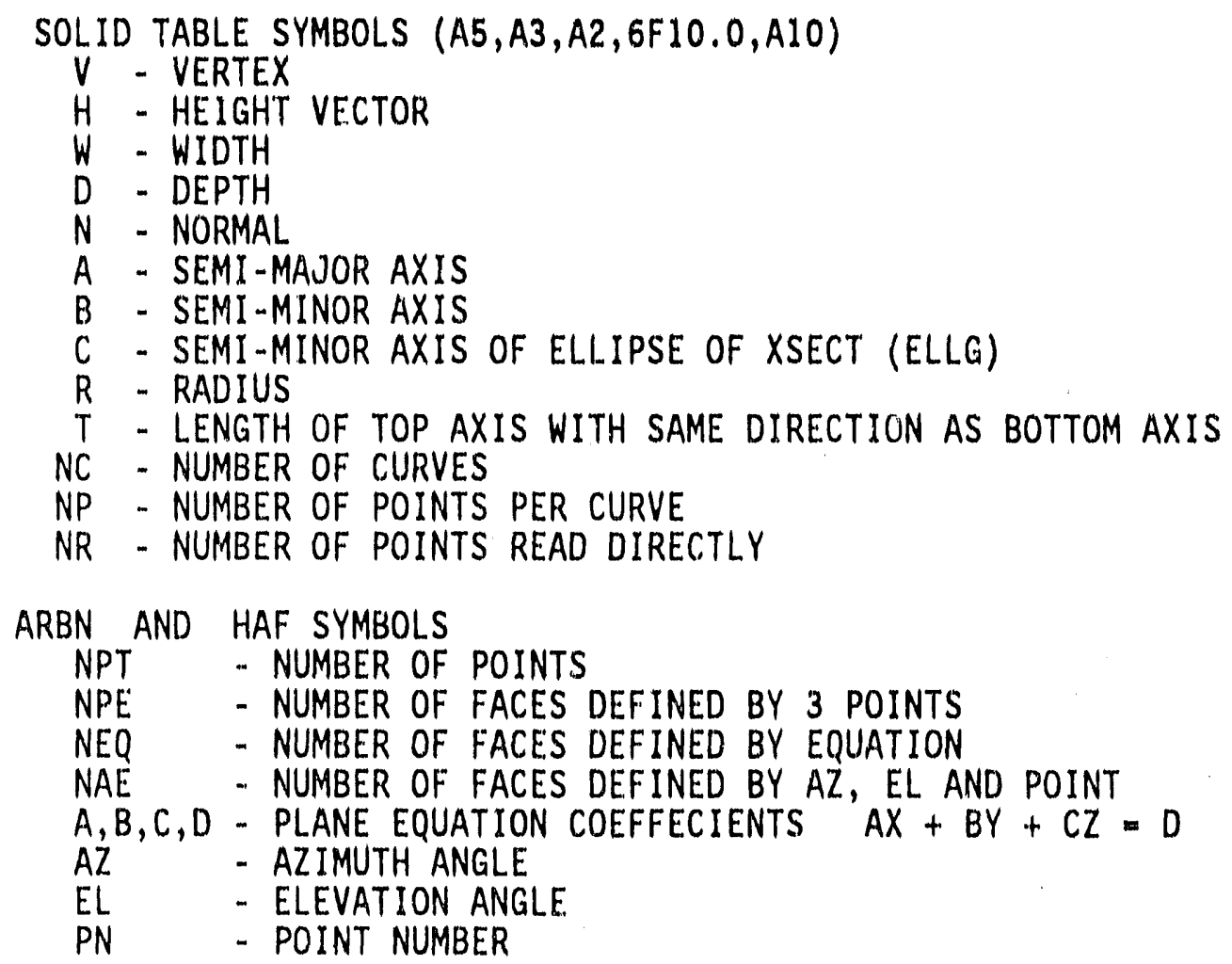

or blank if a continuation card for the soild, the third (Al) field used to designate a variant of the solid type (e.g., a 6 for a six-vertex arbitrary polyhedron or ARBG), the fourth field (6F10.0) used for the solid parameters, and the final (A10) field reserved for user notes or comments.

The parameters required to define the vartous solids are different from one type of solid to another. Usually, however, the parameters can be classified as being one of three types, a "vertex point", a "vector" or a "scalar". The vertex point is specified by its $X, Y, Z$ coordinates, a vector is specified by its components in the $X, Y$, and $Z$ directions, and a scalar is a single numeric value, for example the radius of a sphere.

The summary of the solids table input data given by Table 6-6 will be useful for persons familiar with combinatorial geometry, but a more detalled description of the geometry solid types and their input is necessary for new users and for users using new and more exotic solid types. Therefore a more detailed description of the various geometry solid types, including pictures, has been included. Because of its bulk, this description has been placed in Appendix C. 
CARD GE GIFT5 Regions Table [15, 1X, 9 (A2, I5) 1X, Al0 format]

These input cards are read in subroutine REGIN.

The GIFT5 geometry rogions table is very similar to the zones table in MORSE combinatorial geometry. Via the regions table, the shape and space of several solids can be "combined" to define a component of the system being described. Three operators, a "t", a "-" and an "OR", and combinations thereof, are used to define the "intersection",

"subtraction" or "union" of solids to form a more complex shape. A detailed description of how to use these operators, along with several examples, is given in Appendix B. The un-initiated user of combinatorial geometry should refer to these examples before attempting to model a complex jeometry.

The general format for the regions table is I5, 1X, 9(A2, I5) $1 X, A 10$. The first (I5) field is the region sequence number. Each line has provisions for nine pairs of OR operator fields and solid numbers with + or - sign, followed by 10 spaces for comment. (The $1 X$ means an unused column on the card). Continuation cards can be used for regions describer by the combination of more than nine bodies. For continuation cards, the region (first I5) entry is left blank.

[NOTE: The cards of the above format defining the various regions in the configuration are followed by a card with a -1 in columns 4 and 5 . This "- 1 CARD", as it is called, is the flag that marks the end of the region table.]

\section{CARD GF GIFT5 Region Identification Table [5I5, 5X, A40 format]}

These input cards are read in subroutine IDIN

The identification table is used to assign identification code numbers and additional information to the regions defined in the region table. Entries in the identification tables, in format (5I5, 5X, A40), are as follows:

\begin{aligned} \hline Columns & \multicolumn{1}{c}{ Parameter } \\ \hline & \\ $1-5 &$ Region number \\ $6-10 &$ Component code number $(1-9999) \\ 11-15 &$ Space code number $(1-99) \\ 16-20 &$ Material code \\ $21-25 &$ Percent density \\ $31-70 &$ Alphanumeric region description \end{aligned}


Most of the above information is not used by the GIFT5 package in MORSE; nevertheless the table must be included in input runstreams. For MORSE, only the region number (columns 1-5) need be entered. All the other columns may be left blank (material numbers are assigned in other input to MORSE).

CARD GG GIFT5 Region RPP Table

[NOTE: The Region RPP Table discussed in Appendix $C$ is optional and typically is not input in MASH. The GIFT5 program will automatically produce a Region RPP Table. Consequentiy, the user does not have to enter any data here.]

REMINDER: The remaining sections of input for MASH are read from LOGICAL UNIT 5. This is noted because the previous six sections of the input (Cards GB through Cards GG) were read from LOGICAL UNIT 8.

CARD GH Geometry Region Material Specification Table [14I5 format]

This input card is read in subroutine OVER.

MED material number

NOMED number of regions which are made up of material MED

$\operatorname{IZONE}(I), I=1$, NOMED

NOMED region numbers having the material number MED

[NOTE: These cards are entered (in sets) for each code material number until all regions have designated material. The first two parameters (MED and NOMED) are on a card by themselves. The region numbers are then intered on a second card.]

CARD GI Importance Region Assigntivent Table [14I5 format]

This input card is read in subroutine OVER

$\operatorname{IR}(I), I=1$, NRMAX

the imporiance region assignment for each geometry region is read in this array. 


\subsubsection{Cross-Section Module Input Instructions}

This input is read by subroutine XSEC.

CARD XA Cross Section Title Card [20A4]

[NOTE: This title is also written on tape if a processed tape is written; therefore, it is suggested that the title be definitive.]

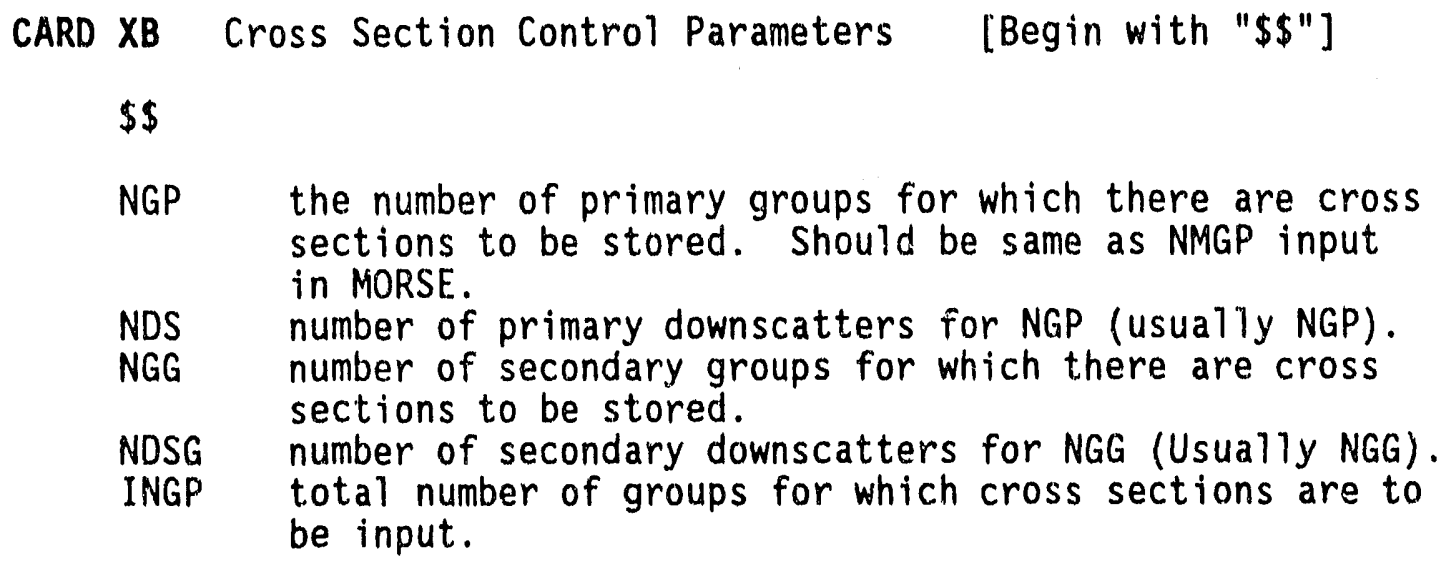

$-5 \cdots$

ITBL table length, i.e., the number of cross sections for each group; (usually equal to number of downscatters + number of upscatters + IHT).

ISGG location of within-group scattering cross sections; (usually equal to number of upscatters plus IHT+1).

NMED number of media for which cross sections are to be stored; should be same as MEDIA input in MORSE.

NELEM number of elements for which cross sections are to be read.

NMIX number of mixing operations (elements times density operations) to be performed (must be $\geq 1$ ).

NCOEF number of coefficients for each element, including $P_{0}$.

NSCT number of discrete angles (usually the integer value of $\mathrm{NCOEF} / 2$ ).

ISTAT flag to store Legendre coefficients if greater than zero.

IHT location of total cross section in the table.

\section{[NOTE: See Table 6-3 in Section 6.2.2 for examples on parameters} NGP, NGG, and INGP.] 


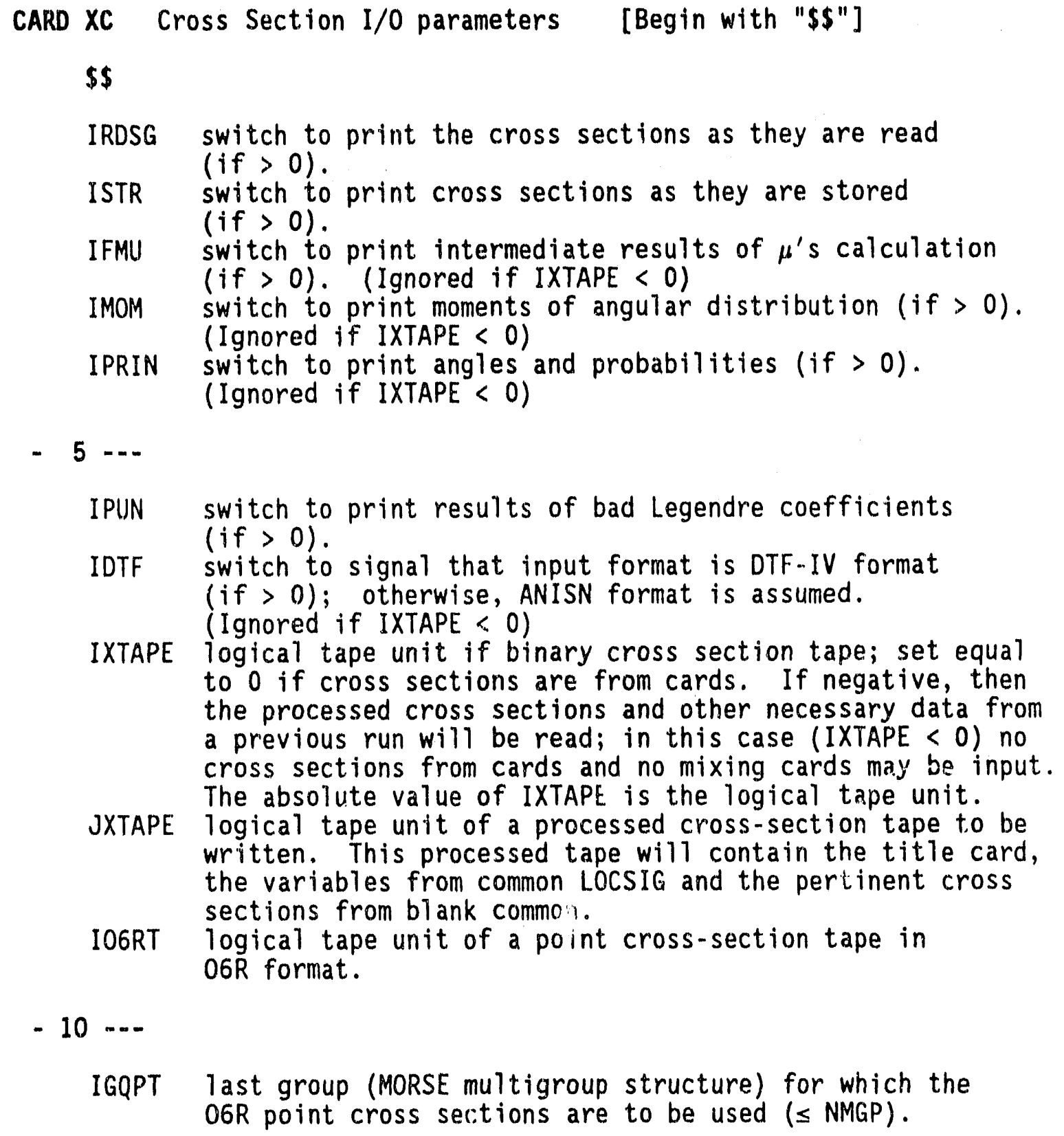

IRDSG switch to print the cross sections as they are read (if $>0$ ).

ISTR switch to print cross sections as they are stored (if $>0$ ).

IFMU switch to print intermediate results of $\mu^{\prime}$ s calculation (if $>0$ ). (Ignored if IXTAPE < 0 )

IMOM switch to print moments of angular distribution (if $>0$ ). (Ignored if IXTAPE < 0 )

IPRIN switch to print angles and probabilities (if $>0$ ). (Ignored if IXTAPE < 0)

$-5-$.

IPUN switch to print results of bad Legendre coefficients (if $>0$ ).

IDTF switch to signal that input format is DTF-IV format (if $>0$ ); otherwise, ANISN format is assumed. (Ignored if IXTAPE $<0$ )

IXTAPE logical tape unit if binary cross section tape; set equal to 0 if cross sections are from cards. If negative, then the processed cross sections and other necessary data from a previous run will be read; in this case (IXTAPE $<0$ ) no cross sections from cards and no mixing cards may be input. The absolute value of IXTAPE is the logical tape unit.

JXTAPE logical tape unit of a processed cross-section tape to be written. This processed tape will contain the title card, the variables from common LOCSIG and the pertinent cross sections from blank commo?.

I06RT logical tape unit of a point cross-section tape in O6R format.

$-10 \cdots$

IGQPT last group (MORSE multigroup structure) for which the O6R point cross sections are to be used ( $\leq$ NMGP).

CARD XD Element Identifiers [Begin with "\$\$"]

[NOTE: Omit if IXTAPE $\leq 0$ ]

Element identifiers for cross-section tape. If element identifiers are in same order as elements on tape, the efficiency of the code is increased due to fewer tape rewinds. 
CARDS XE Cross Sections from Cards [Begin with "**" if free-form]

[NOTE: Omit if IXTAPE *0]

If cross sections are in free-form, a card with "**" in columns 2 and 3 must precede the actual data. ANISN format if IDTF $\leq 0$; otherwise, DTF-IV format. Cross sections for INGP groups with a table length ITBL for NELEM elements each with NCOEF coefficients.

CARDS XF Cross-Section Mixing Table [Use "\$\$" and "**" as indicated]

[NOTE: Omit if IXTAPE < O]

$\$ \$$

KM medium number.

KE element number occurring in medium KM (negative value indicates last mixing operation for that medium). Failure to have a negative value causes code not to generate angular probabilities for that media, i.e. (LEGEND and ANGLE not called).

$* \star$

RHO density of element KE in medium KM in units of atoms/(barn $\mathrm{cm}$ ).

Example Card XF: \$ KM KE ** RHO

[NOTE: NMIX (see Card XB) cards are required.]

CARDS XG 06R Tape Control Parameter [Begin with "\$\$"]

[NOTE: Omit if I06RT $\leq 0$ ]

$\$ \$$

NXPM number of point cross-section sets per medium found on an 06R tape.

$=1$, total cross section only,

$=2$, total + scattering cross section,

$=3$, total, scattering, and $\nu^{\star}$ fission cross section. 


\subsubsection{Special MASH Input Instructions}

In addition to the MORSE input described above, the following input data is required for a MASH run. These parameters are either included on the leakage tape read by DRC, or are used to determine parameters used on the leakage tape.

CARD AA Leakage Tape Tit7e [10A4]

TTL. Title to be placed on leakage tape

CARD BB Region Density Factors [Begin with "**"]

**

$\operatorname{RDEN}(I), I=1, M X R E G$

Density factor for each importance region

CARD CC Air/Ground Media Indicators [Begin with "\$\$"]

$\$ \$$

IGRND Media number of the ground

IAIR Media number of air

Two media numbers must be specified wherein a flag is placed on the product of secondary particle (neutron) generation. The variable names assigned to these numbers indicate that the conventional application of this input is to flag neutrons generated in the ground (IGRND) and the air (IAIR). Any media may be specified, with the understanding that the contribution of the flagged particles will be labeled GROUND and AIR, regardless of their true source.

\subsection{ANISN NUCLIDE FORMAT CROSS-SECTION FILE}

In a MORSE problem, cross sections are read from card input and/or from an external data set in nuclide-organized format, i.e., all the cross sections for one group of one nuclide, followed by other groups for that nuclide, and finally followed by data for other nuclides. Within a group, MORSE expects the ordering of data in the cross section table in the following format: 


\begin{tabular}{|c|c|c|}
\hline able Position & Entry & Cross Section Type \\
\hline 1 & $\sigma^{1}(g)$ & $\begin{array}{l}\text { First activation cross section } \\
\text { (if any) }\end{array}$ \\
\hline \multicolumn{3}{|l|}{. } \\
\hline ; & & \\
\hline IHT -3 & $\sigma^{L}(g)$ & $\begin{array}{l}\text { Last activation cross section } \\
\text { (if any) }\end{array}$ \\
\hline IHT -2 & $\sigma^{\wedge}(g)$ & Absorption \\
\hline IHT-1 & $\nu_{\sigma^{F}}(\mathrm{~g})$ & Neutron Production \\
\hline IHT & $\sigma^{\top}(g)$ & Total removal \\
\hline IHT+1 & $\sigma^{\text {Tus }}(g)$ & $\begin{array}{l}\text { Total upscatter cross section from } \\
\text { group } g \text { (omit this entry if NUS=0) }\end{array}$ \\
\hline IHS-NUS & $\sigma(g+N U S \rightarrow g)$ & Scattering from group $\mathrm{g}+\mathrm{NUS}$ to $\mathrm{g}$ \\
\hline . & . & \\
\hline$\therefore$ & 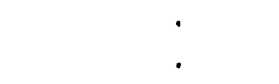 & \\
\hline IHS- 1 & $\sigma(g+1+g)$ & Scattering from group $\mathrm{g}+1$ to $\mathrm{g}$ \\
\hline IHS & $\sigma(g-g)$ & Scattering from group $\mathrm{g}$ to $\mathrm{g}$ \\
\hline IHS+1 & $\sigma(g-1+g)$ & Scattering from group $\mathrm{g}-1$ to $\mathrm{g}$ \\
\hline . & . & \\
\hline : & $\dot{.}$ & \\
\hline IHM & $\sigma(g-N D S+g)$ & Scattering from group $g-N D S$ to $g$ \\
\hline
\end{tabular}

From this, it can be seen that:

NUS $=I H S-I H T-2=$ number of upscatter groups, if greater than 0 , NDS = IHM-IHS = number of downscatter groups,

$L=I H T-3=$ number of activation cross sections. 
Special illustrations of interest are:

$$
\begin{aligned}
& I H M=I G M+3 \\
& I H S=4 \\
& I H T=3
\end{aligned}
$$

Full downscatter; no upscatter; no activation cross section

and,

$$
\begin{aligned}
& I H M=I G M+5+\text { NUS } \\
& I H S=6+\text { NUS } \\
& I H T=4
\end{aligned} \quad \begin{aligned}
& \text { FUII downscatter; NUS upscatter } \\
& \begin{array}{l}
\text { room for an activation cross } \\
\text { section in position } 1 .
\end{array}
\end{aligned}
$$

Thus the parameters IHT, IHS, and IHM completely describe the format of the cross sections. If there are no activity cross sections, IHT=3. If there is no upscatter IHS $=I H T+1$. If there is no downscatter IHM $=$ IHS (i.e. a one group problem). If there is upscatter, MORSE will compute a total upscatter cross section for each group of each material and place that cross section in position IHM +1 . The activity cross sections are not used in the MORSE code.

\subsection{MORSE LEAKAGE TAPE OUTPUT FILE FOR DRC}

Because of the large amount of core storage required for the VISTA freefield fluences, the forward-adjoint coupling could not be accomplished as the Monte Carlo calculation was performed. The coupling involved the calculation of the integral

$$
R=\int \Phi(P) \Phi^{*}(P)(\Omega \cdot n) d P
$$

over the coupling surface $S$.

We define

$$
\begin{aligned}
\Phi(P)= & \text { VISTA free-field fluences in phase space } P . \\
\Phi^{*}(P)= & \text { MORSE adjoint fluence in phase space } P . \\
\Omega \cdot n= & \begin{array}{l}
\text { cosine of angle between particle direction and } \\
\text { inward normal. }
\end{array}
\end{aligned}
$$


The adjoint fluence at the $r_{s}$, the position of the surface $S$, is written on a leakage tape along with other varlables necessary for coupling the MORSE resuits with the VISTA results in DRC. The variables written on tape for each adjoint leakage are as follows:

1. IG - the group number,

2. IGS - the source group,

3. WTBC - the weight of the adjoint particle at leakage,

4. $U$ - X direction cosine,

5. $V$ - Y direction cosine,

6. W - Z direction cosine,

7. $Z$ - height of leakage point,

8. IDET - detector type in which particle is to be scored.

An output buffer was blocked to transfer the data from core to tape efficientiy at the end of each batch. This permitted batch statistics to be calculated in the DRC code which reads the MORSE leakage tape and the VISTA fluence tape and performs the coupling. The leakage tape generated by MORSE contains the following data:

Record 1 - Title and date of MORSE run

Record 2 - Initial Data Record - 24 words

Record 3 - Energy Group Structure, Velocities, Source Spectra and Blasing Function - 4 *NMTG words

Record 4 - Array of Number of Particles Leaking Per Batch NITS+1 words

Record 5 - Array of Source Weights Per Batch - NITS+1 Words

Record 6 - A Block of Leakage Information for 125 Adjoint Particles 1000 words

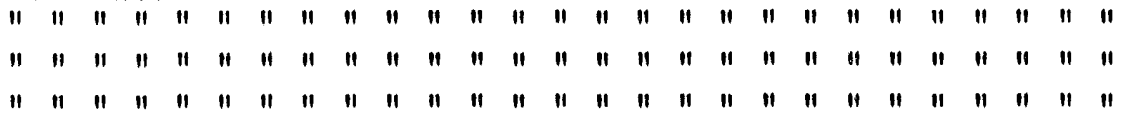

Record N -

A detailed description of the contents of the above records is given below:

1. Record 1 - A 40 character title plus a 32 character date of run

II. Record 2 - The USER labeled common (plus parameter INGB) is written in Record 2. The total number of groups, number of batches, etc., are contained in this record, as defined in Table 6-7. 
III. Record 3 - The multigroup energy structure and group velocities are contained in Record 3 . The data are:

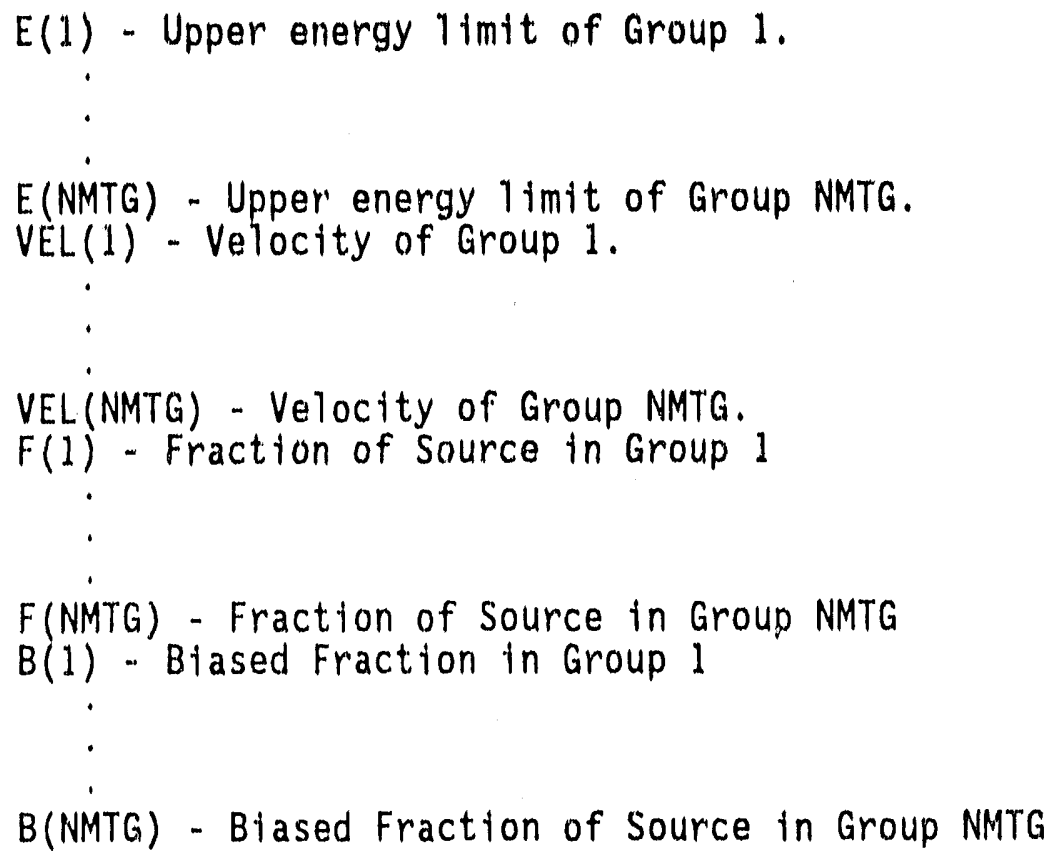

IV. Record 4 . The number of particles that leak per batch are given in Record 4. This data is necessary in order to normalize the batch results per source neutron.

$L(1)$ - Number of leakage particles in batch 1 .

L(NITS) - Number of leakage particles in batch NITS.

$L(N I T S+1)$ - Total number of leakage records written on tape.

V. Record 5 - The sum of the source weights of the particles in a batch.

ASORC (1) - Sum of weights of source particles in batch 1. ASORC(NITS) - Sum of weights of source particles in batch NITS

VI. Record 6 - The adjoint leakage fluence blocked in groups of 125 particles. 


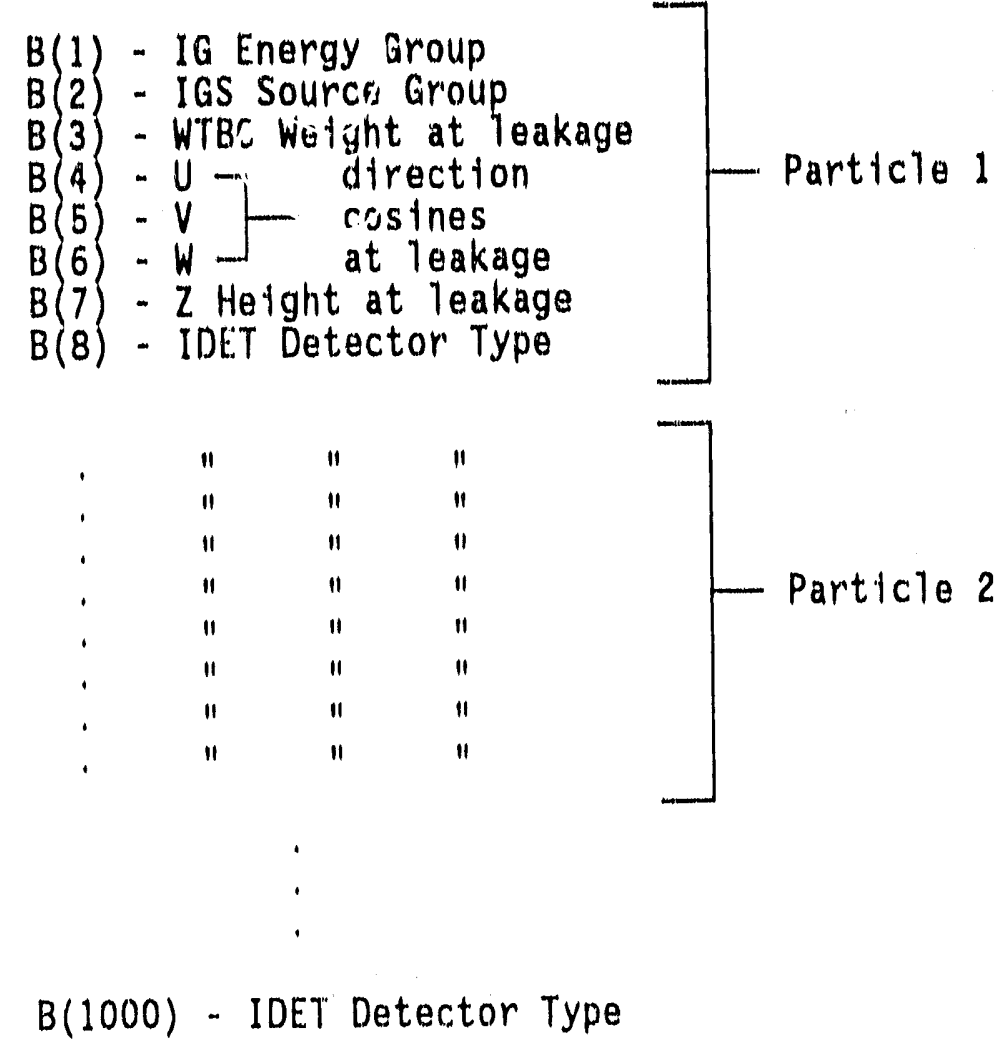

VII. Additional records in the format of Record 6 to a total of $L(N I T S+1)$ leakage records.

\subsection{LOGICAL UNIT REQUIREMENTS}

Below is a listing of the files required to execute a MORSE case along with the default values used in the code. In setting up a MORSE case, efforts must be made for these units to be avallable.

1. Logical Unit IXTAPE - Cross Section Input Flle [Default=10]

2. Logical Unit JXTAPE - Cross Section Output File [Default $=2$ ]

3. Logical Unit FT16 - CG Geometry Scratch Unit [Default=16]

4. Logical Untt ISCU - MORSE Adjoint Leakage Scratch File [Default=23]

5. Logical Unit ICTU - MORSE Adjoint Leakage File [Defau1ta24]

6. Logical Unit 10 - Printed Output [Default=6]

7. Logical Unit I1 - MORSE Card Input File [Default=5]

8. Logical Unit I08 - GIFT5 Card Input File [Default=n8]

9. Logical Unit I09 - GIFT5 Geometry Scratch Unit [Default=9]

[NOTE: Logical Unit FT16 is not used with the GIFT5 Geometry Package. 
Table 6-7. Definitions of Vartables in USER COMMON.

\begin{tabular}{|c|c|c|}
\hline Number & Variable & Definition \\
\hline 1 & AGSTRT & $\begin{array}{l}\text { Initlal chronological age to be assigned to source } \\
\text { particles }\end{array}$ \\
\hline $\begin{array}{l}2 \\
3\end{array}$ & $\begin{array}{l}\text { WTSTRT } \\
\text { XSTRT }\end{array}$ & $\begin{array}{l}\text { Inttial weight to be assigned to source particles } \\
\text { Initial } x \text { position to be assigned to source } \\
\text { particles }\end{array}$ \\
\hline 4 & YSTRT & $\begin{array}{l}\text { Initial y position to be assigned to source } \\
\text { particles }\end{array}$ \\
\hline 5 & ZSTRT & $\begin{array}{l}\text { Inttial } x \text { position to be assigned to source } \\
\text { particles }\end{array}$ \\
\hline $\begin{array}{l}6 \\
7\end{array}$ & $\begin{array}{l}\text { DFF } \\
\text { EBOTN }\end{array}$ & $\begin{array}{l}\text { Normal ization for adjoint problems } \\
\text { Lower energy boundary (eV) of last neutron group } \\
\text { (group NMGP) }\end{array}$ \\
\hline 8 & EBOTG & $\begin{array}{l}\text { Lower energy boundary (eV) of last gamma-ray group } \\
\text { (group NMTG) }\end{array}$ \\
\hline $\begin{array}{l}9 \\
10 \\
11 \\
12 \\
13 \\
14 \\
15 \\
16\end{array}$ & $\begin{array}{l}\text { TCUT } \\
10 \\
\text { I1 } \\
\text { IADJM } \\
\text { NGPQT1 } \\
\text { NGPQT2 } \\
\text { NGPQT3 } \\
\text { NGPQTG }\end{array}$ & $\begin{array}{l}\text { Chronological age limit } \\
\text { Logical unit for output } \\
\text { Logical unit for Input } \\
\text { Adjoint switch ( } 0 \text { for adjoint problem) } \\
\text { Problem-dependent energy group I imit } \\
\text { Problem-dependent energy group } 11 \text { imit } \\
\text { Problem-dependent energy group } 1 \text { imit } \\
\text { Group number of lowest energy gamma-ray group } \\
\text { to be treated }\end{array}$ \\
\hline 17 & NGPQTN & $\begin{array}{l}\text { Group number of lowest energy neutron group to } \\
\text { be treated }\end{array}$ \\
\hline $\begin{array}{l}18 \\
19\end{array}$ & $\begin{array}{l}\text { NITS } \\
\text { NLAST }\end{array}$ & $\begin{array}{l}\text { Number of batches to be run } \\
\text { Last cell in BLANK COMMON used by random walk } \\
\text { package }\end{array}$ \\
\hline $\begin{array}{l}20 \\
21 \\
22 \\
23\end{array}$ & $\begin{array}{l}\text { NLEFT } \\
\text { NMGP } \\
\text { NMTG } \\
\text { NSTRT }\end{array}$ & $\begin{array}{l}\text { Number of cells in BLANK COMMON avatlable to user } \\
\text { Number of primary energy groups } \\
\text { Total number of enlergy groups } \\
\text { Number of source particles for each batch }\end{array}$ \\
\hline
\end{tabular}




\subsection{REFERENCES}

1. M. B. Emmett, "The MORSE Monte Carlo Radiation Transport Code system," ORNL-4972. (1975), ORNL-4972/R1 (1983), ORNL-4972/R2 (1984), Oak Ridge Nattonal Laboratory.

2. K. D. Lathrop, "DTF-IV, A FORTRAN-IV Program for Solving the Multigroup Transport Equation with Ant sotropic Scattering," L.A-3373, Los Alamos National Laboratory, (1965).

3. W. W. Engle, Jr., "A USER'S MANUAL FOR ANISN, A One-Dimensional Discrete-Ordinates Transport Code with Anisotropic Scattering," K-1693, Oak Ridge National Laboratory, (March 1967).

4. W. A. Rhoades and F. R. Mynatt, "The DOT III Two-Dimensional Discrete Ordinates Transport Code," ORNL-TM-4280, Oak Ridge National Laboratory, (September 1973).

5. W. A. Rhoades, D. B. Simpson, R. L. Childs, and W. W. Engle, Jr. "The DOT IV Two-Dimenstonal Discrete Ordinates Transport Code with Space-Dependent Mesh and Quadrature," ORNL/TM-6529, Oak Ridge National Laboratory (January 1979).

6. W. A. Rhoades and R. L. Childs, "An Updated Version of the DOT 4 One- and Two-Dimensional Neutron/Photon Transport Code," ORNL-5851, Oax Ridge National Laboratory (1982).

7. E. A. Straker, W. H. Scott, Jr., and N. R. Byrn, "The MORSE Code with Combinatortal Geometry," Defense Nuclear Agency DNA 2860T, SAI-72-511-LJ, Science Appitcations International Corporation, (May 1972).

8. Lawrence W. Bain, Jr, and Mathew J. Reisinger, "The GIFT Code User Manual; Volume 1. Introduction and Input Requirements," BRL 1802, Ballistic Research Laboratory, (Juty 1975).

9. Gary G. Kueh1, Lawrence W. Bain, Jr. and Mathew J. Reisinger, "The GIFT Code User Manual; Volume II. The Output Options," ARBRLTR-02189, Ballistic Research Laboratory, (September 1979).

10. A. E. Rainis and Ralph E. Rexroad, "MIFT: GIFT Combinatorial Geometry Input to VCS Code," BRL Report No. 1967, Ballistic Research Laboratory, (March 1977).

11. "The GIFT5 Geometry Package", Ballistic Research Laboratory, (No formal documentation exist for GIFT5.)

12. D. C. Irving, R. M. Freestone, Jr. and F. B. K. Kam, "05R, A General Purpose Monte Carlo Neutron Transport Code," ORNL-3622, Oak Ridge National Laboratory, (1965). 
13. C. L. Thompson and E. A. Straker, "06R-ACTIFK, Monte Carlo Neutron Transport Code," ORNL-CF-69-8-36, Oak Ridge National Laboratory, (1969).

14. D. C. Irving, "Description of the CDC-1604 Version of the O6R Neutron Monte Car10 Transport Code," ORNL-TM-3518, Oak Ridge National Laboratory, (1971).

15. C. E. Burgart and E. A. Straker, "XCHEKR - A Multigroup Cross Section Editing and Checking Code, "ORNL-TM-3518, Oak Ridge National Laboratory, (1971).

16. W. H. Scott, Jr., "Vehicle Code System (VCS) Documentation and Uncertainty Analysis, "SAI Report SAI-133-79-977-LJ, Science Applications International Corporation, (December 1979).

17. W. H. Scott, Jr., and V. E. Staggs, "Adjoint Energy Biasing and Thermal Neutron Diffusion in the MORSE and VCS Codes," SAI-133-81-384-LJ Science Applications International Corporation, (November 1981).

18. W. H. Scott, Jr., et a1., "Predictive Algorithm for Radiation Protection," Volume 1, "VCS In-Group Energy Bias, The DACM Code and DOT Calculations," SAIC-85/1710, Sciance Applications International Corporation, (May 1985).

19. J. A. Stoddard, S. D. Egbert, and W. H. Scott, Jr., "The Vehicle Code System with In-Group Energy Bias and GIFT5 Geometry," DNATR-87-23, Science Applications International Corporation, (January 1987).

20. W. A. Rhoades and M. B. Emmett et al., "Vehicle Code System (VCS User's Manual)," ORNL/TM-4648, Oak Ridge National Laboratory (August 1974).

21. W. A. Rhoades et al., "Development of a Code System for Determining Radiation Protection of Armored Vehicles (The VCS Code)," ORNL/TM-4664, Dak Ridge National Laboratory, (October $1974)$.

22. J. 0. Johnson, J. D. Drischler, and J. M. Barnes, "Analys is of the Fal1-1989 Two-Meter Box Test Bed Experiments Performed at the Army Pulse Radiation Facility (APRF)," ORNI./TM-11777, Oak Ridge National Laboratory, (May 1991).

23. K. T. Santoro et a1., "DNA Radiation Environments Program Fall 1989 2-Meter Box Experiments and Analysis," ORNL/TM-11840, Oak Ridge National Laboratory, (May 1991). 


\subsection{SAMPLE PROBLEM}

The sample problem demonstrates the adjoint Monte Carlo analys is of the two-meter box experiments.22,23 The two-meter box, i.e., "NATO standard test bed" is a large cubical steel walled box with a layer of $5.08-\mathrm{cm}-$ thick steel plates mounted on the top, bottom, and all four sides. The exterior set of plates yield overall outside dimensions for the box of $220.32 \mathrm{~cm} \times 220.32 \mathrm{~cm} \times 220.32 \mathrm{~cm}$, and are removable. The interior steel box has outside dimensions $210.16 \mathrm{~cm} \times 210.16 \mathrm{~cm} \times 210.16 \mathrm{~cm}$ with each wall having a thickness of $5.08 \mathrm{~cm}$. This yields an interior air space with dimensions of $200 \mathrm{~cm} \times 200 \mathrm{~cm} \times 200 \mathrm{~cm}$ and thus gives the test bed the common name - "the two-meter box." The box also contains lift tabs (for movement by crane), drainage holes at the base, a cable access hole at the base on the back side of the box, and two hatches. The hatches are located in the center of the top and back faces of the box and the hatch diameters in the interior box and outside plates are staggered to mitigate radiation streaming paths into the box. The hatches are included for loading and unloading experimental equipment, e.g., detectors, phantoms, etc., and for simulating open-hatch vehicle experiments. An isometric view of the two-meter box test bed is shown in Figure 6-1.

A complete ilsting of the input cards for the sample problem is given in Figure 6-2, and Figure 6-3, and some selected output is shown in Figure 6-4. Figure 6-2 represents the input on logical unit 5 which contains the random walk input, the GIFT5 geometry cards (GA, GH, and GI), the cross-section input, and the special MASH input for output to DRC. Figure 6-3 illustrates the GIFT5 geometry input read on logical unit 8 for the Two-Meter Box for geometry Cards GB, GC, GD, GE, and GF). In viewing Figure 6-2, the input illustrates; the descriptive title for the MORSE case, 1000 particle per batch (NSTRT), 50 batches (NITS), and a maximum number of particles to be stored in the banks of 1500 (NMOST). Only one set of 50 batches was to be run (NQUIT). The energy group numbers being analyzed include 46 neutron groups (NGPQTN), 23 gamma groups (NGPQTG), 46 primary particle groups (NMGP), and 69 total groups (NMTG). The adjoint mode switch was set to one (IADJM=1 always), 120 minutes of CPU time was allowed (MAXTIM), and there were four cross section media for the problem (MEDIA).

The source was sampled from the distribution on Cards El (ISOUR=0, and NGPFS $=69$ ). Also, the input source energy spectrum was biased (ISBIAS=1) and the biasing parameters were read from Cards E2. Each starting particle had an initial weight of 1.0 (WTSTRT), and was initiated from the $X, Y, Z$ position corresponding to $(0.0,0.0,110.16 \mathrm{~cm})$ in the geometry. Since UINP, VINP, and WINP were all zero, the source was assumed to be isotropic. The energy groilp boundaries are read from Cards F1. Card G was omitted because no collision tape was requested (NCOLTP $=0$ on Card B). After the energy group boundaries, the starting random number seed is input (Card $H$ ).

The next input card (Card I) indicates the splitting and Russian roulette are to be used (NSPLT and NKILL $=1$ ) on four importance regions 
(MXREG $=4$ ) for al1 69 energy groups (MAXGP=69). Furthermore, in-group energy biasing is turned on (INGB=1).

Four Card J's are inpu't, one for each importance region, to give the weight standards for the splitting and Russian roulette. MXREG could have been defined as 3 since the second and third Card $J$ are identical. Then the second three parameters (NRG1, NDRG, NRG2) on the second Card $J$ would have read $\left(\begin{array}{lll}2 & 1 & 3\end{array}\right)$. This input is terminated by the -1 for NGPI on the last Card $\mathrm{J}$.

Card $K$ is omitted because IEBIAS was equal to zero. All parameters on Card $L$ are zero for this problem and consequently, Card $M$ and Card $N$ are not required. Card 0 is input four times, one for each importance region (MXREG on Card I). In this example, a GWLO parameter was set to 1.0 for all regions and all groups. This has proven to be the most efficient use of this parameter with in-group biasing turned on.

The GIFT5 program control card (Card GA) is next in the input stream, with the first 10 control parameters set to zero, the tolerances set to 1.0 and the final three parameters set to zero. If the last three parameters are set to zero, GIFT5 will use the defaults.

This input is followed by the geometry region material specifications cards (Cards GH) and importance region assignments card (Cards GI). The other GIFT5 geometry input is read from logical unit 8 and is presented in Figure 6-3. There are five sets of material region specification cards, four for the four different cross section materials, and one for the external void (MED=0). Even though there are four importance regions specified on Card 1 , only one region was used in the calculation since the Card GI parameters are all ones. There are 24 entries on the Cards GI corresponding to the number of regions in the GIFT5 geometry.

The next section of input is the cross section Cards $X A$ through $X G$. The energy group parameters (on Card $X B$ ) match the parameters read in Card $B$. There are four cross section media (NMED), 24 elements (NELEM) to be read in from logical unit 10 (IXTAPE), 41 mixing operations (NMIX) to be performed and an output file written to logical unit 2 (JXTAPE). The cross section set was $P_{5}, \quad(N C O E F=6)$, and the number of discrete angles was 3 (NSCAT=NCOEF/2). Al1 of the cross-section print triggers on Card $X C$ are turned off. The element identifiers (a $11 P_{i}$ components) are entered on the Cards XD. There are 144 untries on these cards (NCOEF times NELEM). Card XE is omitted since the input ANISN library is on unit IXTAPE. The next 41 cards correspond to the mixing table on card $X F$. The four different materials are constructed on these cards.

Finally, the input in Figure 6-2 shows the special MASH input for use with DRC. This includes the short title card (40 characters) on Card $A A$, the importance region density factors (a)1 1.0 in this problem) on Card $B B$, and the air and ground identifiers ( 3 and 1 , respectively) on Card CC. 
Figure 6-3 illustrates the five GIFT5 geometry input Cards GB through GF read in on logical unit 8 . The title card indicates units of centimeters and the target. The second card indicates 49 solids in the solid table, and 24 regions in the region table. The 49 solids follow (Cards GD) and then the 24 regions (Cards GE) Note the "-1" card to end the region table. Finally, the input shows the region identification table (24 cards, one for each region) The only information used from this card is the first column.

The coupling surface used in the MORSE sample calculation to tabulate the adjoint leakage current was a RPP centered about the box (solid 1 in the solid table). Other coupling surfaces such as a sphere, right circular cylinder, etc., could be used also. The only assumption is that the VISTA free-field fluence at a given height does not vary between $X-Y$ positions in the vehicle coordinate system. In order to include the parificle leakage from the top and bottom of the vehicle, the RPP extended from below the air-ground interface to several centimeters above the top surface of the venicle. The coupling surface is aiways the exterior boundary of the Monte Carlo geometry, i.e. where the particle enters the external void. Adjoint particles that cross this surface are terminated and recorded on the history data set.

The selected MORSE output shown in Figure 6-4 first illustrates the input parameters read in the first four input data cards from logical unit 5 , followed by tables of the input source spectrum and biasing parameters (Cards El and E'? and the input energy group boundaries (Cards F) This is followed by the splitting and kussian roulette parameters for the different importance regions (Cards I and J) and the secondary production parameters by energy group for the importance regions (Cards $0)$. The next sections of input are from the GIFT5 geometry package and give detailed edits of the geometry input data on logical unit 8 . This print can be suppressed by setting the NOPRNT switch on Card GA to one. The geometry input array edits are followed by a storage summary for the geometry data, and then edits of the material region and importance region specification cards (Cards GH and GI).

The output in Figure 6-4 then edits the processing of the cross section input on Cards $X A$ through $X G$. On this sample problem, this information is suppressed. Large amounts of printout can be generated if the user is not careful with the print triggers on Card XC. It should be noted that the output shows a table of parameters that are read from tape and that the IXTAPE parameter in the "input/output options" table is set to -2 . The input in Figure 6-2 illustrates the input the first time the sample problem was executed. This output reflects that the crosssection set generated in that run was saved and input for this case. The input illustrates what is required for an initial case. Once that case is run, and the cross-section set is saved for future use, only Cards $X A, X B$, and $X C$ are required for the cross section input. Then the output would reflect what is being shown in the sample problem.

The cross section edits are followed by a statement informing the user of the last location used in blank common. This is an indication of how 
much core space the problem required based on the input received from the user. The normalized CDF for the adjoint source sampling is printed next, followed by the adjoint group structure. The next section of printout illustrates the actual random walk (by batch). Each batch will give the starting random number for the batch, the average source particles starting parameters, the time required to execute the batch, and a sequence of counters for the batch which correspond to the 13 positive arguments for subroutine BANKR 11 lustrated in Table 6-1 of Section 6.2.1 of this report. This information is useful in determining how well the user has set the problem up. Unfortunately, the best teacher in properly interpreting these counters is experience. The output also indicates several calls to subroutine RAYCHK, indicating an undefined region(s) in the geometry. This sequence of output is ended by the total time required to process all NITS batches.

The last section of output comes from the analys is routines which summarize the events that occurred in the random walk process for the entire run. The first table is a summary table of the particle deaths, labeled "neutron deaths" followed by a table of scatterings by medium. The next several pages of output are different counters (by importance region and group) for the entire random walk. These tables include the real scattering counters, track length counters, secondary production counters, number of splittings, number of upscatter splittings, number of Russian roulette kills, and the number of Russian roulette survivals. Unfortunately, like the batch summary counters, only experience will increase the users full understanding and interpretation of all these arrays. The last output line informs the user of the total amount of CPU required to run the problem. 


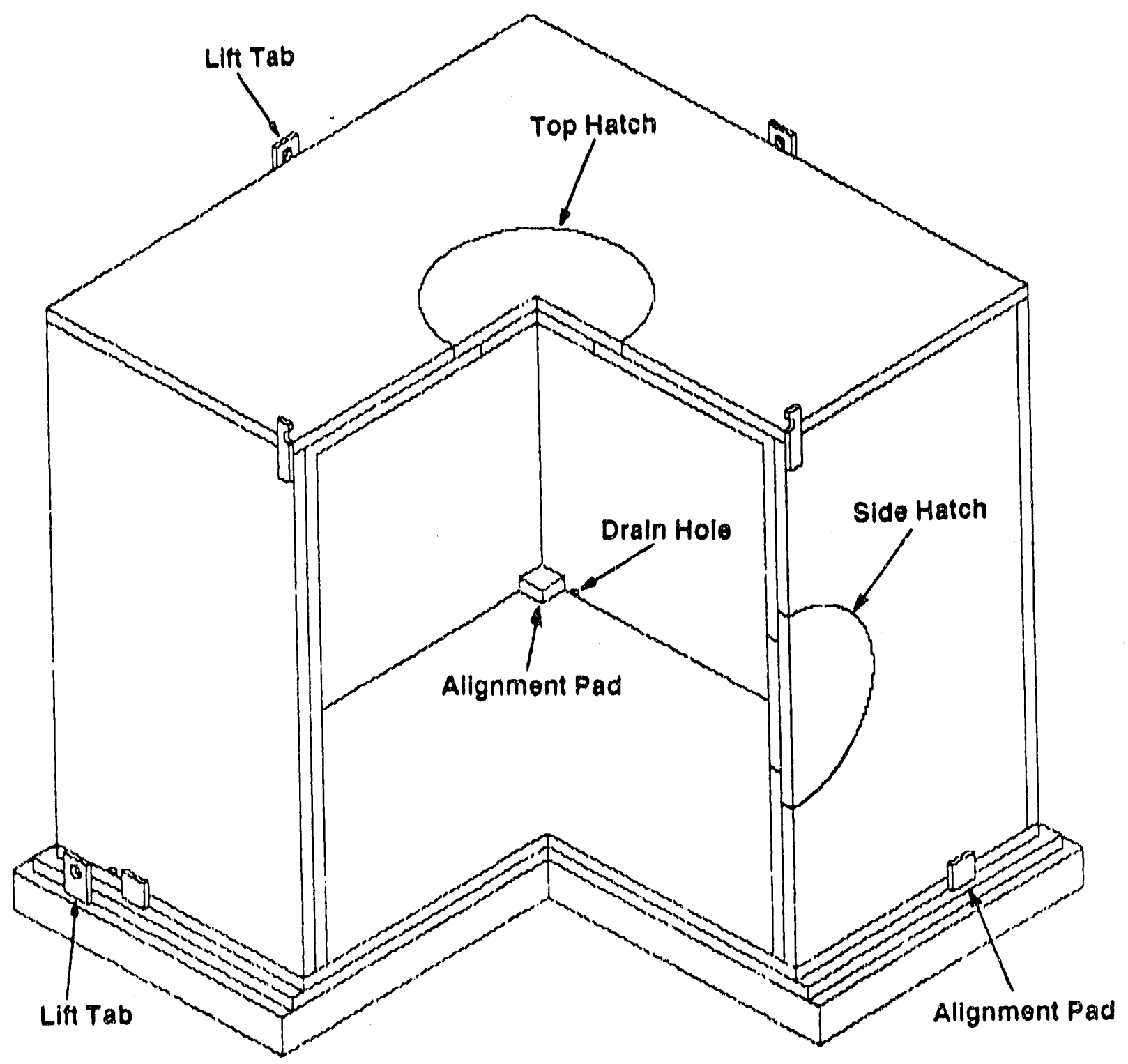

Figure 6-1. Schematic Diagram of the Two-Meter Box Geometry Model Used in the MORSE Analysis. 


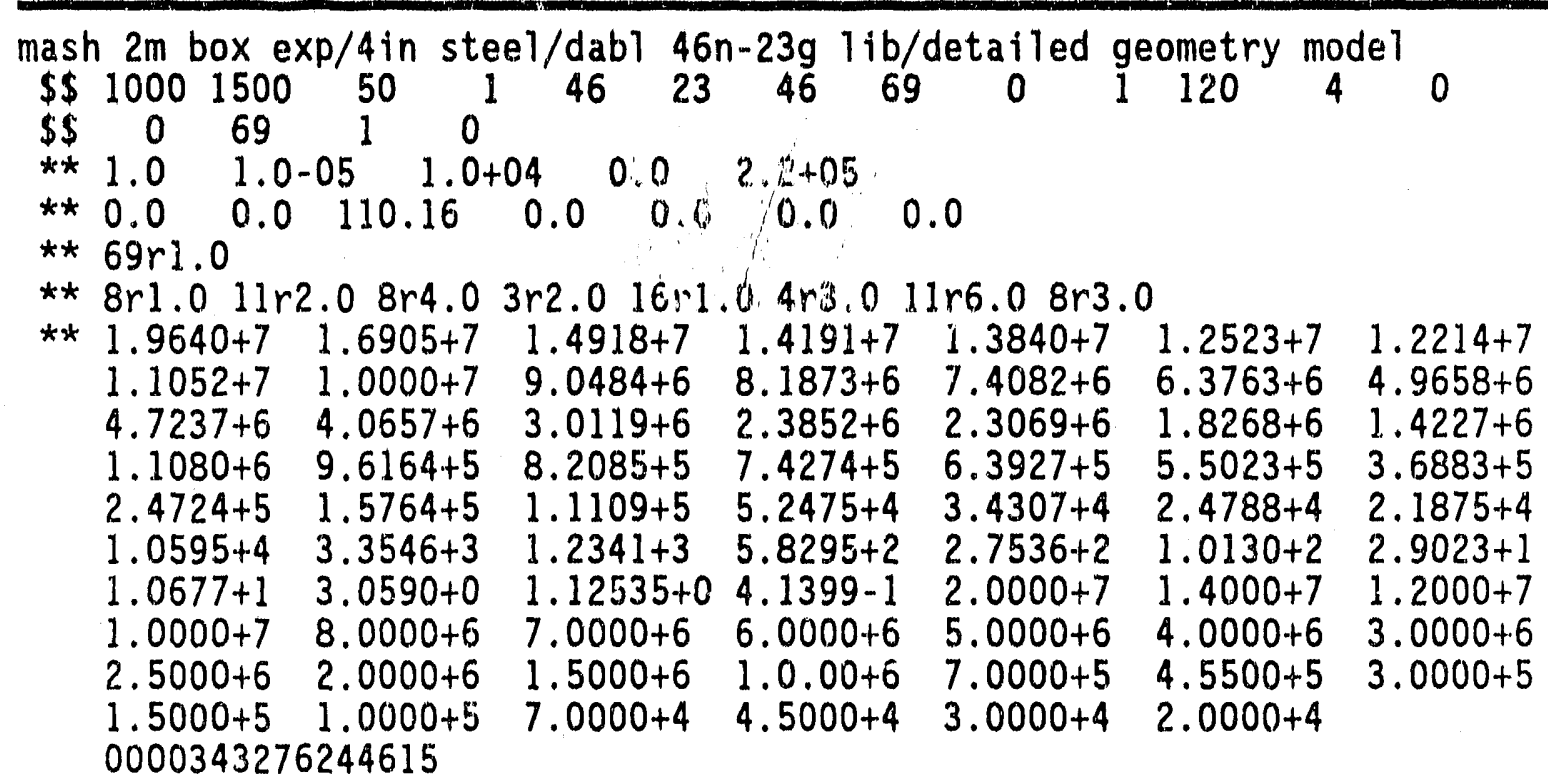

$\begin{array}{rrrrrrrrrrrr}\$ \$ & 1 & 1 & 0 & 0 & 0 & 4 & 69 & 1 & & & \\ \$ \$ & 1 & 1 & 69 & 1 & 1 & 1 & * * & 5.0+00 & 5.0-02 & 2.0-01 & 0.0 \\ \$ \$ & 1 & 1 & 69 & 2 & 1 & 2 & * * & 2.0+00 & 2.0-02 & 1.0-01 & 0.0 \\ \$ \$ & 1 & 1 & 69 & 3 & 1 & 3 & \star \star & 2.0+00 & 2.0-02 & 1.0-01 & 0.0 \\ \$ \$ & 1 & 1 & 69 & 4 & 1 & 4 & * * & 2.0+00 & 2.0-02 & 2.0-01 & 0.0 \\ \$ \$ & -1 & 0 & 0 & 0 & 0 & 0 & * * & 0.0 & 0.0 & 0.0 & 0.0 \\ \$ \$ & 0 & 0 & 0 & 0 & & & & & & & \end{array}$

** $23 r 1.0$

$\star * 23 r 1.0$

$\star * 23 r 1.0$

$\star \star 23 r 1.0$

0000000000

$\begin{array}{lllll}1.0 & 1.0 & 0 & 0 & 0\end{array}$

24

24

23

36

$\begin{array}{llllll}1 & 6 & 9 & 12 & 19 & 20\end{array}$

416

$\begin{array}{llllllllllllll}2 & 3 & 4 & 5 & 7 & 8 & 10 & 11 & 13 & 14 & 15 & 16 & 17 & 18\end{array}$

$21 \quad 22$

$\begin{array}{llllllllllllll}1 & 1 & 1 & 1 & 1 & 1 & 1 & 1 & 1 & 1 & 1 & 1 & 1 & 1\end{array}$

$2 m$ box exp/aprf ground (34\%), borated concrete, 10-24-89(b) air, 1020 stee

$\begin{array}{rrrrrrrrrrrrrrr}\$ \$ & 46 & 46 & 23 & 23 & 59 & 72 & 4 & 4 & 24 & 41 & 6 & 3 & 0 & 3 \\ \$ \$ & 0 & 0 & 0 & 0 & 0 & 0 & 0 & 10 & 2 & 0 & & & & \\ \$ \$ & 1 & 2 & 3 & 4 & 5 & 6 & 43 & 44 & 45 & 46 & 47 & 48 & 49 & 50 \\ & 51 & 52 & 53 & 54 & 55 & 56 & 57 & 58 & 59 & 60 & 61 & 62 & 63 & 64 \\ & 65 & 66 & 67 & 68 & 69 & 70 & 71 & 72 & 79 & 80 & 81 & 82 & 83 & 84 \\ & 85 & 86 & 87 & 88 & 89 & 90 & 91 & 92 & 93 & 94 & 95 & 96 & 97 & 98\end{array}$

Figure 6-2. Sample MORSE Input for the Two-Meter Box Analysis. 


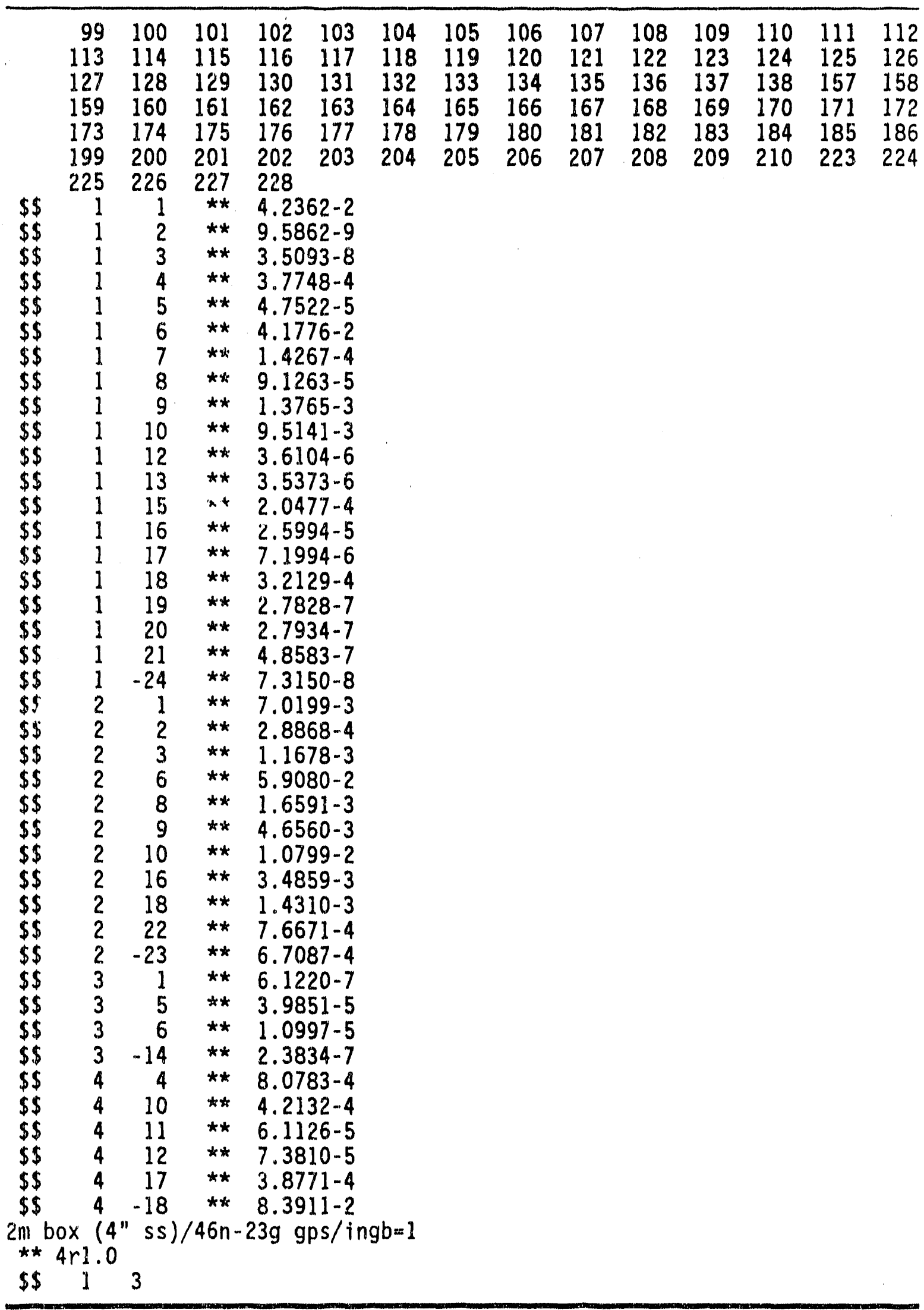

Figure 6-2. (continued) 
cii 2.0 meter cubic box geometry ( 4 inches of steel)

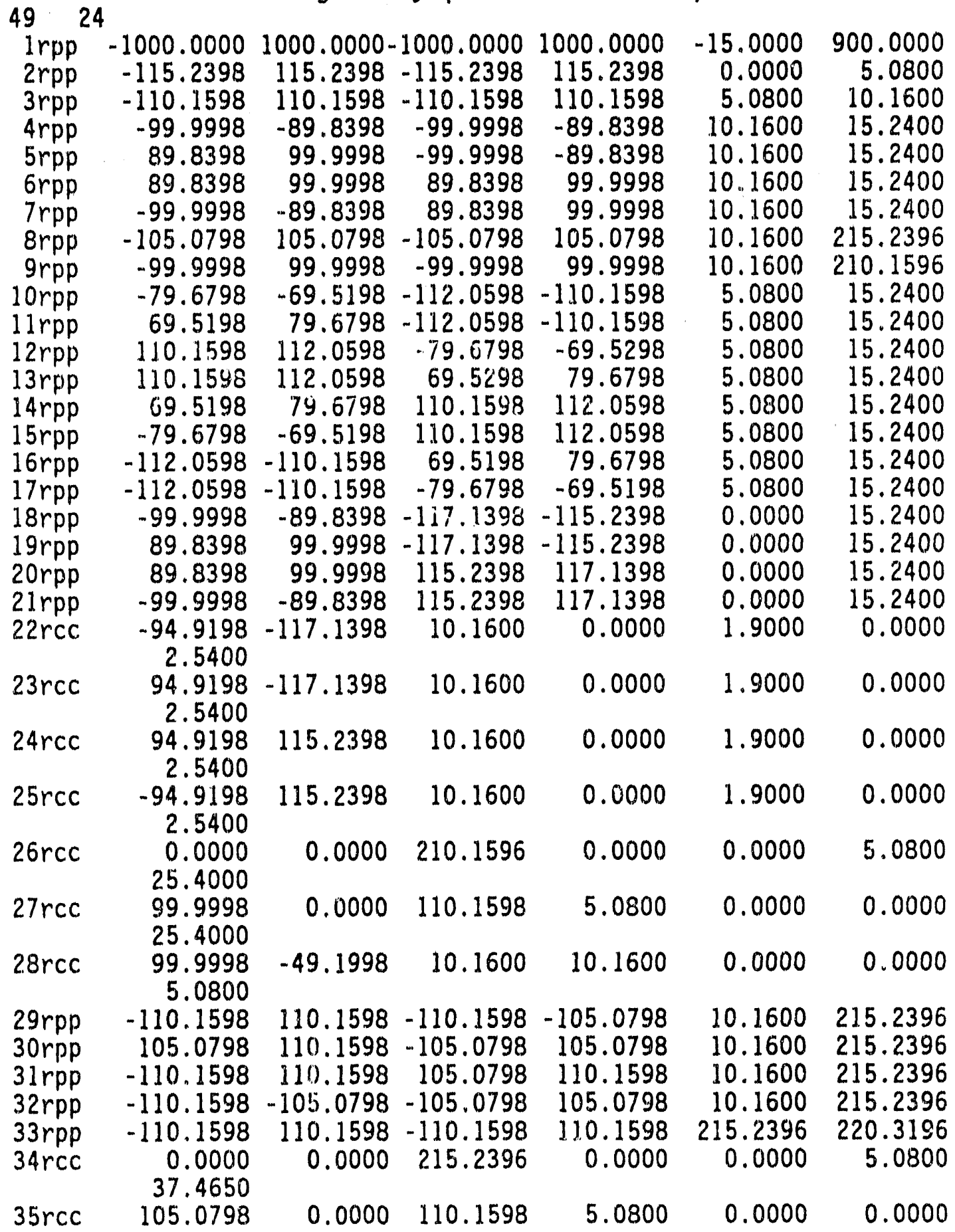

Figure 6-3. Sample GIFT5 Input for the Two-Meter Box Analysis. 


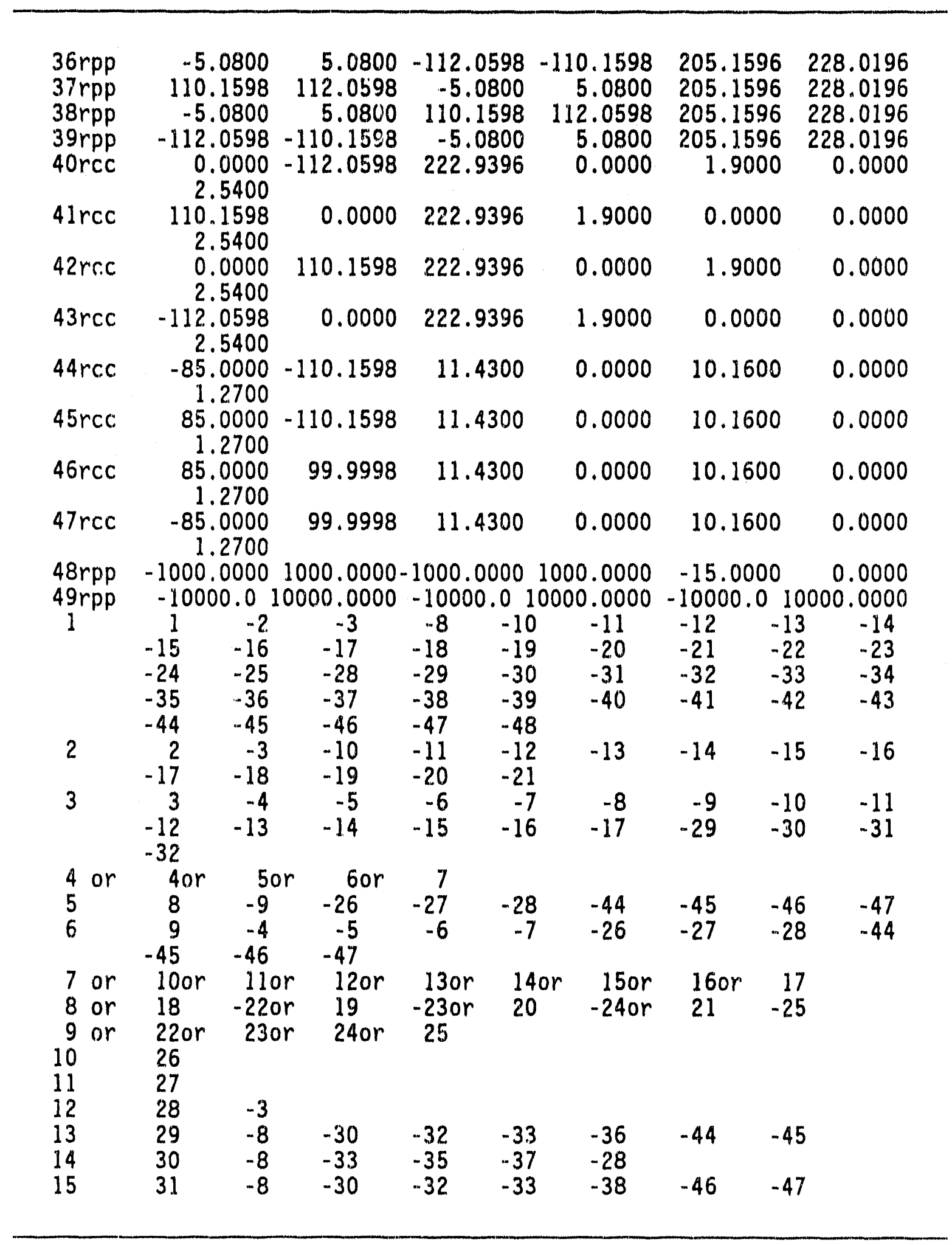

Figure 6-3. (continued) 


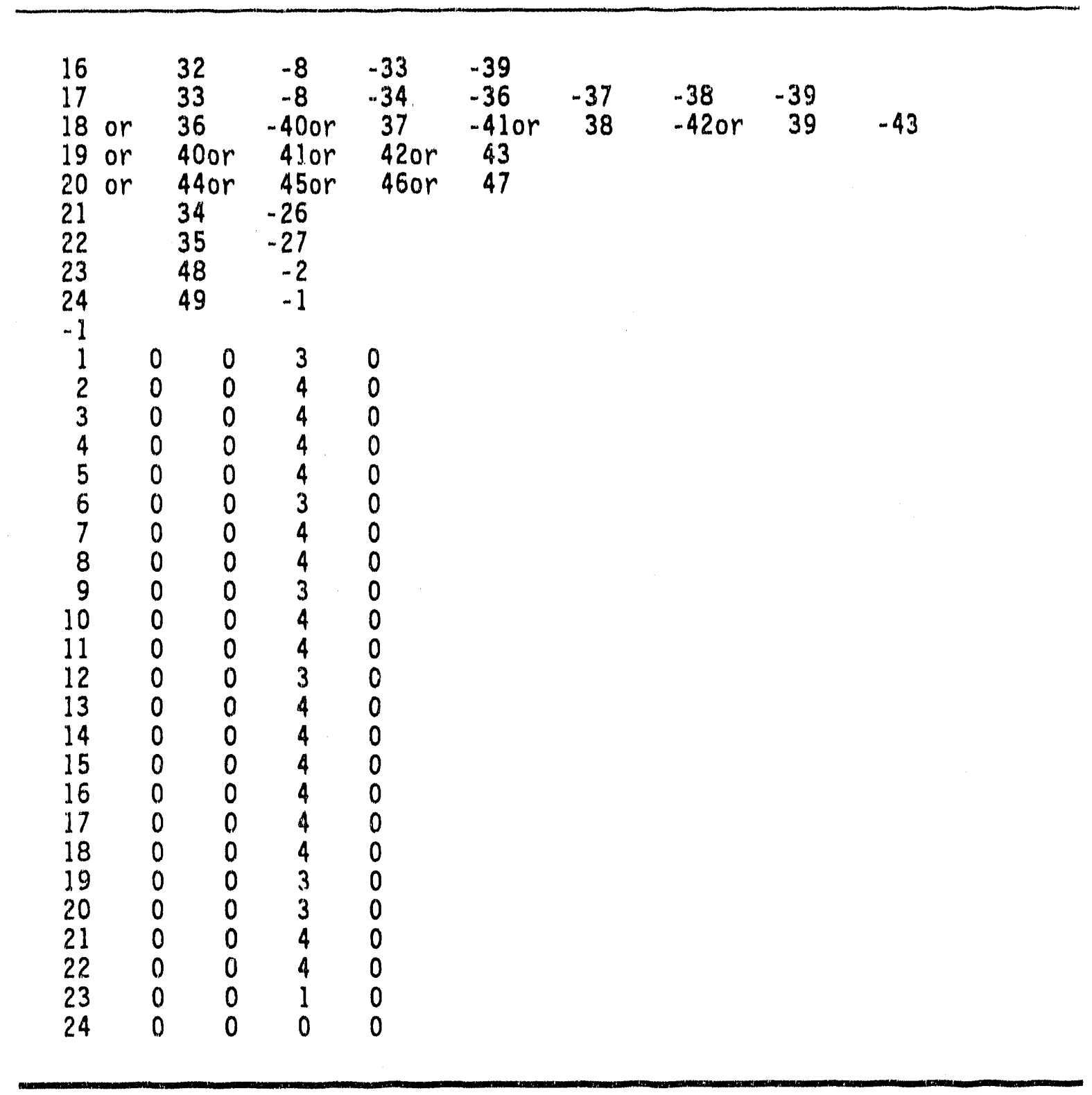

Figure 6-3. (continued) 


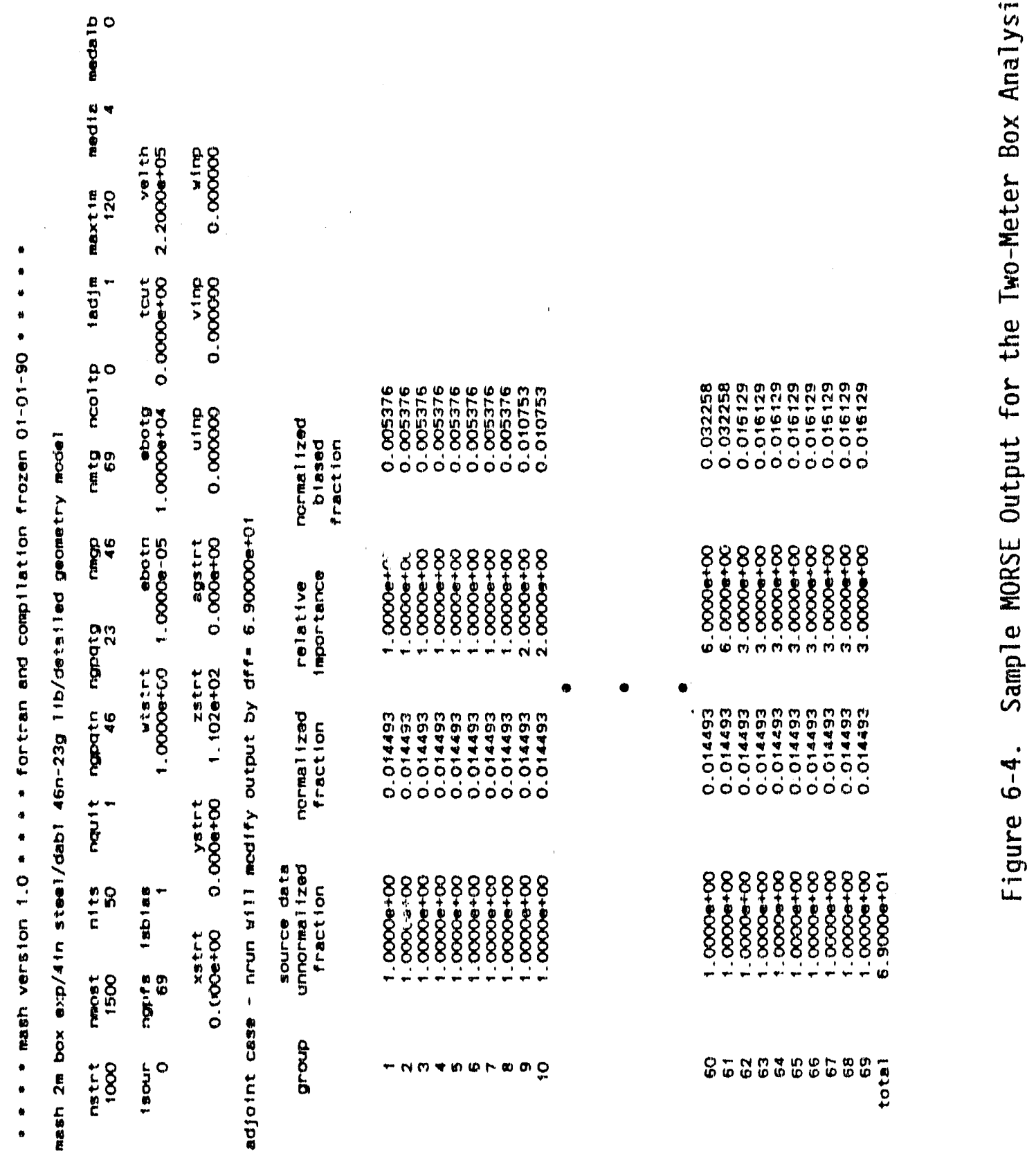





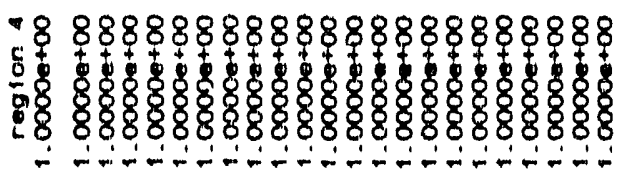

区్త్ర
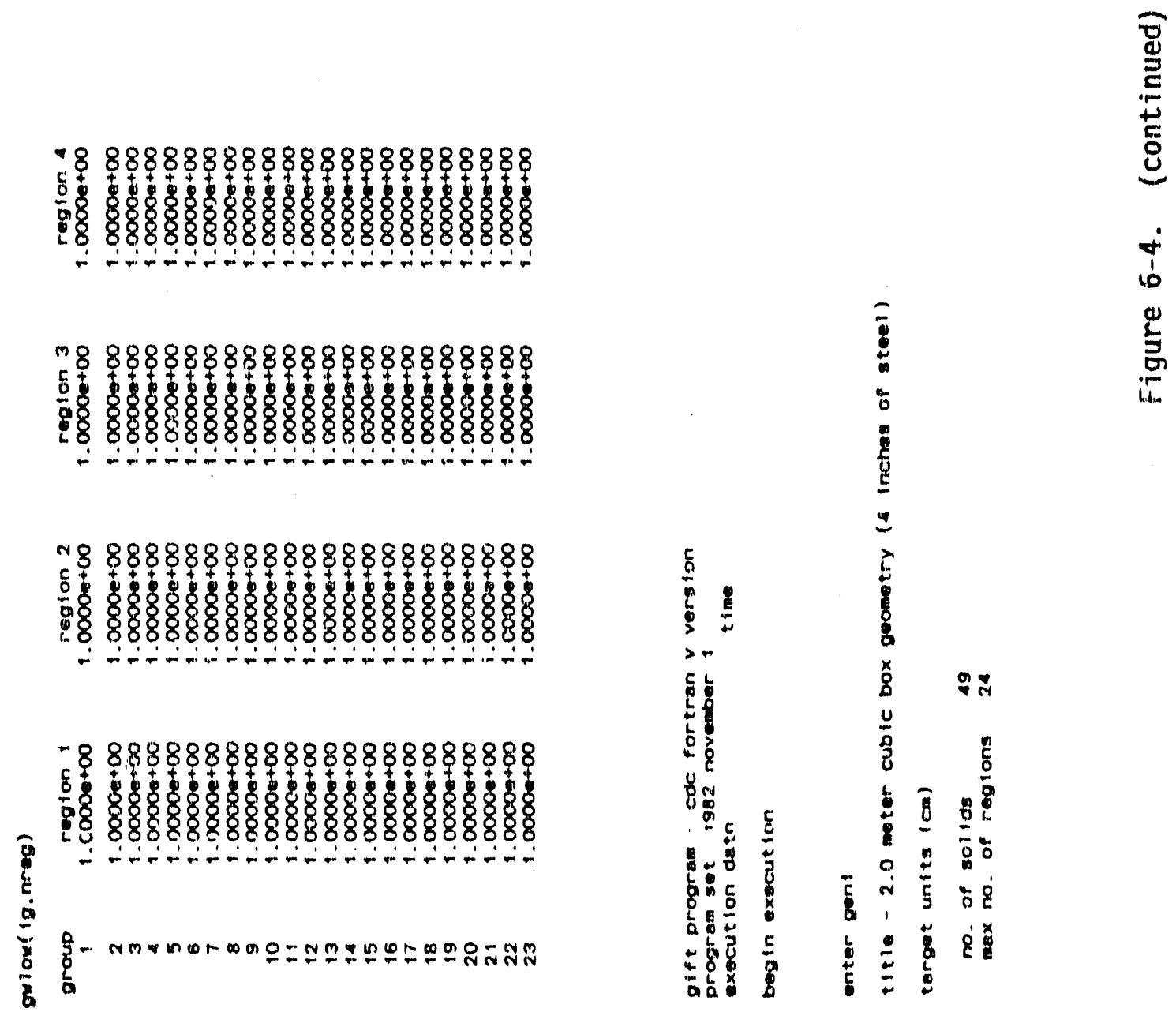


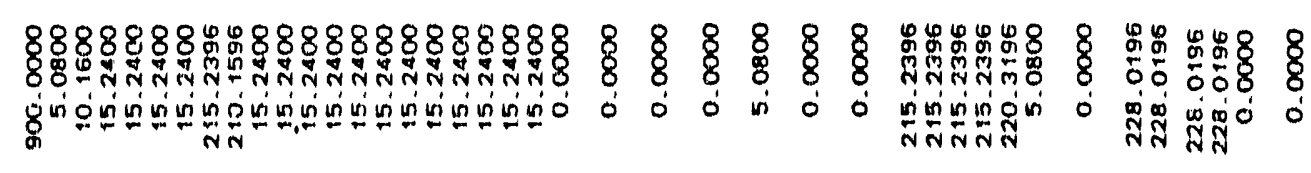

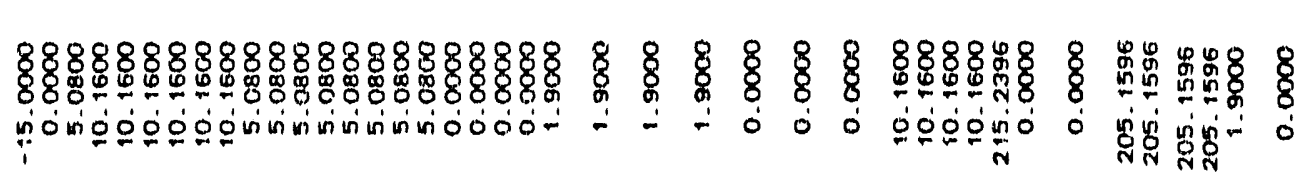

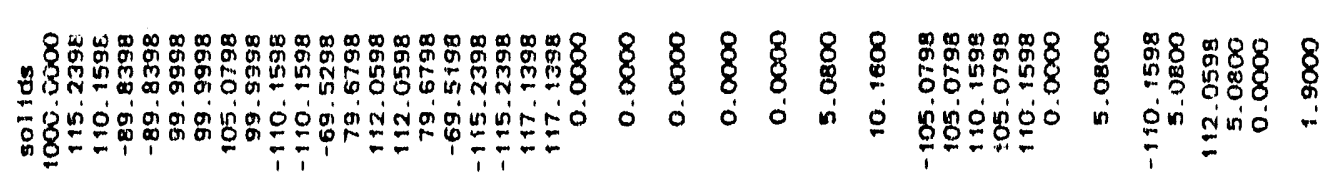
¿

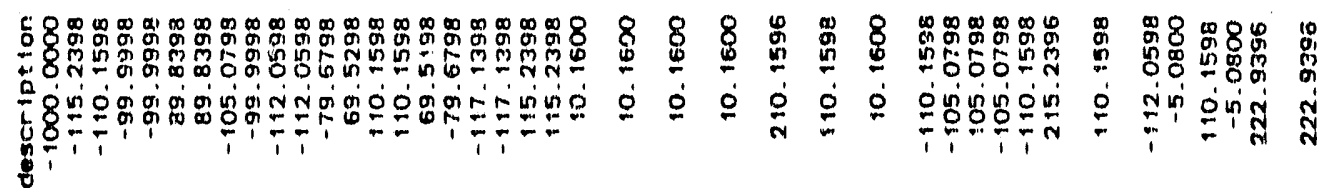

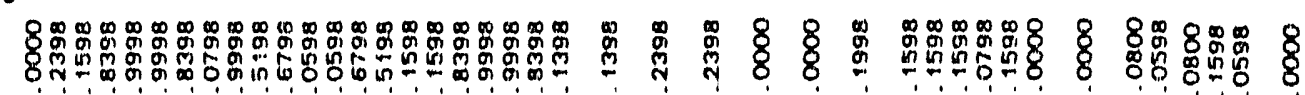

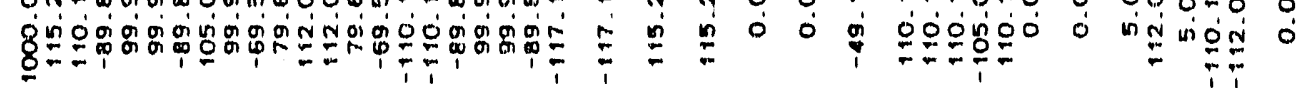

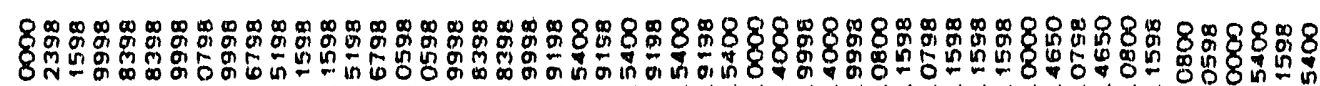
8ํำ

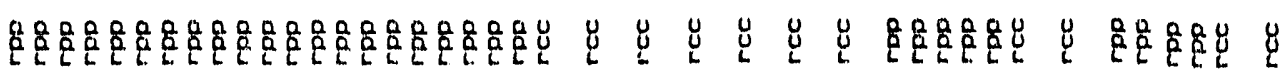

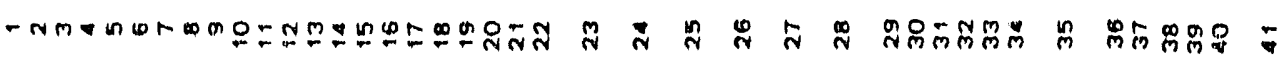

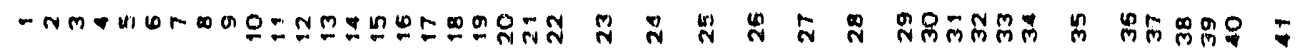




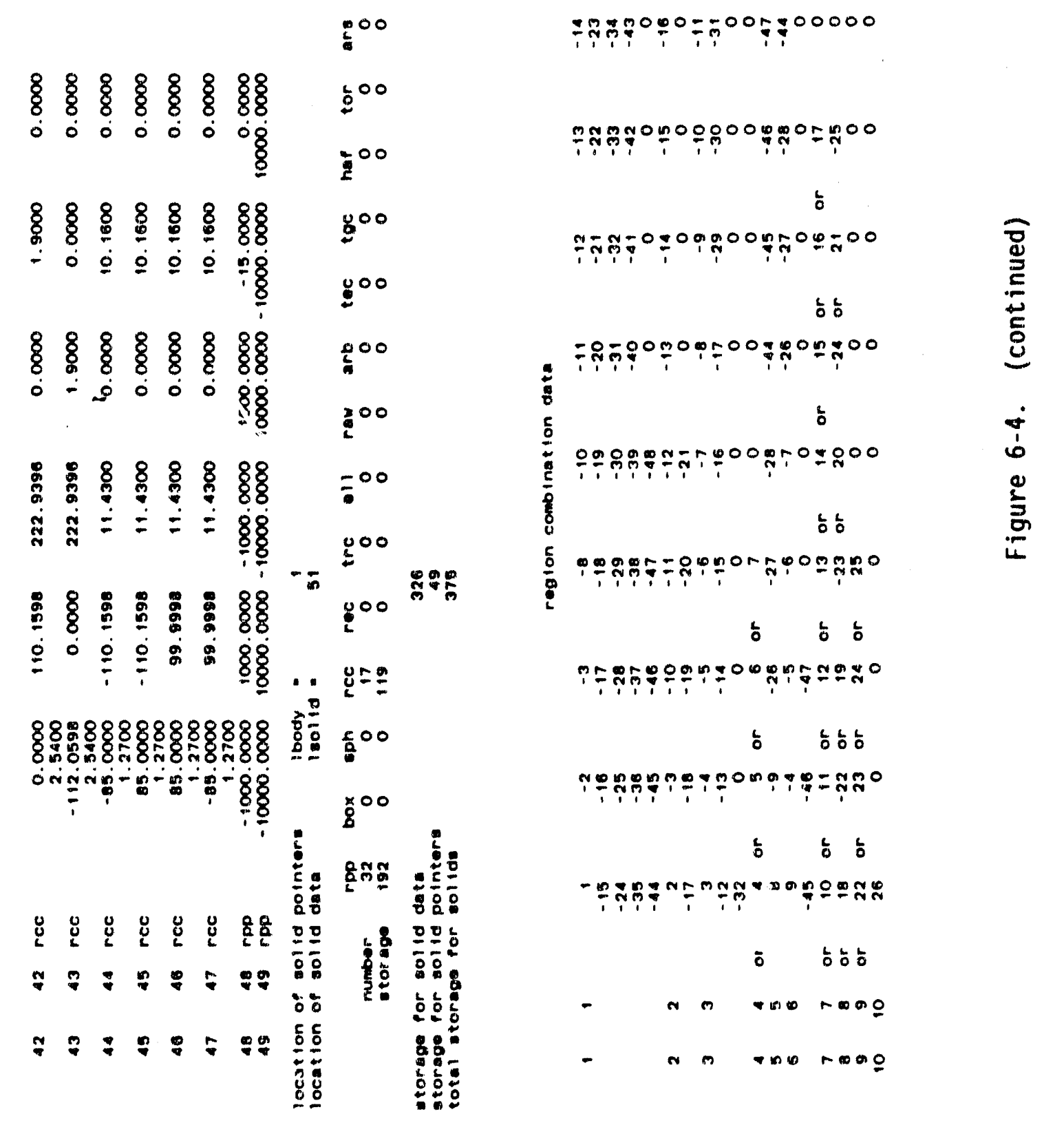


00000000000000

000000000000

00700090000000

L.

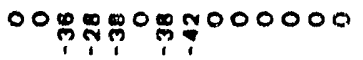

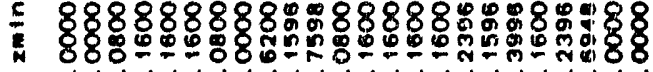

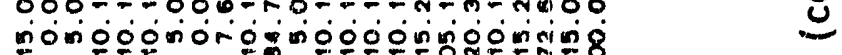

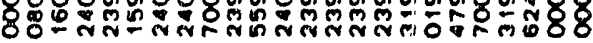

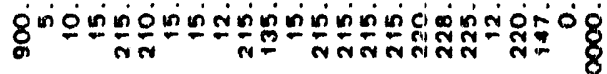

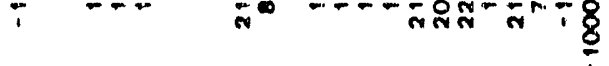

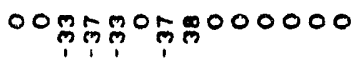

b

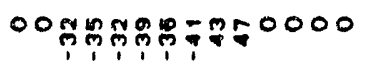

เั

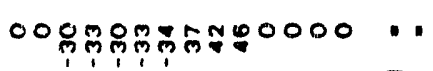

ㄴํำ

8:-

× 8 \%ํํำ

í

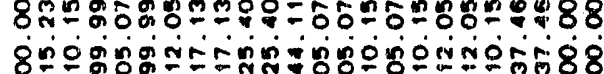

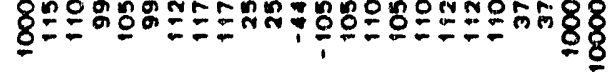

ำำำ

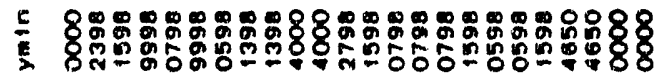

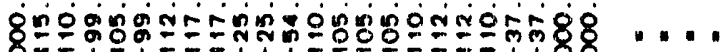

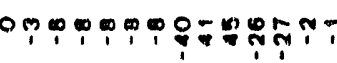

b́

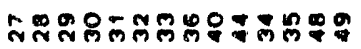

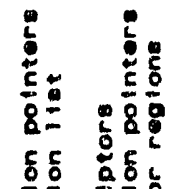

ㄴํㅇ

ช

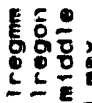

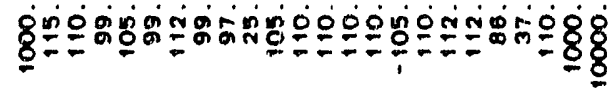

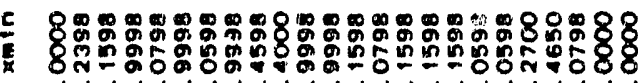

$\frac{5}{5}$

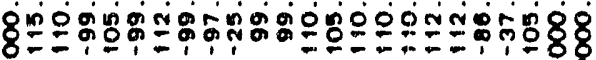

定: $=\frac{0}{9}$

동ำ

薄,

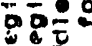

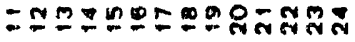

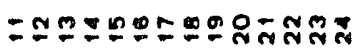

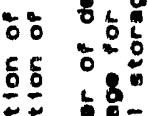

$\frac{6}{8}$

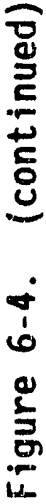

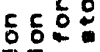

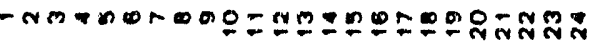




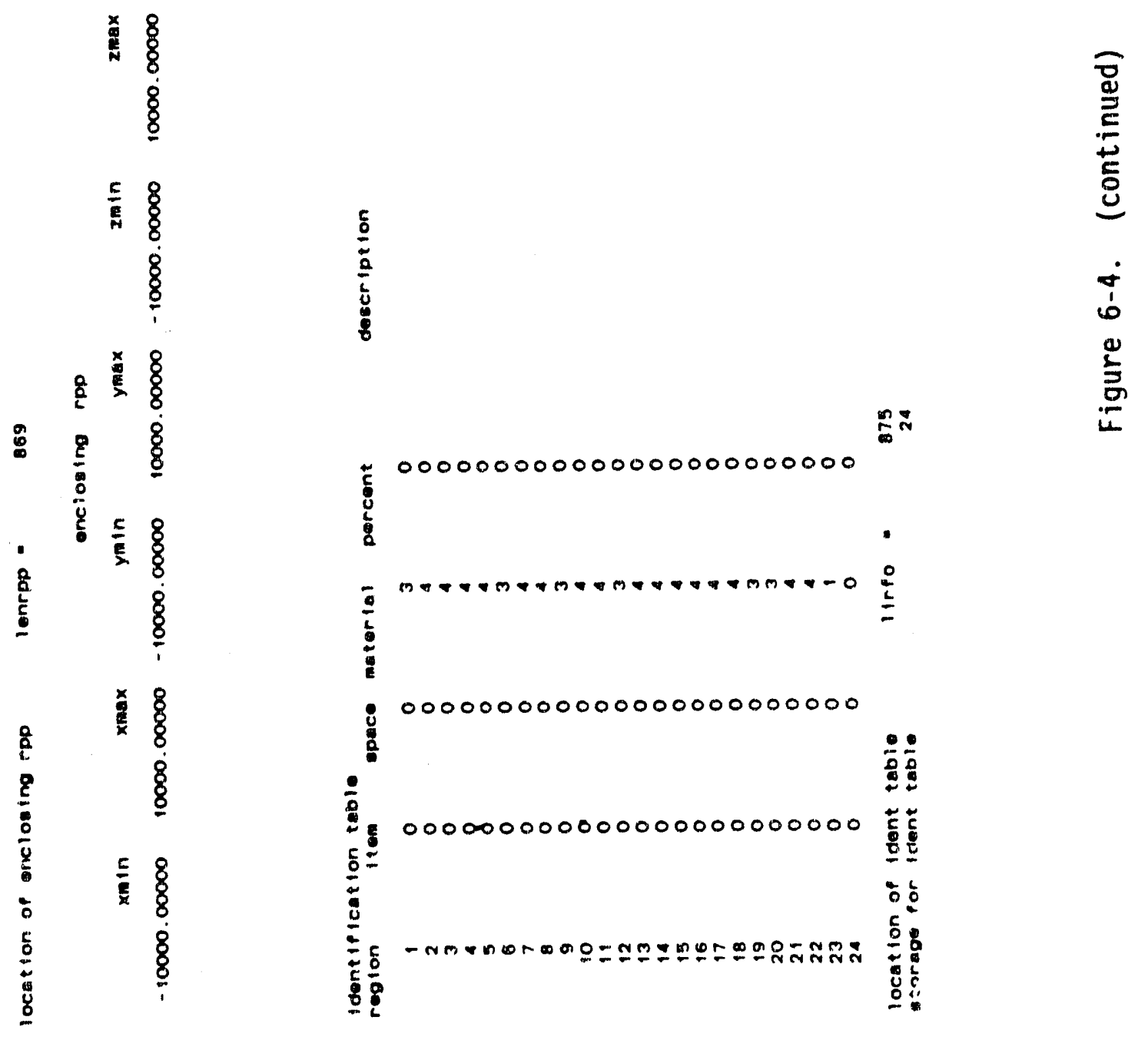




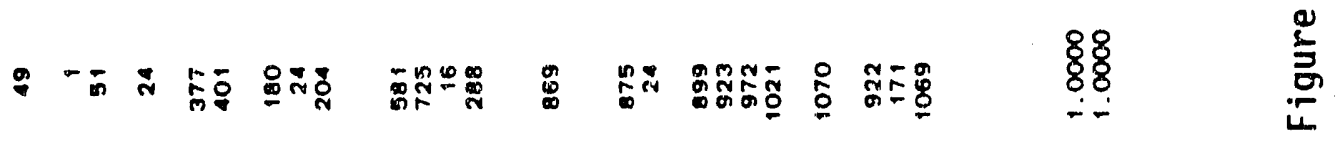

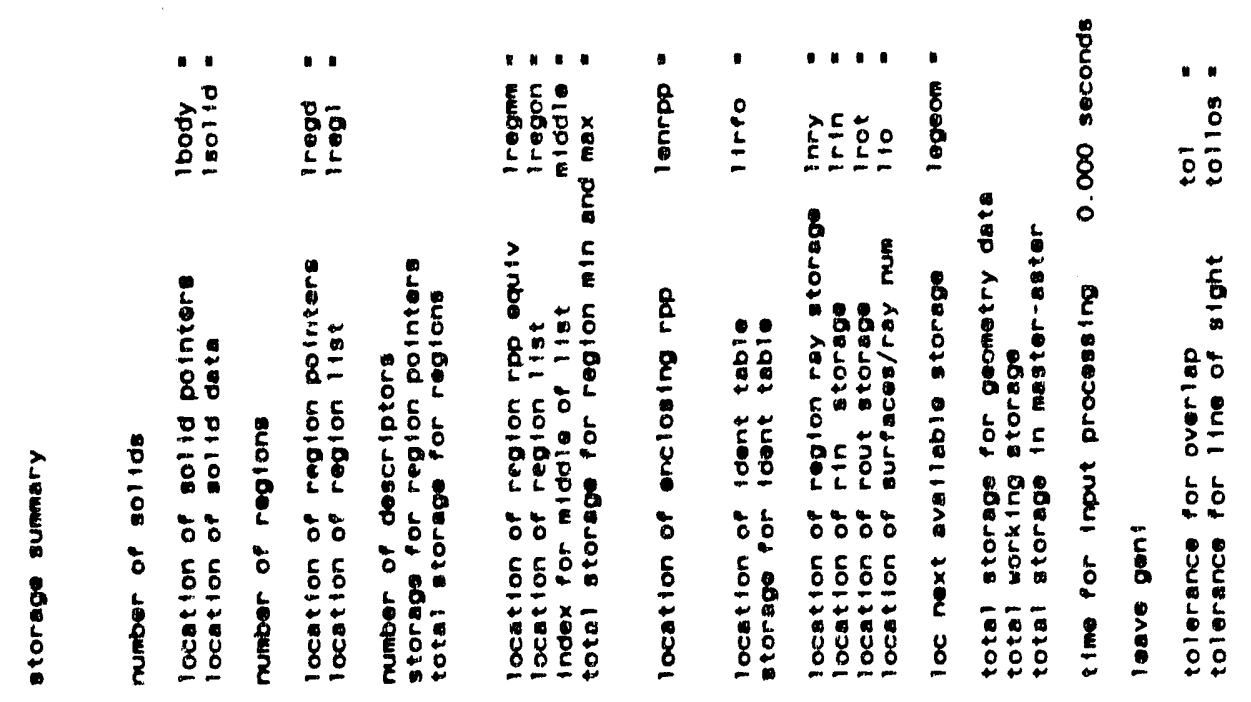




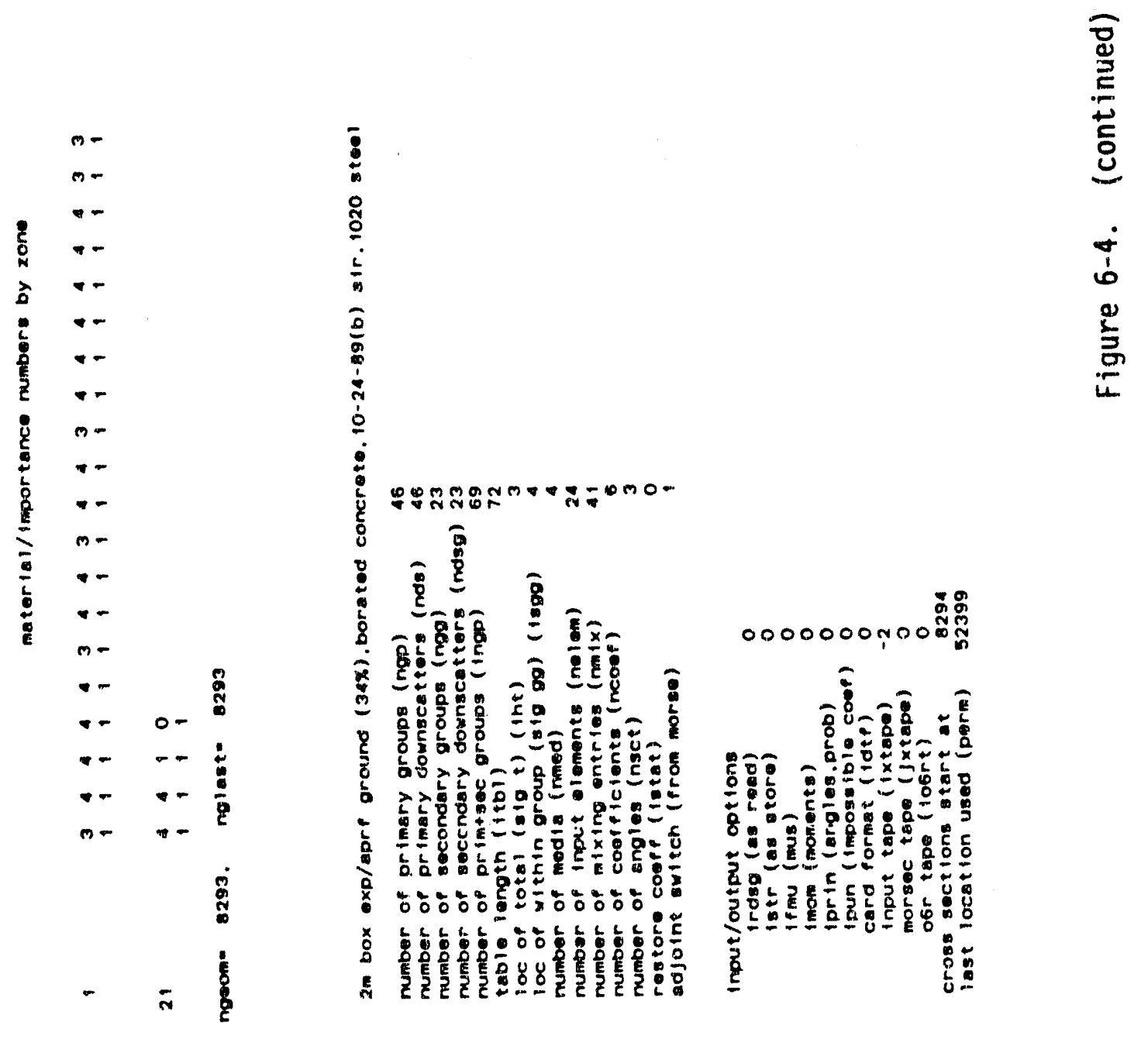




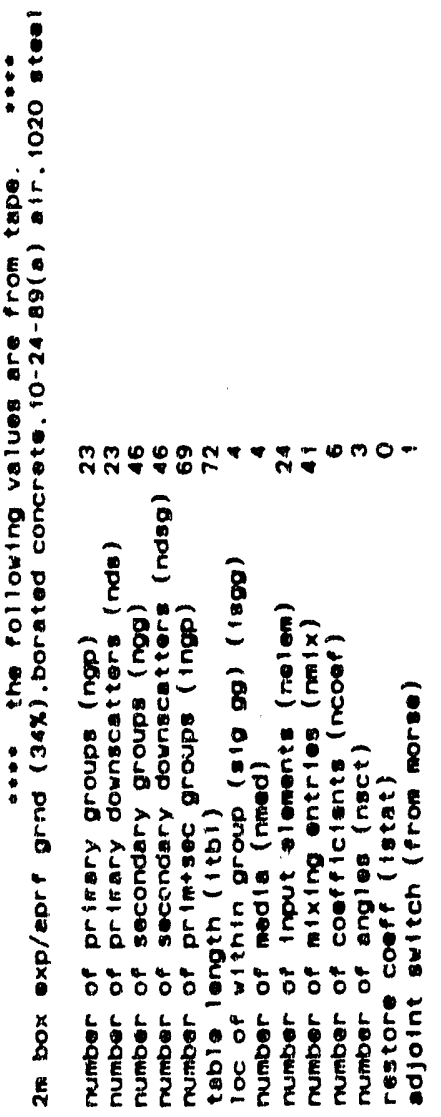

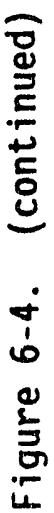

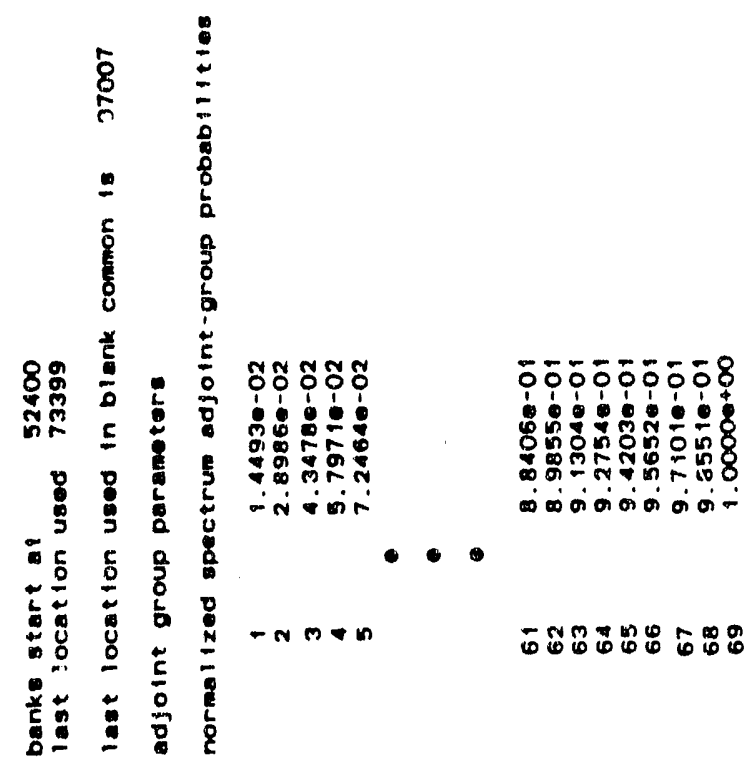



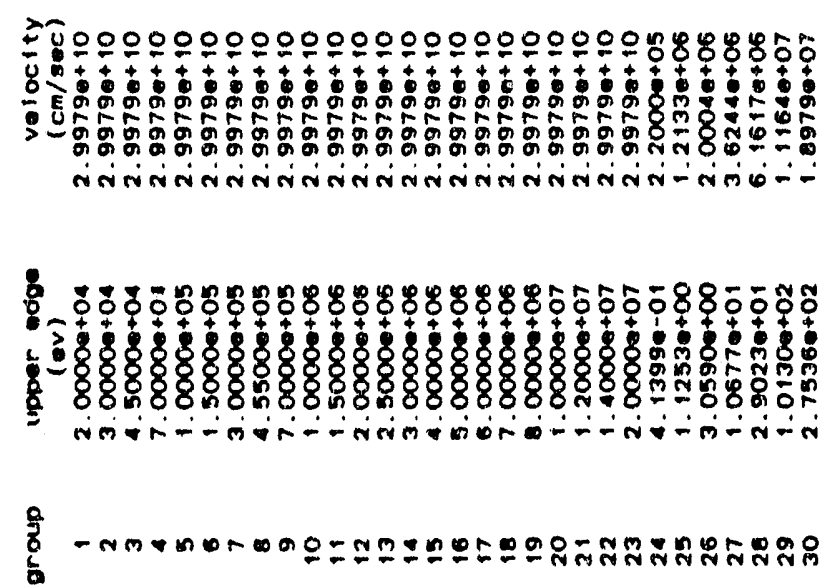

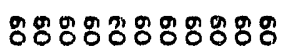

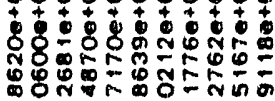

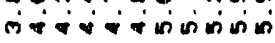

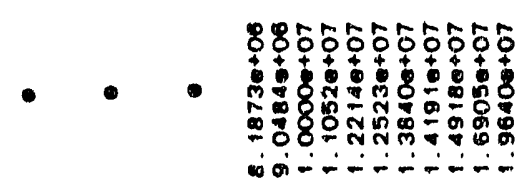

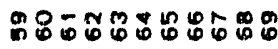




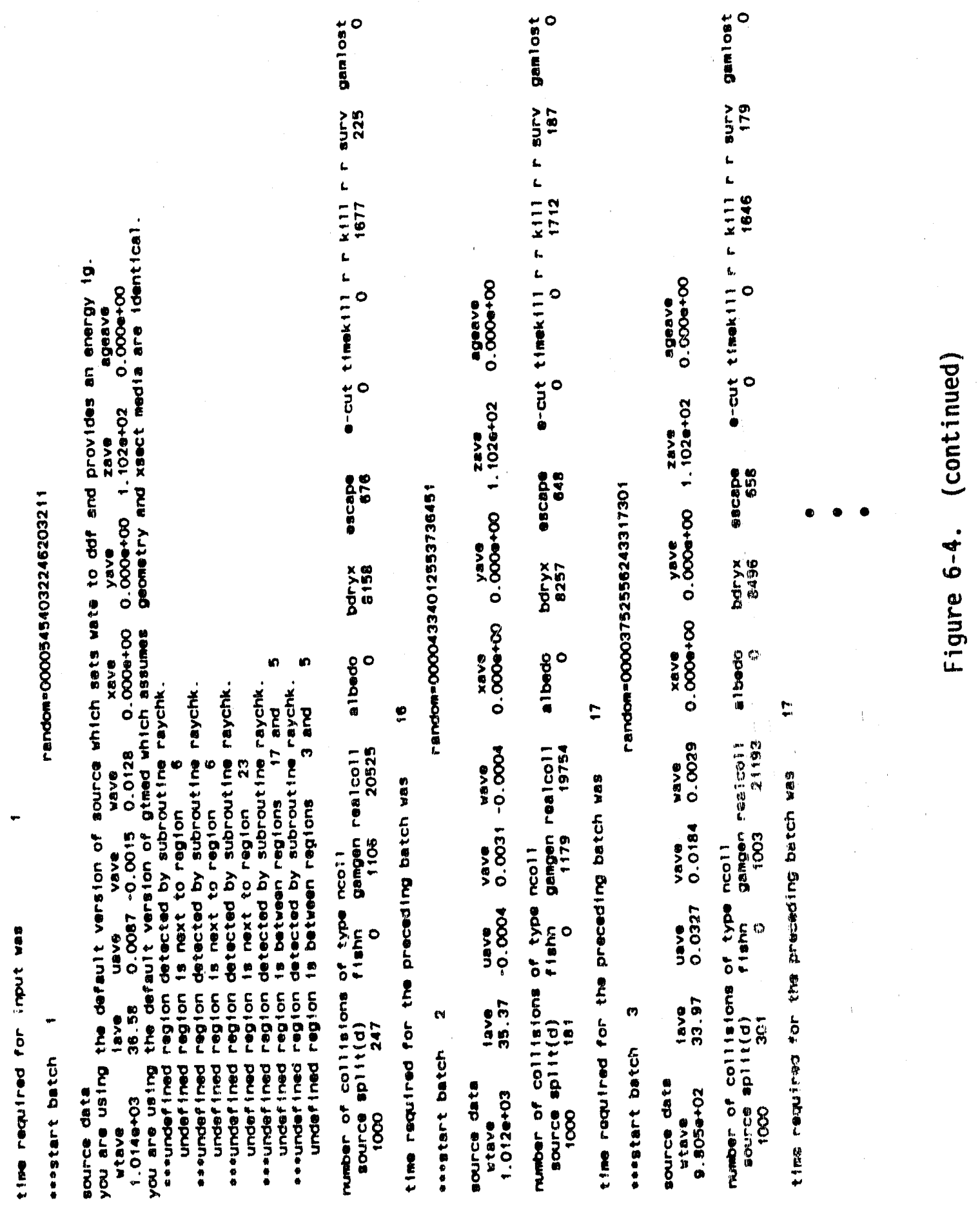




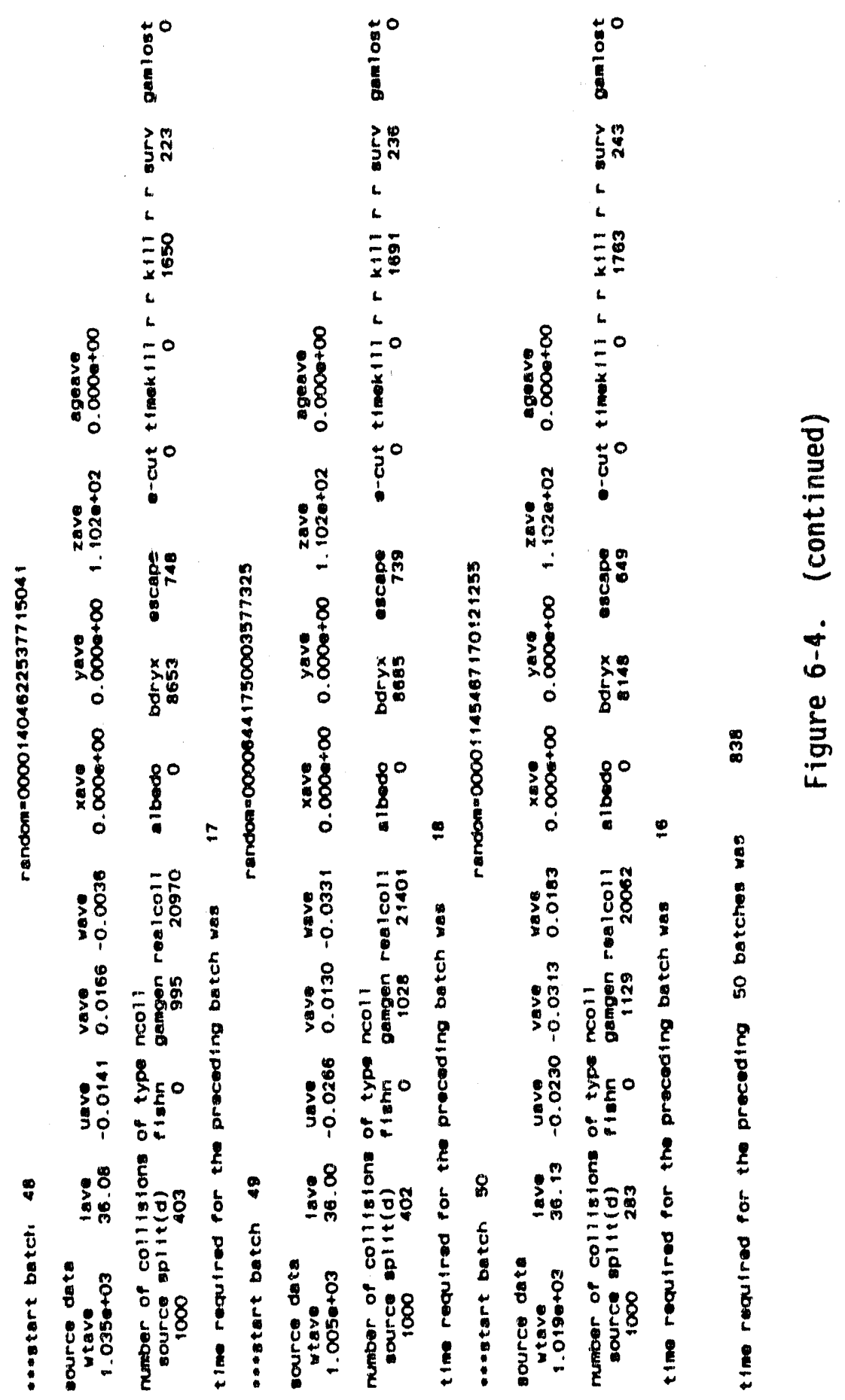




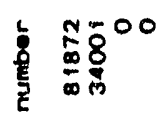

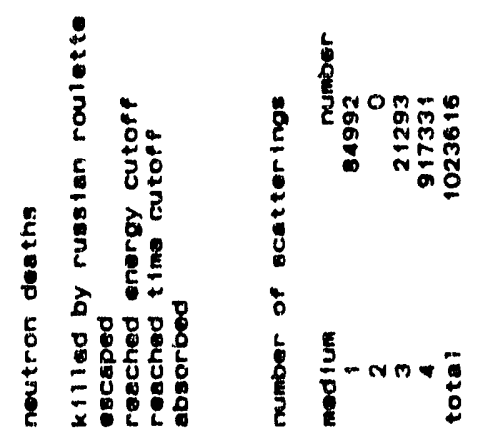



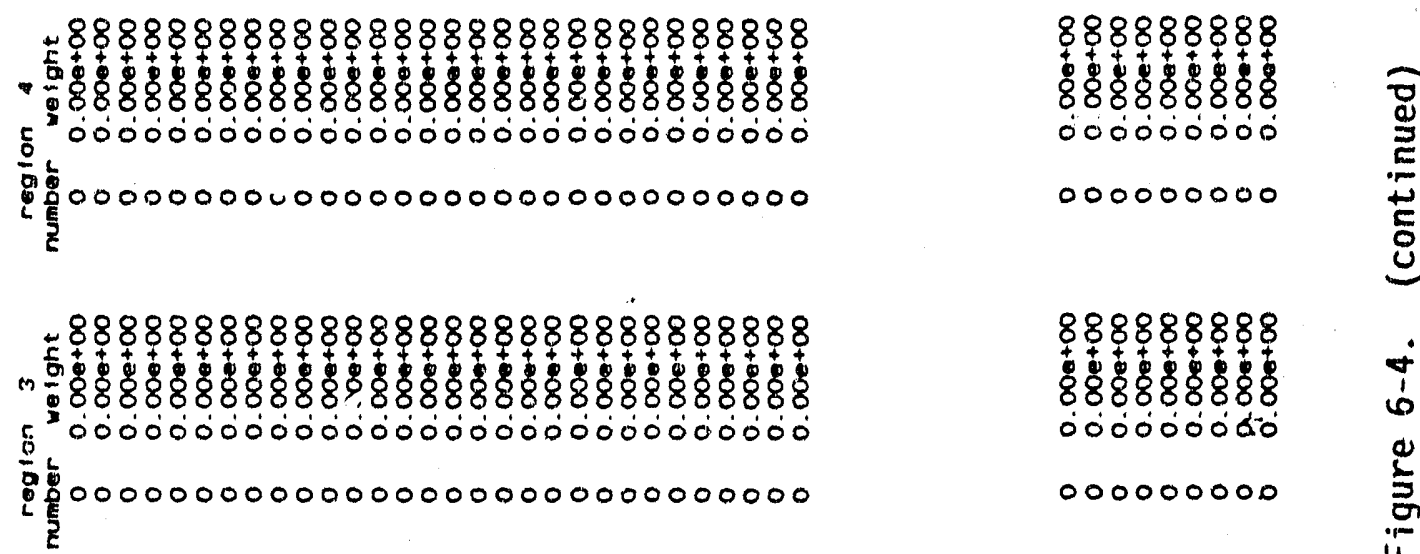

888888888

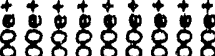

'000 ó0.

000000000

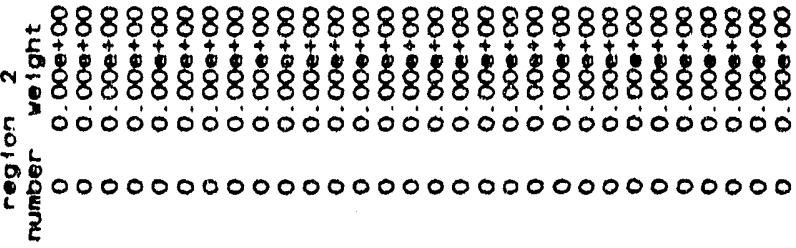

888888888

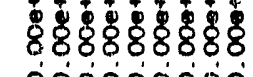

óóóónó

000000000

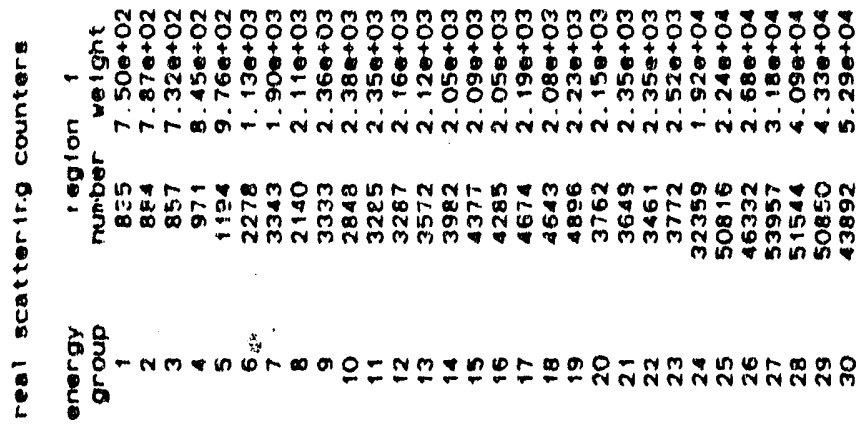

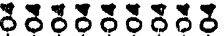

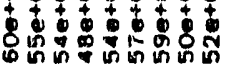

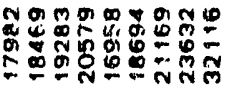

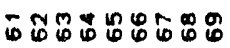




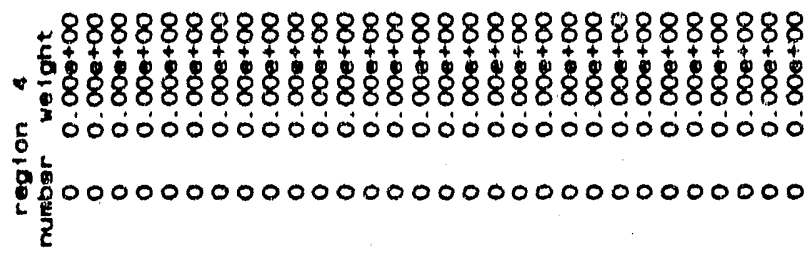

888888888

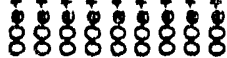

0ं0000000

000000000

888888888

4888888888888888888888888888888 m. 888888888

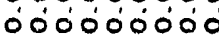
它

000,000000

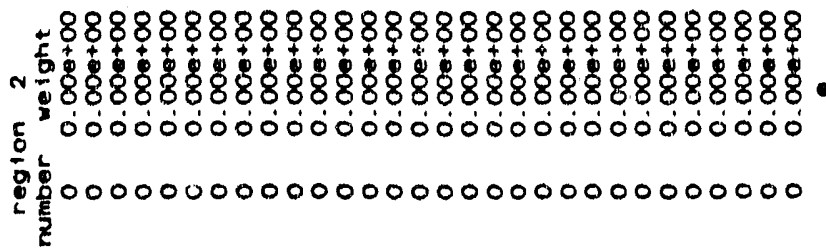

888888858

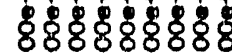

०00000000

000000000

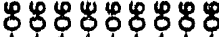

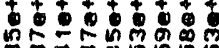

-

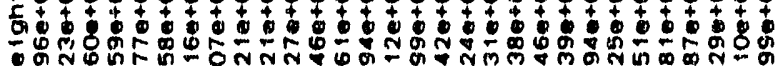

है 更

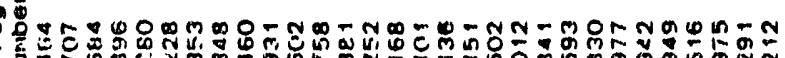

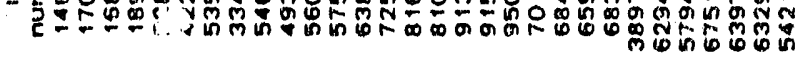

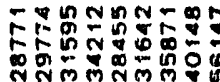

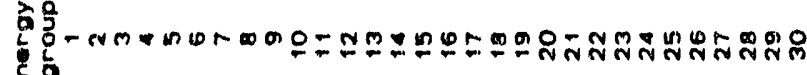

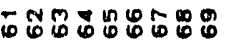




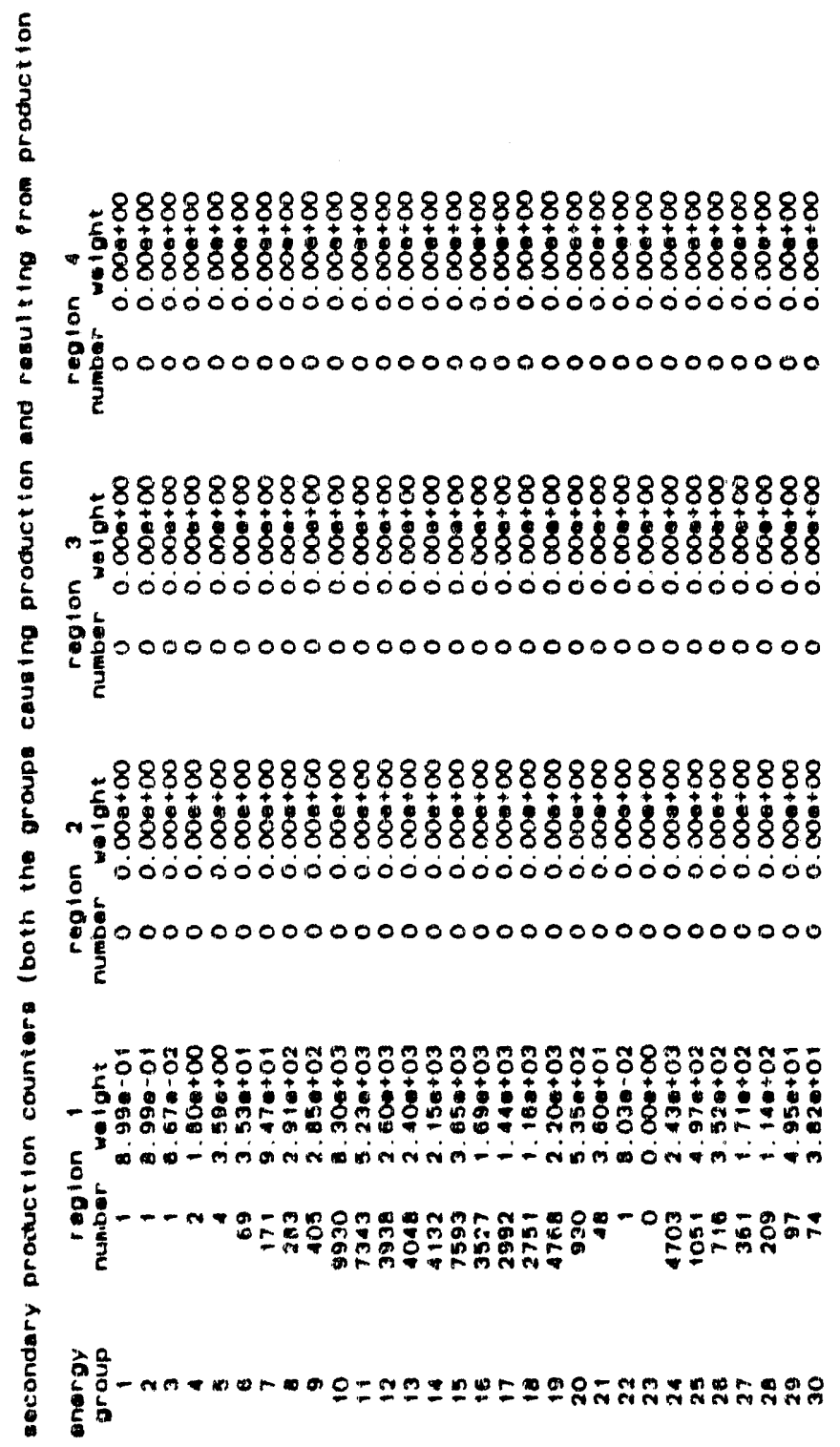

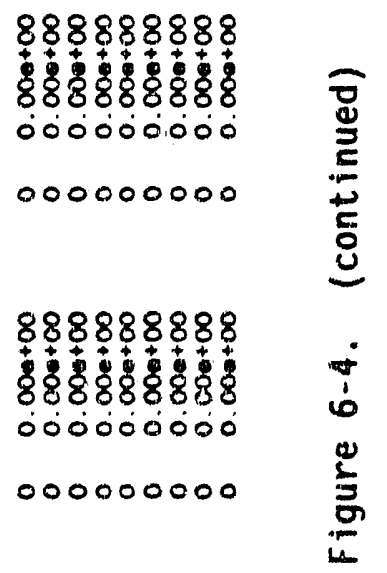

888888888

- 83888880 000000000 000000000

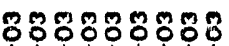

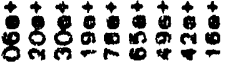

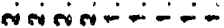

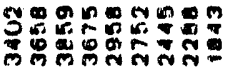

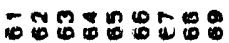




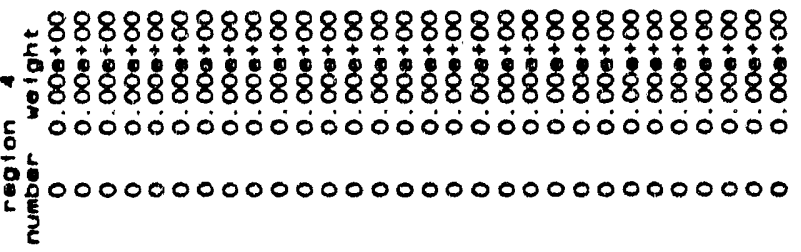

88888.8888

80

ஸं0்000000

000000000

388888888

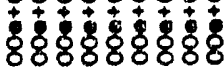

०00000000 (a)

000000000

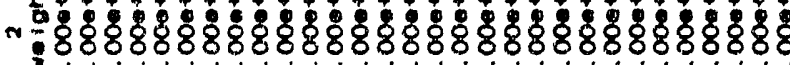

88888888 ₹000000000000000000000000000000

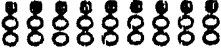

0000000000 : 000000000000000000000000000000

000000000

$-888888888888888888888888888888$

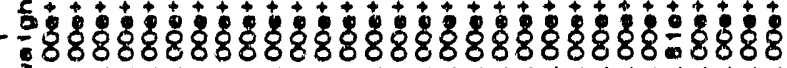

E ó00000000000000000000000000000 t. $0000000000000000000000000-0000$ $-$

है

$:$

莫

0500500008

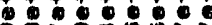

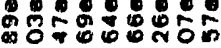

लिंत्र-:-

O=01000. N

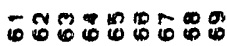



:

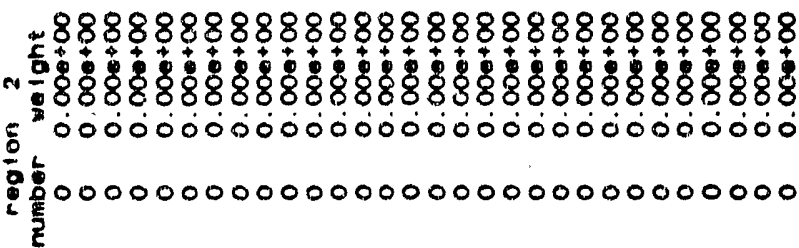

888888888

488888888888888888888888888888

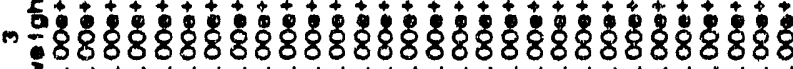
ฮ isor

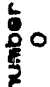

000000000

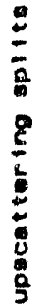

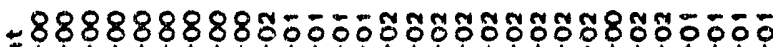

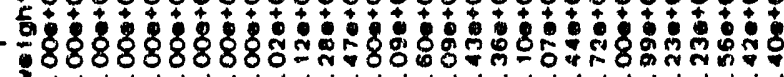
西 है

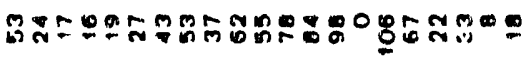

¿

葛客

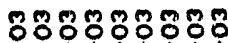

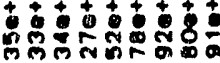

लिल कित

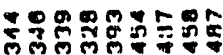

โที่ 

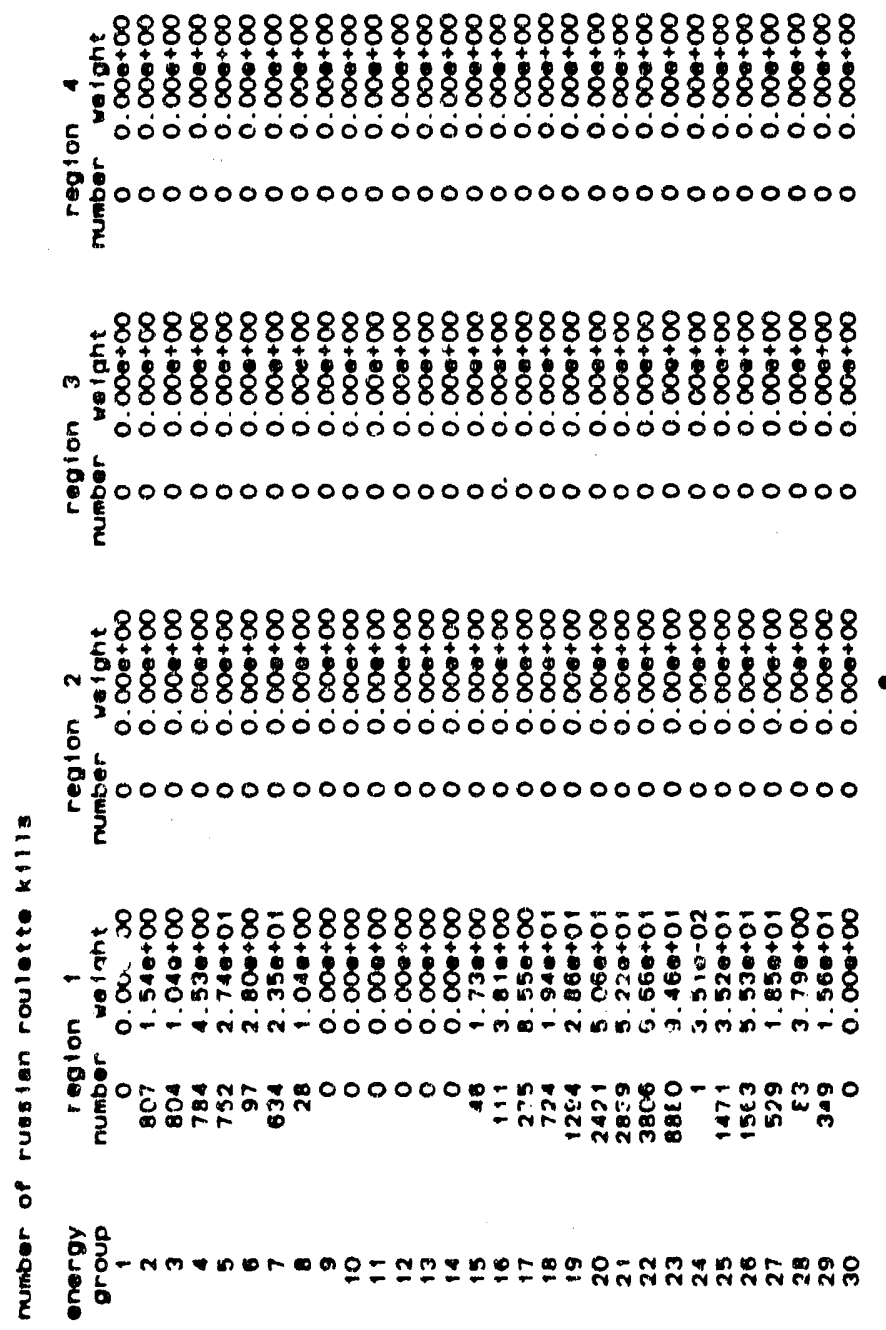

888888888

- 888888888 óó000000

000000000

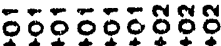

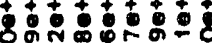
nintoriton

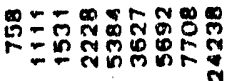

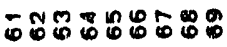



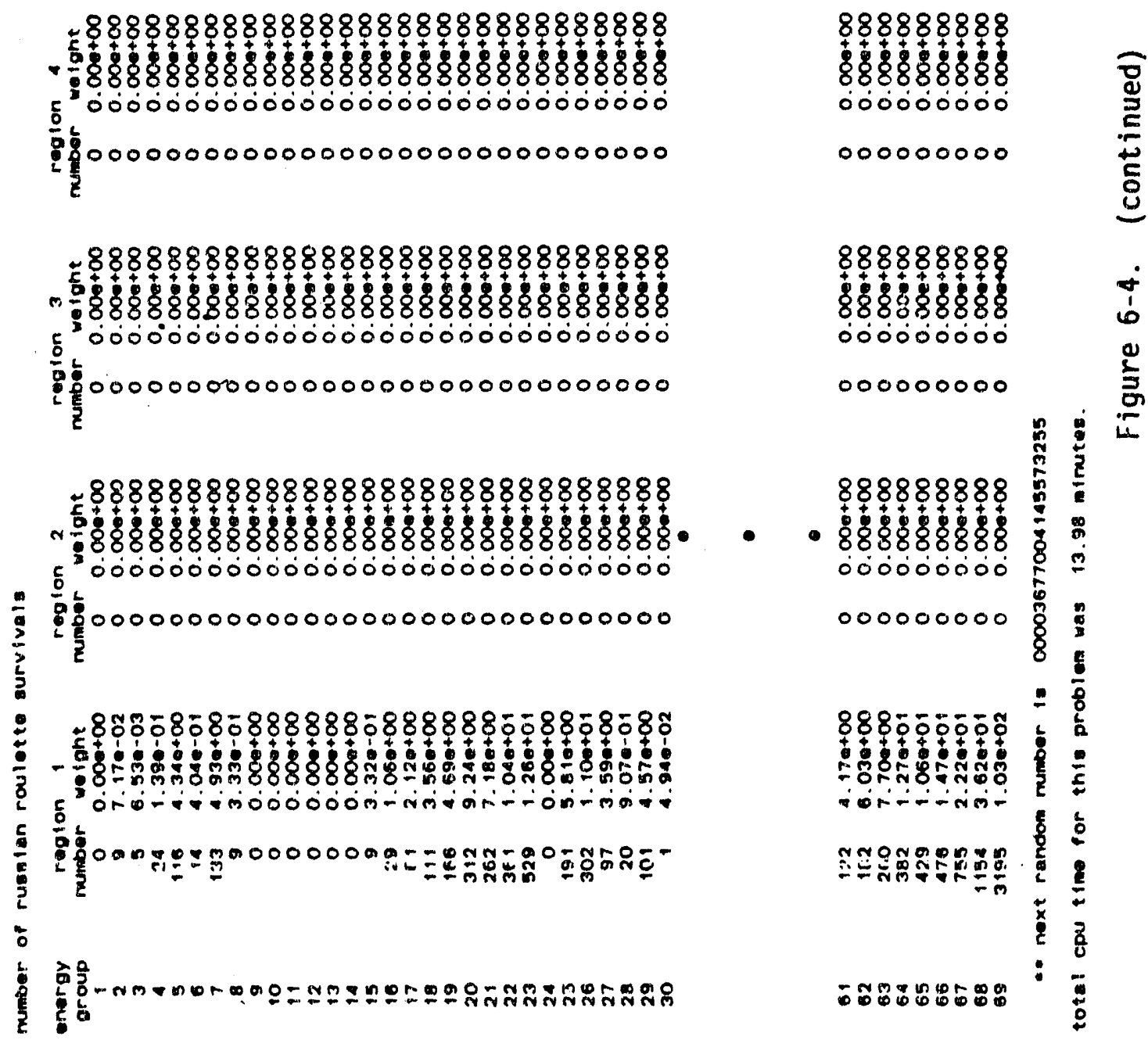

- 883888588

888888888 88808888

०00000000

000000000 óojóióó

000000000

8

8880000000

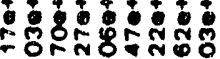

ior--nim-

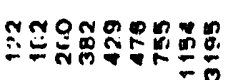

פด

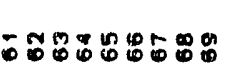

b́

6

苛 


\subsection{DRC: A DETECTOR RESPONSE CODE*}

\subsection{INTRODUCTION TO DRC}

\subsubsection{Background}

The Detector Response Code (DRC) in the MASH code system is used to coup Te the MORSE Monte Carlo adjoint leakage information with the DORT ${ }^{2}$ free-field fluences to compute the estimates of the fluences and doses within the vehicle interior. Furthermore, DRC utilizes the free-field fluences and estimates from the in-vehicle fluences (and doses) to calculate neutron protection factors and reduction factors for the vehicle.

The DRC code in MASH has undergone several modifications from its predecessor in the Vehicle Code System (VCS) ${ }^{3,4}$. The current version of DRC contains the newest SAMBO analys is routines from the MORSE code system. The main difference between this and previous SAMBO versions is that input data is free-form and subroutine ENRGYS defines an array, IARR, which is a key to the correspondence between the DRC analysis group number and the MORSE random walk group number for use by subroutine FLUXST when scoring group-dependent data. This eliminated the requirement in the old VCS-DRC of having the DORT free-field fluences and MORSE adjoint leakages calculated in the same group structure. Furthermore, with the development of the new 69 group $(46 n-23 \gamma)$ cross section set, it was advantageous to modify DRC to couple MORSE adjoint leakage calculations in 69 energy groups with previously calculated DORT free-field data in the old 58 group $(37 n-21 \gamma)$ group structure.

In the current version of DRC, the routines that are unique to DRC have been rewritten with the following characteristics:

1. All arrays are now variably dimensioned. This required the addition of several armays of data to BLANK COMMON.

2. All logical unit numbers are variables within the coding, however, the programming pre-assigns particular numbers to each unit.

3. The common structure is revised, having fewer commons with more variables in each. Two labeled commons, TDT1 and IDRC, contain all the necessary variables.

*Original documentation for the DRC program is found in the following reference: W. A. Rhoades, M. B. Emmett, G. W. Morrison, J. V. Pace, III, and L. M. Petrie, "Vehicle Code System (VCS) User's Manual," ORNL-TM-4648, 
Oak Ridge National Laboratory, (August 1974).

4. Use of only one radial $(r)$-bin is now allowed. Linear interpolation of the DORT fluences in the axial ( $z$ ) direction is used based on adjacent z-bins. Several errors in choosing bins for coupling surfaces, discovered in the old VCS-DRC, have been corrected.

5. Tapes produced with the new VISTA format are used. However, the programming logic is still included to read the old VISA tape formats.

6. A new MORSE collision tape format is used. However, it may be disabled to allow reading the previous format.

7. A new feature (mentioned above) is the ability to couple VISTA results from one group structure with MORSE results from a different group structure. This was added because there are VISTA resuits from the old $37 n-21 \gamma$ group structure which will neel to be coupled with the new $46 n-23 r$ group structure which MOR'SE runs will begin using. The method used is to bring all VISTA data to the MORSE structure at the start of the DRC analysis. This feature requires the energy structure for VISTA; and since it is not present on the VISTA file, it was added to the DRC input data.

\subsubsection{Method Used}

The general structure of the MASH code system illustrated in Section 1.0 of this report shows all data relevant to the calculation of the vehicle protection and reduction factors as input to the DRC module. In particular, the GRTUNCL/DORT air-over-ground analys is of the radiation environment is passed through VISTA for renormalization and reformatting for use in DRC in calculating the free-field fluences and doses and for folding with the adjoint leakage information from MORSE to calculate the in-vehicle fluences and doses along with the vehicle protection and reduction factors as a function of range and vehicle orientation relative to the source. A special feature of MASH is that the MORSE adjoint leakage information contains both the leaking particle's starting energy (source energy) and finat energy (leaking energy). This allows DRC to calculate an energy fluence inside of the vehicle which can then be folded with any input dose response function such as a detector response function or a tissue dose response function.

Consequently, one MORSE calculation can be used to analyze several different possible dose responses and/or several different radiation erivironments (GRTUNCL/DORT calculations). The basic equation used by MASH to calculate the dose inside of a vehicle is given in Equation $1 .^{5}$ 


$$
\lambda=\int_{A} d \vec{x} \int_{4 \pi} d \vec{\Omega} \sum_{I G} \Phi_{I G}(\vec{x}, \vec{\Omega}) \Phi_{I G}^{*}(\vec{x}, \vec{\Omega}) \vec{\Omega} \cdot \vec{n}
$$

where $\lambda$ is the dose,

$\lambda$ is the space variable which is integrated over a closed surface $A$,

$\Omega$ is the angle variable which is integrated over $4 \pi$ steradians,

IG is an energy group index summed over all the energy groups in the transport problem,

$\Phi$ is the forward fluence calculated by the GRTUNCL/DORT codes,

$\Phi^{*}$ is the adjoint fluence calculated by the MORSE code, and

$n$ is the unit vector normal to the area element $A$.

To derive the actual equations used by DRC, it is necessary to consider that the adjoint fluence as calculated by adjoint Monte Carlo is approximated by Equation 2.

$$
\Phi_{I G}^{*}(\vec{x}, \vec{\Omega}) \approx \frac{1}{N} \sum_{i=1}^{N_{l G}} \frac{W_{I G, i}^{*}}{\vec{\Omega}_{i} \cdot \vec{n}_{i}} \delta\left(\vec{x}-\vec{x}_{i}\right) \delta\left(\vec{\Omega}-\vec{\Omega}_{i}\right)
$$

where $N$ is the total number of particles in the adjoint calculation,

$N_{1 G}$ is the total number of particles that leak out of the calculation with energy group IG,

$i \quad$ indicates a leaking particle,

$W_{10,1}^{*}$ is the weight of the $i^{\text {th }}$ adjoint particle that leaks with energy group IG,

$x_{i} \quad$ is the position of the $i^{\text {th }}$ particle when it leaks,

$x_{i} \quad$ is the direction of the $i^{\text {th }}$ particle when it leaks, and

$\delta$ is the Dirac delta function.

Therefore, Equation 2 approximates the adjoint fluence as a summation of Dirac delta functions $(\delta)$ over the weights. The factor of $1 /\left(\Omega_{i} \cdot n_{i}\right)$ converts a leakage current into a leakage fluence. Equation 2 can be substituted into Equation 1, resulting in Equation 3.

$$
\lambda=\frac{1}{N} \sum_{i=1}^{N} \Phi_{I G_{i}}\left(\vec{x}_{i}, \vec{\Omega}_{i}\right) W_{I G, i}^{*}
$$


Here, the dose is calculated by a sum over the products of the weights of leakage particles and the angular fluences at the location and direction of the leaking particles. One of the advantages of DRC is the simplicity of Equation 3. In this equation, the DORT angular fluence is differential in angle as calculated in DORT per unit weight and the $W^{*}$ is the actual particle leakage weight calculated by MORSE. In reality, DRC uses a slightly more complicated form of this equation as shown in Equation 4.

$$
\Phi_{I G S}^{V}=\frac{1}{N \cdot S_{I G S}^{*}} \sum_{i=1}^{N} \Phi_{I G_{i}}\left(\vec{x}_{i}, \vec{\Omega}_{i}\right) W_{I G, I G S, i}^{*}
$$

where IGS corresponds to the source energy of an adjoint particle

$$
\begin{aligned}
& \Phi^{\vee} \text { igs is the energy spectrum calculated by DRC inside the } \\
& \text { vehicle, and } \\
& S^{*} \text { is the energy spectrum sampled when performing the } \\
& \text { MORSE adjoint calculation. }
\end{aligned}
$$

Equation 4 then uses the particle leaking group, IG, and source energy, IGS, to transform Equation 3 into an equation for the energy spectrum inside of the vehicle. The dose inside of the vehicle is then calculated by Equation 5 which folds the internal energy spectrum with a response function of interest.

$$
\lambda=\sum_{I G S} \Phi_{I G S}^{V} R_{I G S}
$$

The solutions of Equations 4 and 5 can be seen in the FOLD and FLUXST subroutines within the programming of DRC.

An additional quantity that is calculated by DRC is the free-field dose which is used to calculate the vehicle protection factors and reduction factors. The free-field dose is calculated from the DORT angular fluences by a different equation as shown in Equation 6 .

$$
\lambda_{f f}(\vec{x}) \sum_{I G} R_{I G} \int_{4 \pi} d \Omega \Phi_{I G}(\vec{x}, \vec{\Omega})
$$

where $\lambda_{f f}(x)$ is the free field dose at a point $x$ which is calculated by summing a dose response over the scalar fluence at that point. 
This is implemented in the code as shown in Equation 7 where the angular integration is replaced by a summation over all the DORT angle points.

$$
\lambda_{f f}(\vec{x})=\sum_{I G} R_{I G} \sum_{K}^{N A N G} \Phi_{I G, K}(\vec{x}) W_{K}
$$

where $W_{k}$ is the angular quadrature weight, $K$ indicates an angle direction, and

NANG is the number of directions.

Equation 7 is performed in Subroutine FREDOS in the DRC code and is subsequently used to calculate protection and reduction factors.

The radiation transport coupling method employed in DRC allows for any complicated Monte Carlo geometry inside the coupling surface to be modeled in three dimensional detail. The two-dimensional GRTUNCL/DORT discrete ordinates air-over-ground environment is defined by an $r-z$ coordinate system, where the radial axis $r$ coincides with the $x$ axis and is perpendicular to the $y$ axis. The three dimensional Monte Carlo geometry system is completely contained within a cylindrical volume element defined by the maximum and minimum values chosen from the radial and axial mesh in VISTA. The coupling between the two systems occurs at the outer leakage surface of the Monte Carlo geometry within the cylindrical volume element. This coupling requires the Monte Carlo coordinate system to be rotated to align the $x, y, z$ axes parallel to and in the same sense of the $x, y, z$ axes of the GRTUNCL/DORT discrete ordinates system. When the adjoint Monte Carlo particle escapes its geometry system at a given point and in a given direction defined by its coordinate system, the coordinates, direction, and energy group can be converted to the discrete ordinates system so that the appropriate forward fluence can be determined in the evaluation of Equation 1. A full Eulerian rotation matrix is incorporated in DRC to allow for the alignment/rotation of the Monte Carlo geometry system within the discrete ordinates system. As presentiy configured, MASH allows for the rotation of the Monte Carlo geometry system in the $x-y$ plane, i.e., rotation abuut the 2 axis. Future versions of MASH may allow full rotational capability. For additional information, a detailed derivation and description of radiation transport coupling methods can be found in Reference 6 .

In order to calculate the protection and reduction factors, the MORSE adjoint leakage tape includes a parameter, IDET, which identifies the detector type in DRC in which a leaking particle is to be scored. Table 7-1 identifies the sever detection types utilized in DRC. The first four detector types are utilized on the MORSE adjoint leakage tape for scoring purposes. The last three detector types in Table 7-1, along with the free-field neutron, gamma ray, and total fluences and/or doses, are used in ORC to calculate the protection and reduction factors defined in Table 7-2. 
In Table 7-2, the difference between the "protection factors" and " reduction factors" is the scoring of the vehicle produced secondary gamma dose (IDET=2 in MORSE or Detector 2 in Table 7-1). The neutron and gamma "protection factors" are a measure of how much the vehicle shielding reduces the dose from free-field neutrons and gammas, respectively, and is a useful parameter in engagement analysis. The "reductions factors," however, are a measure of how much the vehicle shielding alters the neutron or gamma dose as measured by a neutron or gamma sensitive detector. While the "protection factors" are most useful for engagement anaiyses, the "reduction factors" are most useful for comparing calculated results with experimental measurements.

Table 7-1. Detector Response Definitions in DRC.

Detector Response Definition

1 Direct neutron - a neutron entering the vehicle and contributing a neutron dose.

2 Capture gamma rays from vehicle - a gamma ray resulting from a neutron entering the vehicle and contributing a secondary gamma-ray dose.

3 Capture gamma rays from ground - a gamma ray resulting from a neutron entering the ground without passing through the vehicle and generating a secondary gamma-ray dose from a ground interaction. (This dose is already included in the upward-directed gamma rays that enter the vehicle. This generally is negligible.)

4 Direct gamina rays - a gamma ray entering the vehicle and contributing a gamma-ray dose. This source of gamma rays includes both gamma rays originating from the source, and capture gamma rays from the air.

5 Total gamma-ray dose - Sum of detectors 2, 3, and 4.

6 Gamma-ray dose from gamma rays entering the vehicle (usually dominated by direct gamma rays) - Sum of detectors 3 and 4 .

$7 \quad$ Neutron and gamma-ray dose from neutrons incident on the vehicle - Sum of detectors 1 and 2. 
Table 7-2. Definitions of Parameters and

Protection Factors used to Characterize

the Effectiveness of Shields.

\begin{tabular}{|c|c|}
\hline Parameter & Response Definition \\
\hline FFN & Free-Field Neutron Response \\
\hline FFG & Free-Field Gamma Response \\
\hline NPF & $\begin{array}{l}\text { Neutron Protection Factor } \\
\quad N P F=\text { FFN / Det. } 7\end{array}$ \\
\hline GPF & $\begin{aligned} & \text { Gamma Protection Factor } \\
& \text { GPF }=F F G / \text { Det. } 6\end{aligned}$ \\
\hline TPF & $\begin{array}{l}\text { Total Protection Factor } \\
T P F=(\text { FFN }+F F G) /(\text { Det. } 7+\text { Det. } 6)\end{array}$ \\
\hline NRF & $\begin{array}{c}\text { Neutron Reduction Factor } \\
\text { NRF }=F F N / \text { Det. } 1\end{array}$ \\
\hline GRF & $\begin{array}{l}\text { Gamma Reduction Factor } \\
\text { GRF }=F F G / \text { Det. } 5\end{array}$ \\
\hline TRF & $\begin{array}{l}\text { Total Reduction Factor } \\
\text { TRF }=(\text { FFN } F F G) /(\text { Det. } 1+\text { Det. } 5)\end{array}$ \\
\hline
\end{tabular}

\subsection{DRC INPUT REQUIREMENTS}

The following input cards are required to execute DRC. Default values are in brackets ([]).

Card A Job Title Card [18A4]

TITLE 72 alphanumeric character description

Card B Job control parameters [Begin array with "**"]

RAD radius of position of vehicle

AZERO $z$-pOsition of the MORSE coordinate origin in the DORT coordinate system. Equivalent to ZDORT (Ground-Air interface) minus ZMORSE (Ground-Air Interface)

ZBOT lowest $z$ position in MORSE where particles may leak from system 
ZTOP highest $z$ position in MORSE where particles may leak from system

ALPHA rotation of vehicle $x$-axis counterclockwise from DORT $r$-axis

$-5 \ldots$

HGT detector $z$ coordinate in MORSE coordinate system

IPRT print control output flag

- 0 implies print vehicle doses and fluences

$=1$ implies print free field doses and fluences

= 2 implies print both vehicle and free field doses and fluences

WATMX maximum weight allowed on MORSE leakage tape

Card C (ICA(I),I=1,NMTG) [Begin array with "\$\$"]

ICA Integer array giving correspondence between group structures in VISTA and MORSE. $0 \leq I C A \leq N O G$.

Card D NNEUT, NGAM [Begin array with "\$\$"]

NNEUT group number of lowest neutron energy group in VISTA

NGAM group number of lo'vest gamma energy group in VISTA

Card $E$ (VE(I), I=1,NOG+2) [Begin array with "**"]

VE VISTA energy group boundaries in descending magnitude, i.e., neutron energies including bottom energy of lowest neutron group followed by gamma energies including bottom energy of lowest gamma group

NOTE: Input Cards $C, D$, and $E$ are optional. They are required only if the VISTA and MORSE energy group structures do not coincide.

Card $F$ alphanumeric title information [20A4]

Card $G$ title or units for total responses

for all detectors [20A4]

Card $H$ title or units for each total response [20A4]

Card I (RESP(I),I=1,NMTG) [Begin array with $" \star * "]$

RESP response function values in order of decreasing energy [20A4]

Card $J$ units for energy dependent fluence [20A4] 


\subsection{DRC INPUT DATA NOTES}

Except for the title cards, all data are read using the MORSE FIDO input system. A detailed description of the FIDO input system (in general) is given in Appendix $A$. In MORSE FIDO, the array indicators " $* *$ " or "\$" must be entered in columns 2 and 3 . In MORSE FIDO, data arrays are stiTl entered in blocks, but the blocks are not terminated by a " $T$ ". Unused data arrays are not entered and careful attention must be paid to the order of each array, the size of each array, and the requirements for entering (or not entering the array).

Multiple cases can be executed for different vehicle orientations (input parameter ALPHA on Card B) by stacking pairs of Cards A and B for each additional vehicle orientation after the first case. The Cards $C$ through $\mathrm{J}$ need not be repeated after the first case. If this option is chosen, it is suggested the user set IPRT $=0$ to eliminate multiple printouts of the free-field information.

RAD The radius corresponding to the position of the vehicle must match the radius (or one of the radii) on the VISTA fluence tape. An error message will be printed if this condition is not met.

AZERO This parameter allows for the case when the air-ground interface in the DORT calculation does not match the air-ground interface in the MORSE calculation. Typically, the DORT calculation will place the airground interface at $z=0$. The vehicle geometries analyzed, typically place the turret-track interface at $z=0$ and the air-ground interface at some value less than zero. Consequently, the user can make the two airground interfaces match through the parameter AZERO.

ZBOT and ZTOP These two parameters must be within the VISTA axial boundaries read by DRC. The ZBOT and ZTOP boundaries represent the minimum and maximum values a leaking particle from the adjoint MORSE calculation will have. To fold these particles with the forward DORT fluences, the DORT axial mesh must encompass the MORSE axial mesh. In other words, the maximum $z$ boundary written on the VISTA file must be greater than ZTOP and the minimum $z$ boundary on the VISTA file must be less than ZBOT.

ALPHA This parameter allows for different vehicle (or target) orientations relative to the source. The parameter is given in units of degrees and is specified for the rotation of the MORSE positive $x$-axis counterclockwise (as viewed from above) from the DORT positive $r$-axis. For example, a typical vehicle geometry in MORSE has the positive $x$-axis facing forward on the vehicle i.e., in the direction of the gun barrel for a tank. If the user wished to determine the protection factor for the tank facing the source, ALPHA would have to be set to 180 , the degrees rotation of the positive $x$-axis in MORSE with the positive $r$ axis in DORT. A value of 0 for ALPHA would orient the tank with the rear facing the source. Care must be taken when determining the target rotation. 
HGT This parameter must correspond to the detector $z$ position specified on Card $D$ in the MORSE input.

IPRT This parameter controls the amount of print from a DRC case. If IPRT $=0$, only information on the vehicle fluences and doses are printed. This is the recommended option for additional stacked cases for more target orientations. If IPRT $=1$, only the free field fluences and doses are printed. For single DRC cases, IPRT $=2$ is recommended because the user will obtain both free-field and target fluence and dose information.

WATMX This parameter was used to set the maximum weight allowed for an adjoint leaking particle. With the use of in-group biasing, this parameter is obsolete and a value of zero will set the default value of 50 .

Cards $C, D$, and $E$ These cards are input if the VISTA and MORSE energy group structures do not coincide. This option is only valid for cases where the VISTA energy group structure is a subset of the MORSE energy group structure. This option was included in DRC to allow for coupling of new MORSE adjoint leakage tapes in 69 energy groups $(46 n-23 \gamma)$ and old DORT free-field fluence files in 58 energy groups $(37 n-21 \gamma)$. Coupling of artitrary group structures in DRC is not operational. ICA(I)=0 when there is no corresponding data in VISTA for 2 cases: (1) When the MORSE group structure has energy bins below the bottom group of VISTA or (2) when the MORSE structure has one or more energy bins at higher energy than VISTA. For example, if VISTA's highest energy bin is from 14 to 12 $\mathrm{MeV}$ and MORSE's is from 20 to $14 \mathrm{MeV}$, ICA(1)=0. If VISTA has data down to $1 \mathrm{MeV}$ and MORSE to $0.1 \mathrm{MeV}$, then the ICA of any groups below $1 \mathrm{MeV}$ is 0 .

RESP The response function is entered in order of decreasing energy and with the neutron response preceding the gamma-ray response.

\section{y.3.1 Common Block Information}

The input information for DKC is processed into several common block areas for use throughout the code. The common block structure was revised for the current version of DRC resulting in fewer commons with more variables in each. The three main commons used in DRC are BLANK COMMON, Common TDT1, and common IDRC. The variables and structures of these three common blocks are defined in Tables 7-3, 7-4, and 7-5.

\subsection{DRC INPUT FILE FORMATS}

As stated earlier, DRC's primary function is to couple the MORSE Monte Carlo adjoint leakage information with the DORT free-field fluences to compute the estimates of the fluences and doses within the vehicle 
Table 7-3. Location of DRC arrays in BLANK COMMON.

\begin{tabular}{|c|c|c|}
\hline $\begin{array}{l}\text { Mnemonic } \\
\text { name }\end{array}$ & $\begin{array}{l}\text { Location in } \\
\text { BLANK COMMON }\end{array}$ & $\begin{array}{l}\text { Array } \\
\text { Purpose }\end{array}$ \\
\hline $\operatorname{ENER}(I G)$ & $I=1+I G-1$ & Upper limit of MORSE energy group \\
\hline $\begin{array}{l}\text { VEL (IG) } \\
\text { FS(IG) }\end{array}$ & $\begin{array}{l}I=N M T G+I G-1 \\
I=2 * N M T G+I G-1\end{array}$ & $\begin{array}{l}\text { Velocity of MORSE energy group IG } \\
\text { Unnormalized fraction of source } \\
\text { particles in MORSE energy group IG }\end{array}$ \\
\hline$B F S(I G)$ & $I=3 * N M T G+I G-1$ & $\begin{array}{l}\text { Relative importance of MORSE } \\
\text { energy group IG }\end{array}$ \\
\hline $\begin{array}{l}\operatorname{DELE}(\mathrm{IG}) \\
\operatorname{ILEAK}(N B)\end{array}$ & $\begin{array}{l}I=4^{*} N M T G+I G-1 \\
I=L O C L K+N B-1\end{array}$ & $\begin{array}{l}\text { Bin width of energy bin IG } \\
\text { Number of particles leaking from } \\
\text { system in batch NB of MORSE run }\end{array}$ \\
\hline $\begin{array}{l}\text { ASORC (NB) } \\
\text { BUF (IPOS) }\end{array}$ & $\begin{array}{l}I=\text { LOCASC }+ \text { NB }-1 \\
I=\text { LOCBUF }+I P O S-1\end{array}$ & $\begin{array}{l}\text { Total source weight for batch NB } \\
\text { Temporary storage for MORSE } \\
\text { collision tape data }\end{array}$ \\
\hline IVAL (IF) & $I=I V L+I F-1$ & $\begin{array}{l}\text { Index of radial fluence for } \\
\text { fluence point IF }\end{array}$ \\
\hline DWT (IANG) & $I=I W T+I A N G-1$ & $\begin{array}{l}\text { Weight for direction IANG from } \\
\text { VISTA quadrature }\end{array}$ \\
\hline AMU (IANG) & $I=I M U+I A N G-I$ & $\begin{array}{l}\text { Azimuthal angle corresponding to } \\
\text { direction IANG }\end{array}$ \\
\hline ETA(IANG) & $I=I E T+I A N G-1$ & $\begin{array}{l}\text { Polar angle corresponding to } \\
\text { direction IANG }\end{array}$ \\
\hline$R(I N)$ & $I=I R+I N-1$ & $\begin{array}{l}\text { Radial mesh point corresponding to } \\
\text { radial bin IN }\end{array}$ \\
\hline$Z(I N)$ & $I=I Z+I N-1$ & $\begin{array}{l}\text { Axial mesh point corresponding to } \\
\text { height bin IN }\end{array}$ \\
\hline$A(I A)$ & $I=I R O T+I A-1$ & $\begin{array}{l}3 \times 3 \text { rotation matrix for rotating } \\
\text { the vehicle }\end{array}$ \\
\hline $\begin{array}{l}\text { FDOS (IG) } \\
\operatorname{DELU}(I G)\end{array}$ & $\begin{array}{l}I=I F D+I G-1 \\
I=I D U+I G-1\end{array}$ & $\begin{array}{l}\text { Free-field dose for group IG } \\
\text { Logarithm of upper energy } \\
\text { bound/iower energy bound for } \\
\text { group IG }\end{array}$ \\
\hline $\operatorname{ICA}(I G)$ & $I=I C E+I G-1$ & $\begin{array}{l}\text { Correspondence of energy group } \\
\text { numbers in VISTA with those } \\
\text { in MORSE }\end{array}$ \\
\hline$V E(I G G)$ & $I=I N V+I G G-1$ & $\begin{array}{l}\text { VISTA energy group boundaries } \\
\text { followed by NNEUT (number of } \\
\text { neutron groups) and NGAM } \\
\text { (number of ganma groups) }\end{array}$ \\
\hline DELVE(IGG) & $I=I N V+I G G-1$ & $\begin{array}{l}\text { Delta E for VISTA energy group } \\
\text { boundaries }\end{array}$ \\
\hline
\end{tabular}


Table 7-4. Definitions of variables in TDT1 COMMON.

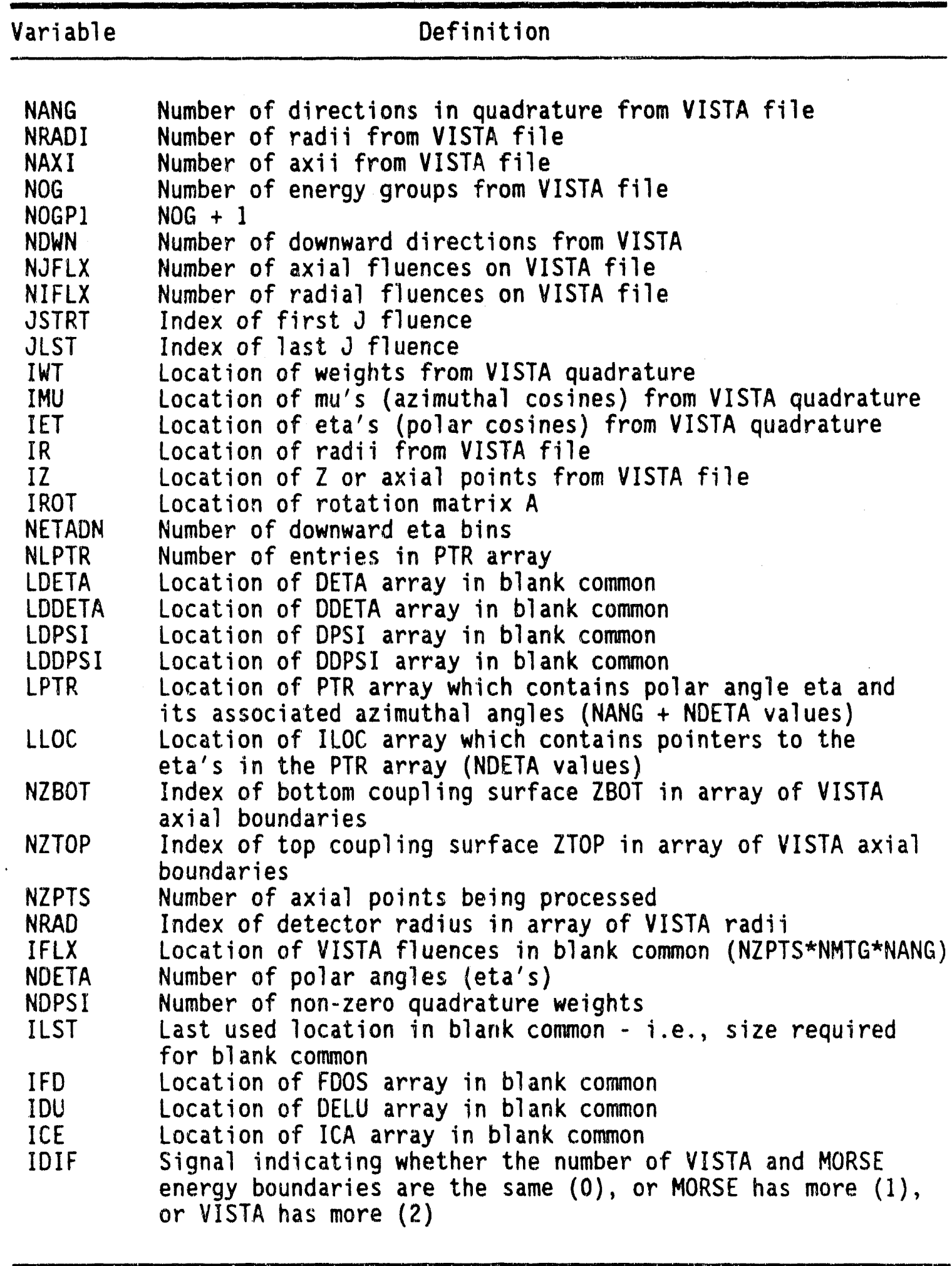


Table 7-5. Definition of variables in IDRC COMMON.

\begin{tabular}{|c|c|}
\hline Variable & Definition \\
\hline LEAK & Leakage value for current batch from MORSE \\
\hline IBUFSZ & $\begin{array}{l}\text { The size of the buffer for the collision parameters } \\
\text { (Set at 1000) }\end{array}$ \\
\hline NGPQTT & Not used \\
\hline MAXSZ & Size of blank common $(100000)$ \\
\hline NMCTP & Logical unit number of MORSE collision tape \\
\hline NSTAP & Logical unit number of VISTA fluence file \\
\hline NIN & Logical unit number of standard input unit \\
\hline NOU & Logical unit number of standard output unit \\
\hline IPRT & $\begin{array}{l}\text { Signal to print detector output only }(0) \text {, free-field } \\
\text { only ( } 1 \text { ), both of the above plus protection factors } \\
\text { and energy-dependent arrays (2). }\end{array}$ \\
\hline IRC & Counter for record number \\
\hline NBTCH & Counter for batch number currently being processed \\
\hline HGT & $\begin{array}{l}\text { Height in } \mathrm{cm} \text { for free-field dose - usually equivalent } \\
\text { to the detector height in the MORSE adjoint run }\end{array}$ \\
\hline TNDOS & Free-field neutron dose \\
\hline TGDOS & Free-field gamma dose \\
\hline WATMX & Maximum weight accepted for processing \\
\hline LOCLK & $\begin{array}{l}\text { Location in blank common of ILEAK array from MORSE } \\
\text { collision tape }\end{array}$ \\
\hline LOCASC & $\begin{array}{l}\text { Location in blank common of ASORC array from MORSE } \\
\text { collision tape }\end{array}$ \\
\hline LOCBUF & $\begin{array}{l}\text { Location in blank common of buffer array for collision } \\
\text { parameters from MORSE }\end{array}$ \\
\hline $\operatorname{TITLM}(18)$ & Alphanumeric title from MORSE collision tape \\
\hline LOCDE & $\begin{array}{l}\text { Location of DELE array, the delta energy for MORSE } \\
\text { energy groups }\end{array}$ \\
\hline
\end{tabular}


interior. Furthermore, DRC utilizes the free-field fluences and estimates from the in-vehicle fluences (and doses) to calculate neutron protection factors and reduction factors for the vehicle.

To accomplish its primary function, DRC reads a VISTA formatted file to obtain the DORT free field fluence data, and a MORSE adjoint leakage tape to obtain the parameters necessary for it to estimate the fluences and doses within the vehicle interior. The format of the VISTA input source file is as follows:

Record 1: TITLE, TDOT - 144 Alphanumeric Characters

Record 2:

MM - Number of quadrature directions
IM $\quad$ - Number of radial intervals
JM $\quad$ Number of axial intervals
IGM $\quad$ Number of energy groups
IGP $\quad$ Number of energy groups plus 1
MMDN - Number of directions downward
NJP - Number of axial fluences
ISH - Height of source point
ISHA - Cosine of source angle (with $Z$-axis)
NIP - Number of radial fluences
JPL - Index of first $J$ interval on file
JPU - Index of last $J$ interval on file
II additional VISTA input values not used in MASH

Record 3: IVAL - Array of radial interval numbers being output

Record 4: [MM values per array; 3 *MM total values]

WT - Weights from DORT quadrature

AMU - Mu's from DORT quadrature

ETA - Eta's from DORT quadrature

Record 5: $\quad$ [NIP + NJP values]

$\mathrm{R}$ - Midpoints of radial bins

$Z$ - Midpoints of axial bins

Record 6 to END: [NANG*NJFLX values per record]

[IGM records per radius, and NIFLX radii]

FLUX - Directional fluences 
Because of the large amount of core storage required for the VISTA freefield fluences, the forward-adjoint coupling could not be accomplished as the Monte Carlo calculation was performed. The coupling involved the calculation of the integral

$$
R=\int \Phi(P) \Phi^{*}(P)(\Omega \cdot n) d P
$$

over the coupling surface $S$.

We define

$\Phi(P)=$ VISTA free-field fluences in phase space $P$.

$\Phi^{*}(P)=$ MORSE adjoint fluence in phase space $P$.

$\Omega \cdot n=$ cosine of angle between particle direction and inward normal.

The adjoint fluence at the $r_{s}$, the position of the surface $S$, was written on a leakage tape along with other variables necessary for coupling the MORSE results with the VISTA results. The variables written on tape for each adjoint leakage were as follows:

1. IG - the group number,

2. IGS - the source group,

3. WTBC - the weight of the adjoint particle at leakage,

4. $U$ - X direction cosine,

5. $V-Y$ direction cosine,

6. W - Z direction cosine,

7. Z - height of leakage point,

8. IDET - detector type in which particle is to be scored.

An output buffer was blocked to transfer the data from core to tape efficiently at the end of each batch. This permitted batch statistics to be calculated in the DRC code which reads the MORSE leakage tape and the VISTA fluence tape and performs the coupling. The leakage tape generated by MORSE contains the following data: 


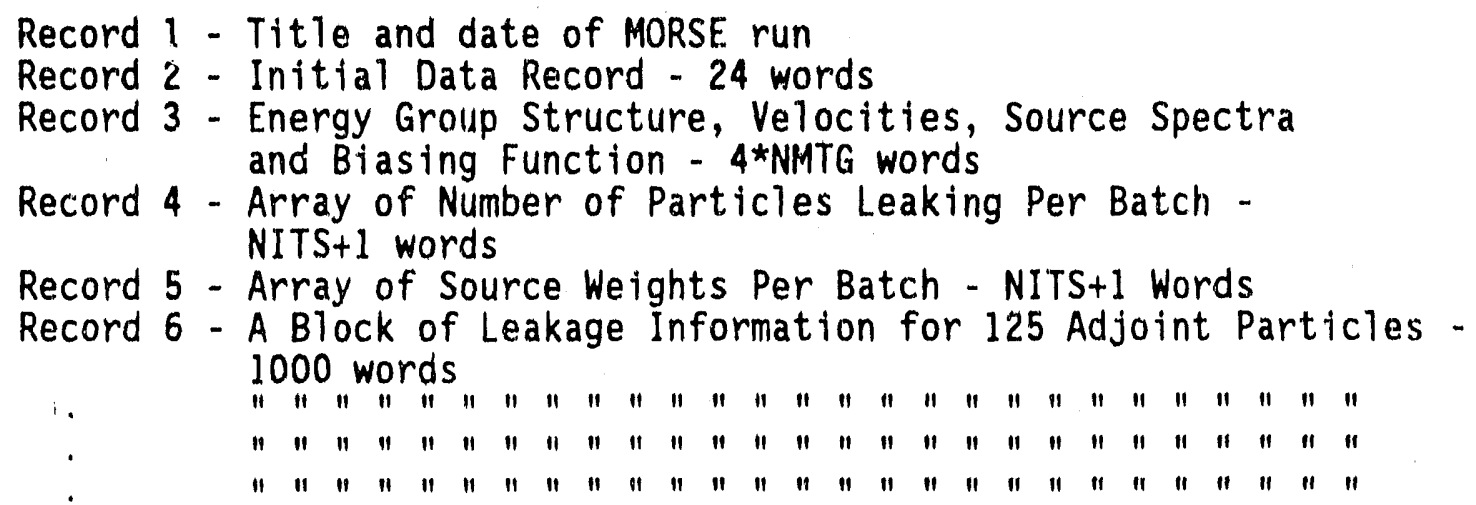

Record N -

A detailed description of the contents of the above records is given below:

I. Record 1 - A 40 character title plus a 32 character date of run

II. Record 2 - The USER labeled common (plus parameter INGB) is written in Record 2. The total number of groups, number of batches, etc., are contained in this record, as defined in Table 7-6.

III. Record 3 - The multigroup energy structure and group velocities are contained in Record 3 . The data are:

$E(1)$ - Upper energy limit of Group 1.

E(NMTG) - Upper energy limit of Group NMTG.

VEL(1) - Velocity of Group 1.

VEL (NMTG) - Velocity of Group NMTG.

$F(1)$ - Fraction of Source in Group 1

F(NMTG) - Fraction of Source in Group NMTG

$B(1)$ - Biased Fraction in Group 1

$B(N M T G)$ - Biased Fraction of Source in Group NMTG 
IV. Record 4 - The number of particles that leak per batch are given in Record 4. This data is necessary in order to normalize the batch results per source neutron.

$L(1)$ - Number of leakage particles in batch 1 .

L(NITS) - Number of leakage particles in batch NITS.

$L(N I T S+1)$ - Total number of leakage records written on tape.

V. Record 5 - The sum of the source weights of the particles in a batch.

ASORC(1) - Sum of weights of source particles in batch 1. ASORC(NITS) - Sum of weights of source particles in batch NITS

VI. Record 6 - The adjoint leakage fluence blocked in groups of 125 particles.

$B(1)$ - IG Energy Group

$B(2)$ - IGS Source Group

$B(3)$ - WTBC Weight at leakage

$B(4)-U 7$ direction

$B(5)-V=$ cosines

$B(6)$ - W at leakage

$B(7)$ - Z Height at leakage

$B(8)$ - IDET Detector Type

$B(1000)$ - IDET Detector Type

VII. Additional records in the format of Record 6 to a total of $L(N I T S+1)$ leakage records. 
Table 7-6. Definitions of Variables in USER COMMON.

\begin{tabular}{|c|c|c|}
\hline Number & Variable & Definition \\
\hline 1 & AGSTRT & $\begin{array}{l}\text { Initial chronological age to be assigned to source } \\
\text { particles }\end{array}$ \\
\hline $\begin{array}{l}2 \\
3\end{array}$ & $\begin{array}{l}\text { WTSTRT } \\
\text { XSTRT }\end{array}$ & $\begin{array}{l}\text { Initial weight to be assigned to source particles } \\
\text { Initial } x \text { position to be assigned to source } \\
\text { particles }\end{array}$ \\
\hline 4 & YSTRT & $\begin{array}{l}\text { Initial y position to be assigned to source } \\
\text { particles }\end{array}$ \\
\hline 5 & ZSTRT & $\begin{array}{l}\text { Initial } x \text { position to be assigned to source } \\
\text { particles }\end{array}$ \\
\hline $\begin{array}{l}6 \\
7\end{array}$ & $\begin{array}{l}\text { DFF } \\
\text { EBOTN }\end{array}$ & $\begin{array}{l}\text { Normal ization for adjoint problems } \\
\text { Lower energy boundary (eV) of last neutron group } \\
\text { (group NMGP) }\end{array}$ \\
\hline 8 & EBOTG & $\begin{array}{l}\text { Lower energy boundary (eV) of last gamma-ray group } \\
\text { (group NMTG) }\end{array}$ \\
\hline $\begin{array}{l}9 \\
10 \\
11 \\
12 \\
13 \\
14 \\
15 \\
16\end{array}$ & $\begin{array}{l}\text { TCUT } \\
\text { I0 } \\
\text { I1 } \\
\text { IADJM } \\
\text { NGPQT1 } \\
\text { NGPQT2 } \\
\text { NGPQT3 } \\
\text { NGPQTG }\end{array}$ & $\begin{array}{l}\text { Chronological age limit } \\
\text { Logical unit for output } \\
\text { Logical unit for input } \\
\text { Adjoint switch ( } 0 \text { for adjoint problem) } \\
\text { Problem-dependent energy group limit } \\
\text { Problem-dependent energy group limit } \\
\text { Problem-dependent energy group limit } \\
\text { Group number of lowest energy gamma-ray group } \\
\text { to be treated }\end{array}$ \\
\hline 17 & NGPQTN & $\begin{array}{l}\text { Group number of lowest energy neutrorl group to } \\
\text { be treated }\end{array}$ \\
\hline $\begin{array}{l}18 \\
19\end{array}$ & $\begin{array}{l}\text { NITS } \\
\text { NLAST }\end{array}$ & $\begin{array}{l}\text { Number of batches to be run } \\
\text { Last ce } 11 \text { in BLANK COMMON used by random walk } \\
\text { package }\end{array}$ \\
\hline $\begin{array}{l}20 \\
21 \\
22 \\
23\end{array}$ & $\begin{array}{l}\text { NLEFT } \\
\text { NMGP } \\
\text { NMTG } \\
\text { NSTRT }\end{array}$ & $\begin{array}{l}\text { Number of cells in BLANK COMMON available to user } \\
\text { Number of primary energy groups } \\
\text { Total number of energy groups } \\
\text { Number of source particles for each batch }\end{array}$ \\
\hline
\end{tabular}

\subsection{DRC OUTPUT FILES}

DRC only produces a printed output file.

\subsection{LOGICAL UNIT REQUIREMENTS}

Below is a listing of the files required to execute a DRC case along with the default values used in the code. In setting up a DRC case, efforts must be made for these units to be available. 
1. Logical Unit NSTAP - VISTA Angular Fluence File [Default=15]

2. Logical Unit NMCPT - MORSE Adjoint Leakage File [Defaultm24]

3. Logical Unit NOU - Printed Output [Default=6]

4. Logical Unit NIN - Card Input [Default=5]

\subsection{REFERENCES}

1. M. B. Emmett, "The MORSE Monte Carlo Radiation Transport Code System," ORNL-4972 (1975), ORNL-4972/R1 (1983), ORNL-4972/R2 (1984), Oak Ridge National Laboratory.

2. W. A. Rhoades and R. L. Childs, "The DORT Two-Dimensional Discrete Ordinates Transport Code," Nuclear Sctence \& Engineering 99, 1, pp. 88-89, (May 1988).

3. W. A. Rhoades, "Development of a Code System for Determining Radiation Protection of Armored Vehicles (The VCS Code)," ORNL. TM-4664, Oak Ridge National Laboratory, (October 1974).

4. W. A. Rhoades, M. B. Emmett, G. W. Morrison, J. V. Pace, III, and L. M. Petrie, "Vehicle Code System (VCS) User's Manual," ORNLTM-4648, Oak Ridge National Laboratory, (August 1974).

5. W. H. Scott, Jr., "Vehicle Code System (VCS) Documentation and Uncertainty Analysis," SAI Report SAI-133-79-977-LJ, Science Applications International Corporation, (December 1979).

6. S. N. Cramer and C. O. Slater, "Investigation of Radiation Effects in Hiroshima and Nagasaki Using a General Monte Carlo-Discrete Ordinates Coupling Scheme," ORNL/TM-11532, Oak Ridge National Laboratory, (May 1990).

7. J. 0. Johnson, J. D. Drischler, and J. M. Barnes, "Analys is of the Fal1-1989 Two-Meter Box Test Bed Experiments Performed at the Army Pulse Radiation Facility (APRF)," ORNL/TM-11777, Oak Ridge National Laboratory, (May 1991).

8. R. T. Santoro et a1., "DNA Radiation Environments Program Fall 1989 2-Meter Box Experiments and Analysis," ORNL/TM-11840, Oak Ridge National Laboratory, (May 1991).

\subsection{SAMPLE PROBLEM}

A complete listing of the input cards for the sample problem is given in Figure 7-1, and some selected output is shown in Figure 7-2. The sample problem demonstrates the processing of the VISTA fluence file for the air-over-ground free-field environment at 400 meters from the source and the MORSE adjoint calculation of the two-meter box experiments at 400 meters.7,8 In viewing Figure $7-1$, the input illustrates; a radius of 
400 meters (RAD $=40000.0 \mathrm{~cm}$ ), the DORT and MORSE air-ground interfaces were equivalent (AZERO=0.0), and a zero degree rotation of the bnx (ALPHA=0.0). The minimum (ZBOT) and maximum (ZTOP) $z$ boundartes in the MORSE geometry were $-15.0 \mathrm{~cm}$ and $900.0 \mathrm{~cm}$, respectively, and the detector height in the MORSE adjoint calculation was $110.16 \mathrm{~cm}$. The print control flag (IPRT) was set to 2 to obtain both free-fleld and invehicle fluence and dose data, and the maximum weight allowed would default to the value pre-set in the code (WATMX $=0)$. The VISTA and MORSE group structures were equivalent, so consequentiy, Cards $C, D$, and $E$ were omitted from the input. The next three title cards are for cards $F, G$, and $H$. The response function utilized in the DRC analysis is the free-in-air tissue kerma response in units of $g y \cdot \mathrm{cm}^{2} / n, \gamma$. The last card is the input for Card $J$. This particular input deck did not exercise the option of stacking cases for different target orientations. Notice that the "**" values are in columns 2 and 3 . DRC w111 not correctly identify the input if these FIDO control parameters are not in the proper columns.

The selected DRC output shown in Figure 7-2 first 1llustrates the input parameters read from Cards $A$ and $B$, the rotation matrix for the parameter alpha, and a series of titles identifying the MORSE calculation, the VISTA calculation file, and the DORT calculation used to create the input files for this DRC case. The titles are followed by parameters relevant to the VISTA fluence flle including some control parameters, the specific quadrature information, and radial and axial mesh points on the VISTA file. The output then contains some arrays used internal to DRC that may not be useful to the user, followed by an indication of the storage used in the DRC case. A title from the MORSE adjoint history file is then printed indicating whether the in-group biasing option in MORSE was invoked. The MORSE input source spectrum and bias function are then printed indicating the source sampling utilized in the adjoint calculation. Next, the output lists the response function title, the values of the response function to be folded with the free-field and in-vehicle fluences, and arrays relevant to the energy bins used in the DRC case. Much of this last set of output is similar to the output of the SAMBO routines in MORSE since DRC contains a modified version of SAMBO.

The next output is the results of the calculations in subroutine FREDOS and represents the GRTUNCL/DORT free field fluence and dose at the detector location in the MORSE geometry. Both the spectra and integral totals are printed. This output is then followed by the DRC detector responses (Table 7-1) along with their fractional standard deviations and protection factors and reduction factors (Table 7-2) for the response function given in the input (Card I). These vaiues are what the entire MASH analysis is geared to determine. Finally, the DRC output gives the energy dependent fluence and dose arrays for the first six detector types 1 isted in Table 7-1 along with the normalized total neutron and photon fluence and dose arrays. The format of these arrays is quite cumbersome, however, useful information can be obtained from them. Future efforts will hopefully improve these output arrays into a more useful format. 
$2 m$ box exp/4in steel/dabl 46n-23g 1/b/aprf sh=16.143m/10-24-89(a) air

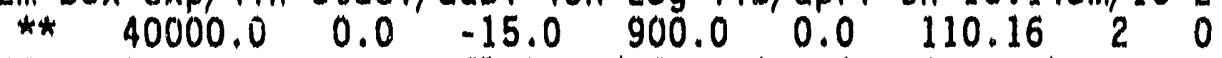
46 neutron - 23 gamma flat and free-in-air tissue kerma response input -.. Imneut 2meh $n-g$ 3mgrd $n-g$ 4mphotons mash $46 n / 23 g$ group free-in-air tissue kerma response $(g y . c m 2 / n, g)$

736532-16 704584-16 685894-16 674499-16 661555-16 638136-16 633529-16 598822-16 57632-15 551514-16 546431-16 $512651-16 \quad 470858-16 \quad 457805-16$ 44359-15 419381-16 357307-16 332494-16 321126-16 291193-16 263438-16 $252468-16 \quad 222507-16 \quad 205934-16 \quad 193682-16 \quad 179065-16 \quad 163746-16 \quad 130075-16$ 102563-16 806538-17 563405-17 359169-17 25917-16 213032-17 $147572-17$ 628401-18 219938-18 925902-19 448136-19 20734-18 103718-19 932428-20 $\begin{array}{lllllllll}135874-19 & 228494-19 & 372646-19 & 129126-18 & 401109-16 & 317559-16 & 27612-15\end{array}$ 23517-15 205102-16 $185083-16 \quad 164356-16 \quad 143343-16 \quad 121273-16 \quad 1036-14$ 902669-17 $755621-17 \quad 585321-17 \quad 42722-16 \quad 296381-17 \quad 192968-17 \quad 105409-17$ 529629-18 348187-18 313231-18 484629-18 $10497-16 \quad 339599-17$ energy group totals

Figure 7-1. Sample DRC Input for the Two-Meter Box Protection Factor Analysis. 


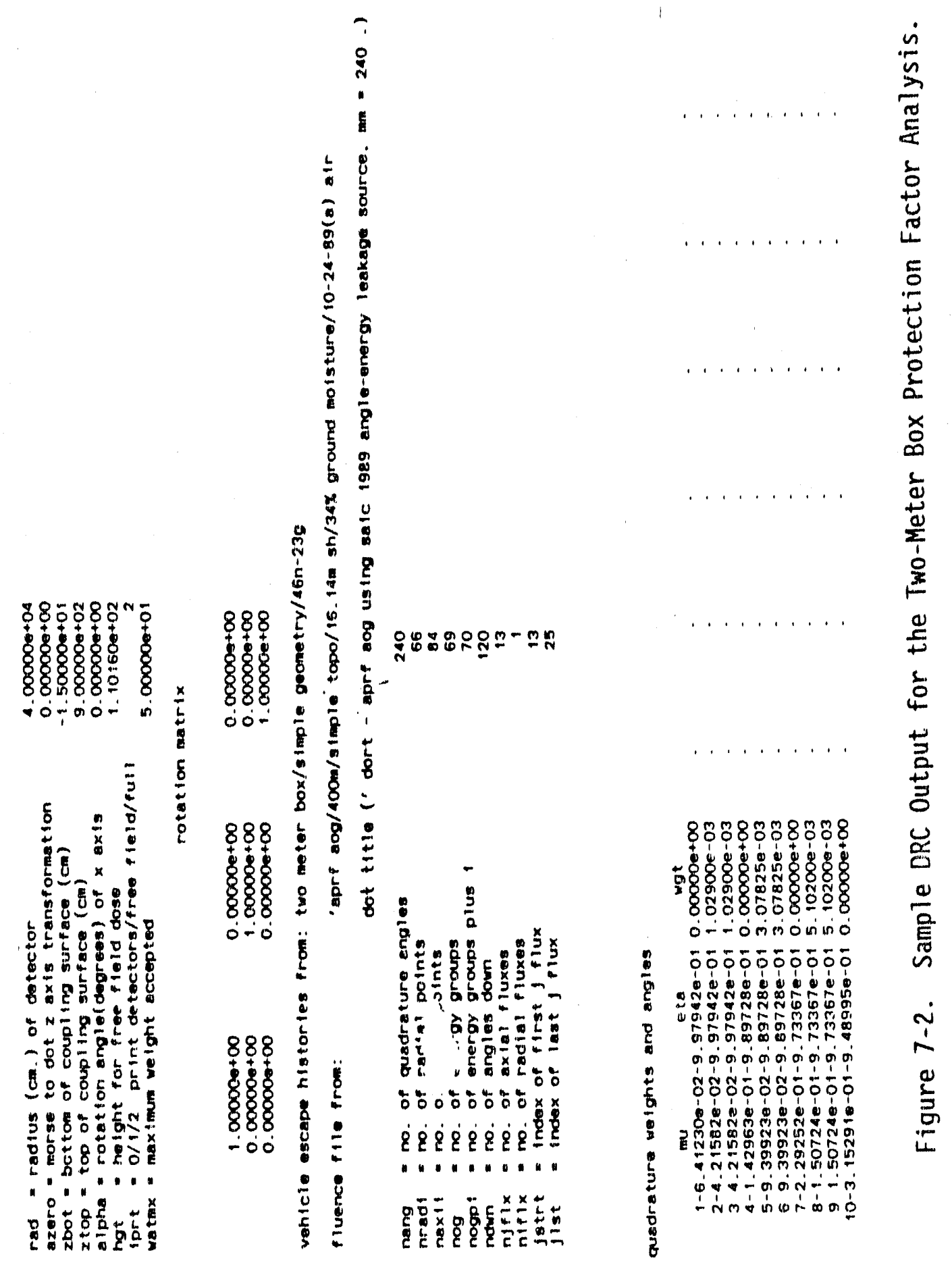




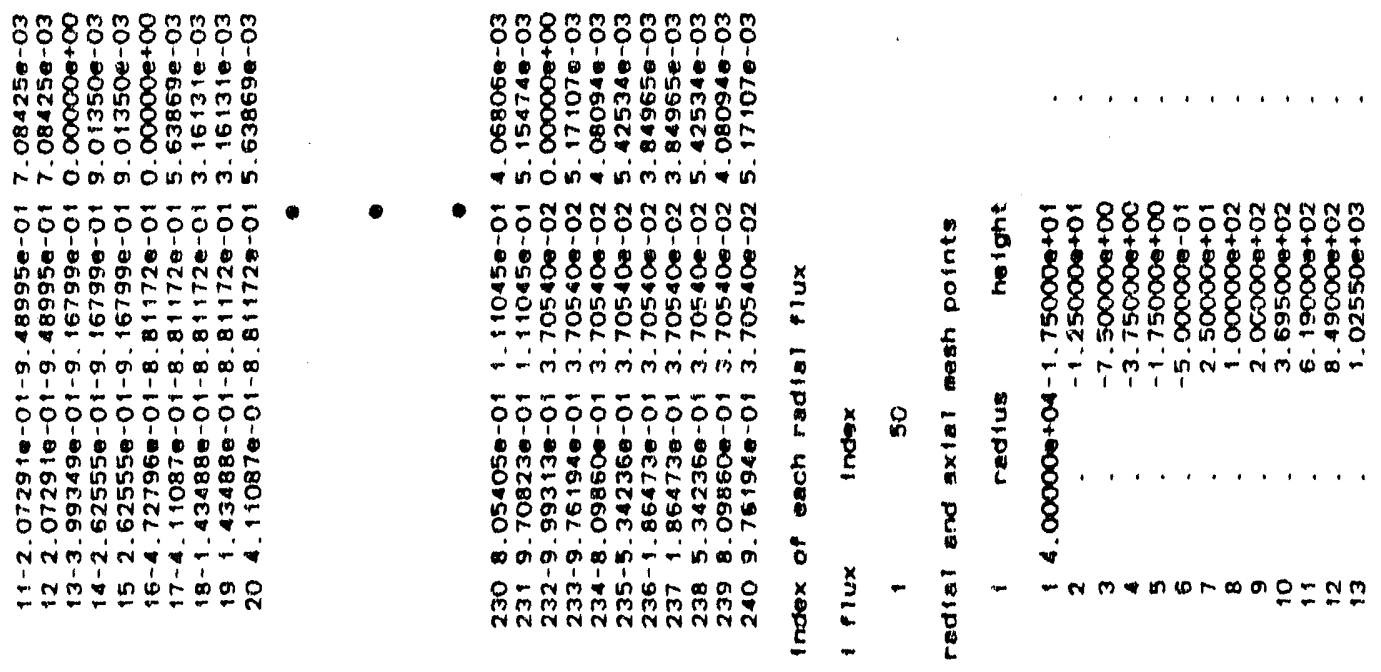




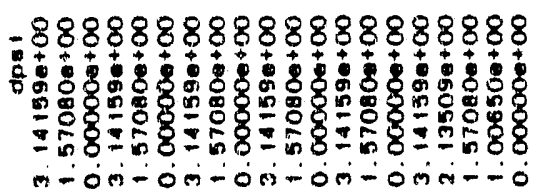
$-88883888888508800880$

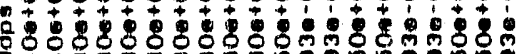

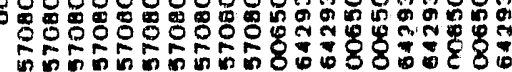

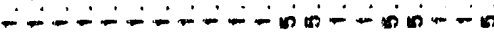

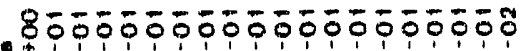
*

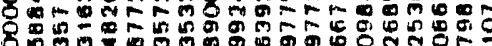

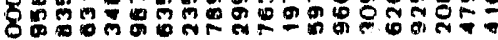

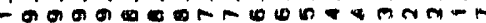

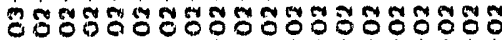
3

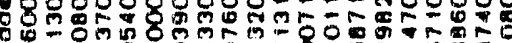

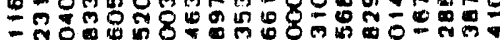
- -rim

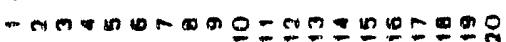

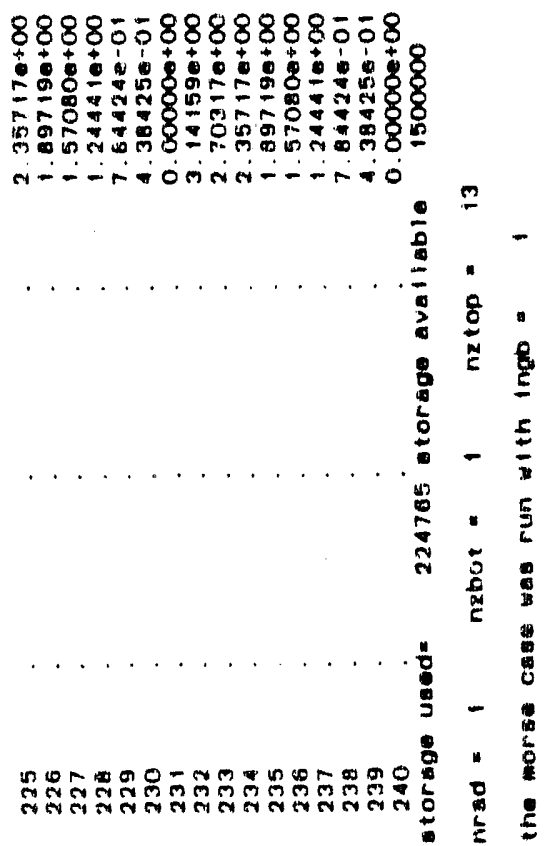

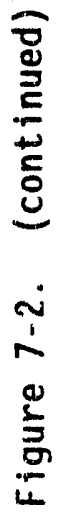




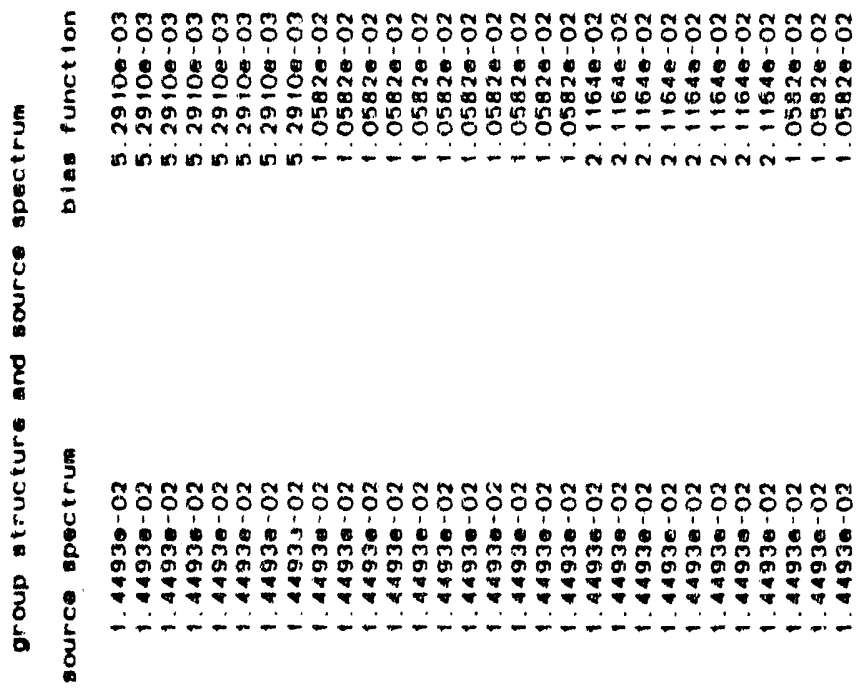

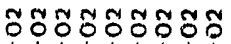

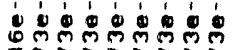

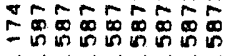
n-- - - - -

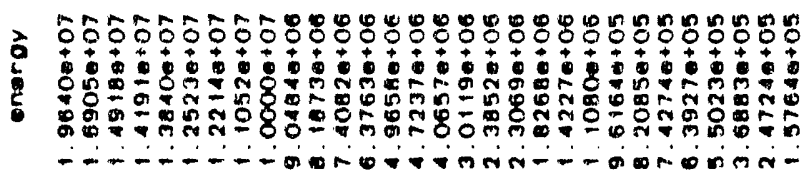




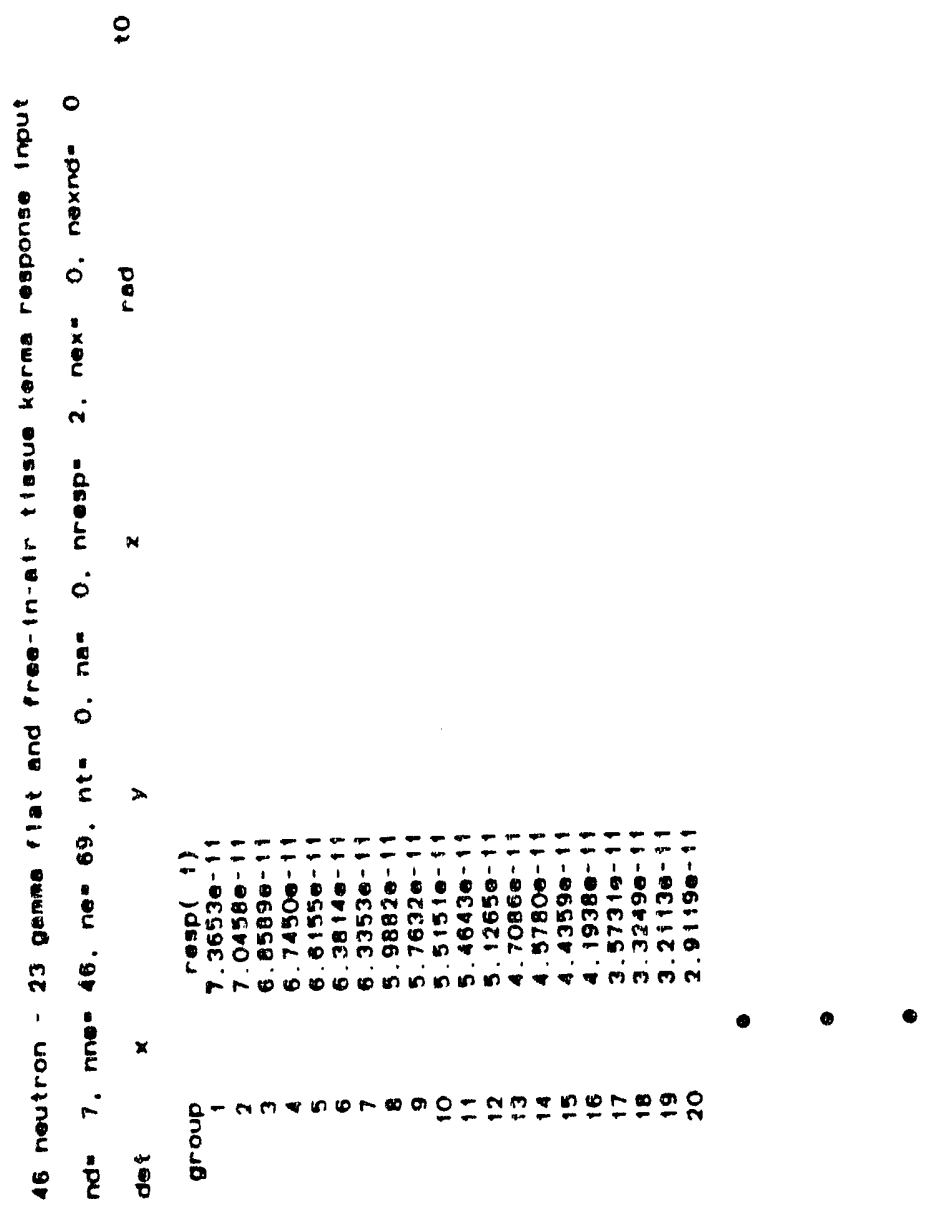

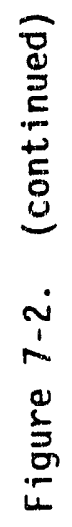




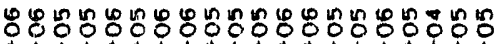

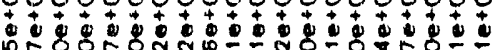

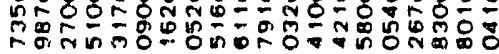

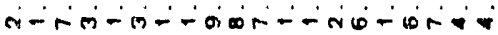

ᄂ T.t.

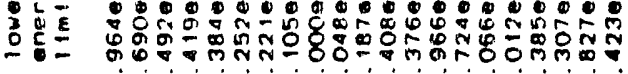

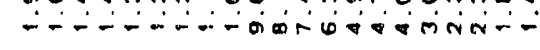

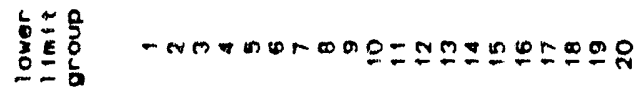

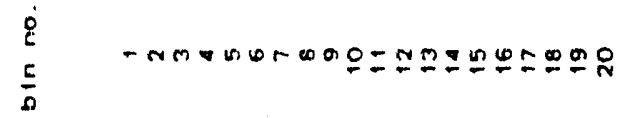

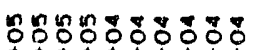

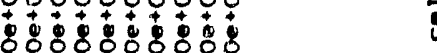
8 品品 88080 $\circ$ 0

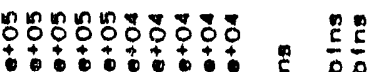

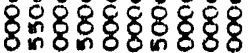

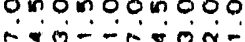

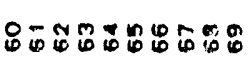

E

N

总 


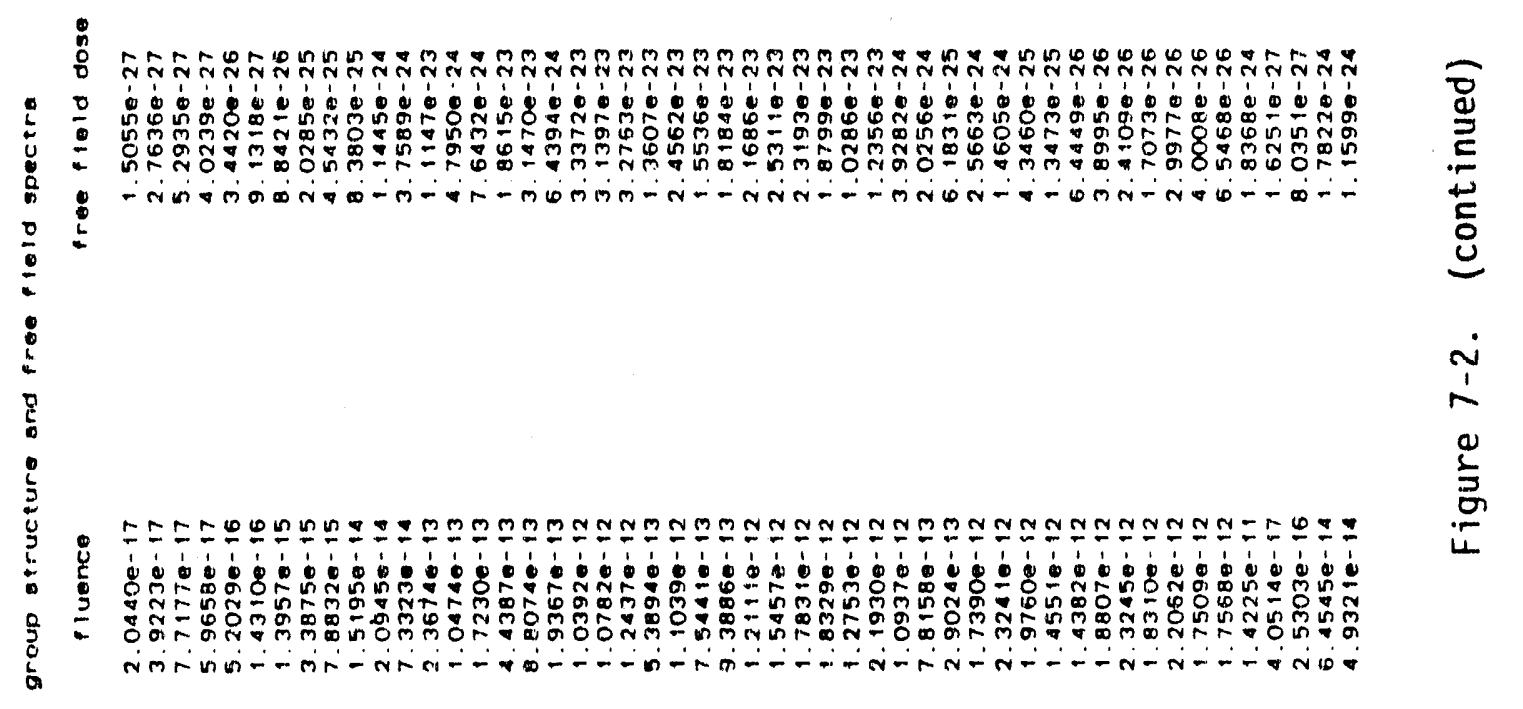

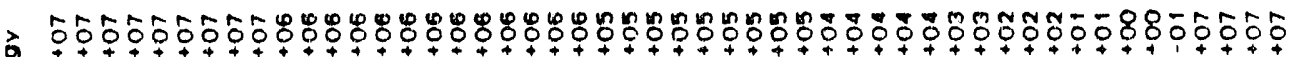

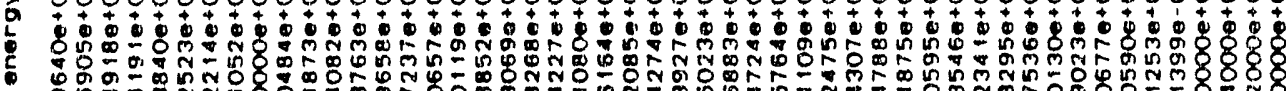

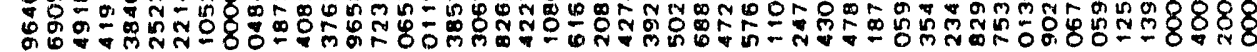
-

$\frac{0}{2}$

- - 

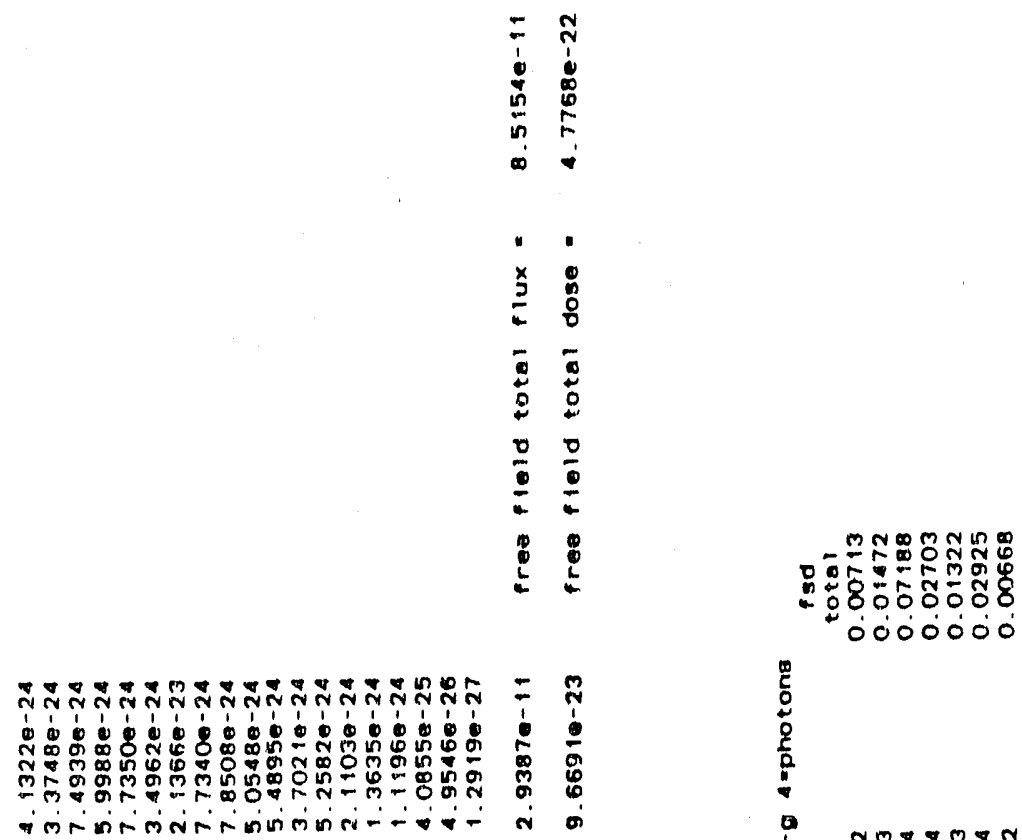

- 000000

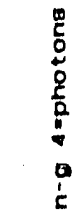

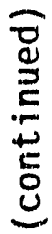

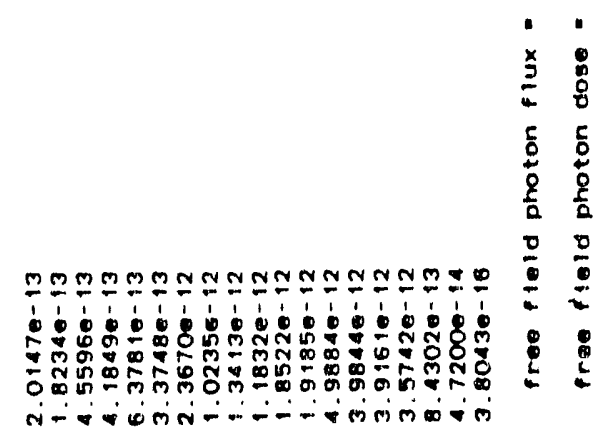

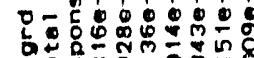

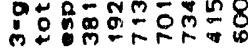

o.

है

5
5
$\vdots$
2

高

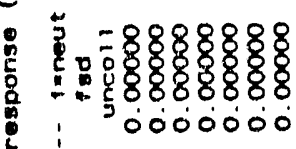

$N$
$\vdots$
$\vdots$
$\frac{1}{2}$
$\frac{1}{4}$
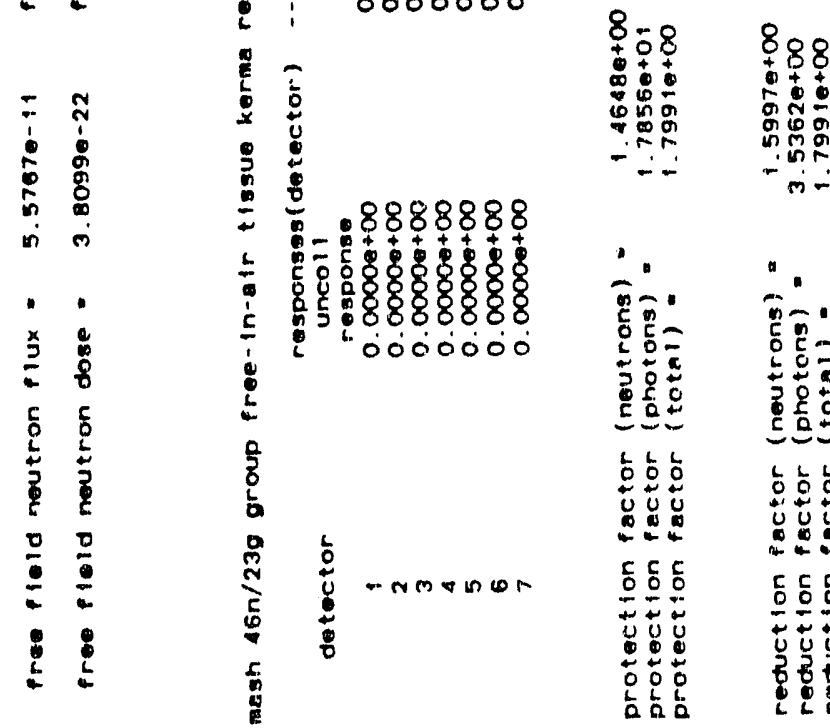

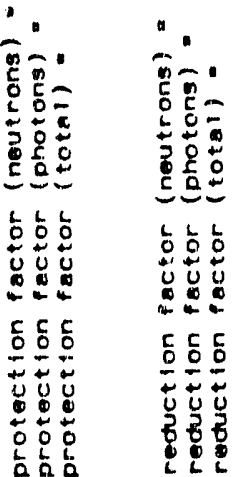




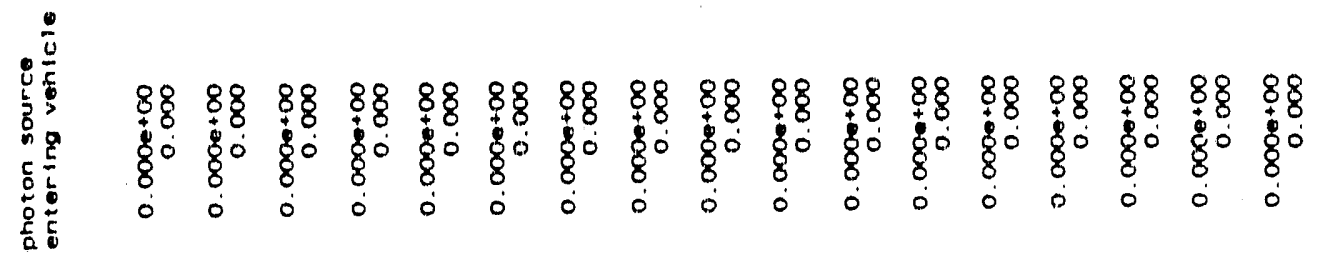

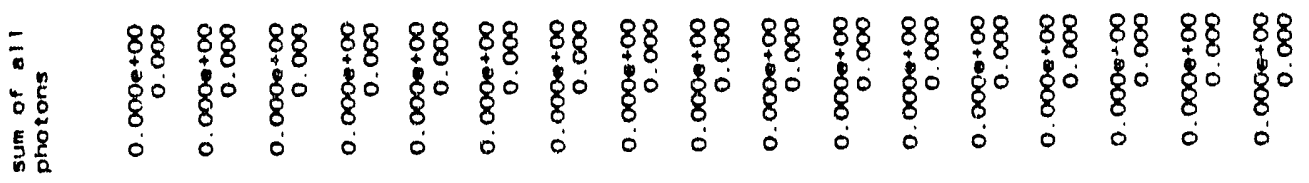

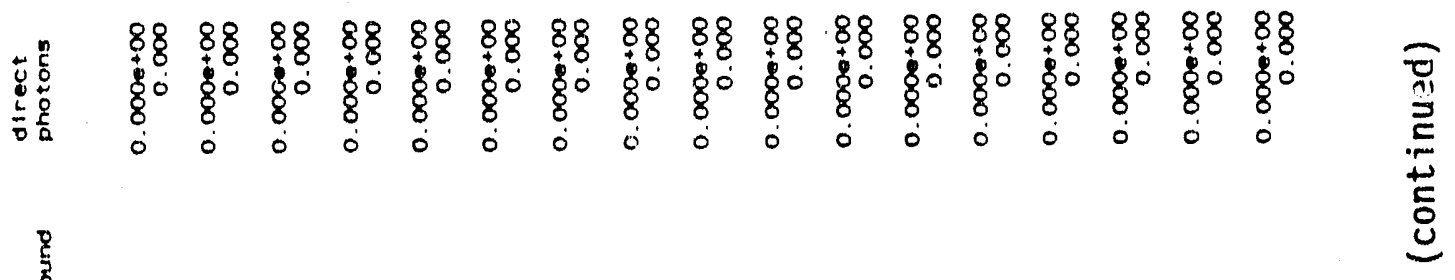

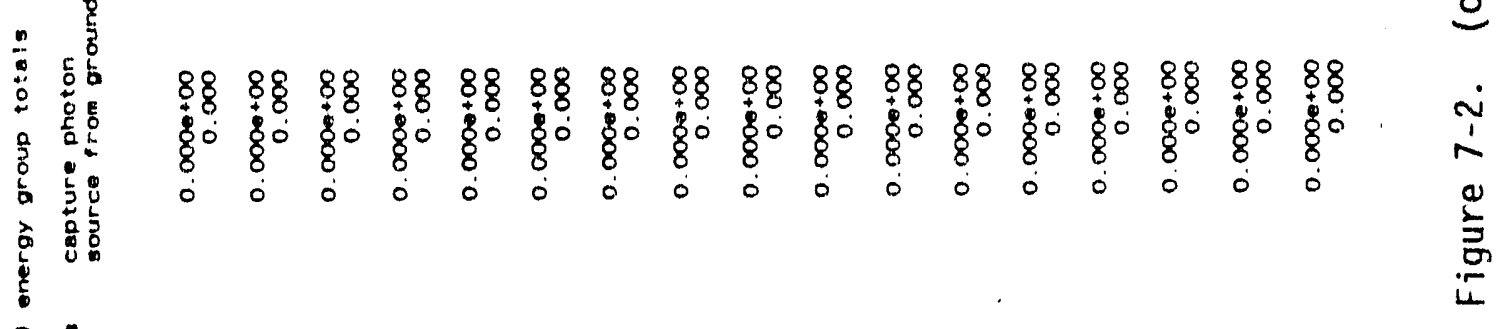

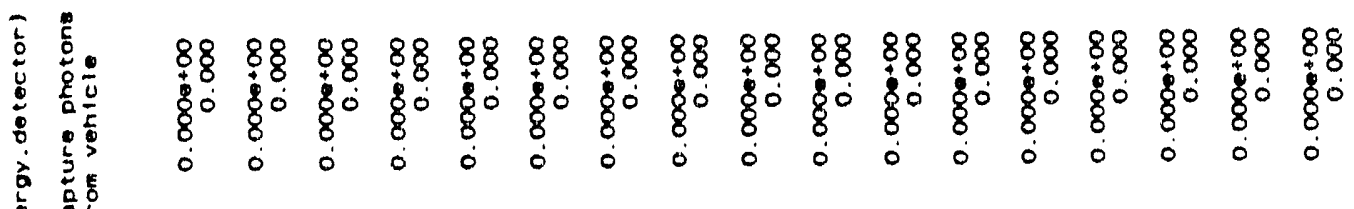

总

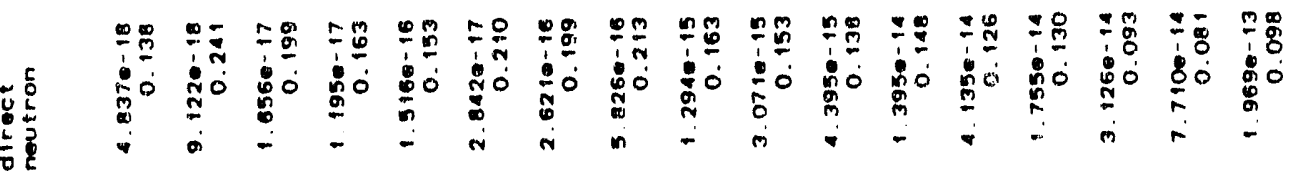

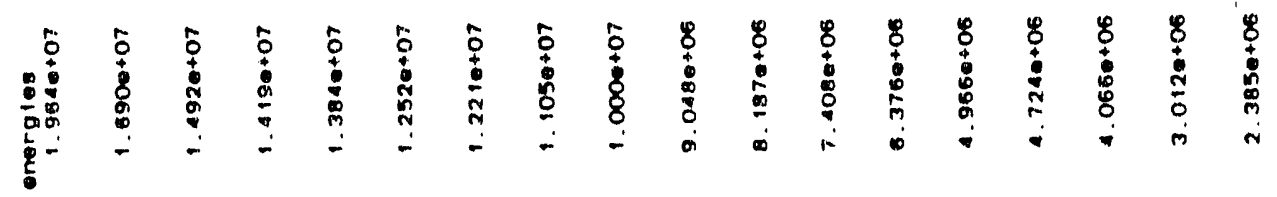




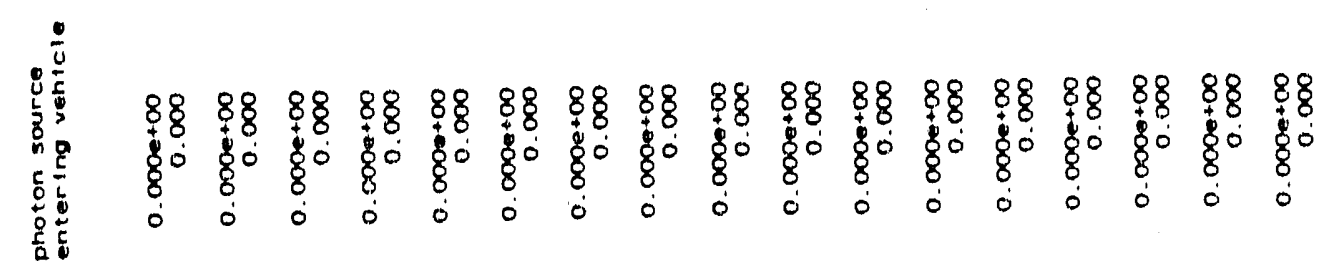

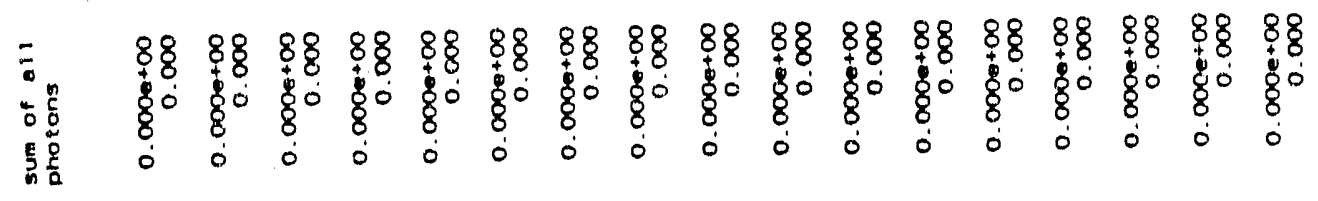

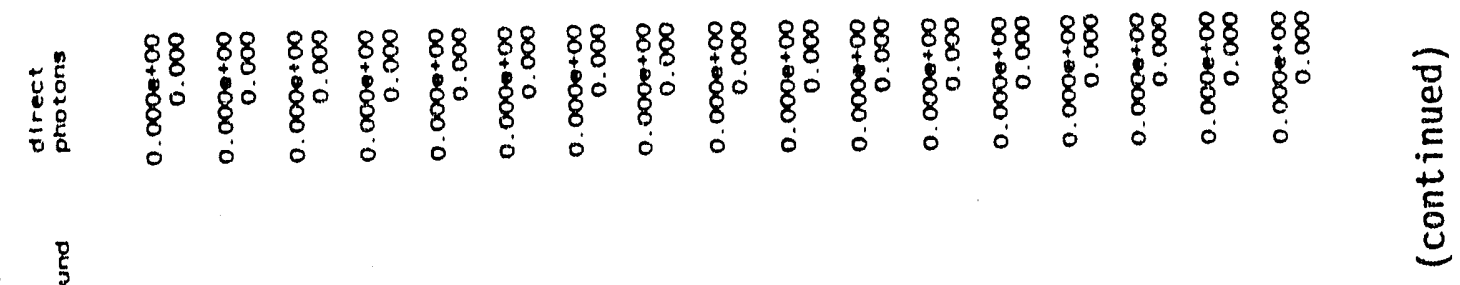

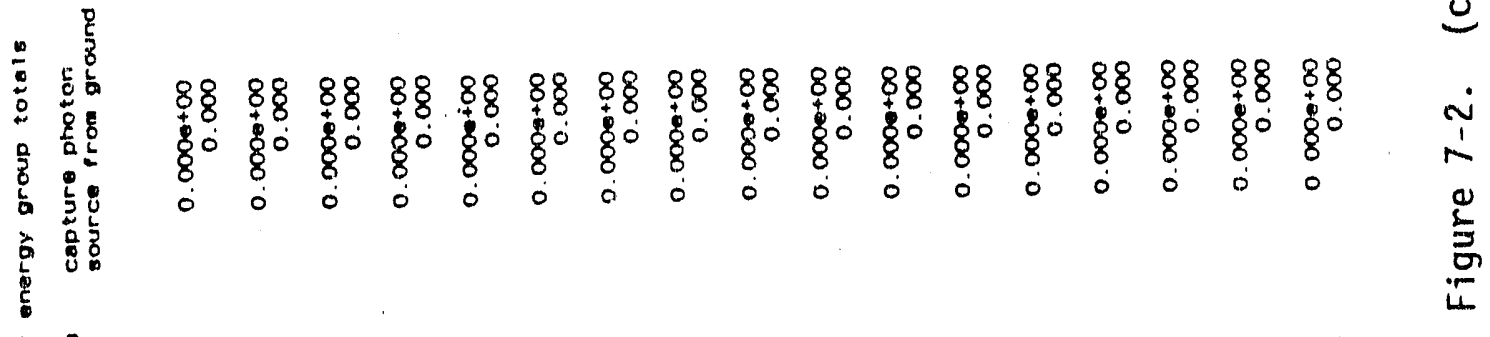

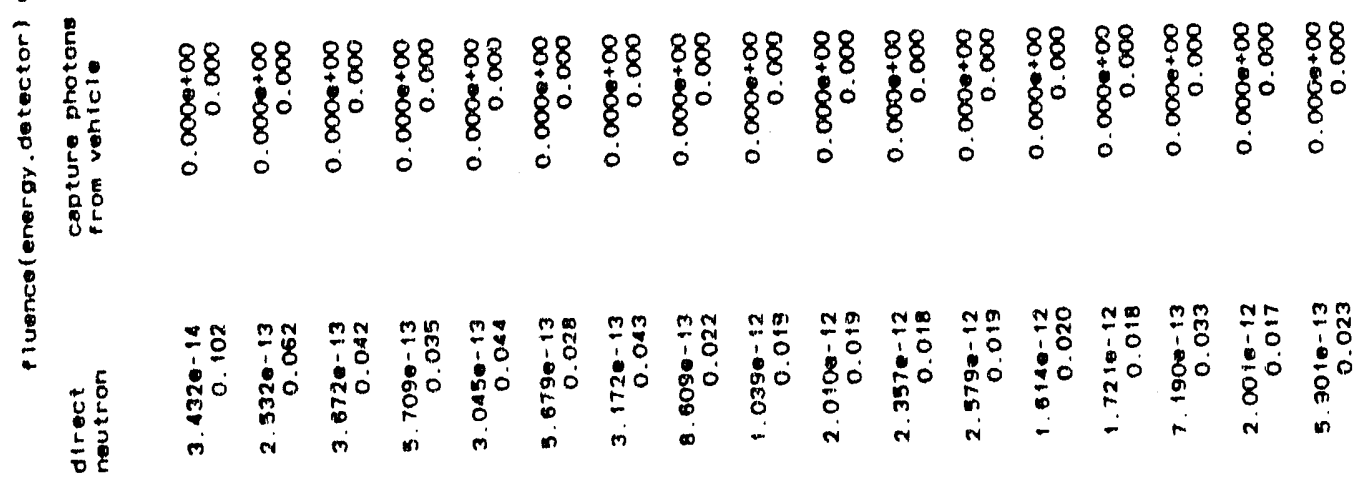

HIIIIII!I!I!! 


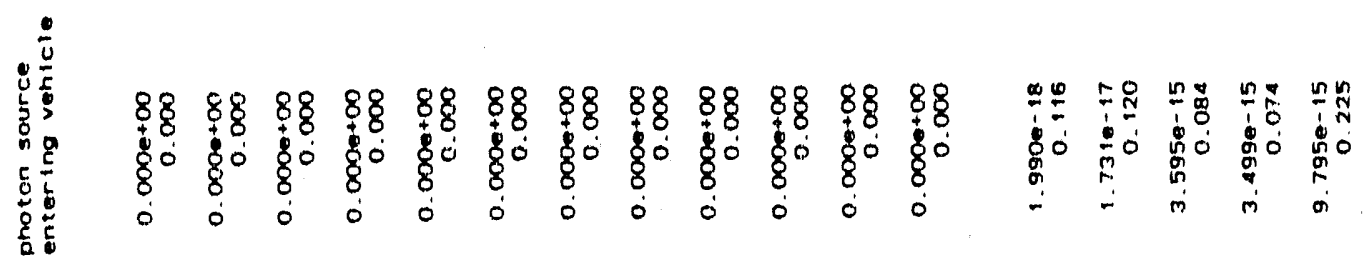

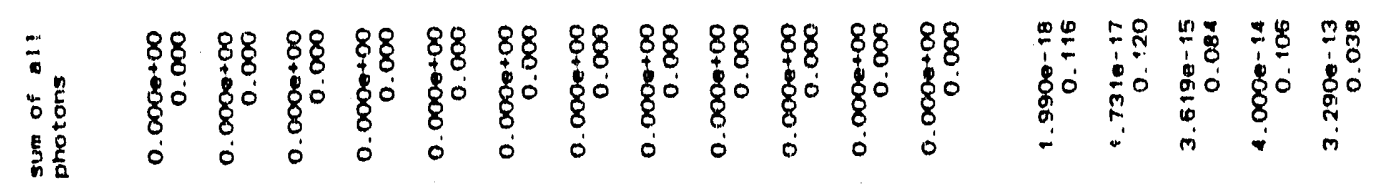

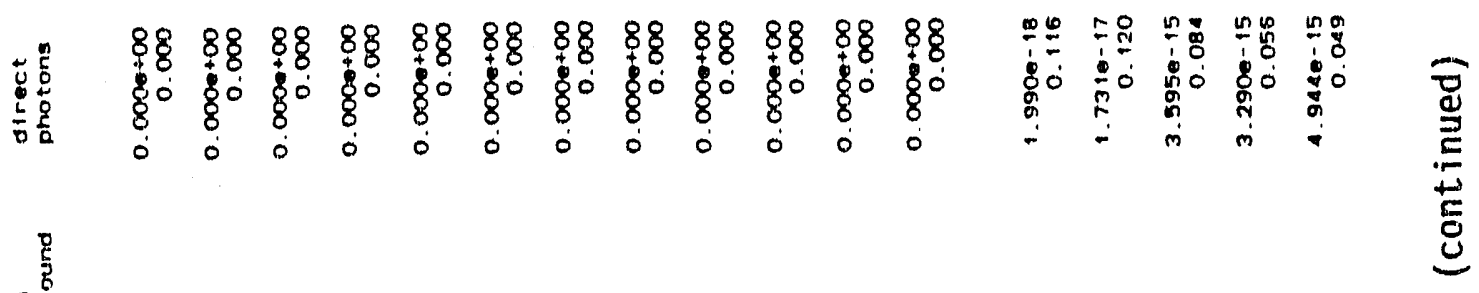

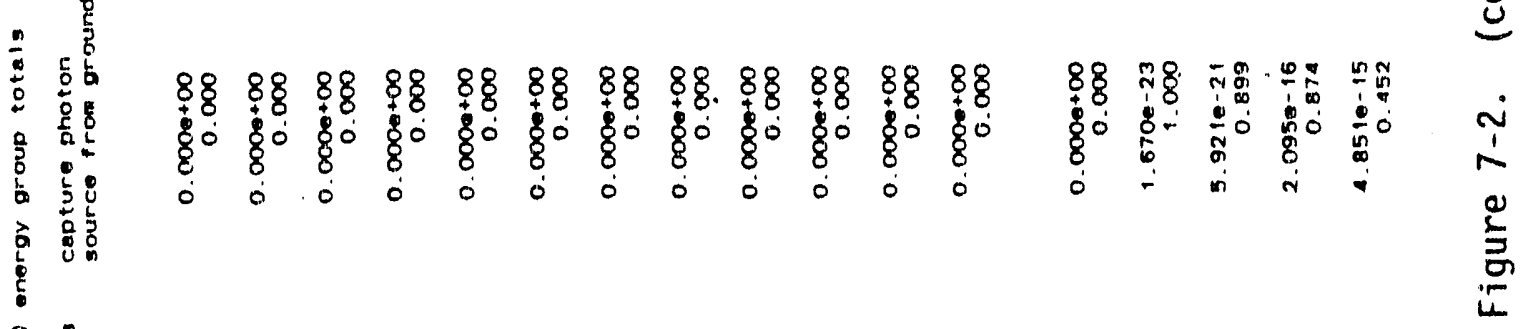

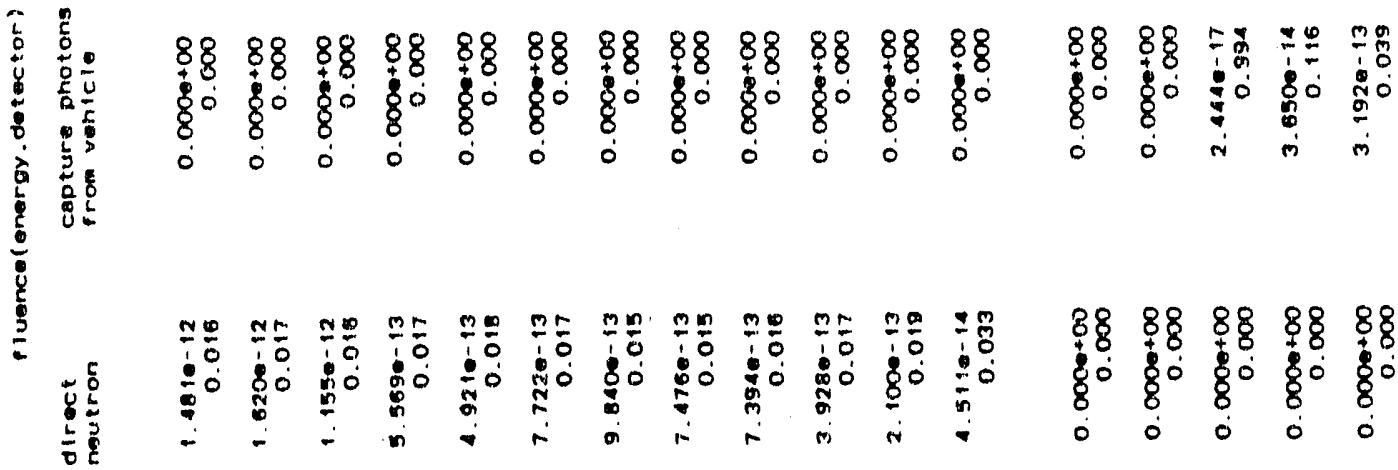

HIII!I!!H!I!! 
\#

If

If if

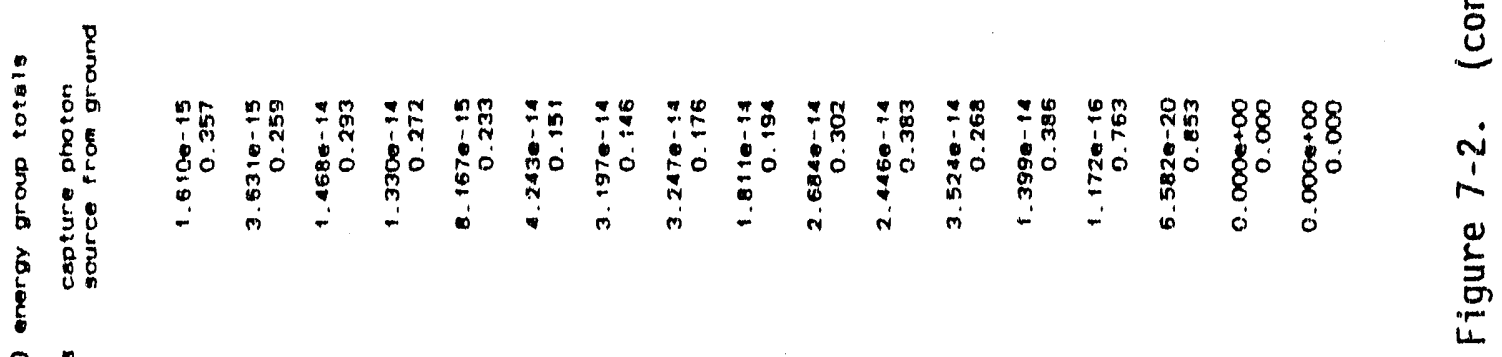

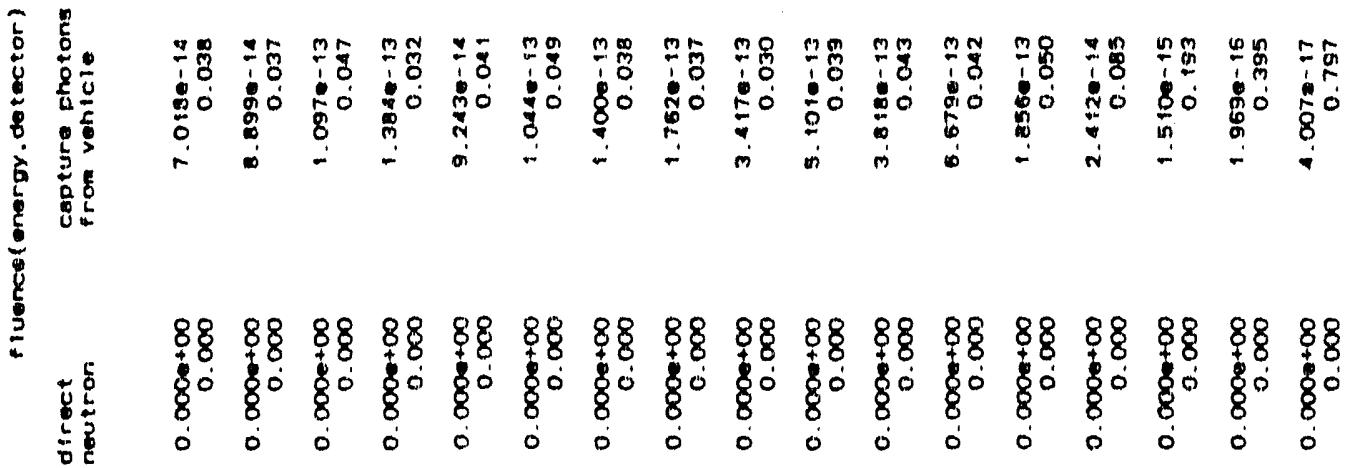

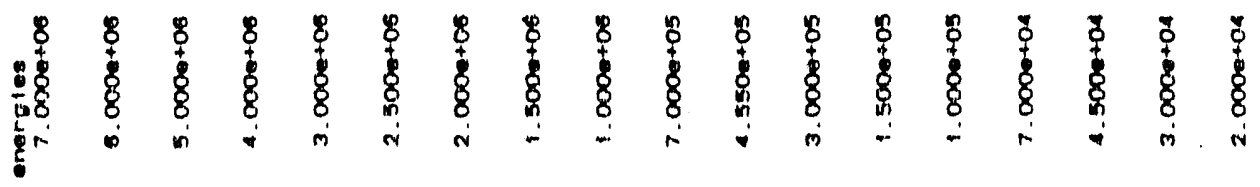




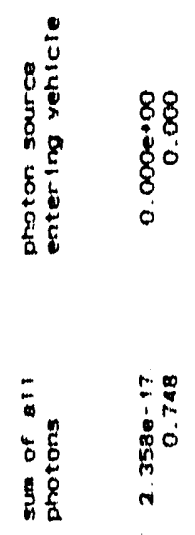

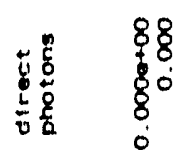

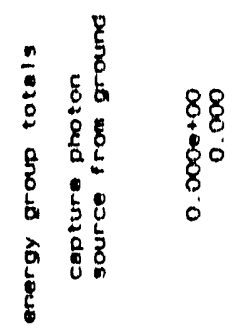

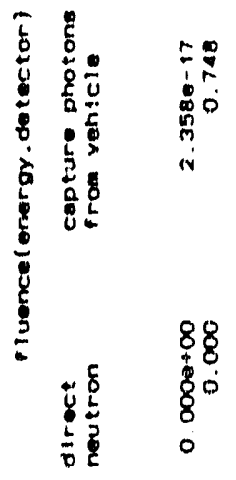

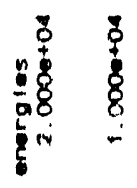

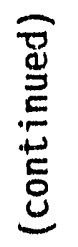

$\frac{1}{r}$

哠

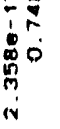




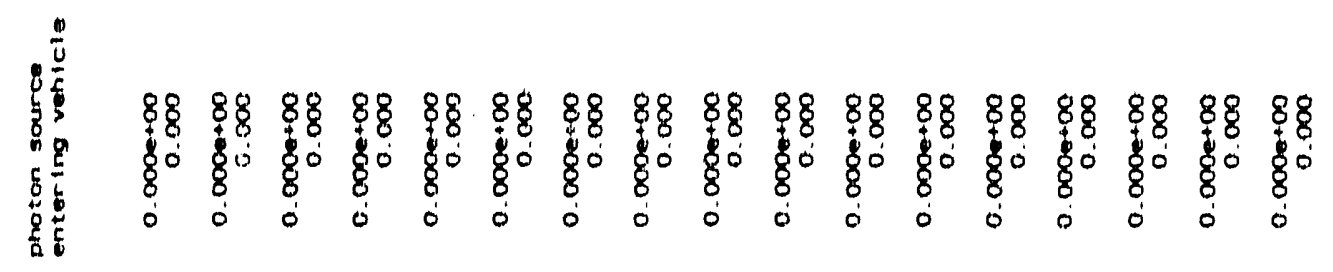
4)

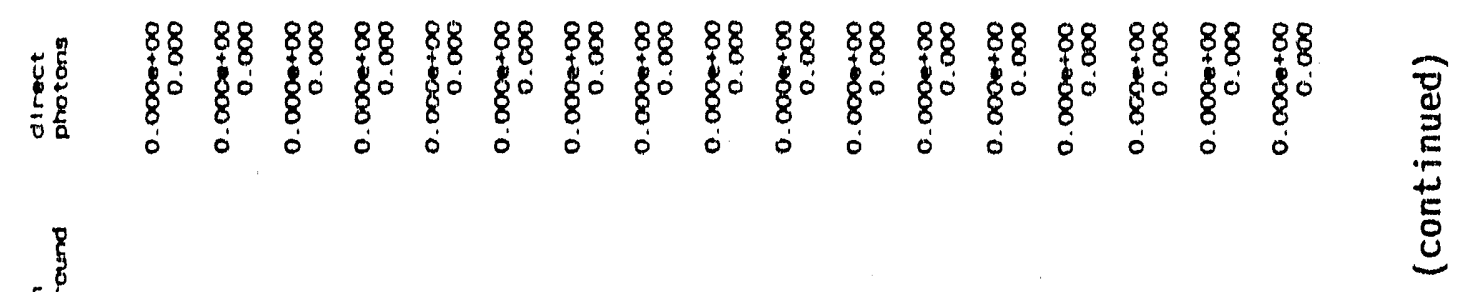

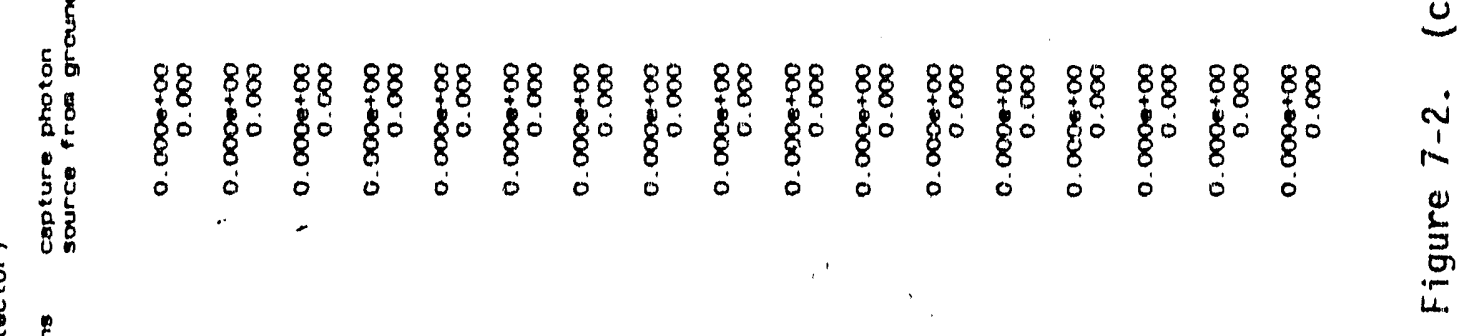

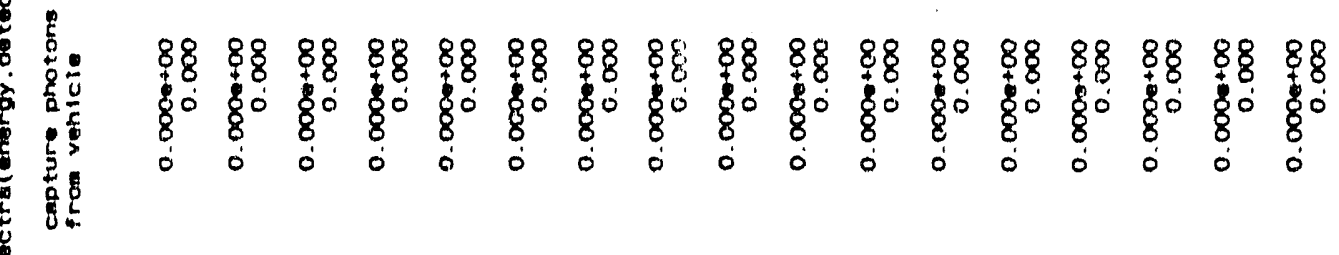

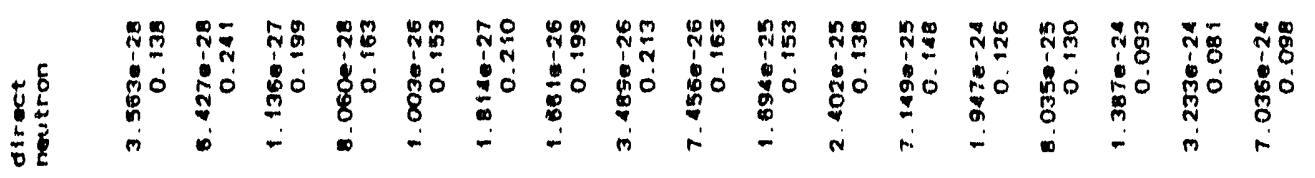
HIIIII!H!H!H 


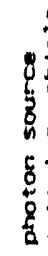

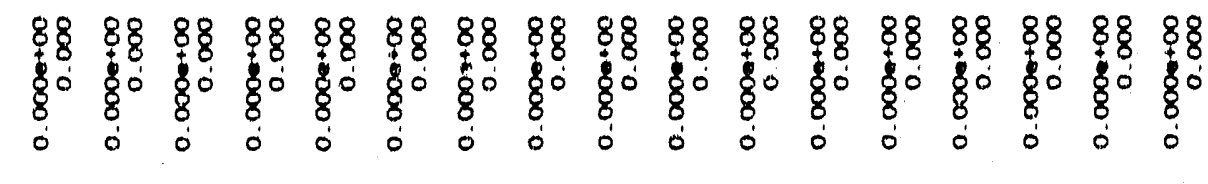

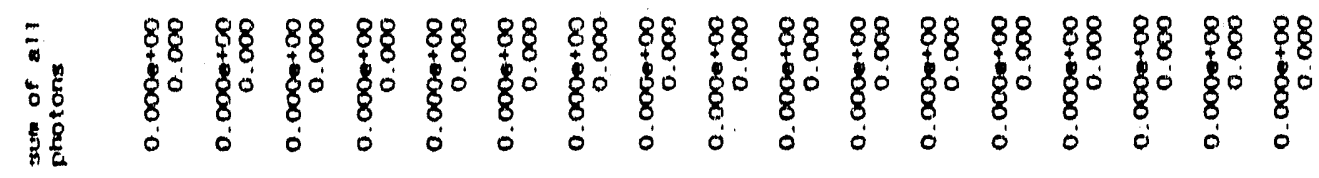

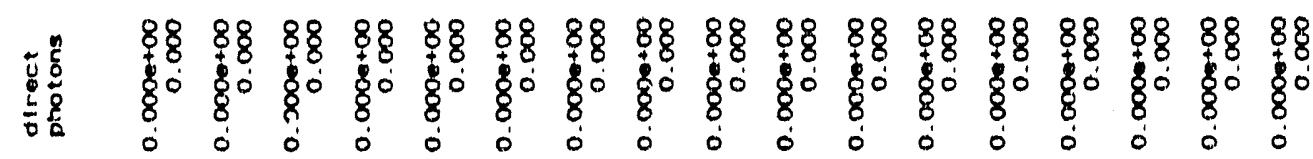

$\begin{array}{lllllllllllllllllll} & & & & & & \\ \end{array}$

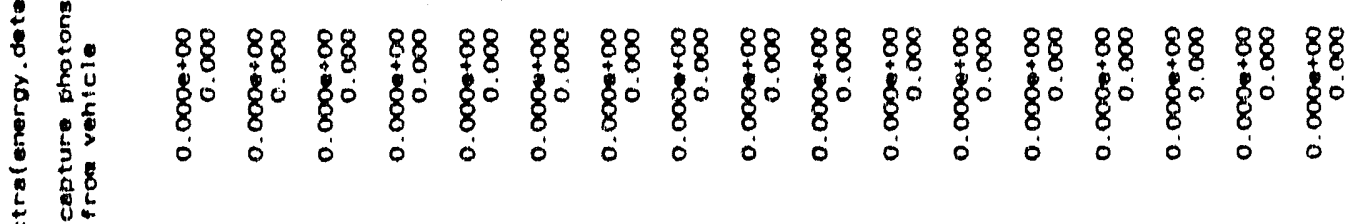

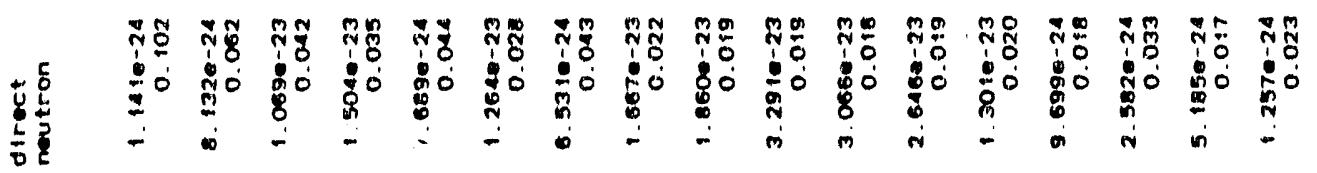

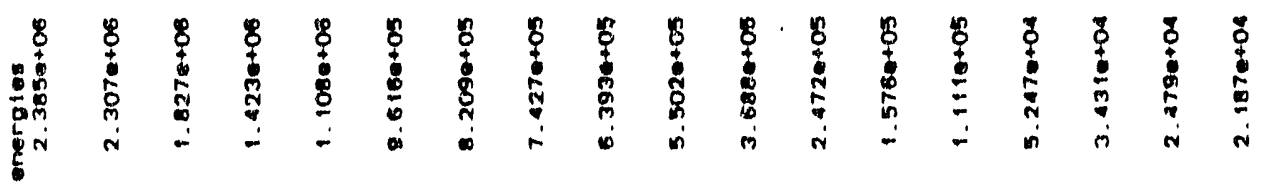




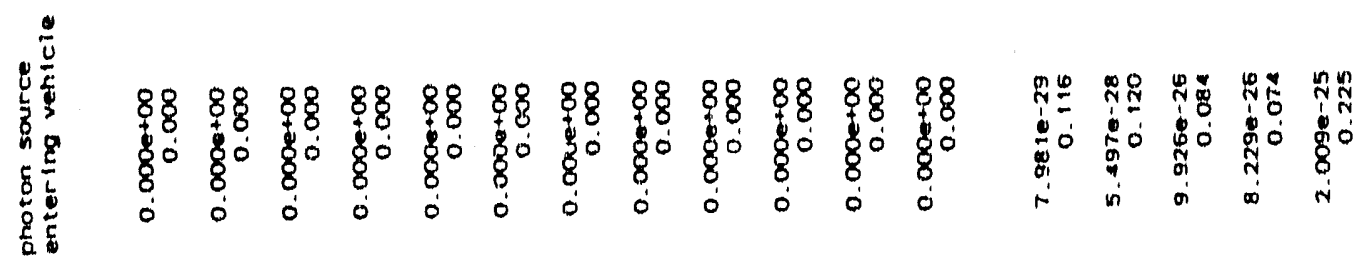

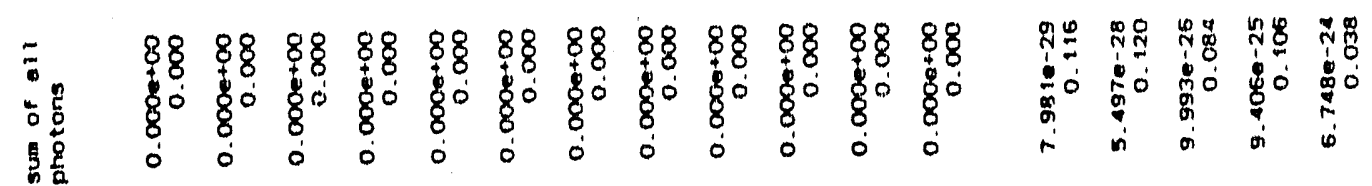

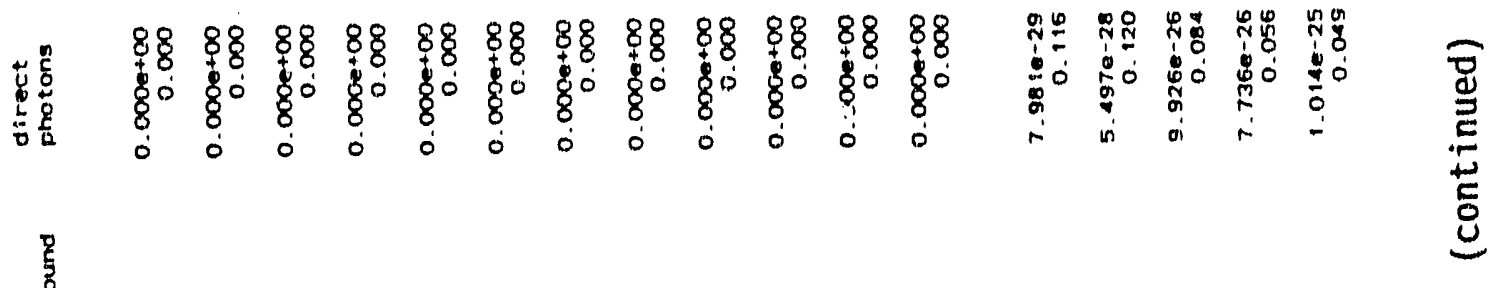

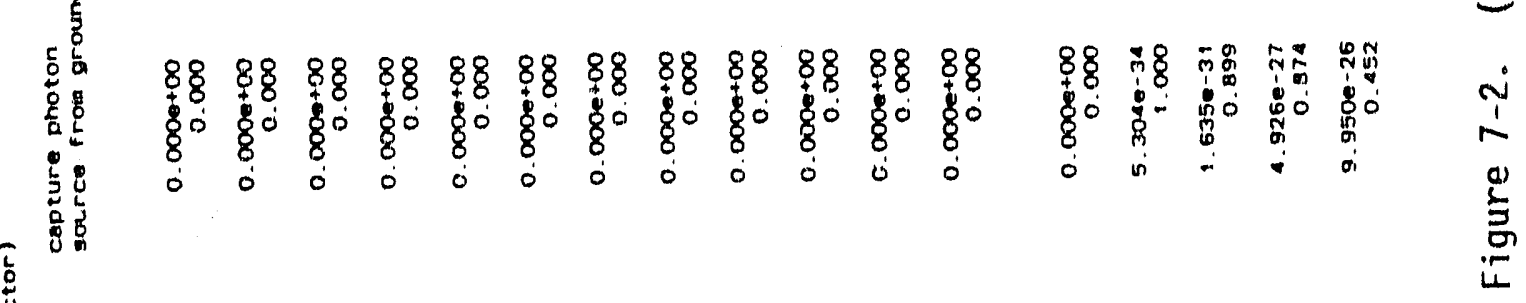

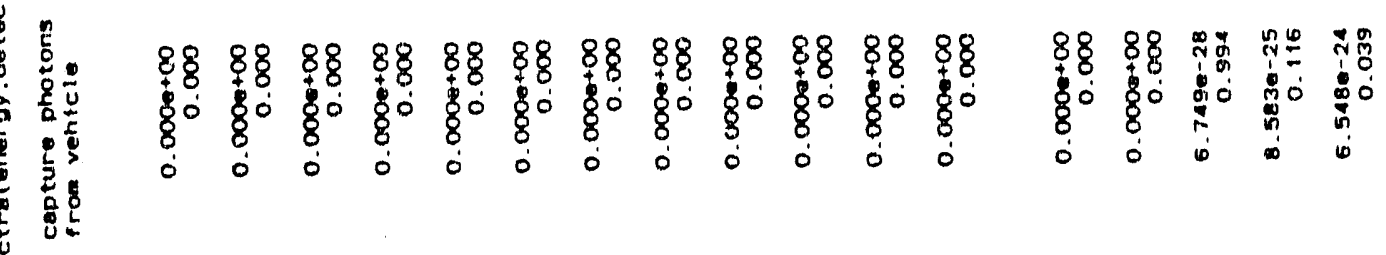

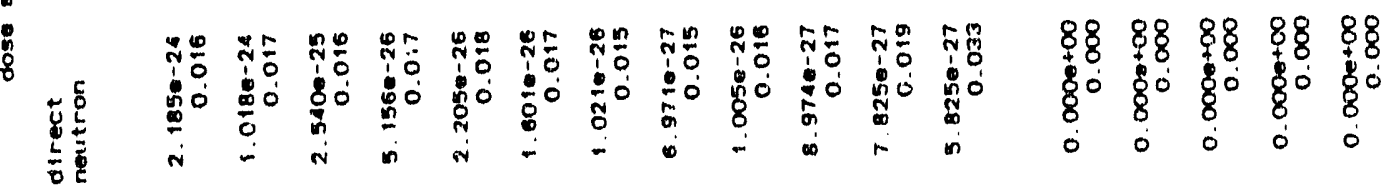

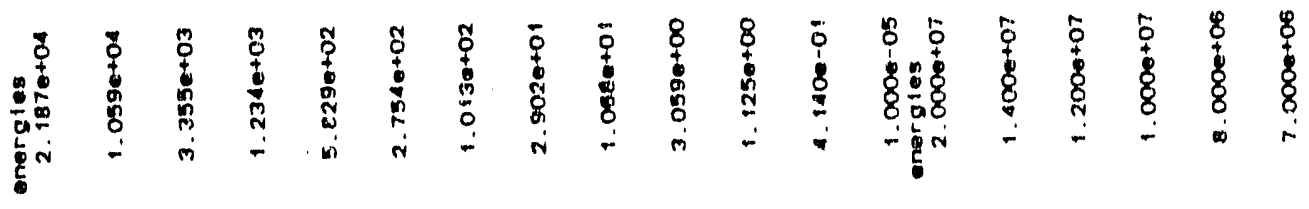




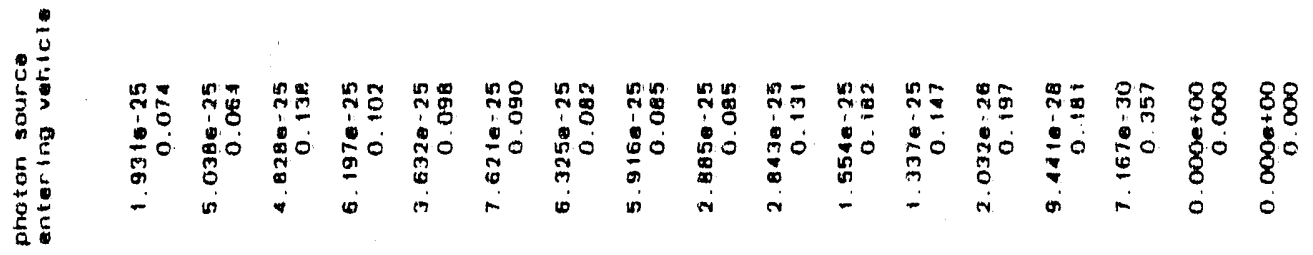

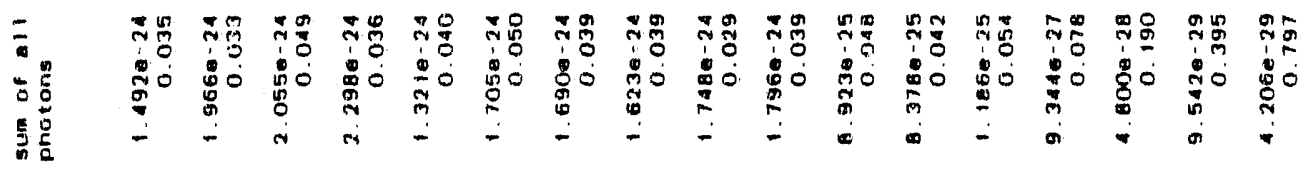

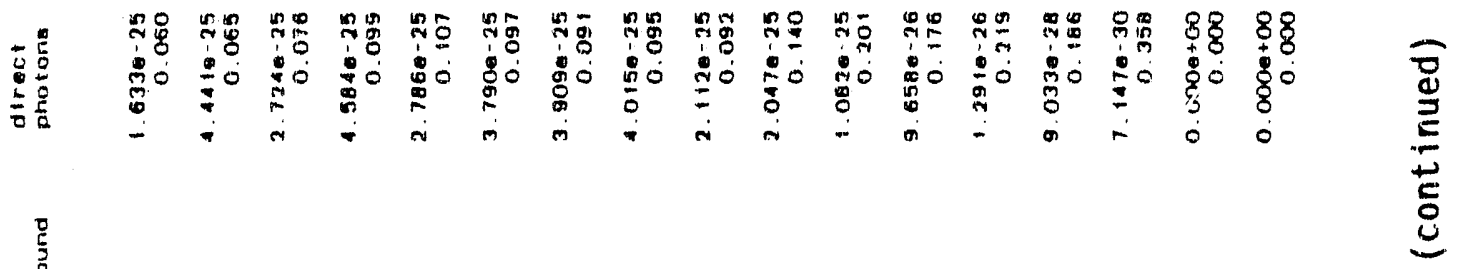

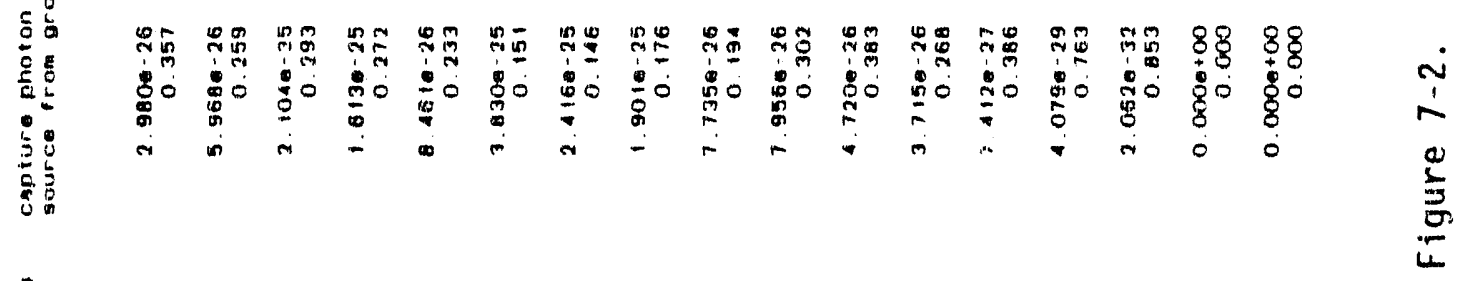

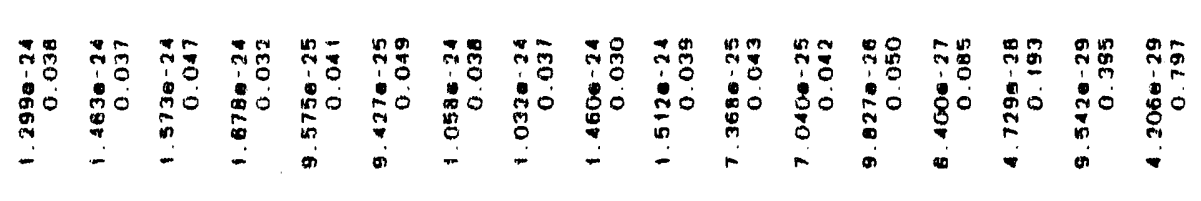

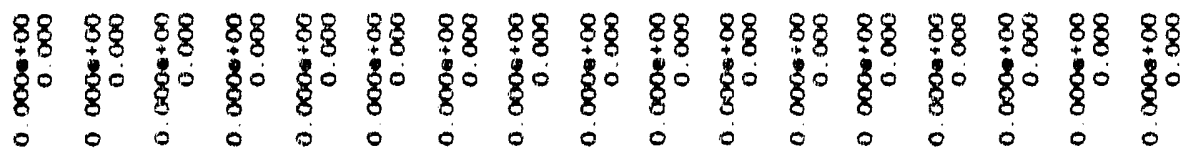

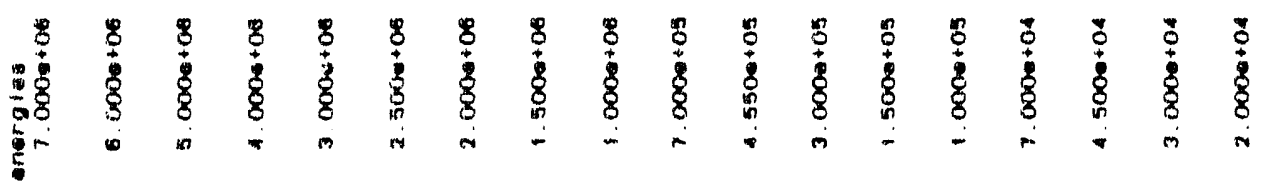




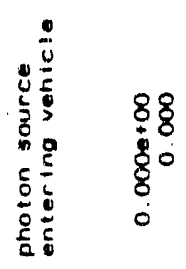

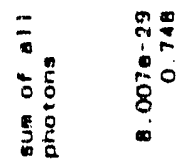

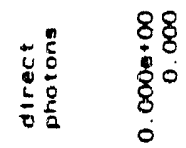

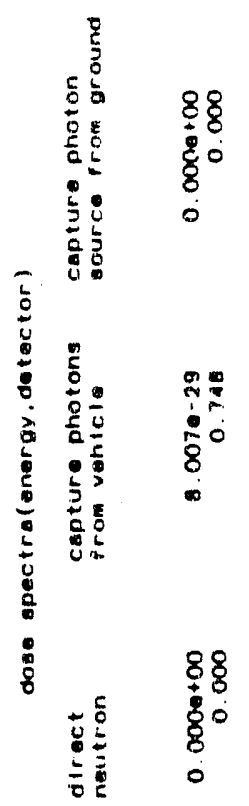

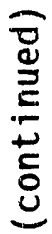

交

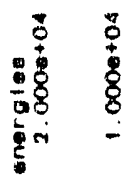




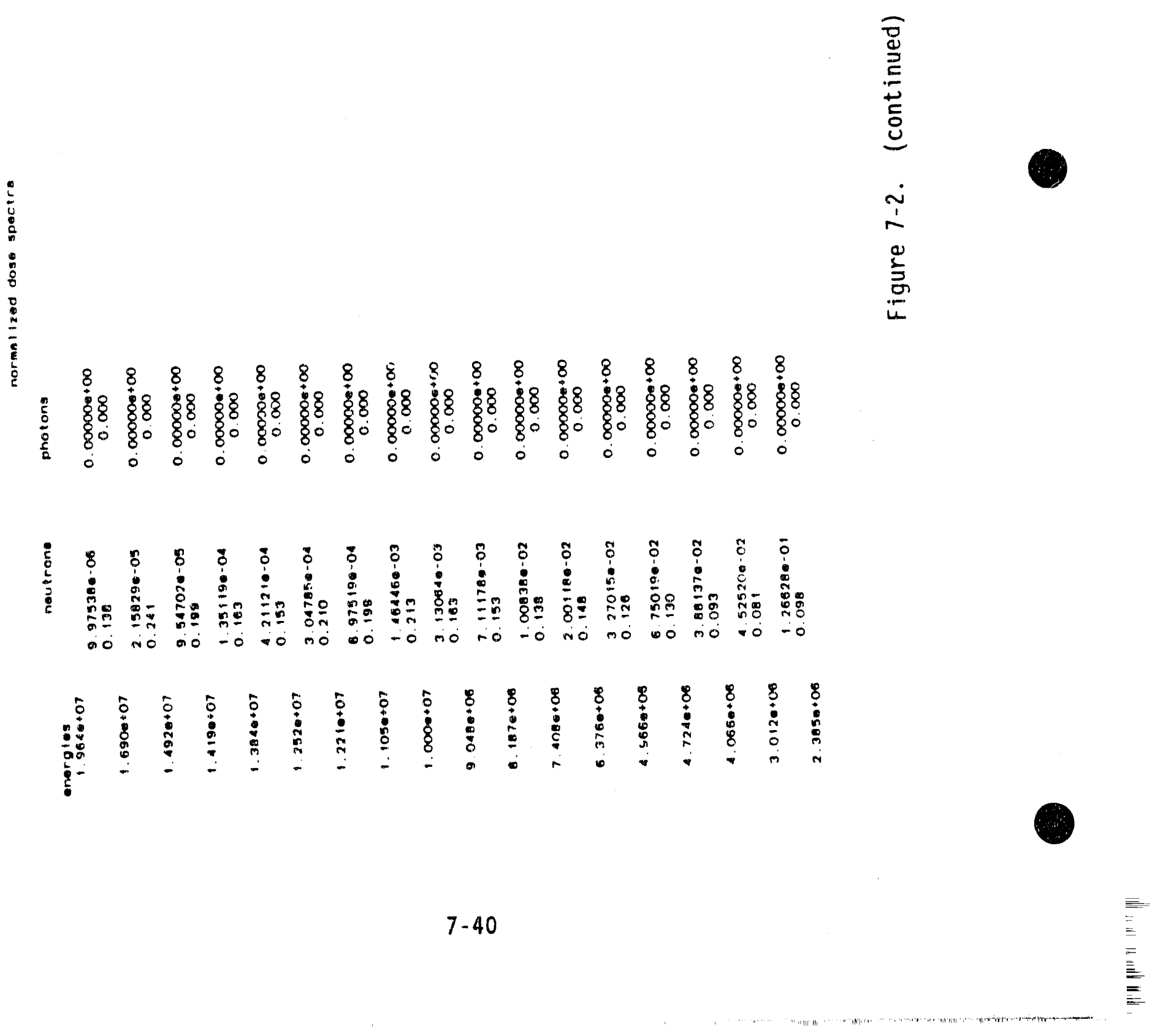




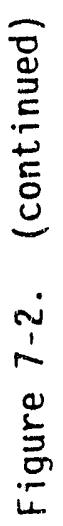

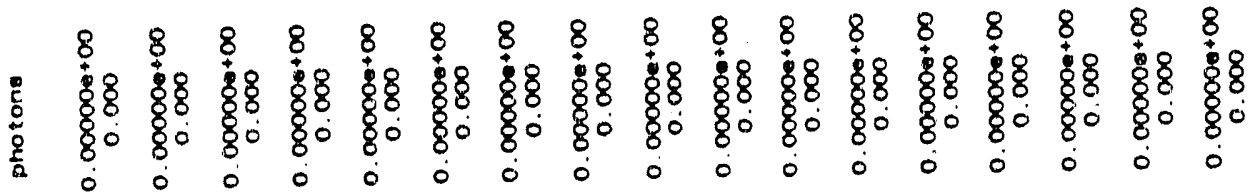

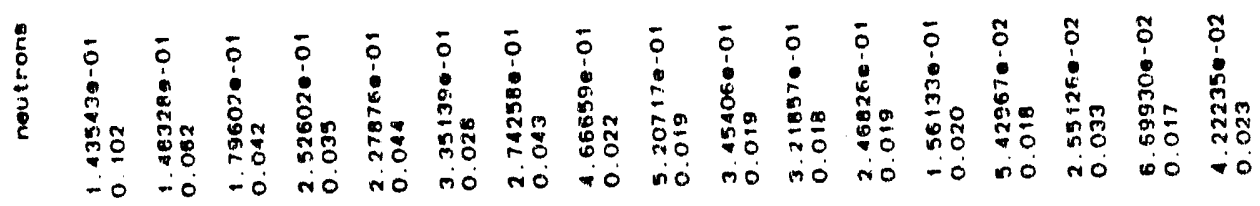

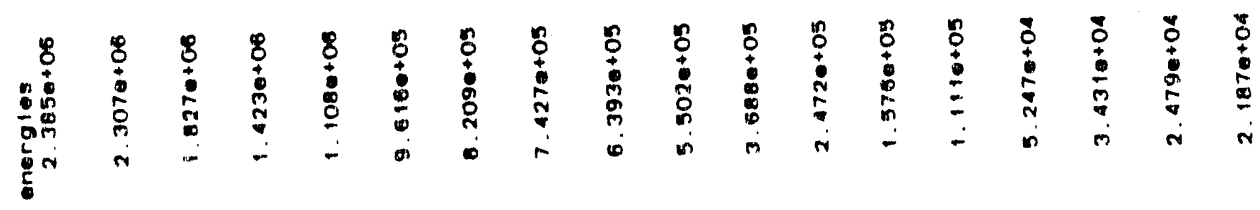




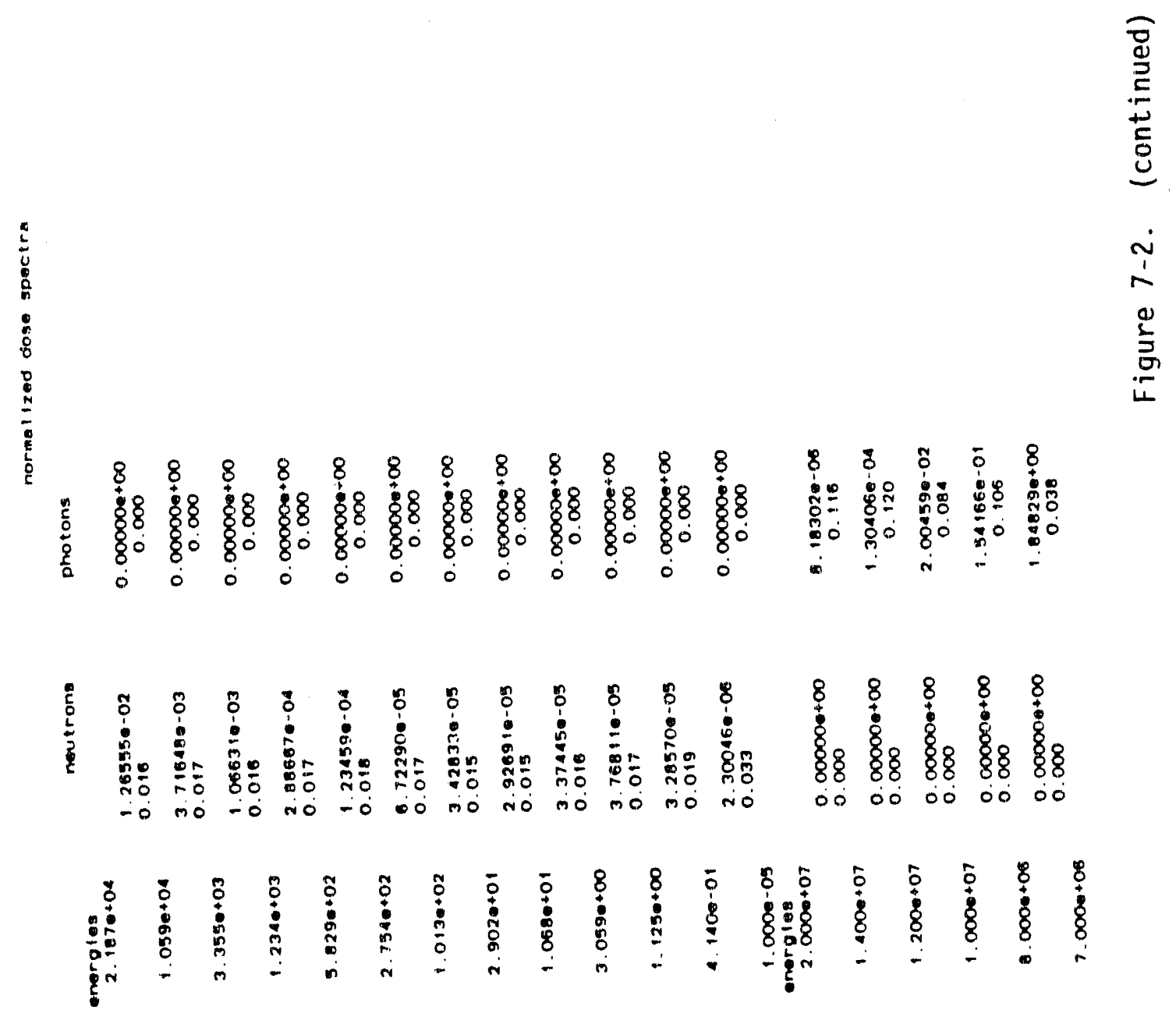

总 芒 
i⿱

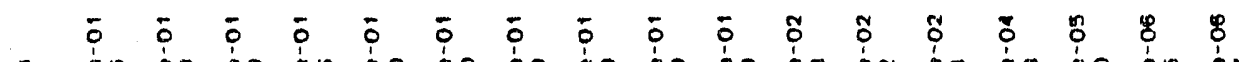

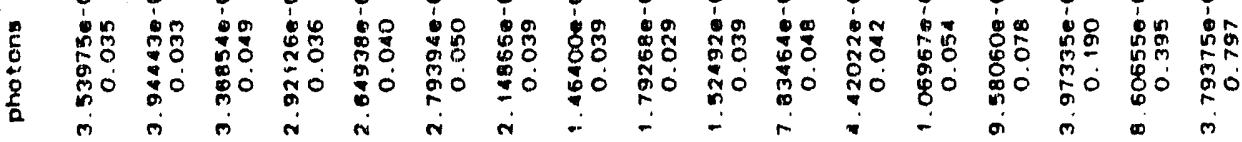

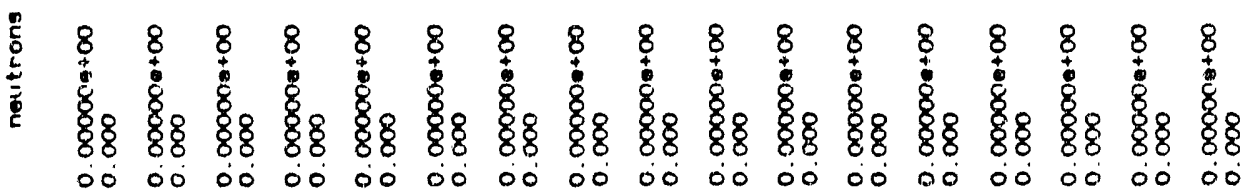

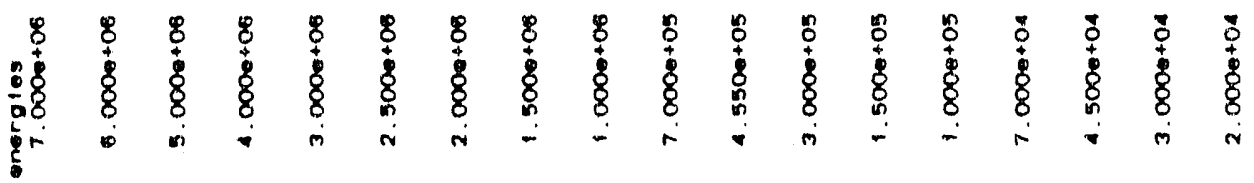




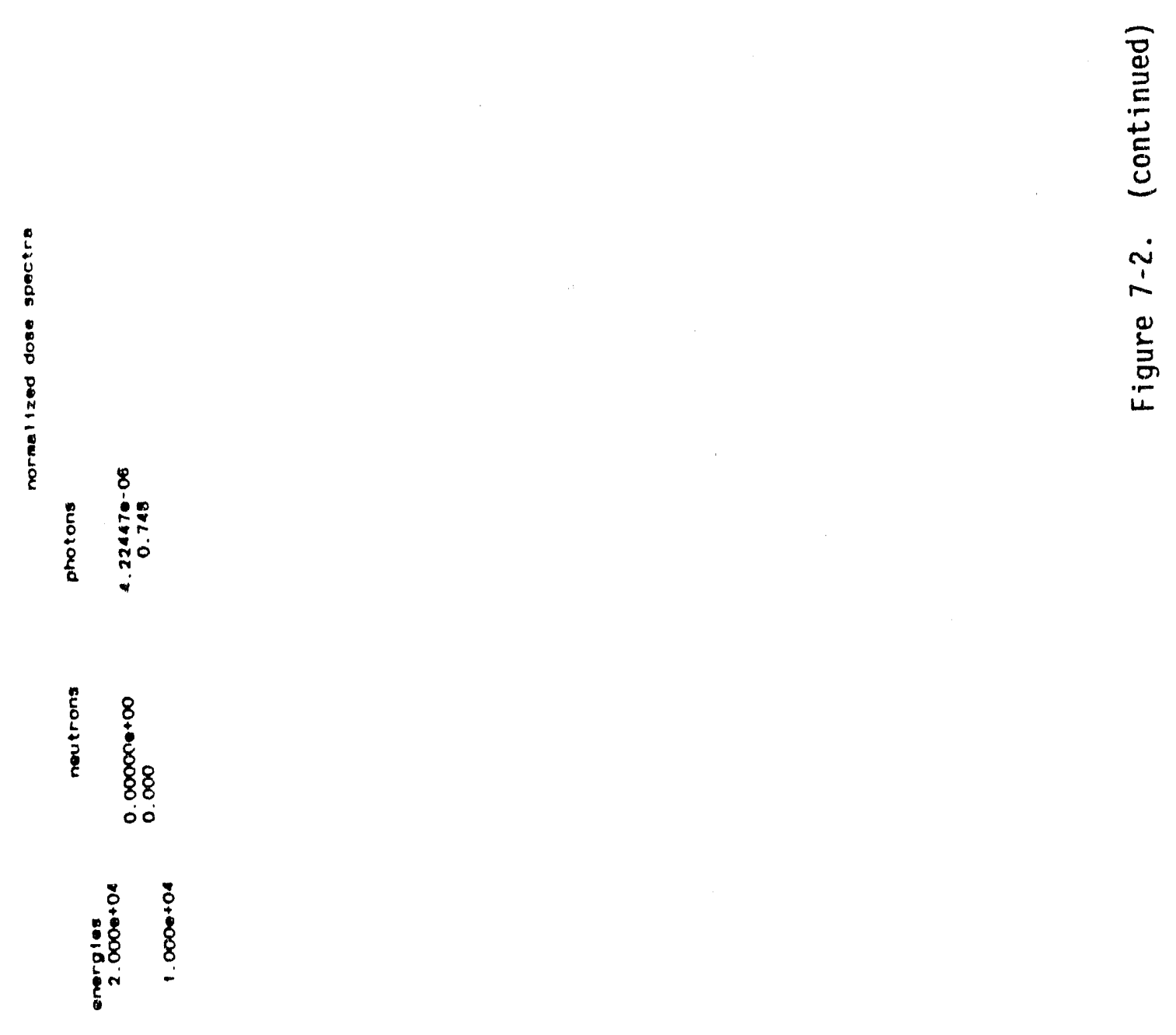

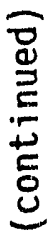

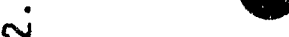

莺

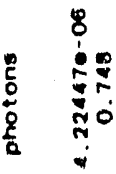

8
$\vdots$
8
8
8
0
0

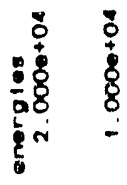

7-44 
APPENDIX A

FIDO INPUT 
The FIDO input method is especially devised to allow the entering or modifying of large data arrays with minimum effort. Special advantage is taken of patterns of repetition or symmetry wherever possible. The FIDO system was patterned after the input method used with the FLOCO coding system at Los ATamos and was first applied by Atomics

International to the DTF-II ${ }^{1}$ code. Since that time, numerous features requested by users have been added, a free-field option has been

developed, and the application of FIDO has spread to innumerable codes.

The data are entered in units called "arrays." An array comprises a group of contiguous storage locations which are to be filled with data at one time. These arrays usually correspond on a one-to-one basis with FORTRAN arrays used in the program. A group of one or more arrays read with a single call to the FIDO package comprises a "block." A special delimiter is required to signify the end of each block. Arrays within a block may be read in any order with respect to each other, but an array belonging to one block must not be shifted to another. The same array can be entered repeatedly within the same block. For example, an array can be filled with zero using a special option, and then a few scattered locations collld be changed by reading in a new set of data for that array. Some arrays can be omitted if no entries to that array are required. If no entries to any of the arrays in a block are required, but the condition requiring the block is met, the delimiter alone satisfic:s the input requirement. Three major types of input are available: free-field input, fixed-field input, and user-field input. Each field includes up to three subfields. Each array is identified by an "array originator field" having two subfields:

Subfield 1: An integer array identifier signals the identity of the array to follow.

Subfield 2: An array type indicator, used as follows:

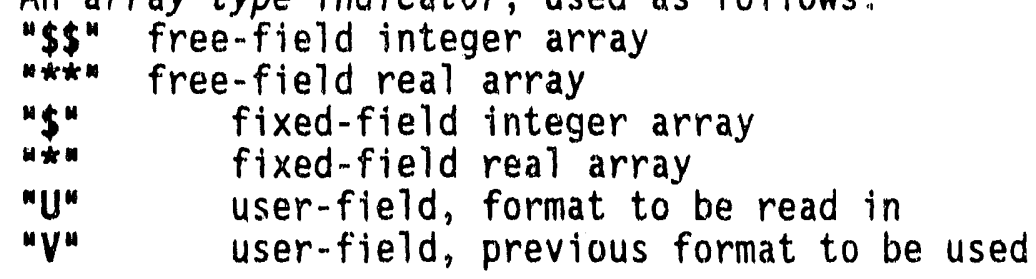

These subfields are written together, without blanks. Data are then placed in successive data fields until the required number of entries has been accounted for.

In entering data, it is convenient to think of an "index" or "pointer" which is under control of the user, and wich specifies the position in the array into which the next data entry is to go. The pointer is always positioned at the first array location by entering the array 
originator field. The pointer subsequently moves according to the data operator chosen.

Free-field input allows an arbitrary number of fields to be entered in columns 1-72, with fields separated by one or more blanks. A field must begin and end in the same record. In general, a data field has up to three flelds:

Subfield 1: The data numerator, $N_{1}$, an integer. Subfield 2: The data operator, $N_{2}$, a character. Subfield 3: The data entry, $\mathrm{N}_{3}$, an integer or real number.

If subfields 1 and 2 are both used in a fleld, they must not be separated by blanks. Blanks may precede subfield 3 , however. All entries following a " $/ "$ in a given record are ignored.

Single data entries use only $N_{3}$, which may be entered with or without a decimal point. The type (integer/real) will be determined by the previous array identifier:

$$
\begin{array}{lll}
1 \$ \$ & 12.3 & \text { /enter integers } 1,2,3 \\
2 * * & 1.1 .12 & \text { /enter reals } 1.0,1.1,2.0
\end{array}
$$

An exponent field may be added, with or without the "E" identifier. No imbedded blanks with in the subfield are allowed. The subfield must have no more than nine columns, including the decimal but not including the exponent field. The pointer is advanced by one for each entry:

$$
\begin{array}{llll} 
& 2^{* *} & 1 . E 0.11+1 \quad 200-2 & \text { / same entries as above } \\
\text { BUT NOT } & 3^{* *} & 1234.5678 E-3 & \text { /8 columns }+ \text { decimal } \\
\text { AND NOT } & 3^{* *} & 1.23456789 & \text { /too many columns } \\
\text { / } & 0.0 E 6 & \text { /produces wrong results }
\end{array}
$$

Multiple data entries use $N_{2}$ and (except the " $F$ " operator) $N_{1}$ together with $\mathrm{N}_{3}$ to enter several items with a single field. The type of operation is indicated by $\mathrm{N}_{2}$.

"R" indicates that data entry $N_{3}$ is to be repeated $N_{1}$ times. The pointer is advanced by $N_{1}$.

"I" indicates linear interpolation. The data numerator, $\mathrm{N}_{1}$, indicates the number of interpolated points to be supplied. The data entry $\mathrm{N}_{3}$ is entered, followed by $N_{1}$ interpolated entries equally spaced between that value and the value in the third subfield of the next field. The next field may be a single.. or multiple-data entry. The pointer is advanced by $N_{1}+1$. The field following an "I" entry is then processed normally, according to its own data operator. The "I" entry is especially valuable for specifying a spatial mesh. In integer arrays, interpolated values will be rounded to the nearest integer. 
"L" indicates logarithmic interpolation. The effect is the same as that of ": except that the resulting data are evenly separated in log-space. This is obviously linited to positive real numbers.

"F" fills the remainder of the array with $N_{3}$. $N_{1}$ is not used. For example, the following are equivalent ways of filling an 8-entry array:

\section{$2 \$ \$ 12346688$ \\ $2 . \$ \$ 2 I 1 \quad 4 \quad 2 R 6 \quad F 8$}

/same as above

Sequence data entries allow entries to be patterned after data entered by previous fieids or previousiy existing in storage. Fields $N_{1}, N_{2}$, and $N_{3}$ are used, except that if $N_{1}$ is omitted, it is taken to be one.

"Q" is used to repeat sequences of numbers without modification. The length of the sequence is given by the third subfield, $N_{3}$. The sequence of $\mathrm{N}_{3}$ previous entries is to be repeated $N_{1}$ times. The pointer is advanced by $N_{1} * N_{3}$. This feature is especially valuable in specifying matrices with repeated or symmetrical columns or rows.

"G" has the same effect as "Q" except that the sign of each entry of the sequence is changed each time it is entered.

"N" has the same effect as "Q." except that the order of the sequence is reversed each time it is entered.

"M" has the same effect as "Q," except that both the sign and the order of the sequerice are reversed each time it is entered.

Options $Q, G$, and $M$ are valuable in entering directional quadrature sets. As examples:

$$
\begin{aligned}
& 81^{\text {t* }} \quad 123103 \quad / 1,2,3,1,2,3 \\
& 82 * * \quad-3-2,-1 M 3 \quad /-3,-2,-1,1,2,3 \\
& 83^{* *} \quad 3 R-2 \text { G3 } \quad /-2,-2,-2,2,2,2
\end{aligned}
$$

Zero data entries use subfields $N_{1}$ and $N_{2}$.

" $Z$ " sets the next $N_{1}$ entries to zero. The pointer is advanced by $N_{1}$. As an example:

$$
1 \$ 3212 \quad / 0,0,0,1,2
$$

Pointer-movement data entries move the pointer without changing the data array. Subfields $N_{1}, N_{3}$, or neither may be required.

"S" indicates that the pointer is to skip forward over $N_{1}$ positions, leaving those array positions unchanged.

"B" moves the pointer backward $\mathrm{N}_{1}$ positions.

"A" moves the pointer to the position $\mathrm{N}_{3}$. 
"E" skips over the renainder" of the array. The array length criterion is satisfled by an "E," uriless too many entries have been spectfied. No more entries to an array may be given following an "E, "except that data entry may be restarted with an "A."

For example, given the following sequence of entries, comments indicate the result of reading an array of length 8 :

$\begin{array}{ll}1 \$ \$ & 123456 E \\ 1 \$ \$ & 21 S 4 A 778 \\ 1 \$ \$ & 2 I 141 B \mathrm{~F} 8\end{array}$

/ E terminates the array, leaving teems 7 and 8 unchanged /now we have $2,2,4,4,5,6,7,8$ /now we have $1,2,3,8,8,8,8,8$

Edit fields control printing within the FIDO subroutines.

"C" causes the position of the last array item entered to be printed. This is the position of the pointer, less 1 . The pointer is not moved.

"O" causes the print trigger to be turned on. The trigger is originally off. When the trigger is on, each card image is listed as it is read.

"p" causes the print trigger to be turned off.

"/" occurring in column 1 causes the entire record to be fgnored as input, but to be printed as a comment in the output stream.

A block termination field consists of a field having only "T" in the second subfteld.

The reading of data to an array is terminated when a new array originator field is supplied, or when the block is terminated by a block termination field. If an incorrect number of positions has been fllled, an error edit is given, and a flag is set which will later abort execution of the problem. FIDO then continues with the next array if an array originator was read. The new array originator need not begin a new record. Otherwise, FIDO returns control to the calling program. For example:

\section{$1 \$ \$ 123 \quad 2 * *$ FO $T$}

User-field input allows the user to specify the input format. If "U" is specified as the array-type indicator, the FORTRAN format to be used must be supplied in columns 1-72 of the next record. The format must be enclosed by parentheses. The data for the entire array must follow on successive records. The rules of ordinary FORTRAN input as to exponents, blanks, etc., appiy. If the array data do not fill the last record, the remainder of the record must be left blank. The user must insure that his format speciftes the correct type of data, i.e., real or integer.

"V" has the same effect as "ll" except that the format read in the last preceding "U" array is used. 
For example, for an array of 4 entrios:

$$
\begin{aligned}
& 100 \\
& \text { (6I2) } \\
& 1234 \\
& \text { IIV } \\
& 4321
\end{aligned}
$$

would enter integers $1,2,3,4$ into the 10th array and $4,3,2,1$ into the 11 th.

Fixed-field input uses 1 to 6 flelds per input record, with fixed, 12column fields. It is thoroughly described in the DOT IV document.? Because it has been almost entirely replaced by the free-field format, its description is not repeated here.

\section{REFERENCES}

1. W. W. Engle, Jr., M. A. Boling, and B. W. Colston, "DTF-II, A OneDimensiona 7, Multigroup Neutron Transport Program," NAA-SR-10951 (March 1966).

2. W. A. Rhoades, D. B. Simpson, R. L. Chllds, and W. W. Engle, Jr., "The DOT'-IV Two-Dimensional Discrete Ordiates Transport Code with Space-Dependent Mesh and Quadrature," ORNL/TM-6529 (January 1979). 


\section{APPENDIX B}

IN-GROUP ENERGY SIASING 


\section{IN-GROUP ENERGY BIASING*}

\section{INTRODUCTION}

This appendix describes the in-group energy biasing methodology' developed for the adjoint MORSE2 calculation utilized in MASH. In-group energy biasing is a methodology developed by $W$. Scott of SAIC to adjust for irregularities observed in adjoint Monte Carlo analyses in thick media where the weights of adjoint particles could, in undergoing multiple s attering events, become very large. Further development of the methodology, discussed herein, suggests that in-group energy bias might have more-general use than initially expected. The in-group biasing procedure was first implemented in the MIFT2 ${ }^{1}$ version of the VCS ${ }^{3,4}$ MORSE code. The in-group biasing procedure has since been implemented in the MASH version of the MORSE code. The bulk of this appendix was extracted from the MIFT2 document ${ }^{1}$ and has been included in this manual for completeness.

In-group energy biasing (or simply in-group biasing or in-group bias) is easy to use. Unlike other Monte Carlo biasing methods, in-group biasing does not require that the user set up and input, based on his judgment, arrays of data to improve the efficiency of the Monte Carlo calculation. This means that a person less skilled in adjoint Monte Carlo can often successfulty use MASH or adjoint MORSE, since use of in-group bias requires only the setting of a switch in MASH. Success in the use of the other biasing methods generally requires considerable adjoint Monte Carlo experience.

Based on results obtained with the MIFT2 and MASH MORSE codes, in-group energy bias appears to generally improve both the answer and the statistics of the calculation. Based on theory, it may be expected that the 7 argest gains from the use of in-group bias occur for fairly thick problems. However, improvements for thinner problems have been seen as well. Based on both the theory and the results of sample cases reported herein, it might be suggested that in-group bias always be used for adjoint Monte Carlo. However, in recognition that users may at times prefer not to use in-group bias or may wish to compare results obtained with and without the use of in-group bias, the methodology has been implemented with a switch so that its use is optional.

This appendix is divided into 6 subsections. Following this introduction section is a background section which traces the short history of the development of the in-group biasing methodology. The

\footnotetext{
J. A. Stoddard, S. D. Egbert, and W. D. Scott, Jr., "The Vehicle Code System with In-Group Energy Bias and GIFT5 Geometry," DNA-TP-8?-23, Science Annlications International Corporation, (Janwary 1987).
} 
next section gives a theoretical basis for in-group bias, followed by a section describing the implementation of the in-group bias methodology into the MASH MORSE code. A section which discusses some comparisons of the results of analyses performed with and without in-group bias with each other and with experimental data, where available, follows. Finally, a list of references pertaining to the discussion given in this appendix is presented.

\section{BACKGROUND}

It has long been recognized that for certain problems adjoint MORSE and MASH are very inefficient at obtaining adequate statistical variances in reasonable run times especially when compared to the efficiency of forward MORSE calculations. In a 1979 SAIC study for the BRL it was recognized that the problem stemmed from a poorly biased energy random walk that occurred in adjoint MORSE whenever the non-absorption probability (PNAB) was much greater than 1.0. At that time it was suggested that a modified adjoint scattering technique, referred to as "in-group energy biasing", would be significantiy more efficient. The defining feature of in-group energy biasing is that for all but the last group, a particle's weight only changes when it scatters to a new group. If the particle stays in the same group (in-group scattering), its weight does not change. Thus, when an adjoint particle has a nonabsorption probability greater than 1.0 , its weight will not increase so long as it scatters in the same group. When it finally upscatters to a new group, its weight will be increased by a fixed amount that is a function only of the cross sections and not of how many times the particle scattered in the same group. With standard adjoint MORSE, such a particle would have its weight increased by PNAB each time that it scattered so that its finai weight upon upscatter would depend upon how many in-group scatterings that it had.

In a 1981 study for the Wehrwissenschaftliche Dienststelle der Bundeswehr fur ABC-Schutz (WWD) Laboratory in the Federal Republic of Germany, SAIC implemented the in-group energy biasing scheme into adjoint MORSE and demonstrated an increase in efficiency of factors of 4 to 5 over standard adjoint MORSE for a $10-\mathrm{cm}$-thick stee? sphere. In that study in-group energy biasing was used for all energy groups.

In a recent effort for the U.S. Army Ballistic Research Laboratory (BRL), the in-group bias scheme was implemented in the BRL version of VCS, and some comparisons of its efficiency were made for other steel and polyethylene configurations. Also, theoretical derivations were made which indicated that in-group energy biasing should always be preferable to the standard MORSE scattering treatment in either forward or adjoint MORSE regardless of the value of the non-absorption probability PNAB. 
In this effort a number of modifications were made to the in-group bias coding to improve its efficiency and clarity. More important, some additional test cases were run wich further demonstrated the advantages of using in-group bias.

\section{THEORETICAL BASIS FOR IN-GROUP ENERGY BIASING}

The theoretical basis for in-group energy biasing is derived by comparing the analytic sample variance of the three-group infinite medium random walk under a variety of possible biasing schemes. Similar conclusions can be reached in two groups with considerably less algebra but with less generality. The procedure is to examine the Markov chains of all possible combinations and permutations of scatterings in three groups and to show that the scattering estimator scores in the random walk sum to the theoretical solution. The corresponding sample variance is then derived, and comparisons are made between the in-group and standard sampling schemes.

Two very important concepts which are stressed in this paper are the "fair game rule" and "particle weightings". The "fair game rule" is descriptive of the Monte Carlo treatment of collisions. If a "fair game" is played in simulating the collision, forward or adjoint, a valid result should be obtained. Particle weightings offer some latitude in playing this "fair game", since tradeoff's can be made between probabilities and particle worth.

A historical example is appropriate here. Original Monte Carlo codes treated absorption events by halting the random walk for a particle whenever sampling indicated that an absorption event had occurred. Later it was recognized that it was more efficient to carry a particle weight and to treat absorption by reducing the weight by the nonabsorption probability at each scatter. Particle histories were then terminated by Russian roulette whenever weights dropped below predetermined cutoffs. This non-absorption treatment avoided losing important histories just before they were about to score. In this example, a "fair game" was played by offsetting the probability of what happened at a scattering event with an appropriate change of particle weight. This concept of adjusting particle weights, within the fair game rule, to improve Monte Carlo statistics is the basis of Monte Carlo biasing schemes. More will be said about the fair game rule in the infinite medium problem described below.

\section{Markov Chain Analysis of the Forward Three-Group Infinite Medium Problem \\ Consider the total dose deposited in an infinite medium due to a source of particles in three energy groups. The source is described by three probabilities $S_{1}, S_{2}$, and $S_{3}$ of beginning particles in the three groups. When the flux in each group is known, the dose is the inner}


product with the response function (or flux to dose conversion factor) $R_{1}, R_{2}$, and $R_{3}$. The scattering properties of the medium are described by total cross sections in each group $\sigma_{1}, \sigma_{2}$, and $\sigma_{3}$, and the downscatter cross section matrix,

$$
\left(\begin{array}{lll}
\sigma_{11} & 0 & 0 \\
\sigma_{12} & \sigma_{22} & 0 \\
\sigma_{13} & \sigma_{23} & \sigma_{33}
\end{array}\right)
$$

where $\sigma_{i j}$ is the cross section for scattering from group $i$ to group $j$. All upscatter cross sections are assumed to be zero. This problem can be solved directly without reference to Monte Carlo or Markov chains by equating the collision density in each group (the product of the flux $\Phi_{i}$ and the total cross section $\sigma_{i}$ to the source in group i) from the original source $S_{i}$ plus the scattering source as shown in Equation (1).

$$
\left(\begin{array}{c}
\sigma_{1} \phi_{1} \\
\sigma_{2} \phi_{2} \\
\sigma_{3} \phi_{3}
\end{array}\right)=\left(\begin{array}{c}
S_{1} \\
S_{2} \\
S_{3}
\end{array}\right)+\left(\begin{array}{ccc}
\sigma_{11} & 0 & 0 \\
\sigma_{12} & \sigma_{23} & 0 \\
\sigma_{13} & \sigma_{23} & \sigma_{33}
\end{array}\right)\left(\begin{array}{l}
\phi_{1} \\
\phi_{2} \\
\phi_{3}
\end{array}\right)
$$

$$
\begin{array}{cc}
\text { Collision } & \text { Originial } \\
\text { Density } & \text { Source }
\end{array}
$$

This equation can be solved directly for the flux in each group, and the dose can be obtained by an irner product with the response,

$$
\lambda=\sum_{i} R_{i} \phi_{i}
$$

so that

$$
\lambda=\left(R_{1}, R_{2}, R_{3}\right)\left(\begin{array}{ccc}
\sigma_{1}-\sigma_{11} & 0 & 0 \\
-\sigma_{12} & \sigma_{2}-\sigma_{22} & 0 \\
-\sigma_{13} & -\sigma_{23} & \sigma_{3}-\sigma_{33}
\end{array}\right)^{-1}\left(\begin{array}{c}
S_{1} \\
S_{2} \\
S_{3}
\end{array}\right)
$$


The solution of the above equation is

$$
\begin{aligned}
\lambda= & \frac{R_{1} S_{1}}{\sigma_{1}-\sigma_{11}}+\frac{S_{1} R_{2} \sigma_{12}}{\left(\sigma_{1}-\sigma_{11}\right)\left(\sigma_{2}-\sigma_{22}\right)} \\
& \left.+\frac{S_{1} R_{3} \sigma_{13}}{\left(\sigma_{1}-\sigma_{11}\right)\left(\sigma_{3}-\sigma_{33}\right.}+\frac{S_{1} R_{3} \sigma_{12} \sigma_{23}}{\left(\sigma_{1}-\sigma_{11}\right)\left(\sigma_{2}-\sigma_{22}\right)\left(\sigma_{3}-\sigma_{33}\right.}\right) \\
& +\frac{S_{2} R_{2}}{\sigma_{2}-\sigma_{22}}+\frac{S_{2} R_{3} \sigma_{23}}{\left(\sigma_{2}-\sigma_{22}\right)\left(\sigma_{3}-\sigma_{33}\right)}+\frac{S_{3} R_{3}}{\sigma_{3}-\sigma_{33}}
\end{aligned}
$$

Notice that the solution for the dose contains seven terms which represent the seven possible means of scattering from a source group to a response group. The first, fifth, and seventh terms result from particles that began and scored in the same group. Each of these particles may have had zero to an infinite number of in-group scatterings. The second, third and sixth terms are the doses from particles that began in one group, had zero to many in-group scatterings, had one transition scattering to a new group and finally had zero to many in-group scatterings in the final group. The fourth term is the dose for particles that began in group one, scattered to group two, and then scattered to group three with any number of in-group scatterings between.

This same result can be derived by Monte Carlo theory considering the probabilities and weight changes of all possible scatterings. In standard forward MORSE the random walk is set up as follows: First, a source group is sampled and the particle weight is set to 1.0. The collision site (which does not matter in the infinite medium problem) is determined by sampling an exponential. The probability of absorption is treated by reducing the particle weight (multiplying the previous weight by the non-absorption probability). Then the probability of scatter to each group is sampled, and the next track proceeds in the new group. At each scattering a score is made equal to the particle weight before the collision times the response function divided by the total cross section.

When this score is made for each scatter, it is called a real scattering estimator. The non-absorption probability (PNAB in MORSE but called W here) is equal to the scattering cross section divided by the total cross section. For group one this is

$$
W_{1}=\frac{\sigma_{11}+\sigma_{12}+\sigma_{13}}{\sigma_{1}}=\frac{\sigma_{1 s}}{\sigma_{1}}
$$


Likewise, the group transfer probability is

$$
P_{i j}=\frac{\sigma_{i j}}{\sum_{j} \sigma_{i j}}=\frac{\sigma_{i j}}{\sigma_{i s}}
$$

Thus, if a particle starts and continues to scatter in group one, its contribution to the dose is

$$
\begin{aligned}
\lambda_{11} & =\frac{S_{1} R_{1}}{\sigma_{1}} \sum_{n=0}^{\infty}\left(W_{1} P_{11}\right)^{n}=\frac{S_{1} R_{1}}{\sigma_{1}} \sum_{n=0}^{\infty}\left(\frac{\sigma_{1 s}}{\sigma_{1}} \frac{\sigma_{11}}{\sigma_{1 s}}\right)^{n} \\
& =\frac{S_{1} R_{1}}{\sigma_{1}} \frac{1}{1-\frac{\sigma_{11}}{\sigma_{1}}}=\frac{S_{1} R_{1}}{\sigma_{1}-\sigma_{11}}
\end{aligned}
$$

Notice that this term is identical to the first term in Equation (4). In a similar manner all combinations of possible sources and scatters can be shown to be equivalent to terms of Equation (4). As another example, particles that begin in group 1, transition to group 2 and finally scatter to group 3 correspond to the fourth term of Equation as follows:

$$
\begin{aligned}
\lambda_{123} & =\frac{S_{1} R_{3}}{\sigma_{3}}\left[\sum_{n=0}^{\infty}\left(W_{1} P_{11}\right)^{n}\right] W_{1} P_{12}\left[\sum_{n=0}^{\infty}\left(W_{2} P_{22}\right)^{n}\right] W_{2} P_{23}\left[\sum_{n=0}^{\infty}\left(W_{3} P_{33}\right)^{n}\right] \\
& =\frac{S_{1} R_{3} \sigma_{12} \sigma_{23}}{\left(\sigma_{1}-\sigma_{11}\right)\left(\sigma_{2}-\sigma_{22}\right)\left(\sigma_{3}-\sigma_{33}\right)}
\end{aligned}
$$

Thus, as expected, the Markov chain analysis of the MORSE random walk for three groups agrees identically with the analytic three-group solution given in Equation (4).

In addition to deriving the dose, the three-group problem can be analyzed for the sample variance of real scattering estimator procedure. The sample variance $V$ is derived by summing the squares of all scores times the probability of making each score. Thus, the sample variance of particles that begin in group one and score in group one, as in Equation (7) for the standard MORSE scattering scheme, is 


$$
\begin{aligned}
V_{11} & =\frac{S_{1} R_{1}^{2}}{\sigma_{1}^{2}} \sum_{n=0}^{\infty}\left(\frac{\sigma_{1 s}^{2} \sigma_{11}}{\sigma_{1}^{2} \sigma_{1 s}}\right)^{n}=\frac{S_{1} R_{1}^{2}}{\sigma_{1}^{2}\left(1-\frac{\sigma_{1} \sigma_{1 s}}{\sigma_{1}^{2}}\right)} \\
& =\frac{S_{1} R_{1}^{2}}{\sigma_{1}^{2}-\sigma_{11} \sigma_{1 s}}
\end{aligned}
$$

Notice that all factors that represent either scores or particle weights are squared while factors representing probabilities are not squared. In this manner all seven terms of the forward MORSE sample variance may be written:

$$
\begin{aligned}
& V_{11}=\frac{S_{1} R_{1}^{2}}{\sigma_{1}^{2}-\sigma_{11} \sigma_{1 s}} \quad V_{12}=\frac{S_{1} R_{2}^{2} \sigma_{12} \sigma_{1 s}}{\left(\sigma_{1}^{2}-\sigma_{11} \sigma_{1 s}\right)\left(\sigma_{2}^{2}-\sigma_{22} \sigma_{2 s}\right)} \\
& V_{13}=\frac{S_{1} R_{3}^{2} \sigma_{13} \sigma_{1 s}}{\left(\sigma_{1}^{2}-\sigma_{11} \sigma_{1 s}\right)\left(\sigma_{3}^{2}-\sigma_{33} \sigma_{3 s}\right)} \\
& V_{123}=\frac{S_{1} R_{3}^{2} \sigma_{12} \sigma_{1 s} \sigma_{23} \sigma_{2 s}}{\left(\sigma_{1}^{2}-\sigma_{11} \sigma_{1 s}\right)\left(\sigma_{2}^{2}-\sigma_{22} \sigma_{2 s}\right)\left(\sigma_{3}^{2}-\sigma_{33}^{2}\right)} \\
& V_{22}=\frac{S_{2} R_{2}^{2}}{\sigma_{2}^{2}-\sigma_{22} \sigma_{2 s}} \quad V_{23}=\frac{S_{2} R_{3}^{2} \sigma_{23} \sigma_{2 s}}{\left(\sigma_{2}^{2}-\sigma_{22} \sigma_{2 s}\right)\left(\sigma_{3}^{2}-\sigma_{33}^{2}\right)} \\
& V_{33}=\frac{S_{3} R_{3}^{2}}{\sigma_{3}^{2}-\sigma_{33}^{2}}
\end{aligned}
$$

All terms have the same form for each combination of transitions, and the denominator terms of $\sigma_{i}{ }^{2}-\sigma_{i j} \sigma_{\text {is }}$ appear in each case. In group 3 it reciuces to $\sigma_{23}-\sigma_{33}^{*}$ because the scattering cross section is equivalent to the in-group cross section. Sometimes in forward MORSE the scattering cross section is greater than the total cross section. This can occur when multiplicity is included as a pseudo-scattering process such as with $(n, 2 n)$ reactions or in gamma ray pair production. If the scattering cross section is sufficiently larger than the total cross section so that $\sigma_{i}^{2}-\sigma_{i j} \sigma_{i s}$ is near-zero, these terms of the variance become large and the calculation may not converge. When this occurs, longer run times may fail to improve MORSE statistics. Also, answers may vary widely, with results for cases with the best statistics tending to be deceivingly low. 
It is important to note that the non-absorption probabllity and group transfer probabilities in the problem solution, $\lambda$, always occur in product form, i.e., $W_{1} P_{1 j}$. This suggests that the paricle weights and transfer probabilities can each be adjusted in btasing schemes provided that the product remains unchanged. Since the answer $\lambda$ depends only on the product, the answer will not be changed. The variance, however, is dependent on the separate $W_{i}$ and $P_{i j}$ terms; thus there is a potential in biasing to improve the variance.

Biasing in forward Monte Carlo calculations by modification of the $W_{1}$ and $P_{i j}$ terms, while retaining a constant product, is a powerful tool in improving Monte Carlo statistics, but that is not the only use of the concept. As will be seen, the concept carries over into adjoint random walks. First the product sufficiency, which is one part of the "fair game" rule, justifies the current treatment of group transfers in adjoint Monte Carlo simulations. Second, it can be used for biasing adjoint Monte Carlo analyses as well as forward calculations.

\section{Adjoint Three-Group Analysis}

An especially interesting feature of the MORSE Monte Carlo code is that the same scattering routines are used to track both forward and adjoint particles by using the transpose of the scattering cross section matrix for adjoint transport. In the adjoint, the non-absorption factor (PNAB) involves a sum of the scattering cross sections for those groups from which scattering could have come. In MORSE, the adjoint energy group indexes are reversed so that the highest energy particles have the highest group numbers. However, the same forward cross section notation is kept here to facilitate comparison with the forward walk. Keeping the same forward cross section group number notation, the adjoint scattering cross section for group three becomes

$$
\sigma_{3}^{*}=\sigma_{33}+\sigma_{23}+\sigma_{13}
$$

Since $\sigma_{23}$ and $\sigma_{13}$ are not a part of the group three total cross section $\sigma_{3}$, this adjoint scattering sum can be much different from the total cross section. For broad neutron groups in the inelastic level regime where there cam be many higher energy groups that scatter into it, the adjoint scattering sum can be much greater than the total cross section. The adjoint non-absorption factor and the scattering probabilities then become

$$
W_{j}=\frac{\sum_{i} \sigma_{i j}}{\sigma_{j}}=\frac{\sigma_{j s}^{*}}{\sigma_{j}}
$$


and

$$
P_{i j}=\frac{\sigma_{i j}}{\sum_{i} \sigma_{i j}}=\frac{\sigma_{i j}}{\sigma_{j s}^{*}}
$$

where the asterisk indicates the adjoint summation of the scattering cross section rather than the forward summation. Note that unlike the forward scattering cross section, the adjoint scattering cross section is not a physical quantity. In fact it depends more upon the energy group structure than the physical scattering process.

Clearly there is no direct physical basis, as with forward Monte Carlo Equations (5) and (6), for using the above weight changes and transfer probabllities for adjoint Monte Carlo. However, the product of the $W_{i} P_{i j}$ in Equations (12) and (13) is equivalent to that for the forward, and the adjoint random walk gets the same answer as the forward walk. In this case, the $\sigma^{*}$ is has no physical meaning, but it has the advantages of giving transfer probabilities $P_{i j}$ which automatically sum to 1.0 and of following the adjoint calculation format in which the transpose of the cross section matrix is used. However, as will be seen below, the non-physical nature of the adjoint scattering cross section (Equation 11) can cause accuracy problems in adjoint Monte Carlo.

Consider the three-group infinite medium Markov chain. As mentioned above, the adjoint random walk will get the same dose as the forward walk. However, the sample variance of the standard MORSE adjoint walk, although similar in form to the forward sample variance, is quite different. When the particle weights are squared and the probabilities are not squared, the non-physical adjoint scattering cross section $\sigma^{*}$ is does not cancel

$$
W_{j}^{2} P_{i j}=\frac{\sigma_{j s}^{*}{ }^{2}}{\sigma_{j}^{2}} \frac{\sigma_{i j}}{\sigma_{j s}^{*}}=\frac{\sigma_{i j} \sigma_{j s}^{*}}{\sigma_{j}^{2}}
$$

and the seven terms of the standard adjoint sample variance become 


$$
\begin{aligned}
& V_{11}=\frac{R_{1} S_{1}^{2}}{\sigma_{1}^{2}-\sigma_{11}^{2}} \quad V_{22}=\frac{R_{22} S_{2}^{2}}{\sigma_{2}^{2}-\sigma_{22} \sigma_{2,}^{*}} \quad V_{33} \frac{R_{3} S_{33}^{2}}{\sigma_{3}^{2}-\sigma_{33} \sigma_{34}^{*}} \\
& V_{21}=\frac{R_{2} S_{1}^{2} \sigma_{12} \sigma_{2,}^{*}}{\left(\sigma_{2}^{2}-\sigma_{22} \sigma_{2,4}^{*}\right)\left(\sigma_{1}^{2}-\sigma_{11}^{2}\right)} \quad V_{31}=\frac{R_{3} S_{1}^{2} \sigma_{13} \sigma_{33}^{*}}{\left(\sigma_{3}^{2}-\sigma_{33} \sigma_{3,4}^{*}\right)\left(\sigma_{1}^{2}-\sigma_{11}^{2}\right)} \\
& V_{32}=\frac{R_{3} S_{2}^{2} \sigma_{23} \sigma_{3 s}^{*}}{\left(\sigma_{3}^{2}-\sigma_{33} \sigma_{3 s}^{*}\right)\left(\sigma_{2}^{2}-\sigma_{22} \sigma_{2 s}^{*}\right)} \\
& V_{321}=\frac{R_{3} S_{1}^{2} \sigma_{23} \sigma_{3,}^{*} \sigma_{12} \sigma_{2 s}^{*}}{\left(\sigma_{3}^{2}-\sigma_{33} \sigma_{3 s}^{*}\right)\left(\sigma_{2}^{2}-\sigma_{22} \sigma_{2 s}^{*}\right)\left(\sigma_{1}^{2}-\sigma_{11}^{2}\right)}
\end{aligned}
$$

The form of these terms is very simllar to the forward sample variance in Equation (10) including terms 1 lke $\left.\sigma_{1}^{2}-\sigma_{1} \sigma^{*}\right]_{s}$ in the denominators. Because of the physical nature of the forward scattering cross section, the forward MORSE random walk is nearly always well behaved. However, since the adjoint scattering cross section $\sigma$ is is often much greater than the total cross section $\sigma_{1}$, these terms can prevent the random walk from converging. In fact in the DLC-31 Data Library Collection?, adjoint iron cross sections have four groups (a1) in the vicinity of 1 $\mathrm{MeV}$ neutrons) where

$$
\sigma_{i i} \sigma_{i s}^{*} \geq \sigma_{i}^{2}
$$

Thus, infinite medium adjoint random walk for the fron with the DLC. -31 cross sections will not converge. More generally, this occurs in adjoint MORSE whenever

$$
P_{i i} P N A B_{i} \geq 1.0
$$

It is perhaps fortunate that the seriousness of this problem is not always observed. For realistic problems not involving deep penetration in thick media, reasonable answers are usually obtained, although with reduced efficiency.

\section{In-Group Energy Bias Analysis}

The major objective of the in-group biasing scheme is to eliminate the instability in the variance for random walks, i.e., to eliminate the $\sigma_{i}^{2}$ - $\sigma_{i j} \sigma^{*}$ is terms which could be zero or negative for physically realistic cross section sets. Although not obvious from the foregoing, this is 
equivalent to eliminating the problem in which the weight of an adjoint particle can grow unbounded by many repeated in-group scatterings.

The approach is to utilize the fair game constratnt in which the product $W_{1} P_{1 j}$ is forced to remain constant while adjustments to certain $W_{1}$ are made. Specifically, as mentioned in the background section, the weight of a particle undergoing in group scattering is forced to remain unchanged. This "stariting condition", along with the constant $W_{1} P_{11}$ "fair game rule" and a couple of other physical requitrements form a recursion-relation-1 lke formulation which redefines all the $W_{1}$ and $P_{11}$. The result is the in-group bias formulation, the derivation and analys is of whtch are described below.

In the standard (not in was always equal to PNAB for group 1 . For in-group blas, this parameter is generalized to also have $f$ dependence. Thus the fair game product rule bocomes, ut $17 \mid z$ ing Equations (12) and (13) (for the standard adjoint MORSE),

$$
P_{i j} W_{i j}=\frac{\sigma_{i j}}{\sigma_{j}}
$$

For in-group scattering for groups other than group 1, it was speciffed that there would be no welght change (W. 1 ); hence from Equation (18),

$$
W_{j j}=1.0 \text { and } P_{j j}=\frac{\sigma_{j j}}{\sigma_{j}}
$$

for in-group scattering other than group 1. An exception is made for group 1, which, being the highest energy group, has no outscattering so that $P_{11}$ must be 1.0 . Thus from Equation (18) for group 1 ,

$$
P_{11}=1.0 \text { and } W_{11}=\frac{\sigma_{11}}{\sigma_{1}}
$$

The above expressions specify the $P$ and $W$ for in-group scattering. The out-of-group scattering $P_{j j}$ and $W_{1 j}$ are specifled by the physical requirement that the scattering probabilities must sum to 1.0 and by the desirability of having the other transfer probabilities be proportional to $\sigma_{i j}$. The result is

$$
i \neq j, P_{i j}=\frac{\sigma_{i j}\left(\sigma_{j}-\sigma_{j j}\right)}{\sigma_{j}\left(\sigma_{j,}^{*}-\sigma_{j j}\right)} \text { and } W_{i j}=\frac{\sigma_{j 0}^{*}-\sigma_{j j}}{\sigma_{j}-\sigma_{j j}}
$$


Thus, in three groups the adjoint in-group scattering and weight factors are specifted as

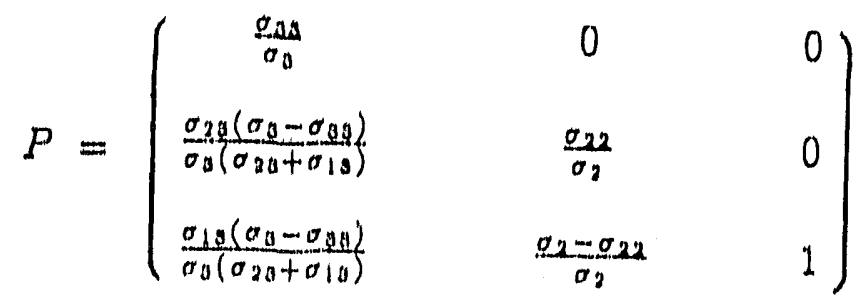

and

$$
W=\left(\begin{array}{ccc}
1 & 0 & 0 \\
\frac{\sigma_{2 u}+\sigma_{13}}{\sigma_{3}-\sigma_{3 a}} & 1 & 0 \\
\frac{\sigma_{2 a}+\sigma_{13}}{\sigma_{3}-\sigma_{3 a}} & \frac{\sigma_{12}}{\sigma_{2}-\sigma_{23}} & \frac{\sigma_{1}}{\sigma_{1}}
\end{array}\right)
$$

From the probability and weight table the total dose can be derived just as in the standard adjoint case. As expected because the product of weight and probabllity is the same for both in-group and standard MORSE, the dose is the same (Equation 4). But the welght squared times the probabllity is different, making the in-group blased sample variance terms, given below, very different from the standard adjoint sample variance of Equation (15).

$$
\begin{aligned}
V_{11} & =\frac{R_{1} S_{1}^{2}}{\sigma_{1}^{2}-\sigma_{11}^{2}} \quad V_{22}=\frac{R_{2} S_{2}^{2}}{\sigma_{2}^{2}-\sigma_{22} \sigma_{2}} \quad V_{33} \frac{R_{3} S_{3}^{2}}{\sigma_{3}^{2}-\sigma_{33} \sigma_{3}} \\
V_{21} & =\frac{R_{2} S_{1}^{2} \sigma_{12}^{2}}{\left(\sigma_{2}-\sigma_{22}\right)^{2}\left(\sigma_{1}^{2}-\sigma_{11}^{2}\right)} \quad V_{31}=\frac{R_{3} S_{1}^{2} \sigma_{13}\left(\sigma_{23}+\sigma_{13}\right)}{\left(\sigma_{3}-\sigma_{33}\right)^{2}\left(\sigma_{1}^{2}-\sigma_{11}^{2}\right)} \\
V_{32} & =\frac{R_{33} S_{2}^{2} \sigma_{23}\left(\sigma_{23}+\sigma_{13}\right)}{\left(\sigma_{3}-\sigma_{33}\right)^{2}\left(\sigma_{2}^{2}-\sigma_{22} \sigma_{2}\right)} \\
V_{321} & =\frac{R_{3} S_{1}^{2} \sigma_{23}\left(\sigma_{23}+\sigma_{13}\right) \sigma_{12}^{2}}{\left(\sigma_{3}-\sigma_{33}\right)^{2}\left(\sigma_{2}-\sigma_{22}\right)^{2}\left(\sigma_{1}^{2}-\sigma_{11}^{2}\right)}
\end{aligned}
$$


In the above terms, all the denominators are positive, non-zero, and well behaved for physical cross sectlons. The possible infintte vartance terms of the standard adjoint MORSE have been eltminated.

At this point the cholce of no welght change on in-group scattering may seem somewhat arbitrary. In fact, this choice can be shown to be the optimum. When the in group weight changes of 1.0 in the weight and probability tables (Equations 22 and 23) are set to variables, new tables are dertved,

$$
W(x, y)=\left(\begin{array}{ccc}
x & 0 & 0 \\
\frac{x\left(\sigma_{y 3}+\sigma_{13}\right)}{2 \sigma_{3}-\sigma_{3 a}} & y & 0 \\
\frac{x\left(\sigma_{y y}+\sigma_{13}\right)}{2 \sigma_{3}-\sigma_{3 y}} & \frac{y \sigma_{12}}{y \sigma_{2} \cdots \sigma_{23}} & \frac{\sigma_{11}}{\sigma_{1}}
\end{array}\right)
$$

and

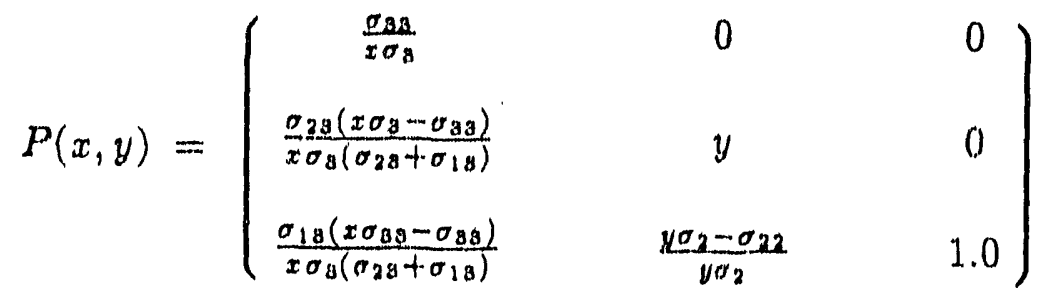

When these probabilities are summed and multiplied by the weights squared over all seven transition possibilities, the terms all have the form of

$$
\frac{x}{\left(2 \sigma_{3}-\sigma_{33}\right)\left(\sigma_{3}-x \sigma_{33}\right)} \text { or } \frac{y}{\left(y \sigma_{2}-\sigma_{22}\right)\left(\sigma_{2}-y \sigma_{22}\right)}
$$

The general features of the above functions are shown in Figure $B-1$. For the region $\sigma_{11} / \sigma_{1} \leq x \leq \sigma_{1} / \sigma_{1 i}$ of interest, the terms are minimized by a value of $x=1.0$ regardless of the value of $\sigma_{1}$ or $\sigma_{11}$.

$$
\frac{1}{\left(\sigma_{3}-\sigma_{33}\right)^{2}} \text { or } \frac{1}{\left(\sigma_{2}-\sigma_{22}\right)^{2}}
$$


Thus, setting the inugroup weight change factor to 1.0 appears to be an optimum in-group blasing scheme.

Having concluded that a weight change factor of 1.0 is optimum for the in group biasing scheme, equtvalent sample variance terms from standard adjoint and in-group adjoint MORSE (Equations 15 and 24) are now compared.

$$
\frac{x}{\left(x \sigma_{i}-\sigma_{i i}\right)\left(\sigma_{i}-x \sigma_{i i}\right)}
$$

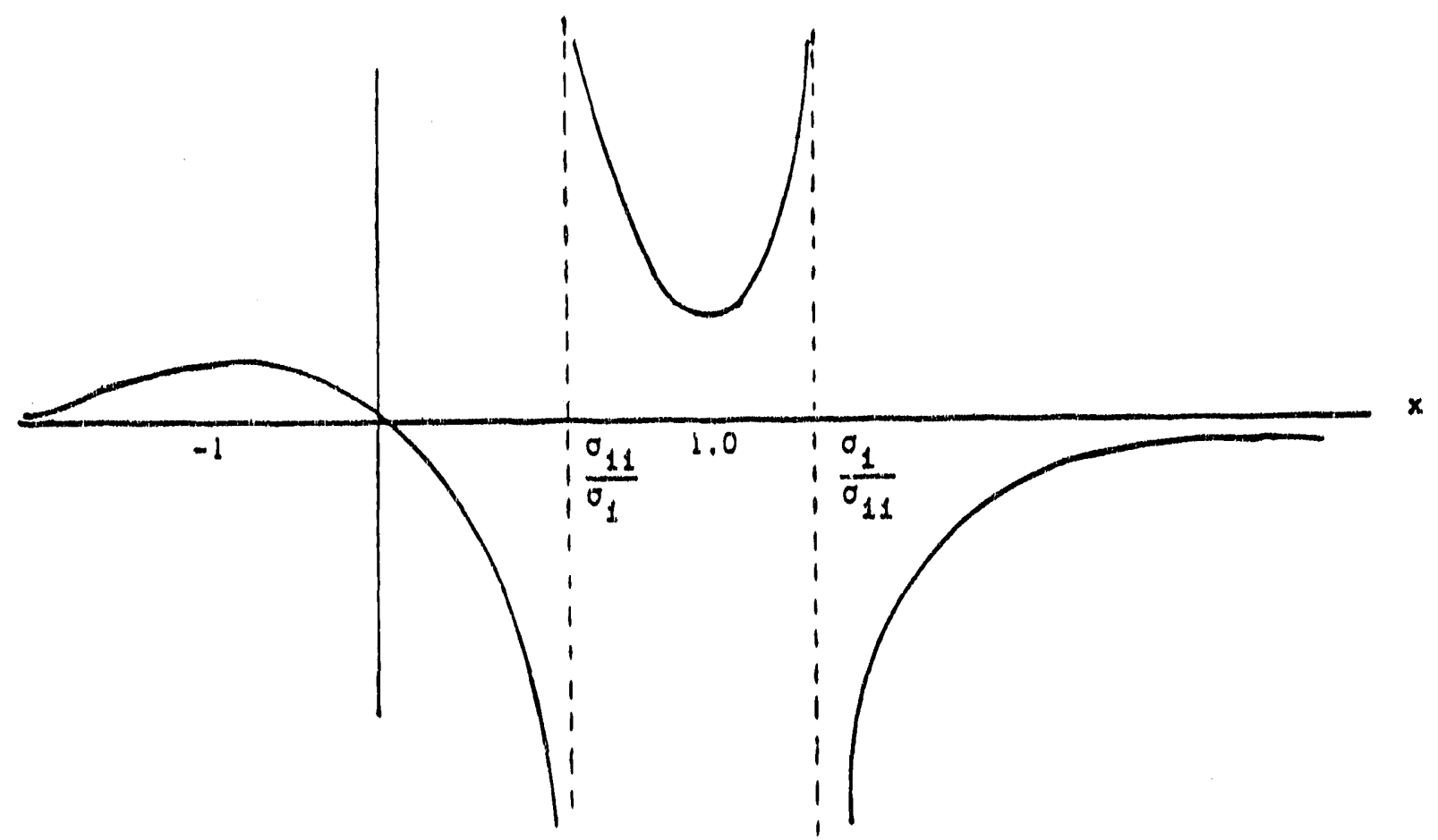

Figure B-1. Characteristics of functions in Equation (27). 
Standard Adjoint In-group Adjoint

$$
\frac{\sigma_{2 y}^{*}}{\sigma_{2}^{2}-\sigma_{22} \sigma_{29}^{*}} \quad \frac{\sigma_{12}}{\left(\sigma_{2}-\sigma_{22}\right)^{2}}=\frac{\sigma_{29}^{*}-\sigma_{22}}{\left(\sigma_{2}-\sigma_{22}\right)^{2}}
$$

or

$$
\frac{\sigma_{3 y}^{*}}{\sigma_{3}^{2}-\sigma_{33} \sigma_{39}^{*}} \quad \frac{\sigma_{23}+\sigma_{13}}{\left(\sigma_{3}-\sigma_{33}\right)^{2}}=\frac{\sigma_{39}^{*}-\sigma_{33}}{\left(\sigma_{3}-\sigma_{33}\right)^{2}}
$$

Analysis of the above terms indicates that the in-group adjoint variance is always less than the standard adjoint variance except at $\sigma_{i}=\sigma^{*}$ is, where the variances are equal. Also, the in-group variances are better behaved for physically valid cross section values, i.e., $\sigma^{*}{ }_{i s} \geq \sigma_{i j}, \sigma_{i} \geq$ $z_{i i}$, and no negative cross sections. This suggests that the in-group biasing techrique is generally superior to the standard methodology for MORSE adjoint analyses with no upscattering. Although in-group bias has not teen exercised with forward MORSE, it appears from this analysis that in-group bias would be superior to the standard treatment in forward Monte Carlo as well as adjoint Monte Carlo.

\section{IN-GROUP BIASING CODE MOOIFICATIONS FOR MORSE}

MORSE is easily modified to incorporate in-group energy bias. Scattering in MORSE is handled in subroutine COLISN Using data in the form of the non-absorption factor $P N A B_{i}$ and the normalized scatterins probabilities $P_{i j}$ which give the probability of scattering to group $j$ provided that a scattering has occurred in group $i$. The original coding is very simple in that the particle weight WATE is multiplied by PNAB where $i$ is the group coming into the collision, and then a random number is compared with the probabilities $f_{i j}$ to select the outgoing group. ints sampling scheme is sasi?y modified to use in-group scattering. Define a bias factor $f$ as follows: 


$$
\begin{array}{ll}
i=j=1 & F=1.0 \\
i=j \neq 1 & F=P N A B_{i} \\
i \neq j & F=\frac{1-P_{i i} P N A B_{i}}{1-P_{i i}}
\end{array}
$$

The same sampling coding is used except that the random number is compared to F.P $P_{i j}$ instead of simply $P_{i j}$ as before. After the outgoing group has been sampled, the weight is corrected by

$$
\text { WATE }=\text { WATE }, \text { PNAB } / F
$$

the above modifications have been implemented in MASH with a switch which permits in-group biasing to be used on option. The implementation correctiy treats in-group biasing with or without $E$ biasing, and includes logic for splitting whenever WATE exceeds WTMAX. Splitting in MORSE is normally performed in subroutine TESTW when a particle's weight is above an importance region- and energy-dependent maximum weight. The particle's weight is split into halves and an identical particle is written to the secondary bank. When the weight increase occurs at upscatter, the excessive weight is not identified until entering subroutine TESTW. Thus, all the splitting secondaries will have the same energy group and the same direction cosines. They will be essentially the same particle. This has been significantly improved by playing the splitting game in subroutine COLISN and actually resampling the upscatter distribution for each splitting secondary particle. In this manner each secondary is independent of the others so that this important probability transition is much more completely represented.

\section{DEMONSTRATION CALCULATIONS}

A number of demonstration calculations have been performed to compare VCS or MORSE results using in-group energy bias with $r$ sults using the standard methodology. Reference 6 describes some results obtained by Siegfried Stuker of Wehrwissenschaftliche Dienststelle der Bundeswehr fur $A B C$-Schutz (WWD), who tested the treatment in a series of $10 \mathrm{~cm}$ iron sphere calculations. Stuker determined that in-group biasing made a significant improvement when it was applied to energy groups with PNAB greater than $2.0^{8}$. He also discovered further improvement when in-group biasing was used for all energy groups including groups with PNAB less inan orie. 
In addition to the calculations performed by Stuker, SAIC has performed calculations on several different test geometries including an iron sphere, a cubical box, and a steel shell resembling a van-like vehicle. These results are discussed in Reference 1. The results for the iron sphere were calculated in a previous effort and are taken from Reference 6. The results for the van-like vehicle have been described in Reference 5.

\section{REFERENCES}

1. J. A. Stoddard, S. D. Egbert, and W. D. Scott, Jr., "The Vehicle Code System with In-Group Energy Bias and GIFT5 Geometry," DNATR-87-23, Science Applications International Corporation, (January $1987)$.

2. M. B. Emmett, "The MORSE Monte Carlo Radiation Transport Code System." ORNL-4972 (February 1975); ORNL-4972/R1 (February 1983); ORNL 4972/R2, Oak Ridge National Laboratory, (July 1984).

3. W. A. Rhoades, "Development of a Code System for Determining Radiation Frotection of Armored Vehicles (The VCS Code), " ORNLTM-466A, Oak Ridge National Laboratory, (October 1974).

4. W. A. Rhoades, et.al., "Vehicle Code System (VCS) User's Manual," ORNL-TM-4648, Oak Ridge National Laboratory, (August 1974).

5. W. H. Scott, Jr., "Vehicle Code System (VCS) Documentation and Uncertainty Analysis," SAI Report SAI-133-79-977-LJ, Science Applications International Corporation, (December 1979).

6. W. H. Scott, Jr., and V.E. Staggs, "Adjoint Energy Biasing and Thermal Neutron Diffusion in the MORSE and VCS Codes, "SAI Report SAI-133-81-384-LJ, Science Applications International Corporation, (November 1981).

7. D. E. Bartine, et.al., "Production and Testing of the DNA FewGroup Coupled Neutron-Gamma Cross-Section Library, " ORNL/TM-4840, Oak Ridge National Laboratory, (1977).

8. S. Stuker and $L$. Schanzler, Wehrwissenschaftliche Dienststelle der Bundeswehr fur ABC-Schutz (WWD), Letter to W.Scott of Science Applications International Corporation, April 28, 1981. 


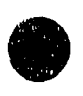

APPENDIX C

THE GIFTS GEOMETRY PACKAGE

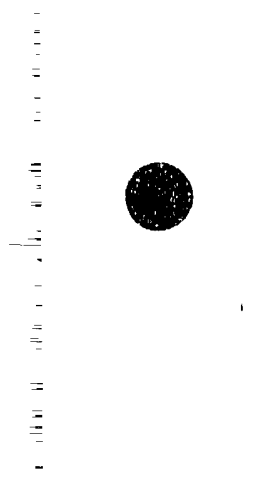




\section{THE GIFT5 GEOMETRY PACKAGE*}

\section{BACKGROUND}

One of the major objectives of earlier efforts in the development of the Vehicle Code System (VCS) 1,2 was to create a version of VCS which could treat vehicle models ueveloped by the Ballistic Research Laboratory (BRL). BRL, a major user of VCS, has developed detailed combinatorial geometry models of a number of arinored vehicles. In order to facilitate the vehicle modeling effort, which often takes a significant fraction of a person year per vehicle, BRL has developed a number of hardware and software tools and has extended the combinatorial geometry models of their codes (other than VCS) to treat a number of new solid "body" types. The result is that BRL has a number of vehicle models which could not be analyzed by VCS unless modified, at considerable time and dollar costs, for use by VCS.

In 1976-1977 BRL removed some of the geometry incompatibilities by installing the geometry package from the BRL GIFT graphics code 3,4 into the VCS MORSE code. BRL named the result "MIFT" for MORSE with GIFT". A review of the MIFT, VCS, GIFT, and GIFT5 codes was performed by Science Applications International Corporation (SAIC) to evaluate and compare the geometry models and to determine the best way to upgrade the geometry treatment in VCS. The GIFT5 geometry treatment was found to be superior to the VCS combinatorial geometry and older GIFT treatment in several ways, including the number of solid "body" types, diagnostics and checks of input data, and a tracking method which could correctly treat overlapping or undefined regions in the geometry. Drawbacks relative to the older VCS combinatorial geometry logic were a siightly larger computer run time for similar problems and increased size and complexity of the resulting VCS code. The GIFTS geometry package was first implemented in the MIFT version of the VCS MORSE code and called MIFT2 ${ }^{6}$. The GIFT5 geometry package has since been implemented in the current MASH version of the MORSE code. The bulk of this appendix was extracted from the GIFT document ${ }^{3}$ to give some background information on the GIFT5 geometry package and has been included in this manual for completeness.

\footnotetext{
"Lawrence W. Bain, Jr. and Mathew J. Reisinger, "The GIFT Code User Manua 1 ; Volume I. Introduction and Input Requirements, "BRL 1802, Ballistic. Research Laboratory, (July 1975).
} 


\section{INTRODUCTION}

GIFT5 geometry is similar to the older MORSE combinatorial geometry in that specified geometric shapes (spheres, cylinders, parallelepipeds, etc.) may be combined to describe complex geometry configurations. As with MORSE combinatorial geometry, a GIFT5 geometry file is comprised largely of a "body" $c r$ "sidid" table followed by a "zones" or "region" table in which the bot ses are combined by $(+),(-)$, or (OR) operators. In GIFT5 geometry, howeves", "bodies" (MORSE terminology) are called "solids" and "zones" (MORSE terminology) are called "regions". In the GIFT5 graphics code there is no equivalent to the "regions" as used in MORSE. In MASH, where the term "region" could have a double meaning, the MORSE equivalent of "region" is called "importance region" to minimize any confusion. In this appendix the GIFT5 terminology, i.e., solids and regions, equivalent to bodies and zones in MORSE, will be used.

\section{TARGET DESCRIPTION DATA}

Target description data defines or models the three-dimensional shape and space of a simple or complex physical structure - the target. The GIFT5 geometry package uses a "combinatorial geometry" or "COM-GEOM" target description technique. This technique defines the shape and space of the components of the target as a single geometric "solid" (a box, a sphere, a cylinder, etc.), or the "combination" of several geometric solids. The parameters which define the spatial locations of the geometric solids used to model the components of the target are recorded in the "Solid Table." Each component (region) of the target is defined as a single solid or a "combination" of solids in the "Region Table."

The Solid and the Region Tables, and the other input data required to create the target description data for the GIFT5 geometry package are defined in the following sections of this appendix.

\section{PRELIMINARY STEPS}

The first step to model a target by any three-dirensional target description technique is to obtain engineering drawings, reports, or any other data which exhibit the physical dimensions of the target. The next step is to define a reference point on the taryet: the origin point ( 0 ) of an $X, Y, Z$ right-handed coordinate system. For example, for tanks, the intersection of the turret datum line and the center lines of the turret is usually selected as the origin point. The parameters within the target description data may be recorded in inches or 
centimeters or any other unit of measure. For example, the $X, Y, Z$ coordinate values for a point "P" can be given as $(X=1 . A, Y=1.0, Z=$ $-1.0)$ inches or $(X=3.5, Y=2.5, Z=-2.5)$ centimeters from the origin point $(X, Y, Z=0)$. Every parameter recorded in the target description data must be measured in the same unit of measure.

\section{SOLIDS}

Table C-1 lists the twenty geometric solids used by the GIFT5 geometry package. The letters under the "Symbol", column are the alphanumeric designation used in this report and on the input cards for the solid. The "Figure" column lists the figure(s) where the input data for the solid is described.

Each solid in a target description is identified by a unique number, 1 , 2,3 , etc. This permits the space of any solid to be distinguished from the space of any other solid. The parameters required to define the twenty solids are different. The parameters can, however, be classified as beiny oither a "vertex point," a "vector," or a "scalar."

A vertex point is a point defined by its $X, Y$ and $Z$ measurements (coordinate values) from the target reference or origin $(0)$ point. For each of the twenty types of solids, at least one vertex point is used to locate the position of the solid in the referenced $X, Y, Z$ space. For example, " $V$ " is the vertex point for a box, while the centerpoint " $C$ " is the vertex point for the sphere solid. Within this report, the dash $(-)$ mark over a letter ( $\mathrm{V}, \mathrm{C}, \mathrm{H}$, etc.) will be used to indicate an $X, Y$ and $Z$ measurement.)

Certain solids require vector parameters to define the three-dimensional shape and space that they occupy. The BOX requires three vectors, $H, W$, $D$, which respectively represent the height, width, and depth of the BoX. Vectors also have an $X, Y$ and $Z$ measurement; however, the measurements of vectors are taken from the vertex point of the solid rather than the target reference or origin point. The $X, Y, Z$ measurements of $H, W$ and $D$ vectors are taken from $V$, not the origin $(0)$ point.

Some solids require scalar parameters to define their shape and space. Scalars are single numeric values. An example of a scalar is the radius parameter used to define the SPH solid.

Figures $C-1$ to $C-20$ illustrate and define the parameters and card input formats for each of the 20 solid types used by the GIFT5 geometry package. The comments on these figures must be given careful attention because the difference between certain solids is slight. 
Table C-1. List of 20 Geometric Solids used by the GIFT5 Geometry Package.

\begin{tabular}{|c|c|c|c|}
\hline & Solid Name & Symbol & Figure \\
\hline 1. & Arbitrary Convex Polyhedron, 4 vertex & ARB4 & $C-1$ \\
\hline 2. & Arbitrary Convex Polyhedron, 5 vertex & ARB5 & $C-2$ \\
\hline 3. & Arbitrary Convex Polyhedron, 6 vertex & ARB6 & $C-3$ \\
\hline 4. & Arbitrary Convex Polyhedron, 7 vertex & ARB7 & $C-4$ \\
\hline 5. & Arbitrary Convex Polyhedron, 8 vertex & ARB8 & $C-5$ \\
\hline 6. & Arbitrary Convex Polyhedron, $\mathrm{N}$ vertex & ARBN & $c-6$ \\
\hline 7. & Triangular Surfaced Polyhedrons & ARS & $C-7$ \\
\hline 8. & Box & $B O X$ & $C-8$ \\
\hline 9. & Ellipsoid of Revolution & ELL & $C-9$ \\
\hline 10. & Ellipsoid with Elliptical Cross Section & ELL G & $C-10$ \\
\hline 11. & Half Space & HAF & $C-11$ \\
\hline 12. & Right Angle Wedge & RAW & $c-12$ \\
\hline 13. & Right Circular Cylinder & RCC & $C-13$ \\
\hline 14. & Right Elliptical Cylinder & REC & $C-14$ \\
\hline 15. & Rectangular Parallelepiped & RPP & $C-15$ \\
\hline 16 . & Sphere & SPH & $C-16$ \\
\hline 17 . & Truncated Elliptical Cone & TEC & $C-17$ \\
\hline 18. & Truncated General Cone & TGC & $C-18$ \\
\hline 19. & Torus & TOR & $C-19$ \\
\hline 20. & Truncated Right Angle Cone & TRC & $C-20$ \\
\hline
\end{tabular}




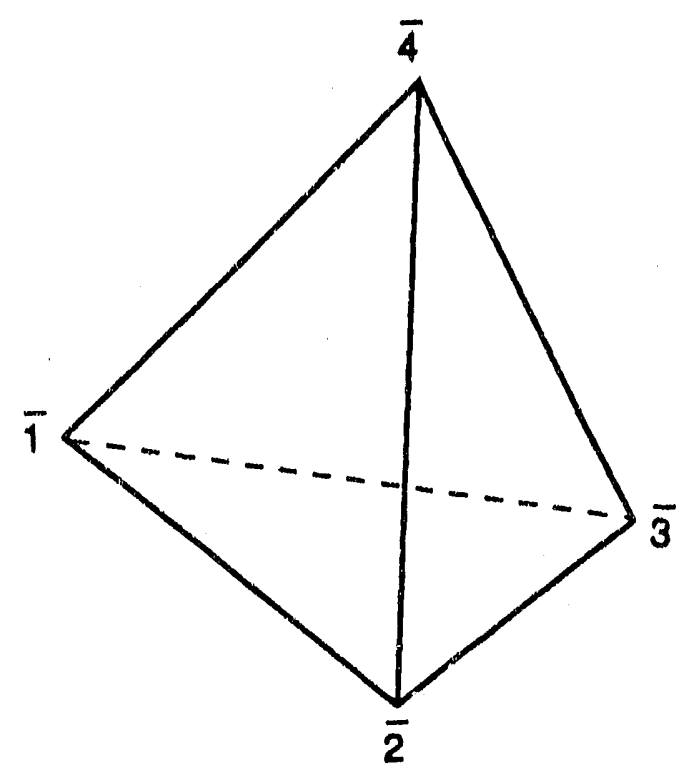

SPECIFY: The $X, Y, Z$ coordinates of the four vertices of the Polyhedron. Vertices are the ordinal numbers 1 to 4 on the figure.

NOTES: This form of the ARB has four ("4" in card column 9) vertices and four faces each defined by three vertices. The GIFT code generates the four faces as: $123,412,423,431$. Card Columns 71-80 may be used for comments.

Card Format: (15, A3, Al, $1 \mathrm{X}, 6 \mathrm{~F} 10.0, \mathrm{~A} 10)$

CARD COLUMNS

\begin{tabular}{|c|c|c|c|c|c|c|c|c|c|}
\hline $1-5$ & $6-8$ & 9 & $11-20$ & $21-30$ & $31-40$ & $41-50$ & $51-60$ & $61-70$ & $\begin{array}{c}\text { Number } \\
\text { of Cards }\end{array}$ \\
\hline $\begin{array}{l}\text { Solid } \\
\text { Number }\end{array}$ & ARB & 4 & $X 1$ & $Y 1$ & $Z 1$ & $X 2$ & $Y 2$ & $Z 2$ & 1 of 2 \\
\hline $\begin{array}{l}\text { Sol id } \\
\text { Number }\end{array}$ & & $X 3$ & $Y 3$ & $Z 3$ & $X 4$ & $Y 4$ & $Z 4$ & 2 of 2 \\
\hline
\end{tabular}

Figure C-1. Four-faced, four vertices, convex polyhedron (ARB4) input. 


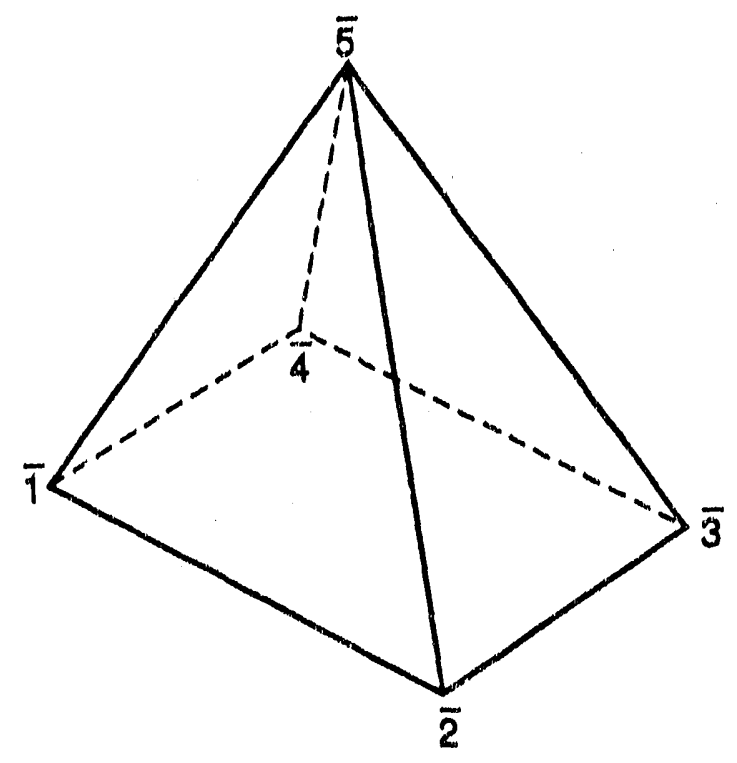

SPECIFY: The $X, Y, Z$ coordinates of the five vertices of the polyhedron. Vertices are the ordinal numbers 1 to 5 on the figure.

NOTES: This form of the ARB has five ("5" in card column 9) vertices and five faces - one defined by four vertices, four faces defined by three vertices. The GIFT code generates the five faces as: $1234,512,523,534,541$ Card Columns 71-80 may be used for comments. Card Format: (15, A3, A1, 1X, 6F10.0, A10)

CARD COLUMNS

\begin{tabular}{|c|c|c|c|c|c|c|c|c|c|}
\hline $1-5$ & $6-8$ & 9 & $11-20$ & $21-30$ & $31-40$ & $41-50$ & $51-60$ & $61-70$ & $\begin{array}{c}\text { Number } \\
\text { of Cards }\end{array}$ \\
\hline $\begin{array}{l}\text { Solid } \\
\text { Number }\end{array}$ & ARB & 5 & $X 1$ & $Y 1$ & $Z 1$ & $X 2$ & $Y 2$ & $Z 2$ & 1 of 3 \\
\hline $\begin{array}{l}\text { Solid } \\
\text { Number }\end{array}$ & & $X 3$ & $Y 3$ & $Z 3$ & $X 4$ & $Y 4$ & 24 & 2 of 3 \\
\hline $\begin{array}{l}\text { Solid } \\
\text { Number }\end{array}$ & $X 5$ & $Y 5$ & $Z 5$ & & & & 3 of 3 \\
\hline
\end{tabular}

Figure C-2. Five-faced, five vertices, convex polyhedron (ARB5) input. 


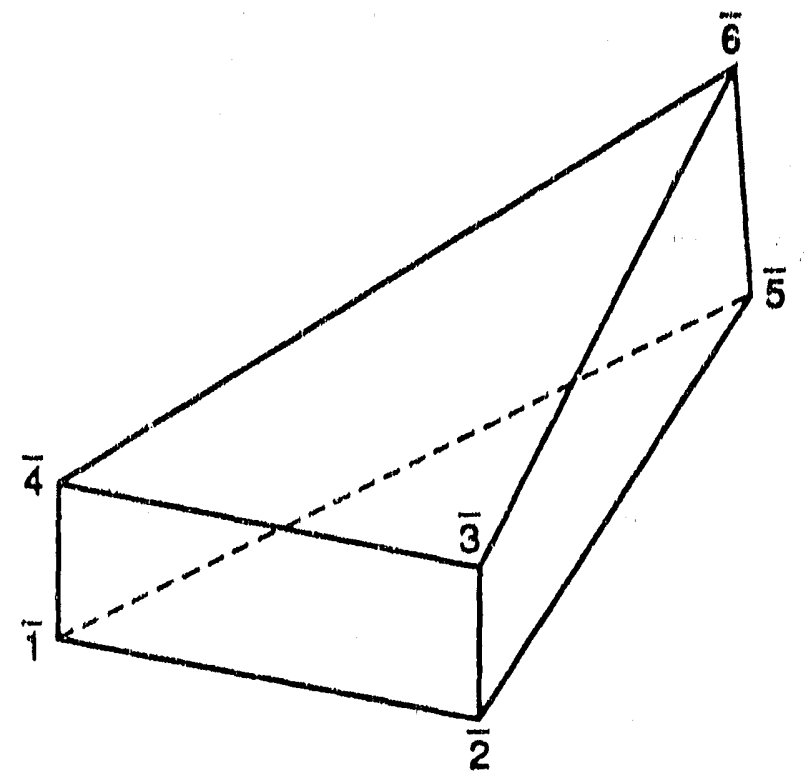

SPECIFY: The $X, Y, Z$ coordinates of the six vertices of the polyhedron. Vertices are the ordinal numbers 1 to 6 on the figure.

WOTES: This form of the ARB has six ("6" in card column 9) vertices and five faces - three faces are defined by four vertices, two faces defined by three vertices. The GIFT code generates the six faces as: $1234,2365,1564,512,634$. Card Columns 71-80 may be used for comments. Caru format: $(15, \mathrm{~A} 3, \mathrm{Al}, 1 \mathrm{X}, 6 \mathrm{~F} 10.0, \mathrm{AlO})$

CARD COLUMNS

\begin{tabular}{|l|c|c|c|c|c|c|c|c|c|}
\hline $1-5$ & $6-8$ & 9 & $11-20$ & $21-30$ & $31-40$ & $41-50$ & $51-60$ & $61-70$ & $\begin{array}{c}\text { Number } \\
\text { of Cards }\end{array}$ \\
\hline $\begin{array}{l}\text { Solid } \\
\text { Number }\end{array}$ & ARB & 6 & $X 1$ & $Y 1$ & 21 & $X 2$ & $Y 2$ & 22 & 1 of 3 \\
\hline $\begin{array}{l}\text { Solid } \\
\text { Number }\end{array}$ & & $X_{3}$ & $Y 3$ & 23 & $X 4$ & $Y 4$ & $Z 4$ & 2 of 3 \\
\hline $\begin{array}{l}\text { Solid } \\
\text { Number }\end{array}$ & $X 5$ & $Y 5$ & $Z 5$ & $X 6$ & $Y 6$ & 26 & 3 of 3 \\
\hline
\end{tabular}

Figure C-3. Five-faced, six vertices, convex polyhedron (ARB6) input. 


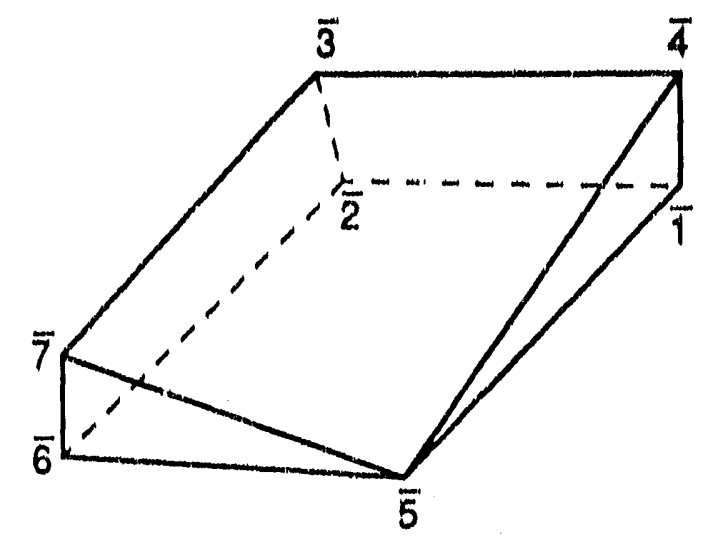

SPECIFY: The $X, Y, Z$ coordinates of the seven vertices of the polyhedron. Vertices are the ordinal number 1 to 7 on the figure.

NOTES: This form of the ARB has seven ("7" in card column 9) vertices and six faces - four faces defined by four vertices, two faces defined by three vertices (triangular faces). The GIFT code generates the six faces as: 1234, 567, 145, 2376, 1265, 4375. Card Columns 71-80 may be used for comments.

Card Format: (I5, A3, A1, IX, 6F10.0, AlO)

CARD COLUMNS

\begin{tabular}{|c|c|c|c|c|c|c|c|c|c|}
\hline $1-5$ & $6-8$ & 9 & $11-20$ & $21-30$ & $31-40$ & $41-50$ & $51-60$ & $61-70$ & $\begin{array}{c}\text { Number } \\
\text { of Cards }\end{array}$ \\
\hline $\begin{array}{l}\text { Solid } \\
\text { Number }\end{array}$ & ARB & 7 & $X 1$ & $Y 1$ & $Z 1$ & $X 2$ & $Y 2$ & $Z 2$ & 1 of 4 \\
\hline $\begin{array}{l}\text { Sol id } \\
\text { Number }\end{array}$ & $\times 3$ & $Y 3$ & $Z 3$ & $X 4$ & $Y 4$ & $Z 4$ & 2 of 4 \\
\hline $\begin{array}{l}\text { Solid } \\
\text { Number }\end{array}$ & $X 5$ & $Y 5$ & $Z 5$ & $X 6$ & $Y 6$ & $Z 6$ & 3 of 4 \\
\hline $\begin{array}{l}\text { Solid } \\
\text { Number }\end{array}$ & $X 7$ & $Y 7$ & $Z 7$ & & & & 4 of 4 \\
\hline
\end{tabular}

Figure C-4. Six-faced, seven vertices, convex polyhedron (ARB7) input. 


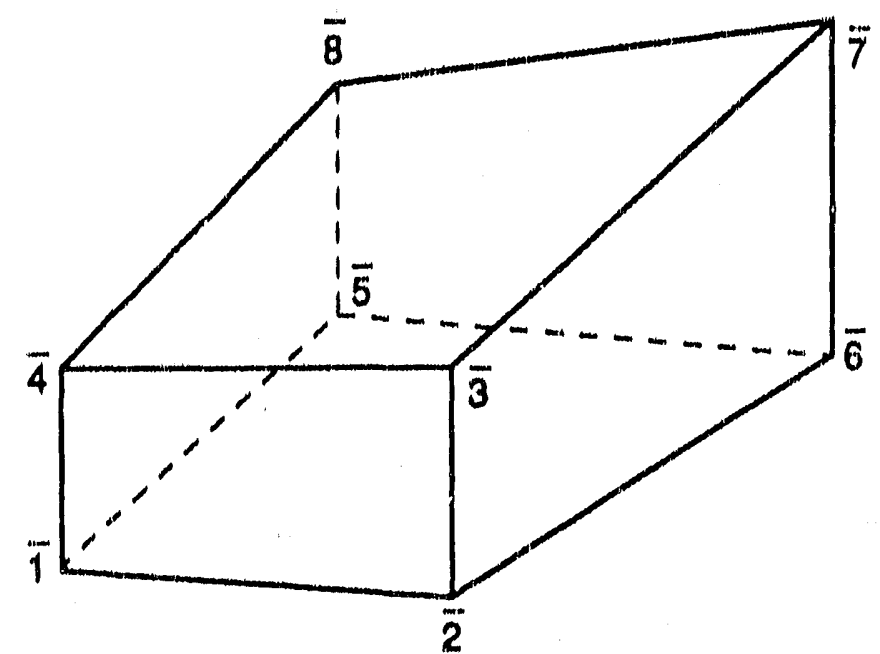

SPECIFY: The $X, Y, Z$ coordinates of the eight vertices of the Polyhedron. Vertices are the ordinal numbers 1 to 8 on the figure.

NOTES: This form of the ARB has eight (" 8 " in card column 9) vertices and six faces each defined by four vertices.

The GIFT code generates the six faces as:

$1234,5678,1584,2376,1265$, and 4378.

Card Columns 71-80 may be used for comments.

Card Format: (I5, A3, A1, IX, 6F10.0, A10)

CARD COLUMNS

\begin{tabular}{|l|c|c|c|c|c|c|c|c|c|}
\hline $1-5$ & $6-8$ & 9 & $11-20$ & $21-30$ & $31-40$ & $41-50$ & $51-60$ & $61-70$ & $\begin{array}{c}\text { Number } \\
\text { of Cards }\end{array}$ \\
\hline $\begin{array}{l}\text { Sol id } \\
\text { Number }\end{array}$ & ARB & 8 & $X 1$ & $Y 1$ & $Z 1$ & $X 2$ & $Y 2$ & $Z 2$ & 1 of 4 \\
\hline $\begin{array}{l}\text { Solid } \\
\text { Number }\end{array}$ & & $X 3$ & $Y 3$ & $Z 3$ & $X 4$ & $Y 4$ & 24 & 2 of 4 \\
\hline $\begin{array}{l}\text { Solid } \\
\text { Number }\end{array}$ & & $X 5$ & $Y 5$ & $Z 5$ & $X 6$ & $Y 6$ & $Z 6$ & 3 of 4 \\
\hline $\begin{array}{l}\text { Solidd } \\
\text { Number }\end{array}$ & & $X 7$ & $Y 7$ & $Z 7$ & $X 8$ & $Y 8$ & 28 & 4 of 4 \\
\hline
\end{tabular}

Figure C-5. Six-faces, eight vertices, convex polyhedron (ARBB) input. 


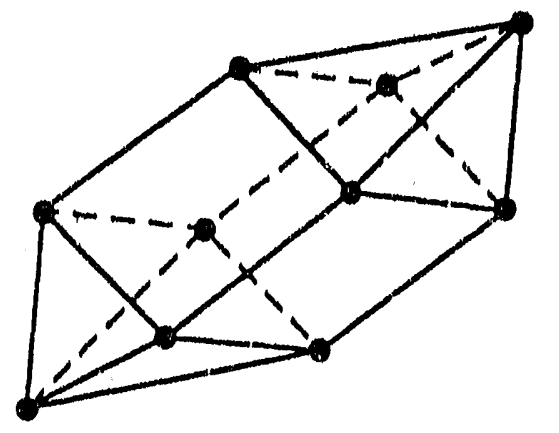

ORNL.DWG $90 M-11878$

SPECIFY: Bounding planes by any or all of three ways: points, equations, or point and vector normal to the plane.

DEFINITIONS: NPT = number of points

NPE = number of faces defined by 3 points

NEQ - number of faces defined by equation

NAE - number of faces defined by point and azimuth and elevation of vector normal to $\mathrm{plane}$

$A, B, C, D=$ plane equation coefficients, $A X+B Y+C Z=D$

$A Z$ = azimuthal angle, degrees

$E L$ - elevation angle, degrees

PN = point number

NOTES: The defined faces must form a totally enclosed region.

This ARB has the letter "N" in column 9.

Columns 71-80 may be used for comments.

Card Format: ( $15, A 3, A 1,1 X, 6 F 10.0, A 10)$ except faces input on $[10 \times, 6(14,213), A 10]$ format.

CARD COLUMNS

\begin{tabular}{|c|c|c|c|c|c|c|c|c|c|}
\hline $1-5$ & $6-8 \mid$ & 9 & $11-20$ & $21-30$ & $31-40$ & $41-50$ & $51-60$ & $61-70$ & $\begin{array}{l}\text { Number } \\
\text { of Cards }\end{array}$ \\
\hline $\begin{array}{l}\text { Solid } \\
\text { Number }\end{array}$ & ARB & $N$ & NPT & NPE & NEQ & NAE & & & 1 of $N$ \\
\hline $\begin{array}{l}\text { Solid } \\
\text { Number }\end{array}$ & & & $x 1$ & $Y 1$ & $\mathrm{Zl}$ & $\times 2$ & $Y_{2}$ & etc. & 2 of $N$ \\
\hline $\begin{array}{l}\text { Solid } \\
\text { Number }\end{array}$ & & & \multicolumn{6}{|c|}{$\begin{array}{c}\text { FACE1, FACE2, FACE3, etc. on } \\
10 X, 6(14,213) \text { format }\end{array}$} & $\begin{array}{l}\text { as needed } \\
\text { for } N P E>0\end{array}$ \\
\hline $\begin{array}{l}\text { Sol id } \\
\text { Number }\end{array}$ & & & Al & B1 & C1 & D1 & (blank) & (b) ank) & $\begin{array}{l}\text { as needed } \\
\text { for } N E Q>0\end{array}$ \\
\hline $\begin{array}{l}\text { Sol id } \\
\text { Number }\end{array}$ & & & $A Z 1$ & EL1 & PNI & $A Z 2$ & EL2 & PN2 & $\begin{array}{l}\text { as needed } \\
\text { for NAE }>0\end{array}$ \\
\hline
\end{tabular}

Figure C-6. N-faces, convex polyhedron (ARBN) input. 


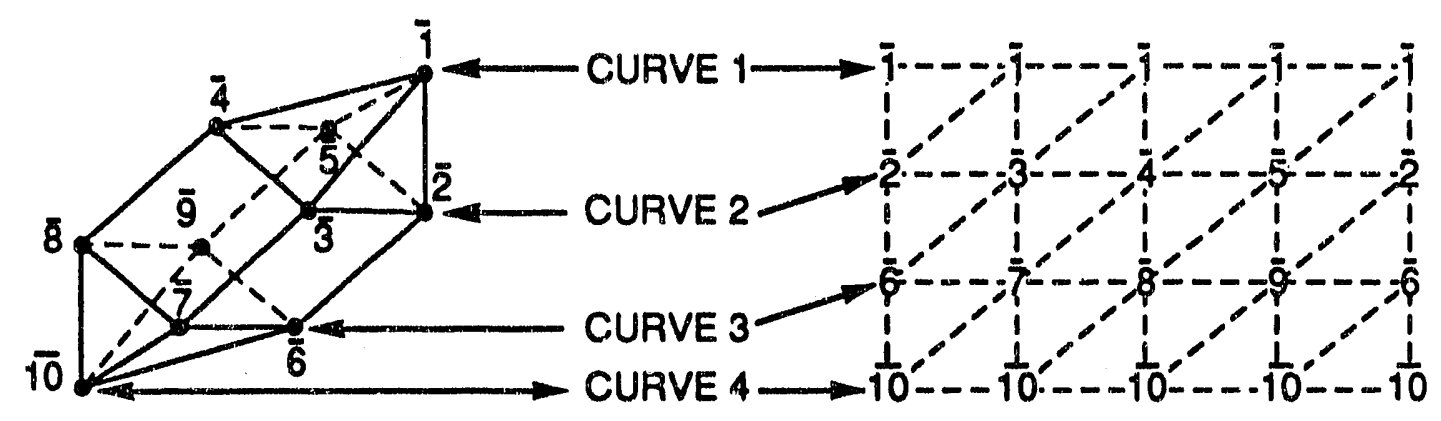

SPECIFY: The $X, Y, Z$ coordinate values of the vertices of the concave or convex polyhedron. Order and record of the vertices by the number of curves $(M)$ and number of points per curve (N) system.

NOTES: The purpose of this solid type is to permit the easy conversion and use of target description data from other BRL codes. BRL recommends that polyhedrons be modeled as a "combination" of ARB's 4, 5, 6, 7, and 8 's. The sample polyhedron has 10 unique vertices $(1,2,3 \ldots 10)$; however, $M=4, N=5$, and $4 \times 5$ or 20 vertices are required and recorded to generate the triangular faces of this polyhedron. The order is illustrated by the $M, N$ matrix above in which the vertices of the generated triangle faces are connected by dashes (--). Each new curve begins on a new card: When $N$ is odd, the card containing the last recorded point of a curve is followed by blanks in card columns 41-70.

Figure C-7. Triangular surfaced (ARS) polyhedron input. 
CARD COLUMNS

\begin{tabular}{|c|c|c|c|c|c|c|c|c|}
\hline $1-5$ & $6-8$ & $11-20$ & $21-30$ & $31-40$ & $41-50$ & $51-60$ & $61-70$ & $\begin{array}{c}\text { Number } \\
\text { of Cards }\end{array}$ \\
\hline $\begin{array}{c}\text { Solid } \\
\text { Number }\end{array}$ & ARS & $M$ & $N$ & & & & & 1 of $n$ \\
\hline $\begin{array}{l}\text { Solid } \\
\text { Number }\end{array}$ & $X(1,1)$ & $Y(1,1)$ & $Z(1,1)$ & $X(1,2)$ & $Y(1,2)$ & $Z(1,2)$ & 2 of $n$ \\
\hline$\vdots$ & $\vdots$ & & & & & & $\vdots$ \\
\hline $\begin{array}{l}\text { Solid } \\
\text { Number }\end{array}$ & $X(1, N)$ & $Y(1, N)$ & $Z(1, N)$ & & & & $\begin{array}{c}1+(N+1) \text { of } n \\
2\end{array}$ \\
\hline $\begin{array}{l}\text { Solid } \\
\text { Number }\end{array}$ & $X(2,1)$ & $Y(2,1)$ & $Z(2,1)$ & $X(2,2)$ & $Y(2,2)$ & $Z(2,2)$ & \\
\hline $\begin{array}{c}\vdots \\
\vdots\end{array}$ & $\vdots$ & & & & & & $\vdots$ \\
\hline $\begin{array}{l}\text { Solid } \\
\text { Number }\end{array}$ & $X(M, 1)$ & $Y(M, 1)$ & $Z(M, 1)$ & $X(M, 2)$ & $Y(M, 2)$ & $Z(M, 2)$ & \\
\hline $\begin{array}{c}\vdots \\
\vdots\end{array}$ & $\vdots$ & & & & & & $\vdots$ \\
\hline $\begin{array}{l}\text { Solid } \\
\text { Number }\end{array}$ & $X(M, N)$ & $Y(M, N)$ & $Z(M, N)$ & & & & $n=1+M(N+1)$ \\
\hline
\end{tabular}

Figure C-7. Triangular surfaces (ARS) polyhedron input (Continued). 


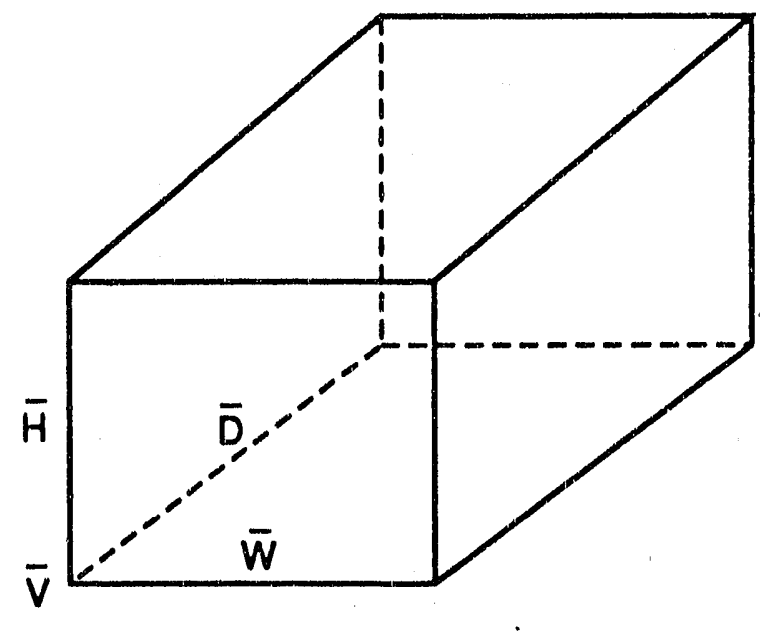

SPECIFY: The vertex ( $V$ ) at one of the corners by giving the $X, Y, Z$ coordinates. The $X, Y, Z$ components of the three mutually perpendicular vectors ( $H, W, D)$ from the vertex point $V$, representing the height, width, and depth of the box.

NOTES: The box may be arbitrarily oriented while the RPP must be parallel to the reference coordinate axes. The vectors $\mathrm{H}, \mathrm{H}$, and $D$ may be interchanged on the card input.

Card Columns 71-80 may be used for comments.

Card Format: $(\mathrm{i5}, \mathrm{A3}, 2 \mathrm{X}, 6 \mathrm{~F} 10.0, \mathrm{Al} 10)$

CARD COLUMNS

\begin{tabular}{|l|c|c|c|c|c|c|c|c|}
\hline $1-5$ & $6-8$ & $11-20$ & $21-30$ & $31-40$ & $41-50$ & $51-60$ & $61-70$ & $\begin{array}{c}\text { Number } \\
\text { of Cards }\end{array}$ \\
\hline $\begin{array}{l}\text { Solid } \\
\text { Number }\end{array}$ & $\mathrm{BOX}$ & $\mathrm{Vx}$ & $\mathrm{Vy}$ & $\mathrm{Vz}$ & $\mathrm{Hx}$ & $\mathrm{Hy}$ & $\mathrm{Hz}$ & 1 of 2 \\
\hline $\begin{array}{l}\text { Sol id } \\
\text { Number }\end{array}$ & & & & & & & $\mathrm{Dz}$ & 2 of 2 \\
\hline
\end{tabular}

Figure C-8. Box (BOX) input. 


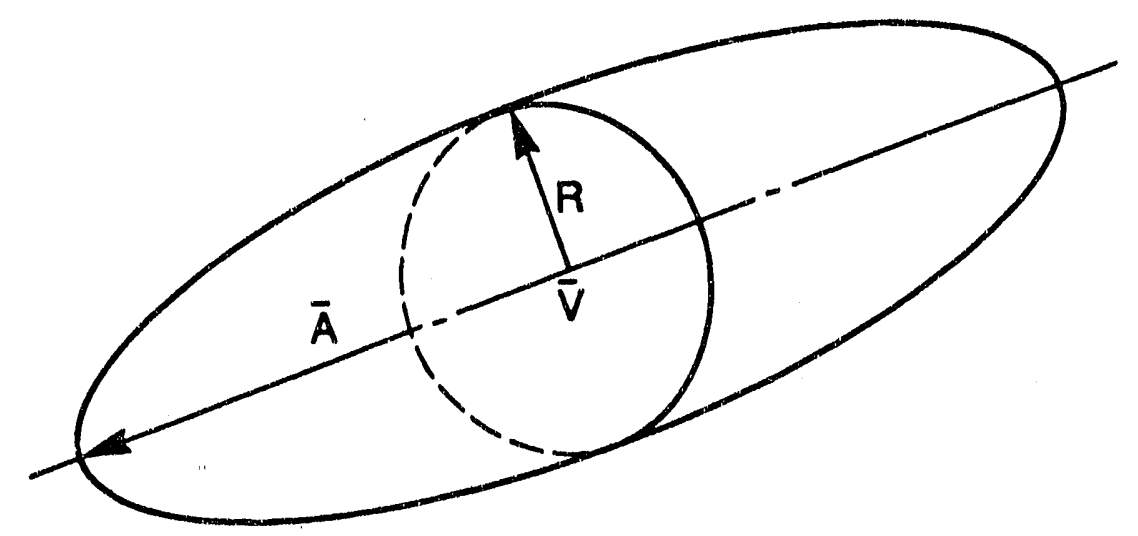

SPECIFY: The $X, Y, Z$ coordinates of the vertex $V$ at the center of the major axis, the vector $A$ defining the semi-major axis, and the scalar $R$ denoting the radius of the circular section taken at the center point $V$.

NOTES: Card Columns 71-80 may be used for comments.

Card Format: $\quad(15, \mathrm{A3}, 2 \mathrm{X}, 6 \mathrm{~F} 10.0, \mathrm{~A} 10)$

CARD COLUMNS

\begin{tabular}{|c|c|c|c|c|c|c|c|c|}
\hline $1-5$ & $6-8$ & $11-20$ & $21-30$ & $31-40$ & $41-50$ & $51-60$ & $61-70$ & $\begin{array}{c}\text { Number } \\
\text { of Cards }\end{array}$ \\
\hline $\begin{array}{l}\text { Solid } \\
\text { Number }\end{array}$ & $E L L$ & $V x$ & $V y$ & $V z$ & $A x$ & $A y$ & $A z$ & 1 of 2 \\
\hline $\begin{array}{l}\text { Solid } \\
\text { Number }\end{array}$ & & $R$ & & & & & & 2 of 2 \\
\hline
\end{tabular}

Figure C-9. Ellipsoid of revolution (ELL) input. 


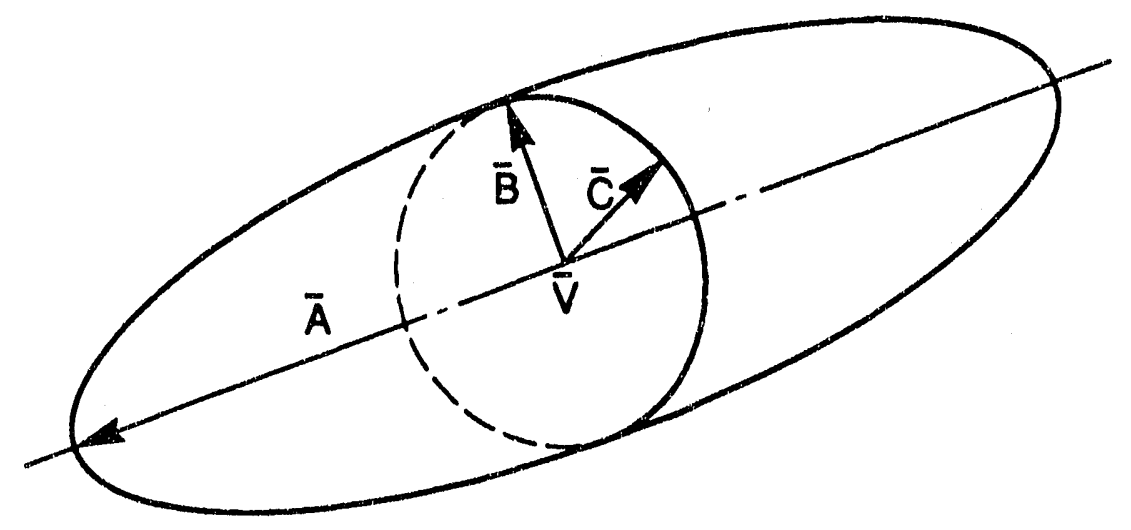

SPECIFY: The $X, Y, Z$ coordinates of the vertex $V$ at the center of the major axis, the vector $A$ defining the semi-major axis, and vectors $B$ and $C$ defining semi-minor axes.

NOTES: The letter "G" in card column " 9 " denotes the second ELL input option. Vectors $A, B$, and $C$ must be perpendicular.

Card Columns 71-80 may be used for comments.

Card Format: $(15, \mathrm{A3}, \mathrm{Al}, 1 \mathrm{X}, 6 \mathrm{~F} 10.0, \mathrm{AlO})$

CARD COLUMNS

\begin{tabular}{|c|c|c|c|c|c|c|c|c|c|}
\hline $1-5$ & $6-8$ & 9 & $11-20$ & $21-30$ & $31-40$ & $41-50$ & $51-60$ & $61-70$ & $\begin{array}{c}\text { Number } \\
\text { of Cards }\end{array}$ \\
\hline $\begin{array}{l}\text { Solid } \\
\text { Number }\end{array}$ & $\mathrm{ELL}$ & $\mathrm{G}$ & $\mathrm{Vx}$ & $\mathrm{Vy}$ & $\mathrm{Vz}$ & $\mathrm{Ax}$ & $\mathrm{Ay}$ & $\mathrm{Az}$ & 1 of 2 \\
\hline $\begin{array}{l}\text { Solid } \\
\text { Number }\end{array}$ & & $\mathrm{Bx}$ & $\mathrm{By}$ & $\mathrm{Bz}$ & $\mathrm{Cx}$ & $\mathrm{Cy}$ & $\mathrm{Cz}$ & 2. of 2 \\
\hline
\end{tabular}

Figure C-10. Input for ellipsoid with elliptical cross section (ELL G). 


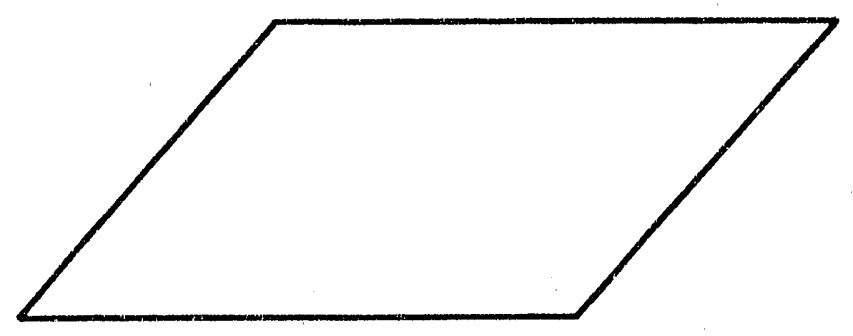

SPECIFY: Coefficients $A, B, C$ and $D$ of $p l a n e$ defined by the equation $A X+B Y+C Z=D$.

NOTES: $\quad A, B, C$ can be viewed as vectors which point away from the desired space. Example: for $A=B=D=0$ and $C=1$, $p l$ ane is $Z=0$. $C=1$ points away from desired space; hence desired space is $Z \leq 0$. Card Columns 71-80 may be used for comments.

Card Format: (I5, A3, 2X, 6F10.0, A10)

CARD COLUMNS

\begin{tabular}{|c|c|c|c|c|c|c|c|c|}
\hline $1-5$ & $6-8$ & $11-20$ & $21-30$ & $31-40$ & $41-50$ & $51-60$ & $61-70$ & $\begin{array}{c}\text { Number } \\
\text { of Cards }\end{array}$ \\
\hline $\begin{array}{l}\text { Solid } \\
\text { Number }\end{array}$ & HAF & A & B & C & D & & & 1 of 1 \\
\hline
\end{tabular}

Figure C-11. Half space (HAF) input. 


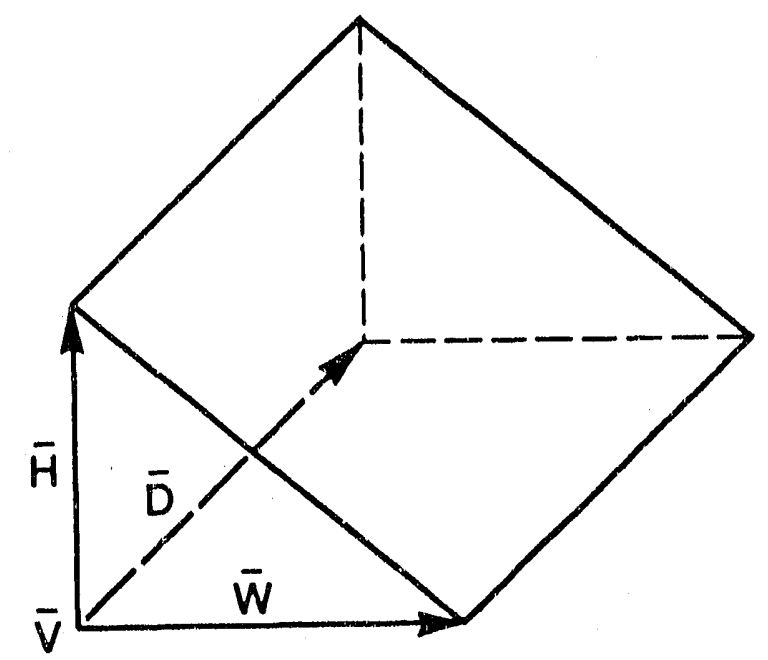

SPECIFY: The vertex (V) at one of the right-angled corners by giving the $X, Y$, and $Z$ coordinate. The components of the three mutually perpendicular vectors $(H, W, D)$, of which two $(H, W)$ are the legs of the right triangle formed while the third (D) is the depth of the wedge.

NOTES: The two legs $H, W$ may be interchanged on card input, but the $D$ vector must remain in position shown.

Card Columns 71-80 may be used for comments.

Card Format: $(15, A 3,2 X, 6 F 10.0, A 10)$

CARD COLUMNS

\begin{tabular}{|c|c|c|c|c|c|c|c|c|}
\hline $1-5$ & $6-8$ & $11-20$ & $21-30$ & $31-40$ & $41-50$ & $51-60$ & $61-70$ & $\begin{array}{c}\text { Number } \\
\text { of Cards }\end{array}$ \\
\hline $\begin{array}{l}\text { Solid } \\
\text { Number }\end{array}$ & RAW & $\mathrm{Vx}$ & $\mathrm{Vy}$ & $\mathrm{Vz}$ & $\mathrm{Hx}$ & $\mathrm{Hy}$ & $\mathrm{Hz}$ & 1 of 2 \\
\hline $\begin{array}{l}\text { Solid } \\
\text { Number }\end{array}$ & $\mathrm{Wx}$ & $\mathrm{Wy}$ & $\mathrm{Wz}$ & $\mathrm{Dx}$ & $\mathrm{Dy}$ & $\mathrm{Dz}$ & 2 of 2 \\
\hline
\end{tabular}

Figure $\mathrm{C}-12$. Right angle wedge (RAW) input. 


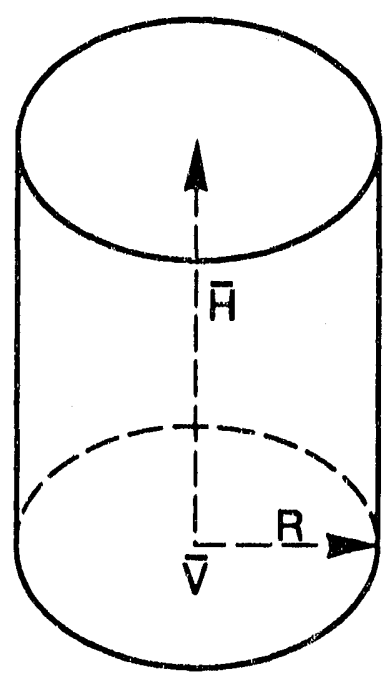

SPECIFY: The vertex point $V$ at the center of one base, height vector $H$ and scalar $R$ denoting the base radius.

NOTES: $\quad$ Card Columins 71-80 may be used for comments.

Card Format: (I5, A3, 2X, 6F10.0, A10)

CARD COLUMNS

\begin{tabular}{|c|c|c|c|c|c|c|c|c|}
\hline $1-5$ & $6-8$ & $11-20$ & $21-30$ & $31-40$ & $41-50$ & $51-60$ & $61-70$ & $\begin{array}{c}\text { Number } \\
\text { of Cards }\end{array}$ \\
\hline $\begin{array}{l}\text { Solid } \\
\text { Number }\end{array}$ & RCC & $V x$ & $V y$ & $V z$ & $H x$ & $H y$ & $H z$ & 1 of 2 \\
\hline $\begin{array}{l}\text { Solid } \\
\text { Number }\end{array}$ & R & & & & & & 2 of 2 \\
\hline
\end{tabular}

Figure C-13. Right circular cylinder (RCC) input. 
ORNL-DWG 9OM-11867

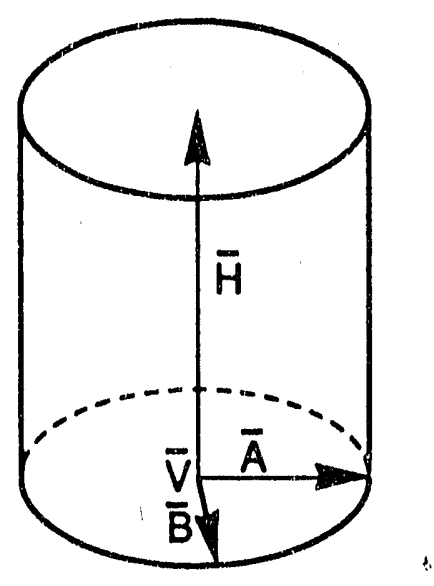

SPECIFY: The $X, Y, Z$ coordinates of the center of the base ellipse $V$, height vector $H$, and vectors $A$ and $B$ in the base plane defining the semi-major and semi-minor axes, respectively.

NOTES: Card Columns 71-80 may be used for comments.

Card Format: (15, A3, 2X, 6F10.0, A10)

CARD COLUMNS

\begin{tabular}{|c|c|c|c|c|c|c|c|c|}
\hline $1-5$ & $6-8$ & $11-20$ & $21-30$ & $31-40$ & $41-50$ & $51-60$ & $61-70$ & $\begin{array}{c}\text { Number } \\
\text { of Cards }\end{array}$ \\
\hline $\begin{array}{l}\text { Solid } \\
\text { Number }\end{array}$ & REC & $\mathrm{Vx}$ & $\mathrm{Vy}$ & $\mathrm{Vz}$ & $\mathrm{Hx}$ & $\mathrm{Hy}$ & $\mathrm{Hz}$ & 1 of 2 \\
\hline $\begin{array}{l}\text { Solid } \\
\text { Number }\end{array}$ & $\mathrm{Ax}$ & $\mathrm{Ay}$ & $\mathrm{Az}$ & $\mathrm{Bx}$ & $\mathrm{By}$ & $\mathrm{Bz}$ & 2 of 2 \\
\hline
\end{tabular}

Figure C-14. Right elliptical cylinder (REC) input. 


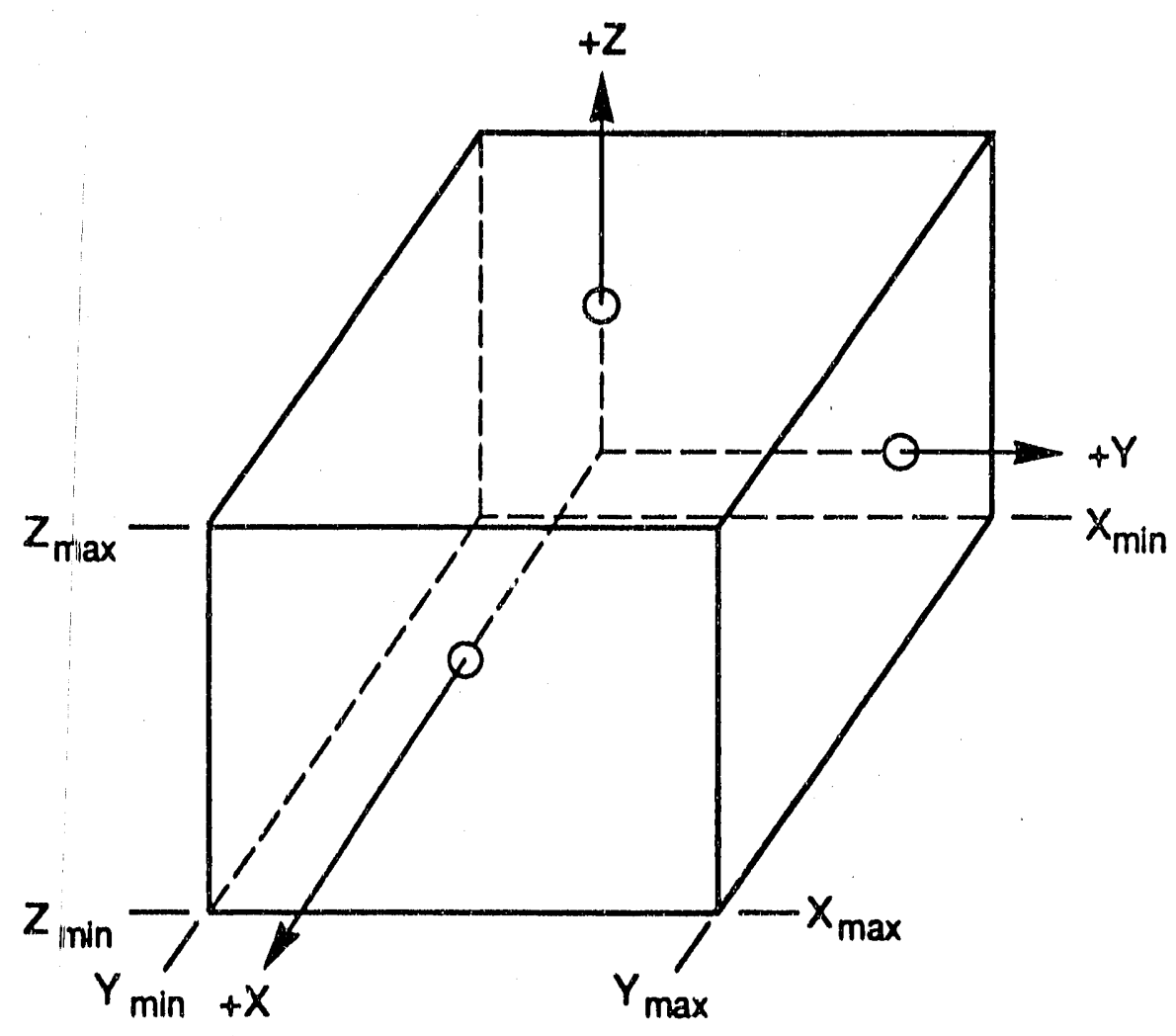

SPECIFY: The maximum (max) and the minimum (min) values of the $X, Y, Z$ coordinates which bound the parallelepiped.

NOTES: The bounding planes must be parallel to the coordinate axes of the target.

Card Columns 71-80 may-be used for comments.

Card Format: $(15, \mathrm{~A} 3,2 \mathrm{X}, 6 \mathrm{~F} 10.0, \mathrm{AlO})$

CARD COLUMNS

\begin{tabular}{|c|c|c|c|c|c|c|c|c|}
\hline $1-5$ & $6-8$ & $11-20$ & $21-30$ & $31-40$ & $41-50$ & $51-60$ & $61-70$ & $\begin{array}{c}\text { Number } \\
\text { of Cards }\end{array}$ \\
\hline $\begin{array}{l}\text { Solid } \\
\text { Number }\end{array}$ & $R P P$ & $X \min$ & $Y \min$ & $Z \min$ & $X \max$ & $Y_{\max }$ & $Z \max$ & 1 of 1 \\
\hline
\end{tabular}

Figure C-15. Rectangular parallelepiped (RPP) input. 


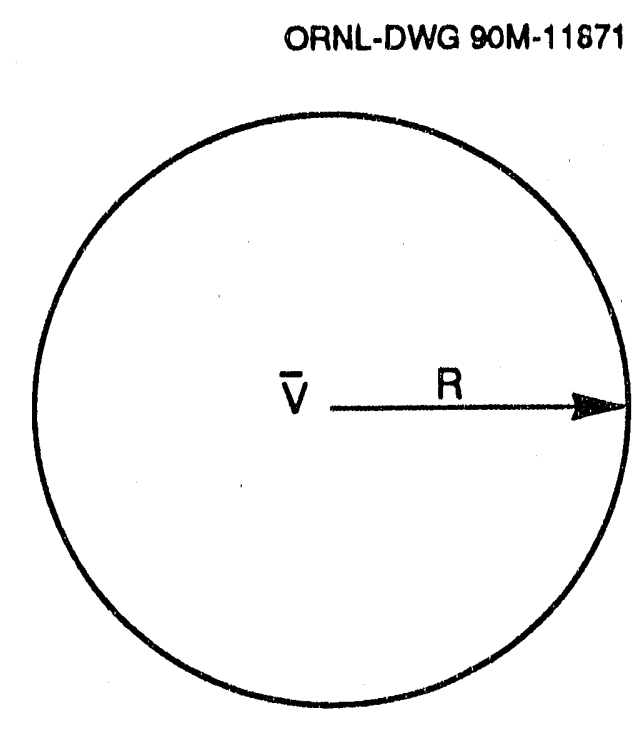

SPECIFY: The center point, $V$, and scalar $R$ denoting the radius.

NOTES: Card Columns 71-80 may be used for comments.

Card Format: $(15, \mathrm{A3}, 2 \mathrm{X}, 6 \mathrm{~F} 10.0, \mathrm{A10})$

CARD COLUMNS

\begin{tabular}{|c|c|c|c|c|c|c|c|c|}
\hline $1-5$ & $6-8$ & $11-20$ & $21-30$ & $31-40$ & $41-50$ & $51-60$ & $61-70$ & $\begin{array}{c}\text { Number } \\
\text { of Cards }\end{array}$ \\
\hline $\begin{array}{l}\text { Solid } \\
\text { Number }\end{array}$ & $\mathrm{SPH}$ & $\mathrm{Vx}$ & $V_{y}$ & $V_{z}$ & $\mathrm{R}$ & & & 1 of 1 \\
\hline
\end{tabular}

Figure C-16. Sphere (SPH' input. 


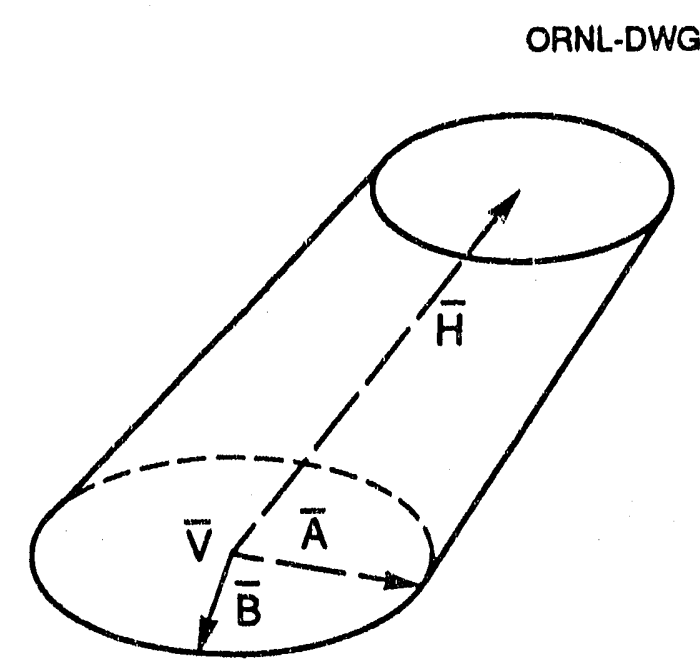

SPECIFY: The coordinates of vertex $V$ at the center of the larger ellipse, and the $X, Y, Z$ components of the height vector $H$ and vectors $A$ and $B$ describing the semi-major and semi-minor axes. The ratio $P$ of the larger to smaller ellipse.

NOTES: The height vector $H$ does not have to be perpendicular to the plane containing vectors $A$ and $B$. The ratio $P$ may be determined by the magnitude (length) of semi-major vector $A$ of the base ellipse divided by the length of semi-major axis of the upper ellipse: $P>1$.

Card Columns 71-80 may be used for comments.

Card Format: (I5, A3, 2X, 6F10.0, A10)

CARD COLUMNS

\begin{tabular}{|l|c|c|c|c|c|c|c|c|}
\hline $1-5$ & $6-8$ & $11-20$ & $21-30$ & $31-40$ & $41-50$ & $51-60$ & $61-70$ & $\begin{array}{c}\text { Number } \\
\text { of Cards }\end{array}$ \\
\hline $\begin{array}{l}\text { Sol id } \\
\text { Number }\end{array}$ & TEC & $\mathrm{Vx}$ & $\mathrm{Vy}$ & $\mathrm{Vz}$ & $\mathrm{Hx}$ & $\mathrm{Hy}$ & $\mathrm{Hz}$ & 1 of 3 \\
\hline $\begin{array}{l}\text { Sol id } \\
\text { Number }\end{array}$ & $\mathrm{Ax}$ & $\mathrm{Ay}$ & $\mathrm{Az}$ & $\mathrm{Bx}$ & $\mathrm{By}$ & $\mathrm{Bz}$ & 1 of 3 \\
\hline $\begin{array}{l}\text { Solid } \\
\text { Number }\end{array}$ & $\mathrm{P}$ & & & & & & 3 of 3 \\
\hline
\end{tabular}

Figure $\mathrm{C}-17$. Truncated elliptic cone (TEC) input. 


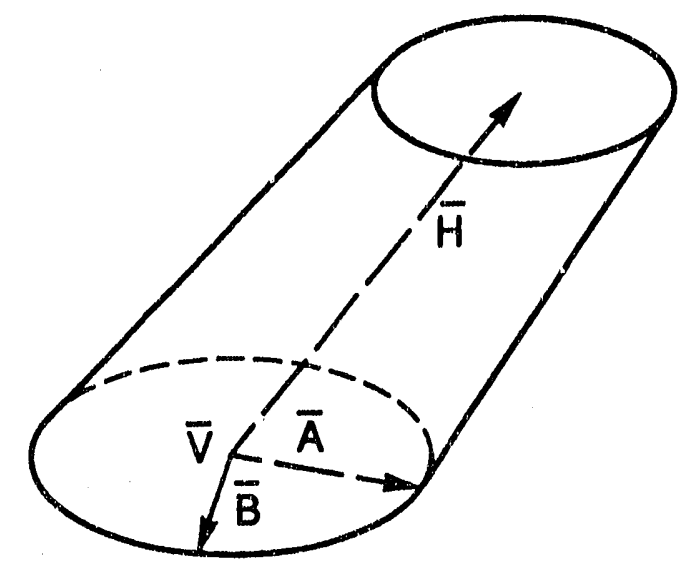

SPECIFY: The coordinates of vertex $V$ at the center of the larger ellipse, and the $X, Y, Z$ components of the height vector $H$ and vectors $A$ and $B$ describing the semi-major and semi-minor axes. The ratios $T(A)$ and $T(B)$ of the semi-major and semi-minor axes for the smaller ellipse.

NOTES: The height vector $H$ does not have to be perpendicular to the plane containing vectors $A$ and $B$. The semi-major and semi-minor axes of the smaller ellipse, $T A * A$ and $T B * B$, respectively, must be parallel to the semi-major and semi-minor axes, respectively, of the larger ellipse.

Card Columns 71-80 may be used for comments.

Card Format: (I5, A3, 2X, 6F10.0, A10)

CARD COLUMNS

\begin{tabular}{|l|c|c|c|c|c|c|c|c|}
\hline $1-5$ & $6-8$ & $11-20$ & $21-30$ & $31-40$ & $41-50$ & $51-60$ & $61-70$ & $\begin{array}{c}\text { Number } \\
\text { of Cards }\end{array}$ \\
\hline $\begin{array}{l}\text { Solid } \\
\text { Number }\end{array}$ & TGC & $\mathrm{Vx}$ & $\mathrm{Vy}$ & $\mathrm{Vz}$ & $\mathrm{Hx}$ & $\mathrm{Hy}$ & $\mathrm{Hz}$ & 1 of 3 \\
\hline $\begin{array}{l}\text { Solid } \\
\text { Number }\end{array}$ & $\mathrm{Ax}$ & $\mathrm{Ay}$ & $\mathrm{Az}$ & $\mathrm{Bx}$ & $\mathrm{By}$ & $\mathrm{Bz}$ & 1 of 3 \\
\hline $\begin{array}{l}\text { Solid } \\
\text { Number }\end{array}$ & $\mathrm{TA}$ & $\mathrm{TB}$ & & & & & 3 of 3 \\
\hline
\end{tabular}

Figure C-18. Truncated general cone (TGC) input. 

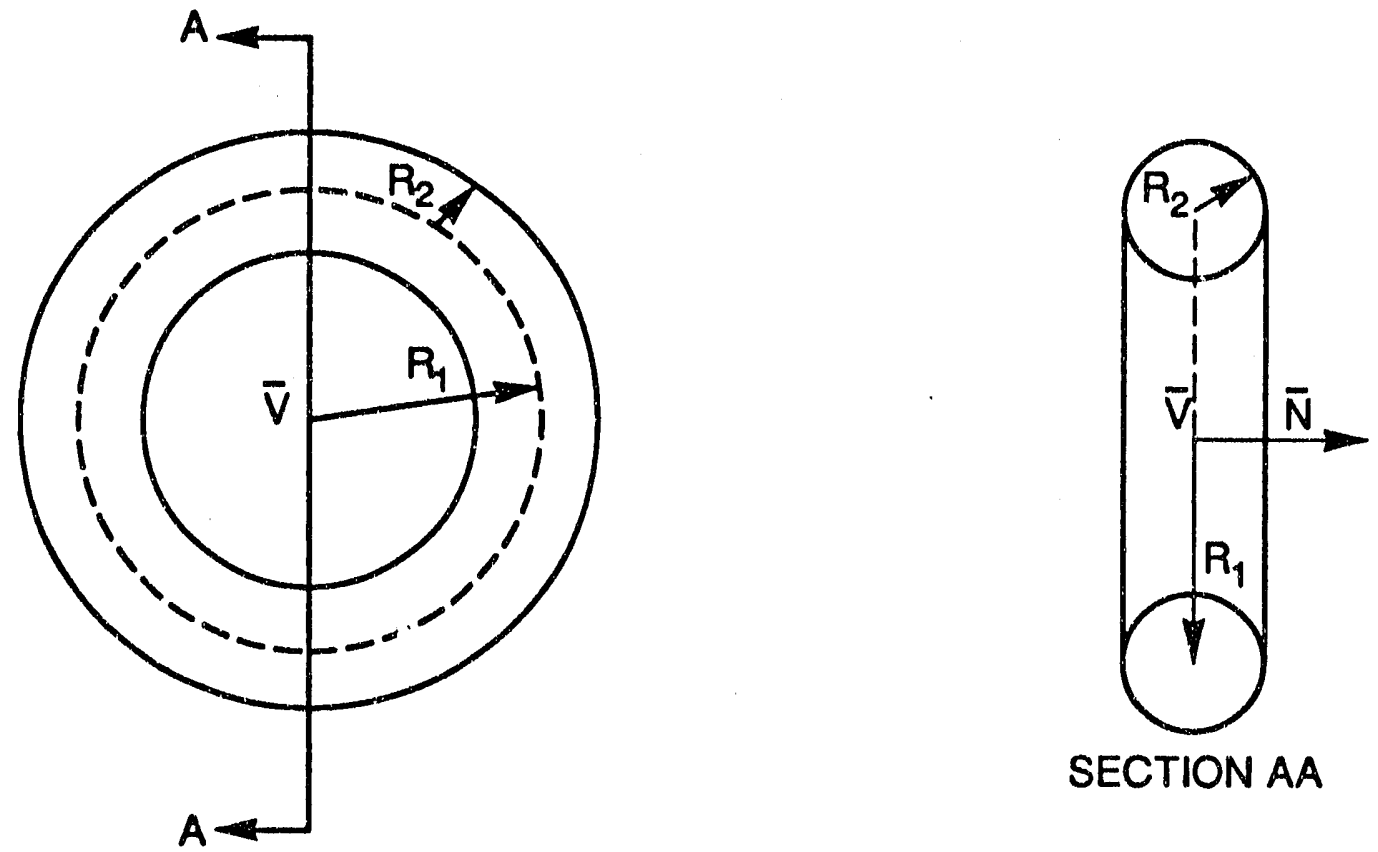

SECTION AA

SPECIFY: The vertex $V$ at the center of the torus, a normal vector $N$ to the plane in which the locus of the mid-points of the circular cross sections lies, and the scalars Rl, the distance from the center $V$ to the mid-point of the circular cross section, and $\mathrm{R} 2$, the radius of the circular cross section.

NOTES: Card Columns 71-80 may be used for comments. Card Format: (I5, A3, 2X, 6F10.0, A10)

CARD COLUMNS

\begin{tabular}{|c|c|c|c|c|c|c|c|c|}
\hline $1-5$ & $6-8$ & $11-20$ & $21-30$ & $31-40$ & $41-50$ & $51-60$ & $61-70$ & $\begin{array}{c}\text { Number } \\
\text { of Cards }\end{array}$ \\
\hline $\begin{array}{l}\text { Solid } \\
\text { Number }\end{array}$ & TOR & $\mathrm{Vx}$ & $\mathrm{Vy}$ & $\mathrm{Vz}$ & $\mathrm{Nx}$ & $\mathrm{Ny}$ & $\mathrm{Nz}$ & 1 of 2 \\
\hline $\begin{array}{l}\text { Solid } \\
\text { Number }\end{array}$ & $\mathrm{R} 1$ & $\mathrm{R} 2$ & & & & & 2 of 2 \\
\hline
\end{tabular}

Figure $\mathrm{C}-19$. Torus (TOR) input. 


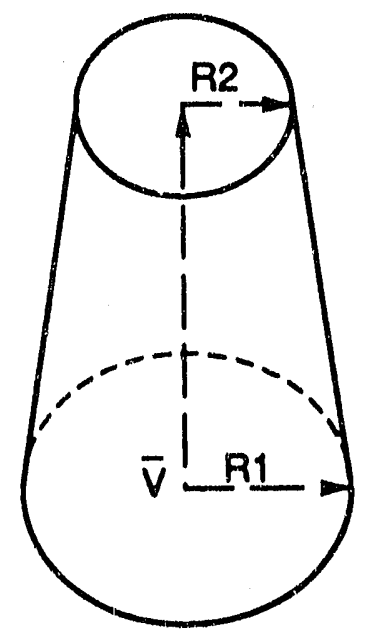

SPECIFY: The vertex $V$ at the center of the larger base, height vector $H$ and scalars $R 1$ and $R 2$ denoting the radii of the larger and smaller bases, respectively.

NOTES: Card Columns 71-80 may be used for comments.

Card Format: (15, A3, 2X, 6F10.0, A10)

CARD COLUMNS

\begin{tabular}{|c|c|c|c|c|c|c|c|c|}
\hline $1-5$ & $6-8$ & $11-20$ & $21-30$ & $31-40$ & $41-50$ & $51-60$ & $61-70$ & $\begin{array}{c}\text { Number } \\
\text { of Cards }\end{array}$ \\
\hline $\begin{array}{l}\text { Sol id } \\
\text { Number }\end{array}$ & TRC & $V x$ & $V y$ & $V z$ & $\mathrm{Nx}$ & $\mathrm{Ny}$ & $\mathrm{Nz}$ & 1 of 2 \\
\hline $\begin{array}{l}\text { Solid } \\
\text { Number }\end{array}$ & $\mathrm{V}$ & $\mathrm{R} 2$ & & & & & 2 of 2 \\
\hline
\end{tabular}

Figure $\mathrm{C}-20$. Truncated right angle cone (TRC) input. 
A Solid Table contains the input cards for every solid used to describe the target. Table $\mathrm{C}-2$ is the Solid Table for a sample target. Twenty solids are used to describe the sample target.

Every solid within a Solid Table must have a unique number. The numbering of the solids in the Solid Table begins with the number "1" and is consecutive $(1,2,3 \ldots)$. No hierarchy exists between the solids; any solid type can be numbered " 1 , or 2 , or $3, \ldots$ "

\section{"COMBINATION" OF SOL.IDS}

The three-dimensional shape and space of several solids can be "combined" to define a component of a target. Figure C-21 illustrates the concepts of "intersection," "subtraction," and "union" which are used to "combine" the space of several solids.

Section A of Figure C-21 exhibits an RPP and SPH which overlap. For discussion, suppose that the RPP is the first solid in a Solid Table; it is therefore numbered "l". If the SPH is the second solid in the Solid Table, it is therefore numbered " 2 ". The "intersection" $(+)$ of the RPP (1) and the SPH (2) solid is represented as "1 2 ". The "1 + 2" symbolization may be interpreted as the space of the first (1) solid in the Solid Table that overlaps or "intersects" $(+)$ the space of the second (2) solid in the Solid Table. The dashed (/) area in section $B$ of Figure C-21 represents the space of " $1+2$ ", the space of the RPP (1) that overlaps the space of the SPH (2).

The "Subtraction" (-) of the space of the SPH (2) from the space of the RPP (1) is represented by "1 - 2". The "1 - 2" symbolization may be interpreted as the space of the first (1) solid removing or "subtracting" the space of the second solid in the Solid Table. The dashed area in section $C$ of the Figure $C-21$ represents the space of "1 $2^{\prime \prime}$ the space of the RPP removing the space of the SPH. Note that the space resuiting from "1+2" and " $2+1$ " (intersect relationship) would be the same; however, "1 - 2" results in a space different from "2 - 1", the space of the SPH removing the space of the RPP.

The "union" (OR) of the space of the RPP (1) and the SPH (2) is represented by "1 OR 2". The "1 OR 2" symbolization is the space of the first (1) solid "and" or "union" (OR) of the space of the second solid (2) in the Solid Table. The dashed area in section $E$ on Figure $C-21$ represents "1 OR 2", the space of the RPP "and" the space of the SPH. As the intersect relationship, the union relationship of "1 OR 2" and "2 OR 1" results in the same space. 
Table C-2. Solid Table for the Sample Target.

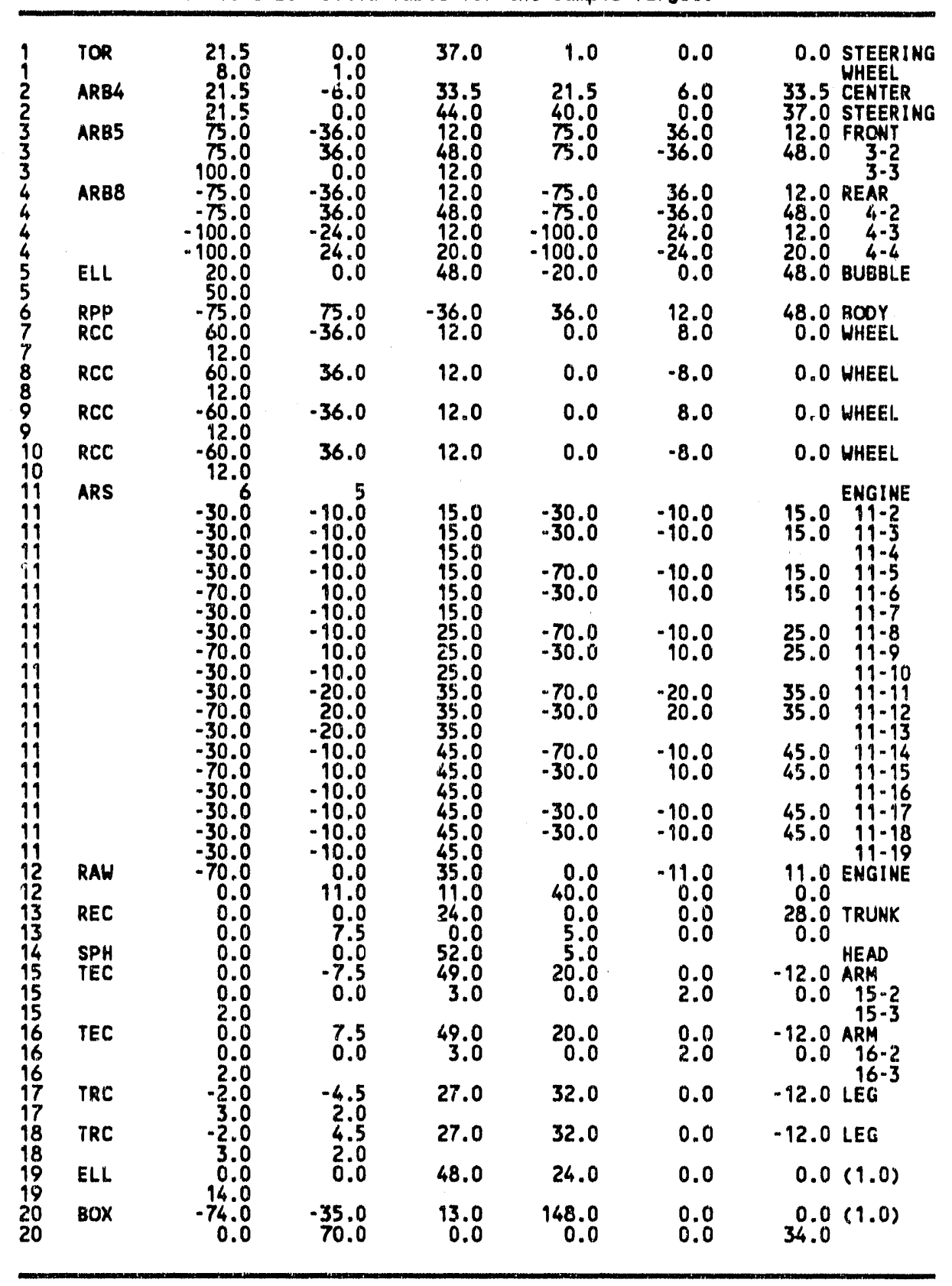




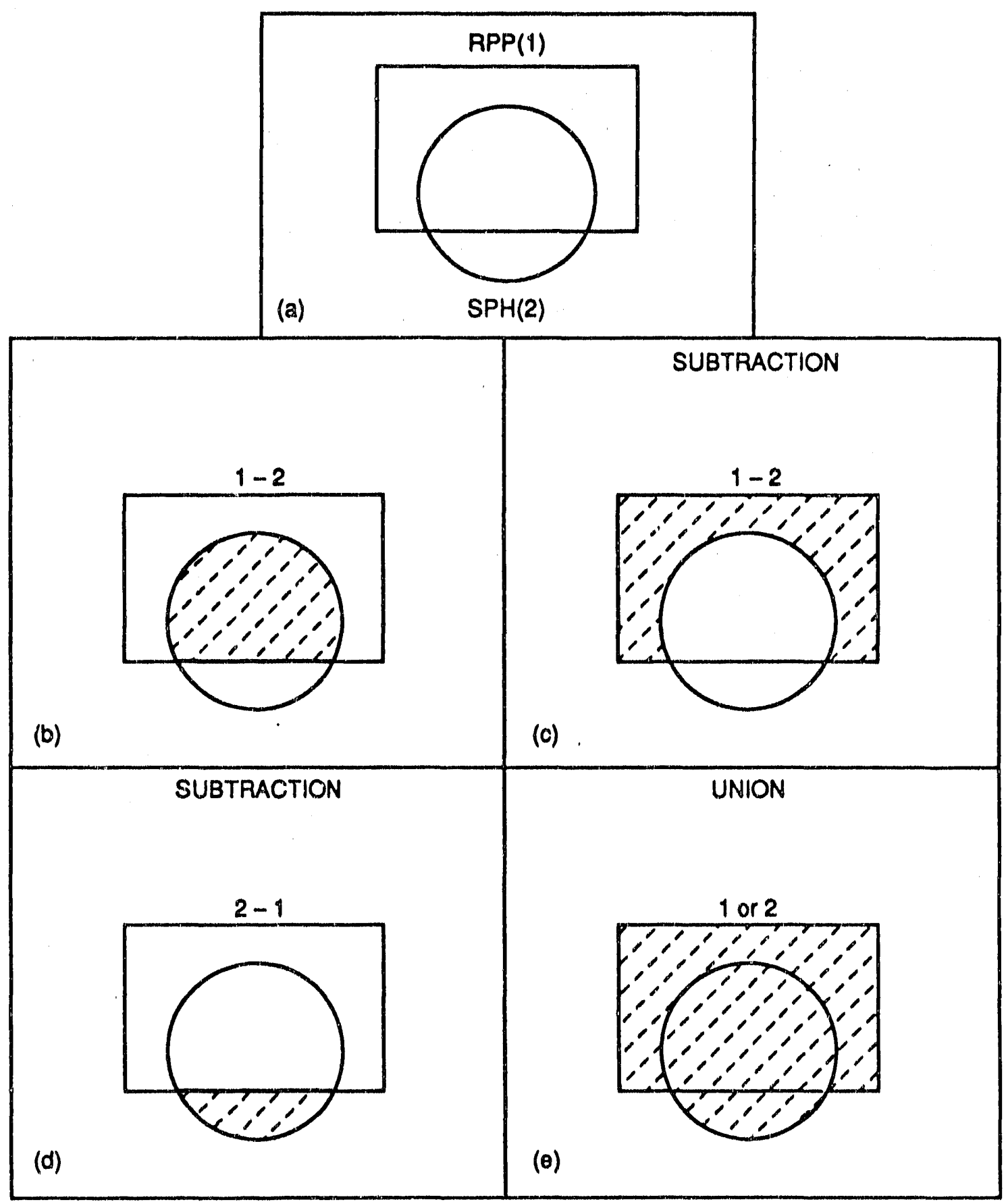

Figure C-21. Intersection, Subtraction, and Union Between Two Solids. 
To model irregular shaped components of a target may require the intersecition, subtraction, and union of many different solids. In section $A$ on Figure C-22 a third solid (numbered " 3 ") has been added to the RPP and SPH configuration used in Figure C-21. The third (3) solid may be a BOX or another RPP.

The dashed area in section $B$ of Figure $\mathrm{C}-22$ represents the space resulting from the intersection of solids 1,2 , and $3(1+2+3)$. A process to arrive at the results of "1+2+3" is to visualize the area resulting from " $1+2$ " (shown in Figure $c-21$ ) and then fird the intersect $(+)$ of the third (3) solid with the area from "1 +2 ".

The dashed area in section $C$ of Figure $\mathrm{C}-22$ represents the space resulting from the relationship "3-1", the space of the third (3) solid removing or subtracting the space of the first (1) solid.

The dashed area in section $D$ represents the space resulting from $1+2$ - 3". Again analyzing the final space by steps: first, visualize the $" 1+2$ " relationship, then remove or subtract the space of solid " 3 ".

The dashed area in section $E$ represents the space resulting from "1 OR 2 OR 3".

The dashed area in section $F$ represents the space of " $1+2-3$ OR 3 1". Note that the space of "1 $+2-3$ OR 3-1" shown in section F is the union of the spaces represented in sections $D$ and $C$. In section $F$, the OR symbol separates the "1 + 2 - 3" portion from the " 3 - 1" portion. The solids and relationships of the first portion $(1+2-3)$ do not influence the solids and relationships of the second portion ( 3 1).

\section{REGION TABLE}

For every component of the target which is being modeled, a region card(s) defines the shape and space of the component as a single solid or combination of several solids. On the region cards, the solids are referred to by their unique solid number and the combination symbols " + , -, OR" are used. The set of region cards which defines the components of the target is called the "Region Table." Figure C-23 displays the input format for the region card(s); a printout of the input cards required for a sample region (111); and a printout of the region cards that comprise the Region Table for the sample target.

The regions in the Region Table, as the solids within the Solid Table, are numbered consecutively; the first region is numbered "1", the second number "2", the third numbered " 3 ", etc.

On Figure $\mathrm{C}-23$, the sample region is numbered "111", implying that it is the one hundred and eleventh region in some Region Table. Two region 
ORNL-DWG 90M-11861

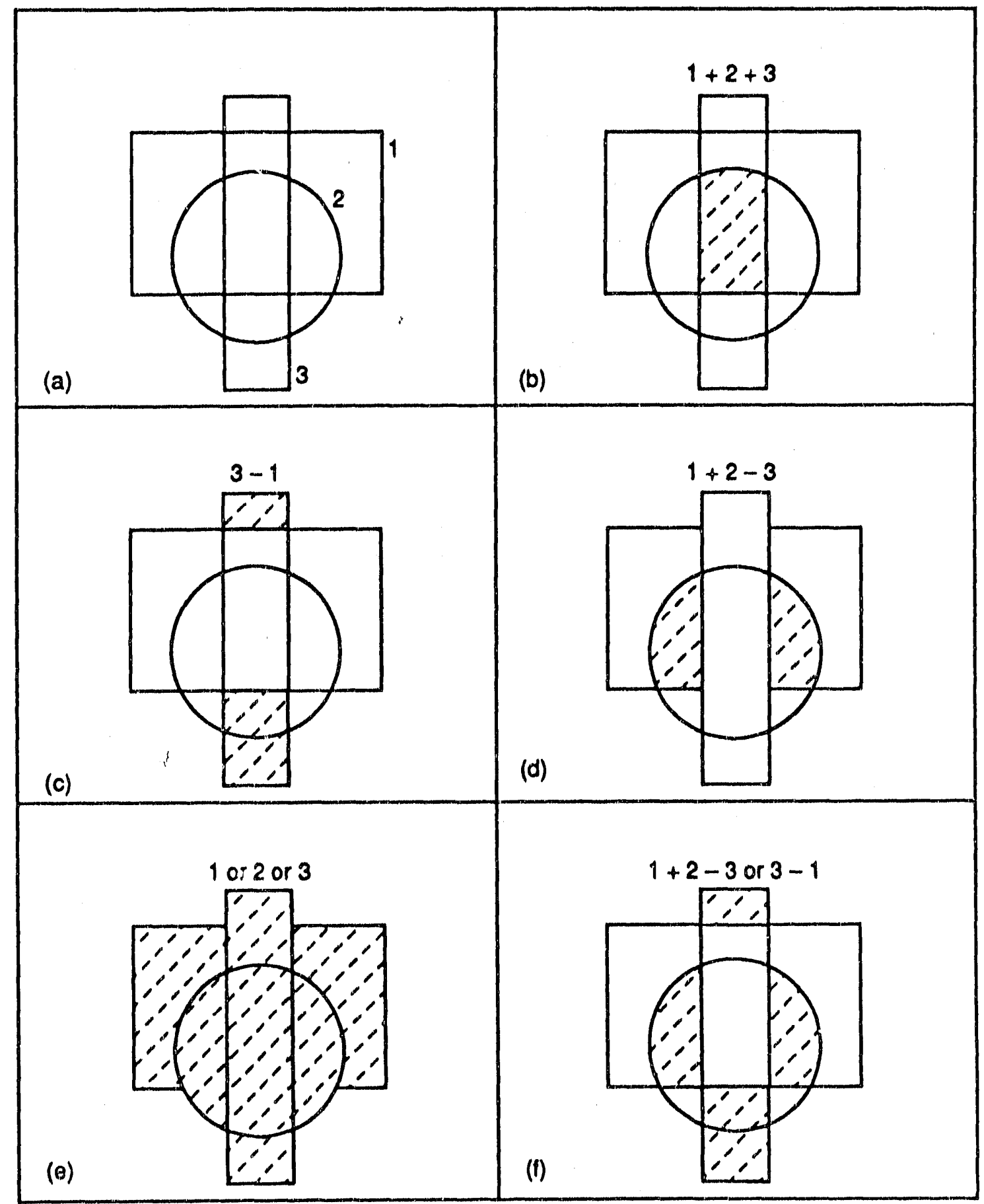

Figure C-22. Intersection, Subtraction, and Union Between Three Solids. 


\begin{tabular}{|l|l|c|c|c|c|c|c|c|c|c|c|c|}
\hline $1-5$ & $7-8$ & $9-13$ & $14-20$ & $21-27$ & $28-34$ & $35-41$ & $42-48$ & $49-55$ & $56-62$ & $63-64$ & $65-69$ & $70-80$ \\
\hline $\begin{array}{l}\text { Region } \\
\text { Number }\end{array}$ & OR & $\begin{array}{c}\text { (t) Solid } \\
\text { Number }\end{array}$ & $*$ & $*$ & $*$ & $*$ & $*$ & $*$ & $*$ & $*$ & $( \pm) \begin{array}{c}\text { Sol id } \\
\text { Number }\end{array}$ & Comments \\
\hline
\end{tabular}

Card Format: $(15,1 X, 9(A 2,15), A 1,2 A 5)$

Note: The first two card columns are reserved for OR if necessary, while the remaining five card columns are used for ( \pm ) Solid Numbers. For example: card columns 14 and 15 are for $O R$ if needed while columns 16-20 are used for $( \pm$ ) Solid Numbers.

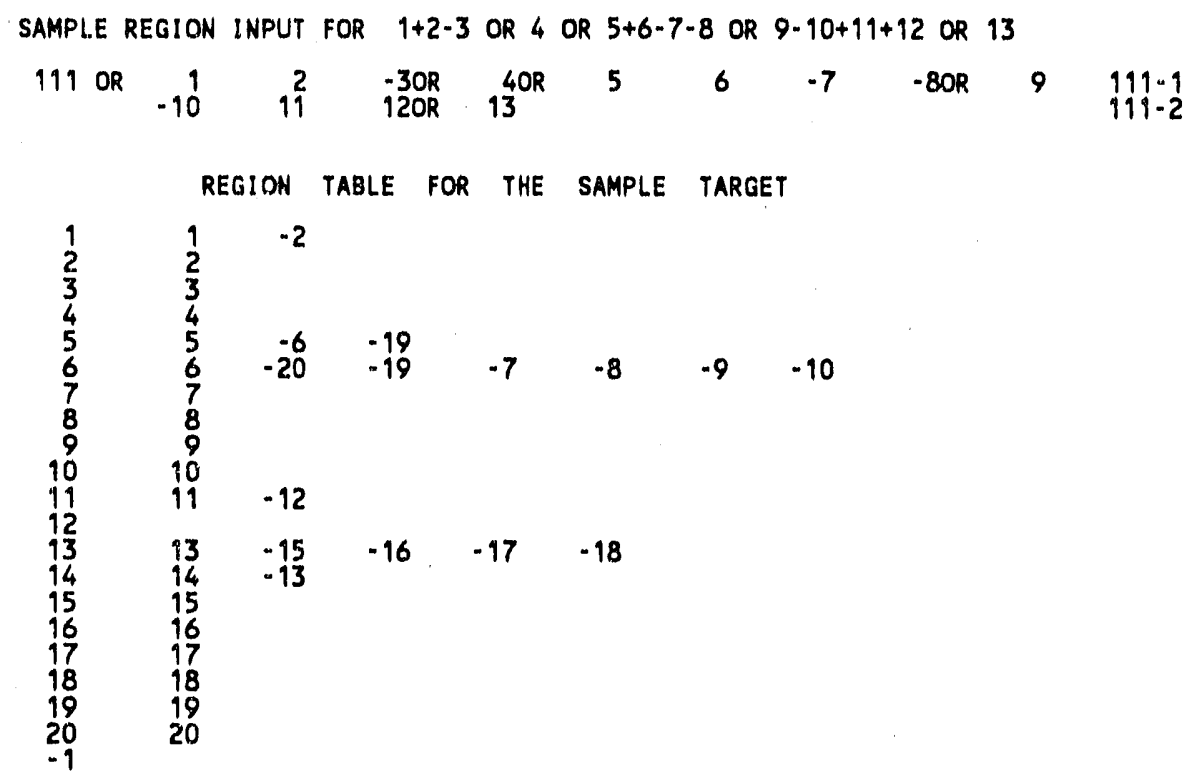

Figure C-23. Card Input for the Region Cards and the Region Table for the Sample Target.

cards are required to define our sample region " $1+2-3$ OR 4 OR $5+6$ - 7 - 8 OR $9-10+11+12$ OR 13". Any number of region cards may be required to define a region; however, only the first card contains the region number. Note that the region number "11" appears in card columns $3,4,5$ only on the first card.

Card columns 70-80 (comments) on the region card may include any comments, but including an identification of the card is recommended when more than one card is required to define a region. For example, the comments "111-1" and "111-2" in columns 70-80 on the sample region cards mean "111" first (1) and second (2) card, respectively. 
When the "OR" relationship is used in the definition of a region, "OR" must be punched in card columns 7-8 on the first region card. Note the "OR" after "111" on the first region card for the sample region. The sample region reads: "OR $1+2-3$ OR $4 \ldots$ "which is the same as "1 +2 - 3 OR $4 \ldots$ "; the introductory "OR" indicates to the GIFT5 geometry package that the "OR" relationship will be used in the description of the region.

On the region cards, the "+" symbol need not be punched. Reviewing the region cards for region 111 and the Region Table for the sample target, note that no " + " symbols are used. A non-punch or blank before a solid number implies the intersection (t) relationship.

There are twenty regions in the Region Tabie for the sample target, and the region numbers in the table are numbered consecutively (1 to 20). The last card (after region number "20" card) in the Region Table contains "-1" in card columns 4 and 5. The "-1 CARD", as it is called, is the flag or mark that indicates the end of the Region Table and must follow the Region Table.

\section{RECCIMENDED PROCEDURES FOR THE REGION TABLE}

The Solid Table for the sample target (Table $\mathrm{C}-2$ ) contains twenty solids and the Region Table contains twenty regions (Figure $(-23)$. The number of solids and the number of regions may be different; however, experience gained from modeling targets containing many solids has proven the usefulness of the following scheme. Note that region number "1" in the Region Table for the sample target begins with the solid number "1"; region number "2" begins with solid number "2"; region " 3 " with solid " 3 " ... region "20" with solid "20". Only region number "12" does not follow the pattern. In fact, region "12" sans any solid numbers is called a "dummy region" because no space is defined by the region. In a Region Table any number of "dummy regions" like region "12" may be used. A "dummy region" is a region card containing only a region number. Because the regions must be numbered consecutively, the purpose of region "12" is to maintain the pattern of region "13, 14, 15 ... 20" beginning with solid number " $13,14,15 \ldots 20 "$ ". When the region number is equal to the first solid number, it is easy to locate the components of the modeled target, to correct errors, and to modify the Solid and Region Tables. Using "dummy regions" and having the first solid and region number for non-dummy regions equal is recommended.

The Region Table for the sample target does not contain any "OR" or union relationships. The OR relationship between solids may be used but its usage is not recommended. (A later section of this appendix presents a technique which is logically equivalent to the "OR" relationship). 


\section{REGION IDENTIFICATION TABLE}

The Region Identification Table assigns an identification (code) number to each region in the Region Table. Table C-3 exhibits a grouping of identification numbers from 1 to 998 used to identify the components of military vehicles - tanks, trucks, etc. Numbers from 1 to 99 are assigned to the regions in the Region Table which represent "personnel and miscellaneous interior components"; numbers from 100 to 199 are assigned to the regions in the Region Table which represent "armor and vehicle structure components";...; numbers from 900 to 998 are used to identify the regions in the Region Table which represent components that are "ammunition."

Regions in the Region Table may also define spaces which represent air within and around a target or vehicle. For example, regions may define the air space where the crew members of a vehicle are (crew compartment air), the air space where the passengers are, and the air space surrounding the engine of the vehicle. Respectively, the identification numbers "02", "03" and "05" (shown in Table C-3) are assigned to these air spaces.

Figure C-24 presents the card input format and a printout of the Region Identification Table for the sample target. On the input cards, the region number is followed by either a component (item) or an air space identification (code) number; a region either models an item or it models an air space. In the Region Identification Table, regions 19 and 20 are identified as "02" air spaces, while region numbers 1 to 18 have item numbers. For example, Region 1 has been identified by component number "40", and represents the "steering wheel" as the comments on the region identification card indicate. The "1-2", also contained in the comment section of this card, is the Region Table description of region 1 where solid 1 is a "TOR". (The verbal and region description, and the solid type are contained in the comment section of the region identification cards for the other regions.) Comments should verbally describe and define the component or air space that the region models.

The user is not required to define any air spaces in the Region and Region Identification Tables. Air spaces are defined if the user wants to identify special air spaces within and around the target such as the crew and engine compartment air. Regions 19 and 20 of the sample target identify the air space (02) inside of the sample target (a car). Regions 19 and 20 were included to illustrate air space usage; otherwise, regions 19 and 20 may have been omitted from the Region and the Region Identification Tables. 
Table C-3. Identification Numbers Used for Vehicles and Air Regions within the Vehicles in the GIFT5 Geometry Package.

\begin{tabular}{cc}
$\begin{array}{c}\text { Identification } \\
\text { Number }\end{array}$ & $\begin{array}{c}\text { Components of the } \\
\text { Target Vehicle }\end{array}$ \\
\hline
\end{tabular}

1 TO 99 PERSONNEL AND MISCELLANEOUS INTERIOR COMPONENTS

100 TO 199 ARMOR AND VEHICLE STRUCTURE COMPONENTS

200 TO 299 FUEL STORAGE AND SUPPLY SYSTEM COMPONENTS

300 TO 399 MISCELLANEOUS EXTERIOR COMPONENTS

400 TO 499 ARMAMENT (NOT AMMO) SYSTEMS COMPONENTS

500 TO 599 TRACK SUSPENSION SYSTEM COMPONENTS

600 TO 699 WHEEL SUSPENSION SYSTEM COMPONENTS

700 TO 799 ENGINE, TRANSMISSION, AND THEIR POWER COMPONENTS

800 TO 899 ENVIRONMENT AND SAFETY COMPONENTS

900 TO 998 AMMUNITION

$\star 501$

$\star 111$

USE FOR TRACK ONLY. GIFTS GENERATES 502 FOR TRACK EDGE.

USE FOR DUMMY REGIONS

*999 NEVER USE. SPECIAL NUMBER FOR GIFT5 GEOMETRY PACKAGE

\begin{tabular}{cc}
\hline $\begin{array}{c}\text { Identification } \\
\text { Number }\end{array}$ & Components of the \\
\hline
\end{tabular}

$\begin{array}{ll}01 & \text { AIR IN GENERAL. GIFT5 ASSIGNS "OI" IN DESCRIPTION GAPS } \\ 02 & \text { CREW COMPARTMENT AIR FOR VEHICLES } \\ 03 & \text { PASSENGER COMPARTMENT AIR FOR VEHICLES } \\ 05 & \text { ENGINE COMPARTMENT AIR FOR VEHICLES } \\ 10 \text { TO } 98 & \text { MAY USE. NO SPECIAL USAGE DEFINED BY GIFT5 } \\ * 09 & \text { NEVER USE. SPECIAL NUMBER FOR GIFT5 GEOMETRY PACKAGE }\end{array}$

* SPECIAL USAGE IDENTIFICATION NUMBERS 
CARD COLUAHS

\begin{tabular}{|c|c|c|c|c|c|c|}
\hline $1-10$ & $6-10$ & $19-15$ & $16-20$ & $21-25$ & $26-30$ & $31-78$ \\
\hline $\begin{array}{l}\text { Region } \\
\text { Number }\end{array}$ & $\begin{array}{c}\text { Ittem Code } \\
\text { Number }\end{array}$ & $\begin{array}{c}\text { Air Space } \\
\text { Code Numbr }\end{array}$ & $\begin{array}{c}\text { Material } \\
\text { Code }\end{array}$ & $\begin{array}{c}\text { Percent } \\
\text { Density }\end{array}$ & & $\begin{array}{c}\text { Alphanumeric Degeription } \\
\text { of the Region }\end{array}$ \\
\hline
\end{tabular}

Card Format: $(515,5 X, A 48)$

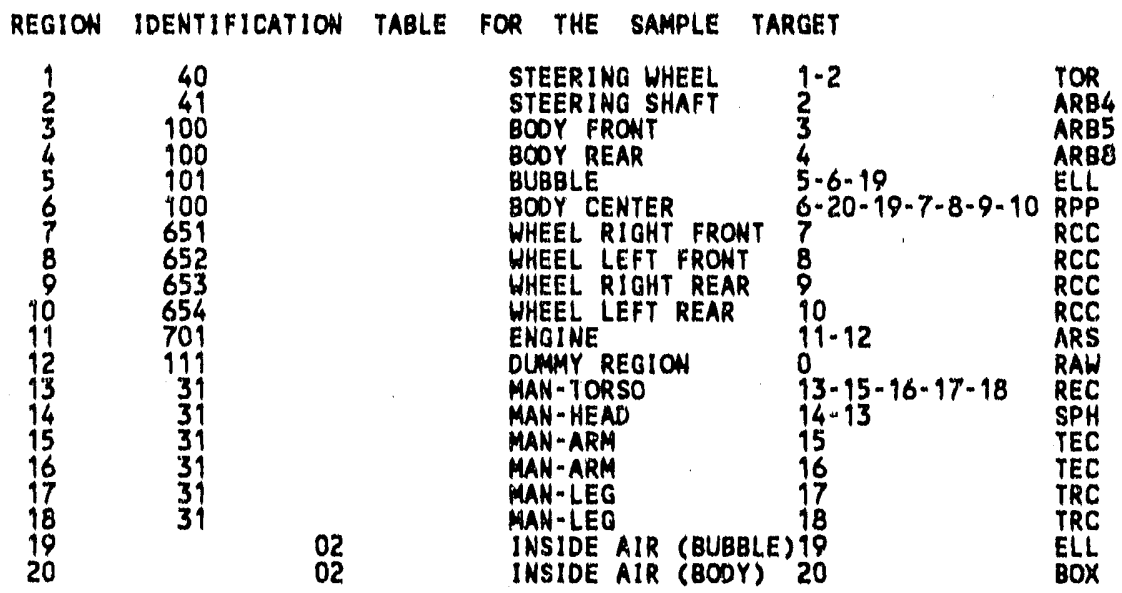

Figure C-24. Card Input for the Region Identification Table and a Region Identification Table for the Sample Target.

\section{SPECIAL REGION IDENTIFICATION NUMBERS}

In Table C-3 certain identification numbers are preceded by an asterisk $\left({ }^{*}\right)$; these identification numbers have specific meanings. Never use number "999" to identify a component and never use "09" to identify an air space.

The number "111" is used to identify the "dummy regions" in the Region Table. For example, for the sample target, region 12 is a dummy region; thus, it is identified by the special number "111". The last special identification number is "501"; this number is only used to identify regions that model the tracks of tracked vehicles. The "501" number is sometimes converted by the GIFT5 geometry package into "502", which indicates the edge of the track.

Users of the GIFT5 geometry package may use the identification system shown in Table C-3 or develop an original grouping scheme, but the usage of the special numbers $(501,111,999$ and 09 air) cannot change. 
In Figure $\mathrm{C}-24$, note that regions 13 to 18 have a common identification number (31), while regions 3,4 and 6 have a common identification number $(100)$. Regions with a common identification number are "combined" as the "OR" relationship combines solids. For example, because regions 13 to 18 are identified by the same code number (31), these regions are equivalent to the following single region: "13 - 15 16 - 17 - 18 OR 14 - 13 OR 15 OR 16 OR 17 OR $18 "$.

It is recommended that the user not use the "OR" relationship, but use common identification numbers to combine regions. There are several reasons why it is more desirable to use the same identification numbers for several simple regions rather than use the OR relationship to create one large region. The computer run-time is increased when the OR relationship is used. It is easier to locate the parts of a component when they are defined by several regions with the same identification number. For example, regions 13 to 18 define the different parts of the man or the driver of the sample vehicle. If a user wanted to place a helmet an the man's head, the man's head is quickly identified to be region "14". If regions 13 to 18 were grouped into a single region by using the $O R$ relationship, it would be harder to locate the head of the man. A user may be required to identify the different parts of the man such as his head, legs, arms, and torso. The users would only have to assign unique identification numbers to regions 13 to 18 . If the man was a single region using the OR relationship, the user would be required to identify the parts of the man and then create new regions and region identification cards. The problems associated with the use of the OR relationship expand as the complexity of the target increases; therefore, it is recommended that the OR relationship not be used.

\section{RULES FOR REGIONS}

The Region Identification Table defines each region in the Region Table as either a component or an air space. Rules exist for regions that model components or items, while different rules apply for regions that define air spaces. Figure $\mathrm{C}-25$ provides illustrations for the rules of the regions.

Section A of Figure $C-25$ exhibits two regions: $R 1$ is a $B O X$ and $R 2$ is a SPH. The SPH and BOX are shown to overlap: the area of overlap contains dashed (/) lines. The three-dimensional SPH and BOX solids are represented on a two-dimensional plane; thus it is assumed that in depth they also overlap. If $R 1$ is defined to be the space of the $B O X$, while R2 is the space of the SPH in a Region Table, and if R1 and R2 were identified in the Region Identification Table as components or items, then the only rule for regions that model components is violated REGIONS THAT MODEL COMPONENTS CANNOT OVERLAP. In the physical world 
ORNL-DWG 9OM-11862

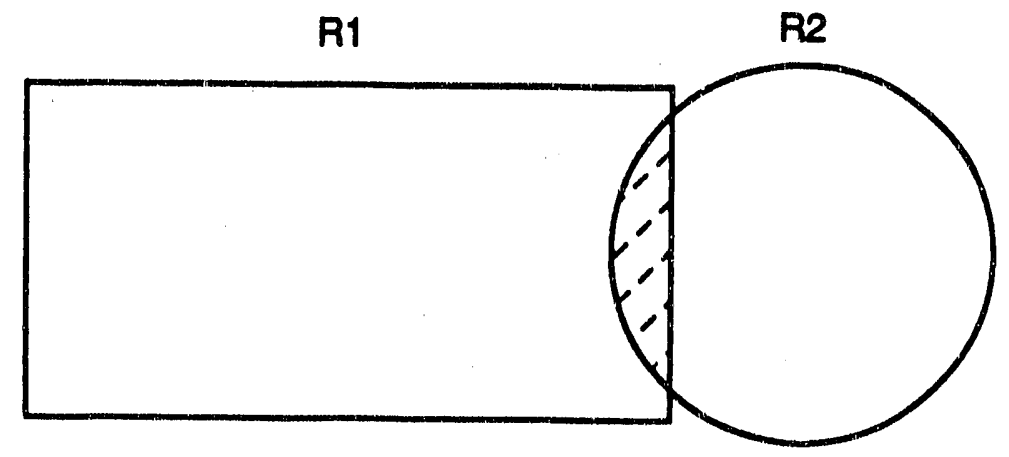

SECTION A

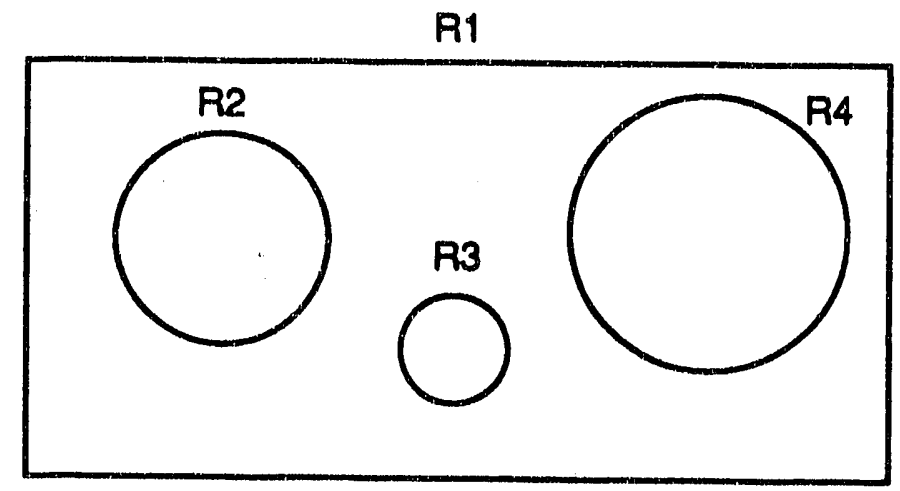

SECTION B

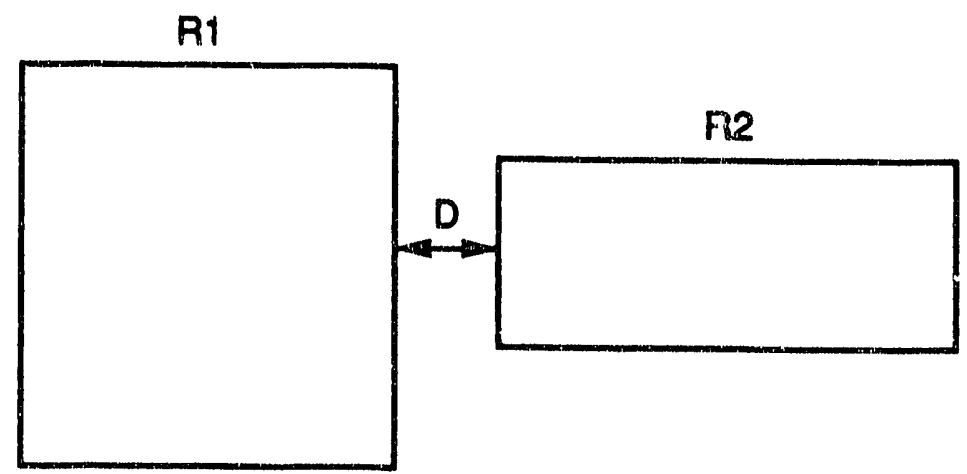

SECTIONC

Figure C-25. Illustrations for Region Rules. 
components cannot overlap or share a common space; thus, R1 and R2 cannot overlap if they model physical components. Regions that model components and overlap indicate errors either in the Solid Table data or in the Region Table data.

If R1 is the space of the BOX while R2 is the space of the SPH, and if R1 and R2 are identified as items, then they must be correctly defined in the Solid Table to assure that they do not overlap. It may be impossible to measure and record the $\mathrm{BOX}, \mathrm{SPH}$, or any solid parameters accurately enough to assure that the solids do not overlap. If the overlap is smal1, the user can modify the model of the regions: R1 can be redefined to be the $B O X$ minus (subtract or less) $(-)$ the space of the $S P H$, removing the space of the SPH that overlaps the BOX from the model of the BOX; or $\mathrm{R} 2$ can be modified in a similar manner to remove the overlap space of the BOX from the SPH. Solids can overlap, but regions identified as components cannot. In the next section of this appendix, it will be explained how the user can specify the amount of overlap between solids to be ignored by the GIFT5 geometry package.

Section B of Figure C-25, displays four regions: $R 1$ is a BOX while R2, R3 and R4 are SPH's. The rules for regions identified in the Region Identification Table as air spaces are different from the rules of the regions identified as components. If $\mathrm{Rl}$ is identified in the Region Identification Table as an air space while R2, R3 and R4 are identified as components, then region RI can be defined as the space of the BOX. REGIONS IDENTIFIED AS AIR SPACES CAN OVERLAP ANY REGIONS IDENTIFIED AS COMPONENTS. Imagine $R I$ as a BOX that defines the air in a rectangular room, while R2, R3, R4 are components within the room. The BOX or R1 would define the space of the air in the room and R2, R3, R4, would be components within the air space (R1) of the room. If $R 1, R 2, R 3$, and $R 4$ were all identified in the Region Identification Table as components, then to avoid overlap, Rl would have to be defined as the space of the BOX less or subtracting $(-)$ the solids that define the space occupied by regions $R 2$, $R 3$, and $R 4$.

Examining the Solid Table, the Region Table and the Region Identification Table for the sample target, note that both regions 19 and 20 are identified as 02 air space and the spaces defined by regions 19 and 20 overlap each other. REGIONS IDENTIFIED AS AIF. SPACES CAN OVERLAP REGIONS WITH THE SAME AIRSPACE IDENTIFICATION CODE NUMBER.

REGIONS WITH AIRSPACE CODE NUMBERS CANNOT OVERLAP REGIONS WITH DIFFERENT AIR SPACE CODE NUMBERS. If region 19 of the sample target was identified in the Region Identification Table with any air space code rumber other than 02 , and region 20 retained its 02 code number, then regions 19 and 20 could not overlap. 


\section{GIFT5 GEOMETRY PACKAGE MEMORY REQUIREMENTS AND REGION TOLERANCES}

The GIFT5 geometry package is not flexibly dimensioned. As such, changes have to be made to the main program to accommodate large target descriptions or different tolerance values for the target description. There are two specific lines within the GIFT5 geometry package that specify the memory size, and two specific lines that give the default values for region tolerances for the target description data.

Lines "COMMON ASTER (5000)" and "NDQ=5000" specify the amount (5000) of words of memory storage reserved for the target description data:

Solid, Region, Region Identification Tables, and other geometric data that are stored in the computer's core memory. The memory size required for a given target description is difficult to compute because a large number of factors must be considered. A crude estimate of the amount of memory words of storage required is 45 times the number of solids in the target description data.

The "5000" memory words indicated on the printout is large enough for the sample target; however, the "5000" words would not be large enough for a target description containing hundred of solids and regions. If a target description has 1000 solids, then 45 times the number of solids $(1000)$, or 45,000 words, is a crude estimate of memory requirements. To run the 1000 solid target description, the user must change the "COMMON ASTER (5000)" I ine to "COMMON ASTER (45000)" and the "NDQ=5000" 1 ine to "ND=45000". If 45,000 words is insufficient, then one of the following statements will be outputted depending upon what data was being stored when the memory was exceeded: 1) NO MORE ROOM FOR SOLID DATA, 2) NO MORE ROOM FOR REGION DATA, 3) NO ROOM FOR IDENTIFICATION TABLE, 4) NO ROOM FOR WORKING STORAGE. Working storage is the memory required to perform the calculations of the GIFT5 geometry package. If one of the above statements is output, then the numeric value of the "COMMON ASTER" and "NDQ=" lines must be enlarged to store the target description data.

Section $C$ of Figure $C-25$ illustrates two regions, $R 1$ and $R 2$, and $a$ distance "D" between the two regions. Suppose that $D$ is a small distance between R1 and R2 that occurred because the input parameters for the solids which define R1 and R2 could not be measured and recorded accurately enough. The GIFT5 geometry package allows the users to specify the gap distance "D" between item and air regions to be ignored or tolerated. TOL is the overlap tolerance, while TOLLOS is the gap tolerance. The value of 0.0001 is one ten-thousandth of the unit of measurement (inches, centimeters, etc.) used in the target description model. For most target descriptions, the "TOL $=0.0001 "$ and TOLLOS $=0.0001$ " 1 ines are replaced by "TOL $=0.01$ " and "TOLLOS $=0.01$ " or one hundredth of the unit of measure is the tolerance used. The user can decide and set the numeric values of TOL and TOLLOS to be used with his target description data. 


\section{REGION RPP TABLE}

An RPP solid (see Figure $C-15$ ) can be defined to enclose or contain the space or volume of any region. The minimum and maximum $X, Y$, and $Z$ coordinate values (XMIN, XMAX, YMIN, YMAX, ZMIN and ZMAX) of the RPP solid that encloses a region may be interpreted as follows: The region is located between XMIN and XMAX, is between YMIN and YMAX, and is between ZMIN and ZMAX. Figure C-26 presents the card input format and a printout of the Region RPP Table for the sample target.

The first card of the Region RPP Table for the sample target states that region number " 1 " is enclosed by an RPP with XMIN=20.0, XMAX $=23.0$, $Y M I N=-10.0$, $Y M A X=10.0, Z M I N=28.0$, and $Z M A X=46.0$. Note that the sample table does not include a card input for region "12" because region "12" is a "dummy region." Region " 2 " modeling the steering shaft or any region may be omitted from the Region RPP Table. In fact, no Region RPP Table is required because the GIFT5 geometry package computes a set of enclosing RPP values (XMIN, XMAX, YMIN ... ZMAX) for any region in the Region Table not included in the Region RPP Table.

The option to input enclosing RPP values for some or every region is provided because the GIFT5 geometry package may not compute a

"desirable" set of RPP values. For example, an RPP with XMIN, YMIN, and ZMIN values of -200.0 and XMAX, YMAX, ZMAX values of 200.0 would enclose every region of the sample target; however, the region RPP values on Figure C-26 define smaller volumes or spaces and are "better fitting" enclosing RPP's and thus more desirable RPP values. For most regions, the GIFT5 geometry package computes a good enclosing RPP; however, the computed enclosing RPP values for a few regions can be improved. The smaller the volume or the better fit of the RPP that encloses a region, the shorter the computer run time for the GIFT5 geometry package.

\section{THE TITLE AND TARGET SPECIFICATION CARDS}

To complete the target description, two additional cards are required the Title Card and the Target Specification Card. Figure C-27 exhibits the card input for these cards.

The Title Card contains the name of the target, the date the model was prepared, the units of measure used in the model, and any other important information on the modeled target. Oniy the first 65 card columns of the Title Card are read by the GIFT5 geometry package; however, card columns 66 to 80 may contain additional information on the target.

The Target Specification Card contains the number of solids and the number of regions used to model the target. 
CARD COLUMNS

\begin{tabular}{|c|c|c|c|c|c|c|c|}
\hline $1-10$ & $11-20$ & $21-30$ & $31-40$ & $41-50$ & $51-60$ & $61-70$ & $\begin{array}{c}\text { Number } \\
\text { of Cards }\end{array}$ \\
\hline $\begin{array}{l}\text { Region } \\
\text { Number }\end{array}$ & $X \min$ & $Y_{\min }$ & $Z \min$ & $X \max$ & $Y_{\max }$ & $Z \max$ & 1 of 1 \\
\hline
\end{tabular}

Card Format: (I10, 6F10.0)

REGION RPP TABLE FOR THE SAMPLE TARGET

$\begin{array}{rrrrrrr}1 & 20.0 & 23.0 & -10.0 & 10.0 & 28.0 & 46.0 \\ 2 & 21.0 & 40.0 & -6.0 & 6.0 & 33.0 & 44.0 \\ 3 & 74.0 & 101.0 & -36.0 & 36.0 & 12.0 & 48.0 \\ 4 & -101.0 & -74.0 & -36.0 & 36.0 & 12.0 & 48.0 \\ 5 & -25.5 & 25.5 & -15.5 & 15.5 & 45.0 & 63.0 \\ 6 & -75.0 & 75.0 & -36.0 & 36.0 & 12.0 & 48.0 \\ 7 & 47.0 & 72.5 & -36.0 & -28.0 & 0.0 & 24.0 \\ 8 & 47.0 & 72.5 & 28.0 & 36.0 & 0.0 & 24.0 \\ 9 & -72.5 & -47.0 & -36.0 & -28.0 & 0.0 & 24.0 \\ 10 & -72.5 & -47.0 & 28.0 & 36.0 & 0.0 & 24.0 \\ 11 & -70.0 & -30.0 & -20.0 & 20.0 & 15.0 & 45.0 \\ 13 & -5.0 & 5.0 & -7.5 & 7.5 & 24.0 & 52.0 \\ 14 & -5.5 & 5.5 & -5.5 & 5.5 & 52.0 & 57.0 \\ 15 & 0.0 & 20.0 & -10.0 & -5.5 & 35.0 & 52.5 \\ 16 & 0.0 & 20.0 & 5.0 & 10.0 & 35.0 & 52.5 \\ 17 & -3.5 & 31.0 & -8.0 & -1.0 & 12.0 & 30.0 \\ 18 & -3.5 & 31.0 & 1.0 & 8.0 & 12.0 & 30.0 \\ 19 & -24.5 & 24.5 & -15.0 & 15.0 & 34.0 & 62.0 \\ 20 & -75.0 & 75.0 & -35.0 & 35.0 & 13.0 & 47.0\end{array}$

Figure C-26. Card Input for the Region RPP Table and the Region RPP Table for the Sample Target. 
TITLE CARD

CARD COLUMNS

\begin{tabular}{|c|c|c|}
\hline $1-2$ & $3-5$ & $6-65$ \\
\hline $\begin{array}{c}\text { Target } \\
\text { Units }\end{array}$ & & $\begin{array}{c}\text { Alphanumeric Description of the target: } \\
\text { including title, date prepared, etc. }\end{array}$ \\
\hline
\end{tabular}

Card Format: (A2, 3X, A60)

TARGET SPECIFICATION CARD

CARD COLUMNS

\begin{tabular}{|c|c|}
\hline $1-5$ & $6-10$ \\
\hline $\begin{array}{c}\text { Number of Solids in } \\
\text { the Solid Table }\end{array}$ & $\begin{array}{c}\text { Number of Regions in } \\
\text { the Region Table }\end{array}$ \\
\hline
\end{tabular}

Card Format: (2I5)

Figure C-27. Card Input for the Title Card and Target Specification Card. 
The order of the card input for the target description data is as follows. The first card is the Title card, followed by the Target Specification card. Next comes the Solid Table - as required by the target description. Be sure the number of solids on the Target Specification card match the number of solids in the Solid Table. Next comes the Region Table - as required by the target description. Again, make sure the number of regions on the Target Specification card match the number of regions in the Region Table. The Region Table is followed by the Region RPP Table. This Table is not required and usually not input. Finally, the Region RPP Table (or Region Table if the Region RPP Table is omitted) is followed by the Region Identification Table. Note that the Region Table is followed by the "-1 Card" while a blank card follows both the Region RPP and the Region Identification Tables. If no Region RPP cards are used, the blank card indicating the end of the Region RPP Table will follow the "-1 Card".

\section{REFERENCES}

1. W. A. Rhoades, "Development of a Code System for Determining Radiation Protection of Armored Vehicles (The VCS Code), " ORNLTM-4664, Oak Ridge National Laboratory, (October 1974).

2. W. A. Rhoades, et.a1., "Vehicle Code System (VCS) User's Manual," ORNL-TM-4648, Oak Ridge National Laboratory, (August 1974).

3. Lawrence W. Bain, Jr. and Mathew J. Reisinger, "The GIFT Code User Manual; Volume I. Introduction and Input Requirements," BRL 1802, Ballistic Research Laboratory, (JuTy 1975).

4. Gary G. Kueh7, Lawrence W. Bain, Jr. and Mathew J. Reisinger, "The GIFT Code User Manual; Volume II. The Output Options," ARBRLTR-02189, Ballistic Research Laboratory, (September 1979).

5. A. E. Rainis and Ralph E. Rexroad, "MIFT: GIFT Combinatorial Geometry Input to VCS Code," BRL. 1967, Ballistic Research Laboratory, (March 1977).

6. J. A. Stoddard, S. D. Egbert, and W. D. Scott, Jr., "The Vehicle Code System with In-Group Energy Bias and GIFT5 Geometry," DNATR-87-23, Science Applications International Corporation, (January 1987). 
APPENDIX D

MASH 1.0 CODE SYSTEM ABSTRACT 
MASH 1.0 CODE SYSTEM ABSTRACT

\section{NAME AND TITLE}

MASH 1.0 - A Monte Carlo Adjoint Shielding Code Syster.1

\section{COMPUTER FOR WHICH PROGRAM IS DESIGNED}

MASH was originally developed on a Cray X-MP, but it ha', al so been checked out on Cray Y-MP and Cray 2 computers as well as an IBM RISC System 6000 workstation. Since it can operate without machine-dependent coding or explicit subroutine library calls, it should be operable on other computers with sufficient memory and disk storage.

\section{NATURE OF PROBLEM SOLVED}

MASH calculates neutron and gamma-ray environments and radiation protection factors for armored military vehicles, structures, trenches, and other shielding configurations. The principal application is to determine the radiation shielding characteristics of armored vehicles from prompt radiation due to a nuclear weapon detonation. The shielding effectiveness can be characterized for both personnel and electronic equipment as a function of weapon detonation height, source/target ground range, and vehicle orientation and configuration.

\section{METHOD OF SOLUTION}

The discrete ordinates calculation determines the fluence on a coupling surface surrounding the shielding geometry due to an external neutron/gamma-ray source. The Monte Carlo calculation determines the effectiveness of the fluence at that surface in causing a response in a detector within the shielding geornetry, i.e., the "dose importance" of the coupling surface fluence. A coupling code folds the fluence together with the dose importance, giving the desired dose response. The coupling code can determine the dose response as a function of the shielding geometry orientation relative to the source, distance from the source, and energy response of the detector.

The code system includes the GRTUNCL and DORT codes for air-nver-ground transport calculations, the MORSE code with the GIFT5 combinatorial geometry package for adjoint shielding calculations, and several peripheral codes that perform the required data preparations, transformations, and coupling functions. MASH is the successor to the Vehicle Code System (VCS) initially developed at Oak Ridge National Laboratory (ORNL). 


\section{RESTRICTIONS OR LIMITATIONS}

MASH is a modular code system made up of several codes, each with their own set of restrictions and limitations. Most of the modules utilize dynamic dimensioning, however, MORSE and DRC use a large container array in Blank Common to accommodate this. Typically, machine size and disk storage space will provide the overall limit.

\section{TYPICAL RUNNING TIME}

Since a typical MASH problem involves running a sequence of codes, quantifying total running time is difficult. The running time varies depending on the complexity of the probiem. The two modules in MASH requiring the bulk of the running time are DORT and MORSE. Typical DORT run times for a 400 meter by 800 meter air-over-ground calculation with 240 angles and 69 energy groups is approximately 3.5 hours CPU on a Cray. A typical adjoint MORSE analysis of a vehicle with approximately 300 bodies and 300 regions requires approximately 2 hours of cray time to obtain adequate statistics on the integral results.

\section{RELATED AND AUXILIARY PROGRAMS}

MASH is a modular code system made up of a collection of programs. These codes include:

\begin{tabular}{|c|c|}
\hline GIP & Cross-section input preparation \\
\hline GRTUNCL & Analytic first collision source code \\
\hline DORT & Two dimensional discrete ordinates transport code \\
\hline VISTA & $\begin{array}{l}\text { Vehicle input sourse transformation and assembly } \\
\text { code }\end{array}$ \\
\hline MORSE & $\begin{array}{l}\text { A general purpose Monte Carlo multigroup neutron } \\
\text { and gamma-ray transport code system }\end{array}$ \\
\hline GIFT & A combinatorial geometry code system \\
\hline DRC & A detector response code \\
\hline
\end{tabular}

\section{STATUS}

MASH Version 1.0 available on Cray CTSS - January 1992

Successful solution of several test problems on the IBM RISC System/6000 workstation was performed by ORNL in November-December 1991. This version will be released at a future date once testing is complete. 


\section{REFERENCES}

1. J. 0. Johnson, editor, "A User's Manual for MASH 1.0 - A Monte Car10 Adjoint Shielding Code System," ORNL/TM-11778, Oak Ridge National Laboratory, (March 1992).

2. W. A. Rhoades and R. L. Childs, "The DORT Two-Dimenstonal

Discrete- Ordinates Transport Code," Nuclear Science \& Engineering 99, 1, pp. 88-89, (May 1988).

3. M. B. Emmett, "The MORSE Monte Carlo Radiatton Transport Code System." ORNL-4972 (February 1975); ORNL-4972/R1 (February 1983); ORNL 4972/R2, Oak Ridge National Laboratory, (July 1984).

4. Lawrence W. Bain, Jr. and Mathew J. Reisinger, "The GIFT Code User Manual; Volume I. Introduction and Input Requirements," BRL 1802, Ballistic Research Laboratory, (July 1975).

\section{COMPUTER HARDWARE REQUIREMENTS}

MASH is a modular code system made up of several codes, each with their own set of computer hardware requitrements. All of the modules are designed to be applicable to most full-scale computers that support direct (random) access disk storage or the equivalent. Machinedependent features such as calls to system-dependent subroutines are restricted to interchangeable interface packages. The code originators maintain a configuration for the cray computer only.

\section{COMPUTER SOFTWARE REQUIREMENTS}

MASH is a modular code system made up of several codes, each with their own set of computer software requirements. The code system can be operated with 100\% FORTRAN language on Cray computers. All of the modules are designed to use the Cray CFT 1.14 and CFT77 Version 3.0 compilers currently in use, but any FORTRAN 77 compller should suffice. External data storage must be provided for up to 9 scratch files, of which 5 must be direct (random) access. User-supplied input and output data files must be supplied on sequential-access devices, i.e., tapes or the equivalent. Library calls are used for job timing, etc., but they can be deleted.

\section{OPERATING SYSTEM}

MASH currently only operates under the Cray CTSS operating system using the standard CFTLIB and FORTLIB 1ibraries. Since the code system is all fortran, operation under the UNICOS operating system should be possible with littie corversion effort. 


\section{CONTRIBUTORS}

MASH was created by:

M. B. Emmett, W. A. Rhoades, R. L. Chllds, and J. O. Johnson Oak Ridge National Laboratory

W. H. Scott, Jr., J. A. Stoddard, D. C. KauT, and S. D. Egbert Sctence Applifations Internattonal Corpuration

Note: There are numerous other contributors to the various codes which make up the MASH code systell. They are recognized in the literature assoctated with each individual code.

\section{POINT OF CONTACT}

R. T. Santoro, Group Leader Engineering Physics and Mathematics Division Oak Ridge National Laboratory

P. 0. Box 2.008, MS-6363

Oak Ridge, Tennessee 37831-6363

\section{CONTENTS OF CODE PACKAGE}

- Source Programs

- User's Manual

16. DATE OF ABSTRACT

March 1992

\section{KEYWORDS}

TRANSPORT, NEUTRONICS, SHIELDING, DISCRETE ORDINATES, MONTE CARLO, CRAY

\section{SPONSOR}

MASH development was sponsored by:

Defense Nuclear Agency, U. S. Department of Defense 
(This page is intentionally left blank.) 
APPENDIX E USER NOTES 


\section{USER NOTES}

\section{INTRODUCTION}

In order to assess the quality of the computational shielding efforts at ORNL, it is first necessary to explore the philosophies used by ORNL to solve problems of the class under consideration. The solution of the air-over-ground problem using the discrete ordinates method requires expert and experienced code users. The same can be said for the solution of the adjoint Monte Carlo formulation for the dose components leaving a complicaced shield geometry.

In this appendix, a series of questions and answers are given in regards to the application of the MASH code system to a particular problem. For illustrative purposes only, the problem alluded to in the discussion is the sample problem included in this manual. When references to the attached input listings are noted in the discussion, the reader should consult that section of this manual to see the example.

The questions come from a novice user of the MASH code system with a background in Nuclear Engineering and a basic understanding of the physics and underlying principles of the methodology. The responses to the questions are those of the editor of this manual only. They are not meant to represent the philosophy of ORNL as a whole. They are simply based on his experience in using the MASH code system.

The purpose of the following questions is to qualify the "rules of thumb" being followed at ORNL, and to try to stimulate critical thought along the lines of discovering flaws or weaknesses in any part of the methodology being followed.

\section{QUESTIONS AND ANSWERS ON AIR-OVER-GROUND ENVIRONMENT CALCULATIONS WITH GRTUNCL/DORT}

1. Q: What library of cross sections is currently being used? What weighting function was chosen for preparing this library? Why was it chosen? Do you have any comments or concerns about the quality of the library? Do you suspect any particil?ar data to be weak?

A: The library currently being utilized in the MASH analys is is the reference DNA DABL69 69 group $(46 \mathrm{n} / 23 \gamma)$ cross-section library. This library has three weighting functions, only one of which was used for all materials. The standard weighting function is a smoothly varying 
function consisting of a $300 \mathrm{~K}$ Maxwellian, a $1.4 \mathrm{MeV}$ fission spectrum, and a $14.07 \mathrm{MeV}$ fusion peak spectrum overlaid on a $1 / E$ slowing-down spectrum. This is the weighting function I use for all my analysis. A fission source through infinite air spectrum was used to collapse additional sets of hydrogen, oxygen, and nitrogen data, and inverse of energy times the total cross section of type-304 stainiess steel spectrum, $1 /\left(E \Sigma_{T}\right)$, was used to collapse the major constituents of steel for use in deep penetration problems involving ferrous materials. Since the cross sections collapsed using the infinite air spectrum were extracted at a distance of 2000 meters, I did not use them for the APRF experiments being performed at 170 and 400 meters. Should I end up doing calculations at distances approaching 2000 meters, I would use these cross sections to mix my air material. Likewise, for most of the armored vehicle analysis (to date), the armor has not been sufficiently thick enough to consider the problem a "deep penetration" problem. Therefore, I use the standard weighted materials for my steel components. Additional information on the details of the library can be obtained from:

D. T. Ingersol1, R. W. Roussin, C. Y. Fu, and J. E. White, "DABL69: A Broad-Group Neutron/Photon Cross-Section Library for Defense Nuclear Applications," ORNL/TM-10568, Oak Ridge National Laboratory, (June 1989).

2. Q: What is your philosophy for choosing radial mesh spaces from the source? How far beyond the target vehicle do you think the calculation should go? Have you checked this out? Do you increase the mesh detail in the vicinity of the target? If so, how? If not, why not? What is your philosophy for choosing axial mesh spaces from lower ground body? How high do you think the calculation should go? Have you checked this out? Do you have a mesh size transition between the ground and the air? Do you increase the mesh detail in the vicinity of the target? If so, how? If not, why not? Please list example data.

A: My current philosophy for choosing radial and axial mesh utilizes a conservative variant of the "square-root of two" rule. In discrete ordinates as you know, you should not let your fluence change by more than a factor of two between mesh cells. Since in air transport, the dominant component of fluence attenuation is geometric attenuation $\left(1 / R^{2}\right)$, you can set your mesh by choosing an initial cell size for the source, and multiplying this cell by the square root of two (i.e., 1.414) to obtain the boundary for the next cell. I use a slightly conservative approach and use 1.3 as my factor. I allow the mesh cell size to increase until it reaches a maximum size of 20 to 30 meters. For radial mesh, this procedure only has to be performed once (for a centrally located point source). For axial mesh, this procedure has to be performed twice (above and below the source location) for a point source. Furthermore, tighter mesh are used at the air/ground interface. I gradually increase the ground axial mesh spacing from a starting value of $0.5 \mathrm{~cm}$ until I reach a maximum size of approximately 5 
$\mathrm{cm}$. I usually extend approximately twice the distance from the source to the target (i.e., 400 meters beyond the 400 meter test site for a total distance from source of 800 meters), with a minimum distance beyond the target of approximately 300 meters. This distance is beyond the longest mean free path in air and more than sufficient. Axially, I usually make the dimension equal to the radial dimension. Consequently, for an 800 meter radial distance, I have an 800 meter axial distance. This will incorporate all the skyshine contribution to the dose at the 400 meter test site. I have run cases with only a 400 meter height since the APRF source is at approximately 16 meters. The difference between using 400 and 800 meter source heights was less than $2 \%$ due to the contribution from low energy gammas not accounted for in the smaller axial case. Likewise, additional cases using more mesh beyond the target showed insignificant changes relative to the currently used 400 meter case. Near the target/experiment positions, I "usually gradually decrease the radial mesh cell until the cell containing the target is only approximately 10 meters wide in the radial direction. The fluence does not change that significantly over the 10 meter interval (at 400 meters) and consequently the single mesh point folding algorithm in DRC will not cause a significant perturbation. In the axial direction, I usually place the first few air intervals above the ground at approximately 1 meter intervals until I reach a few meters above the top of the vehicle or intersect the 1.3 rule coming from the source. If the 1.3 rule does not become a factor, I will gradually increase the axial mesh until it blends in smoothly with that mesh generated with the 1.3 rule. A sample GRTUNCL and DORT listing is attached for the analys is of the Two-meter box at APRF.

3. Q: Which fluence solution option do you use, diamond-difference with negative fluence fix-up, or others? Why? Do you check the solution against any of the other options?

A: I use the $\theta$-weighted fluence solution in DORT. According to $W$. A. Rhoades, this solution has shown the least sensitivity to irregular mesh problems and the most stability. It does not guarantee the fluence solution regardless of mesh, but appears to allow a little more freedom. This has been the only fluence solution option I have used. J. V. Pace, III has used zero-weighted in the past, but I do not know if he did any comparison studies.

4. Q: What angular quadrature set do you use? Who prepared it? What philosophy was used in preparing it?

A: The current angular quadrature set being used is a 240 angle set, derived from a symmetric $S_{8}$ quadrature set, by subdividing each angle into five angles in each of the polar directions. This still yields a symmetric quadrature set, except now there are more polar angles to reduce quadrature streaming effects (ray effects) in the secondary gamma ray component. I believe the originator of this 
quadrature set is J. V. Pace and it came out of his extensive air-overground work on the Hiroshima-Nagasaki dose re-assessment program.

5. Q: What is your philosophy concerning the allowable aspect ratios of the mesh spaces in the radial to axial directions? Do you have any concerns about the accuracy of the diamond-difference average when the aspect ratio is large? If the mesh is variable; do you have any concerns about cumulative truncation errors? Have you ever checked this problem out? Please list some of the aspect ratios used in the vicinity of the target.

A: I have not really developed a philosophy concerning the aspect ratios of the mesh spaces in the axial and radial directions. Based on the rules discussed in regards to questions 2 and 3 , and the use of the $\theta$-weighted fluence option, I look for fluence profiles which appear reasonable for the problem and do not violate the principals governing discrete ordinates calculations. I realize the large radial mesh in the ground will cause the fluence profile to not conform, however, this aspect of the problem remains to be insignificant relative to the fluence profile in the air. I have relied extensively on the experience and expertise of J. V. Pace, III, and W. A. Rhoades since they have been performing air-over-ground analys is for many years and have probably discovered and encountered most of the pitfalls associated with this particular type of analysis through executing many of the perturbations you are enquiring about now. Unfortunately, much of this expertise is inside their head and has not been documented. I believe my worst aspect ratio in the air in the vicinity of the target is ten (radial interval of 10 meters by an axial interval of 1 meter).

6. Q: For purposes of coupling to MORSE, the sidewise and backwards directions are important to the determination of the indirect dose to the target. For the forward-biased quadrature set being used, is there sufficient detail in these other directions to adequately determine this dose component? Has this been checked?

A: The current quadrature set is symmetric and therefore yields the same treatment of the sideways and backward directions as it does the forward directions. I believe there is sufficient detail to model these directions with the quadrature set currently being used. In an unrelated study, I performed a 20 group simulated box problem using a symmetric $S_{2}, S_{4}, S_{6}, S_{8}$, and $S_{10}$ quadrature set. The results showed stability of the neutron dose for all quadrature sets above (and including) $S_{6}$. I did not investigate the stability of the gamma ray dose.

7. Q: At present, the DORT fluence at a single radial position is being used for the entire coupling surface, even though the coupling cylinder may be up to 20 meters in diameter. Are you satisfied with the accuracy of this procedure? Do you expect any significant differences 
to appear if the fluence is interpolated at its correct radial distance to the coupling surface? What about the current entering the top of the coupling surface?

A: As presently configured, DRC assumes the DORT fluence on the "coupling surface" is dependent on energy and elevation only, and not on azimuth. Consequently, DRC only uses the fluence at a single radius in the DORT mesh and does not use the radii encompassing the target. This assumption is valid for small objects at a great distance from the source. Since the size of most of the targets is small relative to the distance from the source, it is felt this assumption is valid for this analysis and would produce an uncertainty within the statistical deviations of the ralculated results. Utilizing the 10 meter radial mesh at the target location and a small air box surrounding the target produced differences less than $2 \%$ in a study on the two-meter box. If the target becomes significantly larger (e.g. a house cluster), you will have violated the "small target relative to the source distance assumption", and the single radial fluence will yield larger discrepancies. Modifying DRC to use multiple radial mesh coupling would be the only true way to assess the effect of the single radial mesh coupling approximation.

8. Q: Do you have any opinion as to the value of doing a several-day CRAY calculation, with much more spatial detail, to provide a benchmark for the normal DORT runs?

A: The best way to answer many of these questions (and probably introduce a few more) would be to set up and execute a large air-overground problem with a consistent axial and radial mesh and with reasonable aspect ratios approaching one. Include sufficient mesh above and beyond the source and target to insure all skyshine and backward directed fluence is accounted for, and possibly, increase the angular quadrature to test the current quadrature set. Re-assess a problem for which the current air-over-ground has been performed and compare the two calculations. Such a calculation could (and probably would) prove useful towards lending credibility to the current problem setup being used.

9. Q: Do you have any concerns about the effects of non-uniform, partially wet and bumpy terrain? If so, do you have any suggestions of how to simulate this effect? Comment similarly with respect to trees and vegetation.

A: Preliminary scoping calculations investigated the effects of the APRF topography out to the 400 meter test site, and the effects of ground moisture and meteorological data (temperature, pressure, and relative humidity) on the air environment during measurements made at APRF. Integral and spectral MASH results showed differences less than $2 \%$ for the topography model analysis. Consequently, a simple topography model of the APRF source-to- 400 meter test site was determined to be 
sufficient for the analysis. Partial wet conditions could not easily be simulated in the 2-D model. The mud puddles in close proximity to the target cause me a little concern (e.g. the free-field phantom was standing right behind and within 5 meters of a huge puddle of water). The only way of modeling the terrain, the trees and vegetation, and to some extent the puddles of water would be to go to a 3-D air-over-ground calculation. This could be performed with either TORT, MORSE, or possibly MCNP. Such an analysis would require a fairly accurate mapping of the environmental conditions at the time a particular measurement was made. Furthermore, standardization of such a model would be extremely difficult in view of changes in between experiments, especially the mud puddles. A better solution would be to backfill the low area where standing water is a problem, and look to standardize the rest of the problem.

\section{QUESTIONS AND ANSWERS ON ADJOINT MONTE CARLO CALCULATIONS WITH MORSE}

1. Q: Where did the geometry model come from that is currently being used? How many bodies, etc.? Has the model been checked for missing regions, holes, etc.? Were any found? What geometry package was used to process the model?

A: The two-meter box geometry was modeled in the MORSE component of the MASH code system using the GIFT geometry package. J. M. Barnes modeled the box by using the engineering blue prints supplied by APRF. The box required 49 bodies, and twenty four regions to model it precisely as the drawings specified. A simpler representation could have been modeled with less bodies and retained the same amount of detail. The geometry was checked by the GIFT debugging tool, converted to MORSECG input and checked with JUNEBUG, and analyzed with the new geometry package being created by T. J. Burns. Results of a preliminary study determined all the details of the box (i.e., lift tabs, drain holes, etc.) made insignificant contributions to the calculation and therefore were omitted in the final computational geometry model. The hatches were retained in the computational model for potential open hatch experiments performed in the future. The walls and roof of the box were comprised of two plates of steel each $5.08-\mathrm{cm}$ thick. This detail was retained in the geometry model for region dependent biasing in the Monte Carlo analysis.

2. Q: What is your philosophy for choosing the number of starters? How many were actually used? What was the number of secondaries created and followed? Have you checked any cases of a similar nature with considerably more starters?

A: The Monte Carlo (MORSE) calculation for the detector position generated and tracked 1,500,000 primary source particles (1500 batches 
of 1000 particles) sampled over the 69 energy groups. All starters were used, approximateiy one secondary particle was generated for each starter, and approximately one particle escaped (to be scored in DRC) for each starter. I do not believe any other cases were run with more starters than what I chose to run. My philosophy for choosing the number of starters depends to some extent on whether I am analyzing integral or spectral experimental data. For integral data, you can obtain a reasonable degree of convergence with much less histories than that required for spectral comparisons. In integral comparisons not all of the energy groups need to be converged; only those making a significant contribution to the total. For spectral comparisons, the majority of the energy groups should be converged (typically less than $5 \%)$ for confidence in the calculated spectrum. I have found that you can obtain statistics on your integral result less than $5 \%$, and have statistics on your differential results (group fluences) greater than $10 \%$. Care must be taken in this situation because you may analyze the same calculation, with the same number of starting particles, using a different starting random number, and obtain an entirely different integral result with a statistical uncertainty less than $5 \%$. In this case, even though your integral result is converged to with in $5 \%$, there is still too much deviation in the contributions from the individual groups. Consequently, your integral result is undersampled even though the fractional standard deviation ( $f s d$ ) says it is converged. One final point should be made about the fsd's printed in the MORSE outpuit. You can converge your MORSE calculation to within $1 \%$ for both your integral and differential data and still not obtain the correct answer. The final result regardless of the level of convergence is only as good as the accuracy of the MASH model.

3. Q: What philosophy do you use for source biasing? Why did you choose this? Please list example data. What exacit geometry was used for the source and where was it positioned?

A: I did not use source biasing in this calculation except for energy biasing discussed in question 5 below.

\section{Q: What source spectrum did you use? Why did you choose it?}

A: The source spectrum was an angle (240 angles) and energy dependent ( 69 groups, $46 n$ and $23 \gamma$ ) source spectrum supplied by SAIC as a result of their recent analysis of the APRF reactor leakage. The input is supplied in the attached GRTUNCL input iisting. Details about the source calculation can be obtained from:

D. C. Kaul and S. D. Egbert, "Radiation Leakage From The Army Pulse Radiation Facility (APRF) Fast Reactor," SAIC-89/1423, Science Applications International Corporation, San Diego, California, (May 1989). 
5. Q: Do you use energy biasing? If so, describe the philosophy used to determine which groups should be biased and by how much. Please list example data.

A: An energy dependent relative importance factor is utilized over the 69 groups to increase the frequency of sampling the adjoint source particle from energy groups which have a significant effect on the dose response function. In this case, the response function is the free-inair tissue dose response function. An example is given in the attached MORSE input stream. Obtaining a good source biasing function can be aided through the use of 1-D ANISN calculations which approximate the 3$D$ calculations as much as possible. The geometry for the source in the adjoint MORSE calculation is just a detector location (a point detector) located in the combinatorial geometry target description. The source in the DORT air-over-ground analys is was modeled as a small cylinder $(5 \mathrm{~cm}$ in height $x 5 \mathrm{~cm}$ in radius) positioned at $R=0.0$, and $Z=16.143$ meters.

6. Q: Do you use region blasing? If so, what philosophy do you use in picking the regions and region thicknesses? How do you set the bias factors and splitting ratios? Why? What weight cutoffs do you use for Russian Roulette? Why? What kill fractions do you use, and why? Please give examples.

A: The secondary particle production probability (GWLO) was set to 1.0 for al1 regions and energy groups in the Monte Carlo calculations, and the in-group energy biasing option in MORSE was switched on. Region dependent and energy independent splitting and Russian Roulette parameters were utilized in the steel to improve the efficiency of the Monte Carlo calculations. This was accomplished by subdividing the 10.16-cm-thickness of steel into two equally thick concentric regions and assigning each of the steel regions different splitting and Russian Roulette parameters which would allow a sufficient number of source particles (and secondary particles) to escape to obtain reasonable statistics. This allowed nominally one escaping particle for each source particle generated. The process is pretty much a trial and error approach. The region thicknesses to some extent were (and will be) set by the geometry data. You do not want to add additional regions to the geometry (unless the geometry is relatively simple) for the sole purpose of performing region dependent biasing. For complicated geometries, this task is impossible. You have to choose an initial kill-to-survival ratio (i.e. 10 to 1,5 to 1 , etc.) and then utilize the geometry data, source data, and energy biasing data to generate a biasing scheme. In MASH, the object is to obtain escaping particles in the energy groups which will make a significant contribution to the dose. Therefore, you want to set up your biasing scheme to maximize your leakages. This will require you to constantly change your biasing parameters to insure you get approximately the same number of leakages as you had startars. You must be careful not to over-bias the problem and create a totally unreal distribution. You also must be carefur that you do not end up with approximately the same number of leaking particles as source particles, but all in a very few groups. The only way to set up a biasing scheme 
for a coupled neutron and gamma ray adjoint calculation with source energy biasing and region depencient biasing is through trial and error because almost everything you do affects everything else. Furthermore, each calculation is sufficiently different enough to render you a novice each time you set up a biasing scheme.

7. Q: What criteria do you use to judge the quality of the resulting calculations? What FSD's do you look at? What values are deemed acceptable or unacceptable, and why? What do you do if you don't like the results?

A: I look at several aspects of the output to determine my confidence in a particular calculation. I will look at the balance tables printed at the end of each batch to see if they are all approximately the same. If they are, the calculation is fairly well behaved and you are not experiencing gross anomalies in the calculation to cause large swings in your batch results. I look at the balance tables (printed by group) to determine how the biasing (if used) is working, how the secondary particle production distribution is looking, and how the collisions are occurring within the different regions in the geometry (if there are different importance regions (relative to the biasing) specified. After the adjoint leakage tape has been folded with the forward fluence on the coupling surface, I look at the integral detector fsd's to determine whether the problem is converged. If the problem is symmetric, you can look at the effect of rotating the geometry through $0,90,180$, and 270 degrees and look at the consistency among the four different answers you will receive. I then look at the individual group fsd's to see how well the group fluences are converged. If I have any misgivings, I may rerun the calculation and double the number of starters to see if the results change. If they do not, then the original calculation probably had an adequate number of starters. If there are significant differences between the two calculations, I begin comparing the two calculations to find out whether it was a case of undersampling, or if some other problems exist. If I cannot resolve the problem as being a "Monte Carlo" problem, I will tear down the entire analysis from the cross section generation on up to make sure I did not make some input error or keypunch error which did not manifest itself in the form of an error message. As I stated earlier, I believe you must have differential (spectral) fsd's in the $5 \%$ to $10 \%$ range for all the groups contributing to the integral response; even though the integral results may yield $5 \%$ fsd's with $20 \%$ fsd's on the differential resuits. Once the fsd's get greater than $15 \%$, I start to have my suspicions about the quality of that result. Unless cost is a factor, I will run the number of histories required to obtain adequate convergence of the differential results to insure adequate convergence of the integral results. This by no means is an iron-clad guarantee, but it helps reduce a source of discrepancy.

8. Q: Do you have any concerns about your current random number generator? 
A: I utilize the CRAY random number generator and do not have any problems with this software.

9. Q: What is your philosophy for picking the size of the couping surface? Have you compared small versus large surfaces? Describe what surface dimensions you use.

A: My current philosophy for picking the coupling surface is to make it a "glove" fit of the outside dimensions of your target geometry. This is primarily due to the single radius fluence coupling algorithm currently in use in DRC. When you use a large coupling surface, you will take into account the transport in the air from the radius at which the fluence is obtained to the edge of the coupling surface twice. This could yield differences greater than $5 \%$ if the coupling surface is large. For instance, in the two-meter box calculations, the coupling surface need only be slightly larger than the box itself since the transport through the air has already been accounted for in the forward DORT calculation. In my initial box calculations, I had a large coupling surface (approximately 20 meters on a side). DRC then folded fluences at 400 meters with escapes at distances between 390 meters and 410 meters. If the source would have been fairly isotropic, the size of the coupling surface would not have mattered. But for the APRF forward directed reactor source at 400 meters, the DRC coupling with a large coupling surface yielded answers lower than those obtained with a small coupling surface. The current coupling surface is only a few centimeters larger than the outside dimension of the box.

10. Q: Should a slightly different spectrum be used inside the vehicle? (To weight the library?) (For example, tuna-can tank DORT run to collapse cross-sections.)

A: Tailoring the cross-sections to fit the particular problem is always a good idea in any analysis when it is feasible to do so. However, for target vehicle analysis utllizing adjoint Monte Carlo and 2-D air-over-ground environments which are not truly indicative of reality, I am not sure the net effect would not be within the statistical uncertainty if not the uncertainty of the parameters utilized in the model. Furthermore, you will be introducing another complicated step into a process that is already complicated for users who may or may not have the background to perform the task. I believe the analysis should be performed for the experimental comparisons exactly the way it will be performed once it is used in the fields. Then you have a true measure of its capabilities and limitations relative to how it will function on a day-to-day basis.

In summary, I must say that the only way to learn how to use MASH is to have a basic understanding of the underlying principals of the theory and then apply them to the problem. To fire tune a problem requires, to 
some extent, a trial and error approach using small test problems. You constantly tweak the input parameters and look at the effect on the summary counters. Understanding these effects takes expertence and invoives a constant learning process. Unfortunately, the information gleaned from one problem may not be applicable or helpful for another problem, even if they are similar. Eventually, you have to cut your losses and proceed with your setup as it is rather than continuously modifying it to obtain better efficiency. 


\section{INTERNAL DISTRIBUTION}

\author{
1. B. R. Appleton \\ 2. J. M. Barnes \\ 3. T. J. Burns \\ 4. S. N. Cramer \\ 5. J. D. Drischler \\ 6. M. B. Emmett \\ 7. W. W. Engle, Jr. \\ 8. C. M. Haaland \\ 9. D. T. Ingersoll \\ 10-14. J. O. Johnson \\ 15. B. L. Kirk \\ 16. R. A. Lillie \\ 17. J. V. Pace, III \\ 18. W. A. Rhoades \\ 19. R. W. Roussin
}

20. R. T. Santoro

21. C. 0. Slater

22. M. S. Smith

23. R. C. Ward

24. L. R. Willitams

25. EPMD Reports Office 26-27. Laboratory Records Department

28. Laboratory Records ORNL-RC

29. Document Reference Section

30. Ceritral Research Library

31. ORNL Patent Section

\section{EXTERNAL DISTRIBUTION}

32. Director, Defense Nuclear Agency

ATTN: RARP (Dr. D. L. Auton)

6801 Telegraph Road

Alexandrta, VA 22310-3398

33. Dr. Roger W. Brockett

Wang Professor of Electrical Engineering and Computer Science

Division of Applied Science

Harvard Universtty

Cambridge, Massachusetts 02138

34. U. S. Military Academy 001Cy ATTN: Dept. of Physics (Co1. Campbe11) West Point, New York 10996 
35. Commander, U.S. Army Nuclear \& Chemical Agency

ATTN: MONA-ZB (Dr. Charles Davidson)

7500 Backltck Rd., Bldg. 2073

Springfield, VA 22150-3198

36. Establissment Technique Central de $I^{\prime}$ Armement

ATTN: Dr. Joel Dhermain

Centre d'Etudes du Boucher

16 bis Avenue Prieur de la Cote d'or

94114 Arcue 11 -Cedex, France

37. Dr. John J. Dorning

Department of Nuclear Engineering \& Engineering Physics

Thorton Hal1, McCormick Road

University of Virginta

Charlottesville, Va 22901

38. Science Applications International Corporation

ATTN: Dr. Stephen Egbert

10260 Campus Point Drive

San Dlego, CA 92121

39. Commander, U.S. Army Combat Systems Test Activity

ATTN: STECS-NE (Mr. John Gerdes)

Aberdeen Proving Ground, MD 21005-50;9

40. Ministry of Defence, Atomic Weapons Establishment

ATTN: Dr. Kevin G. Harrison

Building A72, Aldermaston,

Reading, Berkshire, United Kingdom, RG7 4PR

41. Director, Armed Forces Radiobiology Research Inst ttute

ATTN: MRAD

Bethesda, MD 20814-5145

42. Science Applications International Corporation

ATTN: Mr. Dean C. Kaul

10260 Campus Point Driva

San Diego, CA 92121

43. Director, Defense Nuclear Agency

ATTN: RARP (MAJ Robert Kehlet)

6801 Telegraph Road

Alexandria, VA 22310-3398

44. Director, Harry Diamond Laboratory

ATTN: SLCHD-NW-P (Mr. Klaus Kerris)

2800 Powder Mi11 Road

Adelphi, MD 20783-1197 
45. Dr. James E. Leiss Route 2, Box 142C Broadway, VA 22815

46. Dr. Neville Moray Department of Mechanical and Industrial Engineering University of 111 inois 1206 West Green Street Urbana, II 61801

47. Commander, U.S. Army Foretgn Science \& Techrology Center ATTN: UVA (Dr. Roger Rydin) 2207 th Street NE Charlottesville, VA 22901-5396

48. HEAD, Nuclear Radiation Effects

ATTN: Dr. Ludwig Schaenzler Wehrwissenschaftitiche Dienststelle Postfach 1320 3042 Munster

Federal Republic of Germany

49. Commander, U.S. Army Foreign Science \& Technology Center ATTN: AIFRTA (Mr. Charles Ward)

2207 th Street NE

Charlottesville, VA 22901-5396

50. Dr. Mary F. Wheeler

Department of Mathematical Sciences Rice University

P. 0. Box 1892

Houston, TX 77204-3476

51. Defense Technical Information Center D02Cy ATTN: DTIC/FDAB

Cameron Station

Alexander, Virginia 22304-6145

52. Office of the Assistant Manager for Energy Research and Development Department of Energy, Oak Ridge Operations P.0. Box 2001

Oak Ridge, TN 37831

53-62. Office of Scientific and Technical Information P.0. Box 62

Dak Ridge, TN 37830 